\title{
Rewards-Supply Aggregate Planning in the Management of Loyalty Reward Programs - A Stochastic Linear Programming Approach
}

YUHENG CAO, B.I.B., M.Sc.

A thesis submitted to the Faculty of Graduate and Postdoctoral Affairs in partial fulfillment of the requirements for the degree of

Doctor of Philosophy in Management

Sprott School of Business

Carleton University

Ottawa, Ontario, Canada

September 2011

(C) 2011Yuheng Cao

All Rights Reserved 


\section{Library and Archives} Canada

Published Heritage

Branch

395 Wellington Street Ottawa ON K1A ON4 Canada
Bibliotheque et

Archives Canada

Direction du

Patrimoine de l'édition

395 , rue Wellington

Ottawa ON K1A ON4

Canada
Your file Votre référence

ISBN: 978-0-494-83243-1

Our file Notre référence

ISBN: 978-0-494-83243-1

\section{NOTICE:}

The author has granted a nonexclusive license allowing Library and Archives Canada to reproduce, publish, archive, preserve, conserve, communicate to the public by telecommunication or on the Internet, loan, distribute and sell theses worldwide, for commercial or noncommercial purposes, in microform, paper, electronic and/or any other formats.

The author retains copyright ownership and moral rights in this thesis. Neither the thesis nor substantial extracts from it may be printed or otherwise reproduced without the author's permission.
AVIS:

L'auteur a accordé une licence non exclusive permettant à la Bibliothèque et Archives Canada de reproduire, publier, archiver, sauvegarder, conserver, transmettre au public par télécommunication ou par l'Internet, prêter, distribuer et vendre des thèses partout dans le monde, à des fins commerciales ou autres, sur support microforme, papier, électronique et/ou autres formats.

L'auteur conserve la propriété du droit d'auteur et des droits moraux qui protège cette thèse. $\mathrm{Ni}$ la thèse ni des extraits substantiels de celle-ci ne doivent être imprimés ou autrement reproduits sans son autorisation.
In compliance with the Canadian Privacy Act some supporting forms may have been removed from this thesis.

While these forms may be included in the document page count, their removal does not represent any loss of content from the thesis.
Conformément à la loi canadienne sur la protection de la vie privée, quelques formulaires secondaires ont été enlevés de cette thèse.

Bien que ces formulaires aient inclus dans la pagination, il n'y aura aucun contenu manquant. 


\section{Acknowledgements}

First of all, I would like to thank my supervisor Dr. Aaron L. Nsakanda for his patient guidance, valuable suggestions and enlightening comments during the period of my study in Carleton University. This dissertation owes a lot to his gentle, yet effective guidance. Without his help, this dissertation could not have been finished. His profound knowledge in management science definitely benefited me a lot. Working with him has always been an enjoyable and rewarding experience. I would like to thank all the members of my thesis committee: Dr. Vinod Kumar, Dr. Michael Armstrong, Dr. Akif A. Bulgak, and Dr. Yiqing Zhao with individually and collectively contributed to the thesis.

Secondly, I would like to thank the Sprott School of Business, Carleton University, for providing financial support and necessary research facilities for this thesis. It would have been impossible for me to concentrate on my research without such financial support. Many thanks go to members and staff of the School of Business, who helped me during my studies at Carleton, in particular Melissa Doric, Greg Schmidt, and Jason Holtz. I would like to thank all of the great teachers and researchers I have encountered here at Carleton University, in particular Dr. Shaobo Ji, Dr. Roland Thomas, and Dr. Uma Kumar.

Finally, I would like to thank my parents, whose love, support, and sacrifice made me what I am today. No words can properly express the love, gratitude, and admiration I have for them. 


\begin{abstract}
Loyalty reward programs (LRPs), initially developed as marketing programs to enhance customer retention, have now become an important part of customer-focused business strategies. One of the operational challenges faced by LRP managers is that of planning for the supply of rewards in a given period of time. We have developed three mathematical models for solving this problem under various settings. In each setting, the problem has been formulated as a two-stage stochastic linear programming model with recourse. A heuristic optimization procedure based on sample average approximation (SAA) is proposed for solving each of these models. We carried out extensive computational experiments to demonstrate the viability of the modeling and solution approaches for solving realistically sized (large-scale) problems as well as to evaluate the impacts of changes that internal dynamics and external uncertainties have on the performance of a loyalty reward program operating as a profit center. Findings from these computational studies have led to a number of managerial insights. Our results show that demand variability has negative impacts on LRP performance. As such, adopting an option contract provides good means for mitigation, especially when demand uncertainty is high. Our results have also shown that offering cooperative advertisement through bonus points is a double-edged sword. It may bring in higher LRP profitability, but it also results in higher liability. When demand variability is high, offering bonus points is not preferred in rewards-supply planning. Finally, our results indicate that budget tightness and liability control tightness have an impact on LRP performance to an extent that varies across different system settings.
\end{abstract}

This research contributes to the literature in several ways: it synthesizes and extends the concept of supply chain management in the context of LRPs and it enhances the understanding of LRPs and rewards-supply planning problems through quantitative modeling and stochastic programming. Our findings will help LRP managers to understand the roles of cooperative advertising through bonus points and option contract in planning for the supply of rewards as well as to evaluate the impact of changes in the internal dynamics and external uncertainties on the performance of loyalty reward program operations. 


\section{Table of Contents}

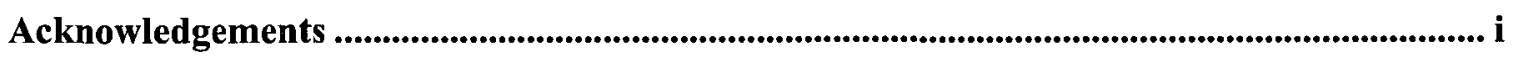

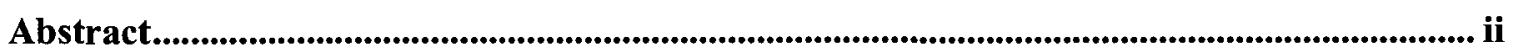

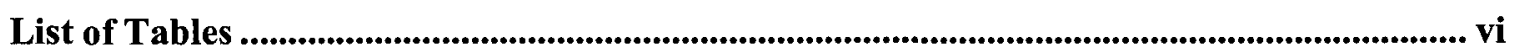

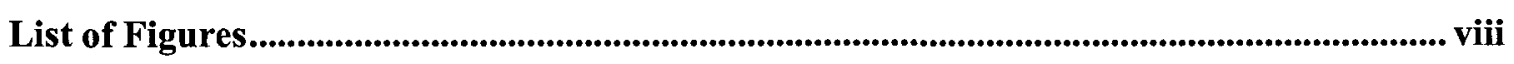

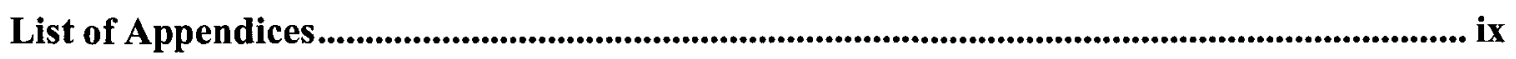

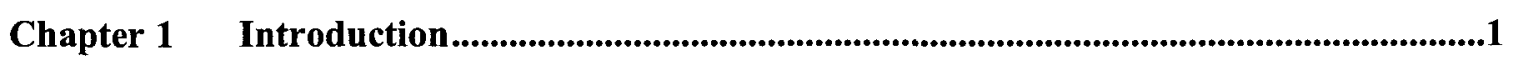

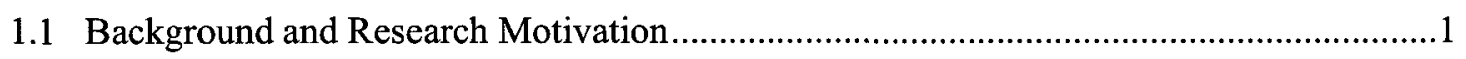

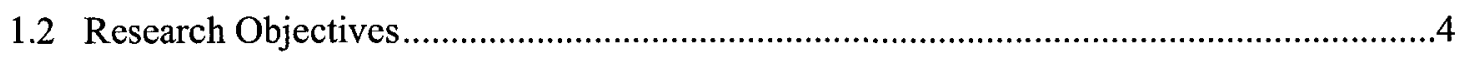

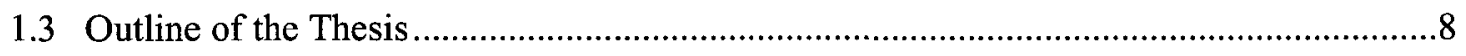

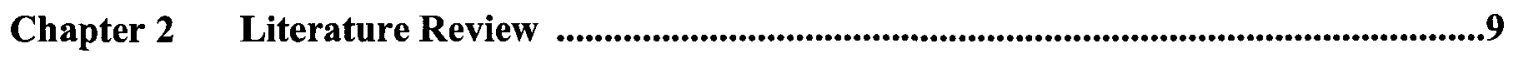

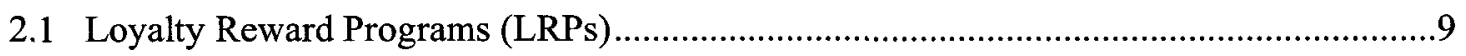

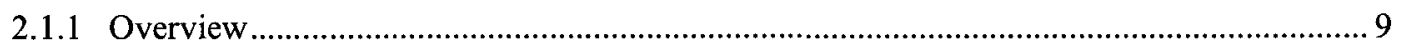

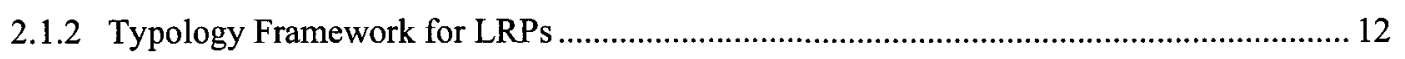

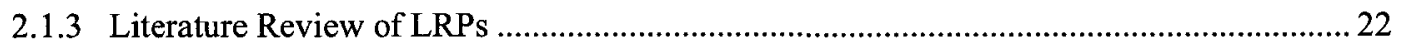

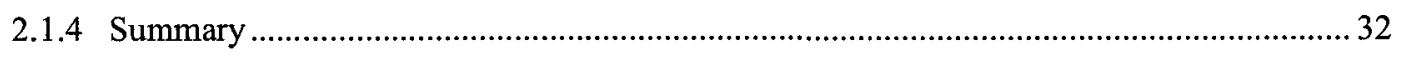

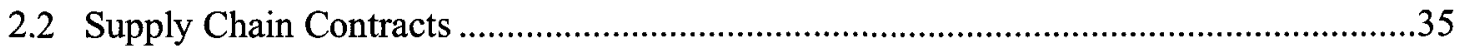

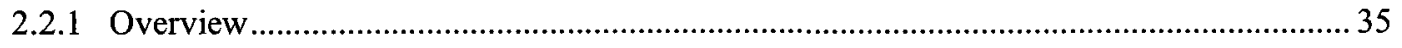

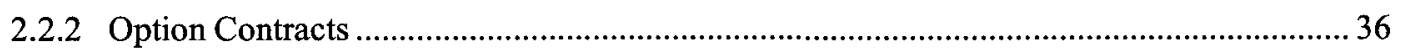

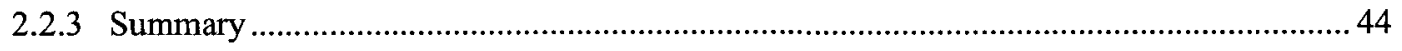

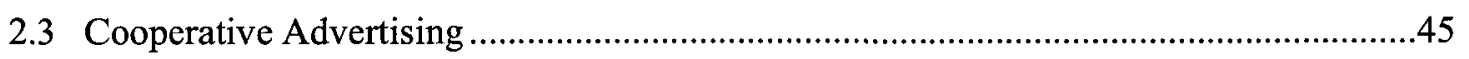

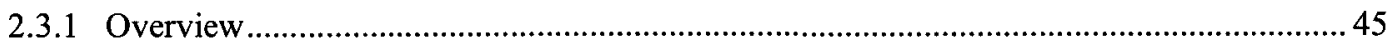

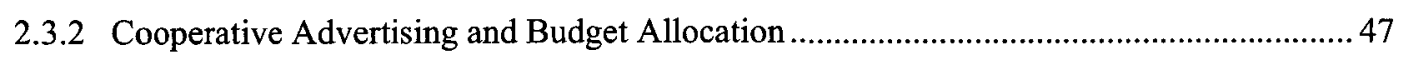

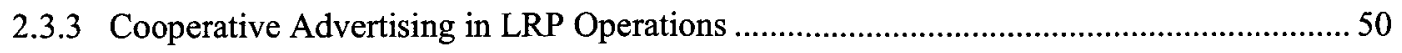

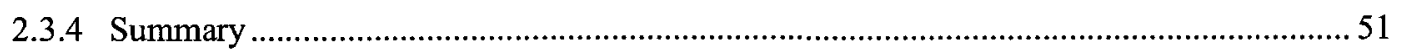

Chapter 3 Research Framework and Mathematical Models ..............................................53

3.1 Loyalty Reward Programs - "Rewards-Points" Supply Chains .....................................53

3.2 LRP Rewards - Supply Aggregate Planning Models ............................................57 
3.2.1 LRP Rewards - Supply Planning Problem without Bonus Points ...................................5 58

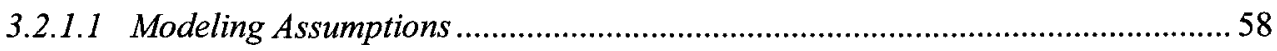

3.2.1.2 Problem Description and Model Formulation...................................................6 60

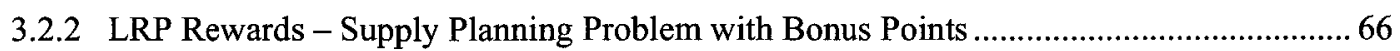

3.2.2.1 Modeling Assumptions ....................................................................................... 67

3.2.2.2 Problem Description and Model Formulation................................................6 68

3.2.3 LRP Rewards - Supply Planning Problem with Option Contracts ...................................... 73

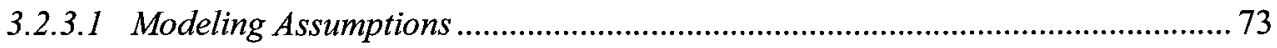

3.2.3.2 Problem Description and Model Formulation................................................ 74

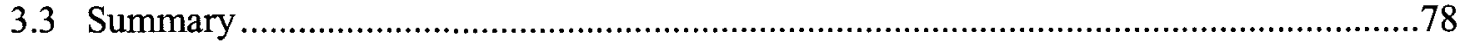

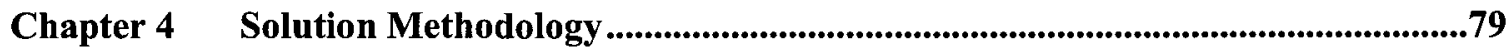

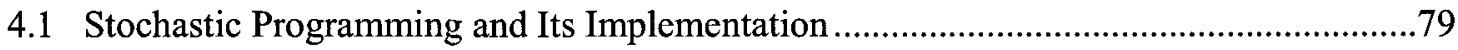

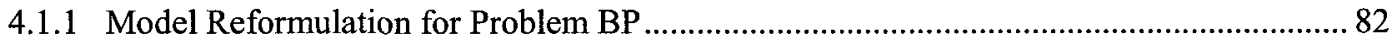

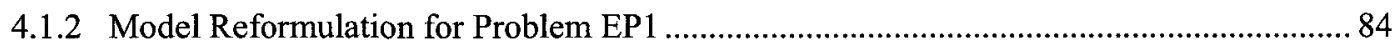

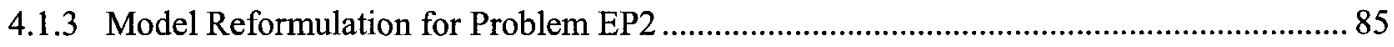

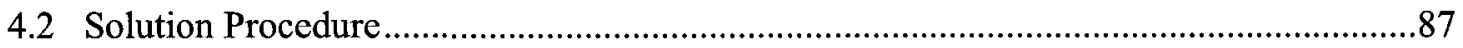

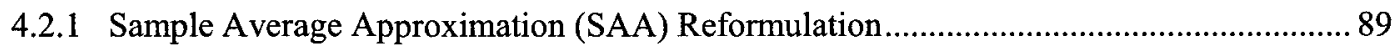

4.2.1.1 SAA Model for Problem BP-2SLPR ………................................................ 90

4.2.1.2 SAA Model for Problem EP1-2SLPR ………..................................................... 91

4.2.1.3 SAA Model for Problem EP2-2SLPR …………………………………….... 92

4.2.2 SAA-based Heuristic Solution Procedure ……..................................................................... 94

4.2.3 Implementation Issues in the Solution Procedure ........................................................ 98

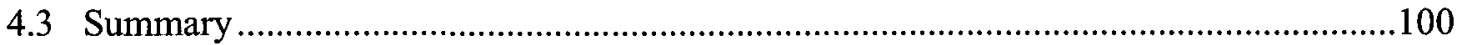

\section{Chapter 5 Design of Numerical Studies ..............................................................................102}

5.1 Procedure for Generating Testing Problems ..........................................................102

5.2 Testing the Effectiveness of the Solution Methodology ...........................................106

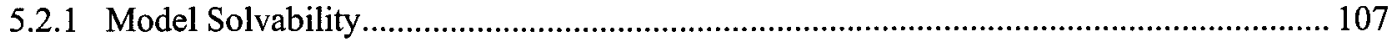

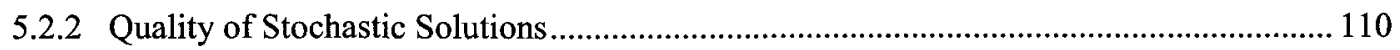

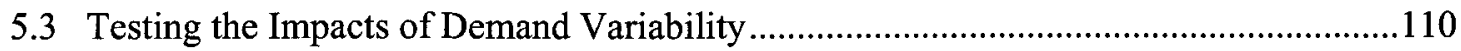

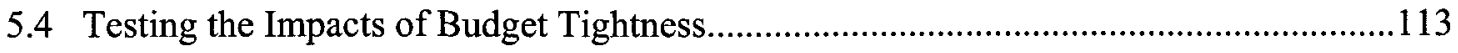

5.5 Testing the Impacts of Liability Control Tightness ...............................................116 
Chapter 6 Results and Analysis .............................................................................................120

6.1 Testing the Effectiveness of the Solution Methodology ...........................................120

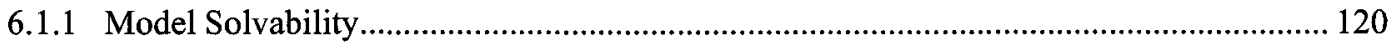

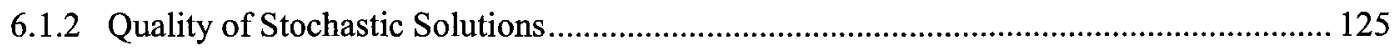

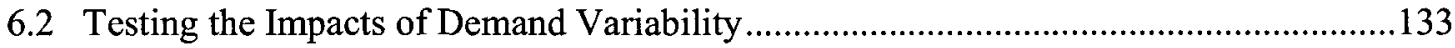

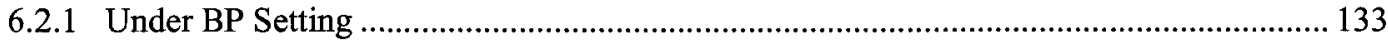

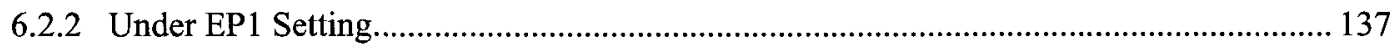

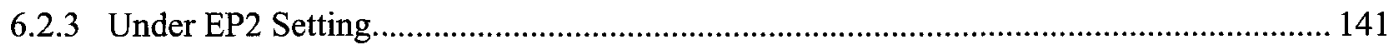

6.2.4 Comparison across BP, EP1, and EP2 Model Settings ..................................................... 146

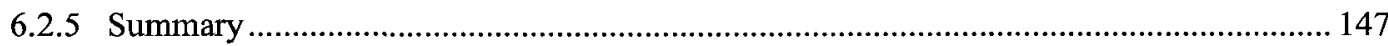

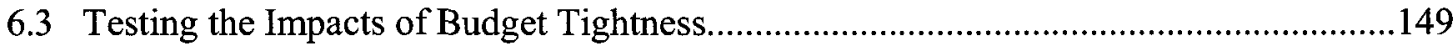

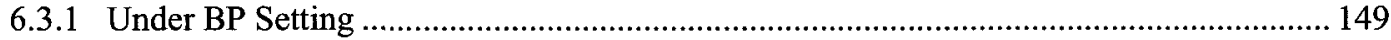

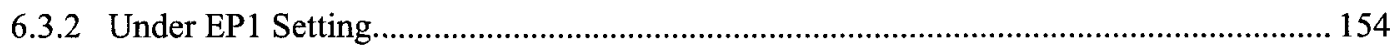

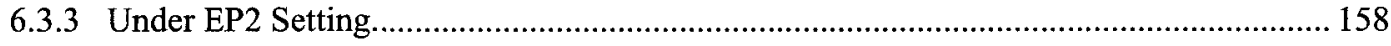

6.3.4 Comparison across BP, EP1, and EP2 Model Settings ................................................. 162

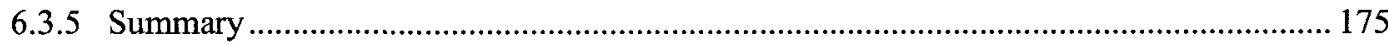

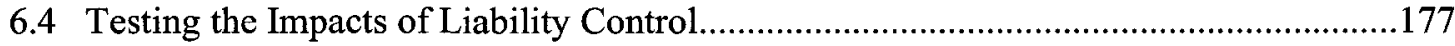

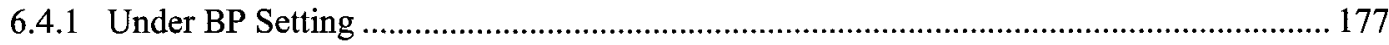

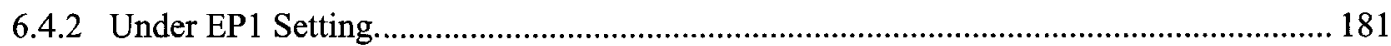

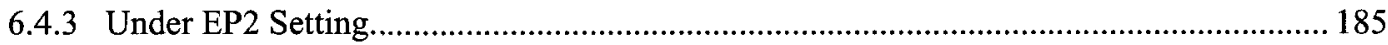

6.4.4 Comparison across BP, EP1, and EP2 Model Settings ................................................... 191

6.4.5 More on Management Insights from Liability Control Analysis...................................... 197

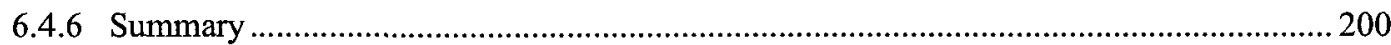

Chapter 7 Conclusions and Future Research Directions ............................................202

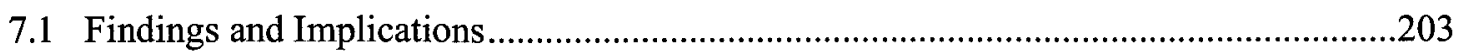

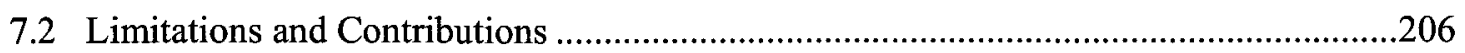

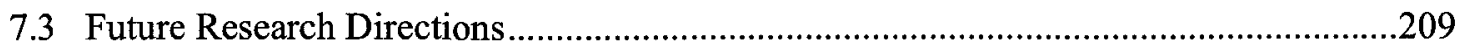

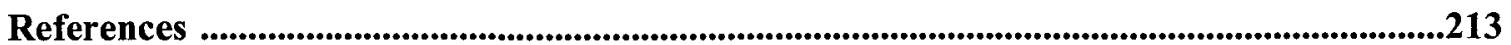

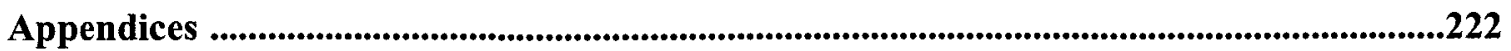




\section{List of Tables}

Table 2.1 Summary of the main streams of LRP literature .27

Table 2.2 Traditional supply chain versus "rewards-points" supply chain 34

Table 2.3 Summary of the main contributions of option contracts in OM/SC literature 42

Table 4.1 Summary of the models 101

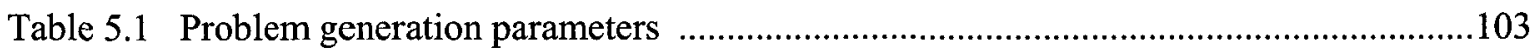

Table 5.2 Problem generation parameters per partner type .....................................................104

Table 5.3 Summary characteristics of the set of random generated problems ...........................109

Table 5.4 Demand variability parameter and its values used for normal distributions ................111

Table 5.5 Demand variability parameter and its values used for uniform distributions .............112

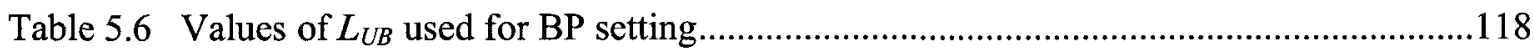

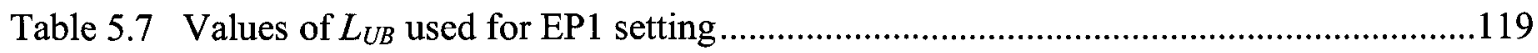

Table 5.8 Values of $L_{U B}$ used for EP2 setting .....................................................................119

Table 6.1 Quality of stochastic solutions of BP model..........................................................127

Table 6.2 Quality of stochastic solutions of EP1 model.........................................................129

Table 6.3 Quality of stochastic solutions of EP2 model .......................................................131

Table 6.4 Summary table of computational results with different levels of demand variability under BP setting

Table 6.5 Summary table of computational results with different levels of demand variability under EP1 setting

Table 6.6 Summary table of computational results with different levels of demand variability under EP2 setting 142

Table 6.7 Impacts of budget tightness under BP setting.....................................................151

Table 6.8 Impacts of budget tightness under EP1 setting ......................................................156

Table 6.9 Impacts of budget tightness under EP2 setting .....................................................160

Table 6.10 Computational results under BP setting............................................................178

Table 6.11 Comparison of the budget usages under BP setting ..........................................180

Table 6.12 Computational results under EP1 setting............................................................182

Table 6.13 Comparison of the budget usages under EP1 setting............................................184

Table 6.14 Computational results under EP2 setting...........................................................186 


\section{List of Tables (Cont.)}

Table 6.15 Comparison of the budget usages under EP2 setting.

190

Table 6.16 Comparison of the impacts of liability control on LRP profitability across different model settings .193

Table 6.17 Comparison of the impacts of liability control on cost of rewards across different model settings

Table 6.18 Comparison of the impacts of liability control on ordering quantity of rewards across different model settings. 


\section{List of Figures}

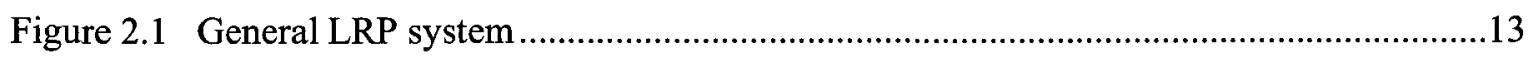

Figure 2.2 Organizational structure models: Type A (I, II, III) .............................................. 14

Figure 2.3 Organizational structure models: Types B (I, II, III, IV) and Type C.......................15

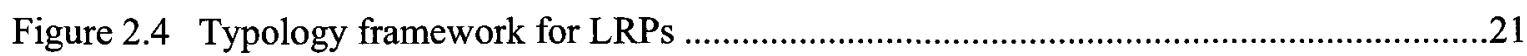

Figure 3.1 Conceptual model of a rewards-points supply chain.............................................53

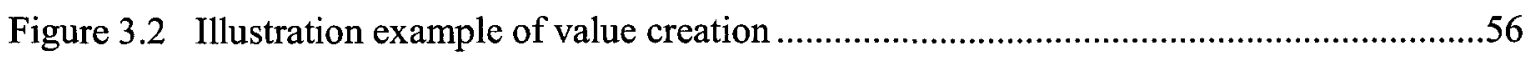

Figure 3.3 Effect of bonus points on accumulation demands .................................................. 70

Figure 6.1 Estimated mean gaps with different sample sizes and sample replications

Figure 6.2 Confidence interval upper bounds of the estimated mean gaps with different sample

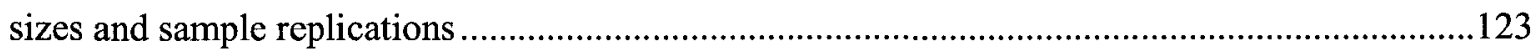

Figure 6.3 CPU times with different sample sizes and sample replications ...............................124

Figure 6.4 LRP profitability under BP, EP1, and EP2 settings ............................................163

Figure 6.5 Liability ratios under BP, EP1, and EP2 settings ................................................167

Figure 6.6 Ordering quantity of rewards under BP, EP1, and EP2 settings .............................171

Figure 6.7 Comparison of budget usage ratios across different model settings .........................195 


\section{List of Appendices}

Appendix A.1 Sample of LRPs in today's marketplace in Canada

Appendix B.1 Computational outputs for examining BP model solvability and determining sample size and sample replications

Appendix B.2 Computational outputs for examining EP1 model solvability and determining sample size and sample replications

Appendix B.3 Computational outputs for examining EP2 model solvability and determining sample size and sample replications

Appendix C.1 Summary table of bonus points to offer with different levels of demand variability under EP1 Setting.

Appendix C.2 Summary table of options to purchase and to exercise with different demand variability under EP2 Setting.

Appendix C.3 LRP profitability comparisons across BP, EP1, and EP2

Appendix C.4 Liability ratio comparisons across BP, EP1, and EP2 ...................................242

Appendix C.5 Budget usage comparisons across BP, EP1, and EP2

Appendix D.1 Comparison of the impacts of budget tightness with a given level of demand variability under BP setting

Appendix D.2 Comparison of the impacts of demand variability with a given level of budget tightness under BP setting

Appendix D.3 Comparison of the impacts of budget tightness with a given level of demand variability under EP1 setting.

Appendix D.4 Comparison of the impacts of demand variability with a given level of budget tightness under EP1 setting

Appendix D.5 Comparison of the impacts of budget tightness with a given level of demand variability under EP2 setting

Appendix D.6 Comparison of the impacts of demand variability with a given level of budget tightness under EP2 setting

Appendix D.7 Comparison of the impacts of the model settings with a given level of budget tightness and a given level of demand variability..... 


\section{List of Appendices (Cont.)}

Appendix E.1 Extended mathematical model for Problem BP with revenue-sharing type of

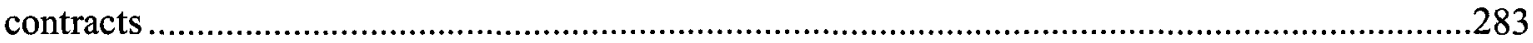

Appendix E.2 Extended mathematical model for Problem EP2 with multi-layer of contracts .286 


\section{Chapter 1 Introduction}

This chapter provides an introduction to the study. It starts by examining the status quo of loyalty reward programs (LRPs) while introducing the concept and management of LRPs. After that, the motivation of the study and the research objectives are discussed. Finally, the organization of the thesis is presented.

\subsection{Background and Research Motivation}

Since the debut of the American Airlines' AAadvantage program in 1981 (Duffy, 1998), LRPs have been employed by a wide range of companies in the consumer goods and service industries. LRPs are today being offered in a number of industries such as airline, retail, hotels, financial services, telecommunications, and gaming and entertainment. In the airline industry alone, more than 130 companies have an LRP, and 163 million people throughout the world collect loyaltybased air miles ${ }^{1}$. LRPs have been quite popular in the United States, United Kingdom, Canada, and a host of other countries. Recent studies (e.g., Berman, 2006) show that no less than 90 percent of the consumers in those countries have at least one loyalty card. Many of them are enrolled in multiple loyalty reward programs. Although various LRPs exist in today's business world, the fundamental business logic behind these programs is the same: to offer consumers incentives or rewards for repeat business. These incentives or rewards, in turn, serve as motivation for consumers to continue buying products from the same product provider. Therefore, LRPs have been widely adopted as an important component of customer relationship

\footnotetext{
1 "Funny Money," Economist, December 24, 2005, 102-103.
} 
management strategy that targets long-term customer profitability (e.g., Jain and Singh, 2002; Liu, 2007; Meyer-Waarden, 2008).

Generally speaking, in a typical LRP system, there are four key players: LRP members, an LRP host, LRP partner(s), and LRP service provider(s). LRP members refer to the end consumers who own a member account and/or a membership card. LRP host is the firm that owns or manages the program. LRP partners refer to the business entities other than the host firm, who join the program to offer accumulation and/or redemption options to LRP members. LRP service providers refer to firms which provide technical support (e.g., maintaining a customer database that stores all LRP members' purchase history) for the LRP business. In such a system, LRP members, based on some specified accumulation scheme, earn loyalty units (e.g., points or miles) along with their purchases of products throughout the network of LRP commercial partners. These units can later be redeemed based on a "reward chart" pre-established by the LRP host. Loyalty units that are not redeemed by LRP members are saved in a "reserve" account and constitute the LRP outstanding balance (referred to as "liability"1). Loyalty units (e.g., points) earned by LRP members during a given period (e.g., a year) constitute the LRP accumulated points (referred to as "accumulation"), whereas loyalty units redeemed by LRP members for rewards during a given period constitute the LRP redeemed points (referred to as "redemption").

In recent years, there has been a general recognition in the industry of a need for more sophisticated loyalty-based systems capable of responding to long-term competitive threats such

\footnotetext{
${ }^{1}$ Liability refers to the value of future redemption obligation of loyalty units (e.g., points or miles) earned by LRP members in an LRP.
} 
as retail overcapacity, spending on mass advertising, and consumer attrition issues. Two new trends of LRP development have been noticed. One trend is that some of the LRP service providers have replaced the traditional LRP host firms (e.g., airline companies or retail firms) to become LRP hosts themselves and treat LRP as their core business. For these loyalty-based service companies, the primary source of their revenue comes from the sale of loyalty units to commercial partners ${ }^{1}$ (referred to as "accumulation partners"). These LRP hosts incur their main costs at the time an LRP member redeems points for rewards, as the LRP host has to purchase the rewards from its commercial partners (referred to as "redemption partners").

Another trend is that in order to compete effectively and to continue contributing to value growth, many existing LRPs have been restructured or expanded in scope to partner with other non-LRP firms to offer new products and/or services. As such, the competition between these LRPs is no longer among individual business entities, but among the networks of business entities involved in these LRPs. The growth of LRPs in recent years has led to a considerable increase in their management and control complexities. For example, Aeroplan ${ }^{\circledR}$, Canada's premier loyalty program, was founded in 1984 by an airline company (Air Canada) as an internal marketing program. Since then, Aeroplan has experienced organizational restructure and expansion several times. Now the program is owned and operated by Group Aeroplan Inc., a loyalty-service-oriented company. At present time, Aeroplan has an accumulation and redemption network of over 75 commercial partners representing more than 150 brands. In 2010 , more than two million rewards were issued to members. The revenue from the sale of Aeroplan Miles was $\$ 1,033$ million, and the total cost of rewards was $\$ 665$ million in the year $2010^{2}$. Air

\footnotetext{
1 http:/www.groupeacroplan.com/PDFS/PDF Reports/2010 Q4 MDA.pdf (page 7, accessed in July, 2011)

2 http://www.groupeaeroplan.com/PDFS/PDF Reports/2010 Q4 MDA.pdf (page 15, accessed in July, 2011)
} 
Miles ${ }^{\circledR}$, a primary competitor of Aeroplan ${ }^{\circledR}$ founded in 1992 , has more than 9.5 million active mile collector accounts, representing approximately two-thirds of all Canadian households. The program is owned and operated by another loyalty-service-oriented company named LoyaltyOne, Inc. Air Miles offers its members more than 1,200 different leisure, entertainment, merchandise, travel, and other lifestyle rewards when members shop at one of more than one hundred brand-name sponsors of the program!

Despite the wide adoption of LRPs in the business world, the increasing economic impacts of these programs, and the increase of the complexities in managing them, there are few academic models that specifically deal with LRPs to support planning and operational decision making. The majority of the existing papers are limited in their coverage of marketing-oriented LRP management problems. Many LRP operational issues have not yet been fully explored. These issues relate to, for instance, prediction and control of liability; short-, medium-, or long-term planning for rewards and points supply; accumulation and redemption demand forecasting; contract design for coordination between LRP host and partners; and revenue assessment. This motivated us to devote our research efforts to addressing operational issues in LRP management.

\subsection{Research Objectives}

A crucial operational issue faced by LRPs is that of planning and managing the supply of rewards (and points) efficiently and effectively in order to achieve management goals - such as meeting customer demand, improving customer satisfaction, lowering operational costs, or generating higher profits - while taking into account both internal (e.g., resource limitations,

${ }^{1}$ http://loyalty.com/business/air-miles-reward-program (accessed in July, 2011) 
management requirements or targets) and external dynamics (e.g., demand uncertainties or competition threats).

In fact, LRP managers rely on good planning for rewards-supply to maintain a balance between the customer service levels and the overall costs of rewarding customers, and to assess the growth of the program and the risk level associated with this growth. The lack of availability of rewards at the time of redemption results in a poor service level and/or an increase of the reward supply costs to meet customer demands, since the LRP host will have to acquire the additional rewards at a higher cost. On the other hand, too much availability will result in a higher cost as well (although the level of customer service would be high in that case). The unused rewards availability will result in a penalty whenever the LRP host decides to reduce or cancel their reservation of rewards or return unused rewards to reward suppliers (i.e., LRP partners). In effect, in setting up long-term contracts with partners, the LRP host must decide the volume of rewards to purchase in advance. Moreover, good planning for rewards-supply provides LRP managers with the ability to develop promotion plans that seek for better management of redemption demand among multiple partners, or for better management policies to mitigate the risks associated with the increases of liability.

It is for these reasons that we explore the planning of the supply of rewards in this study. More specifically, we focus on examining aggregate rewards-supply decisions and the associated LRP performance for a single-period planning horizon. We study the planning issue from only the LRP host point of view. Our research objectives are defined as follows: 
(1) Develop an analytical model to cope with rewards-supply planning decisions in the presence of (a) multiple commercial partners who are involved in the redemption and accumulation business to offer various points collection and redeeming options to LRP members; (b) multiple resource constraints such as budget and capacity constraints; (c) multiple management concerns including LRP profitability, liability control, and demand uncertainties.

(2) Examine the impacts of internal dynamics and external uncertainties on LRP profitability and an LRP host's rewards-supply planning decisions. The internal dynamics that we focus on in this study are the changes in rewards budget and the changes in target liability. The external uncertainties that we focus on are LRP members' accumulation and redemption demand uncertainties.

(3) Investigate the role of cooperative advertising in dealing with internal dynamics and external uncertainties in LRP rewards-supply planning. It is a common practice in LRPs that LRP members can receive "bonus points" when they purchase specified products or services from some LRP partners during a certain time period. This kind of advertising/promotion activities is offered by an LRP host as a type of cooperative advertising between the LRP host and its partners. As pointed out by practitioners" : "the timing and amount of bonus points increase (point) liability, and should be used strategically to drive behaviors that continuously increase spending or create a deeper sense of loyalty"), bonus points not only have an impact on LRP members' accumulation demand but also on liability.

\footnotetext{
${ }^{1}$ Sneed, G.L. 2005. “Points Liability - Enough, or Too Much?" http://newsoct05.maritzloyalty.us/..index.phtml (accessed in July, 2011)
} 
(4) Investigate the role of option contracts in dealing with the internal dynamics and external uncertainties in rewards-supply planning. In the supply chain and operations management (SC/OM) literature, option contracts are widely regarded as a type of SC coordination mechanism between commercial suppliers (e.g., manufacturers) and buyers (e.g., retailers). Researchers have pointed out that option contracts can provide more flexibility in coping with demand uncertainties. In an LRP, redemption partners can be viewed as the suppliers of rewards and the LRP host can be viewed as a buyer of rewards. Therefore, instead of resorting to a traditional supply contract, such as wholesale-priceonly, we assess whether option contracts can provide more flexibility to cope with internal dynamics and external uncertainties in rewards-supply planning problem.

This study contributes to LRP literature and practices by addressing the following research questions:

1) How can the rewards-supply planning problem (RSPP) be formulated mathematically and solved effectively so as to ensure that good quality solutions can be obtained for largescale problems in a reasonable computational time?

2) How does demand variability affect LRP performance in terms of LRP profitability, liability, and cost of rewards? Do the impacts of demand variability vary among different model settings?

3) How does budget tightness affect LRP host decisions as well as LRP profitability and liability? Do the impacts of budget tightness vary among different model settings and under different levels of demand variability? 
4) How does the liability control tightness affect LRP host decisions as well as LRP profitability and reward costs? Do the impacts of liability control tightness vary among different model settings and under different levels of demand variability?

\subsection{Outline of the Thesis}

The thesis is divided into seven chapters. The next chapter reviews the literature that is most relevant to this study. The literature review is based on three areas: loyalty reward programs, option contracts, and cooperative advertising. In addition, a typology framework is presented to provide a guideline for understanding the features of LRPs. Chapter 3 first describes the theoretical background used to develop our analytical models, and then provides a detailed explanation of the mathematical models, including model assumptions, problem description, and model formulation. Chapter 4 reports on the solution methodology. It starts with a brief introduction to stochastic linear programming and then presents a heuristic-based solution procedure for solving our models. The challenges involved in the implementation of the solution procedure are also discussed. Chapter 5 describes the design of our numerical studies carried out through computer simulation. The computational results under each model setting, as well as across the model settings, are presented and analyzed in Chapter 6. Finally, Chapter 7 highlights the main findings and managerial implications, summarizes the contributions and limitations of this study, and discusses future research directions. 


\section{Chapter 2 Literature Review}

This chapter provides an overview of previous studies on three subjects: LRPs, option contracts, and cooperative advertising. It begins by reviewing literature on LRPs and examining the status quo of LRPs in today's marketplace. This leads to proposing a typology framework to capture the variety of LRPs. The type of LRPs that this research focuses on is then presented in detail. Next, the literature on supply chain contracts is discussed, especially option contracts and their modeling in the literature. After that, the concept of cooperative advertising is introduced and a brief review of the literature on advertising budget allocation and advertising-sales response function follows. Each section contains a summary of the relevance of previous studies and of this research work. Because LRPs, cooperative advertising, and option contracts are three broad research areas, we limit our literature review to the concepts and models that are relevant to our study.

\subsection{Loyalty Reward Programs}

In this section, we first introduce the concept of LRP and provide a typology framework of LRPs. Then a survey of thirty-nine well-known LRPs in today's marketplace is provided. After that, we review and discuss the main research streams in the LRP literature. Our view of LRPs in this study is also presented.

\subsubsection{Overview}

LRPs are also known as loyalty programs, rewards programs, "frequent-shopper" programs, "frequent-guest" programs, and "frequent-flier" programs. "Loyalty" and "reward" are the core 
concepts in these programs. More specifically, loyalty is the primary purpose of LRPs and reward is the key instrument for attaining it. According to Oliver (1999), customer loyalty is a deeply held commitment to buy or patronize a preferred product or service consistently in the future. Although academia still debates the meaning of customer loyalty (e.g., Dick and Basu, 1994; Palmer, 1996; O'Malley, 1998), there is no doubt that customer loyalty has become an important asset to a firm (Liu, 2007; O’Brien and Jones, 1995). It has long been proven in psychology studies (e.g., Ebert, 2003) that reward has a strong impact on a person's decision making as well as on behavior modification. In LRPs, rewards refer to all kinds of incentives such as discounts, rebates, free goods, or special services. These rewards are designed to encourage customers to keep doing business with one firm or a group of firms sponsoring the same LRP rather than with competitor firms.

In the literature, there is no universal definition of LRPs because researchers view them in different ways. Some of the researchers (e.g., Taylor and Neslin, 2005; Berman, 2006; Nunes and Drèze, 2006) take a broad view and consider any business/marketing program that uses certain formats of rewards to enhance repeated purchases by customers as LRPs. Using this definition, irrespective of whether a program uses a simple format like "buy a cup of coffee ten times and get one free at the eleventh time" or applies a complex structure as in the case of Aeroplan, it will be designated as LRP. However, other researchers hold the view that some of the simple format programs such as " $20 \%$ off towards your next purchase" are not true LRP programs because these programs do not reward loyal behavior on the basis of a customer's purchase history of products/services. In other words, these programs focus on short-term profits 
rather than long-term customer assets (Shugan, 2005). Therefore, we propose a different definition of LPRs, which is based on literature and our studies.

A business/marketing program may be considered as a loyalty reward program (LRP) when it displays all of the following features:

- The program targets customers' long-term profitability or customers' life-time value.

- The program requires customer enrollment.

- The program collects customer information and records customers' purchase history of products/services through membership cards, co-branded credit cards, or identification numbers (e.g., login ID) that customers use.

- The program rewards repeated customer purchase behavior on the basis of customer's purchasing history.

- The program has clear reward schemes explicitly stating how customers will be rewarded and the benefits that customers can obtain after they join the program.

Despite the increasing use of LRPs worldwide, and proliferation of a large variety of LRPs in recent years, few studies have addressed classification of LRPs, except Kadar and Kotanko (2001), Bagdonienė and Jakštaitė (2006), and Berman (2006). Kadar and Kotanko (2001) studied LRPs in terms of organizational structure and classified LRPs into three categories: exclusive one-company programs, inclusive company-specific programs, and cross-company programs. Exclusive one-company programs refer to programs operated and controlled solely by one firm; inclusive company-specific programs refer to programs which have been extended to include a 
number of partners; and cross-company programs refer to the programs created by a group of companies together. Focusing on the customer perspective, Bagdonienè and Jakštaitè (2006) classified LRPs into open or closed programs, programs for end customers or for intermediate customers, and direct or indirect programs. In an open program all customers are eligible to join the program, as long as they purchase goods or services from the LRP firm. In a closed LRP program only desired or invited customers can join. Programs for end customers are the business to consumers (B2C) LRPs, whereas those for intermediate customers are business to business (B2B) LRPs. Direct LRPs provide customers some financial benefits as rewards, such as permanent discounts, gift cards, or free products. In contrast, indirect LRPs provide customer rewards that are non-financial privileges (e.g., pre-board services or free access to the business lounge at airports) (Bagdonienè and Jakštaitè, 2006). Berman (2006) in his study discussed four types of LRPs in terms of reward scheme: members receive additional discount at register, members receive one unit free when they purchase $n$ units, members receive rebates or points based on cumulative purchases, and members receive targeted offers and mailings.

We found that although these classifications touched different aspects of LRPs, none of them capture the full expanse of the variety of LRPs in today's marketplace. Therefore, we address this issue and propose a comprehensive framework for LRP classification in the next section. Our typology framework was developed based on business to customers (B2C) LRPs only.

\subsubsection{Typology Framework for LRPs}

The typology that we propose here is based on the relationship view of an LRP system. In general, there are five fundamental entities involved in an LRP system (see Figure 2.1). The host 
is the business entity that launches or owns the program. LRP is the program itself. Partners (also known as sponsors) refer to the business entities that join the program to provide redemption and/or accumulation options. Members are the consumers who participate in the program to redeem rewards through purchasing products or services from hosts or partners/sponsors. Service providers are the business entities that provide service or technical support for the program but do not communicate with the members directly (Nsakanda et al., 2006).

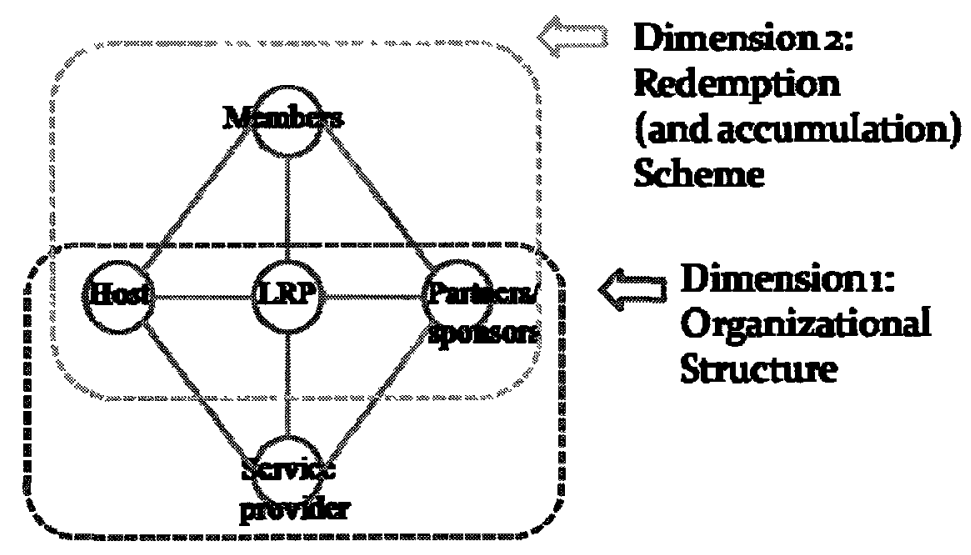

Figure 2.1: General LRP system

(source: Nsakanda et al., 2006)

The variety of LRPs can be viewed as arising from the different relationships among these entities. As both organizational structure and redemption (and accumulation) scheme are the key determinants of the relationships among these entities, we treat them as two dimensions of the framework. Organizational structure defines the relationships among entities in the lower triangle; while the redemption (and accumulation) scheme defines the relationships among the entities in the upper triangle, especially among members and the other three entities (i.e., host, LRP, and partners). 
Typology Dimension One-Organizational Structure

Organizational structure defines the relationships among LRP host, LRP service providers, and LRP partners. Along this dimension, LRPs are grouped into three categories: Type A, single sponsor programs; Type B, multi-sponsor programs; and Type C, joint programs. Under types A and $\mathrm{B}$, there is a subdivision, which we have adopted from Gudmundsson et al. (2002) (see Figure 2.2). Gudmudsson et al. (2002) identified three internal structure models of airline frequent flier programs (FFPs). We find that these structure models are also common in LRPs other than FFPs. The graphs in Figure 2.2 show the differences among these structure models.

Type A

Single-sponsor LRPs
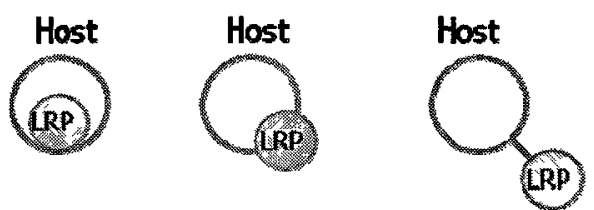

Type A-I Type A-II Type A-III

Figure 2.2: Organizational structure models: Type A (I, II, III) (source: Gudmundsson et al., 2002)

Types A-I, A-II, and A-III illustrate structures where an LRP is fully and solely owned by an LRP host. In Type A-I, the LRP is an internal unit of the LRP host and fully managed by the host. Type A-II represents the structure where an LRP is partially managed by an LRP host and some of the management functions are outsourced to other firms or a third party; whereas in the Type A-III model, all of the management functions of the LRP are outsourced. The "exclusive one-company programs" mentioned in Kadar and Kotanko (2001) are quite similar to the Type A-I LRPs that we define here. 
In the above models, the LRP host is the sole sponsor offering accumulation and redemption to LRP members. In general, such LRPs are limited in flexibility and are narrow in scope. We noticed that in recent years many existing LRPs have been restructured to contribute to value growth. Some of those third-party service agents in Type A-III model have replaced the traditional LRP host enterprises (e.g., airline companies or retail companies) to become LRP hosts themselves. Meanwhile, in order to compete effectively, LRP hosts have started to offer products and services in different categories through partnership with other non-LRP firms (e.g., Hofer, 2008). Therefore, Type B and Type C structure models (see Figure 2.3) have appeared in recent years and have become more and more popular in large-scale LRPs.

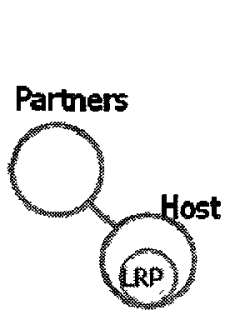

Type B-I
Type B Multi-sponsor LRPs

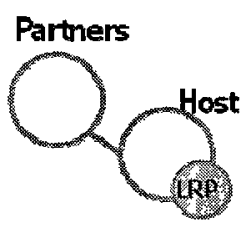

Type B-II

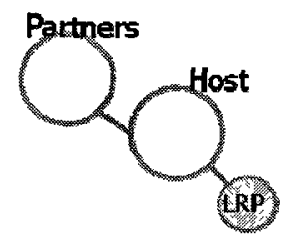

Type B-III
Type C Joint LRPS

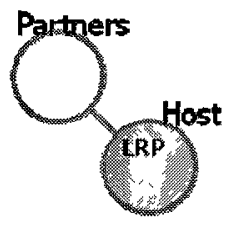

Type B-N

Figure 2.3: Organizational structure models, Types B (I, II, III, IV) and Type C

Type B models (I, II, and III) are extensions of Type A models (I, II, and III). In these Type B models, LRPs follow the same ownership and management structures as those in Type A models, but have multi-redemption and/or multi-accumulation partners/sponsors. Type B-IV model represents the structure where LRP and related services are the focal business of the host firm (e.g., Aeroplan, Air Miles). Type B LRPs are known as multi-sponsor programs or coalition programs. The "more inclusive company-specific program" in Kadar and Kotanko (2001) is similar to a Type B-I LRP. 
In contrast to these structure models, Type $\mathrm{C}$ model represents the structure in which an LRP does not belong to any individual firm (i.e., no sole host) and is formed when a number of firms band together to develop a joint program, known in the literature as joint LRP program ${ }^{1}$ or crosscompany program (e.g., Kadar and Kotanko, 2001).

In Types $\mathrm{B}$ and $\mathrm{C}$, customer loyalty is no longer built around a product or a company but around the LRP program and the associated reward system. Such programs not only possess significant advantages in operational scale and offer a wide range of benefits to members, but more importantly they can leverage their customer bases for cross-selling (Kadar and Kotanko, 2001). In these LRPs, each company brings different capabilities to the table and each may take away a different form of value. In this way, they are structured as win-win solutions for both LRP host and LRP partners.

\section{Typology Dimension Two-Redemption (and Accumulation) Scheme}

The second dimension of the typology framework is based on redemption (and accumulation) scheme. Redemption (and accumulation) scheme primarily defines the relationship between LRP members and the LRP. It is also identified in the literature as an element that is essential to the administration and positioning of LRPs.

Fundamentally, there are two strategies for designing redemption (and accumulation) schemes: static and dynamic. The term 'static' refers to those schemes that do not change over time. They usually take the form of "one scheme applicable for all LRP members". This type of scheme is

\footnotetext{
1 "A tang of bitter-sweet loyalty". Brand Strategy, Oct. 2002.
} 
used quite often in large retailing industries such as The Bay, Shoppers' Drug Mart, and Loblaws. Common rewards offered to LRP members are cash back and gift cards. These firms have a large customer base and they essentially deal with products. Their marginal cost and revenue per customer are low. In these firms, LRPs are viewed purely as marketing tools. Dynamic reward schemes are quite often used by enterprises that specialize in LRPs or large service-industry companies that have a large number of partners. The pricing, type, and timing of redemption (and accumulation) in these schemes change over time, and are structured differently for different member segments. Dynamic reward schemes offer LRP members more accumulation and redemption choices. On the other hand, members need to put in much more effort in order to take advantage of the 'ever-changing' schemes. Although the design and administration costs are higher, dynamic schemes have much more flexibility and capability to improve the profitability of LRPs. Compared to the dynamic schemes, the static schemes are much simpler, requiring lower design and administration costs, and lesser learning effort on the part of members.

Overall, LRPs can be classified as either using a static scheme strategy or using a dynamic scheme strategy. In addition, no matter which design strategy is used, an LRP can be further classified based on four other scheme-related criteria: reward medium, redemption (and accumulation) timing, reward type, and redemption (and accumulation) grid.

Reward medium. In many LRPs, the relationship between a member's purchase effort and a final outcome is mediated by the presence of an intermediate currency known as 'reward medium' or 'loyalty unit' (i.e., points or other exchange units). As Duffy (1998) pointed out, the 
communication between an LRP host and LRP members may get confusing when no proper unified medium is used. Points, miles, or vouchers are the most popular reward mediums that are used to link members' spending to rewards. Most existing LRPs use a single-medium (e.g., expenses $\rightarrow$ points $\rightarrow$ rewards), the rest are either no-medium LRPs (e.g., expenses $\rightarrow$ rewards) or multi-medium LRPs (e.g., expenses $\rightarrow$ points $\rightarrow$ vouchers $\rightarrow$ rewards). Hence, reward medium refers to whether a program currency is used or not and which program currency is used. The paper by Shi and Soman (2004) was the first study to examine the effectiveness of an LRP from the reward-medium perspective. They proposed an analytical model to formulate customer valuation on single-medium (i.e., points only) and multi-medium (i.e., points and vouchers) LRPs and conducted laboratory experiments to compare the impact of single-medium vs. multi-medium on member valuation of LRPs. They found that the multi-medium LRP is more attractive to LRP members, which in turn results in a positive effect on member purchasing behavior.

In real-life LRPs, although most of the programs use at least one reward-medium, there are a few programs in which LRP members are rewarded without using any reward-medium (e.g., M\&M Max, Reservation Rewards, ETR Rewards, see in Appendix A.1). In those programs, LRP members are rewarded directly based on their spending. Therefore, LRPs can be classified based on reward medium as: no-medium LRPs, single-medium LRPs, or multi-medium LRPs.

Reward type. Reward type refers to the type of rewards offered by an LRP. This criterion has been studied in previous LRP literature. Dowling and Uncles (1997) classified LRPs into either direct-reward LRPs or indirect-reward LRPs. A direct-reward LRP refers to an LRP offering 
rewards that directly support the value proposition of the products and/or services that the LRP host or sponsors provide (e.g., gift card, free tickets). The main purpose of offering a directreward is to keep customer loyalty on a single product, one company, or one LRP brand (e.g., Aeroplan). An indirect-reward LRP is defined as an LRP presenting rewards that indirectly cause the LRP members to buy products or services. This type of reward has no linkage with products/services. Usually, this type of reward is money-oriented. Discounts, rebates, and cashback are the typical formats of indirect rewards. As the money that customers get back can be used elsewhere, indirect rewards in most cases cannot lock a customer to further purchases or to use the products or services provided by an LRP. Following Dowling and Uncles' classification, Kim et al. (2001) examined the decisions on selecting direct- or indirect- rewards (i.e. firm's own products/services vs. cash) when the firm faces different customers (heavy vs. light users or price sensitive vs. insensitive users).

In today's marketplace, fewer LRPs offer indirect-reward solely because it is believed that direct-reward is more appropriate for creating loyal customers. Some LRPs offer bundles of direct- and indirect- rewards to their members (Nunes and Park, 2003). Therefore, under this criterion, LRPs can be grouped into LRPs offering indirect rewards, LRPs offering direct rewards, or LRPs offering mixed rewards (i.e. bundles of direct- and indirect- rewards).

Redemption (and accumulation) timing. Redemption (and accumulation) timing is used by some researchers to refer to whether an LRP offers immediate or delayed rewards (e.g., Dowling and Uncles, 1997, Zhang et al., 2000; Yi and Jeon, 2003; Keh and Lee, 2006). Delayed rewards are benefits and incentives that are obtained or are redeemable at a later date from the point of 
sale. Conversely, immediate rewards refer to benefits that are experienced at the point of transaction. Examples of immediate rewards include direct-mail coupons, discounts, or price cuts offered to customers at the point of transaction (Dowling and Uncles, 1997). However, as immediate rewards do not relate to a customer's cumulative purchasing behaviour over time, they are less effective in retaining consumers than delayed rewards (Zhang et al., 2000).

From our point of view, redemption (and accumulation) timing involves the variable of time in the redemption (and accumulation) scheme. For example, in some LRPs, points accumulated or rewards available for redemption expire after some days. Obviously, using redemption (and accumulation) timing can increase flexibility in LRP operations; however, it creates time pressures on LRP members. Members must remain active in order to keep their status in the program. For instance, members are required to acquire or redeem points, sometimes up to a certain minimum level within a certain time period. This type of requirements may have negative impact on members' valuation of the program. Atalik (2005) found that the potential of an LRP to attract members is determined by the value of the rewards it offers, as well as by the timing of the rewards available. In reality, whether to use redemption (and accumulation) timing or not depends on an LRP's overall strategy. Some LRPs use redemption (and accumulation) timing heavily, while others choose not to use it at all ${ }^{1}$. Therefore, based on the redemption (and accumulation) timing criterion, LRP can be classified into two categories: LRPs using reward timing or LPRs not using reward timing.

\footnotetext{
${ }^{1}$ Wells, Jennifer, "Exporting the Loyalty Business: Hoarding, Frustrating, Winning," hitp:/www.globeadvisor.com/servlet/ArticleNews/story/gam/20080823/RCOVER2. [Accessed Sept. 30, 2008]
} 
Redemption (and accumulation) grid. Redemption (and accumulation) grid refers to the detailed reward prices (and accumulation options) offered for members. Redemption (and accumulation) grid can be stated in terms of number of points or miles, amount of LRP members' spending, members' purchase frequency, or members' portfolio. Here portfolio refers to, for instance, the combination of members' spending, product category, and membership 'status' or 'tier'. Therefore, based on this criterion, LRPs can be classified as LRPs using an amount-based grid, LRPs using a frequency-based grid, or LRPs using a portfolio-based grid. To our knowledge, earlier research work has not discussed this criterion formally.

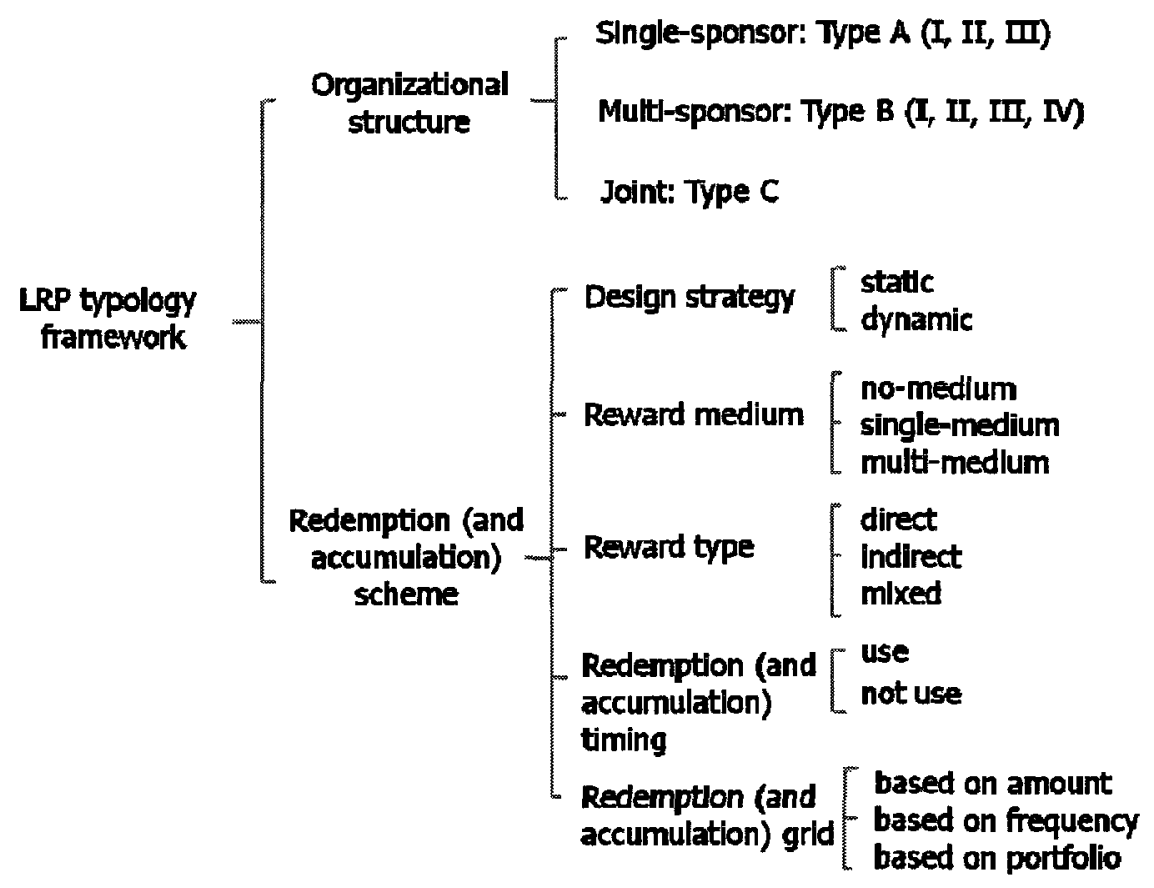

Figure 2.4: Typology framework for LRPs

Based on the above discussion, we present a two-dimensional typology framework in Figure 2.4 below. This framework helps researchers and practitioners to distinguish different facets of LRPs 
systematically and to identify the key characteristics that are important for LRP design and implementation.

Due to the large number of LRPs in today's marketplace, a sample of thirty-nine well-known LRPs in Canada (see Appendix A.1) is surveyed according to this typology framework. Among the thirty-nine LRPs, seventeen programs are of Type A, twenty programs are of Type B and the two remaining programs are of Type C.

In the table of Appendix A.1, we also pointed out whether each LRP runs as a profit center or a cost center. Generally speaking, the LRP that runs as a profit center is an independent business entity and its management focus is on creating revenue directly through the program and the associated business. Taken in this sense, only Type B-IV and Type C LRPs can be profit centers, and the other types of LRPs are either cost centers or semi-cost centers. In a cost center LRP, management focus is on the LRP's contributions to the host firm's focal business. In a semi-cost center LRP, the management focus remains the same as that in a cost center LRP; but the host firm also attempts to create extra revenue through LRP operations directly. For example, the LRP host may sell points or offer LRP-related services to commercial partners (Nunes and Drèze, 2006).

\subsubsection{Literature Review of LRPs}

In this section, we provide a brief review of LRP literature from three perspectives: research topic, research methodology, and types of LRPs that have been examined. 
Researchers have addressed various issues regarding LRPs. However, the majority of these studies are from marketing and economics fields. Economics research have focused on understanding the function of LRPs and the underlining mechanism, such as switching cost, point economics, etc. (e.g., Klemperer 1987, 1995; Caminal and Matutes, 1990; Carlsson and Löfgren, 2006). In marketing literature, there are three main streams of LRP research. One stream of research aims to provide management insights on designing and implementing LRPs (e.g., Kadar and Kotanko, 2001; Shugan, 2005; Berman, 2006). The second stream of research focuses on the short- and/or long-term impact of LRPs on consumer purchase behaviour, attitude, and decision (e.g., Sharp and Sharp 1997; Lewis, 2005; Meyer-Waarden, 2008). The third stream of research explores the influence of LRPs on a firm's market competition (e.g., Caminal and Matutes, 1990; Kim et al., 2001; Singh et al., 2008).

Although all these studies are relevant to the general management of LRPs, the models proposed in these papers do not support planning and operational decision-making. This has left LRP managers trying to address the challenges raised in LRP operations such as revenue or cost optimization, rewards supply planning, accumulation and redemption demands forecasting, etc. by using their experience and intuition rather than analytical-based approaches. However, with the growth in both size and complexity of LRPs, it is impossible for LRP managers to make proper operational decisions without using any analytical approaches.

In recent years, a few studies have appeared in the literature that attempt to address some specific issues in LRP operations. Kim et al. (2004) focused on the adoption and designing issues of LRPs in the context of capacity management. They examined the interdependency between 
excess capacity and price competition in the market. They showed that for those firms who face demand uncertainty and strict short-term capacity constraint, an LRP can be used as a flexible tool to adjust available capacity to market demand. Their analysis includes a discussion on reward amount, but the main purpose is to examine the firms' dynamic price decisions. Gandomi and Zolfaghari (2011) developed a stochastic consumer behavior model to investigate the effectiveness of LRPs. They examined how customers' valuations and satisfaction levels affect LRP profitability in a two-period time horizon. They proposed a customer-oriented model in which customer valuations of the programs and customer satisfaction levels are modeled as stochastic variables. Their computational results show that the effectiveness of LRPs depends on the mean and variance of customer satisfaction levels. Firms may not be always better off when they offer a loyalty program.

The above OM-oriented LRP research shows the increasing attention in the OM literature to explore the dynamics in customer buying behaviour and the triggers behind these dynamics. The authors focused on the value of LRPs on a firm's focal business rather than the management of the LRP itself and LRPs are treated as cost centers. All these studies are deeply rooted in previous marketing or economic theories and analytical models.

In two recent papers, Diaby and Nsakanda (2008) and Nsakanda et al. (2010), a closer look at LRP operations was given from a different angle. The authors considered the LRP as a dynamic system where LRP members are grouped into membership tiers at the beginning of each qualifying period and member accumulation and redemption activities trigger member migration from one tier to another. Diaby and Nsakanda (2008) focused on determining the "breakage 
rate" in Type A-I or Type B-I LRPs. They developed a quantitative model and a simulation process in which the accumulation and redemption of points follow a stochastic process. They also discussed key management issues when an LRP is run as a profit center. Following the same system view of LRPs, Nsakanda et al. (2010) proposed a predictive model of redemption demand and liability level in LRPs that had not been explored in any previous LRP literature. They also discussed practical implementation of the model in the context of a frequent flyer program. As the authors pointed out, although their methodology was developed based on a simple multi-tier LRP (i.e., Type A-I with one accumulation and redemption choice in each tier) it can be expanded to accommodate a more complex LRP structure (e.g., Type B or Type C) and reward scheme (e.g., using timing or dynamic rewards strategy).

With respect to research methodologies, descriptive studies (e.g., Berman, 2006; Shugan, 2005), case studies (e.g., Duffy, 1998; Ho et al., 2009, Ivanuskiene and Auruskeviciene, 2009), empirical studies (e.g., Sharp and Sharp, 1997; Taylor and Neslin, 2005; Park, 2010), and modeling-based studies (e.g., Kim et al., 2001; Lewis, 2005; Labbi and Berrospi, 2007; Singh et al., 2008; Diaby and Nsakanda, 2008; Nsakanda et al., 2010) have all been applied in LRP research. Among modeling-based LRP research, some papers developed game-theoretical models to explore the impact of the LRP on market competition (e.g., Kim et al., 2001, 2004; Singh et al., 2008). Some papers proposed consumer decision and behaviour models to examine the profitability, effectiveness, or attractiveness of LRPs (e.g., Lewis, 2005; Singh et al., 2008; Gandomi and Zolfaghari, 2011). Another set of papers applied the stochastic process in their models to address diverse issues such as allocating promotion budget to targeting LRP members

\footnotetext{
1“"Breakage rate" refers to accumulated points that end up not being redeemed by LRP members In the LRP industry, breakage rate is claimed as part of the host's revenue in the financial statements (e g, Aeroplan annual report 2007, page 24, http //wwu groupederoplan com/pages/nvReports php)
} 
(e.g., Labbi and Berrospi, 2007), calculating breakage rate (e.g., Diaby and Nsakanda, 2008) and estimating redemption and liability (e.g., Nsakanda et al., 2010).

Regarding the types of LRPs, although many researchers realized that LRPs come in great varieties, few of them incorporated this consideration into their studies. The types of LRPs were either set to the most simplified form or ignored. In Table 2.1 , we provide a summary of the main streams of LRP literature in terms of research topics, types of LRPs, research methodologies and key research findings. 
Table 2.1: Summary of the main streams of LRP literature

\begin{tabular}{|c|c|c|c|c|}
\hline Main Streams & $\begin{array}{l}\text { Literature \& } \\
\text { Research Topic }\end{array}$ & $\begin{array}{l}\text { Type of LRP } \\
\left({ }^{*} \text { see Note } 1\right)\end{array}$ & $\begin{array}{c}\text { Research } \\
\text { Methodologies }\end{array}$ & Key Research Findings \\
\hline \multirow[t]{3}{*}{$\begin{array}{l}\text { Customer } \\
\text { switching cost }\end{array}$} & $\begin{array}{l}\text { Klemperer (1987), (1995) } \\
\text { Switching cost }\end{array}$ & $\begin{array}{l}\text { Type A-I, } \\
-/-/ \text { direct/-/- }\end{array}$ & Game theoretical model & $\begin{array}{l}\text { LRPs (e.g. repeat-purchase coupons, frequent flyer } \\
\text { programs) increase switching cost. }\end{array}$ \\
\hline & $\begin{array}{l}\text { Caminal \& Matutes } \\
(1990) \\
\text { Switching cost and } \\
\text { marketing competition }\end{array}$ & $\begin{array}{l}\text { Type A-I, } \\
-/- \text { indirect/-/- }\end{array}$ & Game theoretical model & LRPs tend to decrease the competitiveness of markets. \\
\hline & $\begin{array}{l}\text { Carlsson \& Löfgren } \\
(2006) \\
\text { Switching cost }\end{array}$ & N/A & Empirical study & $\begin{array}{l}\text { LRPs (i.e. frequent flyer programs) increase switching } \\
\text { cost. }\end{array}$ \\
\hline \multirow[t]{3}{*}{$\begin{array}{l}\text { LRP and customer } \\
\text { behaviour }\end{array}$} & $\begin{array}{l}\text { Sharp \& Sharp (1997) } \\
\text { Customer loyalty }\end{array}$ & $\begin{array}{l}\text { Type B-I, } \\
\text { dynamic/one- } \\
\text { medium/ mixed/no } \\
\text { timing/ amount-based }\end{array}$ & Empirical study & $\begin{array}{l}\text { Although to change the fundamental repeat-purchase } \\
\text { patterns of markets is very difficult, it is possible to alter } \\
\text { those patterns at least to a small degree through LRPs. }\end{array}$ \\
\hline & $\begin{array}{l}\text { Zhang et al. }(2000) \\
\text { Optimal choice of } \\
\text { promotion tools }\end{array}$ & $\begin{array}{l}\text { Type A-I, } \\
\text {-/-/indirect/-/- }\end{array}$ & $\begin{array}{l}\text { Consumer behaviour } \\
\text { model and empirical } \\
\text { study }\end{array}$ & $\begin{array}{l}\text { In a market dominated by "switcher" it is more } \\
\text { profitable for a firm to use rear-load incentive (e.g, } \\
\text { LRPs), whereas in a market with high inertia, it is more } \\
\text { profitable for a firm to use front-load incentive. }\end{array}$ \\
\hline & $\begin{array}{l}\text { Kadar \& Kotanko (2001) } \\
\text { LRP design and } \\
\text { implementation }\end{array}$ & $\begin{array}{l}\text { Types A, B and C } \\
-/-/-/-/-\end{array}$ & $\begin{array}{l}\text { Descriptive study \& } \\
\text { Scientific literature } \\
\text { analysis }\end{array}$ & $\begin{array}{l}\text { Properly designed and executed, LRP can bring } \\
\text { tremendous benefits to the firms offering them. }\end{array}$ \\
\hline
\end{tabular}

Note 1: In this column of the table, we listed all the dimensions and criteria that have been discussed or mentioned in the literature in the format of "organization structure, reward design strategy/ medium/ type/use timing or not/ reward grid". We found that in many studies, not all the classification criteria were mentioned. If none of the classification dimensions were mentioned in the literature, "N/A" is used to denote this case; if some of the classification criteria were not mentioned in the literature, then "- $/$ " is used to indicate this case. 
Table 2.1: Summary of the main streams of LRP literature (Cont.)

\begin{tabular}{|c|c|c|c|c|}
\hline Main Streams & $\begin{array}{l}\text { Literature \& } \\
\text { Research Topic }\end{array}$ & $\begin{array}{l}\text { Type of LRP } \\
\text { ("see Note 1) }\end{array}$ & $\begin{array}{l}\text { Research } \\
\text { Methodologies }\end{array}$ & Key Research Findings \\
\hline \multirow[t]{6}{*}{$\begin{array}{l}\text { LRP and customer } \\
\text { behaviour }\end{array}$} & $\begin{array}{l}\text { Kivetz \& Simonson } \\
(2002) \\
\text { Effect of customer effort } \\
\text { level on their preferences } \\
\text { toward LRP rewards }\end{array}$ & $\begin{array}{l}- \\
-/ \text { static / indirect/-/- }\end{array}$ & Empirical study & $\begin{array}{l}\text { Higher program requirements shift preferences in favour } \\
\text { of luxury rewards rather than necessary rewards. This } \\
\text { effect is also observed when the program requirements } \\
\text { are held constant but the individual consumer's effort is } \\
\text { higher. }\end{array}$ \\
\hline & $\begin{array}{l}\text { Yi \& Jeon (2003) } \\
\text { Customer loyalty }\end{array}$ & $\begin{array}{l}- \\
-/-/ \text { direct vs. indirect/ } \\
-/-\end{array}$ & Empirical study & $\begin{array}{l}\text { Effects of LRP on customer loyalty depending on } \\
\text { customer involvements. }\end{array}$ \\
\hline & $\begin{array}{l}\text { Lewis (2004) } \\
\text { LRP and short-term } \\
\text { promotions on customer } \\
\text { retention }\end{array}$ & N/A & $\begin{array}{l}\text { Consumer behaviour } \\
\text { model, simulation and } \\
\text { experiment study }\end{array}$ & LRP effectively increases repeat-purchase rates. \\
\hline & $\begin{array}{l}\text { Atalik (2005) } \\
\text { Impact of LRP elements } \\
\text { on airline customers }\end{array}$ & $\mathrm{N} / \mathrm{A}$ & Empirical study & $\begin{array}{l}\text { LRP elements are of great importance for LRP } \\
\text { members, and also the level of members' perceived } \\
\text { satisfaction has a huge effect on the level of customer } \\
\text { loyalty. }\end{array}$ \\
\hline & $\begin{array}{l}\text { Lewis (2005) } \\
\text { strategic consumer } \\
\text { behaviour }\end{array}$ & $\begin{array}{l}\text { Type A-I, } \\
\text {-/-/indirect/-/- }\end{array}$ & $\begin{array}{l}\text { Consumer behaviour } \\
\text { model and empirical } \\
\text { study }\end{array}$ & $\begin{array}{l}\text { A structural dynamic model is developed to enhance } \\
\text { accurate forecasts of customer value. }\end{array}$ \\
\hline & $\begin{array}{l}\text { Shugan (2005) } \\
\text { LRP design and } \\
\text { implementation }\end{array}$ & $\mathrm{N} / \mathrm{A}$ & $\begin{array}{l}\text { Descriptive study \& } \\
\text { Scientific literature } \\
\text { analysis }\end{array}$ & $\begin{array}{l}\text { Pointed out that many current so-called loyalty } \\
\text { programs appear unrelated to the cultivation of customer } \\
\text { brand loyalty and the creation of customer assets. A true } \\
\text { program should invest in customer with the expectation } \\
\text { of greater future revenue. }\end{array}$ \\
\hline
\end{tabular}


Table 2.1: Summary of the main streams of LRP literature (Cont.)

\begin{tabular}{|c|c|c|c|c|}
\hline Main Streams & $\begin{array}{l}\text { Literature \& } \\
\text { Research Topic }\end{array}$ & $\begin{array}{l}\text { Type of LRP } \\
\text { (*see Note 1) }\end{array}$ & $\begin{array}{c}\text { Research } \\
\text { Methodologies }\end{array}$ & Key Research Findings \\
\hline \multirow[t]{3}{*}{$\begin{array}{l}\text { LRP and customer } \\
\text { behaviour }\end{array}$} & $\begin{array}{l}\text { Liu (2007) } \\
\text { Long-term impact on } \\
\text { consumer purchase } \\
\text { behaviour and loyalty }\end{array}$ & $\begin{array}{l}\text { Type A-I, } \\
\text { static/one-medium/-/- } \\
\text { /amount-based }\end{array}$ & Empirical study & $\begin{array}{l}\text { LRP has positive effects on both light and moderate } \\
\text { buyers' purchase frequency and transaction size. }\end{array}$ \\
\hline & $\begin{array}{l}\text { Meyer-Waarden (2008) } \\
\text { Customer purchase } \\
\text { behaviour }\end{array}$ & $\begin{array}{l}\text { Type A-I, } \\
\text {-/one-medium/ mixed } \\
\text { /-/ amount-based }\end{array}$ & Empirical study & $\begin{array}{l}\text { There is a positive association between LRP and } \\
\text { customer purchase behaviour. }\end{array}$ \\
\hline & $\begin{array}{l}\text { Gandomi and Zolfaghari } \\
\text { (2011) } \\
\text { Impact of consumer } \\
\text { valuations and satisfaction } \\
\text { levels on LRP profitability }\end{array}$ & $\begin{array}{l}\text { Type A-I, } \\
-/-/ \text { indirect/-/ } \\
\text { amount-based }\end{array}$ & $\begin{array}{l}\text { Consumer behaviour } \\
\text { model and Experiment } \\
\text { study }\end{array}$ & $\begin{array}{l}\text { Firm may not always be better off to offer an LRP. } \\
\text { Effectiveness of LRPs depends on the stochastic } \\
\text { parameters: mean and variance of the customer } \\
\text { satisfaction levels. }\end{array}$ \\
\hline \multirow[t]{3}{*}{$\begin{array}{l}\text { Design and } \\
\text { implementation of } \\
\text { LRP }\end{array}$} & $\begin{array}{l}\text { Duffy (1998) } \\
\text { Customer loyalty }\end{array}$ & $\mathrm{N} / \mathrm{A}$ & $\begin{array}{l}\text { Case study, descriptive } \\
\text { study, and scientific } \\
\text { literature analysis }\end{array}$ & $\begin{array}{l}\text { Provided a consistent framework for developing a } \\
\text { loyalty strategy and program. Discussed the selection of } \\
\text { reward types and the design of the reward grid. }\end{array}$ \\
\hline & $\begin{array}{l}\text { Shi \& Soman (2004) } \\
\text { Effectiveness of LRP in } \\
\text { terms of medium usage }\end{array}$ & $\begin{array}{l}\text { Type A-I, } \\
\text { static /one or two- } \\
\text { medium/ mixed /-/ } \\
\text { amount-based }\end{array}$ & $\begin{array}{l}\text { Customer behaviour } \\
\text { model and Experiment } \\
\text { study }\end{array}$ & $\begin{array}{l}\text { Multi-medium LRPs outperform functionally equivalent } \\
\text { single-medium programs. }\end{array}$ \\
\hline & $\begin{array}{l}\text { Banasiewicz (2005) } \\
\text { LRP planning and } \\
\text { analytics }\end{array}$ & N/A & $\begin{array}{l}\text { Descriptive study and } \\
\text { Scientific literature } \\
\text { analysis }\end{array}$ & $\begin{array}{l}\text { A general LRP planning approach is proposed and the } \\
\text { importance of robust customer insights to program } \\
\text { planning and its ongoing management is demonstrated. }\end{array}$ \\
\hline
\end{tabular}


Table 2.1: Summary of the main streams of LRP literature (Cont.)

\begin{tabular}{|c|c|c|c|c|}
\hline Main Streams & $\begin{array}{c}\text { Literature \& } \\
\text { Research Topic }\end{array}$ & $\begin{array}{l}\text { Type of LRP } \\
\text { (*see Note 1) }\end{array}$ & $\begin{array}{c}\text { Research } \\
\text { Methodologies }\end{array}$ & Key Research Findings \\
\hline \multirow[t]{6}{*}{$\begin{array}{l}\text { Design and } \\
\text { implementation of } \\
\text { LRP }\end{array}$} & $\begin{array}{l}\text { Berman (2006) } \\
\text { LRP design and } \\
\text { implement }\end{array}$ & $\begin{array}{l}-, \\
-/-/ \text { mixed } /-/-\end{array}$ & $\begin{array}{l}\text { Descriptive study and } \\
\text { Scientific literature } \\
\text { analysis }\end{array}$ & $\begin{array}{l}\text { Classified LRPs based on reward type and reward grid. } \\
\text { A firm must assess the suitability of each program type } \\
\text { and then carefully plan, evaluate and constantly revise } \\
\text { the program. }\end{array}$ \\
\hline & $\begin{array}{l}\text { Bagdoniené \& Jakštaitė } \\
(2006) \\
\text { LRP purpose, means and } \\
\text { development }\end{array}$ & $\begin{array}{l}\text {-, } \\
\text {-/one-medium vs. no- } \\
\text { medium /direct vs. } \\
\text { indirect /-/- }\end{array}$ & $\begin{array}{l}\text { Descriptive study and } \\
\text { Scientific literature } \\
\text { analysis }\end{array}$ & $\begin{array}{l}\text { There is no absolute constant dependence between } \\
\text { customer loyalty and customer satisfaction. LRPs are } \\
\text { distinguished by great variety. }\end{array}$ \\
\hline & $\begin{array}{l}\text { Keh \& Lee (2006) } \\
\text { Customer satisfaction and } \\
\text { reward preference }\end{array}$ & $\begin{array}{l}- \\
-/-/ \text { direct vs. indirect/- }\end{array}$ & Empirical study & $\begin{array}{l}\text { When customers are satisfied, they prefer delayed, direct } \\
\text { rewards to immediate, direct rewards. When customers } \\
\text { are not satisfied, the conclusion is opposite. }\end{array}$ \\
\hline & $\begin{array}{l}\text { Ivanuskiene \& } \\
\text { Auruskeviciene (2009) } \\
\text { LRP challenges in retail } \\
\text { banking industry }\end{array}$ & $\begin{array}{l}\text { Type A-I, } \\
\text { static/one- } \\
\text { medium/mixed/-/- }\end{array}$ & $\begin{array}{l}\text { Descriptive study and } \\
\text { Case study }\end{array}$ & $\begin{array}{l}\text { The majority of the analyzed LRPs reward their } \\
\text { customers by using only a discount on the transaction } \\
\text { costs, which leads to a constant battle for the price. }\end{array}$ \\
\hline & $\begin{array}{l}\text { Ho et al. (2009) } \\
\text { How to develop an } \\
\text { effective LRP in retail } \\
\text { industry (e.g., grocery } \\
\text { store) }\end{array}$ & $\begin{array}{l}\text { Type A-I, } \\
\text { static /one or two- } \\
\text { medium/ mixed /-/ } \\
\text { amount-based }\end{array}$ & Case study & $\begin{array}{l}\text { Customers prefer different types of rewards. A "hybrid" } \\
\text { reward structure was recommended to incorporating } \\
\text { competition and heterogeneity in customers" } \\
\text { preferences. }\end{array}$ \\
\hline & $\begin{array}{l}\text { Park (2010) } \\
\text { Effect of LRP in airline } \\
\text { industry }\end{array}$ & N/A & $\begin{array}{l}\text { Case study and } \\
\text { Empirical study }\end{array}$ & $\begin{array}{l}\text { Developed a conceptual model to explore the } \\
\text { relationships between LRP and airline service quality, } \\
\text { pricing, passenger satisfaction, airline image, and airline } \\
\text { selection through a case study of the Korean airline } \\
\text { industry. }\end{array}$ \\
\hline
\end{tabular}


Table 2.1: Summary of the main streams of LRP literature (Cont.)

\begin{tabular}{|c|c|c|c|c|}
\hline Main Streams & $\begin{array}{c}\text { Literature \& } \\
\text { Research Topic }\end{array}$ & $\begin{array}{c}\text { Type of LRP } \\
\text { Note 1) }\end{array}$ & $\begin{array}{c}\text { Research } \\
\text { Methodologies }\end{array}$ & Key Research Findings \\
\hline \multirow[t]{4}{*}{$\begin{array}{l}\text { Impact of LRP on } \\
\text { a firm's focal } \\
\text { business and/or } \\
\text { market competition }\end{array}$} & $\begin{array}{l}\text { Kim et al. (2001) } \\
\text { Pricing strategies }\end{array}$ & $\begin{array}{l}\text { Type A-I, } \\
-/-/ \text { indirect } /-/ \text { amount- } \\
\text { based }\end{array}$ & Game theoretical model & $\begin{array}{l}\text { LRPs have ability to weaken price competition and } \\
\text { increase switching cost. }\end{array}$ \\
\hline & $\begin{array}{l}\text { Kim et al. (2004) } \\
\text { Capacity management }\end{array}$ & $\begin{array}{l}\text { Type A-I, } \\
\text {-/-/direct/-/- }\end{array}$ & Game theoretical model & $\begin{array}{l}\text { By offering capacity rewards, firms can effectively } \\
\text { reduce available capacities and set higher price in the } \\
\text { market. }\end{array}$ \\
\hline & $\begin{array}{l}\text { Taylor \& Neslin (2005) } \\
\text { Short and long-term sales } \\
\text { impact }\end{array}$ & $\begin{array}{l}\text { Type A-I, } \\
-/-/ \text { direct/-/- }\end{array}$ & Empirical study & $\begin{array}{l}\text { LRPs create impacts of points-pressure and rewarded- } \\
\text { behaviour on customers' purchases. }\end{array}$ \\
\hline & $\begin{array}{l}\text { Singh et al. (2008) } \\
\text { Profitability \& marketing } \\
\text { competition }\end{array}$ & $\begin{array}{l}-, \\
-/-/ \text { indirect } /-/-\end{array}$ & $\begin{array}{l}\text { Consumer behaviour } \\
\text { model and Game } \\
\text { theoretical model }\end{array}$ & $\begin{array}{l}\text { A market equilibrium exist when one firm offers LRP } \\
\text { and the other doesn't. }\end{array}$ \\
\hline \multirow[t]{3}{*}{ LRP operations } & $\begin{array}{l}\text { Labbi \& Berrospi (2007) } \\
\text { Advertising budget } \\
\text { allocation }\end{array}$ & $\begin{array}{l}\text { Type A-I, } \\
-/-/-/-/-\end{array}$ & $\begin{array}{l}\text { Markov process model } \\
\text { and experimental study }\end{array}$ & $\begin{array}{l}\text { A quantitative methodology is developed to allocation } \\
\text { advertising /promotion budget in LRPs. }\end{array}$ \\
\hline & $\begin{array}{l}\text { Diaby \& Nsakanda (2008) } \\
\text { Breakage rate }\end{array}$ & $\begin{array}{l}\text { Type A-I or Type B-I, } \\
\text {-/one medium / mixed } \\
\text { / use timing /- }\end{array}$ & $\begin{array}{l}\text { Markov process model } \\
\text { and Simulation model }\end{array}$ & $\begin{array}{l}\text { A quantitative approach is proposed to determine } \\
\text { breakage rate in LRPs. }\end{array}$ \\
\hline & $\begin{array}{l}\text { Nsakanda et al. (2010) } \\
\text { Redemption and liability } \\
\text { estimation }\end{array}$ & $\begin{array}{l}\text { Type A-I, } \\
\text {-/one medium /indirect } \\
\text { or direct/ use timing / - }\end{array}$ & $\begin{array}{l}\text { Markov process model } \\
\text { and Simulation model }\end{array}$ & $\begin{array}{l}\text { An analytical model is developed to estimate } \\
\text { redemption and liability in LRPs. }\end{array}$ \\
\hline
\end{tabular}




\subsubsection{Summary}

Our study is complementary to existing LRP literature. The type of LRPs that we focus on is the Type B-IV LRP with static / one-medium / mixed / no timing / amount-based redemption (and accumulation) scheme. Although Type B-IV is one of the most popular LRP structures in the marketplace, it has not been investigated in the previous literature. In Type B-IV, the LRP is an independent business entity operated by a host firm as a profit center. The host's focal business is to provide a brand-name LRP service to LRP members through partnership in both redemption and accumulation operations. There is commonly more than one partner involved in either redemption or accumulation operations in the system to provide goods and/or service in different categories. Points are used as the single universal reward media in the program to record members' purchase efforts. There is no limitation on what types of rewards can be offered in the system. In other words, redemption partners may offer direct, indirect, or mixed rewards. No time variable is used in the redemption (and accumulation) scheme and the redemption (and accumulation) grid is amount-based.

As seen in Appendix A.1, practical applications of Type B-IV LRPs are increasing in recent years. It has become the most popular form of large-scale LRPs in today's marketplace. In this type of LRPs, the host firms run the programs as focal business and attempt to maximize the value created through "rewards and points" business. All LRP partners are linked by the program to provide accumulation and/or redemption services to fulfill customer requirements for points and rewards. Furthermore, through this chain system, the LRP partners also attempt to compete with other non-LRP firms in the market and gain extra profit. Therefore, Type B-IV LRPs can be viewed as rewards-points supply chains (RSCs). 
A rewards-points supply chain (RSC) and a traditional supply chain (TSC) share many common characteristics. Generally speaking, a TSC consists of multiple independent business entities such as vendors, manufacturers, distributors, retailers, etc. Similarly, an RSC typically also consists of independent business entities including an LRP host (i.e., the firm that owns the program), LRP redemption partners (i.e., firms that join the program to provide members with redemption options), LRP accumulation partners (i.e., firms that join the program to provide members with accumulation options), etc. The revenue flows in both the TSC and the RSC are created by end consumers and shared among all business entities in the chain systems. However, important differences exist between TSCs and RSCs. In a TSC, products provided by the business entities are in the same goods/service categories. In an RSC, rewards in different goods/service categories are provided by redemption partners and points are a special type of products provided by an LRP host and distributed by accumulation partners. End consumer demands relate to products in the TSC, while in the RSC, end consumer demands relate to both points and rewards. With regard to the system structure, TSCs are mainly sequentially based. Following the production flow, the business entities involved are operated sequentially. Downstream entities play a key role in interactions with end consumers. In contrast, the structures of RSCs are parallel-based. In an RSC, the LRP host is at the center of the chain system. Redemption and accumulation partners are the LRP host's multiple channel partners and they operate independently. All of these entities in the RSC interact directly with end consumers (i.e., members who join the LRP). Another key difference between a TSC and an RSC is that costs and revenues are generated in a different time order. In the TSC, costs associated with production occur first, and then revenues are generated through selling products. In the RSC, revenues are generated through selling points first (this applies only to an LRP operating as a 
profit center), and then costs associated with rewards occur later when LRP members redeem their points for rewards. Table 2.2 summarizes the similarities and differences between a traditional supply chain (TSC) and a rewards-points supply chain (RSC). The common characteristics between TSCs and RSCs motivate us to apply traditional SC models to study LRPs. Meanwhile, the unique features of LRPs allow us to explore the special problems associated with LRP operations.

Table 2.2: Traditional supply chain versus "rewards-points" supply chain

\begin{tabular}{lll}
\hline $\begin{array}{l}\text { Independent } \\
\text { business entities in } \\
\text { the system }\end{array}$ & $\begin{array}{l}\text { Suppliers, manufacturers, distributors, } \\
\text { retailers, etc. }\end{array}$ & $\begin{array}{l}\text { LRP host, redemption partners, } \\
\text { accumulation partners, etc. }\end{array}$ \\
\hline Information flow & $\begin{array}{l}\text { Product-oriented (e.g., number of } \\
\text { products produced, number of products } \\
\text { sold, each product's inventory level, } \\
\text { demand for each product) }\end{array}$ & $\begin{array}{l}\text { Reward- and point-oriented (e.g., } \\
\text { number of points issued, amount of } \\
\text { rewards, LRP members' accumulation } \\
\text { and redemption demand, liability) }\end{array}$ \\
\hline Product flow & $\begin{array}{l}\text { Products provided by the entities in the } \\
\text { multiple channels are the same (i.e., } \\
\text { either same branded or in the same } \\
\text { goods/service categories) }\end{array}$ & $\begin{array}{l}\text { Rewards in different goods/service } \\
\text { categories are provided by redemption } \\
\text { partners; points are the semi-tangible } \\
\text { product provided by LRP host }\end{array}$ \\
\hline Revenue flow & $\begin{array}{l}\text { Comes from end-consumers and shared } \\
\text { by SC entities }\end{array}$ & $\begin{array}{l}\text { Comes from end-consumers and shared } \\
\text { by SC entities }\end{array}$ \\
\hline Chain Structure & $\begin{array}{l}\text { Sequential-based. Business entities are } \\
\text { operated sequentially following } \\
\text { production flow. Downstream entities } \\
\text { play a key role in the interaction with } \\
\text { end consumers. }\end{array}$ & $\begin{array}{l}\text { Parallel-based, host firm is the center of } \\
\text { the system. Redemption and } \\
\text { accumulation partners are host's } \\
\text { multiple channel members, operated } \\
\text { separately. All entities in the system } \\
\text { plays important role in the interaction } \\
\text { with end consumers. }\end{array}$ \\
\hline $\begin{array}{l}\text { Cost and revenue } \\
\text { occur in a different } \\
\text { time order }\end{array}$ & $\begin{array}{l}\text { Cost associated with production occurs } \\
\text { first, and then revenue is generated } \\
\text { through selling products. }\end{array}$ & $\begin{array}{l}\text { Revenue is generated first through } \\
\text { selling points, and then cost associated } \\
\text { with rewards occurs later when } \\
\text { customers redeem their points. }\end{array}$ \\
\hline system & $\begin{array}{l}\text { End consumer demands are on products } \\
\text { only. }\end{array}$ & $\begin{array}{l}\text { End consumer demands are on both } \\
\text { points and rewards. }\end{array}$ \\
\hline
\end{tabular}




\subsection{Supply Chain Contracts}

Because we view an LRP system as a rewards-points supply chain, exploring the impact of SC contracts on LRP operations and the corresponding decision making becomes another aspect of our research focus.

\subsubsection{Overview}

SC contracts are widely recognized as a necessary means for governing buyer-supplier relationships in the supply chain, coordinating the decisions of the supply chain partners, sharing the risks arising from various sources of uncertainty, or facilitating long-term partnerships (Tsay et al. 1999). Generally speaking, an SC contract is a binding agreement settled partially or fully prior to a B2B exchange that specifies contractual relations among the contract parties. It may also offer suitable incentives, through various terms and conditions, on material, information, and fund flows in order to facilitate the B2B exchange and influence the behaviour and decisions of contract parties along the supply chain (Tirole, 1988; Liu et al., 2005; Park et al., 2006).

A variety of supply chain contracts within and across industries have been investigated in the academic literature and contributions have been reported on a number of issues related to their role. They are critical in coping with internal dynamics and external uncertainties in supply chains, in influencing the behaviour and decisions of contract parties along the supply chain, and in facilitating supply chain coordination. They are also very important in contract selection, design, evaluation, and implementation. 
Wholesale-price contracts, two-part tariff linear price contracts, two-part tariff non-linear price contracts are examples of supply chain contracts that are defined on the basis of pricing policies (e.g., Corbett and Tang, 1999). Buyback or return contracts, quantity-flexibility contracts, backup agreement contracts, revenue-sharing contracts, option contracts, shared-saving contracts and their many variations are other examples of supply chain contracts defined on the basis of incentives or purposes (e.g., Eppen and Iyer, 1997; Li and Kouvelis, 1999; Barnes-Schuster et al., 2002; Tsay, 2002; Cachon and Lariviere, 2005; Corbett et al., 2005). Most existing studies on supply chain contracts have focused on supply chains that deal with tangible products. The analysis in most studies primarily assumes a supply chain involving a single retailer and a single supplier. For comprehensive survey papers on supply chain contracts, we refer readers to Tsay et al. (1999), Lariviere (1999), and Cachon (2003).

Although various SC contracts have been discussed in the literature, in this study, we focus our attention on option contracts. Option contracts are widely viewed as a hedging instrument against supply and demand uncertainties and hence improve overall supply chain performance (e.g., Ritchen and Tapiero, 1986; Wu et al., 2005; Xu and Nozick, 2009; Cheng et al., 2011). Some researchers (e.g., Barnes-Schuster et al., 2002) have showed that quantity flexibility contracts (Anupini and Bassok, 1998), backup agreements (Eppen and Iyer, 1997), and pay-to-delay capacity reservation (Brown and Lee, 1997) are all special cases of option contracts.

\subsubsection{Option Contracts}

Option contracts that were mainly used as a risk management mechanism in the financial area have attracted enormous attention in the recent $\mathrm{OM} / \mathrm{SC}$ literature. In the context of $\mathrm{OM} / \mathrm{SC}$, an 
option contract refers to a contract in which a supplier (e.g., manufacturer) allows a buyer (e.g., retailer) to purchase up to a given quantity of a product during a specified time interval at specified pricing (Xu and Nozick, 2009). In this type of contract, capacity is commonly regarded as an option to be exercised in the future to produce needed goods. A capacity reservation fee (i.e., option price) and execution fee (i.e., exercise price) are the two key parameters in the contracts.

A typical option setting follows a two-phase structure. In the first phase, an option contract specifying a reservation fee, $r$, and an execution fee, $e$, is offered by a supplier. Then, based on these fees, the buyer chooses optional ordering quantity, $Q$, which is matched by the supplier's capacity. At this stage, the actual demand is unknown. While the reservation fee is immediately payable, the execution fee is due when the option is exercised (after demand uncertainty is resolved). In the second phase, the buyer decides on the exercise amount and pays the exercise fee after observing the realized demand.

Option contracts have been widely applied in industries such as semiconductor, aerospace, toys, apparel, electronic, telecommunication, etc. (e.g. Chang, 1996; Cole, 1998; Barnes-Schuster et al., 2002; Jin and $\mathrm{Wu}, 2007)$. Such option contracts are also known as capacity reservation contracts in the literature. Generally speaking, suppliers are motivated to use this type of contracts by "reducing potential cost through early commitments," while buyers are motivated by "ensuring availability during demand upsides" (Wu et al., 2005). Ritchen and Tapiero (1986) are considered to be the first to propose a two-period model to study an option contract to cooperate with uncertain future demands and prices. In their model, the buyer has the choice of 
carrying inventory from the first period to the second and can utilize first-period demand to update the forecast for second-period demand. The buyer then exercises the options based on the updated information at the beginning of the second period. The authors focus their analysis on the buyer's perspective and derive optimal contract policies. They also explored the buyer's ordering decision under different scenarios (i.e., risk neutrality vs. risk aversion and independent vs. dependent price and demand).

Considering a similar option contract, as in Ritchen and Tapiero (1986), other researchers studied various SC management issues. For example, Barnes-Schuster et al. (2002) examined optimal ordering policies and their implementations on SC coordination. The authors discussed the necessary conditions to achieve channel coordination when a return incentive is considered in the contract and the supplier is limited to using a linear pricing policy in contract design. They found that coordination conditions depended not only on the cost and salvage parameters but also on the correlation coefficient parameter of the demand. They showed that options are useful instruments for increasing flexibility and channel profitability. Option contracts in a model with a single capacitated seller and a single buyer were also studied by Serel et al. (2001), Wu et al. (2002), Spinler and Hunchzermeier (2006), Wang and Liu (2007), Cheng et al. (2009) and Zhao et al., (2010). With multiple suppliers and/or multiple buyers, researchers are interested in the strategic interactions between the supplier(s) and the buyer(s) (e.g., Erkoc and Wu, 2005; Jin and $\mathrm{Wu}, 2007)$ or the competitive behavior of independent players with option contracts in an SC system (e.g., Wu and Kleindorfer, 2005; Perakis and Zaretsky, 2008; Xu and Nozick 2009; Martínez-de-Albéniz and Simchi-Levi, 2009). 
Serel et al. (2001) extended the buyer's decision with option contract to a multi-period setting through a periodic review inventory control model. The authors examined the value of the option contract to both buyers and suppliers. In this model, option price and exercise price are not decision variables but predetermined parameters. Jin and Wu (2007) investigated the capacity coordination issue in a high tech manufacturing supply chain. They proposed an option contract in which the buyer's reservation fee (option price) is deductible from the purchasing price (exercise price). They developed a single-period newsvendor-based analytical model for a scenario of one supplier - one buyer. They also examined the capacity allocation issue that arises when one supplier faces two buyers who have the same marginal profit but a different demand distribution in their respective markets. In the same context, Erkoc and $\mathrm{Wu}$ (2005) proposed an option contract integrating partial deduction and cost sharing that can achieve coordination. Cheng et al. (2009) presented a capacity option contract that includes a firm purchase commitment with exogenous wholesale price. The retail price is a constant and demand is random. Similar to Erkoc and Wu (2005), Cheng et al. observed that options will be appealing for the buyer only if the reservation (option) fee is below a certain threshold.

Wu et al. (2002) considered a case in which both supplier and buyer have access to spot markets to sell or to buy non-storable goods. However, both parties embark on a capacity option contract due to uncertainty in the future spot market prices. The paper derived the seller's and buyer's optimal contracting strategies through a game theory framework. Golovachkina and Bradley (2003) also studied a capacity option contract in the presence of a sport market. But unlike Wu et al. who assumed that demand is deterministic, Golovachkina and Bradley considered a case in which the buyer (i.e., a manufacturer) must fulfill periodic stochastic demand. Spinler and 
Hunchzermeier (2006) extended Wu et al.'s framework to value options by taking into account the uncertainty in the buyer's future demand and the seller's future marginal costs. They showed that both parties are better off under option contracts compared to other market schemes. The buyer's demand for options depends on the correlation between buyer demand and spot price. Buzacott et al. (2011) proposed a mean-variance optimization model to examine a supplier's optimal stocking level under option contracts in the presence of demand uncertainty due to spot market price.

Burnetas and Ritchken (2005) studied the option contract in a supply chain where the demand distribution is influenced by retailers' pricing decisions (a downward-sloping demand curve with a stochastic parameter). They found that the introduction of option contracts causes the wholesale price to increase and the volatility of the ratail price to decrease. They also outlined the conditions under which manufacturer and retailer will benefit respectively from option contracts.

In a setting of multiple suppliers, Wu and Kleindorfer (2005) extended the model in $\mathrm{Wu}$ et al. (2002) to examine the situation where several suppliers compete to provide capacity to a single buyer. They investigated the optimal portfolios of contracting and spot market transactions for buyers and the suppliers, and they determined the market equilibrium pricing strategies. Xu and Nozick (2009) provided a solution procedure, a two-stage mixed integer stochastic programming, to optimize supplier selection in a multi-period transportation model using option contracts. In their model, the buyer is required to purchase a fixed amount per period from some of the suppliers (equivalent to minimum purchase requirement). Similar to $\mathrm{Xu}$ and Nozick 
(2009), Perakis and Zaretsky (2008) also considered a competitive SC in a multi-period setting. In their model, they treated the spot market as a dummy supplier with stochastic costs and unlimited capacity. They showed that the equilibrium policies coordinate the SC. Martínez-deAlbéniz and Simchi-Levi (2009) analyzed pricing strategy when suppliers are competing through option price and flexibility. They found that in market equilibrium a variety of suppliers coexist, and these suppliers offer different prices. However, unlike the finding of Perakis and Zaretsky, Martínez-de-Albéniz and Simchi-Levi found that their equilibrium solution cannot achieve SC coordination.

Zhao et al. (2010) included in their work the supply chain members' risk references and negotiating powers. They analyzed how these factors impact the profit allocation between the retailer and the manufacturer in a retailer-led supply chain in which the retailer holds the decision right on pricing and takes the initiative to coordinate the manufacturer's production quantity.

Table 2.3 provides a summary of the main contributions of option contracts in the SC/OM literature in terms of system settings, analytical models, contract structures, and sources of uncertainties. The system settings refer to supply chain structure (i.e., how many suppliers, buyers, and products are involved in the contractual relationships as well as the time period that each contract covers). We found that in the existing literature, the basic system setting in many option contract studies is that of one buyer, one supplier, one product, and one time period ("1/1/1/1"). 
Table 2.3: Summary of the main contributions of option contracts in OM/SC literature

\begin{tabular}{|c|c|c|c|c|}
\hline Literature & $\begin{array}{l}\text { System setting } \\
\text { (supplier / buyer / product / } \\
\text { time period) and from which } \\
\text { perspective }\end{array}$ & $\begin{array}{l}\text { Contract structure } \\
\left({ }^{*} \text { See Note } 2\right)\end{array}$ & $\begin{array}{l}\text { Source of } \\
\text { uncertainty }\end{array}$ & Analytical model \\
\hline Ritchen \& Tapiero (1986) & $\begin{array}{l}1 / 1 / 1 / 1 \\
\text { buyer }\end{array}$ & {$[\mathrm{r}, \mathrm{e}]$} & $\begin{array}{l}\text { demand \& } \\
\text { spot market price }\end{array}$ & $\begin{array}{l}\text { Mean-variance } \\
\text { approximations of } \\
\text { expected utility from } \\
\text { retailer's perspective }\end{array}$ \\
\hline Serel, et al. (2001) & $\begin{array}{l}1+\text { spot } / 1 / 1 / n \\
\text { buyer, Supplier and channel }\end{array}$ & {$[\mathrm{r}, \mathrm{e}]$} & demand & $\begin{array}{l}\text { periodical review } \\
\text { inventory model }\end{array}$ \\
\hline Barnes-Schuster et al. (2002) & $\begin{array}{l}\text { 1/1/1/1 } \\
\text { buyer, supplier and channel }\end{array}$ & {$[\mathrm{r}, \mathrm{e}]$} & demand & $\begin{array}{l}\text { Stochastic inventory } \\
\text { model }\end{array}$ \\
\hline Wu et al. (2002) & $\begin{array}{l}\text { 1/1(n)/1/1 } \\
\text { buyer, supplier }\end{array}$ & {$[\mathrm{r}, \mathrm{e}]$} & spot market price & $\begin{array}{l}\text { Game theoretical } \\
\text { model }\end{array}$ \\
\hline $\begin{array}{l}\text { Golovachkina \& Bradley } \\
(2003)\end{array}$ & $\begin{array}{l}1 / 1 / 1 / 1 \\
\text { supplier }\end{array}$ & {$[\mathrm{r}, \mathrm{e}]$} & $\begin{array}{l}\text { demand, spot } \\
\text { market price }\end{array}$ & $\begin{array}{l}\text { Game theoretical } \\
\text { model }\end{array}$ \\
\hline Erkoc \& Wu (2005) & $\begin{array}{l}1 / 1(2) / 1 / 1 \\
\text { buyer, supplier and channel }\end{array}$ & {$[\mathrm{r}, \mathrm{e}]$} & demand & $\begin{array}{l}\text { Newsvendor-based } \\
\text { model }\end{array}$ \\
\hline Burnetas \& Ritchken (2005) & $\begin{array}{l}1 / 1 / 1 / 1 \\
\text { buyer, supplier }\end{array}$ & {$[\mathrm{r}, \mathrm{e}]$} & demand & $\begin{array}{l}\text { Game theoretical } \\
\text { model }\end{array}$ \\
\hline Wu \& Kleindorfer (2005) & $\begin{array}{l}\mathrm{n} / 1 / 1 / 1 \\
\text { buyer, supplier }\end{array}$ & {$[\mathrm{r}, \mathrm{e}, \mathrm{L}]$} & $\begin{array}{l}\text { demand, spot } \\
\text { market price }\end{array}$ & $\begin{array}{l}\text { Game theoretical } \\
\text { model }\end{array}$ \\
\hline
\end{tabular}


Table 2.3: Summary of the main contributions of option contracts in OM/SC literature (Cont.)

\begin{tabular}{|c|c|c|c|c|}
\hline Literature & $\begin{array}{l}\text { System setting } \\
\text { (supplier / buyer / product / } \\
\text { time period) and from which } \\
\text { perspective }\end{array}$ & $\begin{array}{l}\text { Contract structure } \\
\left({ }^{*} \text { See Note } 2\right)\end{array}$ & $\begin{array}{l}\text { Source of } \\
\text { uncertainty }\end{array}$ & Analytical model \\
\hline $\begin{array}{l}\text { Spinler \& Hunchzermeier } \\
\text { (2006) }\end{array}$ & $\begin{array}{l}1 / 1 / 1 / 1 \\
\text { buyer, supplier }\end{array}$ & {$[\mathrm{r}, \mathrm{e}]$} & $\begin{array}{l}\text { demand, seller's } \\
\text { marginal cost, spot } \\
\text { market price }\end{array}$ & $\begin{array}{l}\text { Game theoretical } \\
\text { model }\end{array}$ \\
\hline Jin \& Wu (2007) & $\begin{array}{l}1 / 1(2) / 1 / 1 \\
\text { buyer, supplier and channel }\end{array}$ & {$[\mathrm{r}, \mathrm{e}, \mathrm{C}]$} & demand & $\begin{array}{l}\text { Newsvendor-based } \\
\text { model and Game } \\
\text { theoretical model }\end{array}$ \\
\hline Perakis \& Zaretsky (2008) & $\begin{array}{l}\mathrm{n} / 1 / 1 / \mathrm{n} \\
\text { buyer, supplier and channel }\end{array}$ & {$[\mathrm{r}, \mathrm{e}]$} & demand & $\begin{array}{l}\text { Game theoretical } \\
\text { model }\end{array}$ \\
\hline $\begin{array}{l}\text { Martínez-de-Albéniz \& } \\
\text { Simchi-Levi (2009) }\end{array}$ & $\begin{array}{l}\mathrm{n} / 1 / 1 / 1 \\
\text { supplier }\end{array}$ & {$[\mathrm{r}, \mathrm{e}]$} & demand & $\begin{array}{l}\text { Game theoretical } \\
\text { model }\end{array}$ \\
\hline Xu \& Nozick, (2009) & $\begin{array}{l}\mathrm{n} / 1 / 1 / \mathrm{n} \\
\text { buyer }\end{array}$ & {$[\mathrm{R}, \mathrm{e}]$} & demand & $\begin{array}{l}\text { Multi-period } \\
\text { transportation model } \\
\text { with option contract }\end{array}$ \\
\hline Cheng et al. (2009) & $\begin{array}{l}1 / 1 / 1 / 1 \\
\text { buyer, supplier and channel }\end{array}$ & $\begin{array}{l}{[r, e] \text { with minimum }} \\
\text { order/purchase commitment }\end{array}$ & demand & $\begin{array}{l}\text { Newsvendor-based } \\
\text { model }\end{array}$ \\
\hline Zhao et al. (2010) & $\begin{array}{l}1 / 1 / 1 / 1 \\
\text { buyer, supplier and channel }\end{array}$ & {$[\mathrm{r}, \mathrm{e}]$} & demand & $\begin{array}{l}\text { Newsvendor-based } \\
\text { model and Game } \\
\text { theoretical model }\end{array}$ \\
\hline Buzacott et al. (2011) & $\begin{array}{l}1 / 1 / 1 / 1 \\
\text { supplier }\end{array}$ & $\begin{array}{l}{[r, e] \text { with minimum }} \\
\text { order/purchase commitment }\end{array}$ & $\begin{array}{l}\text { demand, spot } \\
\text { market price }\end{array}$ & Mean-variance model \\
\hline
\end{tabular}

*Note 2: " $r$ " is reservation fee in the form of unit price, " $e$ " is exercise fee in the form of unit price, " $L$ " is seller's capacity reserved for contract market (vs. spot market), " $C$ " is excess capacity in addition to buyer's reservation amount, and " $R$ " is reservation fee in the form of lump sum payment. 
In Table 2.3, we also listed perspective(s) from which the researchers addressed issues related to option contracts. We found that Ritchen and Tapiero (1986) and Xu and Nozick (2009) studied option contracts only from the buyer's perspective, while Golovachkina and Bradley (2003), Martínezde-Albéniz and Simchi-Levi (2009) and Buzacott et al. (2011) from only the supplier's perspective. In the papers focusing on channel coordination issues, the supplier, the buyer, and the channel perspectives are all included in the analysis (e.g., Erkoc and Wu, 2005; Perakis and Zaretsky, 2008). In most studies, the structures of option contracts are characterized by two parameters: $r$ and $e$. The parameter $r$ refers to the option price, also known as reservation fee. It is paid by a buyer when the buyer reserves the options before knowing actual demand. The parameter $e$ refers to the exercise price, which is paid by the buyer when the buyer exercises the options after knowing the demand.

\subsubsection{Summary}

Since LRP can be viewed as a "rewards and points" supply chain that carries common features of TSC, option contracts are also applicable for LRP management to target demand and/or supply uncertainties. In the existing literature, no one has introduced option contracts in LRP management, which opens an opportunity for us to add contributions to the literature by considering this dimension.

Under the option contract setting, an LRP host (i.e. the reward buyer) and LRP redemption partners (i.e. the reward suppliers) interacts with each other in two phases. In the first phase, an option contract specifying a reservation fee (option price) $r$, an execution fee $e$, and a reservation quantity (options to purchase) $Q$, is agreed upon by both parties. At this stage, the demand is 
unknown. Based on the reservation fee, the host chooses $Q$, which is matched by the redemption partner's capacity. In the second phase, the host decides on the exercise amount (options to exercise) and pays the exercise amount after observing the realized demand.

Rather than examining strategic behavior between the LRP redemption partners and the LRP host in rewards-supply contracting, we focus our attention on the LRP host's rewards-ordering decisions based on the host's own financial and managerial constraints and the option contracts offered by the different rewards suppliers. In other words, option price and exercise price are not decision variables, but predetermined parameters; and the option price is deductable from the exercise price.

\subsection{Cooperative Advertising}

Similar to other marketing programs, advertising and promotion are the common tools used by LRP hosts and LRP partners to increase sales and to target potential customers. In this section, we first present the concept of cooperative advertising (including promotion) (CA), and then provide a brief review of CA literature. We also discuss budget allocation issues and different formulations of sales response functions that are relevant to CA.

\subsubsection{Overview}

CA has been adopted as a business practice for many years. It is essentially a financial arrangement under which two parties agree on how the sponsorship and costs of mutual promotion and advertising will be defrayed (Crimmins, 1984). The three principal categories of 
cooperative advertising are horizontal, ingredient-producer, and vertical (Young and Greyser, 1982). In the SC/OM literature, vertical cooperative advertising (including promotion) is the most prevalent of these types. It refers to an interactive arrangement where a manufacturer pays for some or all of the costs of local advertising implemented by a retailer of the manufacturer's product (Huang et al., 2002). Therefore, $\mathrm{CA}$ in the $\mathrm{SC} / \mathrm{OM}$ literature mainly refers to cost sharing of vertical advertising (e.g., manufacturer shares retailer's advertising and promotion expenses). CA is usually used by a manufacturer to motivate immediate sales at the retail level and to strengthen the image of the brand (Huang et al., 2002).

There are two strategies to realize this cost sharing among SC members (e.g., manufacturers and retailers). The use of side payment is one strategy. Manufacturers decide on the size of a lump sum payment for each retailer, and retailers decide how much of this side payment to use in promoting the manufacturer's product. A portion of this side payment is normally pocketed by the retailer. However, due to a shift in power from manufacturers to retailers and/or the competition in the marketplace, even though the manufacturers know the retailers may pocket much of the payment they are still willing to pay (Kim and Staelin, 1999). It was reported in the research that the percentage pocketed ranges on average from 30 to 50 percent (Armstrong, 1991; MacClaren, 1992; Agrawal, 1996). The use of participation rate is the other strategy. It has been discussed more frequently in the recent CA literature (e.g., Bergen and John, 1997; Huang et al., 2002; Karray and Zaccour, 2007; Xie and Wei, 2009; He et al., 2009, He et al., 2011). Participation rate specifies the proportion of the retailer's advertising and promotion costs that the manufacturer shares. Because participation rate ties to a retailer's actual advertising expenditures, generally speaking, it overcomes the weakness of side payment, and it has become 
the most prominent strategy used by manufacturers. However, since it requires the retailer to self-report on advertising expenditure, it causes more administration costs. In analytical models based on this payment strategy, the manufacturer decides on is the amount of the participation rate while the retailer decides on the amount of advertising spending.

\subsubsection{Cooperative Advertising and Budget Allocation}

In the SC/OM domain, the majority of the research attempts to investigate the role of CA in SC management and SC coordination of suppliers and retailers (e.g., Huang et al., 2002; Yue et al., 2006, Karray and Zaccour, 2007; Xie and Wei, 2009; He et al., 2009). For example, both Huang et al. (2002) and Yue et al., (2006) derived optimal levels of CA expenditures for manufacturers, retailers, and channels under a simple one-to-one SC setting (i.e., one manufacturer and one supplier). But Yue et al. (2006) went one step further. They focused on the situation where a manufacturer offers price deductions directly to customers instead of through a retailer and explored the optimal levels of CA expenditures in this situation. Xie and Wei (2009) examined the impact of the retail price rather than manufacturer's price discount on customer demand in a CA model with the same SC setting as that used in the previous two studies. Karray and Zaccour (2007) considered a SC formed by two suppliers and two retailers. They assessed an efficient CA plan by comparing the scenarios that either no manufacturer or one or both manufacturers offer(s) CA to retailers. Unlike previous studies, He et al. (2009) investigated manufacturers' and retailers' CA decisions through an analytical model that integrates a stochastic sales advertisingresponse function rather than a deterministic advertising-response function. He et al. (2011) extended the model proposed in He et al. (2009) by considering retail competition. We refer 
readers interested in knowing more about CA in a broader context to Neslin (2006), Stewart and Kamins (2002), and Ailawadi et al. (2009) for recent surveys.

Another important decision issue relating to $\mathrm{CA}$ is how to allocate advertising budget among different submarkets or different retailers. These submarkets or retailers are characterized by geographic areas, market segments, product categories, media instruments, or seasonal changes. Extensive studies have examined budget allocation problems.

For example, Holthausen and Assmus (1982) presented an advertising budget allocation model for different market segments from a manufacturer's point of view. They studied the budget allocation issues under sales response uncertainty. Advertising correlations among marketing segments are also considered in the model. Ahmed (1984) investigated a budget allocation and advertising media selection problem in the single-product context. The author developed an efficient integer programming model to solve the problem. Basu and Batra (1988) considered CA for multiple brands. They discussed the issue of how to allocate an advertising budget among individual brands in order to maximize the overall corporate profit when corporate promotional resources are limited. In their analytical model, sales response for each brand is determined by the advertising budget allocated as well as the price elasticity. Doyle and Saunders (1990) focused on allocating retailer's decision on allocating advertising resources to different products with the consideration of influence factors such as manufacturer's allowance, cross-product effects, and store-wide traffic effects. Bockstael et al. (1992), Vande Kamp and Kaiser (2000), and Kinnucan and Myrland (2002) examined the optimal timing of advertising allocations. Seasonal variations in price, demand, and product substitution are the prime concerns in these 
studies. In more recent research, Labbi and Berrospi (2007) proposed an optimization model for budget allocation in the context of LRP. They introduced a customer segmentation approach that is based on customer value and loyalty metrics as well as customers' response behavior. They also used a Markov process to simulate the dynamics of customer status over time, namely the movement of customers among three states: first-time/not frequent, repeated purchase, and loyal customers.

A theoretical underpinning of $\mathrm{CA}$ and budget allocation models is the relationship between advertising inputs (e.g., advertising budget or expenditures) and advertising outputs (e.g., sales response). Simon and Arndt (1980) identified the characteristics of this relationship through a survey of more than one hundred empirical studies of advertising response functions. Their survey showed that the response functions proposed in the majority of research on advertising follow one of the two proposed shapes: (1) a non-negative concave-downward curve and (2) an $\mathrm{S}$-shaped curve. They conclude that the former curve is the one found most often. Based on Simon and Arndt's study, Tull et al. (1986) proposed three different aggregate advertising-sales response functions (namely, a concave saturation sales response function, a diminishing returns function and a quadratic response function) with respect to different operating ranges used by companies for advertising. The authors integrated these functions respectively with a singleperiod profit maximization model to study the decisions on the optimal advertising budget level. Basu and Batra (1988) proposed a general sales response function which allows both concave and S-shaped relationships between the advertising budget and sales response. Mantrala et al. (1992) analyzed budget allocation problem among multiple submarkets. These submarkets are characterized by advertising-sales response functions that are similar to those used in Tull et al. 
(1986). Although the complexity and difficulty in modeling sales-advertising relationship has led to a multitude of research papers on this topic (e.g. Ağrali and Geunes, 2009; Vakratsas et al., 2004), we found that concave saturate-based response functions are the ones most frequently used in the literature (e.g., Naert and Leeflang, 1978; Holthausen and Assmus, 1982; Mantrala et al., 1992; Huang, et al., 2002).

\subsubsection{Cooperative Advertising in LRP Operations}

It is a common practice in LRP management for an LRP host to offer CA to accumulation (and redemption partners) to boost consumers' accumulation (and redemption) demands. In this paper, we focus our attention on a type of CA that is uniquely associated with LRPs. It is known as "bonus points". Bonus points refer to the CA agreement in which the LRP host offers LRP members extra points when the members purchase certain products from the LRP partners or participate in certain partners' promotional activities. Unlike the common CA strategies that we discussed in the previous section, an LRP host doesn't give this benefit (i.e., bonus points) to LRP partners. Instead, the host gives bonus points to LRP members directly. For example, Aeroplan supports his financial partner CIBC by offering 15,000 points to customers on their first application for an Aerogold Visa Card. Air Miles allows members to earn triple reward miles when they shop online through the Air Miles website. In a recent study ${ }^{1}$, several different formats of bonus points were discussed: enrolment-related vs. selected members-related, and flat bonus (i.e. fixed) vs. percentage bonus (i.e. based on accrual points the member earned).

\footnotetext{
1 “Ensuring Customer Loyalty: Designing Next-Generation Loyalty Programs", Oracle White Paper, Feb., 2005. www.oracle.com $/ . . / \mathrm{cm} / . . /$ sicbel-marketing-loyalty-white-paper.pdf
} 
To LRP hosts, 'bonus points' is a two-edged sword. In the short run, bonus points can directly contribute to LRP value growth and encourage LRP partners to promote the LRP besides their own products. But in the long run, bonus points increase redemption costs and points-related liability, which may cause potential risks in the LRP management. Therefore, LRP hosts need to manage bonus-point type of CA properly in order to get the most out of it. Unlike using CA in traditional SCs, in an LRP system, since the LRP host can communicate with LRP members (i.e. consumers) directly through its media network (e.g., e-mails, websites, or call centers), the host doesn't have to rely on the partners' advertising effort and media network to motivate the LRP. In other words, the host can estimate and observe the sales response to bonus-point type CA through tracing LRP members' purchase history. In this sense, the host may control the impact of a bonus-point type of $\mathrm{CA}$ through the proper selection of the amount of bonus points assigned to each partner. This is the essential difference between common CA and LRP CA. To the best of our knowledge, the impact of bonus points on the operational decisions of LRPs has not been explored in the LRP literature.

\subsubsection{Summary}

In this section, we reviewed the literature that is relevant to the second focus of this study bonus points, a unique type of CA associated with LRP management. We first introduced the concept of $\mathrm{CA}$ and the two common $\mathrm{CA}$ strategies (i.e. lump sum payment and percentage rate) that have been examined in the literature. We then focused on the literature related to budget allocation since CA budget allocation is one of the central concerns to a manufacturer/supplier when he plans to offer CA to multiple retailers/buyers. Meanwhile, we overviewed sales advertising-response functions, a key determinant of model validity in the development of CA 
related analytical models. Finally, we pinpointed the differences between bonus-point type of CA and the other common types of CA.

In our study, we examine LRP host's decisions on the amount of bonus points to offer to different LRP accumulation partners. Unlike previous CA and budget allocation research, we integrate this problem into the host's decisions on rewards-ordering by considering the "delayed" cost of offering bonus points. The sales response function proposed in our model is on the same line of the concave saturate-based functions. 


\section{Chapter 3 Research Framework and Mathematical Models}

In this chapter, we first explain the theoretical foundation of this research, and then we discuss the mathematical formulation of the LRP rewards-supply aggregate planning problem under various settings.

\subsection{Loyalty Reward Programs - "Rewards-Points" Supply Chains}

Let us consider an LRP system where a host firm runs it as a focal business that seeks to maximize the value created through the "rewards \& points" business. Multiple LRP partners participate in this system to fulfill the requirements of LRP members for points and rewards. Based on this point of view, the LRP system can be described by the following conceptual model (Figure 3.1):

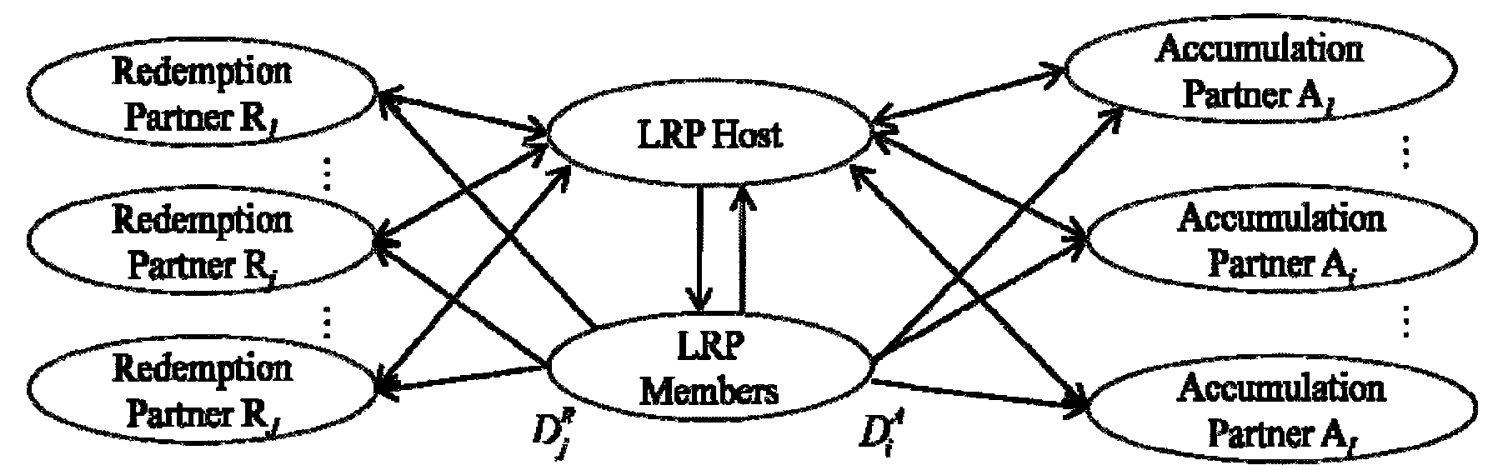

Figure 3.1: Conceptual model of a rewards-points supply chain 
In this conceptual model, one LRP host firm has multiple LRP redemption partners $\left(R_{\jmath}\right.$, $j=1, \ldots, J)$, multiple LRP accumulation partners $\left(A_{l}, i=1, \ldots, I\right)$, and LRP members, the end consumers who collect points and redeem them later for rewards. Hence, LRP members have two types of demand: point-accumulation demand $\left(D_{i}^{A}, i=1, \ldots, I\right)$ and point-redemption demand $\left(D_{j}^{R}, j=1, \ldots, J\right) . D_{t}^{A}$ refers to LRP members' overall (aggregate) demand for collecting points from the LRP accumulation partner $A_{l} . D_{j}^{R}$ refers to LRP members' overall (aggregate) demand for redeeming points for rewards offered by the LRP redemption partner $R_{\jmath}$. LRP members' accumulation demand towards each LRP accumulation partner drives the points business between the LRP host and the LRP accumulation partners (e.g., LRP host sells points to an LRP accumulation partner $A_{l}$ ). Meanwhile, LRP members' redemption demand towards each LRP redemption partner drives the rewards business between the LRP host and the LRP redemption partners (e.g., LRP host purchases rewards from an LRP redemption partner $R_{\jmath}$ ). In this pointsrewards supply chain, points are circulated as LRP currency among all the entities (i.e., LRP host, LRP partners, and LRP members). Meanwhile, points and rewards are the short- and longterm incentives to drive business forward and create value in the whole chain.

How does this rewards-points supply chain create values for all the entities involved in the system? To answer this question, we examine the value creation processes at both accumulation and redemption sides. At the accumulation side, when an LRP member purchases a product or a service from an LRP accumulation partner, the value from points business is created. To the LRP member, the value obtained is the points that were earned (in addition to the product or the service purchased) through the purchase of the product or the service. To the LRP accumulation partner, the value obtained is the revenue generated through the sale of the product or the service. 
However, as the LRP accumulation partner does not directly issue the points earned by the LRP member, those points have to be purchased from the LRP host, the issuer of points. By selling points to the accumulation partner, the LRP host shares the revenue obtained from the purchase of the product or the service by the LRP member. The sum of the revenue shares from all LRP accumulation partners is the value that the LRP host obtains through the points business. At the redemption side, the business starts when an LRP member redeems points for a reward. Since the LRP host does not produce the reward, it has to be purchased from an LRP redemption partner to meet the LRP members' redemption demand. The LPR host charges the LRP member a certain amount of points for the rewards. Therefore, to the LPR host, the value obtained through the rewards business is the difference between the value ${ }^{1}$ of the reward (measured by the value of points that the LRP member gives out for the reward) and the actual amount that the LRP host spends to purchase the reward. To the LRP redemption partner, the value obtained from this redemption business is the revenue generated by selling the reward to the LRP host. The reward that the LRP member receives is the value obtained from redemption.

Figure 3.2 illustrates the value creation process in a rewards-points supply chain. We assume that the accumulation grid is defined as follows:

"For every $\$ 1$ spending at an LRP accumulation partner $\left(A_{l}\right)$, an LRP member can collect one point."

The redemption grid is defined as follows:

“A LRP member obtains a $\$ 0.01$ value of reward per point." (e.g., a reward worth $\$ 150$ requires 15,000 points).

\footnotetext{
${ }^{1}$ The value of rewards quite often depends on the benchmarks, for example, the retail prices of the rewards in the market.
} 
Therefore, the value created with the points business is the revenue generated per point at the accumulation side. Let $p^{A}$ denotes the revenue generated per point at the accumulation side, in this example, $p^{A}=\$ 1$.

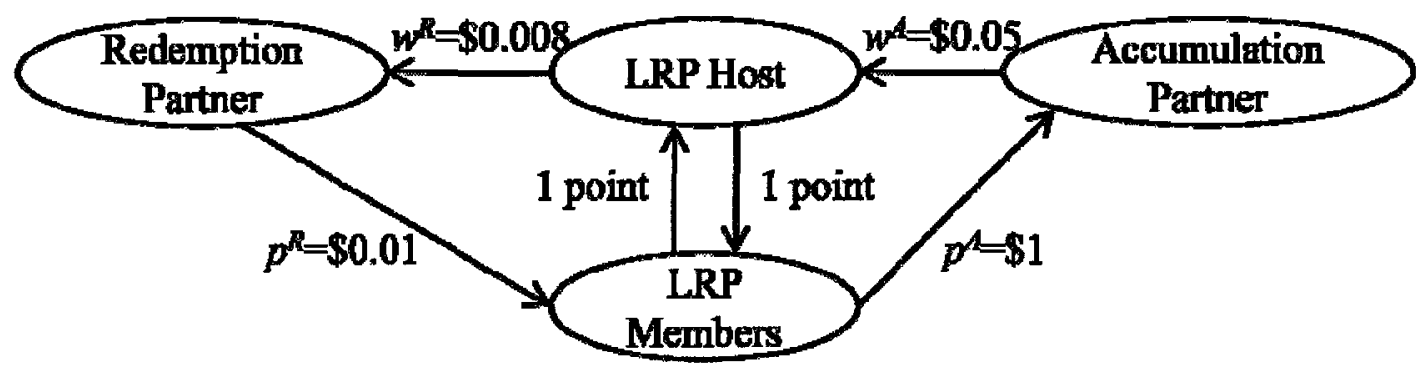

Figure 3.2: Illustration example of value creation

At the accumulation side, the revenue per point will be shared among the LRP accumulation partner $\left(A_{i}\right)$ and the LRP host. The LRP host sells points to the LRP accumulation partner $\left(A_{i}\right)$ at price $w^{A}$ per point, $w^{A}=\$ 0.05$. The accumulation partner $A_{i}$ 's share of the value created with the points business is: $p^{A}-w^{A}$, which is $\$ 0.95$ per point in this example. The LRP host's share of the value is $w^{A}$, which is $\$ 0.05$ per point in the example. At the redemption side, let $p^{R}$ denotes the value of reward per point, $p^{R}=\$ 0.01$. The LRP host buys a reward (transformed to the equivalent value of points) at price $w_{R}$ per point from the LRP redemption partner $\left(R_{j}\right), w^{R}=$ $\$ 0.008$ in the example. As such, the redemption partner $\left(R_{j}\right)$ receives its share of the value created by the rewards business, which is $w^{R}$. When an LRP member redeems points for a reward, the value created through the rewards business is $p^{R}-w^{R}$, from the LRP host point of view, which is $\$ 0.01-\$ 0.008=\$ 0.002$ per point in the example. Redemption business will be profitable only when the redemption cost per point, $w^{R}$ is less than the value of reward per point, 
$p_{R}$. Overall, the LRP value created through transacting 1 point is $\$ 0.05+\$ 0.002=\$ 0.052$ from the LRP host point of view.

As we can see, in this rewards-points supply chain, managing rewards and points plays an important role in the LRP value creation and sustainable development. In the remainder of this study, the overall value that an LRP host obtains through this rewards-points supply chain is called "LRP profitability". It includes the LRP host's shares of values created from both the accumulation and redemption sides of business.

\subsection{LRP Rewards-Supply Aggregate Planning Models}

The LRP host's aggregate planning problem for rewards-supply consists of determining the optimal procurement of rewards from multiple LRP redemption partners to maximize the LRP profitability (as measured by the value creation) subject to system constraints and managerial requirements. The system constraints refer to the capacity constraint that an LRP redemption partner has on offering rewards, and the LRP host's overall budget for purchasing rewards. The managerial requirements mainly refer to the LRP host's control on liability, the outstanding balance of points representing the total value of future financial arrangements (i.e., future redemption obligation).

Liability is widely recognized in the LRP industry as a risk indicator for LRP sustainability. For many LRPs, especially large-size LRPs with a huge customer base, business practices show that a "high liability" results in higher redemption uncertainties, higher management costs, and higher 
risks (e.g., hyperinflation and devaluation of points ${ }^{1}$ ). Therefore, in this study, we explore how to maintain liability within a reasonable range that is most beneficial to the LRP host.

In the following sections, we introduce first a mathematical model for the LRP rewards-supply planning problem without considering cooperative advertising (through bonus points) or option contracts. Then we extend this model to integrate cooperative advertising and option contract decisions. In each section we first explain our modeling assumptions, and then we introduce the model formulation.

\subsubsection{LRP Rewards-Supply Planning Problem without Bonus Points}

In this section, we report the mathematical formulation of the LRP rewards-supply planning problem without bonus points offering.

\subsubsection{Modeling Assumptions}

In our modeling the following assumptions are considered:

Assumption (1): Relationships between LRP partners and an LRP host are governed by contracts.

Assumption(2): LRP redemption partners have capacity limitations on offering rewards.

Assumption (3): LRP host has no capacity limitation on issuing points.

Assumption (4): LRP members' accumulation demands will always be met.

Assumption (5): LRP members' accumulation and redemption demands are not known with certainty but have known probability distributions and both demands are price-independent.

\footnotetext{
${ }^{1}$ Hofer, D. 2008. Reinforcing the value of frequent flyer miles. White paper, Loylogic Inc www.loylogic com
} 
Assumption (6): LRP members' demands towards LRP accumulation partners are independent from each other.

Assumption (7): LRP members' demands towards LRP redemption partners are independent from each other.

Assumption (8): LRP members' demands towards LRP redemption partners are independent from LRP members' demands towards LRP accumulation partners.

Assumption (9): Each LRP accumulation partner's ordering quantity of points is equal to the mean value of LRP members' accumulation demands towards that accumulation partner.

Assumption (10): Static redemption schemes are adopted by LRP redemption partners during the planning time period.

Assumption (11): Static accumulation schemes are adopted by LRP accumulation partners during the planning time period.

Assumption (12): Wholesale-price contracts are used as the governing mechanism between an LRP host and LRP accumulation partners.

Assumption (13): Wholesale-price contracts are used as the governing mechanism between an LRP host and LRP redemption partners.

Assumption (14): Cooperative advertising through bonus points is not considered

Assumption (1) indicates that, similar to the traditional supply chain operations, the business relationships between LRP partners (both for redemption and/or accumulation) and an LRP host are primarily governed by contracts. Although many different types of contracts have been discussed in the literature (e.g., Tsay et al., 1999; Cachon, 2003), we limit our study in this section to the wholesale-price contracts because of its fit for LRPs (see assumptions (12) and (13))

Assumption (2) shows that the amount of rewards (e.g., free airline tickets or vacation packages) provided by redemption partners are limited by the available capacities. 
Assumptions (3) and (4) relate to the unique features of points. As points are a kind of information symbol for recording and counting LRP members' purchase efforts in the LRP system, the LRP host has almost no "production" related costs or "resource" related capacity limitations on offering points. As such, the LRP host has no capacity limitation on issuing points. Furthermore, unlike tangible products, production and movement of points are not limited by time and physical space. Points are never "stock-out" in the sense that there is almost no time lag between producing points and meeting the customer accumulation demand on points. Therefore, LRP members' demands for accumulation will always be met.

Assumptions (5) to (7) indicate that the rewards offered by redemption partners are not substitutable, as well as the accumulation options offered by accumulation partners. Besides that, both accumulation and redemption demands are random parameters following known probability distributions. Assumption (8) indicates that during the planning horizon, LRP members' redemption demands at the aggregate level are not influenced by LRP members' accumulation demands.

Assumption (9) describes that although accumulation demand is a random parameter, an LRP accumulation partner knows the mean value of the accumulation demand and uses it to determine ordering quantity of points. Therefore, this assumption indicates that LRP accumulation partners are risk-neutral.

Assumptions (10) to (11) suggest that the redemption and accumulation schemes are predetermined and do not change over the planning horizon. The schemes are structured through 
formats such as "earn 3 points per dollar spending", "every 1000 points are worth $\$ 1$ value of reward", or "redeeming 5000 points can get $\$ 10$ discount", etc.

\subsubsection{Problem Description and Model Formulation}

The notations used in this section are as follows:

(1) Indices:

$R_{j} \quad$ LRP redemption partners, who offer rewards to LRP members, $j=1,2, \ldots, J$

$A_{t} \quad$ LRP accumulation partners, who allow LRP members to collect points through their purchasing of products or services, $i=1,2, \ldots, I$

$H \quad$ LRP host, who runs an LRP as a profit center.

(2) Decision variables:

$q_{j}^{R} \quad$ LRP host $H$ 's ordering quantity of rewards from LRP partner $R_{j}$.

(3) Parameters in objective function:

$D_{\imath}^{A} \quad$ LPR members' accumulation demand towards LRP accumulation partner $A_{\imath}$.

$q_{t}^{A} \quad$ LRP partner $A_{t}$ 's ordering quantity of points.

$w_{\imath}^{A} \quad$ Wholesale price per unit of points that LRP host $H$ charges to LRP accumulation partner $A_{t}$

$w_{l}^{\prime A} \quad$ Price per unit of points that LPR host $H$ charges to LRP accumulation partner $A_{t}$ when accumulation demand is over $A_{\imath}$ 's ordering quantity.

$w_{J}^{R} \quad$ Wholesale unit price of rewards that LRP redemption partner $R_{J}$ charges to LRP host $H$.

$p_{J}^{R} \quad$ Per unit point value of rewards offered by LRP redemption partner $R_{J}$. 
$D_{J}^{R} \quad$ LRP members' redemption demand towards LRP redemption partner $R_{J}$.

$v_{j}^{R} \quad$ Per unit shortage penalty cost of LRP redemption partner $R_{j}$ 's rewards.

$s_{j}^{R} \quad$ Per unit salvage value of LRP redemption partner $R_{J}$ 's rewards.

(4) Parameters in constraints:

$l_{0} \quad$ Liability in points at the beginning of the planning horizon.

$l \quad$ Target liability in points at the end of the planning horizon.

$l^{\prime} \quad$ Actual liability in points at the end of the planning horizon.

$L_{U B} \quad$ Upper bound of liability control limits for the planning horizon.

$Q_{J}^{R} \quad$ LRP redemption partner $R_{j}$ 's capacity limitation on offering rewards.

$W^{R} \quad$ LRP host $H$ 's budget limitation on purchasing rewards.

The problem that we are concerned with here is to determine, given the ordering quantities of points from LRP accumulation partners $A_{l}$, (i.e., $q_{i}^{A}$ ), the LRP host's optimal ordering quantity of rewards from LRP redemption partner $R_{j}\left(\right.$ i.e., $q_{j}^{R}$ ) in order to maximize the LRP profitability (as measured by its value creation); all of this is subject to the LRP redemption partner $R_{j}$ 's capacity limitations on offering rewards, the LRP host's overall budget for purchasing rewards, and the LRP host's control on liability.

One thing worth mentioning here is that, based on Assumption (4), the LRP host allows the accumulation partners to meet all the accumulation demands (in points) that are above the 
accumulation partners ordering quantities of points. However, the LRP host also has an intention to limit the overall amount of points that the accumulation partners pass to LRP members. Pricing is an effective strategy applied by the LRP host to encourage or discourage the accumulation partners' usage of points and then subsequently control the overall accumulation level. By charging a higher price on the extra ordering quantities of points, the LRP host forces the accumulation partners to share a greater part of the potential cost of points, which will occur later as the redemption cost to the LRP host. Therefore, a reasonable assumption here is that $w_{i}^{A} \geq w_{1}^{A}$

Hence, under the wholesale-price contract setting, the LRP host guarantees each LRP accumulation partner $A_{l}$ a wholesale unit price of points $w_{\imath}^{A}$. The LRP accumulation partner $A_{l}$ decides on the quantity $q_{t}^{A}$ of points to order, which is the mean value of the accumulation demand $D_{1}^{A}$ that accumulation partner $A_{l}$ predicts. At the end of the planning horizon, if the accumulation demand $D_{l}^{A}$ is higher than the LRP accumulation partner $A_{l}$ 's ordering quantity ( $q_{l}^{A}$ ), the per unit excess demand is purchased at $w_{l}^{\prime A}$, the unit price of points given out to meet the excess demand. The LRP host's profitability (i.e., value creation) function at the accumulation side is formulated as follows (BP-A):

$$
\pi_{H(A)}=\sum_{i=1}^{I}\left(w_{i}^{A} \times q_{i}^{A}+w_{i}^{\prime A} \times\left[D_{t}^{A}-q_{i}^{A}\right]_{+}\right)
$$

The first term in (BP-A), denotes the revenue obtained through the selling of points to an accumulation partner $A_{l}$. The second term denotes the extra revenue that the LRP host $H$ gains 
through the accumulation partner $A_{l}$ 's extra ordering of points when $D_{l}^{A}$ is higher than the ordering quantity $q_{i}^{A}$

At the redemption side, each LRP redemption partner $R_{J}$ guarantees the LPR host a wholesale unit cost of points redeemed, $w_{J}^{R}$. The LRP host decides on the quantity of rewards (in points) $q_{J}^{R}$, to order during the planning horizon from LRP redemption partner $R_{J}$ at the given wholesale unit cost, $w_{J}^{R}$. At the end of the planning horizon, if the LRP members' redemption demand $\left(D_{J}^{R}\right.$ ) towards LRP redemption partner $R_{J}$ is higher than the LRP host ordering quantity $q_{J}^{R}$, the excess demand is assumed to be lost and the under-stocking cost $v_{j}^{R}$ per unit of points is incurred. On the contrary, when $D_{J}^{R}$ is less than $q_{j}^{R}$, the excess ordering quantity is sold at a over-stocking unit sale price $s_{J}^{R}$. The LRP host $H$ s profitability (i.e., value creation) function at the redemption side is defined as follows (BP-R):

$\pi_{H(R)}=\sum_{j=1}^{J}\left(p_{J}^{R} \times \min \left\{q_{J}^{R}, D_{J}^{R}\right\}-w_{J}^{R} \times q_{J}^{R}-v_{J}^{R} \times\left[D_{J}^{R}-q_{J}^{R}\right]_{+}\right)+\sum_{j=1}^{J}\left(s_{J}^{R} \times\left[q_{J}^{R}-D_{J}^{R}\right]_{+}\right)$

In (BP-R), $\left[D_{J}^{R}-q_{J}^{R}\right]_{+}$denotes under-stocking quantity when the redemption demand is higher than the LRP host $H$ 's ordering quantity of rewards from a redemption partner $R_{j}$; while $\left[q_{J}^{R}-D_{J}^{R}\right]_{+}$denotes the over-stocking quantity when the redemption demand is lower than the ordering quantity of rewards. Overall, (BP-R) is a linear function consisting of four terms. The first term indicates the value of rewards offered by each partner $R_{j}$. The second term indicates the 
LRP host $H$ 's purchasing cost of rewards. The third term refers to the under-stocking cost of rewards. Finally, the fourth term refers to the salvage value of over-stocking rewards.

Combining (BP-A) and (BP-R), the problem of planning the supply of rewards without bonus points can be formulated as follows (hereafter Problem BP):

$$
\begin{aligned}
& \Pi_{H}\left(q_{\jmath}^{R} ; D_{J}^{R}, D_{\imath}^{A}\right)=\max E\left[\pi_{H(A)}+\pi_{H(R)}\right] \\
& =\max \left(\begin{array}{l}
\sum_{i=1}^{I}\left(w_{\imath}^{A} \times q_{\imath}^{A}\right)-\sum_{J=1}^{J} w_{J}^{R} \times q_{\jmath}^{R}+E\left[\sum_{i=1}^{I} w_{\imath}^{A} \times\left[D_{\imath}^{A}-q_{\imath}^{A}\right]_{+}\right]+ \\
E\left[\sum_{j=1}^{J}\left(p_{J}^{R} \times \min \left\{q_{J}^{R}, D_{J}^{R}\right\}-v_{J}^{R} \times\left[D_{J}^{R}-q_{J}^{R}\right]_{+}+s_{J}^{R} \times\left[q_{J}^{R}-D_{J}^{R}\right]_{+}\right)\right]
\end{array}\right)
\end{aligned}
$$

subject to:

$$
\begin{aligned}
& q_{J}^{R} \leq Q_{J}^{R}, \text { for } j=1, \ldots, J \\
& \sum_{J=1}^{J}\left(w_{j}^{R} \times q_{J}^{R}\right) \leq W^{R} \\
& \frac{l}{l_{0}} \leq L_{U B}, \text { where } l=l_{0}+\sum_{i=1}^{I}\left(q_{i}^{A}+\left[D_{l}^{A}-q_{l}^{A}\right]_{+}\right)-\sum_{j=1}^{J} q_{j}^{R} \\
& q_{J}^{R} \geq 0, \text { for } j=1, \ldots, J
\end{aligned}
$$

Constraints (3.4) indicate that each LRP redemption partner has a capacity limitation on the quantity of rewards offered to an LRP host. Constraint (3.5) indicates that the LRP host has an overall budget limit for purchasing rewards that cannot be exceeded. Constraint (3.6) is the liability control constraint. It is formulated as the ratio of the target liability $(l)$ at the end of the planning horizon and the initial liability $\left(l_{0}\right)$ at the beginning of the planning horizon. As discussed in the previous section, points earned by LRP members are stored in members' accounts. To the LRP host, these points are counted as a liability until they are redeemed by LRP 
members for rewards. Therefore, the overall target liability at the end of the planning horizon $(l)$ is equal to the initial liability $\left(l_{0}\right)$ at the beginning of the planning horizon plus the overall amount of points earned by members during the planning horizon, $\sum_{i=1}^{I}\left(q_{i}^{A}+\left[D_{i}^{A}-q_{t}^{A}\right]_{+}\right)$, minus the overall "target amount" of points redeemed by members for rewards during the same time period, $\sum_{j=1}^{J} q_{j}^{R} . L_{U B}$ is introduced as the upper bound of the liability ratio in Constraint (3.6). If the LRP host wishes to reduce the liability ( $l$ ), the value of $L_{U B}$ should be set to no more than 1 . If the LRP host plans to increase the liability $(l)$ for the planning horizon, the value of $L_{U B}$ should be set to larger than 1 . Otherwise, a value equal to 1 is given to maintain the liability at the same level as before. Constraints (3.7) refer to the non-negativity constraints.

Note that here $\sum_{j=1}^{J} q_{j}^{R}$ is called "target redemption" because the actual amount of redemption depends not only on LRP host $H^{\prime}$ ordering quantities of rewards $q_{J}^{R}$, but also on the redemption demands $D_{J}^{R}$. The actual redemption is equal to $\sum_{J=1}^{J} \min \left(q_{J}^{R}, D_{J}^{R}\right)$, which can be reformulated as: $\sum_{J=1}^{J}\left(q_{J}^{R}-\left[q_{J}^{R}-D_{J}^{R}\right]_{+}\right)$. Therefore, the actual liability $\left(l^{\prime}\right)$ at the end of the planning horizon is computed as follows:

$$
l^{\prime}=l_{0}+\sum_{i=1}^{I}\left(q_{\imath}^{A}+\left[D_{\imath}^{A}-q_{\imath}^{A}\right]_{+}\right)-\sum_{J=1}^{J}\left(q_{J}^{R}-\left[q_{J}^{R}-D_{J}^{R}\right]_{+}\right)
$$




\subsubsection{LRP Rewards-Supply Planning Problem with Bonus Points}

We introduce in this section, the modeling of the LRP rewards-supply planning problem for a case where the LRP host offers cooperative advertising (CA) through bonus points to LRP accumulation partners. Like other promotion and advertising strategies, the purpose of using bonus points is to boost end consumers' demand (i.e., LRP members' accumulation demand) for certain products or services. In the rewards-supply planning problem with bonus points, we transform the CA budget allocation problem into an equivalent bonus points offering decision problem. The amount of bonus points assigned to LRP accumulation partners represents the CA effort.

\subsubsection{Modeling Assumptions}

In the modeling of this problem, assumptions (1) to (13) are still valid, but assumption (14) is relaxed and the following additional assumptions are considered:

Assumption (15): The unit cost of offering bonus points to LRP accumulation partners is a constant.

Assumption (16): The LRP members' accumulation demand is not known with certainty and consists of two parts: the initial random demand and the demand induced by offering bonus points.

Assumption (17): There are no carry-over effects of the bonus points offered (as advertising inputs) in past periods.

Assumption (18): The impact of the CA effort (i.e., bonus points offering) on LRP members' accumulation demand towards each LRP accumulation partner is non-negative and deterministic.

Assumption (19): There are no cross-effects of bonus points offering among LRP accumulation partners. 
Assumption (15) indicates that there are no differences in the costs of offering bonus points to LRP accumulation partners, as the costs associated with issuing points and their transfer are very low (negligible).

Assumption (16) indicates that each accumulation demand consists of two parts. The first part is the initial random demand represented by a probability distribution. This initial unknown demand indicates the accumulation demand obtained at zero level of CA effort (i.e., bonus points are not offered at all). The second part is represented by a saturation-based response function of CA efforts allocated to each accumulation partner. The second part measures the effect of CA (i.e., bonus points) on the initial random demand.

Assumptions (17) to (19) relate to the advertising-response function. They indicate that the analysis of bonus points, similar to Holthansen (1982) and Mantrala et al. (1992), is confined to a single-period decision model as "the effects of advertising input usually do not extend beyond a few months" (Doyle and Saunders 1990). Therefore, we assume that the carry-over effects of advertising inputs (i.e., bonus points) in the past period do not exist. Furthermore, the effect of bonus points on the initial random demand is always nonnegative and there is no randomness in that effect. In other words, demand uncertainties come only from the initial demands. Assumption (19) guarantees that the sales-response function we have proposed is applicable.

\subsubsection{Problem Description and Model Formulation}

The following additional notations are used in our modeling:

(1) Decision variables: 
$b_{l}^{A} \quad$ The amount of bonus points offered to LRP accumulation partner $A_{\imath}$.

(2) Parameters:

$k_{i}^{A} \quad$ Elasticity of LRP host $H$ 's CA effort (i.e., bonus points offering) on accumulation demand towards LRP accumulation partners $A_{\imath}$ 's products.

$c_{\imath}^{A} \quad$ Overhead cost per unit of points for offering bonus points to each accumulation partner $A_{l}$.

$\phi_{t}^{A} \quad$ The maximum proportion of accumulation demand $D_{\imath}^{A}$ that can be reached by CA effort, $0 \leq \phi_{i}^{A} \leq 1$.

$Y_{i}^{A} \quad$ LRP members' overall accumulation demand towards LRP accumulation partner $A_{l}$.

$d_{i}^{A} \quad$ Additional accumulation demand due to the offering of bonus points.

In the setting of an LRP system with bonus points, $Y_{l}^{A}$ is taken to be a function of the initial demand $D_{l}^{A}$, as well as an additional demand $d_{l}^{A}$, due to the offering of bonus points. $Y_{t}^{A}$ is formulated as follows (EP1-Y):

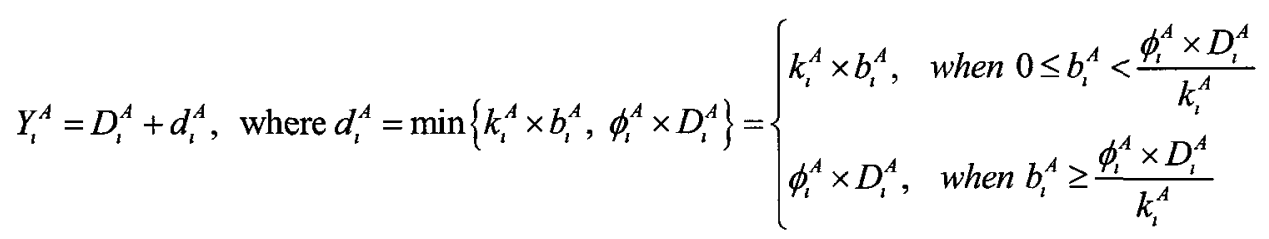

In (EP1-Y), LRP host $H$ 's CA effort is represented by the number of bonus points $\left(b_{\imath}^{A}\right)$ offered to LRP accumulation partners. Therefore, the effect of the CA effort can be captured by a piecewise linear function with a threshold representing the market potential or saturation level of demand 
influenced by bonus points (see Figure 3.3). This threshold is defined as a percentage of initial accumulation demand: $\phi_{i}^{A} \times D_{i}^{A}$.

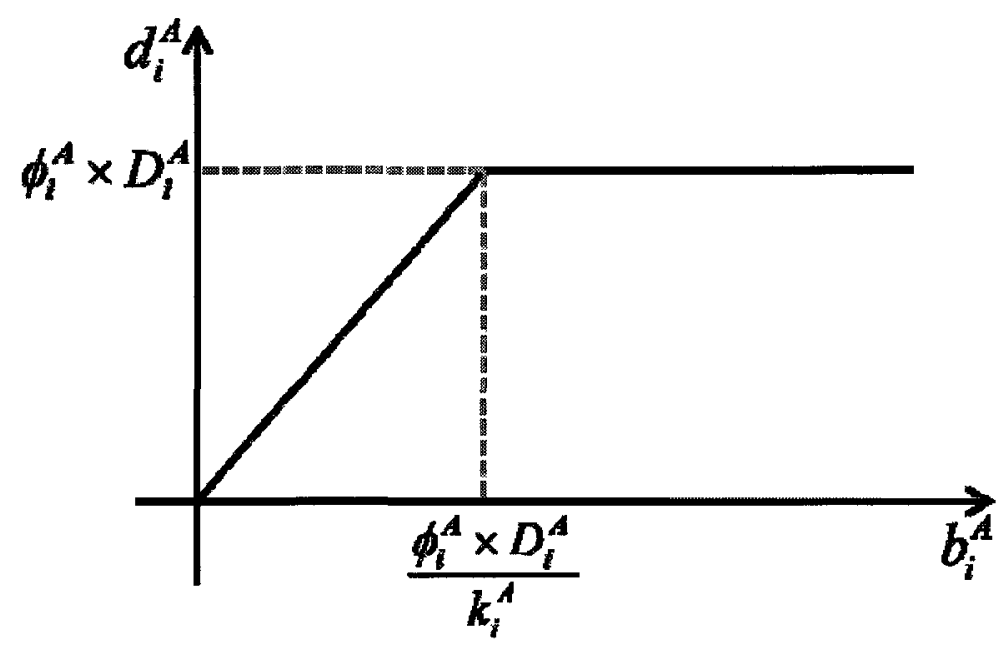

Figure 3.3: Effect of bonus points on accumulation demands

As discussed in the literature (e.g., Mantrala et al., 1992), the type of sales-response functions shown in Figure 3.3 is applicable only when there are no cross-effects of CA budget allocations to individual submarkets and competitive efforts among submarkets can be ignored (hence, Assumption (19) is stated as above). Meanwhile, the competition between LRP accumulation partners is small enough to ignore it in an LRP system because, generally speaking, accumulation options are rarely overlapping or substitutable. As such, we can adopt this type of sales response functions as a part of the LRP member demand function.

Hence, the rewards-supply planning problem we are concerned with here is determining, given the ordering quantities of points from LRP accumulation partners $A_{i}$ (i.e., $q_{i}^{A}, i=1,2, \ldots, I$ ), the 
LRP host's optimal ordering quantities of rewards from LRP redemption partners $R_{\jmath}$ (i.e., $q_{j}^{R}$, $j=1,2, \ldots, J)$ as well as the bonus points offering among LRP accumulation partners $A_{l}$ (i.e., $b_{l}^{A}$, $i=1,2, \ldots, I$ ) in order to maximize the LRP profitability (as measured by its value creation), subject to LRP redemption partners $R_{j}$ 's capacity constraints on offering rewards, the LRP host's overall redemption budget for rewards, and the LRP host's liability control requirement.

The LRP host $H$ 's value creation function considering bonus points offering at the accumulation side can be formulated as follows (EP1-A):

$$
\pi_{H(A)}=\sum_{\imath=1}^{I}\left(w_{\imath}^{A} \times q_{\imath}^{A}+w_{\imath}{ }^{A} \times\left[Y_{\imath}^{A}-q_{\imath}^{A}\right]_{+}-c_{\imath}^{A} \times b_{\imath}^{A}\right)
$$

The meanings of the first and the second terms in the function are the same as in (BP-A), except that the accumulation demand is $Y_{l}^{A}$ and not $D_{l}^{A}$. These terms state that the LPR host revenue from points-business at the accumulation side is obtained through the selling of points, and the LRP accumulation partners' extra ordering of points when $Y_{t}^{A}$ is higher than LRP accumulation partner $A_{l}$ 's ordering quantity of points $q_{l}^{A}$. The third term, $c_{l}^{A} \times b_{l}^{A}$, refers to the costs (e.g. overhead or administration cost) incurred when LRP host $H$ offers bonus points to LRP accumulation partner $A_{l}$.

At the redemption side, the LRP host $H$ 's value creation function remains the same as in the previous model, but it is now restated as (EP1-R): 


$$
\pi_{H(R)}=\sum_{j=1}^{J}\left(p_{J}^{R} \times \min \left\{q_{\jmath}^{R}, D_{\jmath}^{R}\right\}-w_{J}^{R} \times q_{\jmath}^{R}-v_{J}^{R} \times\left[D_{J}^{R}-q_{\jmath}^{R}\right]_{+}\right)+\sum_{\jmath=1}^{J}\left(s_{\jmath}^{R} \times\left[q_{J}^{R}-D_{J}^{R}\right]_{+}\right)
$$

Combining (EP1-A) and (EP1-R), the LRP rewards-supply planning problem with bonus points offerings can be formulated as follow s (hereafter Problem EP1):

$$
\begin{aligned}
& \Pi_{H}\left(q_{J}^{R}, b_{\imath}^{A} ; D_{J}^{R}, D_{\imath}^{A}\right)=\max E\left[\pi_{H(A)}+\pi_{H(R)}\right] \\
& =\max \left\{\begin{array}{l}
\sum_{i=1}^{I}\left(w_{l}^{A} \times q_{\imath}^{A}-c_{\imath}^{A} \times b_{\imath}^{A}\right)+E\left[\sum_{l=1}^{I}\left(w_{l}^{A} \times\left[Y_{l}^{A}-q_{\imath}^{A}\right]_{+}\right)\right]-\sum_{J=1}^{J} w_{J}^{R} \times q_{J}^{R}+ \\
E\left[\sum_{j=1}^{J}\left(p_{J}^{R} \times \min \left\{q_{J}^{R}, D_{J}^{R}\right\}-v_{J}^{R} \times\left[D_{J}^{R}-q_{J}^{R}\right]_{+}+s_{J}^{R} \times\left[q_{J}^{R}-D_{J}^{R}\right]_{+}\right)\right]
\end{array}\right\}
\end{aligned}
$$

subject to:

$$
\begin{aligned}
& q_{J}^{R} \leq Q_{J}^{R}, \text { for } j=1, \ldots, J \\
& \sum_{j=1}^{J}\left(w_{J}^{R} \times q_{J}^{R}\right) \leq W^{R} \\
& \frac{l}{l_{0}} \leq L_{U B}, \text { where } l=l_{0}+\sum_{\imath=1}^{I}\left(q_{\imath}^{A}+\left[Y_{\imath}^{A}-q_{\imath}^{A}\right]_{+}+b_{\imath}^{A}\right)-\sum_{J=1}^{J}\left(q_{J}^{R}\right) \\
& Y_{\imath}^{A}=D_{\imath}^{A}+\min \left\{k_{\imath}^{A} \times b_{\imath}^{A}, \phi_{\imath}^{A} \times D_{\imath}^{A}\right\} \\
& q_{J}^{R} \geq 0, \text { for } j=1, \ldots, J \\
& b_{\imath}^{A} \geq 0, \text { for } i=1, \ldots, I
\end{aligned}
$$

In comparison to Problem $B P$ that deals solely with the ordering quantity decisions, this model also deals with the bonus points offering decisions. Constraint (3.15) in this model is a modification of the constraint (3.6) in Problem BP. In constraint (3.15), the target liability $(l)$ is still equal to the initial liability $\left(l_{0}\right)$ at the beginning of the planning horizon plus the overall amount of points earned by members during the planning horizon (i.e., overall accumulation) minus the overall "target amount" of points redeemed by members for rewards during the same 
time period. The overall accumulation in Problem EPI includes three components: the LRP accumulation partners' ordering quantities of points $\left(q_{l}^{A}\right)$, the extra ordering amount of points ( $\left.\left[Y_{i}^{A}-q_{i}^{A}\right]_{+}\right)$, and the bonus points offered by LRP host $\left(b_{i}^{A}\right)$. The "target" redemption in Constraint (3.15) is the same as that in Constraint (3.6), which is $\sum_{j=1}^{J} q_{j}^{R}$. Constraint (3.16) defines the accumulation demand that includes the initial random demand and the extra demand driven by bonus points. Constraints (3.4), (3.5), and (3.7) in Problem BP are respectively relabeled as constraints (3.13), (3.14), and (3.17) in this model. Constraint (3.18) is the non-negativity constraint for the decision variable $b_{t}^{A}$.

\subsubsection{LRP Rewards-Supply Planning Problem with Option Contracts}

In Problem BP and Problem EPI, we assume that the relationships between LRP redemption partners and the LRP host are governed solely by wholesale-price-only contracts. However, as pointed out in the SC/OM literature (see Chapter 2), this type of contracts cannot offer enough flexibility to cope with demand uncertainties, whereas option contracts have been viewed in the literature as an effective tool that can bring more flexibility into coordination among SC entities. Therefore, as an extension, we introduce in this section the modeling of the LRP rewards-supply planning problem to the case where option contract is used in rewards-supply contracting.

\subsubsection{Modeling Assumptions}

In our modeling, Assumptions (1) to (12) and (14) are still valid, but Assumption (13) is relaxed and the following additional assumption is considered: 
Assumption (20): Option contracts are used as the governing mechanism in rewardssupply contracting.

Assumption (20) indicates that instead of wholesale-price contracts, option contracts are used as the governing mechanism in the business relationships between an LRP host and LRP redemption partners.

\subsubsection{Problem Description and Model Formulation}

The following are the additional notations used in this extension:

(1) Decision variables:

$q_{j}^{R_{0}} \quad$ LRP host $H$ 's initial ordering quantity of rewards from redemption partner $R_{J}$ (transformed into the ordering quantity of points).

$m_{1}^{R_{1}} \quad$ Number of units of options that LRP host $H$ purchases from redemption partner $R_{j}$.

$q_{j}^{R_{1}} \quad$ Number of units of options that LRP host $H$ exercises from redemption partner

(2) Parameters:

$w_{J}^{R_{0}} \quad$ Per unit price that LRP redemption partner $R_{J}$ charges LRP host $H$ to purchase rewards (in points).

$w_{J}^{R_{1}} \quad$ Per unit price that LRP redemption partner $R_{J}$ charges LRP host $H$ to purchase options (in points).

$e_{J}^{R_{1}} \quad$ Per unit price that LRP redemption partner $R$, charges LRP host $H$ to exercise options (in points).

The rewards-supply planning problem with option contracts consists of determining, given the ordering quantities of points from LRP accumulation partners $A_{i}$ (i.e., $q_{t}^{A}, i=1,2, \ldots, I$ ), the LRP 
host $H$ 's optimal ordering quantities of rewards from LRP redemption partners $R_{j}$ (i.e., $q_{J}^{R_{0}}$, $j=1,2, \ldots, J)$, and determining the optimal numbers of options to purchase (i.e., $m_{J}^{R_{0}}, j=1,2, \ldots, J$ ) and to exercise (i.e., $e_{j}^{R_{1}}, j=1,2, \ldots, J$ ) in order to maximize the LRP profitability (as measured by its value creation), subject to LRP redemption partners' capacity constraints on offering rewards, the LRP host's overall redemption budget for rewards, and the LRP host's liability control requirement.

In a typical option contract, the buyer (i.e., the LRP host in the context of this study), is allowed to adjust the ordering quantity after observing the actual demand, whereas the wholesale-price contract doesn't provide this kind of flexibility. More specifically, the LRP host $H$ has to make three types of decisions with regards to rewards ordering quantities. At the initial ordering time (e.g., before sales begin), the LRP host $H$ orders $q_{j}^{R_{0}}$ units of rewards from each partner $R_{J}$ at a unit wholesale-price of $w_{J}^{R_{0}}$. This ordering quantity usually cannot be changed at a later time. It is known as a "firm order" or "minimum purchase commitment". However, the LRP host $H$ is allowed to purchase $m_{J}^{R_{1}}$ units of options up to a certain amount, $M_{J}^{R_{1}}$ (i.e., $0 \leq m_{J}^{R_{1}} \leq M_{J}^{R_{1}}$ ), at a unit option price of $w_{J}^{R_{1}}$. At a later ordering time (e.g., a time closer to the beginning of sales or in the middle of the sales period), the LRP host $H$ may choose to exercise all the units of options or part of them, $q_{J}^{R_{1}}$, at a per unit exercise price of $e_{J}^{R_{1}}$, where $q_{J}^{R_{1}} \leq m_{J}^{R_{1}}$. Therefore, under option contracts, the LRP host $H$ does not get the ordering flexibility for free; instead, the LRP host $H$ has to pay extra money to obtain that flexibility. 
Hence, under the option contracts setting, LRP host $H$ 's value creation function and rewardssupply decisions at the redemption side are modified as (EP2-R):

$$
\pi_{H(R)}=\sum_{j=1}^{J}\left(\begin{array}{l}
p_{J}^{R} \times \min \left\{q_{J}^{R_{0}}+q_{J}^{R_{1}}, D_{J}^{R}\right\}-w_{J}^{R_{0}} \times q_{J}^{R_{0}}-w_{J}^{R_{1}} \times m_{J}^{R_{1}}-e_{J}^{R_{1}} \times q_{J}^{R_{1}} \\
-v_{J}^{R} \times\left[D_{J}^{R}-\left(q_{J}^{R_{0}}+q_{J}^{R_{1}}\right)\right]_{+}+s_{J}^{R} \times\left[q_{J}^{R_{0}}-D_{J}^{R}\right]_{+}
\end{array}\right)
$$

The first term in (EP2-R) indicates the value of rewards offered by each redemption partner $R_{J}$. The second term indicates the LRP host's purchasing cost of rewards. The third and fourth terms indicate the LRP host's costs for purchasing and exercising options. The fifth term refers to the under-stocking cost of rewards. Finally, the sixth term refers to the salvage value of overstocking rewards.

At the accumulation side, the LRP host $H^{\prime}$ 's value creation function remains the same as before, but restated as (EP2-A):

$$
\pi_{H(A)}=\sum_{i=1}^{I}\left(w_{\imath}^{A} \times q_{\imath}^{A}+w_{\imath}^{\prime A} \times\left[D_{\imath}^{A}-q_{t}^{A}\right]_{+}\right)
$$

Combining (EP2-A) and (EP2-R), the rewards-supply planning problem with option contracts is modeled as follows (hereafter Problem EP2):

$$
\begin{aligned}
& \Pi_{H}\left(q_{J}^{R_{0}}, m_{J}^{R_{1}}, q_{J}^{R_{1}} ; D_{J}^{R}, D_{l}^{A}\right)=\max E\left[\pi_{H(A)}+\pi_{H(R)}\right] \\
& =\max \left\{\begin{array}{l}
\sum_{i=1}^{I}\left(w_{l}^{A} \times q_{t}^{A}\right)-\sum_{j=1}^{J}\left[w_{J}^{R_{0}} \times q_{J}^{R_{0}}+w_{J}^{R_{1}} \times m_{J}^{R_{1}}+e_{J}^{R_{1}} \times q_{J}^{R_{1}}\right]+E\left[\sum_{i=1}^{I} w_{l}^{A} \times\left[D_{l}^{A}-q_{i}^{A}\right]_{+}\right]_{+} \\
\left.E\left[\sum_{j=1}^{J} p_{J}^{R} \times \min \left\{q_{J}^{R_{0}}+q_{J}^{R_{1}}, D_{J}^{R}\right\}-\sum_{j=1}^{J} v_{J}^{R} \times\left[D_{J}^{R}-\left(q_{J}^{R_{0}}+q_{J}^{R_{1}}\right)\right]_{+}+\sum_{j=1}^{J} s_{J}^{R} \times\left[q_{J}^{R_{0}}-D_{J}^{R}\right]_{+}\right]\right\}
\end{array}\right.
\end{aligned}
$$

subject to: 


$$
\begin{aligned}
& q_{J}^{R_{0}} \leq Q_{J}^{R_{0}}, \text { for } j=1, \ldots, J \\
& \sum_{J=1}^{J}\left(w_{J}^{R_{0}} \times q_{J}^{R_{0}}+w_{J}^{R_{1}} \times m_{J}^{R_{1}}+e_{J}^{R_{1}} \times q_{J}^{R_{1}}\right) \leq W^{R} \\
& m_{J}^{R_{1}} \leq M_{J}^{R_{1}}, \text { for } j=1, \ldots, J \\
& \frac{l}{l_{0}} \leq L_{U B}, \text { where } l=l_{0}+\sum_{l=1}^{I}\left(q_{\imath}^{A}+\left[D_{l}^{A}-q_{l}^{A}\right]_{+}\right)-\sum_{J=1}^{J}\left(q_{J}^{R_{0}}+q_{J}^{R_{1}}\right) \\
& q_{J}^{R_{1}} \leq m_{J}^{R_{1}}, \text { for } j=1, \ldots, J \\
& q_{J}^{R_{1}} \leq \max \left\{0, D_{J}^{R}-q_{J}^{R_{0}}\right\}, \text { for } j=1, \ldots, J \\
& q_{J}^{R_{0}}, m_{J}^{R_{1}} \geq 0, \text { for } j=1, \ldots, J \\
& q_{J}^{R_{1}} \geq 0, \text { for } j=1, \ldots, J
\end{aligned}
$$

In Problem EP2, Constraint (3.22) indicates that each redemption partner $R_{\jmath}$ has a capacity limitation on the quantity of rewards offered to LRP host $H$. Constraint (3.23) indicates that the LRP host has an overall budget limit for purchasing rewards and options that cannot be exceeded. Constraint (3.24) indicates that each redemption partner has a limitation on the overall quantity of options offered to the LRP host. Constraint (3.25) is the liability constraint. The target redemption in this constraint is different from that in Constraint (3.4) in Problem BP and in Constraint (3.15) in Problem EP1. In Constraint (3.25), the target redemption is the sum of the initial ordering quantity of rewards and the number of options to exercise. Constraint (3.26) represents a logical assumption associated with the option contracts: the number of options $\left(q_{j}^{R_{1}}\right)$ that the LRP host $H$ can exercise should be no more than the number of options that the LRP host $H$ purchased $\left(m_{J}^{R_{1}}\right)$. Constraint (3.27) represents another logical assumption associated with option contracts, which states that, after observing the "true" redemption demand, the options exercised will not exceed the difference between the redemption demand and the initial ordering 
quantity of rewards. In other words, if the "true" redemption demand is less than the initial ordering quantity, then no options will be exercised. Constraints (3.28) and (3.29) refer to the non-negativity constraints.

\subsection{Summary}

In this chapter, we have proposed three mathematical programming models to examine the LRP rewards-supply planning problem under different settings. More specifically, the first model (Problem BP) was developed to cope with rewards-supply planning decisions in the presence of multiple commercial partners offering various redemption and accumulation opportunities to LRP members, and multiple management concerns (e.g., LRP host profitability, liability control, demand uncertainties, budget, and capacity limitations). The second model (Problem EPI) is a generalization of the first model in the sense that it incorporates bonus-point decisions. The third model (Problem EP2) is also a generalization of the first model to consider option contracts in rewards-supply contracting.

Considering the demand uncertainties involved in these models as well as the underlying linearity assumptions, our models lend themselves to stochastic linear programming models. We discuss our solution methodology in the following chapter. 


\section{Chapter 4 Solution Methodology}

In this chapter, we will show how our mathematical models can be solved through the stochastic programming (SP) approach. We begin with a brief introduction of key characteristics of SP and its implementation, and then propose a sampling-based heuristic solution procedure in the context of this study. We also present the reformulations of the rewards-supply planning models (i.e., Problem BP, Problem EP1, and Problem EP2) according to the solution procedure and discuss some computational implementation issues related to our solution procedure.

\subsection{Stochastic Programming and Its Implementation}

$\mathrm{SP}$ is a well-known optimization approach used to incorporate uncertainty in the decision process. The study of SP dates back to the 1950s (e.g., Beale, 1955; Dantzig, 1955). SPs have been applied to a wide range of research problems involving uncertainties. Examples of these research problems include production planning with a random yield (Zanjani et al., 2007), supply chain network design under demand, capacity, transportation cost and supply uncertainties (Santoso et al., 2005), assignment problems with resource-constrained uncertainties (Toktas et al., 2006), resource portfolio planning under demand uncertainty (Wang et al., 2008), and quantitative analysis of multi-periodic supply chain contracts with options (Delft and Vial, 2004).

Most of the models proposed in these studies focus on minimizing (or maximizing) the expectation of the resultant distributions of random components. In this research, we follow the same logic to deal with the randomness involved in our models. As pointed out by many researchers (e.g., Birge and Louveaux, 1997; Ruszczyński and Shapiro, 2003), the advantage of 
using SP to deal with randomness is that the optimal solution obtained is more robust with respect to variations of random elements than the optimal solution of the corresponding deterministic problem in which random elements are replaced by their mean values.

The simplest form of SP results when some of the parameters in a linear program are represented by random variables (either discrete or continuous). Our models are quite similar to that simple form, which is known as stochastic linear programming (SLP) in the literature.

A general format of SLP is defined as follows (hereafter Problem-SLP):

$$
\begin{array}{ll}
\max \pi_{H} & =c^{T} \times z \\
\text { s.t.: } & \\
& A \times z=b \\
& T(\omega) \times z \leq h(\omega) \\
& z \geq 0
\end{array}
$$

where $c^{T}$ is the transpose of the coefficient vector in the objective function (4.1). $z$ is the vector of the decision variables. Constraints (4.2) and (4.3) represent deterministic and stochastic constraints in Problem-SLP, respectively. $A$ and $b$ are the coefficient matrix and the right hand side vector of the deterministic constraints (4.2). $\omega$ is the vector of random parameters and $T(\omega)$ and $h(\omega)$ are the coefficient matrix and right-hand side vector of the stochastic constraints (4.3) involving random parameters. Due to the randomness in the problem data, the value of the objective function in Problem-SLP is no longer deterministic, which brings more challenges in solving it. Various studies have been conducted on SLP formulations, solution techniques, and theoretical solution properties. For an extensive discussion of these topics, 
readers are referred to Kall et al. (1994, 2005), Birge and Louveaux (1997), Sen and Higle (1999), and Ruszczyński and Shapiro (2003).

In our study, due to the special structure, our models lend themselves to a specific type of SLP models, known as two-stage SLP with recourse (2SLPR). The key characteristic of this type of SLP models is that decision variables are classified into two stages according to whether they are implemented before or after an outcome of a (vector valued) random variable is observed. In other words, a set of decisions $(z)$ is taken in the first stage without full information on the random vector. Later, full information is received on the possible realization of the random vector; then a second stage action (also called recourse action) is taken. The second stage decisions $(y)$ allow us to model a response to each of the observed outcomes of the random vector, which constitute the recourse. A 2SLPR format of Problem-SLP is structured as follows (hereafter Problem-2SLPR):

$$
\begin{array}{cl}
\max \pi_{H}=c^{T} \times z+\Phi(z) \\
\text { s.t.: } \\
\qquad \begin{array}{c}
A \times z=b \\
z \geq 0
\end{array}
\end{array}
$$

where $\Phi(z)=E_{\xi}[g(z, \omega)]$ is the expected value of the linear recourse function, and $g(z, \omega)$ is defined by the second stage problem or, in other terminology, by the recourse problem as follows:

$$
\max g(z, \omega)=q(\omega)^{T} \times y(\omega)
$$

s.t.: 


$$
\begin{aligned}
& W(\omega) \times y(\omega)=h(\omega)-T(\omega) \times z \\
& y(\omega) \geq 0
\end{aligned}
$$

where $\xi(\omega)=\{W(\omega), T(\omega), h(\omega), q(\omega)\}$ is the vector of stochastic components in the second stage. Problem-2SLPR is suitable for decision models with a single-period randomness and reaction (e.g., newsvendor-based models). Our models (i.e., Problem BP, Problem EPl, and Problem EP2) satisfy this characteristic. Therefore, they can be rewritten into the 2SLPR formats as shown below in sections 4.1.1, 4.1.2 and 4.1.3, respectively.

\subsubsection{Model Reformulation for Problem BP}

The model considering the rewards-supply planning problem without bonus points (i.e., Problem $B P$ ) can be reformulated into a 2SLPR format as follows (hereafter Problem BP-2SLPR):

$\operatorname{Max} \Pi_{H}=\sum_{i=1}^{I}\left(w_{\imath}^{A} \times q_{i}^{A}\right)+\sum_{j=1}^{J}\left(p_{J}^{R} \times q_{\jmath}^{R}-w_{\jmath}^{R} \times q_{\jmath}^{R}\right)+E_{\xi}\left[g\left(q_{\jmath}^{R}, \omega\right)\right]$

subject to:

$$
\begin{aligned}
& q_{J}^{R} \leq Q_{J}^{R}, \text { for } j=1, \ldots, J \\
& \sum_{J=1}^{J}\left(w_{J}^{R} \times q_{J}^{R}\right) \leq W^{R} \\
& q_{J}^{R} \geq 0, \text { for } j=1, \ldots, J
\end{aligned}
$$

where $g\left(q_{J}^{R}, \omega\right)$ corresponds to solving the following model:

$$
\begin{aligned}
& g\left(q_{J}^{R}, \omega\right)=g\left(q_{J}^{R}, D_{\imath}^{A}, D_{J}^{R}\right)= \\
& \max \sum_{i=1}^{I}\left(w_{l}^{\prime A} \times I_{\imath}^{A-}\right)+\sum_{j=1}^{J}\left(-v_{J}^{R} \times I_{J}^{R-}+s_{J}^{R} \times I_{J}^{R+}-p_{J}^{R} \times I_{J}^{R+}\right)
\end{aligned}
$$


subject to:

$$
\begin{aligned}
& \frac{l}{l_{0}} \leq L_{U B}, \text { where } l=l_{0}+\sum_{i=1}^{I}\left(q_{\imath}^{A}+I_{\imath}^{A-}\right)-\sum_{J=1}^{J} q_{J}^{R} \\
& I_{l}^{A+}-I_{\imath}^{A-}=q_{\imath}^{A}-D_{\imath}^{A}, \text { for } i=1, \ldots, I \\
& I_{J}^{R+}-I_{J}^{R-}=q_{J}^{R}-D_{J}^{R}, \text { for } j=1, \ldots, J \\
& I_{l}^{A+}, I_{\imath}^{A-}, I_{J}^{R+}, I_{J}^{R-} \geq 0, \text { for } i=1, \ldots, I, j=1, \ldots, J
\end{aligned}
$$

In this reformulation, $\omega=\left\{D_{t}^{A}, D_{J}^{R}\right\}$ is the vector of random parameters. The vector of first-stage decision variables is $x=\left\{q_{J}^{R}\right\}$ and the vector of second-stage decision variables is $y(\omega)=\left\{I_{\imath}^{A+}, I_{\imath}^{A-}, I_{\jmath}^{R+}, I_{J}^{R-}\right\}$, where $I_{\imath}^{A+}, I_{\imath}^{A-}, I_{J}^{R+}, I_{J}^{R-}$ are new decision variables defined in the second stage. $I_{\imath}^{A+}$ and $I_{J}^{R+}$ denote the quantities: $\left[q_{l}^{A}-D_{l}^{A}\right]_{+}$and $\left[q_{J}^{R}-D_{J}^{R}\right]_{+}$respectively, whereas, $I_{l}^{A-}$ and $I_{J}^{R_{-}}$denote the quantities: $\left[D_{t}^{A}-q_{1}^{A}\right]_{+}$and $\left[D_{J}^{R}-q_{J}^{R}\right]_{+}$respectively. The values of these second-stage decision variables depend on the variation of demand. The term $\min \left\{q_{J}^{R}, D_{J}^{R}\right\}$ in Problem BP is replaced by $q_{J}^{R}-I_{J}^{R+}$ in Problem BP-2SLPR, for simplicity. Constraint (4.16) is the liability constraint, which has the same formulation as Constraint (3.6) in Problem BP except that $\left[D_{i}^{A}-q_{l}^{A}\right]_{+}$is replaced by $I_{\imath}^{A-}$ in Problem BP-2SLPR. Constraint (4.17) is constructed to define the values of $I_{\imath}^{A-}, I_{\imath}^{A+}$, so that when $D_{\imath}^{A} \geq q_{\imath}^{A}, I_{\imath}^{A-}=\left[D_{\imath}^{A}-q_{l}^{A}\right]_{+}$and $I_{\imath}^{A+}=0$, and when $D_{\imath}^{A}<q_{\imath}^{A}, I_{\imath}^{A+}=\left[q_{\imath}^{A}-D_{\imath}^{A}\right]_{+}$and $I_{\imath}^{A-}=0$. Constraint (4.18) is constructed to define the values of $I_{J}^{R-}$ and $I_{J}^{R+}$, so that when $D_{J}^{R} \geq q_{J}^{R}, I_{J}^{R-}=\left[D_{\jmath}^{R}-q_{\jmath}^{R}\right]_{+}$and $I_{J}^{R+}=0$, and 
when $D_{J}^{R}<q_{J}^{R}, I_{J}^{R+}=\left[q_{J}^{R}-D_{J}^{R}\right]_{+}$and $I_{J}^{R-}=0$. Constraints (4.19) are the non-negativity constraints for the second stage decision variables.

\subsubsection{Model Reformulation for Problem EP1}

The model corresponding to the LRP rewards-supply planning problem with bonus points (i.e., Problem EPI) can be reformulated into 2SLPR format as follows (hereafter Problem EP12SLPR):

$\operatorname{Max} \Pi_{H}=\sum_{i=1}^{I}\left(w_{\imath}^{A} \times q_{\imath}^{A}-c_{i}^{A} \times b_{\imath}^{A}\right)+\sum_{j=1}^{J}\left(p_{J}^{R} \times q_{J}^{R}-w_{J}^{R} \times q_{\jmath}^{R}\right)+E_{\xi}\left[g\left(q_{J}^{R}, b_{\imath}^{A}, \omega\right)\right]$

subject to: constraints $(4.12)-(4.14)$, and

$$
b_{i}^{A} \geq 0, \text { for } i=1, \ldots, I
$$

where $g\left(q_{J}^{R}, b_{\imath}^{A}, \omega\right)$ corresponds to solving the following model:

$$
\begin{aligned}
& g\left(q_{J}^{R}, b_{l}^{A}, \omega\right)=g\left(q_{J}^{R}, b_{l}^{A}, D_{l}^{A}, D_{J}^{R}\right)= \\
& \max \sum_{i=1}^{I}\left(w_{l}^{\prime A} \times I_{\imath}^{A-}\right)+\sum_{J=1}^{J}\left(-v_{J}^{R} \times I_{J}^{R-}+s_{J}^{R} \times I_{J}^{R+}-p_{J}^{R} \times I_{J}^{R+}\right)
\end{aligned}
$$

subject to: constraints (4.18) - (4.19), and

$$
\begin{aligned}
& \frac{l}{l_{0}} \leq L_{U B}, \text { where } l=l_{0}+\sum_{\imath=1}^{I}\left(q_{\imath}^{A}+I_{\imath}^{A-}+b_{\imath}^{A}\right)-\sum_{j=1}^{J} q_{j}^{R} \\
& k_{\imath}^{A} \times b_{\imath}^{A} \leq \phi_{\imath}^{A} \times D_{\imath}^{A}, \text { for } i=1, \ldots, I \\
& I_{\imath}^{A+}-I_{\imath}^{A-}=q_{\imath}^{A}-\left(D_{\imath}^{A}+k_{\imath}^{A} \times b_{\imath}^{A}\right), \text { for } i=1, \ldots, I
\end{aligned}
$$


The first stage model in Problem EPI-2SLPR includes two sets of decision variables: (1) the ordering quantity of rewards $\left(q_{J}^{R}\right)$ and (2) the amount of bonus points $\left(b_{i}^{A}\right)$ offered to each accumulation partner. The impact of bonus points on demand $\left(k_{\imath}^{A} \times b_{\imath}^{A}\right)$ is integrated into the second stage model. Constraint (4.23) is the same as Constraint (3.15) in Problem EP1 except that $\left[Y_{t}^{A}-q_{t}^{A}\right]_{+}$is replaced by $I_{t}^{A-}$. Constraint (4.24) indicates that the maximum impact of bonus points on the random accumulation demand cannot exceed $\phi_{i}^{A} \times D_{\imath}^{A}$. Constraint (4.25) is used to define the values for $I_{t}^{A-}$ and $I_{l}^{A+}$ so that when $D_{t}^{A} \geq q_{t}^{A}, I_{1}^{A-}=\left[Y_{t}^{A}-q_{l}^{A}\right]_{+}$and $I_{t}^{A+}=0$, and when $D_{\imath}^{A}<q_{\imath}^{A}, I_{\imath}^{A+}=\left[q_{\imath}^{A}-Y_{\imath}^{A}\right]_{+}$and $I_{\imath}^{A-}=0$

\subsubsection{Model Reformulation for Problem EP2}

The model corresponding to the LRP rewards-supply planning problem with option contracts (i.e., Problem EP2) can be reformulated into 2SLPR format as follows (hereafter Problem EP22SLPR):

$\operatorname{Max} \Pi_{H}=\sum_{i=1}^{I}\left(w_{t}^{A} \times q_{i}^{A}\right)+\sum_{J=1}^{J}\left(p_{J}^{R}-w_{J}^{R_{0}}\right) \times q_{J}^{R_{0}}-\sum_{j=1}^{J} w_{J}^{R_{1}} \times m_{J}^{R_{1}}+E_{\xi}\left[g\left(q_{J}^{R_{0}}, m_{J}^{R_{1}}, \omega\right)\right]$

subject to:

$$
\begin{aligned}
& q_{J}^{R_{0}} \leq Q_{J}^{R_{0}}, \text { for } j=1, \ldots, J \\
& m_{J}^{R_{1}} \leq M_{J}^{R_{1}}, \text { for } j=1, \ldots, J \\
& q_{J}^{R_{0}}, m_{J}^{R_{1}} \geq 0, \text { for } j=1, \ldots, J
\end{aligned}
$$


where $g\left(q_{j}^{R_{0}}, m_{j}^{R_{1}}, \omega\right)$ corresponds to solving the following model:

$$
\begin{aligned}
& g\left(q_{J}^{R_{0}}, m_{J}^{R_{1}}, \omega\right)=g\left(q_{J}^{R_{0}}, m_{J}^{R_{1}}, D_{l}^{A}, D_{J}^{R}\right) \\
& =\operatorname{Max} \sum_{i=1}^{I}\left(w_{l}^{A} \times I_{\imath}^{A-}\right)+\sum_{J=1}^{J}\left(p_{J}^{R} \times q_{J}^{R_{1}}-e_{J}^{R_{1}} \times q_{J}^{R_{1}}\right)+\sum_{J=1}^{J}\left(-v_{J}^{R} \times I_{J}^{R-}+s_{J}^{R} \times I_{J}^{U+}-p_{J}^{R} \times I_{J}^{R+}\right)
\end{aligned}
$$

subject to: constraints (4.17) and (4.19), and

$$
\begin{aligned}
& \frac{l}{l_{0}} \leq L_{U B}, \text { where } l=l_{0}+\sum_{l=1}^{I}\left(q_{i}^{A}+I_{t}^{A-}\right)-\sum_{J=1}^{J}\left(q_{J}^{R_{0}}+q_{J}^{R_{1}}\right) \\
& \sum_{J=1}^{J}\left(w_{J}^{R_{0}} \times q_{J}^{R_{0}}+w_{J}^{R_{1}} \times m_{J}^{R_{1}}+e_{J}^{R_{1}} \times q_{J}^{R_{1}}\right) \leq W^{R} \\
& I_{J}^{R+}-I_{J}^{R-}=\left(q_{J}^{R_{0}}+q_{J}^{R_{1}}\right)-D_{J}^{R}, \text { for } j=1, \ldots, J \\
& I_{J}^{U+}-I_{J}^{U-}=q_{J}^{R_{0}}-D_{J}^{R}, \text { for } j=1, \ldots, J \\
& q_{J}^{R_{1}} \leq m_{J}^{R_{1}}, \text { for } j=1, \ldots, J \\
& q_{J}^{R_{1}} \leq I_{J}^{U-}, \text { for } j=1, \ldots J \\
& I_{J}^{U+}, I_{J}^{U-} \geq 0, \text { for } j=1, \ldots, J
\end{aligned}
$$

In Problem EP2-2SLPR the first-stage decision variables are $x=\left\{q_{J}^{R_{0}}, m_{j}^{R_{1}}\right\}$ and the second stage decision variables are $y(\omega)=\left\{q_{J}^{R_{1}}, I_{\imath}^{A+}, I_{\imath}^{A-}, I_{J}^{R+}, I_{j}^{R-}, I_{J}^{U+}, I_{J}^{U-}\right\}$. The first-stage model involves optimal decisions on initial ordering quantities of rewards as well as the purchase of options. The second stage model involves optimal decisions on balancing overstocking and under-stocking costs as well as on the number of units of options to be exercised based on initial ordering quantities, purchased options, and observed scenarios of demand realizations. Constraints (3.22) - (3.24), and (3.28) in Problem EP2 are relabeled as constraints (4.27) - (4.29) and (4.32) in this model. Constraint (4.31) is the same as constraint (3.25) except that $\left[D_{t}^{A}-q_{t}^{A}\right]_{+}$is replaced by 
$I_{\imath}^{A-}$. Similar to Constraint (4.18) in Problem BP-2SLPR, Constraint (4.33) is constructed to define the values of $I_{J}^{R-}$ and $I_{J}^{R+}$, so that when $D_{J}^{R} \geq\left(q_{J}^{R_{0}}+q_{J}^{R_{1}}\right), I_{J}^{R-}=\left[D_{J}^{R}-q_{J}^{R_{0}}-q_{J}^{R_{1}}\right]_{+}$, and when $D_{J}^{R}<\left(q_{J}^{R_{0}}+q_{J}^{R_{1}}\right), I_{J}^{R+}=\left[q_{J}^{R_{0}}+q_{J}^{R_{1}}-D_{J}^{R}\right]_{+}$. Constraint (4.34) is constructed to define the values of $I_{J}^{U-}$ and $I_{J}^{U+}$, so that when $D_{J}^{R} \geq q_{J}^{R_{0}}, I_{J}^{U-}=\left[D_{J}^{R}-q_{J}^{R_{0}}\right]_{+}$, and when $D_{J}^{R}<q_{J}^{R_{0}}, I_{J}^{U+}=\left[q_{J}^{R_{0}}-D_{J}^{R}\right]_{+}$. Constraint (4.36) is the reformulation of Constraint (3.27) in Problem EP2. Constraints (4.37) are non-negativity constraints for the second stage decision variables, $I_{j}^{U+}$ and $I_{j}^{U-}$.

\subsection{Solution Procedure}

In this section, we propose and explain the solution procedure used to solve the rewards-supply planning models. Generally speaking, solving an SP model is not an easy task due to the randomness involved in the model. Solution methodologies for solving SLP are divided into two main categories: (1) exact methods that include the L-shaped approach (e.g., Kall et al., 1994 and 2005; Birge and Louveaux, 1997) and the regularized decomposition approach (e.g., Ruszczyński and Swietanowski, 1997), and (2) approximate methods that are based on sampling techniques, such as the sampling average approximation (SAA) approach (e.g., Shapiro et al., 1998; Mak et al., 1999; Kleywegt et al., 2001) or the stochastic decomposition approach (e.g., Higle and Sen, 1996). The exact methods attempt to find exact solutions for an SLP optimization problem. These methods are only feasible for situations where there exists only a finite number of realizations of random parameters (i.e., modeled as discrete random variables). In contrast, approximate approaches attempt to find approximate solutions that are close to the optimal solution within a reasonable error range. One standard approximate approach is to apply the 
Monte Carlo sampling procedure to generate $n$ realizations of random parameters and to solve the approximated problem based on samples of those random parameters.

In our models, redemption and accumulation demands are assumed to be continuous random parameters, and consequently yield an infinite number of demand realizations. In addition to that, with the increase of the number of redemption and accumulation partners, the number of demand parameters increases as well. Each of those demand parameters follows different demand distributions (in terms of the values of the distribution parameters). As such, approximate approach is more suitable for solving our models. We choose the approximate approach that is based on the SAA scheme. This approach is also known as an "external" approximation approach in the literature (e.g., Ruszczyński and Shapiro, 2003; Mak et al., 1999). Compared to other approximation techniques, the external approach has the following advantages, as pointed out by Ruszczyński and Shapiro (2003):

- It is easier to implement because it separates sampling procedures and optimization techniques.

- It is more universal in the sense that any optimization algorithm that is developed for a considered class of SLPs can be applied to the constructed SAA problem in a straightforward way.

- A quite well-developed statistical inference of the SAA method is available that, in turn, gives a possibility of error estimation, validation analysis, and hence stopping rules. 
The structures of our models are complex, and random demands are assumed to be independent and follow different probability distributions. Therefore, it is more appropriate to apply an external approximation approach, such as SAA.

\subsubsection{Sample Average Approximation (SAA) Reformulation}

In the external approach with the SAA scheme, a random sample $\omega^{1}, \omega^{2}, \ldots, \omega^{N}$ of $N$ realizations (scenarios) of the random vector is generated outside of an optimization procedure and the expectation of the second-stage objective function is approximated by the sample average function: $\hat{\Phi}_{N}(z)=\frac{1}{N} \sum_{i=1}^{N} g\left(z, \omega^{t}\right)$. Based on this approach, Problem-2SLPR is approximated by the following SAA problem (hereafter Problem-2SLPR-SAA):

$$
\begin{array}{ll}
\max \hat{\pi}_{H}=c^{T} \times z+\frac{1}{N} \sum_{n=1}^{N} q\left(\omega^{n}\right) \times y\left(\omega^{n}\right) \\
\text { s.t.: } \quad \text { constraints }(4.6),(4.7) \text { and } \\
& W\left(\omega^{n}\right) \times y\left(\omega^{n}\right)=h\left(\omega^{n}\right)-T\left(\omega^{n}\right) \times z, \text { for } n=1, \ldots, N \\
& y\left(\omega^{n}\right) \geq 0, \text { for } n=1, \ldots, N
\end{array}
$$

For a particular realization of the random parameters, Problem-2SLPR-SAA is a deterministic problem that can be solved by an appropriate deterministic algorithm. However, what we obtained through Problem-2SLPR-SAA is an approximate solution rather than the actual solution.

Note that $E\left[\hat{\pi}_{H}\right]=\pi_{H}$. Previous studies have shown that under mild regularity conditions, a fairly good approximate solution to a true SLP problem can be obtained through SAA (Kleywegt et al., 2001; Ruszczyński and Shapiro, 2003). We now show how the SAA scheme applies to the models discussed in this study. 
The SAA model of Problem BP-2SLPR is a deterministic linear programming model that can be stated as follows (hereafter Problem BP-2SLPR-SAA):

$$
\begin{aligned}
\operatorname{Max} \hat{\pi}_{H}= & {\left[\sum_{\imath=1}^{I}\left(w_{\imath}^{A} \times q_{l}^{A}\right)+\frac{1}{N} \sum_{s=1}^{N} \sum_{\imath=1}^{I}\left(w_{\imath}{ }^{A} \times I_{\imath s}^{A-}\right)\right]+\sum_{j=1}^{J}\left(p_{J}^{R}-w_{J}^{R}\right) \times q_{\jmath}^{R} } \\
& +\frac{1}{N} \sum_{s=1}^{N} \sum_{\jmath=1}^{J}\left[-v_{J}^{R} \times I_{\jmath s}^{R-}+\left(s_{J}^{R}-p_{\jmath}^{R}\right) \times I_{\jmath s}^{R+}\right]
\end{aligned}
$$

subject to:

$$
\begin{aligned}
& q_{j}^{R} \leq Q_{\jmath}^{R}, \text { for } j=1, \ldots, J \\
& \sum_{j=1}^{J}\left(w_{J}^{R} \times q_{j}^{R}\right) \leq W^{R} \\
& q_{j}^{R} \geq 0, \text { for } j=1, \ldots, J \\
& \sum_{J=1}^{J}\left(q_{j}^{R}\right) \geq \sum_{i=1}^{I}\left(q_{i}^{A}\right)+\sum_{i=1}^{I}\left(I_{\imath s}^{A-}\right)-\left(L_{U B}-1\right) \times l_{0}, \text { for } s=1, \ldots, N \\
& q_{j}^{R}+I_{\jmath s}^{R-}-I_{j s}^{R+}=D_{j s}^{R}, \text { for } j=1, \ldots, J, s=1, \ldots, N \\
& I_{s s}^{A-}=\max \left\{D_{i s}^{A}-q_{i}^{A}, 0\right\}, \text { for } i=1, \ldots, I, s=1, \ldots, N \\
& I_{\jmath s}^{R+}, I_{\jmath s}^{R-} \geq 0, \text { for } j=1, \ldots, J, s=1, \ldots, N
\end{aligned}
$$

In Problem BP-2SLPR-SAA, the second stage decision variables $I_{t}^{A+}, I_{t}^{A-}, I_{J}^{R+}$, and $I_{j}^{R-}$ are redefined as $I_{t s}^{A+}, I_{t s}^{A-}, I_{j s}^{R+}$, and $I_{j s}^{R-}$ for each sample of random demand realizations. The optimal values of these variables are the sample average of the optimal values of $I_{s s}^{A+}, I_{v s}^{A-}, I_{j s}^{R+}, I_{j s}^{R^{-}}$ obtained under each sample of random demand realizations. Constraints $(4.16)-(4.19)$ in 
Problem BP-2SLPR, which involve the second stage decision variables, are redefined in terms of each sample of random demand realizations as constraints $(4.45)-(4.48)$. Note that, given a sample of random accumulation demand, e.g., $D_{i s}^{A}$, the values of $I_{t s}^{A+}$ and $I_{v s}^{A-}$ are determined before solving the model as $q_{l}^{A}$ are now known parameters. In other words, if $D_{i s}^{A}$ is greater than $q_{i}^{A}$, then $I_{t s}^{A+}=0$ and $I_{t s}^{A-}=D_{t s}^{A}-q_{l}^{A} ;$ otherwise, $I_{t s}^{A+}=q_{l}^{A}-D_{t s}^{A}$ and $I_{t s}^{A-}=0$. Therefore, constraints (4.17) in Problem BP-2SLPR are modified as constraints (4.47) and $I_{\imath s}^{A+}$ and $I_{u s}^{A-}$ are not decision variables any more in Problem BP-2SLPR-SAA.

\subsubsection{SAA Model for Problem EP1-2SLPR}

The SAA model for Problem EP1-2SLPR is also a deterministic linear programming problem formulated as follows (hereafter Problem EPI-2SLPR-SAA):

$$
\begin{aligned}
\operatorname{Max} \hat{\pi}_{H}= & \sum_{\imath=1}^{I}\left(w_{\imath}^{A} \times q_{l}^{A}-c_{\imath}^{A} \times b_{\imath}^{A}\right)+\sum_{J=1}^{J}\left(p_{J}^{R} \times q_{J}^{R}-w_{J}^{R} \times q_{J}^{R}\right)+\frac{1}{N} \sum_{s=1}^{N} \sum_{l=1}^{I}\left(w_{l}^{\prime A} \times I_{t s}^{A-}\right)+ \\
& \frac{1}{N} \sum_{s=1}^{N} \sum_{J=1}^{J}\left(-v_{J}^{R} \times I_{\jmath s}^{R-}+s_{J}^{R} \times I_{\jmath s}^{R+}-p_{J}^{R} \times I_{j s}^{R+}\right)
\end{aligned}
$$

subject to:

$$
\begin{aligned}
& q_{J}^{R} \leq Q_{J}^{R}, \text { for } j=1, \ldots, J \\
& \sum_{j=1}^{J}\left(w_{J}^{R} \times q_{J}^{R}\right) \leq W^{R} \\
& q_{J}^{R} \geq 0, \text { for } j=1, \ldots, J \\
& b_{l}^{A} \geq 0, \text { for } i=1, \ldots, I
\end{aligned}
$$




$$
\begin{aligned}
& \sum_{j=1}^{J}\left(q_{\jmath}^{R}\right)-\sum_{i=1}^{I}\left(I_{\imath s}^{A-}+b_{\imath}^{A}\right) \geq \sum_{\imath=1}^{I}\left(q_{\imath}^{A}\right)-\left(L_{u B}-1\right) \times l_{0}, \text { for } s=1, \ldots, N \\
& k_{\imath}^{A} \times b_{\imath}^{A} \leq \phi_{\imath}^{A} \times D_{\imath s}^{A}, \text { for } i=1, \ldots, I, s=1, \ldots, N \\
& I_{t s}^{A-}-I_{t s}^{A+}-k_{\imath}^{A} \times b_{\imath}^{A}=D_{t s}^{A}-q_{\imath}^{A}, \text { for } i=1, \ldots, I, s=1, \ldots, N \\
& I_{\jmath s}^{R-}-I_{\jmath s}^{R+}+q_{\jmath}^{R}=D_{\jmath s}^{R}, \text { for } j=1, \ldots, J, s=1, \ldots, N \\
& I_{t s}^{A+}, I_{t s}^{A-}, I_{\jmath s}^{R+}, I_{\jmath s}^{R-} \geq 0, \text { for } i=1, \ldots, I, j=1, \ldots, J, s=1, \ldots, N
\end{aligned}
$$

In the same way as in Problem BP-2SLPR-SAA, the second stage decision variables $I_{\imath}^{A+}, I_{\imath}^{A-}, I_{J}^{R+}$, and $I_{J}^{R-}$ in Problem EPI-2SLPR-SAA are redefined as $I_{u s}^{A+}, I_{t s}^{A-}, I_{j s}^{R+}$, and $I_{j s}^{R-}$ for each sample of random demand realizations. The optimal values of these variables are the sample average of the optimal values of $I_{u s}^{A+}, I_{u s}^{A-}, I_{j s}^{R+}, I_{j s}^{R-}$ obtained under each sample of random demand realizations. Constraints (4.18) - (4.19) and (4.23) - (4.25) in Problem EP1-2SLPR are redefined as constraints $(4.54)-(4.58)$ for each sample of random demand realizations because these constraints involve second stage decision variables. The values of $I_{t s}^{A+}$ and $I_{t s}^{A-}$ depend on the random demands $D_{t s}^{A}$ as well as the bonus points $\left(b_{\imath}^{A}\right)$. Since $b_{\imath}^{A}$ is a decision variable in this model, $I_{v}^{A+}$ and $I_{t s}^{A-}$ are decision variables as well.

\subsubsection{SAA Model for Problem EP2-2SLPR}

The SAA problem of (EP2-2SLPR) is a deterministic linear programming problem formulated as follows (hereafter Problem EP2-2SLPR-SAA): 


$$
\begin{aligned}
\operatorname{Max} \hat{\pi}_{H}= & \sum_{i=1}^{I}\left(w_{l}^{A} \times q_{l}^{A}\right)+\sum_{j=1}^{J}\left(p_{J}^{R}-w_{J}^{R_{0}}\right) \times q_{J}^{R_{0}}-\sum_{j=1}^{J} w_{J}^{R_{1}} \times m_{J}^{R_{1}}+\frac{1}{N} \sum_{s=1}^{N} \sum_{l=1}^{I}\left(w_{l}^{\prime A} \times I_{\imath s}^{A-}\right) \\
& +\frac{1}{N} \sum_{s=1}^{N} \sum_{j=1}^{J}\left(p_{J}^{R}-e_{J}^{R_{1}}\right) \times q_{j s}^{R_{1}}+\frac{1}{N} \sum_{s=1}^{N} \sum_{J=1}^{J}\left(-v_{\jmath}^{R} \times I_{\jmath s}^{R-}+s_{J}^{R} \times I_{\jmath s}^{U+}-p_{J}^{R} \times I_{J s}^{R+}\right)
\end{aligned}
$$

subject to:

$$
\begin{aligned}
& q_{J}^{R_{0}} \leq Q_{J}^{R_{0}}, \text { for } j=1, \ldots, J \\
& m_{J}^{R_{1}} \leq M_{J}^{R_{1}}, \text { for } j=1, \ldots, J \\
& q_{j}^{R_{0}}, m_{J}^{R_{1}} \geq 0, \text { for } j=1, \ldots, J \\
& \sum_{J=1}^{J}\left(q_{J}^{R_{0}}+q_{j s}^{R_{1}}\right) \geq \sum_{i=1}^{I}\left(q_{i}^{A}\right)+\sum_{i=1}^{I}\left(I_{\imath s}^{A-}\right)-\left(L_{U B}-1\right) \times l_{0}, \text { for } s=1, \ldots, N \\
& \sum_{j=1}^{J}\left(w_{J}^{R_{0}} \times q_{J}^{R_{0}}+w_{J}^{R_{1}} \times m_{j}^{R_{1}}+e_{J}^{R_{1}} \times q_{j s}^{R_{i}}\right) \leq W^{R} \text {, for } s=1, \ldots, N \\
& q_{j s}^{R_{1}} \leq m_{j}^{R_{1}}, \text { for } j=1, \ldots J, s=1, \ldots, N \\
& q_{j s}^{R_{1}} \leq I_{\jmath s}^{U-}, \text { for } j=1, \ldots J, s=1, \ldots, N \\
& I_{u s}^{A-}=\max \left\{D_{t s}^{A}-q_{l}^{A}, 0\right\}, \text { for } i=1, \ldots, I, s=1, \ldots, N \\
& I_{j s}^{R+}-I_{j s}^{R-}=\left(q_{j}^{R_{0}}+q_{j s}^{R_{1}}\right)-D_{j s}^{R}, \text { for } j=1, \ldots, J, s=1, \ldots, N \\
& I_{\jmath s}^{U+}-I_{\jmath s}^{U-}=q_{\jmath}^{R_{0}}-D_{\jmath s}^{R}, \text { for } j=1, \ldots, J, s=1, \ldots, N \\
& I_{\jmath s}^{R+}, I_{\jmath s}^{R-}, I_{\jmath s}^{U+}, I_{\jmath s}^{U-} \geq 0, \text { for } j=1, \ldots, J, s=1, \ldots, N \\
& q_{j s}^{R_{1}} \geq 0, \text { for } j=1, \ldots, J, s=1, \ldots, N
\end{aligned}
$$

In Problem EP2-2SLPR-SAA, $q_{j s}^{R_{1}}$ is defined as the quantities of exercised options for each sample of random demand realizations. The optimal value of $q_{J}^{R_{1}}$ is estimated as the sample average of the optimal values of $q_{j s}^{R_{1}}$ obtained under each sample of random demand realizations. 
Meanwhile, constraints (4.17), (4.19), and (4.31) - (4.37) in Problem EP2-2SLPR are restructured for each sample of random demand realizations as constraints $(4.63)-(4.71)$.

The solving procedure for obtaining approximate solutions of our models as well as the evaluation procedure for the approximate solutions is presented next.

\subsubsection{SAA-based Heuristic Solution Procedure}

The basic idea of the solving procedure consists of generating an approximate solution, which is the solution of a number of instances, say $M$ of SAA problems, each with $N$ sampled scenarios. The quality of a candidate solution is then tested by bounding the optimality gap between the true objective value and the expected objective value through standard statistical procedures. A sampling evaluation procedure based on common random numbers (CRN) is used to construct the confidence interval for the optimality gap. CRN means that the same $N$ sampled scenarios are used to generate the optimal gap as well as to evaluate the objective function value. According to the work of Mak et al. (1999) and Ruszczyński and Shapiro (2003), CRN can provide a significant variance reduction over naïve sampling. Moreover, $\mathrm{CRN}$ can also eliminate the “negative gap" phenomenon (Mak et al., 1999; Feng et al., 2010). The complete solving procedure is described as follows (hereafter Algorithm 1):

Step 1: Generate i.i.d. batches of samples.

Generate $M$ independent identically distributed (i.i.d.) sample replications of random demand realizations, each of size $\quad N$, i.e., $\left[\omega_{1}^{m} \ldots \omega_{N}^{m}\right]^{T}, \quad m=1, . ., M$; 
$\omega_{n}^{m}=\left[\begin{array}{lllllll}D_{1,1}^{R m} & \ldots & D_{N, J}^{R m} & \ldots & D_{1,1}^{A m} & \ldots & D_{N, I}^{A m}\end{array}\right], n=1, \ldots, N$ where $D_{n, J}^{R m}$ denotes the $n$th sample of redemption demand of partner $R_{J}$ 's in the $m$ th sample replication, and $D_{n, l}^{A m}$ denotes the $n$th sample of accumulation demand of partner $A_{l}$ 's in the $m$ th sample replication.

Step 2: Solve the corresponding SAA problems.

Compute the statistical upper bound estimation of the optimal value of the objective function. Therefore, for each sample replication $m, m=1, \ldots, M$, solve the corresponding SAA problem (e.g., Problem BP-2SLPR-SAA). Let $\hat{\pi}_{N}^{m}$ and $\hat{x}_{N}^{m}$ be the corresponding optimal objective function value and the vector of optimal solution, respectively. It is well known that $\frac{1}{M} \sum_{m=1}^{M} \hat{\pi}_{N}^{m}$ is an unbiased estimator of $\hat{\pi}_{N}\left(\right.$ i.e., $\left.E\left(\hat{x}_{N}\right)=\frac{1}{M} \sum_{m=1}^{M} \hat{\pi}_{N}^{m}\right)$ and meanwhile, $\hat{\pi}_{N}$ is an upwards biased estimator of $\pi^{*}\left(\pi^{*}\right.$ denotes the optimal value of the true problem) in the case of maximization. As the corresponding SAA problems can be seen as relaxation problems (i.e., with fewer feasible constraints/cuts) of the true problem, we have $\hat{\pi}_{N} \geq \pi^{*}$. Hence, $\frac{1}{M} \sum_{m=1}^{M} \hat{\pi}_{N}^{m} \geq \pi^{*}$, which indicates that $\frac{1}{M} \sum_{m=1}^{M} \hat{\pi}_{N}^{m}$ provides a valid statistical upper bound estimation for the optimal value $\pi^{*}$ of the true problem (see Mak et al., 1999, for more details).

Step 3: Generate candidate solutions to compute the statistical lower bound estimation by completing the following two sub-steps: 
Step 3.1: Solve the same SAA problems (e.g., Problem BP-2SLPR-SAA) but use a sample with sample size $N$ ' larger than $N$. Let $\hat{x}_{N}$, be the corresponding optimal solution vector. Here, the sample of size $N^{\prime}$ is generated independently of the samples used to obtain $\hat{x}_{N}^{m}$.

Step 3.2: Estimate the true objective function value $\pi(\bar{x})$ with a candidate solution $\bar{x}=\hat{x}_{N^{\prime}}$ for all replications of samples with sample size $N$ as follows:

$$
\tilde{\pi}_{N}^{m}(\bar{x})=c^{T} \bar{x}+\frac{1}{N} \sum_{s=1}^{N} g\left(\bar{x}, \omega_{s}^{m}\right)=c^{T} \hat{x}_{N^{\prime}}+\frac{1}{N} \sum_{s=1}^{N} g\left(\hat{x}_{N^{\prime}}, \omega_{s}^{m}\right), \text { for } m=1, \ldots, M
$$

This step involves solving $M$ independent second-stage sub-problems $g\left(\hat{x}_{N^{\prime}}, \omega_{s}^{m}\right)$ given $\bar{x}=\hat{x}_{N^{\prime}}$. Let $\tilde{\pi}_{N}^{m}(\bar{x})$ be the corresponding objective function value. If $\bar{x}$ is a feasible solution of the problem shown in the formulation (4.72), then $\tilde{\pi}_{N}^{m}(\bar{x}) \leq \hat{\pi}_{N}^{m}$. In addition, it can be shown that given $\bar{x}, \tilde{\pi}_{N}^{m}(\bar{x})$ is an unbiased estimator of the true objective function value $\pi(\bar{x})$ and $E\left[\tilde{\pi}_{N}^{m}(\bar{x})\right]=\pi(\bar{x}) \leq \pi^{*}$, where $\pi^{*}$ is the true optimal objective function value (see Mak et al., 1999). Therefore, $\frac{1}{M} \sum_{m=1}^{M} \tilde{\pi}_{N}^{m}(\bar{x})$ provides a valid statistical lower bound estimation for the optimal value $\pi^{*}$ of the true problem.

Step 4: Compute the optimality gap for the candidate solution $\bar{x}$ as follows:

$$
G_{N}^{m}(\bar{x})=\hat{\pi}_{N}^{m}-\tilde{\pi}_{N}^{m}(\bar{x})=\hat{\pi}_{N}^{m}-\tilde{\pi}_{N}^{m}\left(\hat{x}_{N^{\prime}}\right), \text { for } m=1, \ldots, M
$$

where $\hat{\pi}_{N}^{m}$ are generated in Step 2 and $\tilde{\pi}_{N}^{m}\left(\hat{x}_{N}\right)$ are generated in Step 3. 
Step 5: Generate the point estimator and the confidence interval (CI) of the optimality gap.

The point estimator of the optimality gap of the candidate solution $\bar{x}=\hat{x}_{N^{\prime}}$ is obtained by calculating the sample mean as follows:

$$
\bar{G}_{N}^{M}(\bar{x})=\frac{1}{M} \sum_{m=1}^{M} G_{N}^{m}(\bar{x})=\frac{1}{M} \sum_{m=1}^{M} G_{N}^{m}\left(\hat{x}_{N^{*}}\right)=\frac{1}{M} \sum_{m=1}^{M} \hat{\pi}_{N}^{m}-\frac{1}{M} \sum_{m=1}^{M} \tilde{\pi}_{N}^{m}\left(\hat{x}_{N^{*}}\right)
$$

Since $\frac{1}{M} \sum_{m=1}^{M} \hat{\pi}_{N}^{m} \geq \frac{1}{M} \sum_{m=1}^{M} \tilde{\pi}_{N}^{m}(\bar{x})$, we have $\bar{G}_{N}^{M}(\bar{x}) \geq 0$. The sample variance of the mean optimality gap can be computed as follows:

$$
s_{G}^{2}(\bar{x})=\frac{1}{M-1} \sum_{m=1}^{M}\left(G_{N}^{m}(\bar{x})-\bar{G}_{N}^{M}(\bar{x})\right)^{2}=\frac{1}{M-1} \sum_{m=1}^{M}\left(G_{N}^{m}(\bar{x})-\bar{G}_{N}^{M}\left(\hat{x}_{N^{*}}\right)\right)^{2}
$$

Therefore, the approximate $100(1-\alpha) \%$ CI for the mean of the optimality gap can be obtained as follows:

$$
\left[0, \bar{G}_{N}^{M}(\bar{x})+\tilde{\varepsilon}_{G}\right], \text { where } \tilde{\varepsilon}_{G}=\frac{t_{M-1, \alpha} \times s_{G}(\bar{x})}{\sqrt{M}}
$$

The algorithm described in steps 1-5 assumes that the candidate solution $\bar{x}=\hat{x}_{N}$, will always be a feasible solution of problem (4.72). However, in Step 3.2, when solving $M$ independent secondstage sub-problems $g\left(\hat{x}_{N^{\prime}}, \omega_{s}^{m}\right)$ with given $\hat{x}_{N^{\prime}}$, we may face an issue of infeasibility. Due to the randomness involved in the models, the optimal solution $\hat{x}_{N^{\prime}}$ of, for example, Problem BP2SLPR-SAA with sample size $N^{\prime}$, may not be a feasible solution of Problem BP-2SLPR-SAA with sample size $N$. In order to deal with this infeasibility issue, the following heuristic procedure is proposed (hereafter Algorithm 2): 
(a) Given a candidate solution $\bar{x}=\hat{x}_{N^{\prime}}^{o}$, solve the second-stage sub-problems $g\left(\hat{x}_{N^{\prime}}, \omega_{s}^{m}\right)$ for $m$ $=1, \ldots, M$ as shown in relation (4.72).

(b) If a feasible solution is found, continue to steps (4) and (5).

(c) If a feasible solution is not found, then identify the constraint(s) that caused the infeasibility, and add them as feasible cuts into the SAA problem used to generate $\hat{x}_{N}^{o}$, in Step 3.1. Solve the SAA problem again as in Step 3.1, but with the extra feasible cuts to generate a new candidate solution. Obviously, if a new candidate solution, for example $\hat{x}_{N}^{1}$, can be found, it must be a feasible solution of all $M$ second-stage sub-problems $g\left(\hat{x}_{N}, \omega_{s}^{m}\right)$

\subsubsection{Implementation Issues of the Solution Procedure}

We found that there are three implementation challenges involved in the above SAA-based heuristic solution procedure.

Challenge 1: In Step 1 of Algorithm 1, an important decision is made about the sample size. According to Kleywegt et al. (2001), theoretically speaking, the sample size can be estimated as:

$$
N \geq \frac{3 \times \sigma_{\max }^{2}}{(\varepsilon-\delta)^{2}} \times \log \left(\frac{|X|}{\alpha}\right)
$$

where $\sigma_{\max }^{2}$ is the maximum variance of a certain function value (see Kleywegt et al., 2001, for more details), $X$ is the feasible set of the true problem, and $|X|$ denotes the number of elements in the set $X$. This estimation guarantees that the SAA solution $\hat{x}$ with an absolute optimal gap of $\delta$ ( 
$\delta \in[0, \varepsilon]$ ) to the SAA problem is a solution with an absolute optimal gap of $\varepsilon$ to the true problem with a probability of at least 1- $\alpha$. In other words, $\left|\hat{\pi}_{N}(\hat{x})-\hat{\pi}^{*}\right| \leq \delta$ $\Rightarrow p\left[\left(\pi(\hat{x})-\pi^{*}\right) \leq \varepsilon\right]=1-\alpha$. However, as the authors pointed out, there are two drawbacks to this estimate: (1) It may be too conservative for practical applications and (2) for many problems either $\sigma_{\max }^{2}$ or $|X|$ or both may be difficult to obtain. Furthermore, the trade-off between the improvement of the solution quality of the SAA problem and the increase of computational complexity for solving the SAA problem should be taken into account in the choice of sample size $N$. Therefore, heuristic approaches are quite often used in the literature to determine the sample size in the numerical tests for various SAA problems (e.g., Kleywegt et al., 2001). In Algorithm 1, higher is the sample size $N$, better is the estimated solution quality of the SAA problem; whereas, higher is the number of replications $M$, smaller is the confidence interval of the optimality gap. The sample size $N$ and the number of replications $M$ in this study will be empirically determined.

Challenge 2: In Step 2 of Algorithm 1, if the SAA problems cannot be solved directly in a reasonable amount of computational time due to the size or the complexity of the problems then some advanced solving techniques, such as decomposition or relaxation methods, will need to be used to reduce the computational time. In our case, in order to determine whether such advanced techniques are necessary or not, a computational simulation is conducted considering different problem sizes (see Chapter 5).

Challenge 3: To implement Algorithm 2, we explore the special structures of our SLP models as there are only a few sets of constraints that may lead to infeasible solutions. We call those 
constraints candidate infeasible constraints. For example, in Problem BP-2SLPR-SAA only the liability control constraints (4.45) are candidate infeasible constraints. Secondly, given a candidate solution $\hat{x}_{N^{\prime}}$, after generating samples of random demand realizations for the secondstage sub-problems, candidate infeasible constraints can immediately be detected without solving the second-stage sub-problems. Therefore, without adding too much extra computational effort, we can easily identify the "infeasible" constraints and add them as feasible cuts into the SAA problem (e.g., Problem BP-2SLPR-SAA) being solved in step 3.1. The number of feasible cuts that we need to add into our SAA problem depends on the number of sample replications $M$ and the sample size $N$. Hence, in the worse case, the maximum number of feasible cuts is $M \times N$.

\subsection{Summary}

In this chapter, we give a brief review of SLP and then discuss its implementation in the context of rewards-supply planning problems. We present the 2SLPR reformulations of the analytical models (i.e., Problem BP, Problem EP1, and Problem EP2). After that, we propose a samplingbased heuristic solution procedure to solve SP models with continuous random parameters. Our solution procedure is a modification of the standard SAA scheme discussed in the literature. In order to implement the solution procedure, we rewrite the 2SLPR-format models into SAA models. The SAA models can provide approximate solutions for the original stochastic models. A summary list of the models developed in Chapter 3 and this chapter is shown in Table 4.1. Finally, some technical challenges involved in the solution procedure are discussed. The computational studies are reported in the next two chapters. 
Table 4.1: Summary of the models

\begin{tabular}{|l|c|c|c|}
\hline \multicolumn{1}{|c|}{ System Setting } & Analytical Model & 2SLPR Format & SAA Model \\
\hline $\begin{array}{l}\text { LRP partnerships are governed by wholesale } \\
\text { price contracts solely without bonus points } \\
\text { offering }\end{array}$ & $\begin{array}{c}\text { Problem BP } \\
\text { Formulation (3.3)- } \\
(3.7)\end{array}$ & $\begin{array}{c}\text { Problem BP-2SLPR } \\
\text { Formulation (4.11)-(4.19) }\end{array}$ & $\begin{array}{c}\text { Problem BP-2SLPR-SAA } \\
\text { Formulation (4.41)-(4.48) }\end{array}$ \\
\hline $\begin{array}{l}\text { LRP partnerships are governed by wholesale } \\
\text { price contracts solely with bonus points offering }\end{array}$ & $\begin{array}{c}\text { Problem EP1 } \\
\text { Formulation (3.12)- } \\
(3.18)\end{array}$ & $\begin{array}{c}\text { Problem EP1-2SLPR } \\
\text { Formulation (4.20)-(4.25) }\end{array}$ & $\begin{array}{c}\text { Problem EP1-2SLPR-SAA } \\
\text { Formulation (4.49)-(4.58) }\end{array}$ \\
\hline $\begin{array}{l}\text { LRP partnerships are governed by wholesale } \\
\text { price contracts at the accumulation side and by } \\
\text { option contracts at the redemption side without } \\
\text { bonus points offering }\end{array}$ & $\begin{array}{c}\text { Problem EP2 } \\
\text { Formulation (3.21)- } \\
(3.29)\end{array}$ & $\begin{array}{c}\text { Problem EP2-2SLPR } \\
\text { Formulation (4.26)-(4.37) }\end{array}$ & $\begin{array}{l}\text { Problem EP2-2SLPR-SAA } \\
\text { Formulation (4.59)-(4.71) }\end{array}$ \\
\hline \multicolumn{1}{|c|}{$\begin{array}{c}\text { single-period } \\
\text { Monstrained news- } \\
\text { vendor models }\end{array}$} & $\begin{array}{c}\text { two stage stochastic } \\
\text { linear programming with } \\
\text { recourse }\end{array}$ & $\begin{array}{c}\text { sample average approximate } \\
\text { models }\end{array}$ \\
\hline
\end{tabular}




\section{Chapter 5 Design of Numerical Studies}

In order to answer our research questions, four sets of numerical studies were carried out. In this chapter, we describe the design of each of them. We first introduce a problem generation procedure that is applicable to all of our numerical studies. Then, we present the design of the studies for examining the effectiveness of our solution methodology, and the design of the studies for investigating the impacts of demand variability, budget tightness, and liability control on an LRP host's decisions and LRP performance. All the results obtained from our numerical studies are reported and analyzed in the next chapter.

From this chapter on, our models previously named as Problem BP-2SLR-SAA, Problem EPI2SLR-SAA, and Problem EP2-2SLR-SAA are abbreviated to BP, EP1, and EP2, respectively.

\subsection{Procedure for Generating Testing Problems}

In the absence of readily available industrial data and academic testing problems, we designed a set of randomly generated problems that are empirically driven.

Table 5.1 reports the main input parameters and their values used in generating the testing problems. Based on these parameters, ten testing cases of different sizes were generated randomly. Furthermore, in each testing case, we also considered the randomness involved in the accumulation and redemption demands. Thus, for each testing case, ten problem instances were carried out and independent demand samples were generated for each problem instance. 
Table 5.1: Problem generation parameters

\begin{tabular}{l|c}
\hline \multicolumn{1}{c|}{ Parameters } & Parameter Value $(s)$ \\
\hline Range of number of LRP redemption partners $(N R)$ & $1-100$ \\
\hline Range of number of LRP accumulation partners $(N A)$ & $1-100$ \\
\hline Average redemption cost of rewards $($ AvgMC) & 8.2 \\
\hline Range of market/retailing price factor of rewards $(M R)$ & $2-10$ \\
\hline Range of back order price factor of points $(B F)$ & $1.5-2$ \\
\hline Range for capacity factor of rewards $(C A)$ & $1-1.5$ \\
\hline Salvage value factor of rewards $(S R)$ & 0.5 \\
\hline Shortage cost factor of rewards $(V R)$ & 1.2 \\
\hline Average wholesale price of points $(A v g M P)$ & 12 \\
\hline Upper bound of liability ratio $\left(L_{U B}\right)$ & 2 \\
\hline Price factor for purchasing options $(O P)$ & 0.2 \\
\hline Price factor for exercising options $(E P)$ & 0.8 \\
\hline
\end{tabular}

In generating each testing case and problem instances, LRP accumulation partners and redemption partners were grouped into three categories (Type A, Type B, and Type C) to reflect the different levels of business activities. Type A refers to the most important partners of the LRP host, Type B refers to the less important partners, and Type C refers to the least important partners. Table 5.2 summarizes the input parameters used to generate additional input data per partner type. We limit our analysis of accumulation and redemption demands following either normal distributions or uniform distributions. For each redemption or accumulation partner, samples of redemption demand realizations $\left(D_{j s}^{R}\right.$ or $\left.D_{i s}^{A}\right)$ are generated randomly based on either normal or uniform distributions with parameter values shown in Table 5.2. 
Table 5.2: Problem generation parameters per partner type

\begin{tabular}{|c|c|c|c|}
\hline & \multicolumn{3}{|c|}{ Partner Type } \\
\hline Parameters & A & $\mathrm{B}$ & $\mathrm{C}$ \\
\hline Probability distribution of partner types & $20 \%$ & $40 \%$ & $40 \%$ \\
\hline Redemption cost factor $(F R)$ & 1.2 & 0.8 & 0.4 \\
\hline $\begin{array}{l}\text { Range of wholesale price factors per unit of } \\
\text { points ordered }(F A)\end{array}$ & $0.8-1$ & $1.0-1.2$ & $1.2-1.4$ \\
\hline $\begin{array}{l}\text { Range for redemption/accumulation demand } \\
\text { distribution (uniform distribution) }\end{array}$ & $40-50$ & $20-30$ & $5-10$ \\
\hline $\begin{array}{l}\text { Demand variability parameter (DV) for } \\
\text { uniform distribution }\end{array}$ & \multicolumn{3}{|c|}{$0.05,0.10,0.15,6.20,0.25$} \\
\hline $\begin{array}{l}\text { Mean and standard deviation for } \\
\text { redemption/accumulation demand (normal } \\
\text { distribution) }\end{array}$ & $(45,6)$ & $(25,3)$ & $(7.5,1)$ \\
\hline DV for normal distribution & \multicolumn{3}{|c|}{$0.1,0.5,1.0,1.5,1.8$} \\
\hline $\begin{array}{l}\text { Maximum percentage of accumulation } \\
\text { demand increase caused by CA effort } \\
\text { through bonus points offering }\left(\phi_{l}^{A}\right)\end{array}$ & 0.1 & 0.1 & 0.1 \\
\hline $\begin{array}{l}\text { Elasticity of CA effort through bonus points } \\
\text { offering on accumulation demand }\left(k_{t}^{A}\right)\end{array}$ & 1.2 & 1 & 0.8 \\
\hline
\end{tabular}

The other input parameters for our problems are obtained according to the schemes described below:

a) Wholesale price $\left(w_{J}^{R}\right)$ of rewards in terms of dollar value per unit points is the product of redemption cost factor $(F R)$ and the average redemption cost of rewards in terms of dollar value per unit points $(A v g M C)$.

b) Market/Retail price $\left(p_{J}^{R}\right)$ of rewards in terms of dollar value per unit points is the product of wholesale price of rewards $\left(w_{J}^{R}\right)$ and the market price factor (MR) that is generated randomly within the given range.

c) Salvage value $\left(s_{j}^{R}\right)$ of rewards is the product of wholesale price of rewards $\left(w_{\jmath}^{R}\right)$ and salvage value factor $(S R)$. 
d) Shortage cost $\left(v_{J}^{R}\right)$ of rewards is the product of wholesale price of rewards $\left(w_{J}^{R}\right)$ and shortage cost factor $(V R)$.

e) Wholesale price $\left(w_{l}^{A}\right)$ per unit points ordered by accumulation partners is the product of wholesale price factor of points $(F A)$ that is generated randomly within the given range and the average wholesale price of points in terms of dollar value per unit points ( $A v g M P)$.

f) Back order price $\left(w_{1}^{\prime A}\right)$ per unit points requested by accumulation partners is the product of the wholesale price $\left(w_{1}^{A}\right)$ and the back order price factor of points $(B F)$ that is generated randomly within the given range.

g) The right hand side of the capacity constraint $\left(Q_{J}^{R}\right)$ for each redemption partner is generated based on the ranges of the corresponding redemption demand parameters $(D R)$ from the redemption partner and the capacity factor of the rewards. The capacity factor $(C A)$ is generated randomly within the given range.

h) The available budget $\left(W^{R}\right)$ for purchasing rewards is required to be determined carefully to ensure problem feasibility and to guarantee a certain level of computational complexity in solving our problem instances. As the maximum possible ordering quantity of rewards from each redemption partner is limited by the capacity of rewards offered by the corresponding redemption partner $\left(Q_{J}^{R}\right)$, we use $Q_{J}^{R}$ and the wholesale price $\left(w_{J}^{R}\right)$ to determine the value of $W^{R}$

i) The initial liability $\left(l_{0}\right)$ is determined based on the information of accumulation demands. We assume that before the planning horizon of the testing problems, only accumulation occurs and no redemption, and the accumulation demand follows the same demand distribution as the current planning horizon. Therefore, the initial liability is determined by 
the sum of accumulation demands of points from all accumulation partners. Since the accumulation demand is a random parameter in the model, we use the sum of the upper bounds of the accumulation demands from all accumulation partners as the value of the initial liability.

j) In EP2, the option purchase price $\left(w_{J}^{R_{1}}\right)$ is the product of the price factor for purchasing options $(O P)$ and the wholesale price of rewards $\left(w_{J}^{R}\right)$. The option exercise price, $e_{J}^{R_{1}}$ is the product of the price factor for exercising options $(E P)$ and wholesale price of rewards $\left(w_{J}^{R}\right)$.

\subsection{Testing the Effectiveness of the Solution Methodology}

In this section, a set of numerical studies is developed to evaluate the effectiveness of our solution methodology for solving realistically sized rewards-supply planning problems in the range of LRPs that exist in today's marketplace, considering both the number of LRP redemption partners and the number of LRP accumulation partners.

The effectiveness of the proposed solution procedure is examined from two perspectives: model solvability and quality of stochastic solutions. Model solvability refers to the extent to which our solution procedure can solve problems of various sizes within a reasonable computation times (i.e., computational efficiency) while providing approximated solutions of good quality (ie., a smaller solution gap). In order to evaluate the model solvability, three model outputs are generated: (1) the estimated optimality gap (in percentage) between a candidate solution and an estimated true optimal solution, (2) the $95 \%$ confidence internal of the estimated optimal gap (in percentage), and (3) the computational time required to solve each testing problem (CPU time). 
These outputs are the key indicators of model solvability (Mak et al., 1999; Kleywegt et al., 2000; Ruszczyński \& Shapiro, 2003). With smaller solution gaps and shorter computational times, the model solvability is better. Meanwhile, these outputs are also used to determine the sample size and sample replications for the rest of the numerical studies. The sample size and sample replications are determined by the tradeoff between the solution gap and the computational efficiency.

Quality of stochastic solutions seeks to examine whether using a stochastic programming approach is appropriate and worthwhile. In order to evaluate the quality of stochastic solutions, solutions of the stochastic optimization models are quite often compared to those resulting from deterministic optimization models involving mean values of the uncertain problem parameters (e.g., Santoso et al., 2005; Zanjani et al., 2007; Chen-Ritzo et al., 2010). The difference between the expected values of stochastic models and the corresponding mean-value models is known as the value of the stochastic solution (VSS) (Birge and Louveaux, 1997). As long as the VSS is greater than zero, the stochastic models are no worse than the deterministic models. This indicates that stochastic models are worthwhile to resort to. Higher is the VSS, more appropriate are the stochastic models when compared to the deterministic models.

\subsubsection{Model Solvability}

The characteristics of the testing problems generated for examining the model solvability are shown in Table 5.3, where $T C$ refers to the problem case designed to represent real life LRP systems of different sizes. $N R$ refers to the number of LRP redemption partners, $N A$ refers to the number of accumulation partners. The size of an LRP system is determined by $N R$ and $N A . N$ 
refers to the number of sample demand realizations (i.e., sample size), $M$ refers to the number of sample replications, $N D$ refers to the number of decision variables, and $N C$ refers to the number of constraints. In order to determine the proper sample size and the proper number of replications, we consider three candidate sample sizes: $N=30,60$, and 90 ; and two candidate sample replications: $M=30$ and 45 for each testing case.

As listed in Table 5.3, ten problem cases were developed. The number of LRP accumulation partners and the number of LRP redemption partners in each problem case were arbitrarily determined based on our study on LRPs in the Canadian marketplace (see Chapter 2). For a problem case, four testing problems were generated to represent the different combinations of the candidate sample sizes and the candidate sample replications. For a testing problem, the number of decision variables and the number of constraints, as shown in Table 5.3, are different for each model (i.e., BP, EP1, and EP2). For instance, Problem No.1.1 has 61 decision variables and 62 constraints in BP model, 122 decision variables and 122 constraints in the EP1 model, and 152 decision variables and 182 constraints in the EP2 model. The largest testing problem for our models has 45,200 decision variables and 36,380 constraints (i.e., EP2 model, Problem No.10.4). 
Table 5.3: Summary characteristics of the set of random generated problems

\begin{tabular}{|c|c|c|c|c|c|c|c|c|c|c|c|}
\hline \multirow{2}{*}{$\begin{array}{l}\text { TC } \\
\text { No. }\end{array}$} & \multirow{2}{*}{$N R$} & \multirow{2}{*}{$N A$} & \multirow{2}{*}{$\begin{array}{l}\text { Problem } \\
\text { No. }\end{array}$} & \multirow{2}{*}{$N$} & \multirow{2}{*}{$M$} & \multicolumn{3}{|c|}{$N D$} & \multicolumn{3}{|c|}{$N C$} \\
\hline & & & & & & BP & EP1 & EP2 & BP & EP1 & EP2 \\
\hline \multirow{4}{*}{1} & 1 & 1 & 1.1 & 30 & 30 & 61 & 122 & 152 & 62 & 122 & 182 \\
\hline & 1 & 1 & 1.2 & 60 & 30 & 121 & 242 & 302 & 122 & 242 & 362 \\
\hline & 1 & 1 & 1.3 & 60 & 45 & 121 & 242 & 302 & 122 & 242 & 362 \\
\hline & 1 & 1 & 1.4 & 90 & 45 & 181 & 362 & 452 & 182 & 362 & 542 \\
\hline \multirow{4}{*}{2} & 3 & 5 & 2.1 & 30 & 30 & 183 & 488 & 456 & 124 & 424 & 426 \\
\hline & 3 & 5 & 2.2 & 60 & 30 & 363 & 968 & 906 & 244 & 844 & 846 \\
\hline & 3 & 5 & 2.3 & 60 & 45 & 363 & 968 & 906 & 244 & 844 & 846 \\
\hline & 3 & 5 & 2.4 & 90 & 45 & 543 & 1448 & 1356 & 364 & 1264 & 1266 \\
\hline \multirow{4}{*}{3} & 10 & 10 & 3.1 & 30 & 30 & 610 & 1220 & 1520 & 341 & 941 & 1280 \\
\hline & 10 & 10 & 3.2 & 60 & 30 & 1210 & 2420 & 3020 & 671 & 1871 & 2540 \\
\hline & 10 & 10 & 3.3 & 60 & 45 & 1210 & 2420 & 3020 & 671 & 1871 & 2540 \\
\hline & 10 & 10 & 3.4 & 90 & 45 & 1810 & 3620 & 4520 & 1001 & 2801 & 3800 \\
\hline \multirow{4}{*}{4} & 20 & 15 & 4.1 & 30 & 30 & 1220 & 2135 & 3040 & 651 & 1551 & 2500 \\
\hline & 20 & 15 & 4.2 & 60 & 30 & 2420 & 4235 & 6040 & 1281 & 3081 & 4960 \\
\hline & 20 & 15 & 4.3 & 60 & 45 & 2420 & 4235 & 6040 & 1281 & 3081 & 4960 \\
\hline & 20 & 15 & 4.4 & 90 & 45 & 3620 & 6335 & 9040 & 1911 & 4611 & 7420 \\
\hline \multirow{4}{*}{5} & 25 & 30 & 5.1 & 30 & 30 & 1525 & 3355 & 3800 & 806 & 2606 & 3110 \\
\hline & 25 & 30 & 5.2 & 60 & 30 & 3025 & 6655 & 7550 & 1586 & 5186 & 6170 \\
\hline & 25 & 30 & 5.3 & 60 & 45 & 3025 & 6655 & 7550 & 1586 & 5186 & 6170 \\
\hline & 25 & 30 & 5.4 & 90 & 45 & 4525 & 9955 & 11300 & 2366 & 7766 & 9230 \\
\hline \multirow{4}{*}{6} & 40 & 40 & 6.1 & 30 & 30 & 2440 & 4880 & 6080 & 1271 & 3671 & 4940 \\
\hline & 40 & 40 & 6.2 & 60 & 30 & 4840 & 9680 & 12080 & 2501 & 7301 & 9800 \\
\hline & 40 & 40 & 6.3 & 60 & 45 & 4840 & 9680 & 12080 & 2501 & 7301 & 9800 \\
\hline & 40 & 40 & 6.4 & 90 & 45 & 7240 & 14480 & 18080 & 3731 & 10931 & 14660 \\
\hline \multirow{4}{*}{7} & 60 & 55 & 7.1 & 30 & 30 & 3660 & 7015 & 9120 & 1891 & 5191 & 7380 \\
\hline & 60 & 55 & 7.2 & 60 & 30 & 7260 & 13915 & 18120 & 3721 & 10321 & 14640 \\
\hline & 60 & 55 & 7.3 & 60 & 45 & 7260 & 13915 & 18120 & 3721 & 10321 & 14640 \\
\hline & 60 & 55 & 7.4 & 90 & 45 & 10860 & 20815 & 27120 & 5551 & 15451 & 21900 \\
\hline \multirow{4}{*}{8} & 65 & 70 & 8.1 & 30 & 30 & 3965 & 8235 & 9880 & 2046 & 6246 & 7990 \\
\hline & 65 & 70 & 8.2 & 60 & 30 & 7865 & 16335 & 19630 & 4026 & 12426 & 15850 \\
\hline & 65 & 70 & 8.3 & 60 & 45 & 7865 & 16335 & 19630 & 4026 & 12426 & 15850 \\
\hline & 65 & 70 & 8.4 & 90 & 45 & 11765 & 24435 & 29380 & 6006 & 18606 & 23710 \\
\hline \multirow{4}{*}{9} & 80 & 80 & 9.1 & 30 & 30 & 4880 & 9760 & 12160 & 2511 & 7311 & 9820 \\
\hline & 80 & 80 & 9.2 & 60 & 30 & 9680 & 19360 & 24160 & 4941 & 14541 & 19480 \\
\hline & 80 & 80 & 9.3 & 60 & 45 & 9680 & 19360 & 24160 & 4941 & 14541 & 19480 \\
\hline & 80 & 80 & 9.4 & 90 & 45 & 14480 & 28960 & 36160 & 7371 & 21771 & 29140 \\
\hline \multirow{4}{*}{10} & 100 & 95 & 10.1 & 30 & 30 & 6100 & 11895 & 15200 & 3131 & 8831 & 12260 \\
\hline & 100 & 95 & 10.2 & 60 & 30 & 12100 & 23595 & 30200 & 6161 & 17561 & 24320 \\
\hline & 100 & 95 & 10.3 & 60 & 45 & 12100 & 23595 & 30200 & 6161 & 17561 & 24320 \\
\hline & 100 & 95 & 10.4 & 90 & 45 & 18100 & 35295 & 45200 & 9191 & 26291 & 36380 \\
\hline
\end{tabular}




\subsubsection{Quality of Stochastic Solutions}

In order to calculate the value of stochastic solution (VSS) for each of our models (i.e., BP, EP1, and EP2), the following steps are followed:

Step 1: Replace random parameters $D_{\jmath}^{R}, D_{l}^{A}$ with the mean values of $D_{\jmath}^{R}, D_{\imath}^{A}$ respectively. Solve the corresponding models (i.e., BP, EP1, and EP2) and find the optimal solutions $x^{M V P}$.

Step 2: Compute the estimated true objective function values, $\hat{\pi}_{N}\left(x^{M V P}\right)$ for $x^{M V P}$.

Step 3: Let $\hat{\pi}_{N}(\bar{x})$ be the objective function value of the SAA reformulation problem for the solution $\bar{x}$. The VSS for each candidate solution $(\bar{x})$ is calculated as:

$$
\mathrm{VSS}=\hat{\pi}_{N}(\bar{x})-\hat{\pi}_{N}\left(x^{M V P}\right)
$$

VSS in terms of percentage is calculated as:

$$
\operatorname{VSS}(\%)=\frac{\hat{\pi}_{N}(\bar{x})-\hat{\pi}_{N}\left(x^{M V P}\right)}{\hat{\pi}_{N}\left(x^{M V P}\right)} \times 100
$$

The quality of stochastic solutions was evaluated on the set of randomly generated problems shown in Table 5.3 with sample size of $N=60$ and sample replications of $M=30$ (i.e., Problem No. $1.2,2.2,3.2,4.2,5.2,6.2,7.2,8.2,9.2$, and 10.2).

\subsection{Testing the Impacts of Demand Variability}

Our second set of numerical studies aims to analyze the situation where internal constraints remain the same, but external uncertainty (i.e., demand uncertainty) varies. In this section, we 
describe the design of the studies for testing the impacts of demand variability on LRP performance.

In LRP management, demand uncertainty is one of the primary challenges that LRP managers have to face. Unlike daily goods (e.g., grocery) on which the overall customer demand is generally quite stable, LRP members' demands on accumulation and redemption vary due to various reasons. Therefore, some of the questions of interest to managers are: Does demand variability have an impact on LRP performance? If yes, is that impact negative or positive? Does that impact remain the same or not under different model settings (i.e., BP, EP1 and EP2)?

Given a demand distribution, demand variability (i.e., the degree of demand randomness) is quite often measured by the variance of the demand distribution. In order to change the degree of demand randomness, we define a demand variability parameter, DV, and use it to change the variances of demand distributions in our testing problems. With the increase in the values of DV, demand variability increases. The values of the demand variability parameter (DV) and the resulting changes in the demand distributions with the values of DV are listed in Tables 5.4 5.5.

Table 5.4: Demand variability parameter and its values used for normal distributions

\begin{tabular}{|c|c|c|c|c|c|c|c|c|}
\hline & \multicolumn{2}{|c|}{ Normal } & \multicolumn{7}{|c|}{$\sigma^{2}=\sigma_{0}^{2} \times D V$} \\
\cline { 2 - 9 } & $\mu_{0}$ & $\sigma_{0}$ & $\mathrm{DV}=0.1$ & $\mathrm{DV}=0.5$ & $\mathrm{DV}=1.0$ & $\mathrm{DV}=1.5$ & $\mathrm{DV}=1.8$ & $\mathrm{DV}=2$ \\
\hline Type A & 45 & 6 & 0.6 & 3.0 & 6.0 & 9.0 & 10.8 & 12.0 \\
\hline Type B & 25 & 3 & 0.3 & 1.5 & 3.0 & 4.5 & 5.4 & 6.0 \\
\hline Type C & 7.5 & 1 & 0.1 & 0.5 & 1.0 & 1.5 & 1.8 & 2.0 \\
\hline
\end{tabular}


Table 5.5: Demand variability parameter and its values used for uniform distributions

\begin{tabular}{|c|c|c|c|c|c|c|c|}
\hline & \multicolumn{2}{|c|}{ Uniform } & \multicolumn{4}{|c|}{$a=\mu+\sqrt{3} \times \sigma, b=\mu-\sqrt{3} \times \sigma$, where $\sigma=\mu \times D V$} \\
\cline { 2 - 8 } & $\left(a_{0}, b_{0}\right)$ & $\mu$ & $\mathrm{DV}=5 \%$ & $\mathrm{DV}=10 \%$ & $\mathrm{DV}=15 \%$ & $\mathrm{DV}=20 \%$ & $\mathrm{DV}=25 \%$ \\
\hline Type A & $(40,50)$ & 45 & $(41.1,48.9)$ & $(37.2,52.8)$ & $(33.3,56.7)$ & $(29.4,60.6)$ & $(25.5,64.5)$ \\
\hline Type B & $(20,30)$ & 25 & $(22.8,27.2)$ & $(20.7,29.3)$ & $(18.5,31.5)$ & $(16.3,33.7)$ & $(14.2,35.8)$ \\
\hline Type C & $(5,10)$ & 7.5 & $(6.9,8.1)$ & $(6.2,8.8)$ & $(5.6,9.4)$ & $(4.9,10.1)$ & $(4.3,10.7)$ \\
\hline
\end{tabular}

In Table 5.4, $\mu_{0}$ denotes the initial values of the mean of the normal distribution for each type of LRP accumulation and redemption partners, and $\sigma_{0}^{2}$ denotes the initial values of the variance of the normal distribution for each type of accumulation and redemption partners. With a value of DV, the variances of the normal distributions $\left(\sigma^{2}\right)$ are changed by using the relation $\sigma^{2}=\sigma_{0}^{2} \times D V$, and the means of the normal distributions remain the same. In Table 5.5, $a_{0}$ and $b_{0}$ denotes the initial lower bound and upper bound values of the uniform distributions for different types of LRP partners. With a value of DV, the variance of a uniform distribution $\left(\sigma^{2}\right)$ is obtained as follows:

$$
\sigma^{2}=(\mu \times D V)^{2}
$$

and then the lower bound $(a)$ and the upper bound $(b)$ of the uniform distribution is obtained as follows:

$$
a=\mu+\sqrt{3} \times \sigma, \text { and } b=\mu-\sqrt{3} \times \sigma
$$




\subsection{Testing the Impacts of Budget Tightness}

To evaluate the impact of budget tightness, the upper bound of the budget constraint (i.e., $W^{R}$ in constraint (4.43), constraint (4.51), and constraint (4.64)) in our models is redefined as follows:

$$
W^{R} \times(1-\alpha)
$$

where $\alpha$ denotes the budget tightness parameter, $0 \leq \alpha<1 . \alpha=0$ indicates that there is no change in the budget availability. $\alpha<1$ indicates that there is a change in the budget availability (i.e., reduction). As such, the level of budget tightness is determined by the budget tightness parameter $\alpha$. The higher is the value of $\alpha$, the tighter is the budget availability.

We develop our analysis by comparing the outputs of our models with a low level of budget tightness to the outputs of the models with a high level of budget tightness. The outputs include LRP profitability, actual liability ratio, and ordering quantities of rewards. In order to compare with the previous analysis in this chapter, we limit our study to three testing cases (i.e., TC No.4: $\mathrm{NR}=20$ and $\mathrm{NA}=40$, TC No. $6: N R=40$ and $N A=40$, and TC No.8: $N R=65$ and $N A=70$ ), and three levels of demand variability (low, medium, and high). These three test cases represent the most common sizes of LRPs in real world applications. With a normally distributed demand, the demand variability parameter $\mathrm{DV}=0.1$ represents a low level of demand variability, $\mathrm{DV}=1.0$ represents a medium level of demand variability, and DV $=1.8$ represents a high level of demand variability. With a uniformly distributed demand, $D V=0.05$ represents a low level of demand variability, $\mathrm{DV}=0.15$ represents a medium level of demand variability, and $\mathrm{DV}=0.25$ represents a high level of demand variability. 
For each testing problem, at each level of the demand variability (i.e., low, medium, and high), we perform hypothesis tests on the differences in the mean values of LRP profitability, liability ratio, and ordering quantities of rewards in the tight budget and loose budget cases. We also perform hypothesis tests on the differences in the mean values of profitability, liability ratio, and ordering quantities of rewards across different levels of demand variability (i.e., low, medium, and high) for cases of both tight and loose budgets. In addition, we perform hypothesis tests on the differences in the mean values of profitability, liability ratio, and ordering quantities of rewards across the model settings (i.e., BP, EP1, and EP2). The purpose of conducting these hypothesis tests is to examine whether there are significant impacts of budget tightness, demand variability, and model settings on LRP performance and the LRP host's decisions. The null hypothesis for each test is stated as follows:

H 1: With a given level of demand variability (i.e., low, medium, and high) there is no significant difference in the mean LRP profitability between a setting with low budget tightness and a setting with high budget tightness.

H 2: With a given level of demand variability (i.e., low, medium, and high), there is no significant difference in the mean liability ratio between a setting with low budget tightness and a setting with high budget tightness.

H 3: With a given level of demand variability (i.e., low, medium, and high), there is no significant difference in the mean rewards ordering quantity between a setting with low budget tightness and a setting with high budget tightness.

H 4: With a given level of budget tightness (i.e., low and high), there is no significant difference in the mean values of LRP profitability between settings operating under different demand variability levels. 
H 5: With a given level of budget tightness (i.e., low and high), there is no significant difference in the mean liability ratio between settings operating under different demand variability levels.

H 6: With a given level of budget tightness (i.e., low and high), there is no significant difference in the mean rewards ordering quantities between settings operating under different demand variability levels.

H 7: With a given level of demand variability (i.e., low, medium, and high) as well as a level of budget tightness (i.e., low and high) there is no significant difference in the mean LRP profitability between different model settings (i.e., BP, EP1, and EP2).

H 8: With a given level of demand variability (i.e., low, medium, and high) as well as a level of budget tightness (i.e., low and high), there is no significant difference in the mean liability ratio between different model settings (i.e., BP, EP1, and EP2).

H 9: With a given level of demand variability (i.e., low, medium, and high) as well as a level of budget tightness (i.e., low and high), there is no significant difference in the mean rewards ordering quantities between different model settings (i.e., BP, EP1, and EP2).

Hypothesis tests $\mathrm{H} 1$ - H6 are conducted under each model setting given both uniformly distributed demand and normally distributed demand. Hypothesis tests H7 - H9 are conducted across the model settings given both uniformly distributed demand and normally distributed demand. Overall, 378 hypothesis tests are conducted by employing all pair-wise comparisons technique using SPSS (PASW Statistics 18). The confidence level (CL) is set to $95 \%$ for all hypothesis tests. Intuitively, we would like to see that the null hypothesis would be rejected, which indicates that the budget tightness does have an impact on LRP performance and LRP host rewards-supply decisions. 


\subsection{Testing the Impacts of Liability Control Tightness}

As discussed in Chapter 3, the liability associated with unredeemed points is a key concern in the management of LRPs. In reality, many companies have experienced a dramatic increase of the liability with the growth of LRPs. For example, Aeroplan stated that the estimated future redemption cost for the liability amounts to $\$ 1,277$ million. According to its annual report for the fiscal year 2010, this amount is even higher than the revenue from selling points in the year 2010 (\$1,033 million). Marriott International, Inc. also reported that their liability for the Marriott Rewards program was $\$ 1,536$ million at year-end $2008^{1}$. Hence, one of the challenges in LRP operations is to plan for the supply of rewards while keeping the liability within a certain range.

To evaluate the impacts of liability control, the upper bound of the liability ratio $\left(L_{U B}\right)$ in liability constraints (i.e., constraints (4.45), (4.54), and (4.63) in BP, EP1, and EP2) is redefined as follow:

$$
L_{U B}=\gamma \times r_{0}
$$

where $\gamma$ denotes the liability tightness parameter, and $r_{0}$ denotes the initial liability ratio.

A four-step procedure described below is developed to conduct our numerical studies on liability control:

\footnotetext{
${ }^{1}$ Marriott International annual report 2008. http:' files shareholder.com/downloads/MAR/1350812803×0x283218'C30CB1C0-3AD4-4331-A4E35A6FI2D41AE7/Marroott 2008_AR.pdf (accessed in July, 2011)
} 
Step 1: With the model outputs from BP, EP1, and EP2, generate the initial liability ratio $\left(r_{0}\right)$ for each test case and each level of demand variability by calculating $\frac{l}{l_{0}}$, i.e., set $r_{0}=\frac{l}{l_{0}}$

Step 2: Based on the values of $\gamma$ and $r_{0}$, reset the values of the upper bound of the liability ratio $\left(L_{U B}\right)$ using the above formulation (5.6).

Step 3: Relax the budget and capacity constraints in BP, EP1, and EP2 to solve each model with the new upper bound of the liability ratio as obtained in Step 2.

Step 4: Generate the model outputs based on the solutions obtained in Step 3. The model outputs include: (1) LRP profitability, (2) cost of rewards, (3) ordering quantity of rewards, and (4) budget usage ratio of the current budget usage (obtained from the model without the budget and capacity constraints) to the initial budget usage (obtained from the model with the budget and capacity constraints), and to the maximum available budget ( $\left.W^{R}\right)$, respectively.

In Step 2, in order to understand the sole impact of liability control, we relax the capacity as well as the budget constraints (i.e., constraints $4.42,4.43,4.50,4.51,4.61,4.64$ ) in our models and then generated the model outputs given various values of $L_{U B}$. The values of $L_{U B}$ used in the numerical study for each model are listed in Tables (5.6) - (5.8). As shown in those tables, in each of these models $\gamma=0.7$ represents the situation where the liability control level is tight (i.e., the upper bound of the liability ratio in the liability constraint is less than the initial liability ratio). $\gamma=1.3$ represents the situation where the liability control level is loose (i.e., the upper 
bound of the liability ratio in the liability constraint is larger than the initial liability ratio). As in Section 5.4, we limit our analysis to three problem testing cases: TC No. 4, 6, and 8 .

Table 5.6: Values of $L_{U B}$ used for BP setting

(a) with Uniformly Distributed

Demand

Case 4
\begin{tabular}{|c|c|c|c|}
\hline \multicolumn{2}{|c|}{} & \multicolumn{2}{c|}{$\gamma$} \\
\hline DV & $\mathrm{r}_{0}$ & 0.7 & 1.3 \\
\hline 0.05 & 0.538 & 0.377 & 0.699 \\
0.15 & 0.455 & 0.319 & 0.592 \\
0.25 & 0.396 & 0.277 & 0.515 \\
\hline
\end{tabular}

Case 6

\begin{tabular}{|c|c|c|c|}
\hline & \multicolumn{3}{|c|}{ Case } \\
\hline & & & \\
\hline DV & $\mathrm{r}_{0}$ & 0.7 & 1.3 \\
\hline 0.05 & 0.953 & 0.667 & 1.239 \\
\hline 0.15 & 0.900 & 0.630 & 1.170 \\
\hline 0.25 & 0.881 & 0.617 & 1.145 \\
\hline
\end{tabular}

Case 8

\begin{tabular}{|c|c|c|c|}
\multicolumn{5}{|c|}{ Case 8} \\
\hline DV & $\mathrm{r}_{0}$ & 0.7 & 1.3 \\
\hline 0.05 & 1.109 & 0.776 & 1.442 \\
0.15 & 1.071 & 0.750 & 1.392 \\
0.25 & 1.061 & 0.743 & 1.379 \\
\hline
\end{tabular}

(b) with Normally Distributed

Demand

\begin{tabular}{|c|c|c|c|}
\hline \multicolumn{4}{|c|}{ Case 4} \\
\hline & & & \\
\hline DV & $r_{0}$ & 0.7 & 1.3 \\
\hline 0.1 & 0.572 & 0.400 & 0.744 \\
\hline 1.0 & 0.467 & 0.327 & 0.607 \\
\hline 1.8 & 0.404 & 0.283 & 0.525 \\
\hline
\end{tabular}

Case 6

\begin{tabular}{|c|c|c|c|}
\hline & & & \\
\hline DV & $r_{0}$ & 0.7 & 1.3 \\
\hline 0.1 & 0.981 & 0.687 & 1.275 \\
\hline 1.0 & 0.911 & 0.638 & 1.184 \\
\hline 1.8 & 0.885 & 0.620 & 1.151 \\
\hline
\end{tabular}

Case 8

\begin{tabular}{|c|c|c|c|}
\hline \multicolumn{2}{|c|}{} & \multicolumn{2}{c|}{$\gamma$} \\
\hline $\mathrm{DV}$ & $\mathrm{r}_{0}$ & 0.7 & 1.3 \\
\hline 0.1 & 1.128 & 0.790 & 1.466 \\
1.0 & 1.075 & 0.753 & 1.398 \\
1.8 & 1.063 & 0.744 & 1.382 \\
\hline
\end{tabular}


Table 5.7: Values of $L_{U B}$ used for EP1 setting

$\begin{array}{ll}\text { (a) with Uniformly Distributed } & \text { (b) with Normally Distributed }\end{array}$ Demand

\begin{tabular}{|c|c|c|c|}
\multicolumn{5}{|c|}{ Case 4} \\
\hline DV & $\mathrm{r}_{0}$ & 0.7 & 1.3 \\
\hline 0.05 & 0.610 & 0.427 & 0.763 \\
0.15 & 0.507 & 0.355 & 0.659 \\
0.25 & 0.435 & 0.305 & 0.566 \\
\hline
\end{tabular}

\begin{tabular}{|c|c|c|c|}
\multicolumn{5}{|c|}{ Case 6 } \\
\begin{tabular}{|c|c|c|c|}
\hline \multicolumn{2}{|c|}{} & \multicolumn{2}{c|}{$v$} \\
\hline 0.05 & 1.085 & 0.760 & 1.411 \\
0.15 & 0.994 & 0.696 & 1.292 \\
0.25 & 0.952 & 0.666 & 1.238 \\
\hline
\end{tabular}
\end{tabular}

\begin{tabular}{|c|c|c|c|}
\hline \multicolumn{4}{|c|}{ Case 8} \\
\hline & & & \\
\hline DV & $r_{0}$ & 0.7 & 1.3 \\
\hline 0.05 & 1.242 & 0.869 & 1.615 \\
\hline 0.15 & 1.167 & 0.817 & 1.517 \\
\hline 0.25 & 1.133 & 0.793 & 1.473 \\
\hline
\end{tabular}

Table 5.8: Values of $L_{U B}$ used for EP2 setting

(a) with Uniformly Distributed Demand

(b) with Normally Distributed Demand

\begin{tabular}{|c|c|c|c|}
\multicolumn{5}{|c|}{ Case 4} \\
\begin{tabular}{|c|c|c|c|}
\hline \multicolumn{2}{|c|}{} & \multicolumn{2}{c|}{$\gamma$} \\
\hline 0.05 & 0.564 & 0.395 & 0.733 \\
0.15 & 0.520 & 0.360 & 0.676 \\
0.25 & 0.480 & 0.336 & 0.624 \\
\hline
\end{tabular}
\end{tabular}

Case 4
\begin{tabular}{|c|c|c|c|}
\hline \multicolumn{3}{|c|}{} & \multicolumn{2}{c|}{$\gamma$} \\
\hline $\mathrm{DV}$ & $\mathrm{r}_{0}$ & 0.7 & 1.3 \\
\hline 0.1 & 0.581 & 0.407 & 0.755 \\
1.0 & 0.539 & 0.377 & 0.701 \\
1.8 & 0.482 & 0.337 & 0.627 \\
\hline
\end{tabular}

Case 6
\begin{tabular}{|c|c|c|c|}
\hline \multicolumn{3}{|c|}{} & \multicolumn{2}{c|}{$\gamma$} \\
\hline DV & $\mathrm{r}_{0}$ & 0.7 & 1.3 \\
\hline 0.05 & 0.977 & 0.684 & 1.270 \\
0.15 & 0.950 & 0.665 & 1.235 \\
0.25 & 0.927 & 0.649 & 1.205 \\
\hline
\end{tabular}

Case 6
\begin{tabular}{|c|c|c|c|}
\hline \multicolumn{2}{|c|}{} & \multicolumn{2}{c|}{$y$} \\
\hline $\mathrm{DV}$ & $\mathrm{r}_{0}$ & 0.7 & 1.3 \\
\hline 0.1 & 0.988 & 0.692 & 1.284 \\
1.0 & 0.967 & 0.677 & 1.257 \\
1.8 & 0.937 & 0.656 & 1.218 \\
\hline
\end{tabular}

\begin{tabular}{|c|c|c|c|}
\multicolumn{5}{|c|}{ Case 8 } \\
\begin{tabular}{|c|c|c|c|}
\hline \multicolumn{2}{|c|}{} & \multicolumn{2}{c|}{$\gamma$} \\
\hline DV & $\mathrm{r}_{0}$ & 0.7 & 1.3 \\
\hline 0.05 & 1.128 & 0.790 & 1.466 \\
0.15 & 1.113 & 0.779 & 1.447 \\
0.25 & 1.100 & 0.770 & 1.430 \\
\hline
\end{tabular}
\end{tabular}

\begin{tabular}{|c|c|c|c|}
\hline \multicolumn{4}{|c|}{ Case 8} \\
\hline & & & \\
\hline DV & $r_{0}$ & 0.7 & 1.3 \\
\hline 0.1 & 1.135 & 0.795 & 1.476 \\
\hline 1.0 & 1.121 & 0.785 & 1.457 \\
\hline 1.8 & 1.199 & 0.769 & 1.429 \\
\hline
\end{tabular}




\section{Chapter 6 Results and Analysis}

In this chapter, we present and discuss the results obtained from our numerical studies, which are about: (1) the effectiveness of our models and solution methodology, and (2) the impacts of demand uncertainty, budget tightness, and liability control on LRP performance and LRP host's decisions, respectively. We discuss our findings for each model setting (i.e., BP, EP1, and EP2) as well as across model settings.

All of our numerical studies were carried out through computer simulations on a Toshiba Tecra M10 notebook equipped with $2.26 \mathrm{GHZ}$ processor. A computer simulation (coded in $\mathrm{C}++$ ) was developed to generate testing problems and implement the solution procedure for our SLP models (the $\mathrm{C}++$ code is available upon request). The IBM Optimization Subroutine Library (OSL, version 3.0) was used to solve the corresponding SAA problems.

\subsection{Testing the Effectiveness of the Solution Methodology}

Our first set of numerical studies sought to evaluate the effectiveness of our solution methodology for solving realistically sized (large-scale) rewards-supply planning problems.

\subsubsection{Model Solvability}

Figures $6.1-6.3$ illustrate the model outputs (i.e., optimality gaps, confidence intervals of the optimality gaps, and CPU times) for each testing problem defined in Table 5.3 (see Section 5.2 in Chapter 5). "MeanG(\%)" in Figure 6.1 refers to the sample average of the optimality gap of a 
candidate solution in terms of percentage (i.e., $\operatorname{MeanG}(\%)=\frac{\bar{G}_{N}^{M}(\bar{x})}{\frac{1}{M} \sum_{m=1}^{M} \hat{\pi}_{N}^{m}} \times 100$, where $\bar{G}_{N}^{M}(\bar{x})$ is the mean optimality gap for a candidate solution $\bar{x}$, and $\frac{1}{M} \sum_{m=1}^{M} \hat{\pi}_{N}^{m}$ is the upper bound estimation for the optimal objective value of the true problem). "CIU(\%)" in Figure 6.2 refers to the average upper bound of the confidence interval of the optimality gap in terms of percentage (i.e., CIU(\%) $=\frac{\bar{G}_{N}^{M}(\bar{x})+\tilde{\varepsilon}_{G}}{\frac{1}{M} \sum_{m=1}^{M} \hat{\pi}_{N}^{m}} \times 100$, where $\tilde{\varepsilon}_{G}$ is the half-width of the confidence interval. "CPU (mins)" in Figure 6.3 refers to the average computational time (in minutes) spent to solve each testing problem. The value of demand variability parameter (DV) is set to 1.8 for normally distributed demand and 0.25 for uniformly distributed demand. 
Figure 6.1: Estimated mean gaps with different sample sizes and sample replications

(a) Under BP Setting
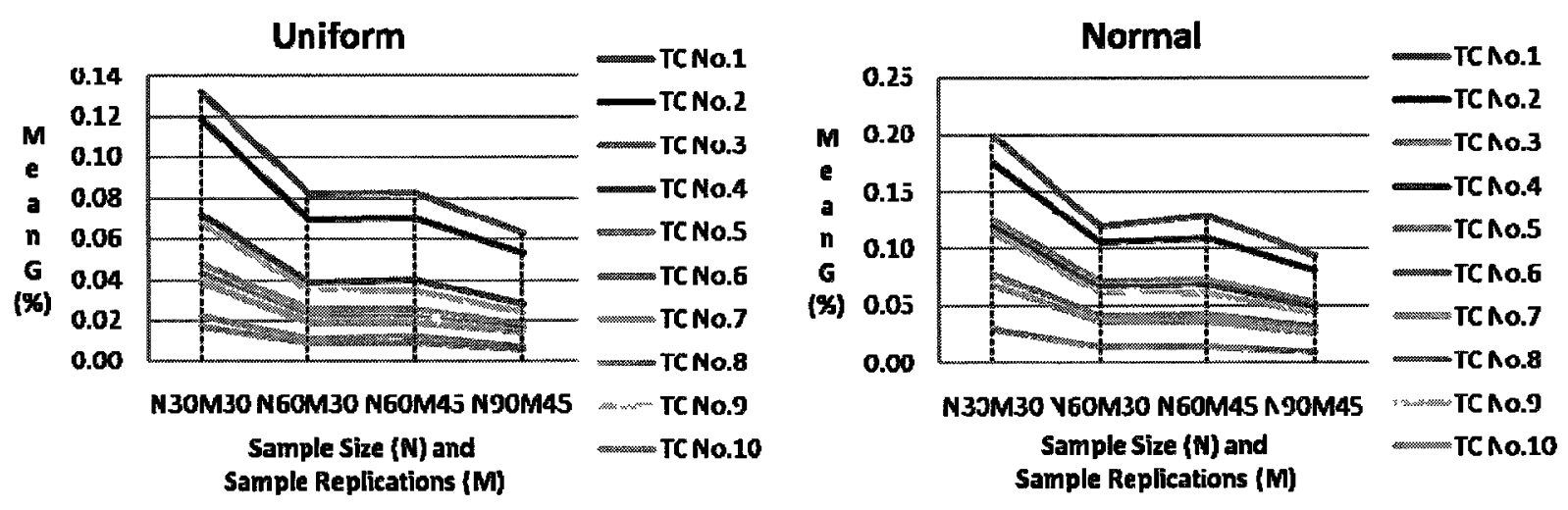

(b) Under EP1 Setting
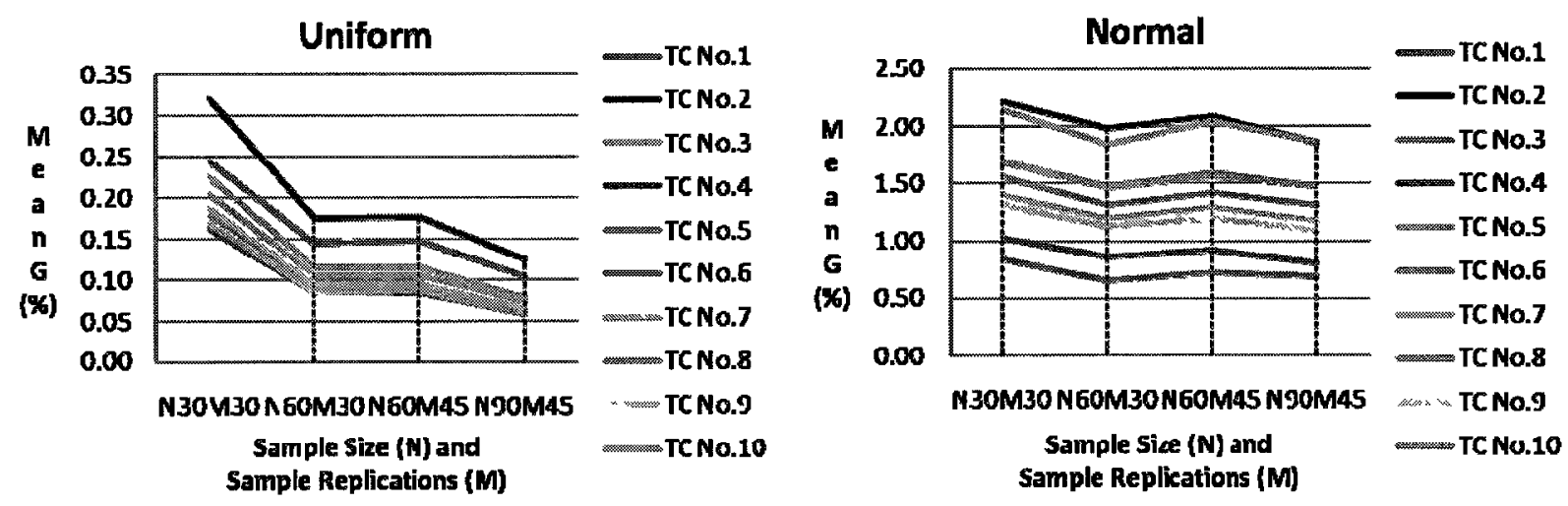

(c) Under EP2 Setting
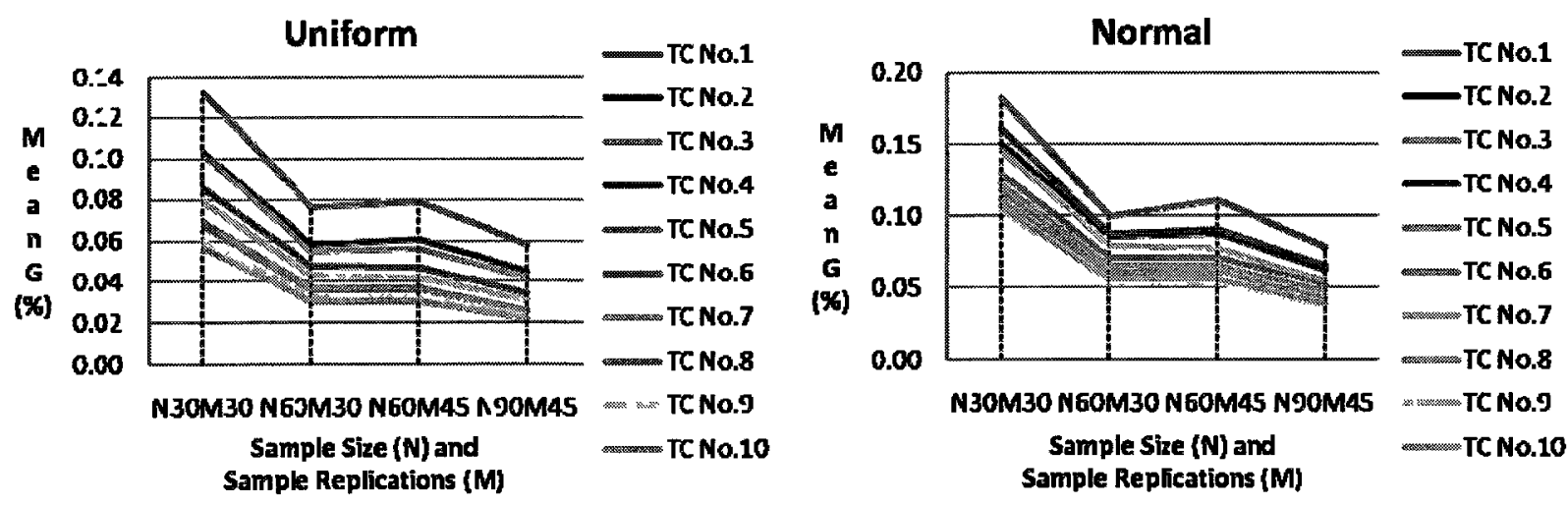
Figure 6.2: Confidence interval upper bounds of the estimated mean gaps with different sample sizes and sample replications

(a) Under BP Setting
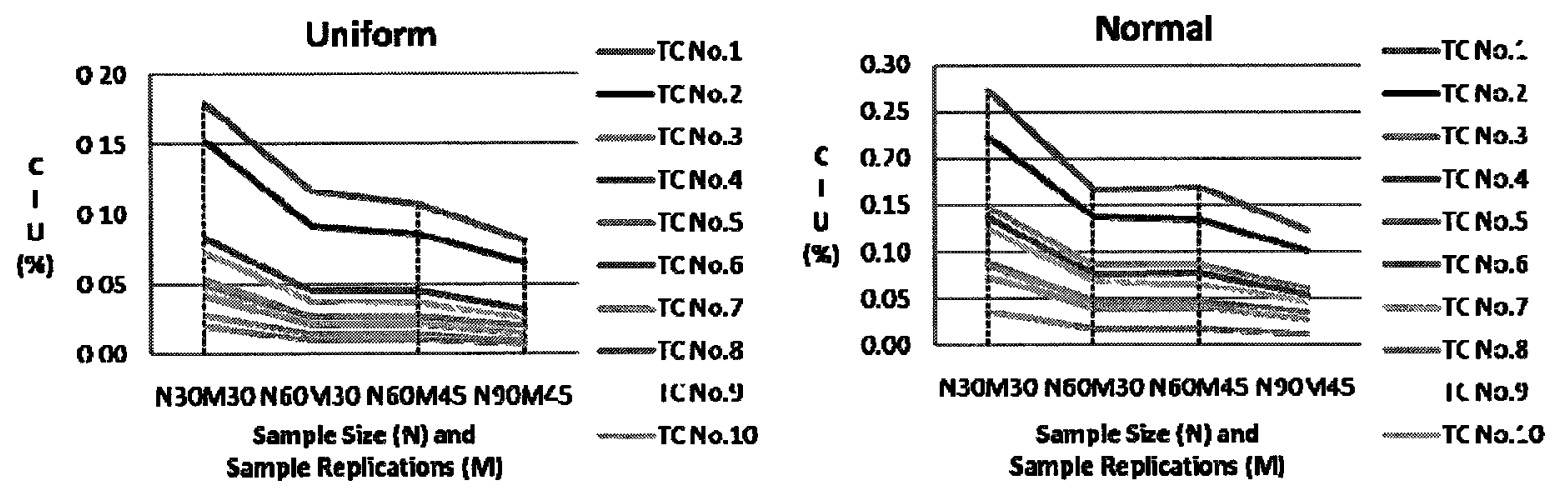

(b) Under EP1 Setting
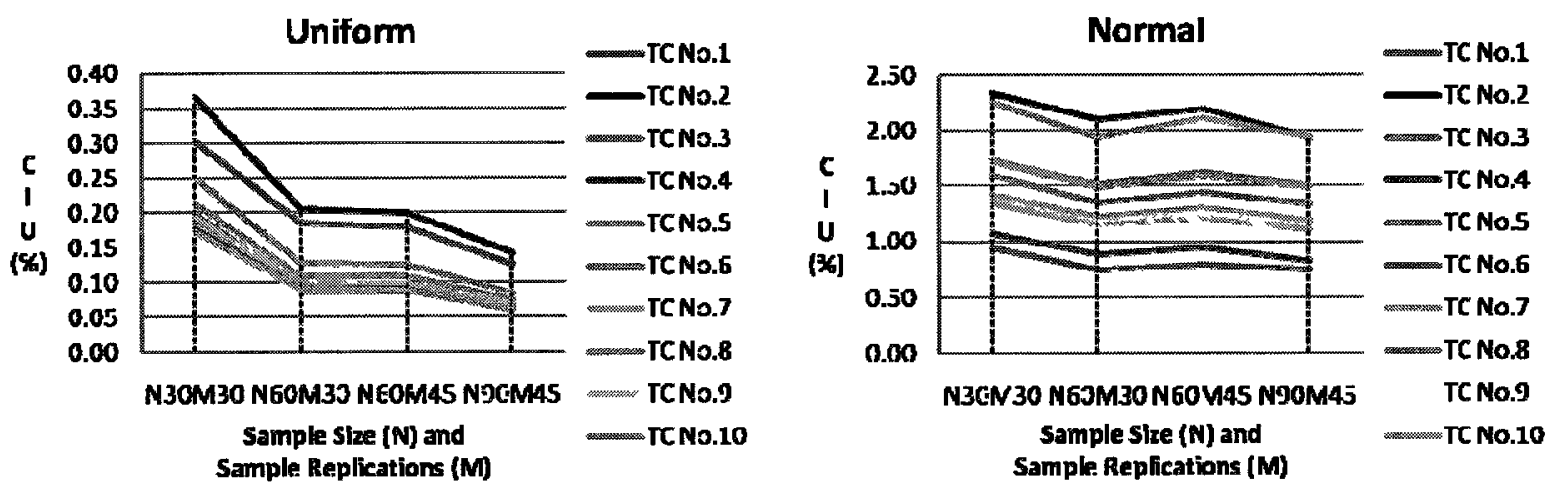

(c) Under EP2 Setting
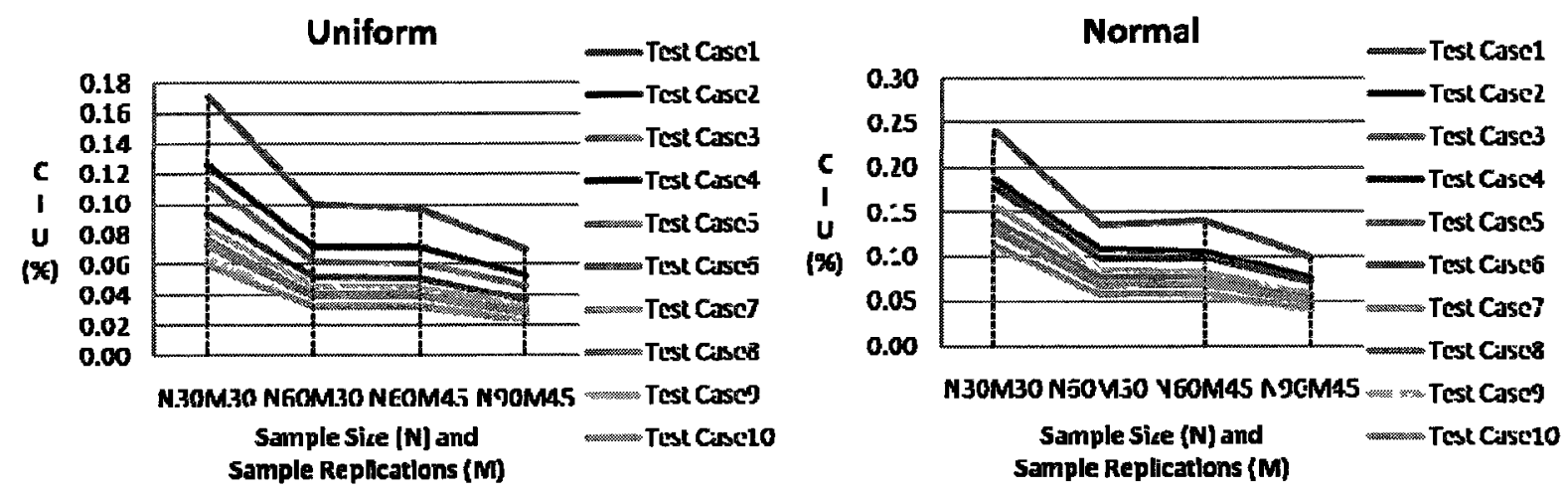
Figure 6.3: CPU times with different sample sizes and sample replications

(a) Under BP Setting
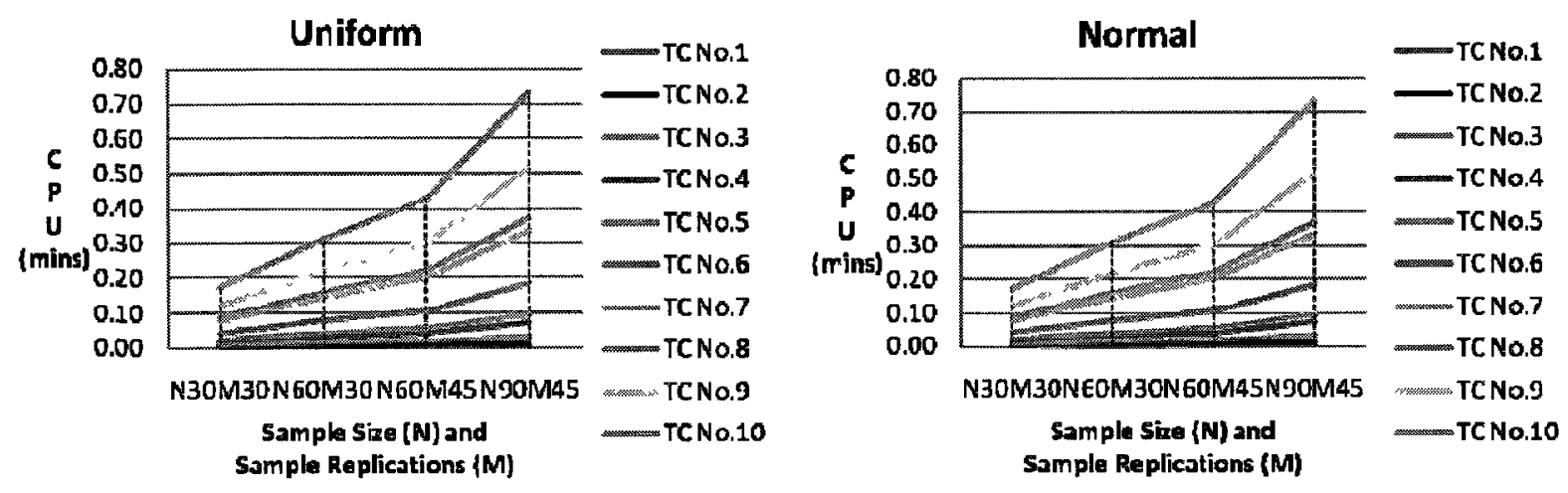

(b) Under EP1 Setting
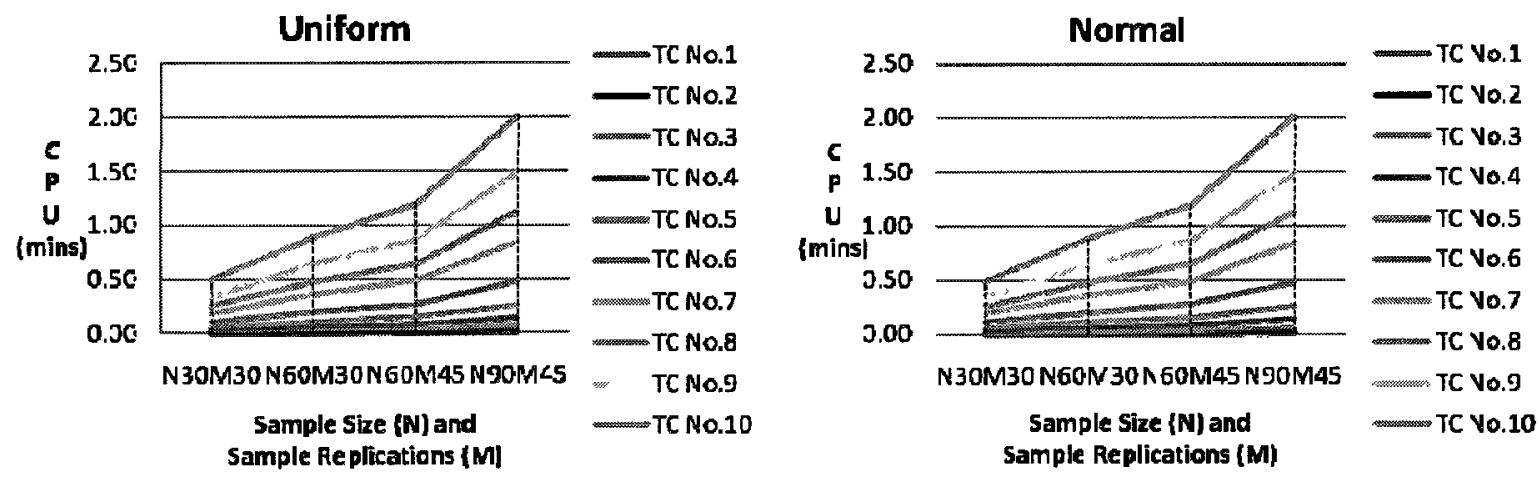

(c) Under EP2 Setting
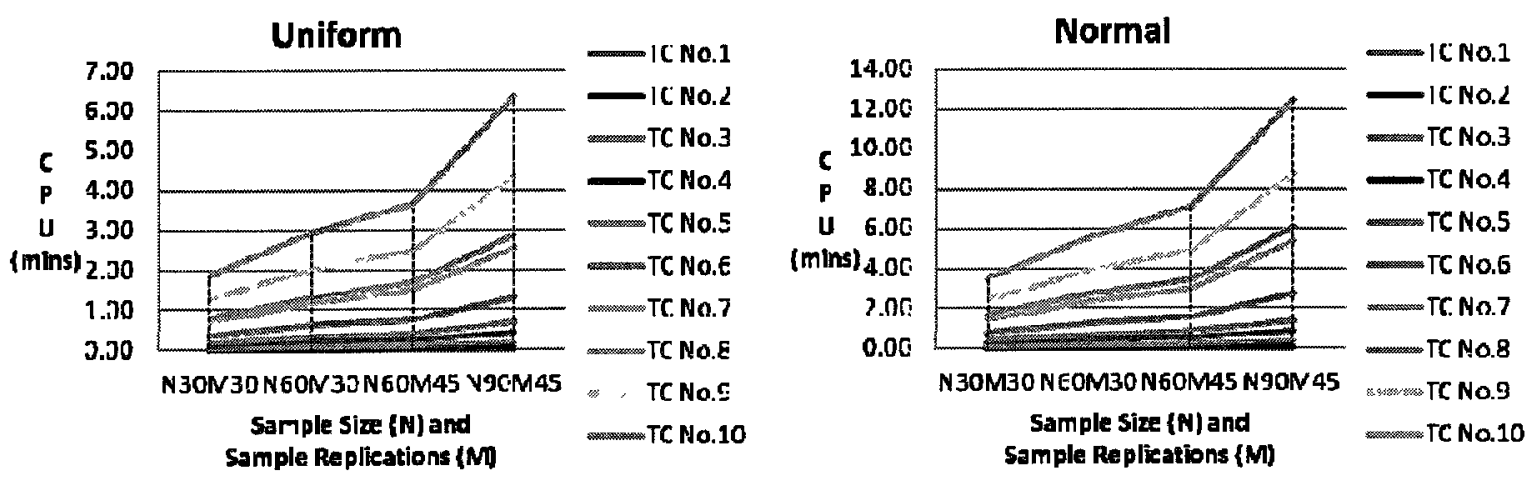
According to the model outputs illustrated in Figures $6.1-6.3$ (for more detailed model outputs please refer to Appendices B.1 - B.3), with only a modest number of samples (e.g. $N=30$ ), the proposed solution procedure provides high quality estimated SAA solutions to the true stochastic programming problems (i.e., Problem BP-2SLPR, Problem-EP1-2SLPR, and Problem-EP2$2 S L P R)$. For instance, with sample size $N=30$, and number of replications $M=30$, the estimated mean optimal gaps (MeanG(\%)) and the estimated upper bound of the confidence interval $(\mathrm{CIU}(\%))$ of the mean optimal gaps are lower than $0.25 \%$ in BP and EP2, and these values are lower than $2.5 \%$ in EP1. Regarding the determination of the sample size and sample replications for solving BP, EP1, and EP2 models, we found that higher improvement of the optimality gaps in terms of MeanG(\%) and $\mathrm{CIU}(\%)$ occurs when sample size $N$ increases from 30 to 60 , compared to the improvement of the optimal gap when $N$ changes from 60 to 90 and $M$ changes from 30 to 45 . On the other hand, comparing the increase in CPU times when $N$ changes from 60 to 90 and $M$ changes from 30 to 45 , lower increase in CPU times occurs when sample size $N$ increases from 30 to 60 and $M$ remains at 30 . These trends remain the same regardless of the demand distribution (uniform versus normal). Hence, in the remainder of this work, all numerical studies are based on a sample size of $N=60$ and a sample replication of $M=30$. In comparison to other tested sample sizes and sample replications, these offer a better tradeoff between the quality of solutions and the computational efficiency.

\subsubsection{Quality of Stochastic Solutions}

The comparison between candidate solutions of stochastic models and the solutions of the meanvalue models are reported in Tables $6.1-6.3$ considering normal distribution (with demand 
variability parameter $\mathrm{DV}=0.1,1.0$, and 1.8 ) and uniform distribution (with demand variability parameter $\mathrm{DV}=0.05,0.15$, and 0.25 ), respectively.

Results from Tables 6.1-6.3 show that VSS(\%) in all the testing problems are greater than zero, which indicates that the estimated optimal values of the objective function (i.e. LRP profitability) for all candidate solutions of the stochastic programming models are greater than those of the mean-value-based models (see the calculation of $\operatorname{VSS}(\%)$ in the formulation (5.2)). This reveals that stochastic programming solutions are superior to the corresponding mean-value solutions in terms of the objective function values. These solutions also lead to significantly smaller mean gaps (i.e., MeanG(\%)) and a smaller variability of the gaps (i.e., StdG). Moreover, with the increases in demand variability, the quality of the stochastic solutions is more superior to the quality of the mean-value model solutions. The value of the stochastic solution in percentage (i.e., VSS(\%)) increases from less than $0.5 \%$ in the case of low demand variability to more than $10 \%$ in the case of high demand variability. All of these results show that the stochastic model is an effective tool to solve rewards-supply planning problems with the presence of random demand.

Overall, the results from all testing problems show that our solution methodology can solve realistically sized rewards-supply planning problems in reasonable time and it can generate estimated optimal solutions with an optimality gap that is small enough. Moreover, the expected values of our stochastic models are better than the expected values of the corresponding meanvalue models, especially when the variability of the stochastic parameter in our models (i.e., redemption and accumulation demands) increases. 
Table 6.1: Quality of stochastic solutions of BP model

(a) with Uniformly Distributed Demand

\begin{tabular}{|c|c|c|c|c|c|c|}
\hline \multirow[b]{2}{*}{$\begin{array}{c}\text { TC } \\
\#\end{array}$} & \multirow[b]{2}{*}{ DV } & \multicolumn{2}{|c|}{ Stochastic Model } & \multicolumn{2}{|c|}{ Mean Value Model } & \multirow{2}{*}{$\begin{array}{l}\text { VSS } \\
(\%)\end{array}$} \\
\hline & & StdG & $\begin{array}{c}\text { MeanG } \\
(\%)\end{array}$ & StdG & $\begin{array}{c}\text { MeanG } \\
(\%)\end{array}$ & \\
\hline \multirow{3}{*}{1} & 0.05 & 0.492 & 0.011 & 15.725 & 1.887 & 2.16 \\
\hline & 0.15 & 1.476 & 0.032 & 47.175 & 5.660 & 6.76 \\
\hline & 0.25 & 2.460 & 0.053 & 78.625 & 9.434 & 11.80 \\
\hline \multirow{3}{*}{2} & 0.05 & 0.530 & 0.013 & 11.181 & 1.441 & 2.12 \\
\hline & 0.15 & 1.590 & 0.037 & 33.590 & 4.235 & 6.46 \\
\hline & 0.25 & 2.650 & 0.061 & 56.008 & 6.939 & 10.99 \\
\hline \multirow{3}{*}{3} & 0.05 & 0.362 & 0.011 & 6.733 & 1.094 & 2.01 \\
\hline & 0.15 & 0.976 & 0.024 & 19.705 & 3.062 & 5.75 \\
\hline & 0.25 & 0.857 & 0.012 & 26.343 & 4.427 & 8.47 \\
\hline \multirow{3}{*}{4} & 0.05 & 1.122 & 0.011 & 26.094 & 1.659 & 1.92 \\
\hline & 0.15 & 2.834 & 0.026 & 74.984 & 4.689 & 5.65 \\
\hline & 0.25 & 4.155 & 0.029 & 116.372 & 7.427 & 9.30 \\
\hline \multirow{3}{*}{5} & 0.05 & 1.027 & 0.012 & 18.266 & 1.250 & 1.93 \\
\hline & 0.15 & 2.130 & 0.016 & 50.296 & 3.410 & 5.44 \\
\hline & 0.25 & 1.535 & 0.010 & 68.130 & 4.858 & 7.95 \\
\hline \multirow{3}{*}{6} & 0.05 & 1.240 & 0.013 & 24.769 & 1.439 & 2.09 \\
\hline & 0.15 & 3.222 & 0.029 & 70.917 & 3.954 & 5.96 \\
\hline & 0.25 & 4.205 & 0.027 & 109.508 & 5.918 & 9.23 \\
\hline
\end{tabular}

(b) with Normally Distributed Demand

\begin{tabular}{|c|c|c|c|c|c|c|}
\hline & & Stocha: & ic Model & Mean V & ue Model & \\
\hline $\begin{array}{c}\text { TC } \\
\#\end{array}$ & DV & StdG & $\begin{array}{c}\text { MeanG } \\
(\%)\end{array}$ & StdG & $\begin{array}{c}\text { MeanG } \\
(\%)\end{array}$ & $(\%)$ \\
\hline \multirow{3}{*}{1} & 0.1 & 0.279 & 0.006 & 3.802 & 0.422 & 0.47 \\
\hline & 1.0 & 2.788 & 0.056 & 38.021 & 4.240 & 4.94 \\
\hline & 1.8 & 4.808 & 0.099 & 68.330 & 7.663 & 9.32 \\
\hline \multirow{3}{*}{2} & 0.1 & 0.239 & 0.006 & 2.543 & 0.313 & 0.45 \\
\hline & 1.0 & 2.385 & 0.057 & 25.419 & 3.058 & 4.55 \\
\hline & 1.8 & 4.281 & 0.101 & 45.762 & 5.411 & 8.32 \\
\hline \multirow{3}{*}{3} & 0.1 & 0.149 & 0.005 & 1.412 & 0.246 & 0.44 \\
\hline & 1.0 & 1.372 & 0.041 & 13.702 & 2.255 & 4.14 \\
\hline & 1.8 & 1.216 & 0.021 & 21.046 & 3.632 & 6.84 \\
\hline \multirow{3}{*}{4} & 0.1 & 0.640 & 0.006 & 7.704 & 0.405 & 0.46 \\
\hline & 1.0 & 5.690 & 0.056 & 66.310 & 3.510 & 4.13 \\
\hline & 1.8 & 7.781 & 0.065 & 108.763 & 5.989 & 7.31 \\
\hline \multirow{3}{*}{5} & 0.1 & 0.460 & 0.005 & 4.788 & 0.286 & 0.43 \\
\hline & 1.0 & 3.643 & 0.036 & 45.130 & 2.554 & 3.99 \\
\hline & 1.8 & 2.977 & 0.019 & 66.856 & 4.038 & 6.51 \\
\hline \multirow{3}{*}{6} & 0.1 & 0.573 & 0.006 & 6.377 & 0.311 & 0.44 \\
\hline & 1.0 & 4.716 & 0.046 & 60.660 & 2.912 & 4.29 \\
\hline & 1.8 & 6.921 & 0.049 & 101.079 & 4.788 & 7.29 \\
\hline
\end{tabular}


Table 6.1: Quality of stochastic solutions of BP model (Cont.)

(a) with Uniformly Distributed Demand

\begin{tabular}{|c|c|c|c|c|c|c|}
\hline & & \multicolumn{2}{|c|}{ Stochastic Model } & \multicolumn{2}{c|}{ Mean Value Model } & \multirow{2}{*}{$\begin{array}{c}\text { VSS } \\
(\%)\end{array}$} \\
\hline $\begin{array}{c}\text { TC } \\
\#\end{array}$ & DV & StdG & $\begin{array}{c}\text { MeanG } \\
(\%)\end{array}$ & StdG & $\begin{array}{c}\text { MeanG } \\
(\%)\end{array}$ & \\
\hline \multirow{4}{*}{7} & 0.05 & 2.095 & 0.014 & 38.709 & 1.544 & 2.09 \\
\cline { 2 - 7 } & 0.15 & 5.787 & 0.034 & 112.002 & 4.310 & 6.05 \\
\cline { 2 - 7 } & 0.25 & 6.876 & 0.038 & 176.970 & 6.760 & 10.24 \\
\hline \multirow{4}{*}{8} & 0.05 & 1.702 & 0.012 & 40.179 & 1.374 & 2.04 \\
\cline { 2 - 7 } & 0.15 & 4.526 & 0.025 & 116.233 & 3.845 & 5.93 \\
\cline { 2 - 7 } & 0.25 & 6.103 & 0.027 & 171.042 & 5.734 & 9.12 \\
\hline \multirow{4}{*}{9} & 0.05 & 2.022 & 0.012 & 45.220 & 1.464 & 1.99 \\
\cline { 2 - 7 } & 0.15 & 5.232 & 0.023 & 117.790 & 3.717 & 5.23 \\
\cline { 2 - 7 } & 0.25 & 6.620 & 0.022 & 180.675 & 5.511 & 8.01 \\
\hline \multirow{4}{*}{10} & 0.05 & 2.015 & 0.011 & 50.143 & 1.484 & 2.01 \\
\cline { 2 - 7 } & 0.15 & 4.385 & 0.020 & 121.046 & 3.764 & 5.29 \\
\cline { 2 - 7 } & 0.25 & 5.989 & 0.021 & 174.391 & 5.395 & 7.80 \\
\hline
\end{tabular}

(b) with Normally Distributed Demand

\begin{tabular}{|c|c|c|c|c|c|c|}
\hline & & \multicolumn{2}{|c|}{ Stochastic Model } & \multicolumn{2}{c|}{ Mean Value Model } & \multirow{2}{*}{$\begin{array}{c}\text { VSS } \\
(\%)\end{array}$} \\
\hline $\begin{array}{c}\text { TC } \\
\#\end{array}$ & DV & StdG & $\begin{array}{c}\text { MeanG } \\
(\%)\end{array}$ & StdG & $\begin{array}{c}\text { MeanG } \\
(\%)\end{array}$ & \\
\hline \multirow{4}{*}{7} & 0.1 & 0.962 & 0.007 & 10.937 & 0.355 & 0.47 \\
\cline { 2 - 7 } & 1.0 & 9.088 & 0.058 & 102.348 & 3.249 & 4.46 \\
\cline { 2 - 7 } & 1.8 & 12.452 & 0.068 & 171.690 & 5.530 & 7.89 \\
\hline \multirow{4}{*}{8} & 0.1 & 0.962 & 0.006 & 10.413 & 0.314 & 0.45 \\
\cline { 2 - 7 } & 1.0 & 8.175 & 0.044 & 99.524 & 2.911 & 4.40 \\
\cline { 2 - 7 } & 1.8 & 10.166 & 0.045 & 158.037 & 4.757 & 7.43 \\
\hline \multirow{4}{*}{9} & 0.1 & 1.031 & 0.006 & 11.951 & 0.340 & 0.45 \\
\cline { 2 - 6 } & 1.0 & 8.195 & 0.039 & 95.413 & 2.831 & 3.91 \\
\cline { 2 - 6 } & 1.8 & 10.639 & 0.041 & 155.508 & 4.529 & 6.45 \\
\hline \multirow{4}{*}{10} & 0.1 & 1.077 & 0.006 & 12.051 & 0.338 & 0.45 \\
\cline { 2 - 6 } & 1.0 & 7.257 & 0.033 & 100.861 & 2.884 & 3.98 \\
\cline { 2 - 6 } & 1.8 & 10.312 & 0.035 & 147.234 & 4.473 & 6.35 \\
\hline
\end{tabular}


Table 6.2: Quality of stochastic solutions of EP1 model

(a) with Uniformly Distributed Demand

\begin{tabular}{|c|c|c|c|c|c|c|}
\hline \multirow[b]{2}{*}{$\begin{array}{c}\text { TC } \\
\#\end{array}$} & \multirow[b]{2}{*}{ DV } & \multicolumn{2}{|c|}{ Stochastic Model } & \multicolumn{2}{|c|}{ Mean Value Model } & \multirow{2}{*}{$\begin{array}{l}\text { VSS } \\
(\%)\end{array}$} \\
\hline & & StdG & $\begin{array}{c}\text { MeanG } \\
(\%)\end{array}$ & StdG & $\begin{array}{c}\text { MeanG } \\
(\%)\end{array}$ & \\
\hline \multirow{3}{*}{1} & 0.05 & 0.552 & 0.017 & 15.752 & 1.866 & 2.12 \\
\hline & 0.15 & 1.678 & 0.052 & 47.261 & 5.678 & 6.69 \\
\hline & 0.25 & 2.806 & 0.089 & 78.771 & 9.606 & 11.71 \\
\hline \multirow{3}{*}{2} & 0.05 & 0.635 & 0.027 & 11.177 & 1.403 & 2.01 \\
\hline & 0.15 & 1.942 & 0.084 & 33.580 & 4.278 & 6.25 \\
\hline & 0.25 & 3.251 & 0.145 & 55.992 & 7.282 & 10.72 \\
\hline \multirow{3}{*}{3} & 0.05 & 0.582 & 0.031 & 6.740 & 1.058 & 1.82 \\
\hline & 0.15 & 1.767 & 0.093 & 19.737 & 3.126 & 5.42 \\
\hline & 0.25 & 2.637 & 0.133 & 26.438 & 4.794 & 8.12 \\
\hline \multirow{3}{*}{4} & 0.05 & 1.203 & 0.017 & 26.097 & 1.640 & 1.89 \\
\hline & 0.15 & 3.199 & 0.046 & 75.009 & 4.707 & 5.59 \\
\hline & 0.25 & 4.972 & 0.063 & 116.398 & 7.581 & 9.22 \\
\hline \multirow{3}{*}{5} & 0.05 & 1.304 & 0.027 & 18.361 & 1.216 & 1.81 \\
\hline & 0.15 & 3.308 & 0.069 & 50.551 & 3.458 & 5.23 \\
\hline & 0.25 & 4.904 & 0.102 & 68.628 & 5.157 & 7.73 \\
\hline \multirow{3}{*}{6} & 0.05 & 1.588 & 0.027 & 24.796 & 1.401 & 1.98 \\
\hline & 0.15 & 4.517 & 0.075 & 71.054 & 3.996 & 5.77 \\
\hline & 0.25 & 7.145 & 0.108 & 109.882 & 6.221 & 9.01 \\
\hline
\end{tabular}

(b) with Normally Distributed Demand

\begin{tabular}{|c|c|c|c|c|c|c|}
\hline \multirow{2}{*}{$\begin{array}{c}\mathrm{TC} \\
\#\end{array}$} & \multirow[b]{2}{*}{ DV } & \multicolumn{2}{|c|}{ Stochastic Model } & \multicolumn{2}{|c|}{ Mean Value Model } & \multirow{2}{*}{$\begin{array}{l}\text { VSS } \\
(\%)\end{array}$} \\
\hline & & StdG & $\begin{array}{c}\text { MeanG } \\
(\%)\end{array}$ & StdG & $\begin{array}{c}\text { MeanG } \\
(\%)\end{array}$ & \\
\hline \multirow{3}{*}{1} & 0.1 & 0.578 & 0.033 & 3.819 & 0.440 & 0.46 \\
\hline & 1.0 & 6.035 & 0.350 & 38.225 & 4.511 & 4.89 \\
\hline & 1.8 & 10.961 & 0.655 & 68.730 & 8.301 & 9.28 \\
\hline \multirow{3}{*}{2} & 0.1 & 0.706 & 0.087 & 2.653 & 0.377 & 0.42 \\
\hline & 1.0 & 7.429 & 0.955 & 26.627 & 3.908 & 4.41 \\
\hline & 1.8 & 13.302 & 1.599 & 47.976 & 7.061 & 8.21 \\
\hline \multirow{3}{*}{3} & 0.1 & 1.098 & 0.122 & 1.793 & 0.342 & 0.38 \\
\hline & 1.0 & 11.609 & 1.351 & 17.965 & 3.502 & 3.91 \\
\hline & 1.8 & 20.074 & 2.169 & 29.154 & 5.949 & 6.69 \\
\hline \multirow{3}{*}{4} & 0.1 & 1.234 & 0.045 & 7.804 & 0.434 & 0.45 \\
\hline & 1.0 & 12.593 & 0.485 & 67.775 & 3.913 & 4.08 \\
\hline & 1.8 & 19.406 & 0.674 & 111.271 & 6.677 & 7.27 \\
\hline \multirow{3}{*}{5} & 0.1 & 1.844 & 0.091 & 5.019 & 0.354 & 0.39 \\
\hline & 1.0 & 18.990 & 0.991 & 48.232 & 3.458 & 3.84 \\
\hline & 1.8 & 33.762 & 1.647 & 74.096 & 5.779 & 6.40 \\
\hline \multirow{3}{*}{6} & 0.1 & 2.148 & 0.085 & 6.537 & 0.372 & 0.41 \\
\hline & 1.0 & 22.275 & 0.925 & 62.963 & 3.742 & 4.16 \\
\hline & 1.8 & 40.113 & 1.498 & 108.084 & 6.365 & 7.19 \\
\hline
\end{tabular}


Table 6.2: Quality of stochastic solutions of EP1 model (Cont.)

(a) with Uniformly Distributed Demand

\begin{tabular}{|c|c|c|c|c|c|c|}
\hline & & Stoch & c Model & Mean V & Model & \\
\hline $\begin{array}{c}\mathrm{TC} \\
\#\end{array}$ & DV & StdG & $\begin{array}{c}\text { MeanG } \\
(\%)\end{array}$ & StdG & $\begin{array}{c}\text { MeanG } \\
(\%)\end{array}$ & $(\%)$ \\
\hline \multirow{3}{*}{7} & 0.05 & 2.358 & 0.025 & 38.609 & 1.511 & 2.00 \\
\hline & 0.15 & 6.685 & 0.072 & 111.689 & 4.345 & 5.90 \\
\hline & 0.25 & 9.570 & 0.109 & 176.427 & 7.036 & 9.68 \\
\hline \multirow{3}{*}{8} & 0.05 & 2.119 & 0.026 & 40.102 & 1.337 & 1.93 \\
\hline & 0.15 & 6.113 & 0.074 & 116.043 & 3.890 & 5.73 \\
\hline & 0.25 & 9.326 & 0.113 & 170.894 & 6.047 & 8.89 \\
\hline \multirow{3}{*}{9} & 0.05 & 2.323 & 0.023 & 45.281 & 1.432 & 1.91 \\
\hline & 0.15 & 6.511 & 0.062 & 117.968 & 3.754 & 5.10 \\
\hline & 0.25 & 9.506 & 0.092 & 180.807 & 5.757 & 7.85 \\
\hline \multirow{3}{*}{10} & 0.05 & 2.411 & 0.023 & 50.179 & 1.452 & 1.93 \\
\hline & 0.15 & 6.019 & 0.059 & 121.162 & 3.800 & 5.16 \\
\hline & 0.25 & 9.173 & 0.091 & 174.555 & 5.634 & 7.66 \\
\hline
\end{tabular}

(b) with Normally Distributed Demand

\begin{tabular}{|c|c|c|c|c|c|c|}
\hline & & \multicolumn{2}{|c|}{ Stochastic Model } & \multicolumn{2}{|c|}{ Mean Value Model } & \multirow{2}{*}{$\begin{array}{c}\text { VSS } \\
(\%)\end{array}$} \\
\hline $\begin{array}{c}\text { TC } \\
\#\end{array}$ & DV & StdG & $\begin{array}{c}\text { MeanG } \\
(\%)\end{array}$ & StdG & $\begin{array}{c}\text { MeanG } \\
(\%)\end{array}$ & \\
\hline \multirow{4}{*}{7} & 0.1 & 2.554 & 0.073 & 11.242 & 0.405 & 0.44 \\
\cline { 2 - 7 } & 1.0 & 26.675 & 0.792 & 105.679 & 3.947 & 4.35 \\
\cline { 2 - 7 } & 1.8 & 45.950 & 1.280 & 176.503 & 6.885 & 7.81 \\
\hline \multirow{4}{*}{8} & 0.1 & 3.182 & 0.088 & 11.096 & 0.377 & 0.42 \\
\cline { 2 - 7 } & 1.0 & 32.730 & 0.950 & 107.454 & 3.772 & 4.25 \\
\cline { 2 - 7 } & 1.8 & 56.296 & 1.584 & 171.000 & 6.444 & 7.32 \\
\hline \multirow{4}{*}{9} & 0.1 & 3.284 & 0.074 & 12.303 & 0.393 & 0.42 \\
\cline { 2 - 7 } & 1.0 & 34.185 & 0.797 & 101.638 & 3.560 & 3.82 \\
\cline { 2 - 7 } & 1.8 & 58.814 & 1.273 & 166.487 & 5.896 & 6.39 \\
\hline \multirow{3}{*}{10} & 0.1 & 3.272 & 0.072 & 12.463 & 0.389 & 0.42 \\
\cline { 2 - 7 } & 1.0 & 33.638 & 0.769 & 107.129 & 3.587 & 3.89 \\
\cline { 2 - 7 } & 1.8 & 59.748 & 1.245 & 161.939 & 5.795 & 6.29 \\
\hline
\end{tabular}


Table 6.3: Quality of stochastic solutions of EP2 model

(a) with Uniformly Distributed Demand

\begin{tabular}{|c|c|c|c|c|c|c|}
\hline & & Stoch & c Model & Mean V & Model & \\
\hline $\begin{array}{c}\mathrm{TC} \\
\#\end{array}$ & DV & StdG & $\begin{array}{c}\text { MeanG } \\
(\%)\end{array}$ & StdG & $\begin{array}{c}\text { MeanG } \\
(\%)\end{array}$ & $(\%)$ \\
\hline & 0.05 & 0.372 & 0.010 & 16.157 & 2.104 & 2.32 \\
\hline 1 & 0.15 & 1.115 & 0.031 & 48.472 & 6.302 & 7.27 \\
\hline & 0.25 & 1.782 & 0.049 & 81.002 & 10.485 & 12.70 \\
\hline & 0.05 & 0.372 & 0.011 & 11.748 & 1.712 & 2.38 \\
\hline 2 & 0.15 & 1.116 & 0.032 & 35.246 & 5.066 & 7.31 \\
\hline & 0.25 & 1.774 & 0.049 & 58.934 & 8.317 & 12.49 \\
\hline & 0.05 & 0.236 & 0.010 & 7.257 & 1.417 & 2.41 \\
\hline 3 & 0.15 & 0.697 & 0.029 & 21.730 & 4.145 & 7.22 \\
\hline & 0.25 & 1.179 & 0.040 & 35.361 & 6.610 & 11.86 \\
\hline & 0.05 & 0.804 & 0.011 & 30.384 & 2.133 & 2.37 \\
\hline 4 & 0.15 & 2.252 & 0.029 & 90.224 & 6.384 & 7.44 \\
\hline & 0.25 & 3.249 & 0.038 & 142.091 & 10.296 & 12.54 \\
\hline & 0.05 & 0.671 & 0.011 & 20.207 & 1.662 & 2.40 \\
\hline 5 & 0.15 & 1.966 & 0.029 & 60.293 & 4.893 & 7.36 \\
\hline & 0.25 & 5.743 & 0.058 & 86.124 & 7.595 & 12.03 \\
\hline & 0.05 & 0.939 & 0.012 & 26.313 & 1.777 & 2.40 \\
\hline 6 & 0.15 & 2.760 & 0.035 & 78.845 & 5.267 & 7.44 \\
\hline & 0.25 & 3.896 & 0.040 & 130.376 & 8.547 & 12.60 \\
\hline
\end{tabular}

(b) with Normally Distributed Demand

\begin{tabular}{|c|c|c|c|c|c|c|}
\hline \multirow{2}{*}{$\begin{array}{c}\mathrm{TC} \\
\#\end{array}$} & \multirow[b]{2}{*}{ DV } & \multicolumn{2}{|c|}{ Stochastic Model } & \multicolumn{2}{|c|}{ Mean Value Model } & \multirow{2}{*}{$\begin{array}{c}\text { VSS } \\
(\%)\end{array}$} \\
\hline & & StdG & $\begin{array}{c}\text { MeanG } \\
(\%)\end{array}$ & StdG & $\begin{array}{c}\text { MeanG } \\
(\%)\end{array}$ & \\
\hline \multirow{3}{*}{1} & 0.1 & 0.267 & 0.006 & 4.039 & 0.489 & 0.53 \\
\hline & 1.0 & 2.623 & 0.064 & 40.387 & 4.899 & 5.55 \\
\hline & 1.8 & 3.623 & 0.076 & 72.223 & 8.771 & 10.27 \\
\hline \multirow{3}{*}{2} & 0.1 & 0.206 & 0.006 & 2.804 & 0.390 & 0.53 \\
\hline & 1.0 & 2.046 & 0.059 & 28.043 & 3.848 & 5.47 \\
\hline & 1.8 & 3.137 & 0.080 & 50.429 & 6.776 & 9.89 \\
\hline \multirow{3}{*}{3} & 0.1 & 0.131 & 0.006 & 1.607 & 0.335 & 0.57 \\
\hline & 1.0 & 1.262 & 0.050 & 15.839 & 3.213 & 5.56 \\
\hline & 1.8 & 2.098 & 0.065 & 27.842 & 5.434 & 9.48 \\
\hline \multirow{3}{*}{4} & 0.1 & 0.608 & 0.008 & 8.835 & 0.496 & 0.54 \\
\hline & 1.0 & 5.615 & 0.066 & 86.360 & 4.938 & 5.64 \\
\hline & 1.8 & 8.510 & 0.080 & 144.137 & 8.577 & 10.13 \\
\hline \multirow{3}{*}{5} & 0.1 & 0.389 & 0.006 & 5.469 & 0.383 & 0.54 \\
\hline & 1.0 & 3.719 & 0.056 & 54.636 & 3.742 & 5.53 \\
\hline & 1.8 & 6.170 & 0.063 & 79.982 & 5.448 & 8.93 \\
\hline \multirow{3}{*}{6} & 0.1 & 0.527 & 0.007 & 7.057 & 0.397 & 0.53 \\
\hline & 1.0 & 5.185 & 0.064 & 70.438 & 3.919 & 5.44 \\
\hline & 1.8 & 7.130 & 0.072 & 124.758 & 6.863 & 9.81 \\
\hline
\end{tabular}


Table 6.3: Quality of stochastic solutions of EP2 model (Cont.)

(a) with Uniformly Distributed Demand

\begin{tabular}{|c|c|c|c|c|c|c|}
\hline & & \multicolumn{2}{|c|}{ Stochastic Model } & \multicolumn{2}{c|}{ Mean Value Model } & \multirow{2}{*}{$\begin{array}{c}\text { VSS } \\
(\%)\end{array}$} \\
\hline \multirow{4}{*}{$\begin{array}{c}\text { TC } \\
7\end{array}$} & DV & StdG & $\begin{array}{c}\text { MeanG } \\
(\%)\end{array}$ & StdG & $\begin{array}{c}\text { MeanG } \\
(\%)\end{array}$ & \\
\hline & 0.05 & 1.451 & 0.014 & 42.809 & 1.947 & 2.44 \\
\cline { 2 - 7 } & 0.15 & 4.220 & 0.038 & 127.778 & 5.798 & 7.65 \\
\cline { 2 - 7 } & 0.25 & 6.342 & 0.050 & 206.932 & 9.412 & 12.99 \\
\hline \multirow{4}{*}{8} & 0.05 & 1.194 & 0.012 & 43.440 & 1.739 & 2.42 \\
\cline { 2 - 7 } & 0.15 & 3.554 & 0.032 & 129.795 & 5.137 & 7.46 \\
\cline { 2 - 7 } & 0.25 & 5.341 & 0.040 & 213.609 & 8.287 & 12.51 \\
\hline \multirow{4}{*}{9} & 0.05 & 1.523 & 0.012 & 51.318 & 1.906 & 2.43 \\
\cline { 2 - 7 } & 0.15 & 4.156 & 0.032 & 152.365 & 5.661 & 7.58 \\
\cline { 2 - 7 } & 0.25 & 6.000 & 0.035 & 234.685 & 8.982 & 12.63 \\
\hline \multirow{3}{*}{10} & 0.05 & 1.402 & 0.012 & 55.093 & 1.886 & 2.40 \\
\cline { 2 - 7 } & 0.15 & 3.935 & 0.030 & 164.144 & 5.602 & 7.50 \\
\cline { 2 - 7 } & 0.25 & 5.346 & 0.031 & 254.704 & 8.916 & 12.50 \\
\hline
\end{tabular}

(b) with Normally Distributed Demand

\begin{tabular}{|c|c|c|c|c|c|c|}
\hline & & \multicolumn{2}{|c|}{ Stochastic Model } & \multicolumn{2}{c|}{ Mean Value Model } & \multirow{2}{*}{$\begin{array}{c}\text { VSS } \\
(\%)\end{array}$} \\
\hline $\begin{array}{c}\text { TC } \\
\#\end{array}$ & DV & StdG & $\begin{array}{c}\text { MeanG } \\
(\%)\end{array}$ & StdG & $\begin{array}{c}\text { MeanG } \\
(\%)\end{array}$ & \\
\hline \multirow{4}{*}{7} & 0.1 & 0.818 & 0.008 & 11.940 & 0.451 & 0.56 \\
\cline { 2 - 7 } & 1.0 & 7.952 & 0.069 & 118.935 & 4.482 & 5.82 \\
\cline { 2 - 7 } & 1.8 & 13.269 & 0.088 & 210.874 & 7.853 & 10.55 \\
\hline \multirow{4}{*}{8} & 0.1 & 0.801 & 0.006 & 11.600 & 0.404 & 0.55 \\
\cline { 2 - 7 } & 1.0 & 7.753 & 0.058 & 115.522 & 3.977 & 5.69 \\
\cline { 2 - 7 } & 1.8 & 11.041 & 0.067 & 204.277 & 6.915 & 10.18 \\
\hline \multirow{4}{*}{10} & 0.1 & 0.887 & 0.007 & 13.353 & 0.434 & 0.54 \\
\cline { 2 - 6 } & 1.0 & 8.373 & 0.060 & 132.225 & 4.298 & 5.64 \\
\cline { 2 - 6 } & 1.8 & 11.487 & 0.057 & 217.831 & 7.386 & 10.07 \\
\hline \multirow{4}{*}{10} & 0.1 & 0.977 & 0.007 & 13.189 & 0.429 & 0.54 \\
\cline { 2 - 6 } & 1.0 & 8.763 & 0.059 & 131.072 & 4.248 & 5.58 \\
\cline { 2 - 6 } & 1.8 & 11.304 & 0.054 & 220.858 & 7.314 & 9.96 \\
\hline
\end{tabular}




\subsection{Testing the Impacts of Demand Variability}

In this section, we report the results of the numerical studies that we carried out to investigate the impacts of demand variability on LRP performance. Ten problem testing cases (TC No. 1 - 10) are used in the studies to represent realistically sized planning problems.

\subsubsection{Under BP Setting}

In Table 6.4, we report LRP profitability (i.e. host firm's value creation), actual liability ratio $\left(l^{\prime}\right)$, and budget usage (in percentage) under BP setting with demand variability changing from small to large.

As shown in Table 6.4, in one half of the testing cases (i.e., TC No. 2, 3, 5, 6, and 8), LRP profitability increases with the increases of demand variability, while in the other half of the testing cases (i.e., TC No.1, 4, 7, 9, and 10), LRP profitability decreases with the increase of demand variability. The reason is that profitability from the accumulation side always increases with the increase of variability in accumulation demand due to the assumption that accumulation demand will always met. Meanwhile, profitability from the redemption side always decreases with the increase of variability in redemption demand due to the increased cost of purchasing rewards (i.e., the need to order more rewards when demand variability is higher). When the increase in the accumulation-side profitability is higher than the decrease in the redemption-side profitability, the overall profitability will increase. This is more likely to happen when the number of LRP redemption partners $(N R)$ is less than the number of LRP accumulation partners $(N A)$. When the increase in the accumulation-side profitability is lower than the decrease in the 
redemption-side profitability, the overall profitability will decrease. This is more likely to happen when $N R$ is greater than $N A$. However, the average increasing or decreasing rates of LRP profitability among different testing cases are quite similar (e.g., the average increasing rate is $1.623 \%$ and the average decreasing rate is $1.191 \%$ with uniformly distributed demand; $1.763 \%$ and $1.212 \%$ with normally distributed demand).

Table 6.4: Summary table of computational results with different levels of demand variability under BP setting

(a) with Uniformly Distributed Demand

\begin{tabular}{|c|c|c|c|c|}
\hline $\begin{array}{l}\text { TC } \\
\text { No. }\end{array}$ & DV & $\begin{array}{c}\text { LRP } \\
\text { Profitability }\end{array}$ & $\begin{array}{c}\text { Liability } \\
\text { Ratio }\end{array}$ & $\begin{array}{l}\text { Budget } \\
\text { Used(\%) }\end{array}$ \\
\hline \multirow{5}{*}{1} & 0.05 & 3982.53 & 1.02 & 72.09 \\
\hline & 0.10 & 3982.04 & 1.04 & 77.23 \\
\hline & 0.15 & 3981.56 & 1.06 & 82.37 \\
\hline & 0.20 & 3981.07 & 1.08 & 87.51 \\
\hline & 0.25 & 3980.59 & 1.10 & 92.66 \\
\hline \multirow{5}{*}{2} & 0.05 & 4313.95 & 1.27 & 74.21 \\
\hline & 0.10 & 4338.98 & 1.29 & 79.05 \\
\hline & 0.15 & 4363.27 & 1.31 & 83.83 \\
\hline & 0.20 & 4387.35 & 1.33 & 88.61 \\
\hline & 0.25 & 4411.36 & 1.35 & 93.40 \\
\hline \multirow{5}{*}{3} & 0.05 & 6048.34 & 1.25 & 84.17 \\
\hline & 0.10 & 6111.25 & 1.27 & 88.79 \\
\hline & 0.15 & 6169.31 & 1.29 & 92.88 \\
\hline & 0.20 & 6220.19 & 1.32 & 95.37 \\
\hline & 0.25 & 6257.19 & 1.34 & 96.90 \\
\hline \multirow{5}{*}{4} & 0.05 & 26003.79 & 0.60 & 83.32 \\
\hline & 0.10 & 25884.58 & 0.61 & 87.95 \\
\hline & 0.15 & 25745.84 & 0.62 & 92.27 \\
\hline & 0.20 & 25582.89 & 0.64 & 96.00 \\
\hline & 0.25 & 25394.13 & 0.65 & 99.08 \\
\hline
\end{tabular}

(b) with Normally Distributed Demand

\begin{tabular}{|c|c|c|c|c|}
\hline $\begin{array}{c}\text { TC } \\
\text { No. }\end{array}$ & DV & $\begin{array}{c}\text { LRP } \\
\text { Profitability }\end{array}$ & $\begin{array}{c}\text { Liability } \\
\text { Ratio }\end{array}$ & $\begin{array}{c}\text { Budget } \\
\text { Used(\%) }\end{array}$ \\
\hline \multirow{4}{*}{1} & 0.1 & 3980.64 & 1.01 & 68.39 \\
\cline { 2 - 5 } & 0.5 & 3971.13 & 1.03 & 74.14 \\
\cline { 2 - 5 } & 1.0 & 3959.24 & 1.05 & 81.34 \\
\cline { 2 - 5 } & 1.5 & 3947.36 & 1.08 & 88.54 \\
\cline { 2 - 5 } & 1.8 & 3940.23 & 1.10 & 92.86 \\
\hline \multirow{4}{*}{2} & 0.1 & 4293.20 & 1.26 & 70.55 \\
\cline { 2 - 5 } & 0.5 & 4311.82 & 1.28 & 75.66 \\
\cline { 2 - 5 } & 1.0 & 4333.60 & 1.30 & 81.84 \\
\cline { 2 - 5 } & 1.5 & 4354.90 & 1.33 & 88.02 \\
\cline { 2 - 5 } & 1.8 & 4368.03 & 1.35 & 91.72 \\
\hline \multirow{4}{*}{3} & 0.1 & 5999.51 & 1.24 & 80.27 \\
\cline { 2 - 5 } & 0.5 & 6062.32 & 1.26 & 85.01 \\
\cline { 2 - 5 } & 1.0 & 6136.65 & 1.29 & 90.37 \\
\cline { 2 - 5 } & 1.5 & 6205.61 & 1.32 & 94.02 \\
\cline { 2 - 5 } & 1.8 & 6242.72 & 1.34 & 95.06 \\
\hline \multirow{4}{*}{4} & 0.1 & 26073.03 & 0.59 & 79.58 \\
\hline & 0.5 & 25949.75 & 0.60 & 84.91 \\
\hline & 1.0 & 25768.85 & 0.62 & 90.81 \\
\hline & 1.5 & 25558.23 & 0.64 & 95.71 \\
\hline & 1.8 & 25420.08 & 0.65 & 98.19 \\
\hline
\end{tabular}


Table 6.4: Summary table of computational results with different levels of demand variability under BP setting (Cont.)

(a) with Uniformly Distributed Demand

\begin{tabular}{|c|c|c|c|c|}
\hline $\begin{array}{l}\text { TC } \\
\text { No. }\end{array}$ & DV & $\begin{array}{c}\text { LRP } \\
\text { Profitability }\end{array}$ & $\begin{array}{c}\text { Liability } \\
\text { Ratio }\end{array}$ & $\begin{array}{c}\text { Budget } \\
\text { Used(\%) }\end{array}$ \\
\hline \multirow{5}{*}{5} & 0.05 & 24702.56 & 1.20 & 88.52 \\
\hline & 0.10 & 24819.33 & 1.22 & 93.17 \\
\hline & 0.15 & 24902.59 & 1.24 & 96.55 \\
\hline & 0.20 & 24936.42 & 1.27 & 98.30 \\
\hline & 0.25 & 24927.07 & 1.30 & 99.25 \\
\hline \multirow{5}{*}{6} & 0.05 & 36666.07 & 1.01 & 83.44 \\
\hline & 0.10 & 36789.67 & 1.03 & 88.09 \\
\hline & 0.15 & 36863.62 & 1.05 & 91.88 \\
\hline & 0.20 & 36889.60 & 1.08 & 94.87 \\
\hline & 0.25 & 36861.70 & 1.11 & 97.08 \\
\hline \multirow{5}{*}{7} & 0.05 & 59578.89 & 0.85 & 81.63 \\
\hline & 0.10 & 59576.17 & 0.87 & 86.00 \\
\hline & 0.15 & 59544.94 & 0.89 & 90.00 \\
\hline & 0.20 & 59466.84 & 0.91 & 93.45 \\
\hline & 0.25 & 59316.55 & 0.93 & 96.33 \\
\hline \multirow{5}{*}{8} & 0.05 & 65567.84 & 1.16 & 83.73 \\
\hline & 0.10 & 65844.95 & 1.18 & 88.17 \\
\hline & 0.15 & 66072.34 & 1.20 & 92.00 \\
\hline & 0.20 & 66184.62 & 1.23 & 94.92 \\
\hline & 0.25 & 66199.26 & 1.25 & 97.37 \\
\hline \multirow{5}{*}{9} & 0.05 & 84760.01 & 0.91 & 87.15 \\
\hline & 0.10 & 84657.97 & 0.93 & 90.85 \\
\hline & 0.15 & 84413.78 & 0.96 & 93.96 \\
\hline & 0.20 & 84078.08 & 0.98 & 96.54 \\
\hline & 0.25 & 83652.86 & 1.01 & 98.44 \\
\hline \multirow{5}{*}{10} & 0.05 & 99565.95 & 0.9 & 86.69 \\
\hline & 0.10 & 99511.84 & 0.92 & 90.59 \\
\hline & 0.15 & 99254.77 & 0.94 & 93.64 \\
\hline & 0.20 & 98821.74 & 0.97 & 96.06 \\
\hline & 0.25 & 98258.78 & 1.00 & 97.99 \\
\hline
\end{tabular}

(b) with Normally Distributed Demand

\begin{tabular}{|c|c|c|c|c|}
\hline $\begin{array}{l}\text { TC } \\
\text { No. }\end{array}$ & DV & $\begin{array}{c}\text { LRP } \\
\text { Profitability }\end{array}$ & $\begin{array}{c}\text { Liability } \\
\text { Ratio }\end{array}$ & $\begin{array}{c}\text { Budget } \\
\text { Used(\%) }\end{array}$ \\
\hline \multirow{5}{*}{5} & 0.1 & 24595.88 & 1.18 & 84.52 \\
\hline & 0.5 & 24696.73 & 1.20 & 89.79 \\
\hline & 1.0 & 24799.21 & 1.23 & 95.03 \\
\hline & 1.5 & 24856.23 & 1.26 & 98.03 \\
\hline & 1.8 & 24864.56 & 1.29 & 98.90 \\
\hline \multirow{5}{*}{6} & 0.1 & 36541.35 & 1.00 & 79.57 \\
\hline & 0.5 & 36671.37 & 1.02 & 84.50 \\
\hline & 1.0 & 36794.06 & 1.05 & 89.82 \\
\hline & 1.5 & 36862.55 & 1.08 & 93.92 \\
\hline & 1.8 & 36877.88 & 1.10 & 95.80 \\
\hline \multirow{5}{*}{7} & 0.1 & 59507.29 & 0.83 & 78.09 \\
\hline & 0.5 & 59488.76 & 0.85 & 82.74 \\
\hline & 1.0 & 59389.32 & 0.88 & 88.10 \\
\hline & 1.5 & 59246.24 & 0.91 & 92.62 \\
\hline & 1.8 & 59129.67 & 0.92 & 94.77 \\
\hline \multirow{5}{*}{8} & 0.1 & 65306.44 & 1.14 & 80.01 \\
\hline & 0.5 & 65551.02 & 1.16 & 84.93 \\
\hline & 1.0 & 65796.13 & 1.19 & 90.27 \\
\hline & 1.5 & 65936.77 & 1.22 & 94.08 \\
\hline & 1.8 & 65964.39 & 1.24 & 95.97 \\
\hline \multirow{5}{*}{9} & 0.1 & 84673.54 & 0.90 & 83.45 \\
\hline & 0.5 & 84680.46 & 0.92 & 88.10 \\
\hline & 1.0 & 84458.14 & 0.95 & 92.34 \\
\hline & 1.5 & 84097.06 & 0.98 & 95.85 \\
\hline & 1.8 & 83837.28 & 1.00 & 97.44 \\
\hline \multirow{5}{*}{10} & 0.1 & 99417.19 & 0.89 & 82.93 \\
\hline & 0.5 & 99479.74 & 0.91 & 87.72 \\
\hline & 1.0 & 99287.42 & 0.94 & 92.05 \\
\hline & 1.5 & 98848.16 & 0.97 & 95.15 \\
\hline & 1.8 & 98502.04 & 0.99 & 96.62 \\
\hline
\end{tabular}


With respect to the actual liability ratio $\left(l^{\prime}\right)$, in some testing cases (i.e., TC No. $2,3,5$, and 8 ) the liability ratio is strictly larger than 1.0 with different DVs, while in other testing cases the liability ratio is around 1.0 (i.e., cases $1,6,9$, and 10) or strictly less than 1.0 (i.e., cases 4 and 7 ), with different DVs. Overall, with the increase of demand variability, the liability ratio always increases in each of the testing cases. This is because when demand variability increases, the overall accumulation will increase, while the overall redemption quantity will decrease. For example, in the testing case 10 , the accumulation (i.e., the number of accumulated points) increases from 2040.792 at $\mathrm{DV}=0.05$ to 2213.96 at $\mathrm{DV}=0.25$ given uniformly distributed demand and from 2008.456 at $\mathrm{DV}=0.1$ to 2194.924 at $\mathrm{DV}=1.8$ given normally distributed demand. The redemption (i.e., the number of redeemed points) decreases from 2313.632 to 2227.693 given uniformly distributed demand, and from 2318. 269 to 2233.97 given normally distributed demand. When demand variability changes from small to large, the increasing rates of the liability ratio in terms of percentage are between $6 \%$ and $11 \%$ in the case of uniformly distributed demand, and between $7 \%$ and $12 \%$ in the case of normally distributed demand.

In regards to the budget usage, it increases with the demand variability in all problem testing cases given both normally distributed and uniformly distributed demand. With increasing demand variability, the increasing rate of the budget usage (in percentage) is between $12 \%$ and $29 \%$ in the case of uniformly distributed demand, and between $16 \%$ and $36 \%$ in the case of normally distributed demand. Compared to the changes in the profitability and in the liability ratio, changes in the budget usage are remarkably larger due to the increases in overall rewards ordering quantity when demand variability increases. In effect, the overall rewards ordering quantity increases with the increase of demand variability, which results in an increase of budget 
usage for each testing case. For example, in TC No.1, the total ordering quantity of rewards increases from 48.456 at $\mathrm{DV}=0.05$ to 62.28 at $\mathrm{DV}=0.25$ in uniformly distributed cases and from 45.967 at $\mathrm{DV}=0.1$ to 62.414 at $\mathrm{DV}=1.8$ in normally distributed cases.

The above computational results and analysis indicate that under BP setting, demand variability does have a negative impact on LPR profitability (i.e., higher DVs, lower profitability), especially when the number of redemption partners is greater than the number of accumulation partners (i.e., $N R>N A$ ). The negative impact of demand variability on an LRP host firm's liability ratios (i.e., higher DVs, higher liability) seems more noticeable compared to the LRP profitability, as the host firm's liability ratio increases in all testing cases, with the increases in demand variability given normally distributed demand or uniformly distributed demand. Also, demand variability has a negative impact on budget usage (i.e., higher DVs, higher budget usage). The increases in budget usage in terms of percentage have reached up to $35 \%$ in the testing cases. We also find that the impacts of demand variability on LRP performance in terms of profitability, liability ratio, and budget usage are consistent between normally distributed demand and uniformly distributed demand.

\subsubsection{Under EP1 Setting}

In Table 6.5, we report LRP profitability, actual liability ratio $\left(l^{\prime}\right)$, and budget usage (in percentage) under EP1 setting with demand variability changing from small to large. 
Table 6.5: Summary table of computational results with different levels of demand variability under EP1 setting

\begin{tabular}{|c|c|c|c|c|}
\hline \multicolumn{5}{|c|}{ (a) with Uniformly Distributed Demand } \\
\hline $\begin{array}{l}\text { TC } \\
\text { No. }\end{array}$ & DV & $\begin{array}{c}\text { LRP } \\
\text { Profitability }\end{array}$ & $\begin{array}{l}\text { Liability } \\
\text { Ratio }\end{array}$ & $\begin{array}{l}\text { Budget } \\
\text { Used(\%) }\end{array}$ \\
\hline \multirow{5}{*}{1} & 0.05 & 4039.82 & 1.15 & 72.09 \\
\hline & 0.10 & 4023.91 & 1.15 & 77.23 \\
\hline & 0.15 & 4016.06 & 1.15 & 82.37 \\
\hline & 0.20 & 4010.23 & 1.16 & 87.51 \\
\hline & 0.25 & 4005.19 & 1.17 & 92.66 \\
\hline \multirow{5}{*}{2} & 0.05 & 4472.99 & 1.40 & 74.21 \\
\hline & 0.10 & 4454.92 & 1.40 & 79.05 \\
\hline & 0.15 & 4458.79 & 1.40 & 83.83 \\
\hline & 0.20 & 4468.06 & 1.41 & 88.61 \\
\hline & 0.25 & 4479.43 & 1.42 & 93.40 \\
\hline \multirow{5}{*}{3} & 0.05 & 6377.41 & 1.38 & 84.17 \\
\hline & 0.10 & 6351.02 & 1.38 & 88.79 \\
\hline & 0.15 & 6366.51 & 1.39 & 92.88 \\
\hline & 0.20 & 6386.59 & 1.40 & 95.37 \\
\hline & 0.25 & 6397.51 & 1.41 & 96.90 \\
\hline \multirow{5}{*}{4} & 0.05 & 26400.97 & 0.67 & 83.32 \\
\hline & 0.10 & 26173.98 & 0.67 & 87.95 \\
\hline & 0.15 & 25984.05 & 0.68 & 92.27 \\
\hline & 0.20 & 25784.06 & 0.68 & 96.00 \\
\hline & 0.25 & 25563.89 & 0.69 & 99.08 \\
\hline \multirow{5}{*}{5} & 0.05 & 25720.90 & 1.33 & 88.52 \\
\hline & 0.10 & 25562.46 & 1.33 & 93.17 \\
\hline & 0.15 & 25514.22 & 1.34 & 96.55 \\
\hline & 0.20 & 25452.87 & 1.35 & 98.30 \\
\hline & 0.25 & 25362.93 & 1.37 & 99.25 \\
\hline \multirow{5}{*}{6} & 0.05 & 38007.51 & 1.14 & 83.44 \\
\hline & 0.10 & 37769.25 & 1.14 & 88.09 \\
\hline & 0.15 & 37670.12 & 1.15 & 91.88 \\
\hline & 0.20 & 37570.64 & 1.16 & 94.87 \\
\hline & 0.25 & 37436.32 & 1.18 & 97.08 \\
\hline
\end{tabular}

\begin{tabular}{|c|c|c|c|c|}
\hline \multicolumn{5}{|c|}{ (b) with Normally Distributed Demand } \\
\hline $\begin{array}{l}\text { TC } \\
\text { No. }\end{array}$ & DV & $\begin{array}{c}\text { LRP } \\
\text { Profitability }\end{array}$ & $\begin{array}{c}\text { Liability } \\
\text { Ratio }\end{array}$ & $\begin{array}{l}\text { Budget } \\
\text { Used(\%) }\end{array}$ \\
\hline \multirow{5}{*}{1} & 0.1 & 4055.35 & 1.16 & 68.39 \\
\hline & 0.5 & 4016.62 & 1.14 & 74,14 \\
\hline & 1.0 & 3985.72 & 1.12 & 81.34 \\
\hline & 1.5 & 3962.05 & 1.12 & 88.54 \\
\hline & 1.8 & 3948.97 & 1.12 & 92.86 \\
\hline \multirow{5}{*}{2} & 0.1 & 4499.44 & 1.41 & 70.55 \\
\hline & 0.5 & 4435.02 & 1.38 & 75.66 \\
\hline & 1.0 & 4403.02 & 1.37 & 81.84 \\
\hline & 1.5 & 4390.85 & 1.37 & 88.02 \\
\hline & 1.8 & 4390.31 & 1.37 & 91.72 \\
\hline \multirow{5}{*}{3} & 0.1 & 6424.25 & 1.39 & 80.27 \\
\hline & 0.5 & 6311.27 & 1.36 & 85.01 \\
\hline & 1.0 & 6274.51 & 1.35 & 90.37 \\
\hline & 1.5 & 6275.96 & 1.35 & 94.02 \\
\hline & 1.8 & 6283.85 & 1.36 & 95.06 \\
\hline \multirow{5}{*}{4} & 0.1 & 26583.35 & 0.67 & 79.58 \\
\hline & 0.5 & 26242.15 & 0.66 & 84.91 \\
\hline & 1.0 & 25924.65 & 0.66 & 90.81 \\
\hline & 1.5 & 25633.73 & 0.66 & 95.71 \\
\hline & 1.8 & 25466.15 & 0.66 & 98.19 \\
\hline \multirow{5}{*}{5} & 0.1 & 25918.07 & 1.34 & 84.52 \\
\hline & 0.5 & 25494.61 & 1.31 & 89.79 \\
\hline & 1.0 & 25254.70 & 1.30 & 95.03 \\
\hline & 1.5 & 25104.29 & 1.31 & 98.03 \\
\hline & 1.8 & 25017.56 & 1.31 & 98.90 \\
\hline \multirow{5}{*}{6} & 0.1 & 38276.45 & 1.15 & 79.57 \\
\hline & 0.5 & 37702.85 & 1.12 & 84.50 \\
\hline & 1.0 & 37372.80 & 1.11 & 89.82 \\
\hline & 1.5 & 37165.54 & 1.11 & 93.92 \\
\hline & 1.8 & 37061.49 & 1.12 & 95.80 \\
\hline
\end{tabular}


Table 6.5: Summary table of computational results with different levels of demand variability under EP1 setting (Cont.)

(a) with Uniformly Distributed Demand

\begin{tabular}{|c|c|c|c|c|}
\hline $\begin{array}{c}\text { TC } \\
\text { No. }\end{array}$ & DV & $\begin{array}{c}\text { LRP } \\
\text { Profitability }\end{array}$ & $\begin{array}{c}\text { Liability } \\
\text { Ratio }\end{array}$ & $\begin{array}{c}\text { Budget } \\
\text { Used(\%) }\end{array}$ \\
\hline \multirow{4}{*}{7} & 0.05 & 61364.47 & 0.96 & 81.63 \\
\cline { 2 - 5 } & 0.10 & 60880.35 & 0.96 & 86.00 \\
\cline { 2 - 5 } & 0.15 & 60619.24 & 0.96 & 90.00 \\
\cline { 2 - 5 } & 0.20 & 60374.31 & 0.97 & 93.45 \\
\cline { 2 - 5 } & 0.25 & 60082.33 & 0.99 & 96.33 \\
\hline \multirow{5}{*}{8} & 0.05 & 68095.71 & 1.29 & 83.73 \\
\cline { 2 - 5 } & 0.10 & 67689.66 & 1.29 & 88.17 \\
\cline { 2 - 5 } & 0.15 & 67591.30 & 1.30 & 92.00 \\
\cline { 2 - 5 } & 0.20 & 67467.41 & 1.31 & 94.92 \\
\cline { 2 - 5 } & 0.25 & 67281.61 & 1.32 & 97.37 \\
\hline \multirow{5}{*}{10} & 0.05 & 87366.57 & 1.03 & 87.15 \\
\cline { 2 - 5 } & 0.10 & 86560.48 & 1.03 & 90.85 \\
\cline { 2 - 5 } & 0.15 & 85980.61 & 1.04 & 93.96 \\
\cline { 2 - 5 } & 0.20 & 85401.45 & 1.06 & 96.54 \\
\cline { 2 - 5 } & 0.25 & 84769.57 & 1.07 & 98.44 \\
\hline \multirow{4}{*}{10} & 0.05 & 102565.78 & 1.02 & 86.69 \\
\cline { 2 - 5 } & 0.10 & 101700.54 & 1.02 & 90.59 \\
\cline { 2 - 5 } & 0.15 & 101056.97 & 1.03 & 93.64 \\
\hline & 0.20 & 100343.94 & 1.04 & 96.06 \\
\cline { 2 - 5 } & 0.25 & 99543.22 & 1.06 & 97.99 \\
\hline
\end{tabular}

(b) with Normally Distributed Demand

\begin{tabular}{|c|c|c|c|c|}
\hline $\begin{array}{c}\text { TC } \\
\text { No. }\end{array}$ & DV & $\begin{array}{c}\text { LRP } \\
\text { Profitability }\end{array}$ & $\begin{array}{c}\text { Liability } \\
\text { Ratio }\end{array}$ & $\begin{array}{c}\text { Budget } \\
\text { Used(\%) }\end{array}$ \\
\hline \multirow{5}{*}{7} & 0.1 & 61816.47 & 0.96 & 78.09 \\
\cline { 2 - 5 } & 0.5 & 60860.36 & 0.94 & 82.74 \\
\cline { 2 - 5 } & 1.0 & 60157.98 & 0.93 & 88.10 \\
\cline { 2 - 5 } & 1.5 & 59645.41 & 0.94 & 92.62 \\
\cline { 2 - 5 } & 1.8 & 59371.22 & 0.94 & 94.77 \\
\hline \multirow{5}{*}{8} & 0.1 & 68585.85 & 1.30 & 80.01 \\
\cline { 2 - 5 } & 0.5 & 67514.14 & 1.27 & 84.93 \\
\cline { 2 - 5 } & 1.0 & 66909.12 & 1.26 & 90.27 \\
\cline { 2 - 5 } & 1.5 & 66529.72 & 1.26 & 94.08 \\
\cline { 2 - 5 } & 1.8 & 66322.74 & 1.27 & 95.97 \\
\hline \multirow{5}{*}{9} & 0.1 & 88039.46 & 1.03 & 83.45 \\
\cline { 2 - 5 } & 0.5 & 86665.99 & 1.01 & 88.10 \\
\cline { 2 - 5 } & 1.0 & 85564.03 & 1.01 & 92.34 \\
\cline { 2 - 5 } & 1.5 & 84663.48 & 1.01 & 95.85 \\
\cline { 2 - 5 } & 1.8 & 84180.86 & 1.02 & 97.44 \\
\hline \multirow{5}{*}{10} & 0.1 & 103298.58 & 1.02 & 82.93 \\
\cline { 2 - 5 } & 0.5 & 101786.43 & 1.00 & 87.72 \\
\cline { 2 - 5 } & 1.0 & 100581.82 & 0.99 & 92.05 \\
\hline & 1.5 & 99524.36 & 1.00 & 95.15 \\
\hline & 1.8 & 98915.22 & 1.01 & 96.62 \\
\hline
\end{tabular}

Based on the computational outputs, we observe that under EP1 setting, LRP profitability decreases with the increases in demand variability in all testing cases with a few exceptions (i.e., TC No. 2 and 3). The average rate of decrease (in percentage) of LRP profitability is $2.02 \%$ with uniformly distributed demand and $3.4 \%$ with normally distributed demand. In testing cases 2 and 3, with uniformly distribution demands, the LRP profitability decreases with DV changes from 0.05 to 0.10 and then increases with DV changes from 0.10 to 0.25 . As explained in Section 6.2.1, LRP profitability from the accumulation side always increases with the increase of 
variability in accumulation demand and LRP profitability from the redemption side always decreases with the increase of variability in redemption demand. When the increase in the accumulation-side profitability is higher than the decrease in the redemption-side profitability, the overall profitability will increase. On the other hand, when the increase in the accumulationside profitability is lower than the decrease in the redemption-side profitability, the overall profitability will increase.

In regards to the actual liability ratio, in some of the testing cases (i.e., TC No.1, 2, 3, 5, 6, and 8) the liability ratio is strictly larger than 1.0 , while in the rest of the testing cases the liability ratio is either strictly less than 1.0 (i.e., TC No. 4) or around 1.0 (i.e., TC No. 7, 9, and 10). Under EP1 setting, the impact of demand variability on liability ratio seems much smaller than that under BP setting. With the changes in demand variability, the maximum change in liability ratio is around 0.05 in $\mathrm{EP} 1$, whereas in $\mathrm{BP}$, the maximum change in the liability ratio is around 0.1 .

With respect to the impact of demand variability on budget usage, the same phenomena are observed as those under BP setting. The increases in budget usage in terms of percentage are between $12 \%$ and $29 \%$ with uniformly distributed demand, and between $16 \%$ and $36 \%$ with normally distributed demand. This indicates that increases in demand variability force the LRP host to increase the ordering quantities of rewards to maximize LRP profitability, which in turn increases the budget usage.

In this setting, in addition to the ordering quantities of rewards, the LRP host will also need to decide on the number of bonus points to offer to each LRP accumulation partner. The 
computational results for bonus points are reported in Appendix C.1. In the table, "TQA" refers to the total volume of points ordered by LRP accumulation partners in each testing case. As we mentioned in Section 5.1, Chapter 5, TQA is one of the input parameters in our models. "TBA" refers to the total volume of bonus points to offer to the accumulation partners. In each testing case, the overall volume of bonus points to offer decreases with the increases in demand variability. With uniformly distributed demand, when the value of demand variability parameter (DV) changes from 0.05 to 0.25 , TBA reduces from around $8.5 \%$ of TQA to around $5.5 \%$ of TQA. With normally distributed demand, when the value of demand variability parameter (DV) changes from 0.1 to 1.8 , TBA reduces from around $9 \%$ of TQA to $1.7 \%$ of TQA. Those results indicate that when demand variability increases, offering bonus points is not beneficial for improving LRP profitability in rewards-supply planning.

Based on the above computational results and analysis, we observe that demand variability has a negative impact on LRP profitability, liability, and budget usage under EP1 setting. The increase in demand variability forces an LRP host to consider purchasing more rewards from redemption partners and offering fewer bonus points to accumulation partners, which indicates that demand variability does influence the LRP host's decisions on both rewards ordering quantities and offering bonus points.

\subsubsection{Under EP2 Setting}

In Table 6.6, we report LRP profitability, actual liability ratio ( $\left.l^{\prime}\right)$, and budget usages under EP2 setting with demand variability changing from small to large. 
Table 6.6: Summary table of computational results with different levels of demand variability under EP2 Setting

(a) with Uniformly Distributed Demand

\begin{tabular}{|c|c|c|c|c|}
\hline $\begin{array}{l}\mathrm{TC} \\
\text { No. }\end{array}$ & DV & Profitability & $\begin{array}{c}\text { Liability } \\
\text { Ratio }\end{array}$ & $\begin{array}{c}\text { Budget } \\
\text { Used }(\%)\end{array}$ \\
\hline \multirow{5}{*}{1} & 0.05 & 3988.24 & 1.02 & 70.04 \\
\hline & 0.10 & 3993.46 & 1.04 & 73.13 \\
\hline & 0.15 & 3998.68 & 1.06 & 76.22 \\
\hline & 0.20 & 4003.90 & 1.08 & 79.31 \\
\hline & 0.25 & 4009.12 & 1.10 & 82.40 \\
\hline \multirow{5}{*}{2} & 0.05 & 4321.42 & 1.27 & 72.44 \\
\hline & 0.10 & 4354.34 & 1.29 & 75.64 \\
\hline & 0.15 & 4387.33 & 1.31 & 78.82 \\
\hline & 0.20 & 4420.39 & 1.32 & 81.97 \\
\hline & 0.25 & 4452.92 & 1.34 & 85.08 \\
\hline \multirow{5}{*}{3} & 0.05 & 6061.41 & 1.25 & 82.68 \\
\hline & 0.10 & 6139.52 & 1.27 & 86.31 \\
\hline & 0.15 & 6217.30 & 1.29 & 89.91 \\
\hline & 0.20 & 6293.90 & 1.31 & 93.35 \\
\hline & 0.25 & 6367.79 & 1.33 & 95.79 \\
\hline \multirow{5}{*}{4} & 0.05 & 26102.01 & 0.60 & 81.70 \\
\hline & 0.10 & 26114.80 & 0.61 & 85.23 \\
\hline & 0.15 & 26123.92 & 0.62 & 88.67 \\
\hline & 0.20 & 26103.00 & 0.63 & 91.87 \\
\hline & 0.25 & 26048.32 & 0.64 & 95.00 \\
\hline \multirow{5}{*}{5} & 0.05 & 24778.14 & 1.20 & 86.94 \\
\hline & 0.10 & 24990.78 & 1.22 & 90.71 \\
\hline & 0.15 & 25201.29 & 1.23 & 94.38 \\
\hline & 0.20 & 25403.55 & 1.25 & 97.11 \\
\hline & 0.25 & 25547.23 & 1.28 & 97.94 \\
\hline \multirow{5}{*}{6} & 0.05 & 36744.12 & 1.01 & 81.91 \\
\hline & 0.10 & 36982.92 & 1.03 & 85.48 \\
\hline & 0.15 & 37222.90 & 1.05 & 89.01 \\
\hline & 0.20 & 37453.94 & 1.07 & 92.39 \\
\hline & 0.25 & 37654.63 & 1.09 & 95.62 \\
\hline
\end{tabular}

(b) with Normally Distributed Demand

\begin{tabular}{|c|c|c|c|c|}
\hline $\begin{array}{l}\text { TC } \\
\text { No. }\end{array}$ & DV & Profitability & $\begin{array}{c}\text { Liability } \\
\text { Ratio }\end{array}$ & $\begin{array}{l}\text { Budget } \\
\text { Used(\%) }\end{array}$ \\
\hline \multirow{5}{*}{1} & 0.1 & 3982.68 & 1.01 & 67.77 \\
\hline & 0.5 & 3981.35 & 1.03 & 71.05 \\
\hline & 1.0 & 3979.68 & 1.05 & 75.14 \\
\hline & 1.5 & 3976.30 & 1.08 & 79.82 \\
\hline & 1.8 & 3970.64 & 1.09 & 83.85 \\
\hline \multirow{5}{*}{2} & 0.1 & 4295.63 & 1.26 & 70.05 \\
\hline & 0.5 & 4324.10 & 1.27 & 73.29 \\
\hline & 1.0 & 4359.71 & 1.30 & 77.30 \\
\hline & 1.5 & 4394.05 & 1.32 & 81.27 \\
\hline & 1.8 & 4411.79 & 1.34 & 84.26 \\
\hline \multirow{5}{*}{3} & 0.1 & 6003.52 & 1.24 & 79.90 \\
\hline & 0.5 & 6084.43 & 1.26 & 83.31 \\
\hline & 1.0 & 6183.23 & 1.28 & 87.45 \\
\hline & 1.5 & 6275.55 & 1.31 & 91.79 \\
\hline & 1.8 & 6330.02 & 1.32 & 94.44 \\
\hline \multirow{5}{*}{4} & 0.1 & 26090.70 & 0.59 & 79.04 \\
\hline & 0.5 & 26093.67 & 0.60 & 82.66 \\
\hline & 1.0 & 26095.38 & 0.61 & 87.03 \\
\hline & 1.5 & 26052.86 & 0.63 & 91.62 \\
\hline & 1.8 & 25999.64 & 0.64 & 94.55 \\
\hline \multirow{5}{*}{5} & 0.1 & 24613.97 & 1.18 & 84.09 \\
\hline & 0.5 & 24806.33 & 1.20 & 87.83 \\
\hline & 1.0 & 25041.36 & 1.23 & 92.44 \\
\hline & 1.5 & 25255.36 & 1.25 & 96.49 \\
\hline & 1.8 & 25360.08 & 1.27 & 97.48 \\
\hline \multirow{5}{*}{6} & 0.1 & 36563.78 & 1.00 & 79.14 \\
\hline & 0.5 & 36791.79 & 1.02 & 82.55 \\
\hline & 1.0 & 37075.97 & 1.04 & 86.79 \\
\hline & 1.5 & 37345.19 & 1.06 & 91.12 \\
\hline & 1.8 & 37482.86 & 1.08 & 93.87 \\
\hline
\end{tabular}


Table 6.6 Summary table of computational results with different levels of demand variability under EP2 Setting (Cont.)

(a) with Uniformly Distributed Demand

\begin{tabular}{|c|c|c|c|c|}
\hline $\begin{array}{c}\text { TC } \\
\text { No. }\end{array}$ & DV & Profitability & $\begin{array}{c}\text { Liability } \\
\text { Ratio }\end{array}$ & $\begin{array}{c}\text { Budget } \\
\text { Used(\%) }\end{array}$ \\
\hline \multirow{5}{*}{7} & 0.05 & 59735.47 & 0.85 & 80.34 \\
\cline { 2 - 5 } & 0.10 & 59974.75 & 0.86 & 83.79 \\
\cline { 2 - 5 } & 0.15 & 60217.29 & 0.88 & 87.17 \\
\cline { 2 - 5 } & 0.20 & 60426.33 & 0.89 & 90.36 \\
\cline { 2 - 5 } & 0.25 & 60578.63 & 0.91 & 93.51 \\
\hline \multirow{5}{*}{8} & 0.05 & 65730.73 & 1.16 & 82.30 \\
\cline { 2 - 5 } & 0.10 & 66226.32 & 1.17 & 85.84 \\
\cline { 2 - 5 } & 0.15 & 66722.78 & 1.19 & 89.29 \\
\cline { 2 - 5 } & 0.20 & 67192.34 & 1.21 & 92.52 \\
\cline { 2 - 5 } & 0.25 & 67605.01 & 1.23 & 95.74 \\
\hline \multirow{5}{*}{9} & 0.05 & 85028.66 & 0.91 & 85.83 \\
\cline { 2 - 5 } & 0.10 & 85418.32 & 0.93 & 89.47 \\
\cline { 2 - 5 } & 0.15 & 85814.35 & 0.94 & 92.94 \\
\cline { 2 - 5 } & 0.20 & 86134.35 & 0.96 & 95.98 \\
\cline { 2 - 5 } & 0.25 & 86305.89 & 0.98 & 98.21 \\
\hline \multirow{5}{*}{10} & 0.05 & 99849.83 & 0.90 & 85.30 \\
\hline & 0.10 & 100327.26 & 0.91 & 88.95 \\
\cline { 2 - 5 } & 0.15 & 100810.84 & 0.93 & 92.44 \\
\hline & 0.20 & 101215.82 & 0.95 & 95.56 \\
\hline & 0.25 & 101438.54 & 0.97 & 98.19 \\
\hline \multirow{4}{*}{} & & & & \\
\hline
\end{tabular}

(b) with Normally Distributed Demand

\begin{tabular}{|c|c|c|c|c|}
\hline $\begin{array}{c}\text { TC } \\
\text { No. }\end{array}$ & DV & Profitability & $\begin{array}{c}\text { Liability } \\
\text { Ratio }\end{array}$ & $\begin{array}{c}\text { Budget } \\
\text { Used(\%) }\end{array}$ \\
\hline \multirow{4}{*}{7} & 0.1 & 59547.60 & 0.83 & 77.67 \\
\cline { 2 - 5 } & 0.5 & 59734.32 & 0.85 & 81.11 \\
\cline { 2 - 5 } & 1.0 & 59968.57 & 0.87 & 85.33 \\
\cline { 2 - 5 } & 1.5 & 60156.80 & 0.89 & 89.52 \\
\cline { 2 - 5 } & 1.8 & 60218.49 & 0.91 & 92.30 \\
\hline \multirow{5}{*}{8} & 0.1 & 65349.54 & 1.14 & 79.57 \\
\cline { 2 - 5 } & 0.5 & 65793.85 & 1.16 & 83.10 \\
\cline { 2 - 5 } & 1.0 & 66350.07 & 1.19 & 87.44 \\
\cline { 2 - 5 } & 1.5 & 66856.72 & 1.21 & 91.88 \\
\cline { 2 - 5 } & 1.8 & 67117.20 & 1.23 & 94.60 \\
\hline \multirow{5}{*}{10} & 0.1 & 84731.09 & 0.90 & 83.00 \\
\cline { 2 - 5 } & 0.5 & 85075.23 & 0.92 & 86.61 \\
\cline { 2 - 5 } & 1.0 & 85505.44 & 0.94 & 91.02 \\
\cline { 2 - 5 } & 1.5 & 85849.92 & 0.96 & 94.95 \\
\cline { 2 - 5 } & 1.8 & 85953.12 & 0.98 & 97.22 \\
\hline \multirow{4}{*}{10} & 0.1 & 99483.72 & 0.89 & 82.46 \\
\hline & 0.5 & 99902.41 & 0.90 & 86.06 \\
\hline & 1.0 & 100427.16 & 0.93 & 90.46 \\
\hline & 1.5 & 100841.15 & 0.95 & 94.66 \\
\hline & 1.8 & 100987.79 & 0.96 & 96.99 \\
\hline
\end{tabular}

Our computational results show that LRP profitability increases with the increases in demand variability in all testing cases except testing TC No.4. In that testing case, the profitability increases slightly and then decreases slightly with the increasing demand variability. The reason behind these results is the same as that under BP setting: profitability from the accumulation business always increases with the increase of demand variability due to the assumption that the accumulation demand will always been met. Meanwhile, profitability from the redemption business always decreases with the increase of demand variability due to the increased cost of 
purchasing rewards (i.e., the need to order more rewards when demand variability is higher). When the increase in the accumulation-side profitability is higher (lower) than the decrease in the redemption-side profitability, the overall profitability will increase (decrease). However, unlike in BP model, in EP2 model the increases in demand variability most of the time enhance LRP profitability rather than lower it. The average and maximum increases in LRP profitability among all the testing cases are $2.14 \%$ and $5.05 \%$ with uniformly distributed demand. With normally distributed demand they are $1.98 \%$ and $5.44 \%$.

With regard to liability ratio, overall it increases with the increases in demand variability. This indicates that when demand variability is larger, LRP liability is expected to increase due to increased accumulation and decreased redemption. These results are very similar to those that we obtained under BP setting. When demand variability increases, the increases in the liability ratio in terms of percentage are between $5 \%$ and $8 \%$ with uniformly distributed demand, and between $6 \%$ and $9 \%$ with normally distributed demand.

Similar results are obtained with budget usage: with the increases in demand variability, the budget usage increases in all testing cases with normally distributed or uniformly distributed demands. The changes in budget usage are much larger when demand variability is larger. With the highest level of demand variability, the increases in budget usage in terms of percentage are between $12 \%$ and $18 \%$ when demand is uniformly distributed and between $16 \%$ and $24 \%$ when demand is normally distributed. 
Under EP2 setting, in addition to the initial ordering quantity of rewards, an LRP host has to decide on the total number of options to purchase and to exercise. We find that an LRP host firm's best decision, when facing an increase in demand variability, is to increase the overall rewards ordering quantity (including both the initial ordering quantity of rewards and the total number of options to purchase). This also results in increases in budget usage. Appendix C.2 reports the computational results related to options to purchase and to exercise. In this table, "TQR" refers to the total number of rewards (in points) to order, "TOR" refers to the total number of options (in points) to purchase, and "TOE" refers to the total number of options (in points) to exercise. With uniformly distributed demand, when the value of demand variability parameter (DV) changes from 0.05 to 0.25 , TOR increases from around $5 \%$ of TQR to around $21 \%$ of TQR. With normally distributed demand, when the value of demand variability parameter (DV) changes from 0.1 to 1.8 , TOR increases from around $1.7 \%$ of TQR to around $22 \%$ of TQR. Meanwhile, the number of options to exercise is around $18 \%-26 \%$ of the number of options to purchase when demand is uniformly distributed, and $13 \%-20 \%$ when demand is normally distributed. These results suggest that option contract does play a role in dealing with demand variability.

Based on the above computational results and analysis, we find that demand variability does have negative impacts on LRP liability and budget usage (i.e., higher demand variability results in higher LRP liability and budget usage). Meanwhile, the increases in demand variability also force the LRP host to consider purchasing more rewards from the redemption partners (i.e., both the initial ordering quantity and the number of options to purchase have increased). This suggests that demand variability does influence an LRP host's decisions on both rewards ordering 
quantities and the number of options to purchase. Another interesting finding is that demand variability also causes changes in LRP profitability, but higher demand variability results in higher LRP profitability under EP2 setting. This indicates that using option contracts can mitigate the negative impacts of demand variability on LRP profitability.

\subsubsection{Comparisons across BP, EP1, and EP2 Model Settings}

In Sections 6.2.1 - 6.2.3 we examine the impacts of demand variability under BP, EP1, and EP2 model settings, respectively. This section investigates the impacts of demand variability on LRP performance (in terms of LRP profitability, liability ratio, and budget usage) across BP, EP1, and EP2 model settings.

Appendix C.3 shows that, although the LRP profitability curves of BP, EP1, and EP2 models are slightly different in different testing cases, the overall pattern seems quite similar. When demand variability is lower (e.g., $\mathrm{DV}=0.1$, or $\mathrm{DV}=0.05$ ), $\mathrm{BP}$ and $\mathrm{EP} 2$ models generate almost the same profitability, while EP1 model generates much higher profitability due to bonus points offering. However, with the increases in demand variability, the advantage of EP1 model in terms of profitability is weakening. In contrast to that, in almost all the testing cases EP2 model results in higher profitability than BP and EP1 models under high demand variability (e.g., DV=1.8, or $\mathrm{DV}=0.25$ ). These results suggest that adopting option contracts is more attractive when demand variability is higher. 
Appendix C. 4 shows that the liability ratio is quite different among different testing cases (e.g., the liability ratio is less than 0.8 in testing case 4 , while it is more than 1.2 in testing case 1 ). Overall, in each of the testing cases, the liability ratios under BP and EP2 settings are almost the same. However, due to the bonus points offering (i.e., creating a higher accumulation), EP1 model always generates a higher liability ratio than BP and EP2 models do. However, when demand variability increases, in comparison to EP1 model, the advantage of BP and EP2 models in terms of liability ratio is not very obvious. These results suggest that an option contract does play a role in keeping liability relatively low and generating relatively high profitability, especially when the LRP host faces high demand variability.

With regards to budget usage, as reported in Appendix C.5, we observe that it increases with the increases in demand variability. In BP and EP1 models, budget usage is exactly the same in each of our testing cases. However, EP2 model results in lower budget usage than BP and EP1 models, and the differences in budget usage are more notable with higher demand variability (e.g., see TC No. 1, 2, 4, 7, and 8). In addition to that, EP2's advantage in budget usage seems to be weakened in larger-sized problems (e.g., see TC No.9 and 10). These results suggest that option contracts do play a role in keeping budget usage relatively lower when the LRP host faces higher demand variability. However, the LRP host does need to pay more attention to the design of option contracts in the presence of quite a large number of LRP partners.

\subsubsection{Summary}

The computational results from the numerical experiments reveal that high demand variability does have some negative impacts, especially under BP setting, on LRP performance (in terms of 
profitability, liability ratio, and budget usage). Although EP1 setting (i.e., offering bonus points) can achieve higher profitability when demand variability is relatively low, it also results in higher liability and higher budget usage than EP2 setting (i.e., using option contracts). Therefore, overall, using option contracts one can achieve better LRP performance than not using option contracts and/or offering bonus points, especially in a situation where demand variability is high. LRP managers should pay some attention to the negative impacts of demand variability and take some actions to reduce the negative impacts through either matching demand variability (e.g., using option contract as in EP2 setting), especially when facing relatively high demand variability or leveraging demand variability (e.g., using promotions such as bonus points, as in EP1 setting) when facing relatively low demand variability. 


\subsection{Testing the Impacts of Budget Tightness}

This set of numerical studies has been conducted to examine the impacts of redemption budget tightness on LRP performance in terms of LRP profitability and liability, and the LRP host's decisions. We also investigate whether the impacts vary among different model settings (i.e., BP, EP1, and EP2) or under different demand variability levels. The redemption budget is one of the internal dynamics in LRPs.

Given that the budget usage in all our original testing problems (i.e., those considered in Section 6.2) is lower than $100 \%$, we used them as basis for comparison with budget tightness parameter $\alpha=0$ (i.e., low budget tightness). We set the tightness parameter $\alpha=0.3$ for high budget tightness, since at that level, the budget usage in most of our testing problems reaches to or above $100 \%$.

\subsubsection{Under BP Setting}

Table 6.7 shows the computational outputs from both tight and loose budget limits under BP setting. We find that no matter what the demand distribution (uniform or normal) is, the average LRP profitability and the average ordering quantity of rewards with a tight budget (i.e., $\alpha=0.3$ ) are always lower than those with loose budget. The average actual liability ratio $\left(l^{\prime}\right)$ in each testing case is higher in the tight budget case than in the loose budget case. Moreover, given different level of demand variability, the decrease in LRP profitability with the increase in budget tightness (i.e., $\alpha$ changes from 0 to 0.3 ) is within the range of $4.4 \%$ to $11.6 \%$. Hence, when demand variability is higher, LRP profitability is lower. On the other hand, the increases 
in liability ratio due to the increase in budget tightness are within the range of $7.4 \%-23.6 \%$, and the increases in ordering quantity of rewards are within the range of $12.9 \%-31.2 \%$. Overall, with a tight budget (i.e., $\alpha=0.3$ ) the LRP profitability decreases with the increases in demand variability, while the liability ratio and the ordering quantity of rewards increase. In addition to that, with the increases in demand variability, the decreasing rate of the profitability and the increasing rate of liability ratio and ordering quantity of rewards are higher in the case of a tight budget compared to the case of a loose budget. 
Table 6.7: Impacts of budget tightness under BP setting

(a) with Uniformly Distributed Demand

\begin{tabular}{|c|c|c|c|c|c|c|c|c|c|c|c|c|c|}
\hline & & \multicolumn{4}{|c|}{ Case 4 (NR20NA15) } & \multicolumn{4}{|c|}{ Case 6 (NR40NA40) } & \multicolumn{4}{|c|}{ Case 8 (NR65NA70) } \\
\hline & & \multicolumn{2}{|c|}{ BG-L(0.0) } & \multicolumn{2}{|c|}{ BG-H(0.3) } & \multicolumn{2}{|c|}{ BG-L(0.0) } & \multicolumn{2}{|c|}{ BG-H(0.3) } & \multicolumn{2}{|c|}{ BG-L(0.0) } & \multicolumn{2}{|c|}{$\mathrm{BG}-\mathrm{H}(0.3)$} \\
\hline & DV & Mean & $\operatorname{Std}(\%)$ & Mean & $\operatorname{Std}(\%)$ & Mean & $\operatorname{Std}(\%)$ & Mean & $\operatorname{Std}(\%)$ & Mean & $\operatorname{Std}(\%)$ & Mean & $\operatorname{Std}(\%)$ \\
\hline \multirow{3}{*}{$\begin{array}{c}\text { LRP } \\
\text { Profitability }\end{array}$} & $\mathrm{L}(0.1)$ & 26003.790 & 0.033 & 24272.866 & 0.009 & 36666.071 & 0.015 & 34744.045 & 0.015 & 65567.844 & 0.005 & 62195.351 & 0.007 \\
\hline & $\mathrm{M}(1.0)$ & 25745.837 & 0.095 & 23399.437 & 0.029 & 36863.618 & 0.045 & 34270.721 & 0.045 & 66072.339 & 0.014 & 61551.359 & 0.021 \\
\hline & $\mathrm{H}(1.8)$ & 25394.129 & 0.159 & 22468.701 & 0.057 & 36861.704 & 0.072 & 33792.458 & 0.074 & 66199.264 & 0.026 & 60907.177 & 0.037 \\
\hline \multirow{3}{*}{$\begin{array}{c}\text { Liability } \\
\text { Ratio }\end{array}$} & $\mathrm{L}(0.1)$ & 0.598 & 0.051 & 0.717 & 0.098 & 1.01 & 0.021 & 1.121 & 0.029 & 1.156 & 0.014 & 1.252 & 0.019 \\
\hline & $M(1.0)$ & 0.624 & 0.143 & 0.754 & 0.075 & 1.054 & 0.06 & 1.191 & 0.081 & 1.201 & 0.038 & 1.319 & 0.054 \\
\hline & $\mathrm{H}(1.8)$ & 0.654 & 0.224 & 0.808 & 0.118 & 1.106 & 0.086 & 1.262 & 0.15 & 1.251 & 0.064 & 1.386 & 0.081 \\
\hline \multirow{3}{*}{$\begin{array}{l}\text { Rewards } \\
\text { Ordering Q }\end{array}$} & $\mathrm{L}(0.1)$ & 531.059 & 0.044 & 433.313 & 0.05 & 969.734 & 0.04 & 808.633 & 0.02 & 1547.79 & 0.06 & 1289.345 & 0.018 \\
\hline & $\mathrm{M}(1.0)$ & 590.089 & 0.113 & 437.123 & 0.054 & 1066.687 & 0.117 & 809.841 & 0.066 & 1698.329 & 0.147 & 1289.382 & 0.054 \\
\hline & $\mathrm{H}(1.8)$ & 635.552 & 0.182 & 437.326 & 0.14 & 1125.889 & 0.139 & 810.784 & 0.08 & 1793.485 & 0.182 & 1288.837 & 0.089 \\
\hline
\end{tabular}


Table 6.7: Impacts of budget tightness under BP setting (Cont.)

(b) with Normally Distributed Demand

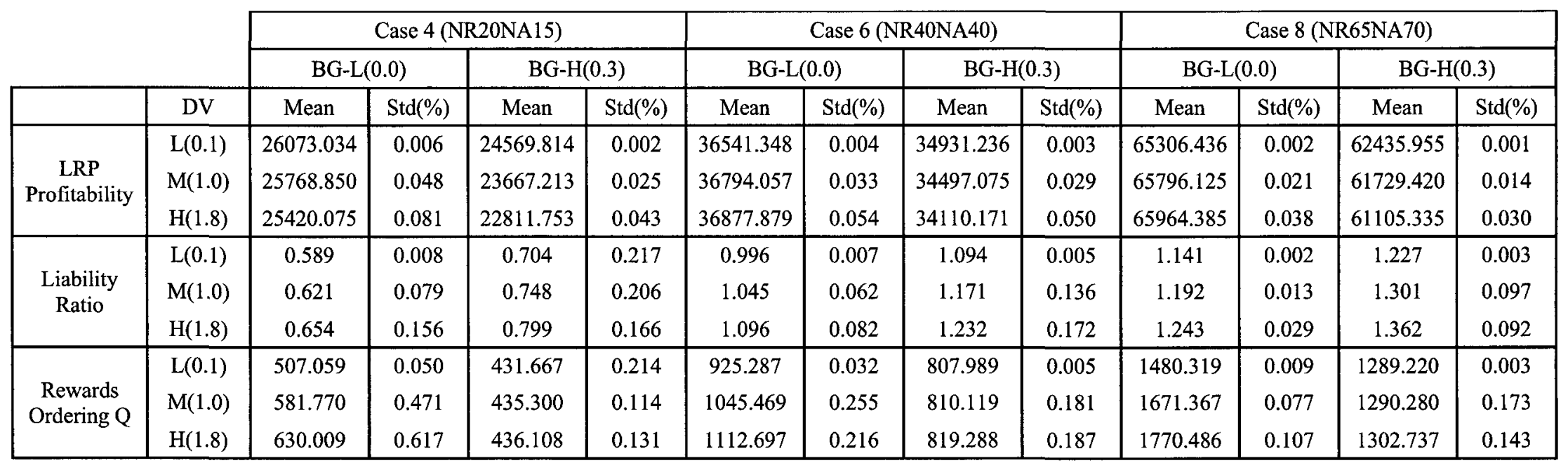


The results of the hypothesis tests related to BP model are summarized in Appendices D.1 and D.2, in which we provide the confidence intervals generated for all pair-wise comparisons. In Appendix D.1, i2 refers to high budget tightness (i.e., BG-H), and i1 refers to low budget tightness (i.e., BG-L). The confidence intervals are reported as:

$$
\left(U_{i 2}-U_{l 1}\right) \pm \text { half-width, }
$$

where $U_{l}$ refers to the mean values of our computational results (e.g., LRP profitability, liability ratio, and ordering quantity). For instance, in Appendix D.1(a), testing case 4, the confidence interval for LPR profitability is $-1503.22 \pm 1.079$. “-1503.22" refers to the difference of the mean LRP profitability with a tight budget limit from that of a loose budget limit. "1.079" refers to the half-width of the confidence interval associated with the difference of the mean LRP profitability. Similarly, in Appendix D.2, $j 2$ refers to the demand variability level, either medium (i.e., DV-M) or high (i.e., DV-H), and $j 1$ also refers to the demand variability level, either low (i.e., DV-L) or medium (i.e., DV-M).

On one hand, the testing results (as reported in Appendix D.1) show that, given a level of demand variability (i.e., low, medium, or high), the mean values of LRP profitability, liability ratio, and ordering quantity of rewards are significantly different (at 95\% CL) in both the tight and loose budget cases. This confirms that budget tightness does have impacts on LRP profitability, liability ratios, and the LRP host's rewards-supply decisions. On the other hand, with either low or high budget tightness, the mean values of LRP profitability, liability ratio, and LRP host firm's rewards-ordering decisions (as reported in Appendix D.2) are significantly different (at 95\% CL) across different levels of demand variability, except in the testing case 8 (i.e., TC No.8). In that testing case, with the tight budget (i.e., $\alpha=0.3$ ), the LRP host's overall 
ordering quantity of rewards seems not significantly different among various levels of demand variability (i.e., the confidence interval includes zero, as highlighted by "**" in Appendix D.2). These results indicate that, statistically speaking, demand variability does have a significant impact on LRP profitability, liability ratio, and the LRP host firm's rewards-ordering decisions. However, in some cases, when the budget is very tight, the optimal ordering quantity decisions will be limited by the available amount of budget (one of the internal constraints), and the LRP system cannot fully respond to external uncertainties (e.g., demand variability).

Based on the above computational results and statistical analyses, we find that budget tightness does have a negative impact on LRP profitability and liability ratio (i.e., higher budget tightness, lower LRP profitability, and higher liability ratio) regardless any changes in demand variability. However, it seems that BP model is more sensitive to budget tightness when the demand variability is higher. Furthermore, through hypothesis tests and all pair-wise comparisons, we statistically show that the impacts of budget tightness on LRP profitability, the liability ratio, and the LRP host's ordering decisions are significant at a 95\% confidence level considering different levels of demand variability. We also statistically show that the changes in demand variability have a significant impact on LRP profitability, the liability ratio, and the LRP host's ordering decisions at a $95 \%$ confidence level in most of the testing cases.

\subsubsection{Under EP1 Setting}

As shown in Table 6.8, in each testing case, the mean LRP profitability and the mean ordering quantity of rewards are lower, and the mean liability ratio is higher in the case of tight budget (i.e., $\alpha=0.3$ ), compared to the case of loose budget (i.e., $\alpha=0$ ). The decrease in the mean LRP 
profitability due to the increase in budget tightness is within the range of $4.2 \%-11.5 \%$. On the other hand, the increase in the mean liability ratio due to the increase in budget tightness is within the range of $6.5 \%-22.5 \%$, and the increase in the mean ordering quantity of rewards is within the range of $12.6 \%-31.5 \%$. Overall, with a tight budget (e.g., $\alpha=0.3$ ), LRP profitability decreases with the increase in demand variability, while the liability ratio and the ordering quantity of rewards increase. In addition, with the increase in demand variability, the decreasing rate of the mean LRP profitability and the increasing rates of the mean liability ratio and the mean ordering quantity of rewards are higher in the case of a tight budget compared to the case of a loose budget. 
Table 6.8: Impacts of budget tightness under EP1 setting

(a) with Uniformly Distributed Demand

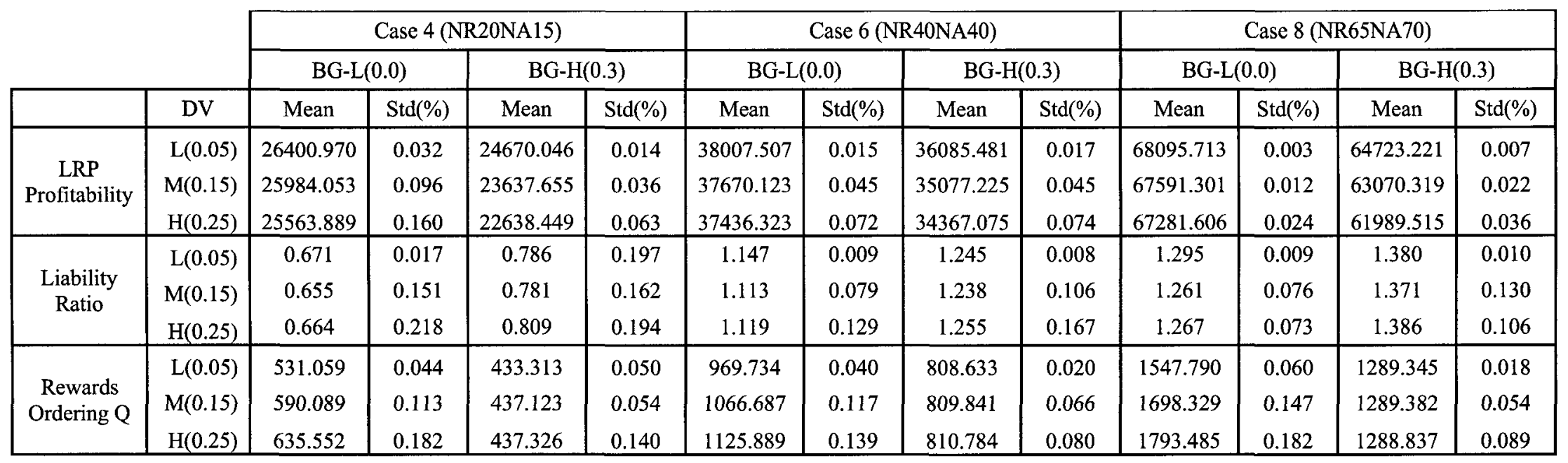


Table 6.8: Impacts of budget tightness under EP1 setting (Cont.)

(b) with Normally Distributed Demand

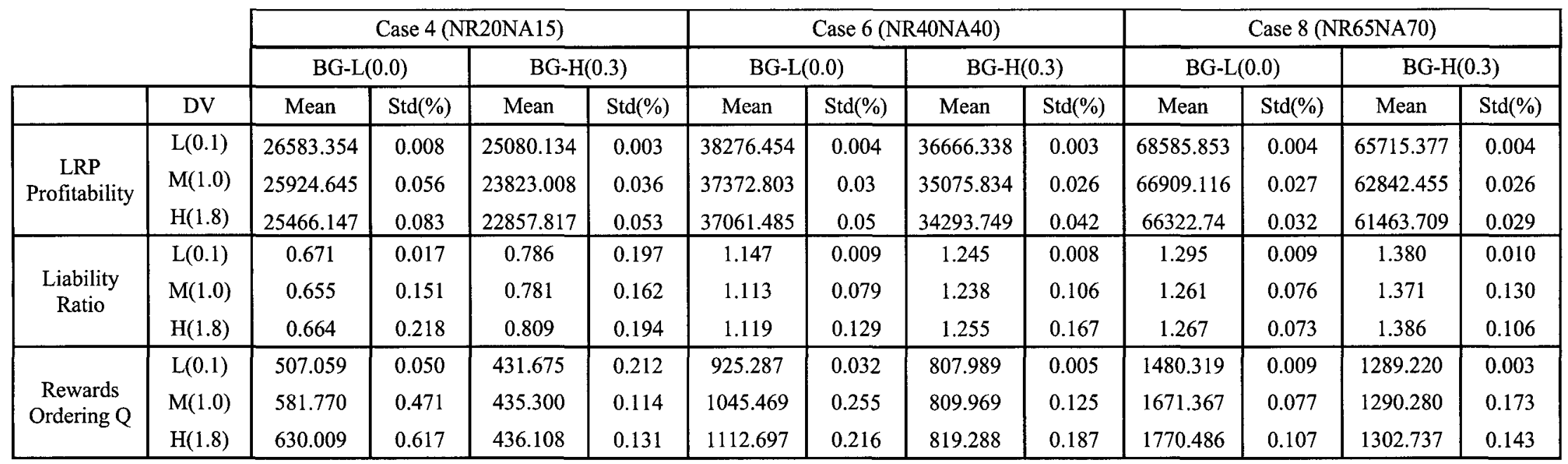


The results of the hypothesis tests related to EP1 model are summarized in Appendix D.3 and Appendix D.4. We find that the same conclusions can be drawn under EP1 setting as were drawn under BP setting. All testing problems show that there are significant differences among the mean values of LRP profitability, liability ratio, and ordering quantities of rewards in tight and loose budget cases and among different levels of demand variability, with a few exceptions (as highlighted by “*” in Appendix D.4).

Overall, with regard to the impacts of budget tightness under EP1 setting, we find that budget tightness does have negative impacts on LRP profitability and liability ratio, no matter what the changes in demand variability. In addition, through hypotheses tests and all pair-wise comparisons, we statistically show that the impacts of budget tightness on LRP profitability, liability ratio, and LRP host's decisions are significant at a $95 \%$ confidence level considering different levels of demand variability. We also statistically show that the changes in demand variability have a significant impact on LRP profitability, liability ratio, and the LRP host's decisions at a $95 \%$ confidence level in most of the testing cases.

\subsubsection{Under EP2 Setting}

As shown in Table 6.9, the mean LRP profitability and the mean ordering quantity of rewards are lower, and the mean liability ratio is higher in the case of tight budget (i.e., $\alpha=0.3$ ) compared to the case of loose budget (i.e., $\alpha=0$ ). The decrease in the mean LRP profitability due to the increase in the budget tightness is within the range of $4.2 \%-10.6 \%$. On the other hand, the increase in the mean liability ratio due to the increase in the budget tightness is within the range of $6.8 \%-23.3 \%$, and the increases in the mean ordering quantity of rewards are within the range 
of $10.7 \%-38.5 \%$. Overall, when the budget is tight (i.e., $\alpha=0.3$ ), the mean LRP profitability decreases with the increases in demand variability, while the mean liability ratio and the mean ordering quantity of rewards increase. In addition to that, with the increases in demand variability, the decreasing rate of the LRP profitability and the increasing rates of liability ratios and ordering quantity of rewards are higher in the case of tight budget compared to the case of loose budget. 
Table 6.9: Impacts of budget tightness under EP2 setting

(a) with Uniformly Distributed Demand

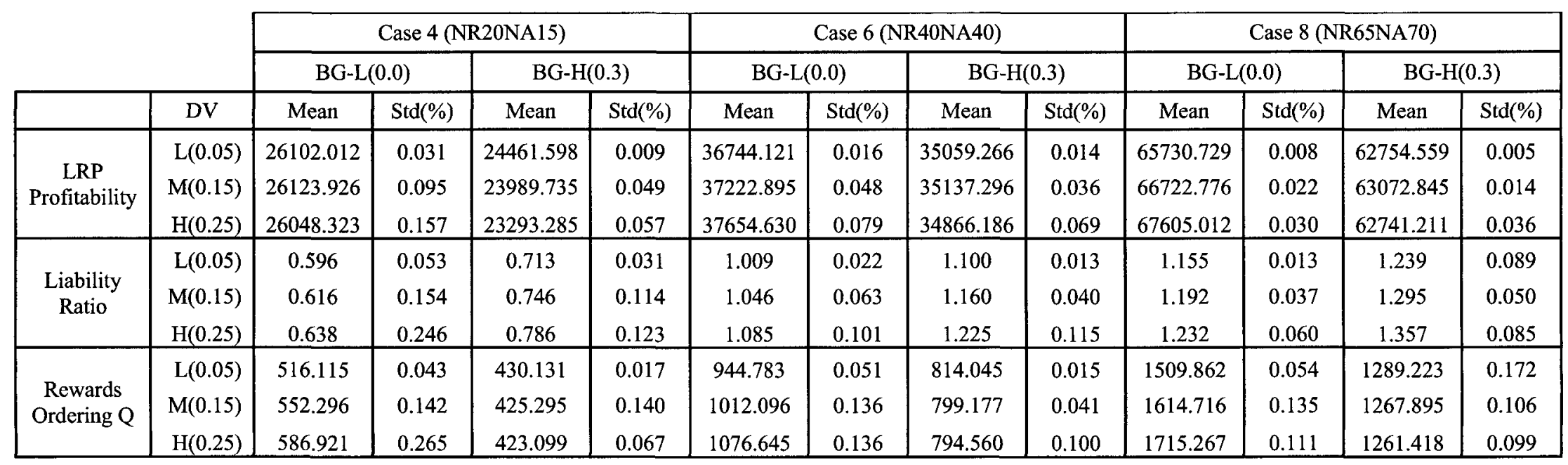


Table 6.9: Impacts of budget tightness under EP2 setting (Cont.)

(b) with Normally Distributed Demand

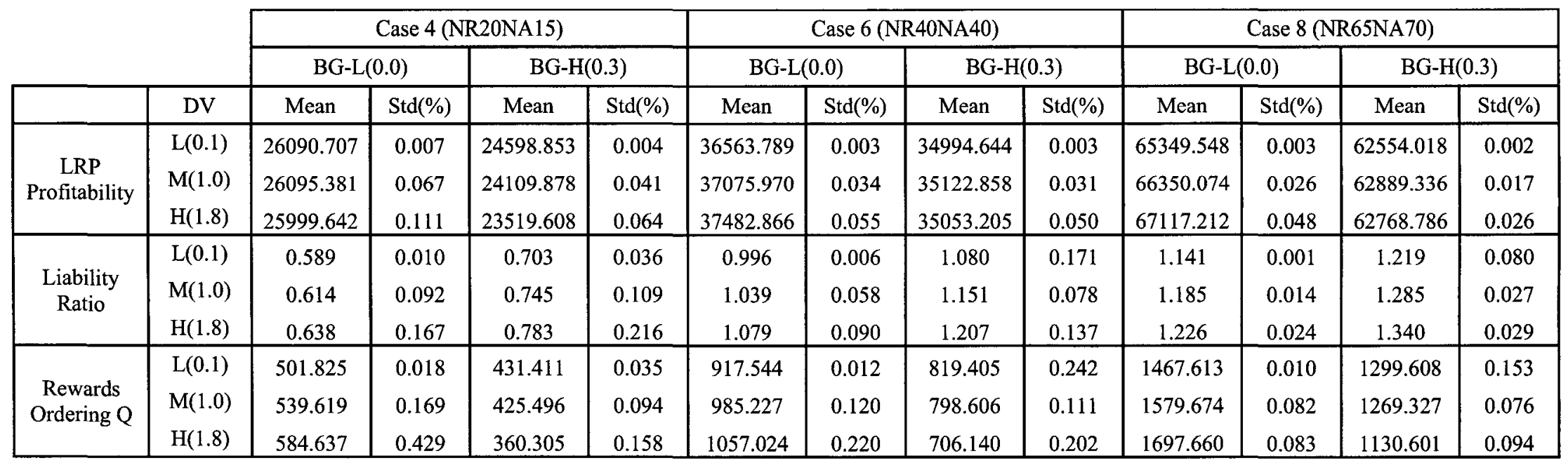


The results of the hypothesis tests related to EP2 model are summarized in Appendix D.5 and Appendix D.6. We find that same conclusions can be drawn under EP2 setting as those drawn under BP and EP1 settings. All testing results show that there are significant differences among the mean values of LRP profitability, liability ratio, and ordering quantities of rewards in cases of both tight and loose budget as well as among different levels of demand variability, with a few exceptions (as highlighted by "**" in Appendix D.6).

Overall, we can draw the same conclusions as those under BP and EP1 settings: budget tightness does have significant negative impacts on LRP profitability and liability ratios at a $95 \%$ confidence level with the changes in demand variability. However, it seems that EP2 model is more sensitive to budget tightness when demand variability is higher. Furthermore, through hypothesis tests and all pair-wise comparisons, we statistically show that the impacts of budget tightness on LRP profitability, liability ratios and the LRP host's decisions are significant at a $95 \%$ confidence level with different levels of demand variability. We also statistically show that the changes in demand variability have a significant impact on LRP profitability, liability ratio, and the LRP host's decisions at a 95\% confidence level in most of the testing problems.

\subsubsection{Comparison across BP, EP1, and EP2 Model Settings}

As shown in Figure 6.4, LRP profitability decreases under each model setting when budget tightness increases. It seems that LRP profitability is slightly more sensitive to demand variability in the case of tight budget. With a lower level of demand variability, BP and EP2 models generate similar LRP profitability, but EP1 model generates higher LRP profitability than BP and EP2 models. On the other hand, when demand variability level is high, EP2 model 
generates higher LRP profitability, but BP and EP1 models generate similar LRP profitability in both tight and loose budget cases.

Figure 6.4: LRP profitability under BP, EP1, and EP2 settings

(a) Case 4: NR20NA15

(with uniformly distributed demand) (b) Case 4: NR20NA15 (with normally distributed demand)

\section{LRP Profitability}
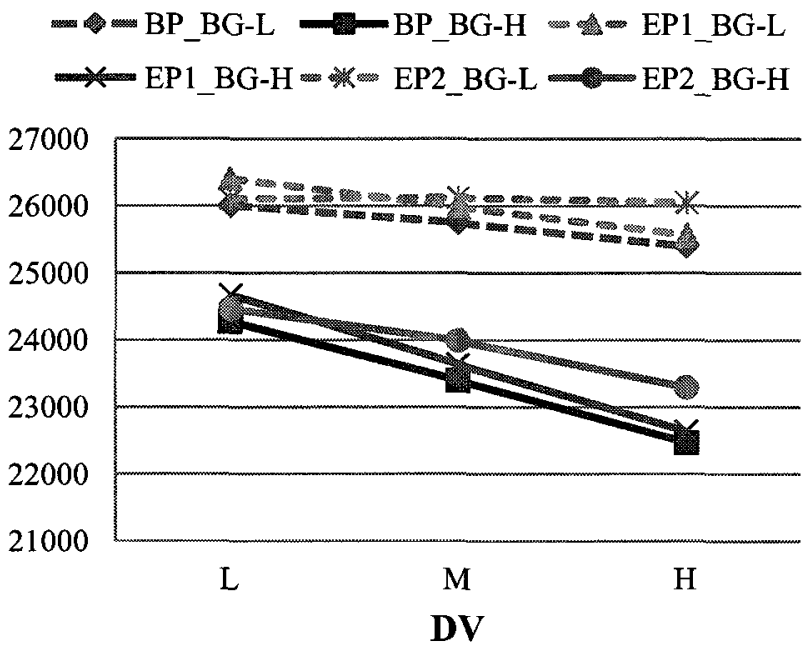

\section{LRP Profitability}
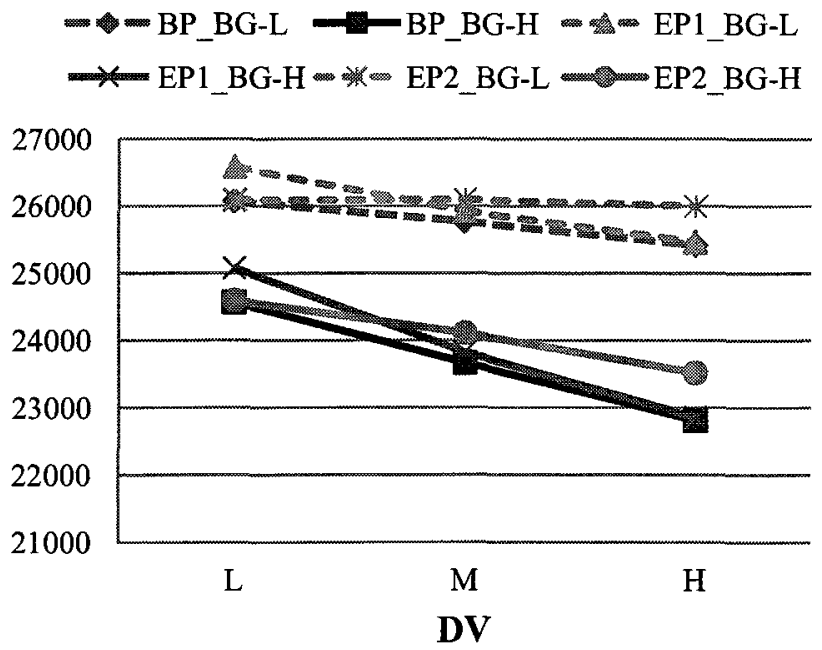
Figure 6.4: LRP profitability under BP, EP1, and EP2 settings (Cont.)

(c) Case 6: NR40NA40

(with uniformly distributed demand) (d) Case 6: NR40NA40 (with normally distributed demand)

\section{LPR Profitability}

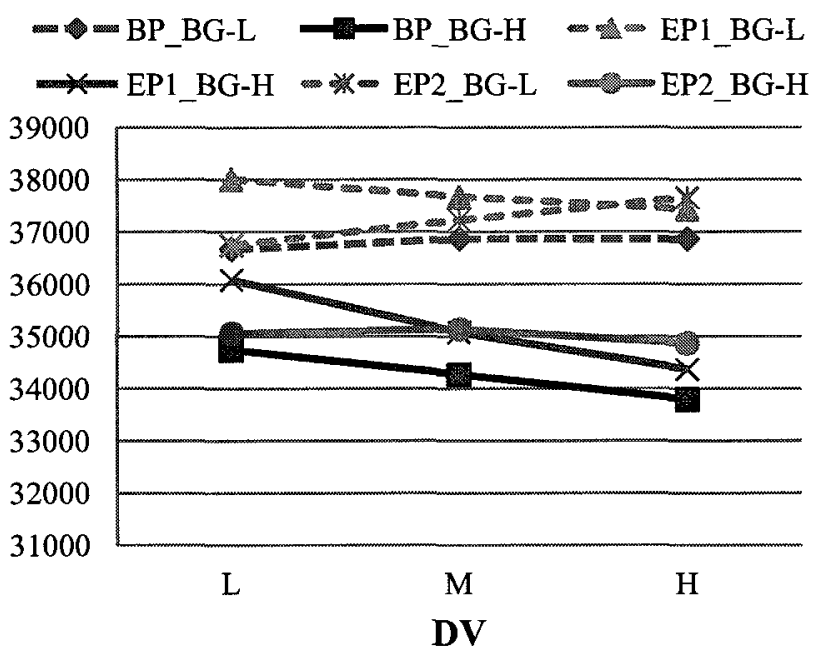

LPR Profitability
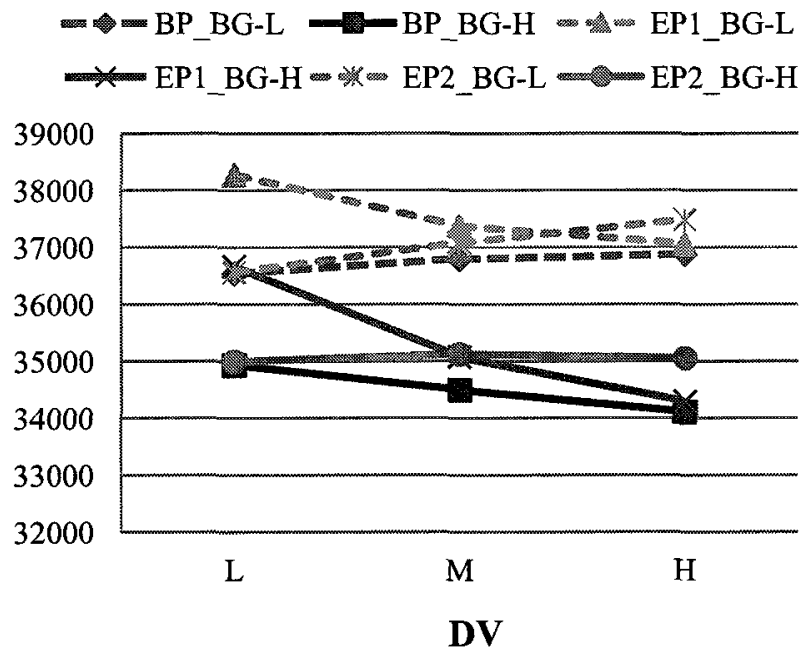
Figure 6.4: LRP profitability under BP, EP1, and EP2 settings (Cont.)

(e) Case 8: NR65NA70

(with uniformly

distributed demand)

\section{LRP Profitability}
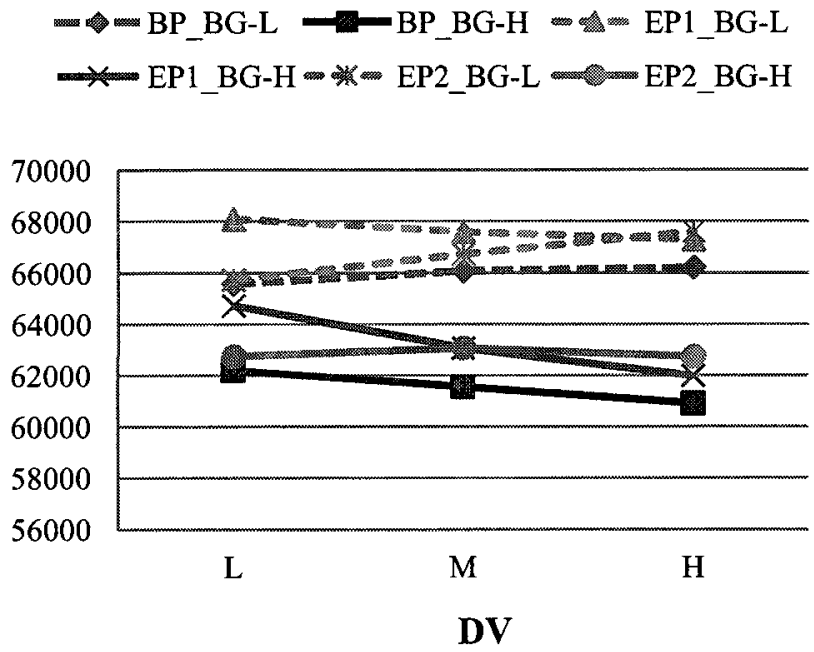

(f) Case 8: NR65NA70

(with normally distributed demand)

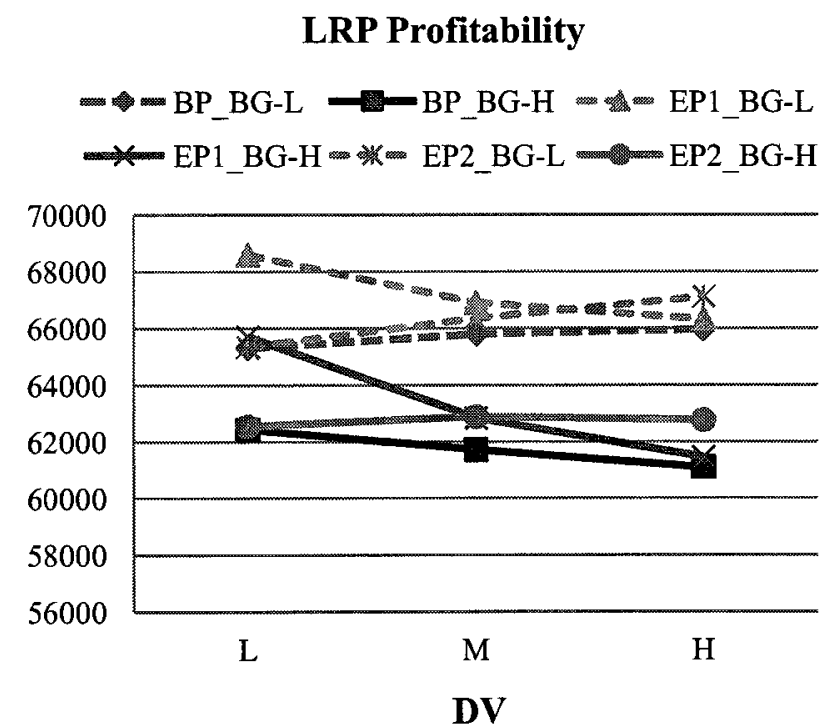

With respect to actual liability ratio, it increases with the increase in budget tightness and the demand variability under each model setting. EP1 model always incurs a higher liability ratio than the other two models. Furthermore, when demand variability increases, the liability ratio in 
BP and EP2 models seems to get closer to the liability ratio in EP1 model, no matter what the changes in budget tightness. This indicates that in comparison to EP1 model, the advantages of BP and EP2 models (in terms of liability ratio) are mitigated when the demand variability level is high. These phenomena are observed in all testing cases (see Figure 6.5). 
Figure 6.5: Liability ratios under BP, EP1, and EP2 settings

(a) Case 4: NR20NA15

(with uniformly distributed demand)

\section{Liability Ratio}

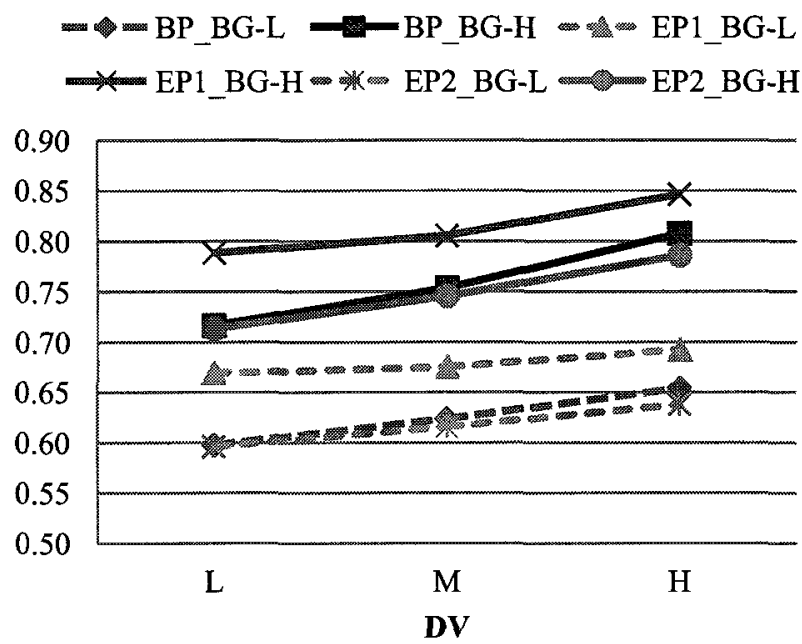

(b) Case 4: NR20NA15

(with normally distributed demand)

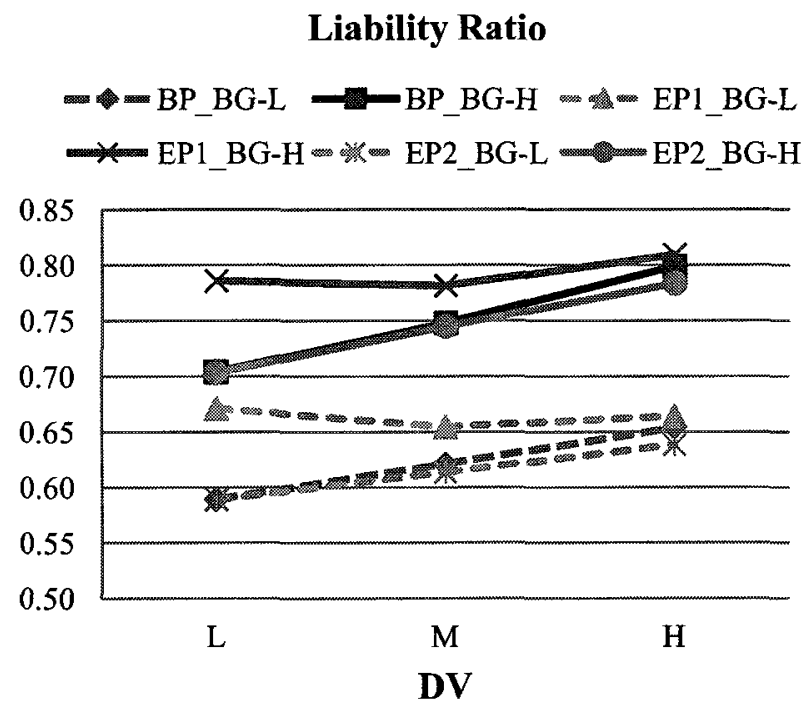


Figure 6.5: Liability ratios under BP, EP1, and EP2 settings (Cont.)

(c) Case 6: NR40NA40 (with uniformly distributed demand)

\section{Liability Ratio}

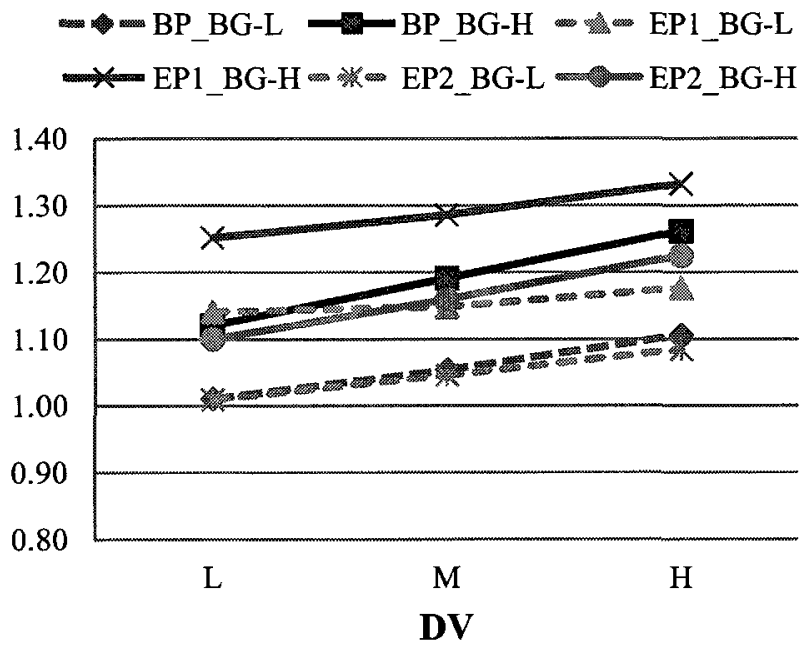

Liability Ratio
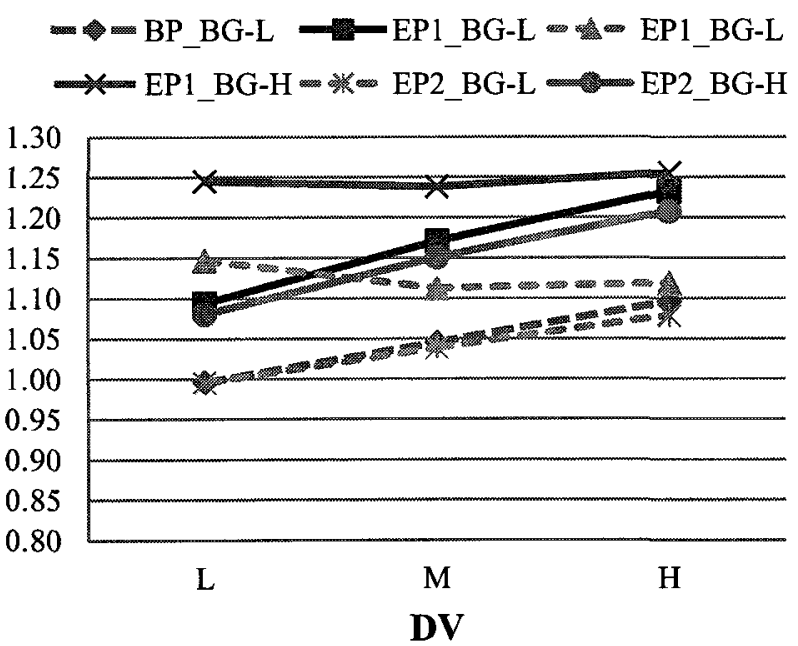
Figure 6.5: Liability ratios under BP, EP1, and EP2 settings (Cont.)

(e) Case 8: NR65NA70 (with uniformly distributed demand)

(f) Case 8: NR65NA70 (with normally distributed demand)

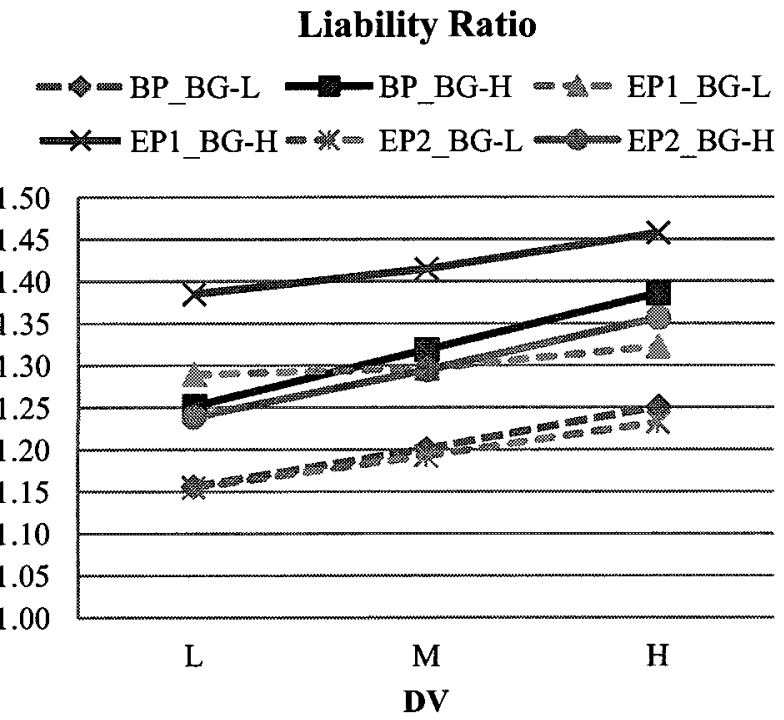

Liability Ratio
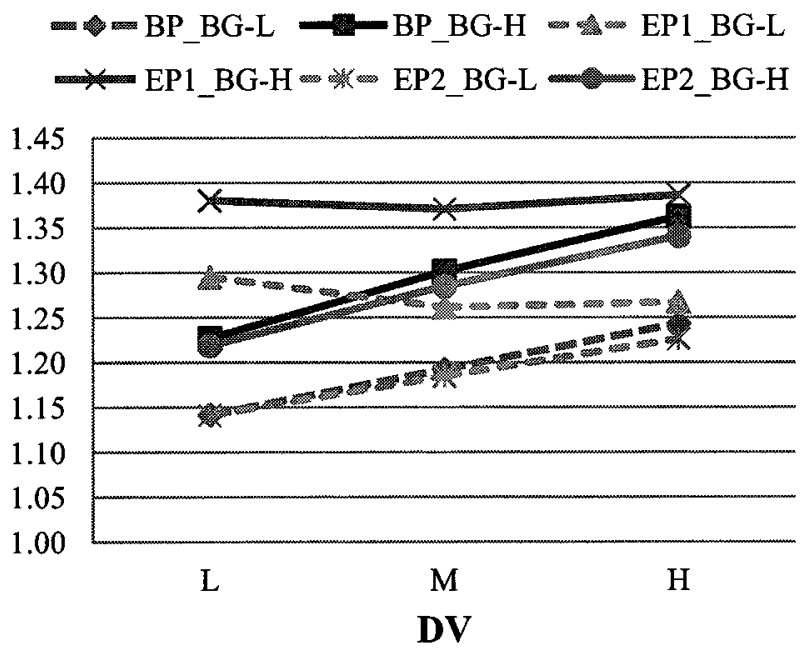
With respect to the ordering quantity of rewards, it is the same under BP and EP1 settings. As shown in Figure 6.6, when the level of budget tightness is low, the ordering quantities of rewards may increase with the increase in demand variability under BP, EP1, and EP2 settings. However, when the level of budget tightness is high, the ordering quantity of rewards either remains the same (i.e., in BP and EP1 models) or decrease slightly (i.e., in EP2 model). This is because the ordering quantity of rewards is limited by the available amount of budget. Ordering quantities of rewards determined under EP2 setting are never more than those under BP and EP1 settings, no matter what the changes in budget tightness and demand variability. 
Figure 6.6: Ordering quantity of rewards under BP, EP1, and EP2 settings

(a) Case 4: NR20NA15

(with uniformly

distributed demand)
Ordering Quantity of Rewards

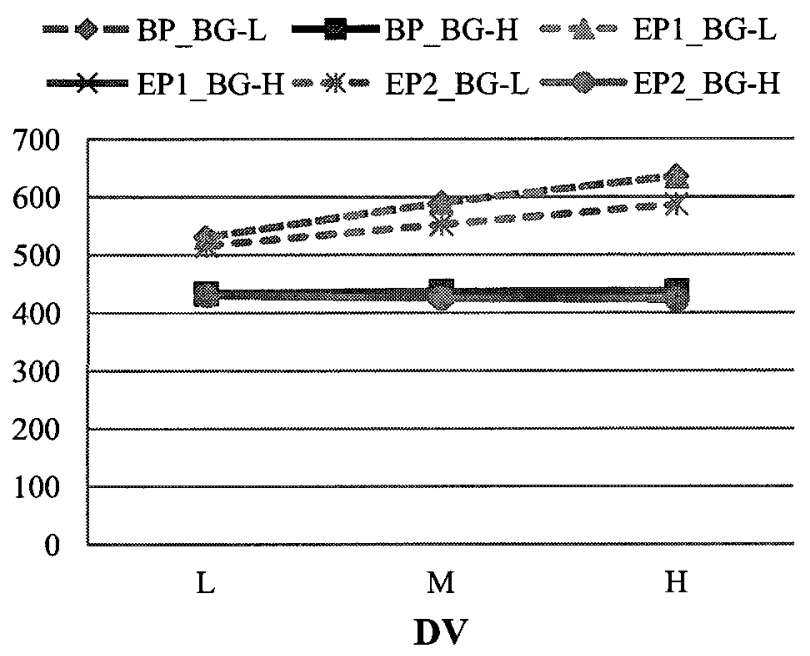

Ordering Quantity of Rewards
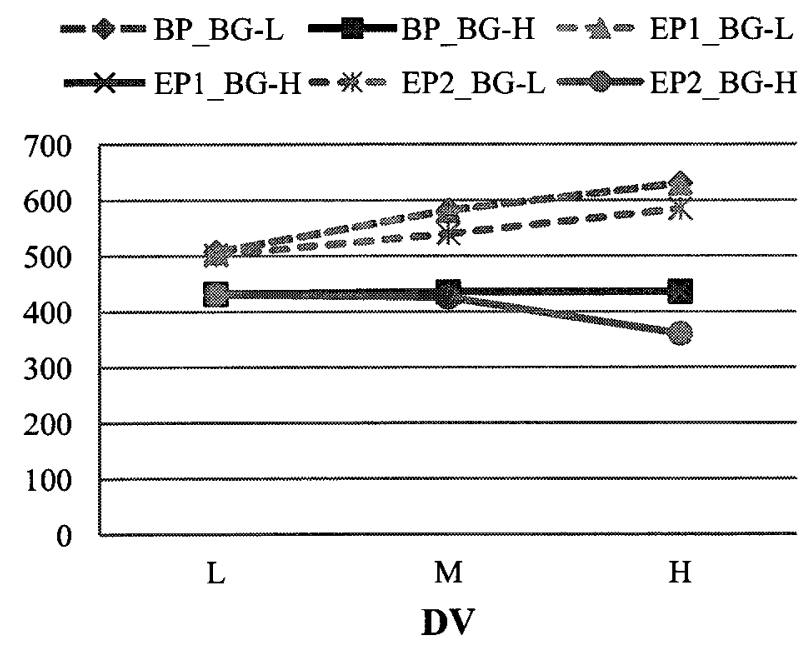

(b) Case 4: NR20NA15

(with normally distributed demand) 
Figure 6.6: Ordering quantity of rewards under BP, EP1, and EP2 settings (Cont.)

(c) Case 6: NR40NA40 (with uniformly distributed demand) (d) Case 6: NR40NA40 (with normally distributed demand)

\section{Ordering Quantity of Rewards}
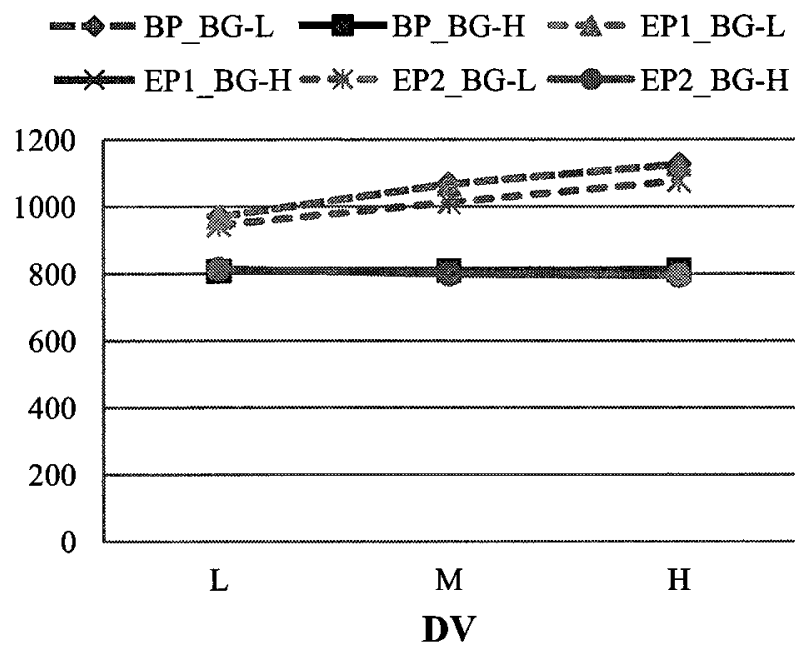

Ordering Quantity of Rewards
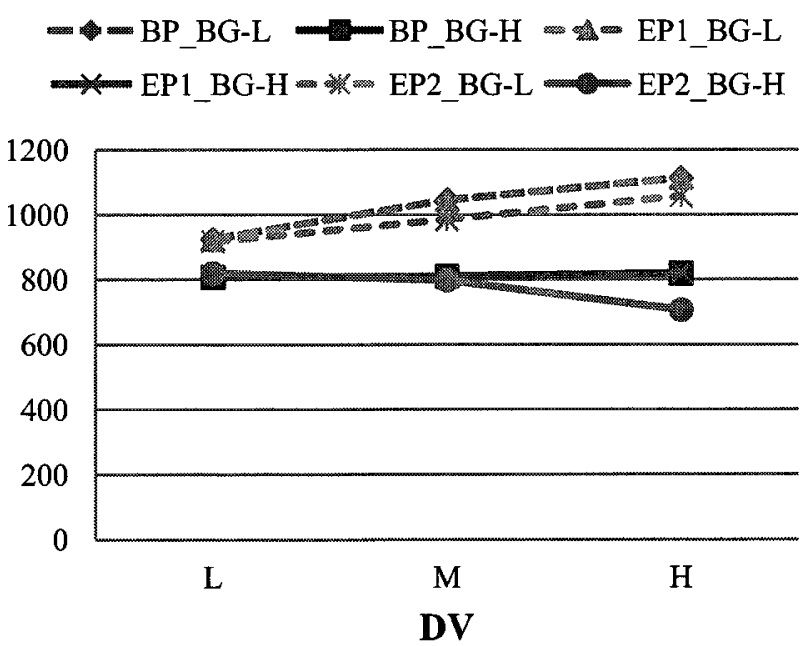
Figure 6.6: Ordering quantity of rewards under BP, EP1, and EP2 settings (Cont.)

(e) Case 8: NR65NA70

(with uniformly distributed demand)

\section{Ordering Quantity of Rewards}

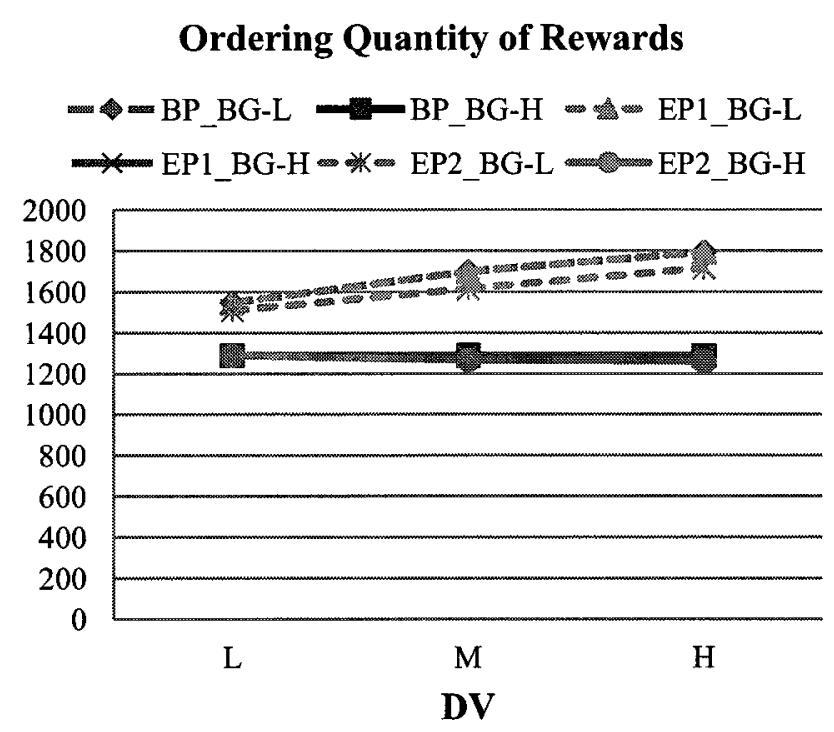

Ordering Quantity of Rewards

(f) Case 8: NR65NA70 (with normally distributed demand)

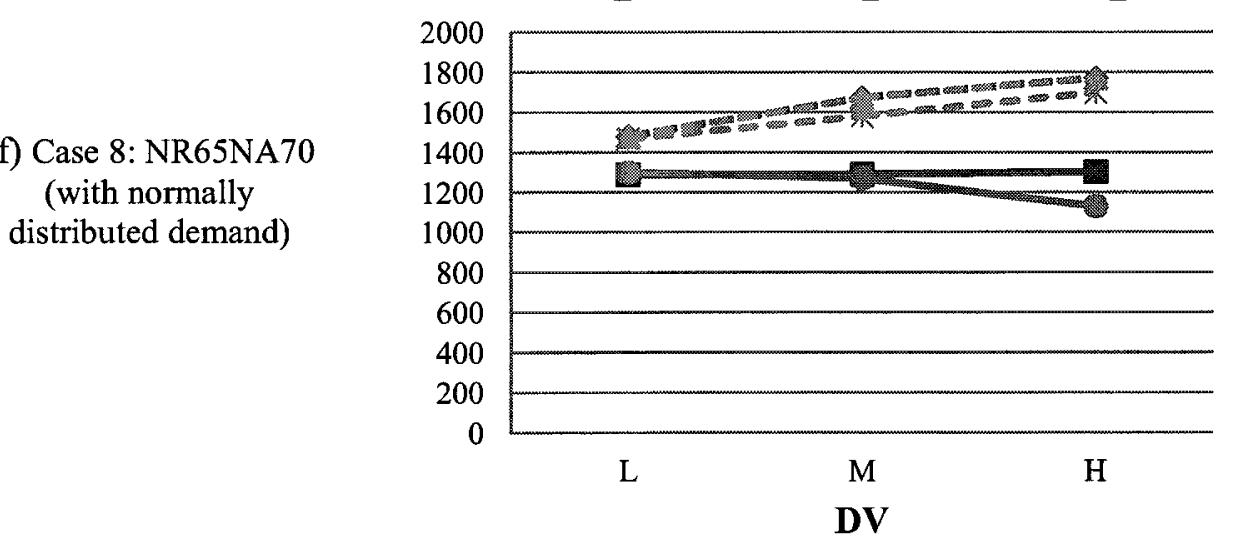


The results for all pair-wise comparisons and hypothesis tests (see Appendix D.7) show that given the changes in budget tightness and demand variability, the impacts of the model settings (i.e., BP, EP1, and EP2) on LRP profitability, liability ratio, and ordering quantities of rewards are significantly different in most of the testing cases with an exception in the testing case 4 (i.e., TC No.4) with normally distributed demand. In that testing case, with a low level of demand variability (e.g., $\mathrm{DV}=0.1$ ) and a high level of budget tightness (e.g., $\alpha=0.3$ ), the ordering quantities of rewards across the three models have no significant differences. In addition, the ordering quantities of rewards are the same under BP and EP1 settings and the corresponding confidence intervals include zero (as highlighted by “*” in Appendices D.7). These results indicate that offering bonus points doesn't affect the LRP host's rewards-ordering decisions.

According to these computational outputs and statistical analyses, we conclude that with a high level of budget tightness, EP2 model still achieves higher LRP profitability than BP and EP1 models when demand variability increases, whereas EP1 model seems better than BP and EP2 models when the demand variability level is low. With respect to liability, EP1 model generates a higher liability than BP and EP2 models given a level of budget tightness. BP and EP2 models seem to be better than EP1 model in terms of liability, but this advantage is mitigated when demand variability increases. With regard to an LRP host's decisions on ordering quantities of rewards, under BP and EP1 settings the ordering quantities of rewards are the same; whereas under EP2 setting, the ordering quantities are slightly lower than those under BP and EP1 settings. 


\subsubsection{Summary}

In this section, we investigate the impacts of budget tightness on LRP profitability, liability, and LRP host firm's rewards-ordering decisions. We conduct hypothesis tests through all pair-wise comparisons under each model setting and across model settings. In addition to that, we also examine the impacts of budget tightness under different demand variability levels. We observe that:

(1) Budget tightness does have a negative impact on LRP profitability and LRP liability (i.e., higher budget tightness, lower LRP profitability and higher liability ratio) under BP, EP1, and EP2 settings, respectively.

(2) Budget tightness does have negative impacts on LRP profitability and LRP liability (i.e., higher budget tightness, lower LRP profitability and higher liability ratios) at all levels of demand variability (i.e., low, medium, and high).

(3) With a level of budget tightness, EP2 model always achieves a higher LRP profitability when demand variability level is high; however, EP1 model seems to be better than BP and EP2 models in terms of LPR profitability when demand variability level is low.

(4) With a level of budget tightness, EP1 model always generates a higher liability than BP and EP2 models. BP and EP2 models seem to be better than EP1 model in terms of LRP liability, but this advantage is mitigated when demand variability increases.

(5) With a level of budget tightness, under BP and EP1 settings, ordering quantities of rewards are always the same; however, under EP2 model setting the ordering quantities are slightly lower than those under BP and EP1 model settings. 
These observations are consistent with what we expect to see (i.e., null hypotheses are rejected). Overall, internal dynamics such as budget tightness do have an impact on LRP profitability, liability, and LRP host firm's rewards-ordering decisions. Given the model inputs, through our analytical models we can examine how likely and at which level the impact will occur. In terms of LRP profitability and liability, using option contracts still seems better than using wholesaleprice-only contracts in rewards-supply contracting when the level of budget tightness is high. Meanwhile, the advantages of adopting option contracts are more obvious when demand variability increases. Offering bonus points doesn't affect LRP host's rewards-ordering decisions and it achieves higher LRP profitability only when the level of demand variability is low, no matter what the changes in budget tightness. 


\subsection{Testing the Impacts of Liability Control}

Through the set of numerical experiments in this section, we seek to examine the impacts of liability control, another important internal dynamic, on the LRP host's ordering decisions, LRP profitability, and budget usage. We also investigate whether the impacts varies between different model settings and under different demand variability levels.

\subsubsection{Under BP Setting}

Table 6.10 summarizes the results of BP when budget constraint (4.43) and capacity constraints (4.42) are relaxed and the liability control becomes tight (i.e., $\gamma=0.7$ ). We observe that LRP profitability decreases with the increase of liability control tightness (i.e., $\gamma$ changes from 1.3 to 0.7). When the liability control is tight (i.e., $\gamma=0.7$ ), higher target redemption is required. In other words, the estimated optimal ordering quantities of rewards and the cost of purchasing rewards (i.e., budget usage) are expected to increase, which results in decrease in the LRP profitability at the redemption side. As such, the overall LRP profitability decreases. On the other hand, just as in the case of the loose liability control (i.e., $\gamma=1.3$ ), with the increase in demand variability, the LRP profitability decreases in the testing case 4 but increases in the testing cases 6 and 8 when liability control is tight. 
Table 6.10: Computational results under BP Setting

(with uniformly distributed demand)

(with normally distributed demand)

\begin{tabular}{|c|c|c|c|c|c|c|}
\hline \multirow{2}{*}{} & \multicolumn{2}{|c|}{ Case 4 } & \multicolumn{2}{c|}{ Case 6 } & \multicolumn{2}{c|}{ Case 8 } \\
\cline { 2 - 7 } & \multicolumn{2}{|c|}{ LC tightness $(\gamma)$} & \multicolumn{2}{c|}{ LC tightness $(\gamma)$} & \multicolumn{2}{c|}{ LC tightness $(\gamma)$} \\
\hline $\mathrm{DV}$ & $\mathrm{H}(0.7)$ & $\mathrm{L}(1.3)$ & $\mathrm{H}(0.7)$ & $\mathrm{L}(1.3)$ & $\mathrm{H}(0.7)$ & $\mathrm{L}(1.3)$ \\
\hline $\mathrm{L}(0.05)$ & 25893.42 & 26053.63 & 36149.06 & 36674.10 & 64488.31 & 65609.06 \\
$\mathrm{M}(0.15)$ & 25843.55 & 25980.27 & 36515.64 & 37008.69 & 65286.49 & 66349.89 \\
$\mathrm{H}(0.25)$ & 25809.68 & 25906.92 & 36924.43 & 37343.28 & 66149.67 & 67090.72 \\
\hline
\end{tabular}

\begin{tabular}{|c|c|c|c|c|c|c|}
\hline \multirow{2}{*}{} & \multicolumn{2}{|c|}{ Case 4 } & \multicolumn{2}{c|}{ Case 6 } & \multicolumn{2}{c|}{ Case 8 } \\
\cline { 2 - 7 } & \multicolumn{2}{|c|}{ LC tightness $(\gamma)$} & \multicolumn{2}{c|}{ LC tightness $(\gamma)$} & \multicolumn{2}{c|}{ LC tightness $(\gamma)$} \\
\hline DV & $\mathrm{H}(0.7)$ & $\mathrm{L}(1.3)$ & $\mathrm{H}(0.7)$ & $\mathrm{L}(1.3)$ & $\mathrm{H}(0.7)$ & $\mathrm{L}(1.3)$ \\
\hline $\mathrm{L}(0.1)$ & 25908.26 & 26074.50 & 36015.31 & 36541.92 & 64182.80 & 65310.30 \\
$\mathrm{M}(1.0)$ & 25792.48 & 25932.22 & 36357.52 & 36857.98 & 64901.85 & 65955.28 \\
$\mathrm{H}(1.8)$ & 25716.11 & 25811.36 & 36716.09 & 37144.76 & 65634.67 & 66543.62 \\
\hline
\end{tabular}

(b) Costs of Rewards

(with uniformly distributed demand)

(with normally distributed demand)

\begin{tabular}{|c|c|c|c|c|c|c|}
\hline \multirow{2}{*}{} & \multicolumn{2}{|c|}{ Case 4 } & \multicolumn{2}{c|}{ Case 6 } & \multicolumn{2}{c|}{ Case 8 } \\
\cline { 2 - 7 } & \multicolumn{2}{|c|}{ LC tightness $(\gamma)$} & \multicolumn{2}{c|}{ LC tightness $(\gamma)$} & \multicolumn{2}{c|}{ LC tightness $(\gamma)$} \\
\hline DV & H $(0.7)$ & L $(1.3)$ & H $(0.7)$ & L $(1.3)$ & H $(0.7)$ & L $(1.3)$ \\
\hline L(0.05) & 4606.27 & 4270.61 & 8095.63 & 7005.97 & 14286.85 & 11981.71 \\
M(0.15) & 5177.84 & 4857.84 & 9005.40 & 7897.89 & 15833.28 & 13509.92 \\
H(0.25) & 5717.44 & 5445.07 & 9830.85 & 8789.82 & 17249.86 & 15038.13 \\
\hline
\end{tabular}

\begin{tabular}{|c|c|c|c|c|c|c|}
\hline \multirow{2}{*}{} & \multicolumn{2}{|c|}{ Case 4 } & \multicolumn{2}{c|}{ Case 6 } & \multicolumn{2}{c|}{ Case 8 } \\
\cline { 2 - 7 } & \multicolumn{2}{|c|}{ LC tightness $(\gamma)$} & \multicolumn{2}{c|}{ LC tightness $(\gamma)$} & \multicolumn{2}{c|}{ LC tightness $(\gamma)$} \\
\hline DV & H $(0.7)$ & L $(1.3)$ & H $(0.7)$ & L $(1.3)$ & H $(0.7)$ & L $(1.3)$ \\
\hline L(0.1) & 4397.76 & 4055.29 & 7741.01 & 6668.47 & 13696.65 & 11409.45 \\
$\mathrm{M}(1.0)$ & 5143.13 & 4759.85 & 8850.40 & 7644.74 & 15589.67 & 13136.07 \\
$\mathrm{H}(1.8)$ & 5764.62 & 5385.18 & 9741.11 & 8514.50 & 17116.27 & 14666.01 \\
\hline
\end{tabular}


Table 6.10: Computational results under BP setting (Cont.)

(c) Ordering quantity of rewards

(with uniformly distributed demand)

\begin{tabular}{|c|c|c|c|c|c|c|}
\hline \multirow{2}{*}{} & \multicolumn{2}{|c|}{ Case 4 } & \multicolumn{2}{c|}{ Case 6 } & \multicolumn{2}{c|}{ Case 8 } \\
\cline { 2 - 7 } & \multicolumn{2}{|c|}{ LC tightness $(\gamma)$} & \multicolumn{2}{c|}{ LC tightness $(\gamma)$} & \multicolumn{2}{c|}{ LC tightness $(\gamma)$} \\
\hline DV & $\mathrm{H}(0.7)$ & $\mathrm{L}(1.3)$ & $\mathrm{H}(0.7)$ & $\mathrm{L}(1.3)$ & $\mathrm{H}(0.7)$ & $\mathrm{L}(1.3)$ \\
\hline $\mathrm{L}(0.05)$ & 633.20 & 534.07 & 1296.23 & 972.16 & 2243.74 & 1554.30 \\
$\mathrm{M}(0.15)$ & 695.16 & 607.20 & 1409.70 & 1096.47 & 2421.21 & 1752.90 \\
$\mathrm{H}(0.25)$ & 747.37 & 680.33 & 1497.46 & 1220.78 & 2559.09 & 1951.51 \\
\hline
\end{tabular}

(with normally distributed demand)

\begin{tabular}{|c|c|c|c|c|c|c|}
\hline \multirow{2}{*}{} & \multicolumn{2}{|c|}{ Case 4 } & \multicolumn{2}{c|}{ Case 6 } & \multicolumn{2}{c|}{ Case 8 } \\
\cline { 2 - 7 } & \multicolumn{2}{|c|}{ LC tightness $(\gamma)$} & \multicolumn{2}{c|}{ LC tightness $(\gamma)$} & \multicolumn{2}{c|}{ LC tightness $(\gamma)$} \\
\hline DV & H $(0.7)$ & L $(1.3)$ & H $(0.7)$ & L $(1.3)$ & H $(0.7)$ & L $(1.3)$ \\
\hline L(0.1) & 609.77 & 507.44 & 1248.54 & 925.47 & 2171.78 & 1480.85 \\
$\mathrm{M}(1.0)$ & 692.94 & 596.94 & 1393.03 & 1064.73 & 2397.70 & 1713.50 \\
H(1.8) & 754.00 & 676.42 & 1491.75 & 1188.75 & 2550.26 & 1919.80 \\
\hline
\end{tabular}

Shown in Table 6.11 are the budget usage ratios generated in Step 4 of the procedure designed for conducting the numerical studies in this section (see Section 5.5 in Chapter 5 for more details of the procedure). In Table 6.11 , the budget ratio of Type I refers to the ratio of the current budget usage (obtained in the model without budget and capacity constraints) to the initial budget usage (obtained in the model with budget and capacity constraints). The budget ratio of Type II refers to the ratio of the current budget usage to the maximum budget available $\left(W^{R}\right)$. With respect to the budget usage, we observe that increased liability control tightness results in a higher budget usage. As such, the budget ratio of Type I in each testing case is greater than 1.0. We also observe that the budget ratio of Type I increases with the increase in liability control tightness (i.e., $\gamma$ changes from 1.3 to 0.7 ). Furthermore, in some cases, the current budget usage (obtained in the model without budget and capacity constraints) is even larger than the maximum amount of budget (i.e., the right-hand side of the budget constraint: $W^{R}$ ) (see budget ratio of Type 
II in Table 6.11), especially when the liability control is tight (i.e., $\gamma=0.7$ ) and the demand variability level is high.

Table 6.11 Comparison of the budget usages under BP setting

(with uniformly distributed demand)

(a) Budget Ratio (Type I)

(with normally distributed demand)

\begin{tabular}{|c|c|c|c|c|c|c|}
\hline \multirow{2}{*}{} & \multicolumn{2}{|c|}{ Case 4 } & \multicolumn{2}{c|}{ Case 6 } & \multicolumn{2}{c|}{ Case 8 } \\
\cline { 2 - 7 } & \multicolumn{2}{|c|}{ LC tightness $(\gamma)$} & \multicolumn{2}{c|}{ LC tightness $(\gamma)$} & \multicolumn{2}{c|}{ LC tightness $(\gamma)$} \\
\hline $\mathrm{DV}$ & $\mathrm{H}(0.7)$ & $\mathrm{L}(1.3)$ & $\mathrm{H}(0.7)$ & $\mathrm{L}(1.3)$ & $\mathrm{H}(0.7)$ & $\mathrm{L}(1.3)$ \\
\hline $\mathrm{L}(0.05)$ & 1.086 & 1.007 & 1.158 & 1.002 & 1.197 & 1.004 \\
$\mathrm{M}(0.15)$ & 1.102 & 1.034 & 1.170 & 1.026 & 1.207 & 1.030 \\
$\mathrm{H}(0.25)$ & 1.133 & 1.079 & 1.208 & 1.080 & 1.243 & 1.083 \\
\hline
\end{tabular}

\begin{tabular}{|c|c|c|c|c|c|c|}
\hline \multirow{2}{*}{} & \multicolumn{2}{|c|}{ Case 4 } & \multicolumn{2}{c|}{ Case 6 } & \multicolumn{2}{c|}{ Case 8 } \\
\cline { 2 - 7 } & \multicolumn{2}{|c|}{ LC tightness $(\gamma)$} & \multicolumn{2}{c|}{ LC tightness $(\gamma)$} & \multicolumn{2}{c|}{ LC tightness $(\gamma)$} \\
\hline $\mathrm{DV}$ & $\mathrm{H}(0.7)$ & $\mathrm{L}(1.3)$ & $\mathrm{H}(0.7)$ & $\mathrm{L}(1.3)$ & $\mathrm{H}(0.7)$ & $\mathrm{L}(1.3)$ \\
\hline $\mathrm{L}(0.1)$ & 1.085 & 1.001 & 1.161 & 1.000 & 1.201 & 1.000 \\
$\mathrm{M}(1.0)$ & 1.112 & 1.030 & 1.176 & 1.016 & 1.211 & 1.021 \\
$\mathrm{H}(1.8)$ & 1.153 & 1.077 & 1.214 & 1.061 & 1.251 & 1.072 \\
\hline
\end{tabular}

(b) Budget Ratio (Type II)

(with uniformly distributed demand)

\begin{tabular}{|c|c|c|c|c|c|c|}
\hline \multirow{2}{*}{} & \multicolumn{2}{|c|}{ Case 4 } & \multicolumn{2}{c|}{ Case 6 } & \multicolumn{2}{c|}{ Case 8 } \\
\cline { 2 - 7 } & \multicolumn{2}{|c|}{ LC tightness $(\gamma)$} & \multicolumn{2}{c|}{ LC tightness $(\gamma)$} & \multicolumn{2}{c|}{ LC tightness $(\gamma)$} \\
\hline $\mathrm{DV}$ & $\mathrm{H}(0.7)$ & $\mathrm{L}(1.3)$ & $\mathrm{H}(0.7)$ & $\mathrm{L}(1.3)$ & $\mathrm{H}(0.7)$ & $\mathrm{L}(1.3)$ \\
\hline $\mathrm{L}(0.05)$ & 0.905 & 0.839 & 0.966 & 0.836 & 1.002 & 0.840 \\
$\mathrm{M}(0.15)$ & 1.017 & 0.954 & 1.075 & 0.943 & 1.111 & 0.948 \\
$\mathrm{H}(0.25)$ & 1.123 & 1.070 & 1.173 & 1.049 & 1.210 & 1.055 \\
\hline
\end{tabular}

(with normally distributed demand)

\begin{tabular}{|c|c|c|c|c|c|c|}
\hline \multirow{2}{*}{} & \multicolumn{2}{|c|}{ Case 4 } & \multicolumn{2}{c|}{ Case 6 } & \multicolumn{2}{c|}{ Case 8 } \\
\cline { 2 - 7 } & \multicolumn{2}{|c|}{ LC tightness $(\gamma)$} & \multicolumn{2}{c|}{ LC tightness $(\gamma)$} & \multicolumn{2}{c|}{ LC tightness $(\gamma)$} \\
\hline $\mathrm{DV}$ & $\mathrm{H}(0.7)$ & $\mathrm{L}(1.3)$ & $\mathrm{H}(0.7)$ & $\mathrm{L}(1.3)$ & $\mathrm{H}(0.7)$ & $\mathrm{L}(1.3)$ \\
\hline $\mathrm{L}(0.1)$ & 0.864 & 0.797 & 0.924 & 0.796 & 0.961 & 0.800 \\
$\mathrm{M}(1.0)$ & 1.010 & 0.935 & 1.056 & 0.912 & 1.094 & 0.921 \\
$\mathrm{H}(1.8)$ & 1.132 & 1.058 & 1.162 & 1.016 & 1.201 & 1.029 \\
\hline
\end{tabular}


This examination of the budget usage, considering the target liability control requirement (e.g., defined by liability control parameter $\gamma$ ), provides a means of determining whether or not the budget constraint would be violated. In other words, if the model results show that the budget usage is lower than the maximum budget available (i.e., $W^{R}$ ), then we know that we can meet the target liability control requirement by purchasing more rewards. If the model results show that the budget usage is larger than the maximum budget available, then we can tell what the extra amount of budget required to meet the target liability control requirement will be.

\subsubsection{Under EP1 Setting}

Table 6.12 summarizes the results of EP1 when budget constraint (4.51) and capacity constraints (4.50) are relaxed and the liability control becomes tight (i.e., $\gamma=0.7$ ). We observe that LRP profitability decreases with the increase of liability control tightness (i.e., $\gamma$ changes from 1.3 to 0.7). When liability control is tight (e.g., $\gamma=0.7$ ), higher target redemption is expected. In other words, the estimated optimal ordering quantities of rewards and the cost for purchasing rewards (i.e., budget usage) increase, which results in decrease in LRP profitability at the redemption side. As such, the overall LRP profitability decreases. On the other hand, just as in the case that the liability control is loose (i.e., $\gamma=1.3$ ), with the increase in demand variability, the LRP profitability decreases in the testing case 4 , and decreases first (e.g., DV=0.15) and then increases (e.g., $\mathrm{DV}=0.25$ ) in testing cases 6 and 8 in the case that liability control is tight. This result suggests that as long as the budget and the capacities for rewards are not limited, the LRP host may reduce the negative impact of demand uncertainty on LRP profitability with a larger number of accumulation and redemption partners (e.g., in Case 6, there are 40 accumulation partners, and 40 redemption partners; and in Case 8 , there are 70 accumulation partners, and 65 
redemption partners), even when the host attempts to implement a tighter liability control requirement.

Table 6.12 Computational results under EP1 setting

(a) LRP Profitability

(with uniformly distributed demand)

(with normally distributed demand)

\begin{tabular}{|c|c|c|c|c|c|c|}
\hline \multirow{2}{*}{} & \multicolumn{2}{|c|}{ Case 4 } & \multicolumn{2}{c|}{ Case 6 } & \multicolumn{2}{c|}{ Case 8 } \\
\cline { 2 - 7 } & \multicolumn{2}{|c|}{ LC tightness $(\gamma)$} & \multicolumn{2}{c|}{ LC tightness $(\gamma)$} & \multicolumn{2}{c|}{ LC tightness $(\gamma)$} \\
\hline DV & $\mathrm{H}(0.7)$ & $\mathrm{L}(1.3)$ & $\mathrm{H}(0.7)$ & $\mathrm{L}(1.3)$ & $\mathrm{H}(0.7)$ & $\mathrm{L}(1.3)$ \\
\hline $\mathrm{L}(0.05)$ & 26262.89 & 26450.81 & 37403.69 & 38015.03 & 66856.38 & 68136.93 \\
$\mathrm{M}(0.15)$ & 26056.79 & 26218.49 & 37246.98 & 37815.19 & 66673.21 & 67868.85 \\
$\mathrm{H}(0.25)$ & 25960.22 & 26076.68 & 37442.39 & 37917.90 & 67131.84 & 68173.06 \\
\hline
\end{tabular}

\begin{tabular}{|c|c|c|c|c|c|c|}
\hline \multirow{2}{*}{} & \multicolumn{2}{|c|}{ Case 4 } & \multicolumn{2}{c|}{ Case 6 } & \multicolumn{2}{c|}{ Case 8 } \\
\cline { 2 - 7 } & \multicolumn{2}{|c|}{ LC tightness $(\gamma)$} & \multicolumn{2}{c|}{ LC tightness $(\gamma)$} & \multicolumn{2}{c|}{ LC tightness $(\gamma)$} \\
\hline DV & H $(0.7)$ & L $(1.3)$ & H $(0.7)$ & L $(1.3)$ & H $(0.7)$ & L $(1.3)$ \\
\hline L(0.1) & 26393.36 & 26584.82 & 37665.59 & 38277.03 & 67302.10 & 68589.72 \\
M(1.0) & 25929.80 & 26088.02 & 36879.95 & 37436.73 & 65912.47 & 67068.27 \\
H(1.8) & 25753.62 & 25857.43 & 36873.07 & 37328.37 & 65938.03 & 66901.97 \\
\hline
\end{tabular}

(b) Costs of Rewards

\begin{tabular}{|c|c|c|c|c|c|c|}
\hline \multirow{2}{*}{} & \multicolumn{2}{|c|}{ Case 4 } & \multicolumn{2}{c|}{ Case 6 } & \multicolumn{2}{c|}{ Case 8 } \\
\cline { 2 - 7 } & \multicolumn{2}{|c|}{ LC tightness $(\gamma)$} & \multicolumn{2}{c|}{ LC tightness $(\gamma)$} & \multicolumn{2}{c|}{ LC tightness $(\gamma)$} \\
\hline DV & $\mathrm{H}(0.7)$ & $\mathrm{L}(1.3)$ & $\mathrm{H}(0.7)$ & $\mathrm{L}(1.3)$ & $\mathrm{H}(0.7)$ & $\mathrm{L}(1.3)$ \\
\hline L(0.05) & 4661.61 & 4270.61 & 8269.33 & 7005.97 & 14606.07 & 11981.71 \\
$\mathrm{M}(0 \quad 15)$ & 5227.74 & 4857.84 & 9156.05 & 7897.89 & 16097.44 & 13509.92 \\
$\mathrm{H}(0.25)$ & 5755.84 & 5445.07 & 9944.04 & 8789.82 & 17449.95 & 15038.13 \\
\hline
\end{tabular}

(with uniformly distributed demand)

(with normally distributed demand)

\begin{tabular}{|c|c|c|c|c|c|c|}
\hline \multirow{2}{*}{} & \multicolumn{2}{|c|}{ Case 4 } & \multicolumn{2}{c|}{ Case 6 } & \multicolumn{2}{c|}{ Case 8 } \\
\cline { 2 - 7 } & \multicolumn{2}{|c|}{ LC tightness $(\gamma)$} & \multicolumn{2}{c|}{ LC tightness $(\gamma)$} & \multicolumn{2}{c|}{ LC tightness $(\gamma)$} \\
\hline DV & $\mathrm{H}(0.7)$ & $\mathrm{L}(1.3)$ & $\mathrm{H}(0.7)$ & $\mathrm{L}(1.3)$ & $\mathrm{H}(0.7)$ & $\mathrm{L}(1.3)$ \\
\hline $\mathrm{L}(0.1)$ & 4448.15 & 4055.29 & 7910.67 & 6668.47 & 14016.50 & 11409.45 \\
$\mathrm{M}(1.0)$ & 5180.04 & 4759.85 & 8962.94 & 7644.74 & 15794.25 & 13136.07 \\
$\mathrm{H}(1.8)$ & 5781.72 & 5385.18 & 9794.42 & 8514.50 & 17226.13 & 14666.01 \\
\hline
\end{tabular}


Table 6.12 Computational results under EP1 setting (Cont.)

(with uniformly distributed demand)

(c) Ordering quantity of rewards

\begin{tabular}{|c|c|c|c|c|c|c|}
\hline \multirow{2}{*}{} & \multicolumn{2}{|c|}{ Case 4 } & \multicolumn{2}{c|}{ Case 6 } & \multicolumn{2}{c|}{ Case 8 } \\
\cline { 2 - 7 } & \multicolumn{2}{|c|}{ LC tightness $(\gamma)$} & \multicolumn{2}{c|}{ LC tightness $(\gamma)$} & \multicolumn{2}{c|}{ LC tightness $(\gamma)$} \\
\hline $\mathrm{DV}$ & $\mathrm{H}(0.7)$ & $\mathrm{L}(1.3)$ & $\mathrm{H}(0.7)$ & $\mathrm{L}(1.3)$ & $\mathrm{H}(0.7)$ & $\mathrm{L}(1.3)$ \\
\hline $\mathrm{L}(0.05)$ & 650.07 & 534.07 & 1349.15 & 972.16 & 2341.07 & 1554.30 \\
$\mathrm{M}(0.15)$ & 710.37 & 607.20 & 1455.52 & 1096.47 & 2501.75 & 1752.90 \\
$\mathrm{H}(0.25)$ & 759.07 & 680.33 & 1531.97 & 1220.78 & 2620.10 & 1951.51 \\
\hline
\end{tabular}

\begin{tabular}{|c|c|c|c|c|c|c|}
\hline \multirow{2}{*}{} & \multicolumn{2}{|c|}{ Case 4 } & \multicolumn{2}{c|}{ Case 6 } & \multicolumn{2}{c|}{ Case 8 } \\
\cline { 2 - 7 } & \multicolumn{2}{|c|}{ LC tightness $(\gamma)$} & \multicolumn{2}{c|}{ LC tightness $(\gamma)$} & \multicolumn{2}{c|}{ LC tightness $(\gamma)$} \\
\hline DV & H $(0.7)$ & L $(1.3)$ & H $(0.7)$ & L $(1.3)$ & H $(0.7)$ & L $(1.3)$ \\
\hline L(0.1) & 625.13 & 507.44 & 1300.24 & 925.47 & 2269.30 & 1480.85 \\
M(1.0) & 704.20 & 596.94 & 1427.33 & 1064.73 & 2460.07 & 1713.50 \\
H(1.8) & 759.21 & 676.42 & 1508.00 & 1188.75 & 2583.75 & 1919.80 \\
\hline
\end{tabular}

(with normally distributed demand)

(d) Bonus points offering

(with uniformly distributed demand)

\begin{tabular}{|c|c|c|c|c|c|c|}
\hline \multirow{2}{*}{} & \multicolumn{2}{|c|}{ Case 4 } & \multicolumn{2}{c|}{ Case 6 } & \multicolumn{2}{c|}{ Case 8 } \\
\cline { 2 - 7 } & \multicolumn{2}{|c|}{ LC tightness $(\gamma)$} & \multicolumn{2}{c|}{ LC tightness $(\gamma)$} & \multicolumn{2}{c|}{ LC tightness $(\gamma)$} \\
\hline $\mathrm{DV}$ & $\mathrm{H}(0.7)$ & $\mathrm{L}(1.3)$ & $\mathrm{H}(0.7)$ & $\mathrm{L}(1.3)$ & $\mathrm{H}(0.7)$ & $\mathrm{L}(1.3)$ \\
\hline $\mathrm{L}(0.05)$ & 23.69 & 23.69 & 78.79 & 78.79 & 148.44 & 148.44 \\
$\mathrm{M}(0.15)$ & 19.21 & 19.21 & 63.86 & 63.86 & 120.32 & 120.32 \\
$\mathrm{H}(0.25)$ & 14.72 & 14.72 & 48.94 & 48.94 & 92.20 & 92.20 \\
\hline
\end{tabular}

(with normally distributed demand)

\begin{tabular}{|c|c|c|c|c|c|c|}
\hline \multirow{2}{*}{} & \multicolumn{2}{|c|}{ Case 4 } & \multicolumn{2}{c|}{ Case 6 } & \multicolumn{2}{c|}{ Case 8 } \\
\cline { 2 - 7 } & \multicolumn{2}{|c|}{ LC tightness $(\gamma)$} & \multicolumn{2}{c|}{ LC tightness $(\gamma)$} & \multicolumn{2}{c|}{ LC tightness $(\gamma)$} \\
\hline DV & $\mathrm{H}(0.7)$ & $\mathrm{L}(1.3)$ & $\mathrm{H}(0.7)$ & $\mathrm{L}(1.3)$ & $\mathrm{H}(0.7)$ & $\mathrm{L}(1.3)$ \\
\hline $\mathrm{L}(0.1)$ & 24.57 & 24.57 & 82.11 & 82.11 & 154.82 & 154.82 \\
$\mathrm{M}(1.0)$ & 12.22 & 12.22 & 44.85 & 44.85 & 85.74 & 85.74 \\
$\mathrm{H}(1.8)$ & 3.90 & 3.90 & 15.94 & 15.94 & 30.87 & 30.87 \\
\hline
\end{tabular}

As shown in Table 6.12, we also observe that changes in liability control parameter have no impact on the LRP host's decisions on offering bonus points. The estimated optimal decisions on 
offering bonus points remain the same, regardless any changes in the liability control requirement.

Table 6.13 Comparison of the budget usage under EP1 setting

(with uniformly distributed demand)

(with normally distributed demand)

(a) Budget Ratio (Type I)

\begin{tabular}{|c|c|c|c|c|c|c|}
\hline \multirow{2}{*}{} & \multicolumn{2}{|c|}{ Case 4 } & \multicolumn{2}{c|}{ Case 6 } & \multicolumn{2}{c|}{ Case 8 } \\
\cline { 2 - 7 } & \multicolumn{2}{|c|}{ LC tightness $(\gamma)$} & \multicolumn{2}{c|}{ LC tightness $(\gamma)$} & \multicolumn{2}{c|}{ LC tightness $(\gamma)$} \\
\hline $\mathrm{DV}$ & $\mathrm{H}(0.7)$ & $\mathrm{L}(1.3)$ & $\mathrm{H}(0.7)$ & $\mathrm{L}(1.3)$ & $\mathrm{H}(0.7)$ & $\mathrm{L}(1.3)$ \\
\hline $\mathrm{L}(0.05)$ & 1.099 & 1.007 & 1.183 & 1.002 & 1.224 & 1.004 \\
$\mathrm{M}(0.15)$ & 1.113 & 1.034 & 1.189 & 1.026 & 1.227 & 1.030 \\
$\mathrm{H}(0.25)$ & 1.141 & 1.079 & 1.222 & 1.080 & 1.257 & 1.083 \\
\hline
\end{tabular}

\begin{tabular}{|c|c|c|c|c|c|c|}
\hline \multirow{2}{*}{} & \multicolumn{2}{|c|}{ Case 4 } & \multicolumn{2}{c|}{ Case 6 } & \multicolumn{2}{c|}{ Case 8 } \\
\cline { 2 - 7 } & \multicolumn{2}{|c|}{ LC tightness $(\gamma)$} & \multicolumn{2}{c|}{ LC tightness $(\gamma)$} & \multicolumn{2}{c|}{ LC tightness $(\gamma)$} \\
\hline $\mathrm{DV}$ & $\mathrm{H}(0.7)$ & $\mathrm{L}(1.3)$ & $\mathrm{H}(0.7)$ & $\mathrm{L}(1.3)$ & $\mathrm{H}(0.7)$ & $\mathrm{L}(1.3)$ \\
\hline $\mathrm{L}(0.1)$ & 1.098 & 1.001 & 1.186 & 1.000 & 1.229 & 1.000 \\
$\mathrm{M}(1.0)$ & 1.120 & 1.030 & 1.191 & 1.016 & 1.227 & 1.021 \\
$\mathrm{H}(1.8)$ & 1.157 & 1.077 & 1.220 & 1.061 & 1.259 & 1.072 \\
\hline
\end{tabular}

(b) Budget Ratio (Type II)

(with uniformly distributed demand)

\begin{tabular}{|c|c|c|c|c|c|c|}
\hline \multirow{2}{*}{} & \multicolumn{2}{|c|}{ Case 4 } & \multicolumn{2}{c|}{ Case 6 } & \multicolumn{2}{c|}{ Case 8 } \\
\cline { 2 - 7 } & \multicolumn{2}{|c|}{ LC tightness $(\gamma)$} & \multicolumn{2}{c|}{ LC tightness $(\gamma)$} & \multicolumn{2}{c|}{ LC tightness $(\gamma)$} \\
\hline $\mathrm{DV}$ & $\mathrm{H}(0.7)$ & $\mathrm{L}(1.3)$ & $\mathrm{H}(0.7)$ & $\mathrm{L}(1.3)$ & $\mathrm{H}(0.7)$ & $\mathrm{L}(1.3)$ \\
\hline $\mathrm{L}(0.05)$ & $\mathbf{0 . 9 1 6}$ & 0.839 & 0.987 & 0.836 & 1.025 & 0.840 \\
$\mathrm{M}(0.15)$ & 1.027 & 0.954 & 1.093 & 0.943 & 1.129 & 0.948 \\
$\mathrm{H}(0.25)$ & 1.131 & 1.070 & 1.187 & 1.049 & 1.224 & 1.055 \\
\hline
\end{tabular}

(with normally distributed demand)

\begin{tabular}{|c|c|c|c|c|c|c|}
\hline \multirow{2}{*}{} & \multicolumn{2}{|c|}{ Case 4 } & \multicolumn{2}{c|}{ Case 6 } & \multicolumn{2}{c|}{ Case 8 } \\
\cline { 2 - 7 } & \multicolumn{2}{|c|}{ LC tightness $(\gamma)$} & \multicolumn{2}{c|}{ LC tightness $(\gamma)$} & \multicolumn{2}{c|}{ LC tightness $(\gamma)$} \\
\hline $\mathrm{DV}$ & $\mathrm{H}(0.7)$ & $\mathrm{L}(1.3)$ & $\mathrm{H}(0.7)$ & $\mathrm{L}(1.3)$ & $\mathrm{H}(0.7)$ & $\mathrm{L}(1.3)$ \\
\hline $\mathrm{L}(0.1)$ & 0.874 & 0.797 & 0.944 & 0.796 & 0.983 & 0.800 \\
$\mathrm{M}(1.0)$ & 1.017 & 0.935 & 1.070 & 0.912 & 1.108 & 0.921 \\
$\mathrm{H}(1.8)$ & 1.136 & 1.058 & 1.169 & 1.016 & 1.208 & 1.029 \\
\hline
\end{tabular}


The budget usage ratio for each testing cases is reported in Table 6.13. We observe that increased liability control tightness results in higher budget usage. As such, the budget ratio of Type-I in most testing problems is greater than 1.0. We also observe that the budget ratio of Type-II increases with the increase in liability control tightness (i.e., $\gamma$ changes from 1.3 to 0.7 ). Moreover, in some cases the current budget usage (obtained in the model without budget and capacity constraints) is even larger than the maximum amount of budget (i.e., the right hand side of the budget constraint: $W^{R}$ ) (see the budget ratio of Type-II in Table 6.13), especially when the liability control is tight (i.e., $\gamma=0.7$ ) and the demand variability level is high. The same type of budget-related analysis as that discussed in Section 6.4.1 can be done under EP1 setting.

\subsubsection{Under EP2 Setting}

Table 6.14 provides a summary of EP2 model results obtained when the budget constraint (4.64) and the capacity constraint (4.61) are relaxed and the liability control becomes tight (i.e., $\gamma=0.7$ ). Similar to what has been observed and discussed under BP and EP1 settings, higher redemption is required when the liability control gets tighter. We also observe that under EP2 setting, a higher redemption results in an increase in the optimal ordering quantity of rewards and in the optimal number of options to purchase and to exercise. As such, the corresponding costs for purchasing rewards and options and for exercising options increase and the overall LRP profitability is reduced due to the decreases in the redemption side LRP profitability. Meanwhile, when the liability control is tight (i.e., $\gamma=0.7$ ), the number of options to purchase as well as the number of options to exercise increase considerably. In Table 6.14(d), for example, in the testing case 4 , given $\mathrm{DV}=0.05$, the optimal solution shows that the number of options to purchase increases from $5.19 \%$ of the initial ordering quantity of rewards $(=511.09)$ when $\gamma=1.3$ to 
$17.25 \%$ of the initial ordering quantity of rewards $(=531.06)$ when $\gamma=0.7$ (see Table $6.14(d)$ and (e)). Meanwhile, the number of options to exercise increases from $18.97 \%$ of the options to purchase $(=26.51)$ when $\gamma=1.3$ to $90.58 \%$ of the options to purchase $(=91.63)$ when $\gamma=0.7$ (see Table 6.14(f) and (g)).

Table 6.14 Computational results under EP2 setting

(a) LRP Profitability

(with uniformly distributed demand)

\begin{tabular}{|c|c|c|c|c|c|c|}
\hline \multirow{2}{*}{} & \multicolumn{2}{|c|}{ Case 4 } & \multicolumn{2}{c|}{ Case 6 } & \multicolumn{2}{c|}{ Case 8 } \\
\cline { 2 - 7 } & \multicolumn{2}{|c|}{ LC tightness $(\gamma)$} & \multicolumn{2}{c|}{ LC tightness $(\gamma)$} & \multicolumn{2}{c|}{ LC tightness $(\gamma)$} \\
\hline DV & $\mathrm{H}(0.7)$ & $\mathrm{L}(1.3)$ & $\mathrm{H}(0.7)$ & $\mathrm{L}(1.3)$ & $\mathrm{H}(0.7)$ & $\mathrm{L}(1.3)$ \\
\hline $\mathrm{L}(0.05)$ & 25793.31 & 26102.01 & 35552.92 & 36744.12 & 63130.63 & 65730.73 \\
$\mathrm{M}(0.15)$ & 25865.52 & 26127.03 & 36251.64 & 37223.11 & 64459.57 & 66725.78 \\
$\mathrm{H}(0.25)$ & 25933.31 & 26157.59 & 36790.42 & 37708.46 & 65603.96 & 67731.81 \\
\hline
\end{tabular}

(with normally distributed demand)

\begin{tabular}{|c|c|c|c|c|c|c|}
\hline \multirow{2}{*}{} & \multicolumn{2}{|c|}{ Case 4 } & \multicolumn{2}{c|}{ Case 6 } & \multicolumn{2}{c|}{ Case 8 } \\
\cline { 2 - 7 } & \multicolumn{2}{|c|}{ LC tightness $(\gamma)$} & \multicolumn{2}{c|}{ LC tightness $(\gamma)$} & \multicolumn{2}{c|}{ LC tightness $(\gamma)$} \\
\hline DV & $\mathrm{H}(0.7)$ & $\mathrm{L}(1.3)$ & $\mathrm{H}(0.7)$ & $\mathrm{L}(1.3)$ & $\mathrm{H}(0.7)$ & $\mathrm{L}(1.3)$ \\
\hline $\mathrm{L}(0.1)$ & 25763.76 & 26090.71 & 35274.95 & 36563.79 & 62623.07 & 65349.55 \\
$\mathrm{M}(1.0)$ & 25835.22 & 26098.21 & 36114.58 & 37077.70 & 64137.71 & 66352.97 \\
$\mathrm{H}(1.8)$ & 25863.09 & 26111.84 & 36605.81 & 37543.11 & 65102.10 & 67269.32 \\
\hline
\end{tabular}

(b) Costs of Rewards (including purchasing and exercising costs of options)

(with uniformly distributed demand)

\begin{tabular}{|c|c|c|c|c|c|c|}
\hline \multirow{2}{*}{} & \multicolumn{2}{|c|}{ Case 4 } & \multicolumn{2}{c|}{ Case 6 } & \multicolumn{2}{c|}{ Case 8 } \\
\cline { 2 - 7 } & \multicolumn{2}{|c|}{ LC tightness $(\gamma)$} & \multicolumn{2}{c|}{ LC tightness $(\gamma)$} & \multicolumn{2}{c|}{ LC tightness $(\gamma)$} \\
\hline DV & $\mathrm{H}(0.7)$ & $\mathrm{L}(1.3)$ & $\mathrm{H}(0.7)$ & $\mathrm{L}(1.3)$ & $\mathrm{H}(0.7)$ & $\mathrm{L}(1.3)$ \\
\hline $\mathrm{L}(0.05)$ & 4532.30 & 4159.42 & 8156.57 & 6863.38 & 14496.86 & 11731.83 \\
$\mathrm{M}(0.15)$ & 4947.28 & 4517.58 & 8680.58 & 7458.54 & 15426.96 & 12731.26 \\
$\mathrm{H}(0.25)$ & 5322.80 & 4865.75 & 9235.63 & 8036.70 & 16338.47 & 13701.37 \\
\hline
\end{tabular}

(with normally distributed demand)

\begin{tabular}{|c|c|c|c|c|c|c|}
\hline \multirow{2}{*}{} & \multicolumn{2}{|c|}{ Case 4 } & \multicolumn{2}{c|}{ Case 6 } & \multicolumn{2}{c|}{ Case 8 } \\
\cline { 2 - 7 } & \multicolumn{2}{|c|}{ LC tightness $(\gamma)$} & \multicolumn{2}{c|}{ LC tightness $(\gamma)$} & \multicolumn{2}{c|}{ LC tightness $(\gamma)$} \\
\hline DV & H $(0.7)$ & L $(1.3)$ & H $(0.7)$ & L $(1.3)$ & H $(0.7)$ & L (1.3) \\
\hline L(0.1) & 4373.06 & 4023.95 & 7956.85 & 6631.53 & 14131.49 & 11343.88 \\
M(1.0) & 4886.76 & 4436.02 & 8503.74 & 7272.47 & 15171.19 & 12466.16 \\
H(1.8) & 5328.08 & 4797.57 & 9131.59 & 7834.55 & 16237.16 & 13436.59 \\
\hline
\end{tabular}


Table 6.14 Computational results under EP2 setting (Cont.)

(with uniformly distributed demand)

(c) Initial ordering quantity of rewards

\begin{tabular}{|c|c|c|c|c|c|c|}
\hline \multirow{2}{*}{} & \multicolumn{2}{|c|}{ Case 4 } & \multicolumn{2}{c|}{ Case 6 } & \multicolumn{2}{c|}{ Case 8 } \\
\cline { 2 - 7 } & \multicolumn{2}{|c|}{ LC tightness $(\gamma)$} & \multicolumn{2}{c|}{ LC tightness $(\gamma)$} & \multicolumn{2}{c|}{ LC tightness $(\gamma)$} \\
\hline $\mathrm{DV}$ & $\mathrm{H}(0.7)$ & $\mathrm{L}(1.3)$ & $\mathrm{H}(0.7)$ & $\mathrm{L}(1.3)$ & $\mathrm{H}(0.7)$ & $\mathrm{L}(1.3)$ \\
\hline $\mathrm{L}(0.05)$ & 531.06 & 511.09 & 969.73 & 936.22 & 1547.79 & 1495.61 \\
$\mathrm{M}(0.15)$ & 590.09 & 536.60 & 1066.69 & 985.13 & 1698.33 & 1569.13 \\
$\mathrm{H}(0.25)$ & 635.55 & 559.99 & 1125.89 & 1029.03 & 1793.49 & 1634.42 \\
\hline
\end{tabular}

(with normally distributed demand)

\begin{tabular}{|c|c|c|c|c|c|c|}
\hline \multirow{2}{*}{} & \multicolumn{2}{|c|}{ Case 4 } & \multicolumn{2}{c|}{ Case 6 } & \multicolumn{2}{c|}{ Case 8 } \\
\cline { 2 - 7 } & \multicolumn{2}{|c|}{ LC tightness $(\gamma)$} & \multicolumn{2}{c|}{ LC tightness $(\gamma)$} & \multicolumn{2}{c|}{ LC tightness $(\gamma)$} \\
\hline DV & H $(0.7)$ & L $(1.3)$ & H $(0.7)$ & L $(1.3)$ & H $(0.7)$ & L (1.3) \\
\hline L $(0.1)$ & 507.06 & 500.43 & 925.29 & 915.12 & 1480.32 & 1463.51 \\
$\mathrm{M}(1.0)$ & 581.77 & 524.45 & 1045.47 & 960.15 & 1671.37 & 1535.71 \\
$\mathrm{H}(1.8)$ & 630.01 & 544.64 & 1112.70 & 997.51 & 1770.49 & 1590.09 \\
\hline
\end{tabular}

(d) Number of options to purchase

(with uniformly distributed demand)

\begin{tabular}{|c|c|c|c|c|c|c|}
\hline \multirow{2}{*}{} & \multicolumn{2}{|c|}{ Case 4 } & \multicolumn{2}{c|}{ Case 6 } & \multicolumn{2}{c|}{ Case 8 } \\
\cline { 2 - 7 } & \multicolumn{2}{|c|}{ LC tightness $(\gamma)$} & \multicolumn{2}{c|}{ LC tightness $(\gamma)$} & \multicolumn{2}{c|}{ LC tightness $(\gamma)$} \\
\hline DV & H $(0.7)$ & L $(1.3)$ & H $(0.7)$ & L $(1.3)$ & H $(0.7)$ & L (1.3) \\
\hline L(0.05) & 91.63 & 26.51 & 308.35 & 45.18 & 669.09 & 73.40 \\
M(0.15) & 83.03 & 81.20 & 305.21 & 139.06 & 663.50 & 227.90 \\
H(0.25) & 95.81 & 138.01 & 367.56 & 237.96 & 726.98 & 390.63 \\
\hline
\end{tabular}

(with normally distributed demand)

\begin{tabular}{|c|c|c|c|c|c|c|}
\hline \multirow{2}{*}{} & \multicolumn{2}{|c|}{ Case 4 } & \multicolumn{2}{c|}{ Case 6 } & \multicolumn{2}{c|}{ Case 8 } \\
\cline { 2 - 7 } & \multicolumn{2}{|c|}{ LC tightness $(\gamma)$} & \multicolumn{2}{c|}{ LC tightness $(\gamma)$} & \multicolumn{2}{c|}{ LC tightness $(\gamma)$} \\
\hline DV & H (0.7) & L $(1.3)$ & H $(0.7)$ & L $(1.3)$ & H $(0.7)$ & L (1.3) \\
\hline L(0.1) & 99.49 & 9.77 & 317.96 & 15.53 & 681.57 & 26.01 \\
M(1.0) & 93.24 & 100.06 & 305.67 & 156.27 & 661.29 & 264.45 \\
H(1.8) & 123.60 & 181.52 & 384.09 & 284.08 & 771.63 & 484.87 \\
\hline
\end{tabular}


Table 6.14 Computational results under EP2 setting (Cont.)

(with uniformly distributed demand)

(e) Ratio of number of options to purchase and initial ordering quantity of rewards (\%)

\begin{tabular}{|c|c|c|c|c|c|c|}
\hline \multirow{2}{*}{} & \multicolumn{2}{|c|}{ Case 4 } & \multicolumn{2}{c|}{ Case 6 } & \multicolumn{2}{c|}{ Case 8 } \\
\cline { 2 - 7 } & \multicolumn{2}{|c|}{ LC tightness $(\gamma)$} & \multicolumn{2}{c|}{ LC tightness $(\gamma)$} & \multicolumn{2}{c|}{ LC tightness $(\gamma)$} \\
\hline $\mathrm{D}$ & $\mathrm{H}(0.7)$ & $\mathrm{L}(1.3)$ & $\mathrm{H}(0.7)$ & $\mathrm{L}(1.3)$ & $\mathrm{H}(0.7)$ & $\mathrm{L}(1.3)$ \\
\hline $\mathrm{L}(0.05)$ & $17.25 \%$ & $5.19 \%$ & $31.80 \%$ & $4.83 \%$ & $43.23 \%$ & $4.91 \%$ \\
$\mathrm{M}(0.15)$ & $14.07 \%$ & $15.13 \%$ & $28.61 \%$ & $14.12 \%$ & $39.07 \%$ & $14.52 \%$ \\
$\mathrm{H}(0.25)$ & $15.08 \%$ & $24.64 \%$ & $32.65 \%$ & $23.12 \%$ & $40.53 \%$ & $23.90 \%$ \\
\hline
\end{tabular}

(with normally distributed demand)

\begin{tabular}{|c|c|c|c|c|c|c|}
\hline \multirow{2}{*}{} & \multicolumn{2}{|c|}{ Case 4 } & \multicolumn{2}{c|}{ Case 6 } & \multicolumn{2}{c|}{ Case 8 } \\
\cline { 2 - 7 } & \multicolumn{2}{|c|}{ LC tightness $(\gamma)$} & \multicolumn{2}{c|}{ LC tightness $(\gamma)$} & \multicolumn{2}{c|}{ LC tightness $(\gamma)$} \\
\hline DV & $\mathrm{H}(0.7)$ & $\mathrm{L}(1.3)$ & $\mathrm{H}(0.7)$ & $\mathrm{L}(1.3)$ & $\mathrm{H}(0.7)$ & $\mathrm{L}(1.3)$ \\
\hline $\mathrm{L}(0.1)$ & $19.62 \%$ & $1.95 \%$ & $34.36 \%$ & $1.70 \%$ & $46.04 \%$ & $1.78 \%$ \\
$\mathrm{M}(1.0)$ & $16.03 \%$ & $19.08 \%$ & $29.24 \%$ & $16.28 \%$ & $39.57 \%$ & $17.22 \%$ \\
$\mathrm{H}(1.8)$ & $19.62 \%$ & $33.33 \%$ & $34.52 \%$ & $28.48 \%$ & $43.58 \%$ & $30.45 \%$ \\
\hline
\end{tabular}

(f) Number of options to exercise

(with uniformly distributed demand)

\begin{tabular}{|c|c|c|c|c|c|c|}
\hline \multirow{2}{*}{} & \multicolumn{2}{|c|}{ Case 4 } & \multicolumn{2}{c|}{ Case 6 } & \multicolumn{2}{c|}{ Case 8 } \\
\cline { 2 - 7 } & \multicolumn{2}{|c|}{ LC tightness $(\gamma)$} & \multicolumn{2}{c|}{ LC tightness $(\gamma)$} & \multicolumn{2}{c|}{ LC tightness $(\gamma)$} \\
\hline DV & H $(0.7)$ & L $(1.3)$ & H $(0.7)$ & L $(1.3)$ & H $(0.7)$ & L $(1.3)$ \\
\hline L(0.05) & 83.00 & 5.03 & 291.14 & 8.56 & 644.64 & 14.25 \\
$\mathrm{M}(0.15)$ & 52.91 & 15.07 & 253.58 & 26.99 & 590.15 & 45.70 \\
$\mathrm{H}(0.25)$ & 35.02 & 27.31 & 250.75 & 47.30 & 588.21 & 80.35 \\
\hline
\end{tabular}

\begin{tabular}{|c|c|c|c|c|c|c|}
\hline \multirow{2}{*}{} & \multicolumn{2}{|c|}{ Case 4 } & \multicolumn{2}{c|}{ Case 6 } & \multicolumn{2}{c|}{ Case 8 } \\
\cline { 2 - 7 } & \multicolumn{2}{|c|}{ LC tightness $(\gamma)$} & \multicolumn{2}{c|}{ LC tightness $(\gamma)$} & \multicolumn{2}{c|}{ LC tightness $(\gamma)$} \\
\hline DV & $\mathrm{H}(0.7)$ & $\mathrm{L}(1.3)$ & $\mathrm{H}(0.7)$ & $\mathrm{L}(1.3)$ & $\mathrm{H}(0.7)$ & $\mathrm{L}(1.3)$ \\
\hline $\mathrm{L}(0.1)$ & 96.04 & 1.40 & 312.71 & 2.43 & 674.14 & 4.11 \\
$\mathrm{M}(1.0)$ & 51.77 & 14.87 & 252.69 & 24.67 & 587.03 & 42.72 \\
$\mathrm{H}(1.8)$ & 38.75 & 27.34 & 247.54 & 45.51 & 593.86 & 80.12 \\
\hline
\end{tabular}


Table 6.14 Computational results under EP2 setting (Cont.)

(with uniformly distributed demand)

(g) Ratio of number of options to exercise and Number of options to purchase (\%)

\begin{tabular}{|c|c|c|c|c|c|c|}
\hline \multirow{2}{*}{} & \multicolumn{2}{|c|}{ Case 4 } & \multicolumn{2}{c|}{ Case 6 } & \multicolumn{2}{c|}{ Case 8 } \\
\cline { 2 - 7 } & \multicolumn{2}{|c|}{ LC tightness $(\gamma)$} & \multicolumn{2}{c|}{ LC tightness $(\gamma)$} & \multicolumn{2}{c|}{ LC tightness $(\gamma)$} \\
\hline $\mathrm{DV}$ & $\mathrm{H}(0.7)$ & $\mathrm{L}(1.3)$ & $\mathrm{H}(0.7)$ & $\mathrm{L}(1.3)$ & $\mathrm{H}(0.7)$ & $\mathrm{L}(1.3)$ \\
\hline $\mathrm{L}(0.05)$ & $90.58 \%$ & $18.97 \%$ & $94.42 \%$ & $18.95 \%$ & $96.35 \%$ & $19.42 \%$ \\
$\mathrm{M}(0.15)$ & $63.72 \%$ & $19.42 \%$ & $83.08 \%$ & $19.41 \%$ & $88.94 \%$ & $20.05 \%$ \\
$\mathrm{H}(0.25)$ & $36.55 \%$ & $19.79 \%$ & $68.22 \%$ & $19.88 \%$ & $80.91 \%$ & $20.57 \%$ \\
\hline
\end{tabular}

\begin{tabular}{|c|c|c|c|c|c|c|}
\hline \multirow{2}{*}{} & \multicolumn{2}{|c|}{ Case 4 } & \multicolumn{2}{c|}{ Case 6 } & \multicolumn{2}{c|}{ Case 8 } \\
\cline { 2 - 7 } & \multicolumn{2}{|c|}{ LC tightness $(\gamma)$} & \multicolumn{2}{c|}{ LC tightness $(\gamma)$} & \multicolumn{2}{c|}{ LC tightness $(\gamma)$} \\
\hline DV & $\mathrm{H}(0.7)$ & $\mathrm{L}(1.3)$ & $\mathrm{H}(0.7)$ & $\mathrm{L}(1.3)$ & $\mathrm{H}(0.7)$ & $\mathrm{L}(1.3)$ \\
\hline $\mathrm{L}(0.1)$ & $96.54 \%$ & $14.29 \%$ & $98.35 \%$ & $15.64 \%$ & $98.91 \%$ & $15.79 \%$ \\
$\mathrm{M}(1.0)$ & $55.52 \%$ & $14.86 \%$ & $82.67 \%$ & $15.78 \%$ & $88.77 \%$ & $16.16 \%$ \\
$\mathrm{H}(1.8)$ & $31.35 \%$ & $15.06 \%$ & $64.45 \%$ & $16.02 \%$ & $76.96 \%$ & $16.52 \%$ \\
\hline
\end{tabular}

Under EP2 setting, the budget usage ratio for each testing case is reported in Table 6.15. We find that the results under EP2 setting are similar to those obtained under BP and EP1 settings. The increase in liability control tightness results in a higher budget usage. As such, the budget ratio of Type $I$ in all testing problems is greater than or equal to 1.0 . We also observe that the budget ratio of Type II increases with the increase in liability control tightness (i.e., $\gamma$ changes from 1.3 to 0.7 ). Moreover, in some cases, the current budget usage (obtained in the model without budget and capacity constraints) is even larger than the maximum budget available (i.e., the right hand side of the budget constraint: $W^{R}$ ) (see budget ratio of Type II in Table 6.15), especially when the liability control is tight (i.e., $\gamma=0.7$ ) and demand variability level is high. The same type of budget related analysis that was discussed in section 6.4.1 can be done under EP2 setting. 
Table 6.15 Comparison of the budget usage under EP2 setting

(with uniformly distributed demand)

(with normally distributed demand)

\begin{tabular}{|c|c|c|c|c|c|c|}
\hline \multirow{2}{*}{} & \multicolumn{2}{|c|}{ Case 4 } & \multicolumn{2}{c|}{ Case 6 } & \multicolumn{2}{c|}{ Case 8 } \\
\cline { 2 - 7 } & \multicolumn{2}{|c|}{ LC tightness $(\gamma)$} & \multicolumn{2}{c|}{ LC tightness $(\gamma)$} & \multicolumn{2}{c|}{ LC tightness $(\gamma)$} \\
\hline $\mathrm{DV}$ & $\mathrm{H}(0.7)$ & $\mathrm{L}(1.3)$ & $\mathrm{H}(0.7)$ & $\mathrm{L}(1.3)$ & $\mathrm{H}(0.7)$ & $\mathrm{L}(1.3)$ \\
\hline $\mathrm{L}(0.05)$ & 1.090 & 1.000 & 1.188 & 1.000 & 1.236 & 1.000 \\
$\mathrm{M}(0.15)$ & 1.096 & 1.001 & 1.164 & 1.000 & 1.212 & 1.000 \\
$\mathrm{H}(0.25)$ & 1.101 & 1.006 & 1.153 & 1.003 & 1.197 & 1.004 \\
\hline
\end{tabular}

\begin{tabular}{|c|c|c|c|c|c|c|}
\hline \multirow{2}{*}{} & \multicolumn{2}{|c|}{ Case 4 } & \multicolumn{2}{c|}{ Case 6 } & \multicolumn{2}{c|}{ Case 8 } \\
\cline { 2 - 7 } & \multicolumn{2}{|c|}{ LC tightness $(\gamma)$} & \multicolumn{2}{c|}{ LC tightness $(\gamma)$} & \multicolumn{2}{c|}{ LC tightness $(\gamma)$} \\
\hline $\mathrm{DV}$ & $\mathrm{H}(0.7)$ & $\mathrm{L}(1.3)$ & $\mathrm{H}(0.7)$ & $\mathrm{L}(1.3)$ & $\mathrm{H}(0.7)$ & $\mathrm{L}(1.3)$ \\
\hline $\mathrm{L}(0.1)$ & 1.087 & 1.000 & 1.200 & 1.000 & 1.246 & 1.000 \\
$\mathrm{M}(1.0)$ & 1.103 & 1.001 & 1.169 & 1.000 & 1.217 & 1.000 \\
$\mathrm{H}(1.8)$ & 1.107 & 0.997 & 1.161 & 0.996 & 1.204 & 0.996 \\
\hline
\end{tabular}

(b) Budget Ratio (Type II)

(with uniformly distributed demand)

\begin{tabular}{|c|c|c|c|c|c|c|}
\hline \multirow{2}{*}{} & \multicolumn{2}{|c|}{ Case 4 } & \multicolumn{2}{c|}{ Case 6 } & \multicolumn{2}{c|}{ Case 8 } \\
\cline { 2 - 7 } & \multicolumn{2}{|c|}{ LC tightness $(\gamma)$} & \multicolumn{2}{c|}{ LC tightness $(\gamma)$} & \multicolumn{2}{c|}{ LC tightness $(\gamma)$} \\
\hline $\mathrm{DV}$ & $\mathrm{H}(0.7)$ & $\mathrm{L}(1.3)$ & $\mathrm{H}(0.7)$ & $\mathrm{L}(1.3)$ & $\mathrm{H}(0.7)$ & $\mathrm{L}(1.3)$ \\
\hline $\mathrm{L}(0.05)$ & 0.890 & 0.817 & 0.973 & 0.819 & 1.017 & 0.823 \\
$\mathrm{M}(0.15)$ & 0.972 & 0.887 & 1.036 & 0.890 & 1.082 & 0.893 \\
$\mathrm{H}(0.25)$ & 1.046 & 0.956 & 1.102 & 0.959 & 1.146 & 0.961 \\
\hline
\end{tabular}

(with normally distributed demand)

\begin{tabular}{|c|c|c|c|c|c|c|}
\hline \multirow{2}{*}{} & \multicolumn{2}{|c|}{ Case 4 } & \multicolumn{2}{c|}{ Case 6 } & \multicolumn{2}{c|}{ Case 8 } \\
\cline { 2 - 7 } & \multicolumn{2}{|c|}{ LC tightness $(\gamma)$} & \multicolumn{2}{c|}{ LC tightness $(\gamma)$} & \multicolumn{2}{c|}{ LC tightness $(\gamma)$} \\
\hline $\mathrm{DV}$ & $\mathrm{H}(0.7)$ & $\mathrm{L}(1.3)$ & $\mathrm{H}(0.7)$ & $\mathrm{L}(1.3)$ & $\mathrm{H}(0.7)$ & $\mathrm{L}(1.3)$ \\
\hline $\mathrm{L}(0.1)$ & 0.859 & 0.790 & 0.950 & 0.791 & 0.990 & 0.796 \\
$\mathrm{M}(1.0)$ & 0.960 & 0.871 & 1.015 & 0.868 & 1.064 & 0.874 \\
$\mathrm{H}(1.8)$ & 1.047 & 0.942 & 1.090 & 0.935 & 1.139 & 0.943 \\
\hline
\end{tabular}

Overall, the impacts of liability control are quite consistent under BP, EP1, and EP2 settings. A summary is provided as follows: 
(1) When the liability control gets tighter, LRP profitability seems to be reduced due to the increasing costs for purchasing rewards.

(2) When the liability control gets tighter, higher target redemption is required. This causes the estimated optimal ordering quantities of rewards to increase. Under EP2 setting, the initial ordering quantities and the number of options to purchase and to exercise increase with the increase in liability control tightness. Under EP1 setting, the LRP host's decisions on bonus points are not influenced by the changes in liability control requirement.

(3) When the liability control gets tighter, the budget usage increases. Compared to the budget usage under the original model settings (with budget and capacity constraints, and loose liability control), and under the current model settings (i.e., tight liability control), the budget usage is always larger than that under the original model setting. Especially when the demand variability level is high, to meet a liability control requirement, the budget usage may exceed the maximum budget available (i.e., $W^{R}$ ).

\subsubsection{Comparison across BP, EP1, and EP2 Model Settings}

In order to compare the impacts of liability control across our model settings (BP, EP1 and EP2), we examine the changes in LRP profitability, cost of rewards, and ordering quantity of rewards in terms of percentage when the liability control parameter changes from loose (i.e., $\gamma=1.3$ ) to tight (i.e., $\gamma=0.7$ ), and budget and capacity constraints are relaxed. Let $P(0.7), C(0.7), Q(0.7)$ denote the LRP profitability, cost of rewards, and the ordering quantity of rewards respectively, given $\gamma=0.7$. Let $P(1.3), C(1.3), Q(1.3)$ denote the LRP profitability, cost of rewards, and the 
ordering quantity of rewards, respectively, given $\gamma=1.3$. The following three measures of the impact of the liability control are calculated:

Changes in LRP profitability $(\%)=\frac{P(0.7)-P(1.3)}{P(1.3)} \times 100$,

Changes in cost of rewards $(\%)=\frac{C(0.7)-C(1.3)}{C(1.3)} \times 100$, and

Changes in ordering quantity of rewards $(\%)=\frac{Q(0.7)-Q(1.3)}{Q(1.3)} \times 100$

Tables 6.16-6.18 report on these changes under BP, EP1, and EP2 settings. Given a level of demand variability, higher decrease (in percentage) in the LRP profitability and higher increase (in percentage) in the cost of rewards are observed under EP2 setting than under BP and EP1 settings. Regarding the ordering quantities of rewards (including the options to exercise under EP2 setting), when the level of demand variability is high, a higher increase (in percentage) in the ordering quantity of rewards is observed under EP2 setting than under BP and EP1 settings. However, when the level of demand variability is low, EP1 model is observed to have a higher increase (in percentage) than BP and EP2 models. These results suggest that EP2 model seems more sensitive to changes in the liability control parameter. In other words, EP2 model does not show any advantage in controlling the liability. 
Table 6.16 Comparison of the impacts of liability control on LRP profitability across different model settings

(a) with Uniformly Distributed Demand

\begin{tabular}{|c|c|c|c|c|c|c|c|c|c|}
\hline & \multicolumn{3}{|c|}{ Case 4 } & \multicolumn{3}{c|}{ Case 6 } & \multicolumn{3}{c|}{ Case 8 } \\
\cline { 2 - 9 } DV & BP & EP1 & EP2 & BP & EP1 & EP2 & BP & EP1 & EP2 \\
\hline $\mathrm{L}(0.05)$ & $-0.61 \%$ & $-0.71 \%$ & $-1.18 \%$ & $-1.43 \%$ & $-1.61 \%$ & $-3.24 \%$ & $-1.71 \%$ & $-1.88 \%$ & $-3.95 \%$ \\
$\mathrm{M}(0.15)$ & $-0.53 \%$ & $-0.62 \%$ & $-1.00 \%$ & $-1.33 \%$ & $-1.50 \%$ & $-2.61 \%$ & $-1.60 \%$ & $-1.76 \%$ & $-3.40 \%$ \\
$\mathrm{H}(0.25)$ & $-0.38 \%$ & $-0.45 \%$ & $-0.86 \%$ & $-1.12 \%$ & $-1.25 \%$ & $-2.43 \%$ & $-1.40 \%$ & $-1.53 \%$ & $-3.14 \%$ \\
\hline
\end{tabular}

(b) with Normally Distributed Demand

\begin{tabular}{|c|c|c|c|c|c|c|c|c|c|}
\hline & \multicolumn{3}{|c|}{ Case 4 } & \multicolumn{3}{c|}{ Case 6 } & \multicolumn{3}{c|}{ Case 8 } \\
\cline { 2 - 9 } DV & BP & EP1 & EP2 & BP & EP1 & EP2 & BP & EP1 & EP2 \\
\hline L(0.1) & $-0.64 \%$ & $-0.72 \%$ & $-1.18 \%$ & $-1.44 \%$ & $-1.60 \%$ & $-3.24 \%$ & $-1.73 \%$ & $-1.88 \%$ & $-3.95 \%$ \\
M(1.0) & $-0.54 \%$ & $-0.61 \%$ & $-1.00 \%$ & $-1.36 \%$ & $-1.49 \%$ & $-2.61 \%$ & $-1.60 \%$ & $-1.72 \%$ & $-3.40 \%$ \\
H(1.8) & $-0.37 \%$ & $-0.40 \%$ & $-0.86 \%$ & $-1.15 \%$ & $-1.22 \%$ & $-2.43 \%$ & $-1.37 \%$ & $-1.44 \%$ & $-3.14 \%$ \\
\hline
\end{tabular}

Table 6.17 Comparison of the impacts of liability control on cost of rewards across different model settings

(a) with Uniformly Distributed Demand

\begin{tabular}{|c|c|c|c|c|c|c|c|c|c|}
\hline \multirow{2}{*}{ DV } & \multicolumn{3}{|c|}{ Case 4 } & \multicolumn{3}{c|}{ Case 6 } & \multicolumn{3}{c|}{ Case 8 } \\
\cline { 2 - 10 } & BP & EP1 & EP2 & BP & EP1 & EP2 & BP & EP1 & EP2 \\
\hline L(0.05) & $7.86 \%$ & $9.16 \%$ & $8.97 \%$ & $15.55 \%$ & $18.03 \%$ & $18.84 \%$ & $19.24 \%$ & $21.90 \%$ & $23.57 \%$ \\
$\mathrm{M}(0.15)$ & $6.59 \%$ & $7.61 \%$ & $9.51 \%$ & $14.02 \%$ & $15.93 \%$ & $16.38 \%$ & $17.20 \%$ & $19.15 \%$ & $21.17 \%$ \\
$\mathrm{H}(0.25)$ & $5.00 \%$ & $5.71 \%$ & $9.39 \%$ & $11.84 \%$ & $13.13 \%$ & $14.92 \%$ & $14.71 \%$ & $16.04 \%$ & $19.25 \%$ \\
\hline
\end{tabular}

(b) with Normally Distributed Demand

\begin{tabular}{|c|c|c|c|c|c|c|c|c|c|}
\hline & \multicolumn{3}{|c|}{ Case 4 } & \multicolumn{3}{c|}{ Case 6 } & \multicolumn{3}{c|}{ Case 8 } \\
\cline { 2 - 10 } DV & BP & EP1 & EP2 & BP & EP1 & EP2 & BP & EP1 & EP2 \\
\hline L(0.1) & $8.45 \%$ & $9.69 \%$ & $8.68 \%$ & $16.08 \%$ & $18.63 \%$ & $19.99 \%$ & $20.05 \%$ & $22.85 \%$ & $24.57 \%$ \\
M(1.0) & $8.05 \%$ & $8.83 \%$ & $10.16 \%$ & $15.77 \%$ & $17.24 \%$ & $16.93 \%$ & $18.68 \%$ & $20.24 \%$ & $21.70 \%$ \\
H(1.8) & $7.05 \%$ & $7.36 \%$ & $11.06 \%$ & $14.41 \%$ & $15.03 \%$ & $16.56 \%$ & $16.71 \%$ & $17.46 \%$ & $20.84 \%$ \\
\hline
\end{tabular}


Table 6.18 Comparison of the impacts of liability control on ordering quantity of rewards across different model settings

(a) with Uniformly Distributed Demand

\begin{tabular}{|c|c|c|c|c|c|c|c|c|c|}
\hline & \multicolumn{3}{|c|}{ Case 4 } & \multicolumn{3}{c|}{ Case 6 } & \multicolumn{3}{c|}{ Case 8 } \\
\cline { 2 - 9 } DV & BP & EP1 & EP2 & BP & EP1 & EP2 & BP & EP1 & EP2 \\
\hline L(0.05) & $18.56 \%$ & $21.72 \%$ & $18.98 \%$ & $33.34 \%$ & $38.78 \%$ & $33.46 \%$ & $44.36 \%$ & $50.62 \%$ & $45.21 \%$ \\
$\mathrm{M}(0.15)$ & $14.49 \%$ & $16.99 \%$ & $16.41 \%$ & $28.57 \%$ & $32.75 \%$ & $30.45 \%$ & $38.13 \%$ & $42.72 \%$ & $41.72 \%$ \\
$\mathrm{H}(0.25)$ & $9.85 \%$ & $11.57 \%$ & $14.18 \%$ & $22.67 \%$ & $25.49 \%$ & $27.90 \%$ & $31.13 \%$ & $34.26 \%$ & $38.89 \%$ \\
\hline
\end{tabular}

(b) with Normally Distributed Demand

\begin{tabular}{|c|c|c|c|c|c|c|c|c|c|}
\hline & \multicolumn{3}{|c|}{ Case 4 } & \multicolumn{3}{c|}{ Case 6 } & \multicolumn{3}{c|}{ Case 8 } \\
\cline { 2 - 9 } DV & BP & EP1 & EP2 & BP & EP1 & EP2 & BP & EP1 & EP2 \\
\hline L(0.1) & $20.17 \%$ & $23.19 \%$ & $20.18 \%$ & $34.91 \%$ & $40.49 \%$ & $34.92 \%$ & $46.66 \%$ & $53.24 \%$ & $46.80 \%$ \\
M(1.0) & $16.08 \%$ & $17.97 \%$ & $17.47 \%$ & $30.83 \%$ & $34.06 \%$ & $31.82 \%$ & $39.93 \%$ & $43.57 \%$ & $43.08 \%$ \\
H(1.8) & $11.47 \%$ & $12.24 \%$ & $16.92 \%$ & $25.49 \%$ & $26.86 \%$ & $30.41 \%$ & $32.84 \%$ & $34.58 \%$ & $41.39 \%$ \\
\hline
\end{tabular}

Figure 6.7 illustrates the ratio of budget usage under EP1 and EP2 settings and the budget usage under BP model setting in each testing case. Let $\mathrm{B}(\mathrm{BP}), \mathrm{B}(\mathrm{EP} 1)$, and $\mathrm{B}(\mathrm{EP} 2)$ denote the budget usages under BP, EP1, and EP2 settings, respectively. Let $R(B P), R(E P 1)$, and R(EP2) denote the ratio of budget usage under different model settings. The ratio illustrated in Figure 6.7 is calculated by using the follow relations:

$$
\begin{aligned}
& \mathrm{R}(\mathrm{BP})=\mathrm{B}(\mathrm{BP}) / \mathrm{B}(\mathrm{BP}) \\
& \mathrm{R}(\mathrm{EP} 1)=\mathrm{B}(\mathrm{EP} 1) / \mathrm{B}(\mathrm{BP}), \text { and } \\
& \mathrm{R}(\mathrm{EP} 2)=\mathrm{B}(\mathrm{EP} 2) / \mathrm{B}(\mathrm{BP})
\end{aligned}
$$

Under different levels of liability control (i.e., $\gamma=0.7$ or 1.3 ), we observed that in most cases, EP1 model generates the highest budget usage among the three models, whereas EP2 model generates the lowest budget usage. However, when the demand variability level is low, EP2 
model requires more budget than EP1 and BP models (e.g., in testing cases 6 and 8, DV=0.05 or $\mathrm{DV}=0.1$ ). When the demand variability level is high, EP2 models seems better than both BP and EP1 models because it achieves the same liability control requirement while requiring a lower budget.

Figure 6.7 Comparison of budget usage ratios across different model settings

(a) with Uniformly Distributed Demand

Case 4 (NR20NA15)

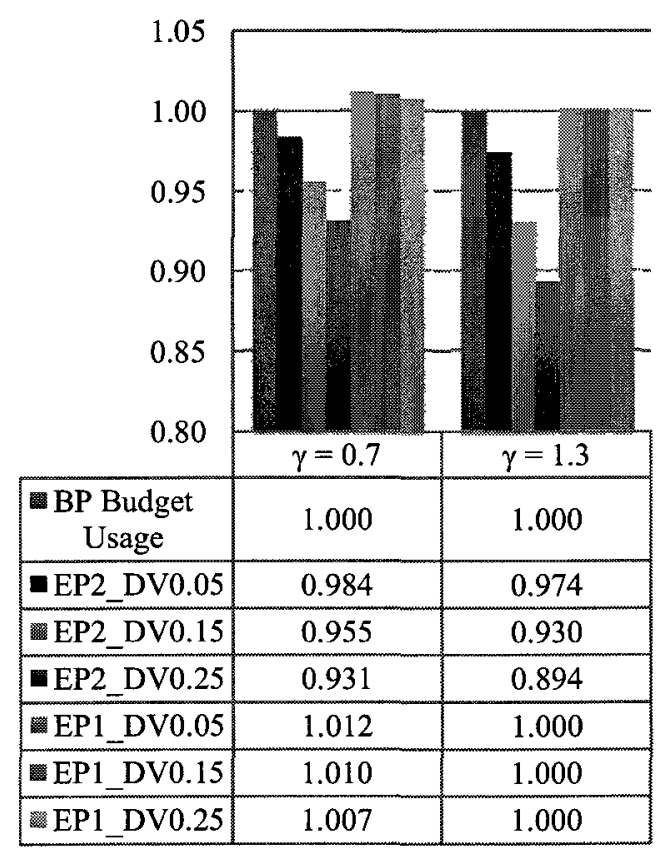

(b) with Normally Distributed Demand

\section{Case 4 (NR30NA15)}

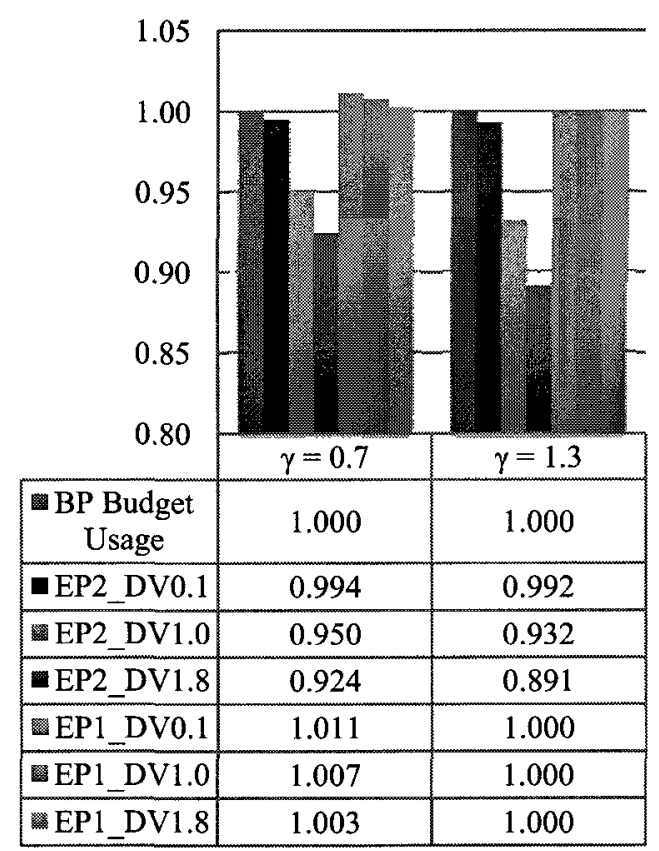


Figure 6.7 Comparison of budget usage ratios across different model settings (Cont.)

(a) with Uniformly Distributed Demand

\section{Case 6 (NR40NA40)}

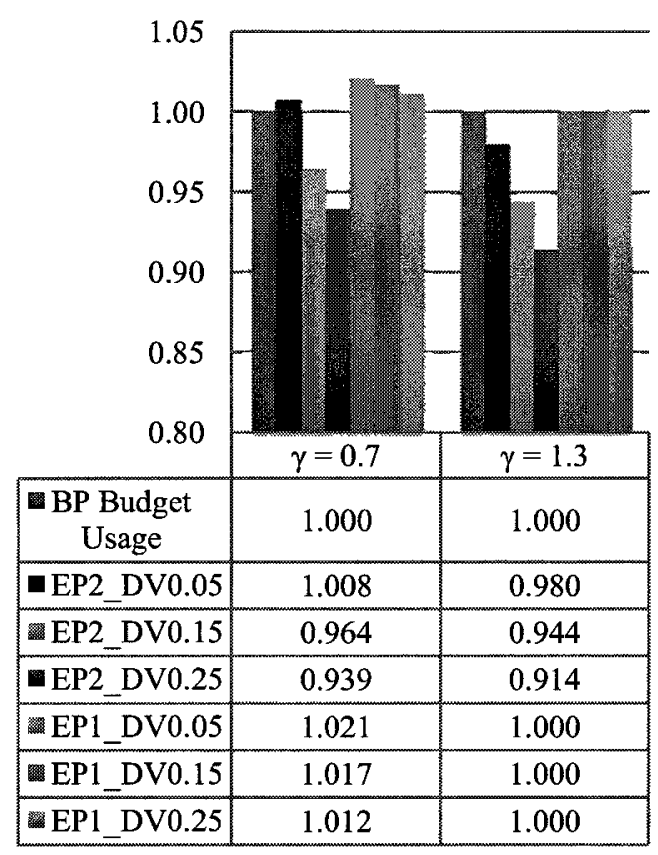

(b) with Normally Distributed Demand

\section{Case 6 (NR40NA40)}

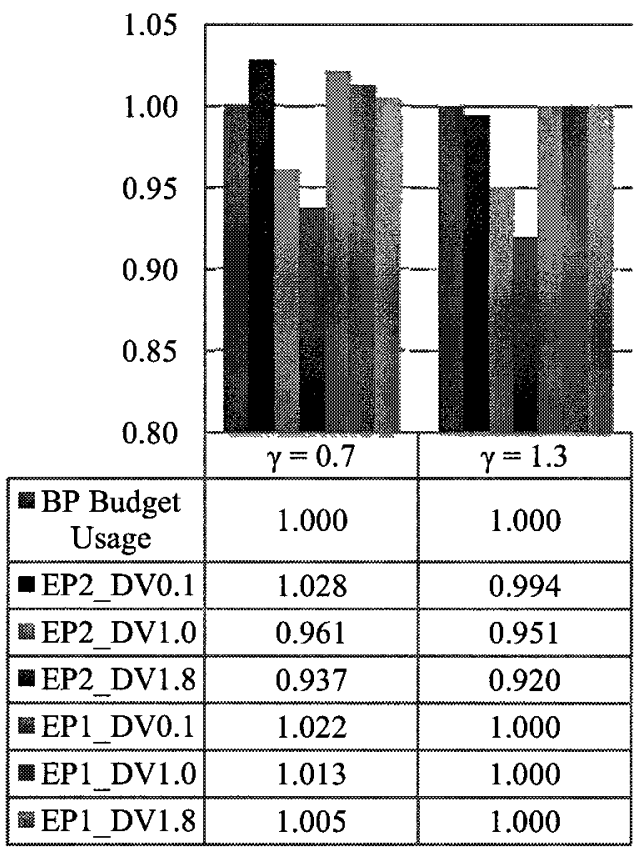


Figure 6.7 Comparison of budget usage ratios across different model settings (Cont.)

(a) with Uniformly Distributed Demand

\section{Case 8 (NR65NA70)}

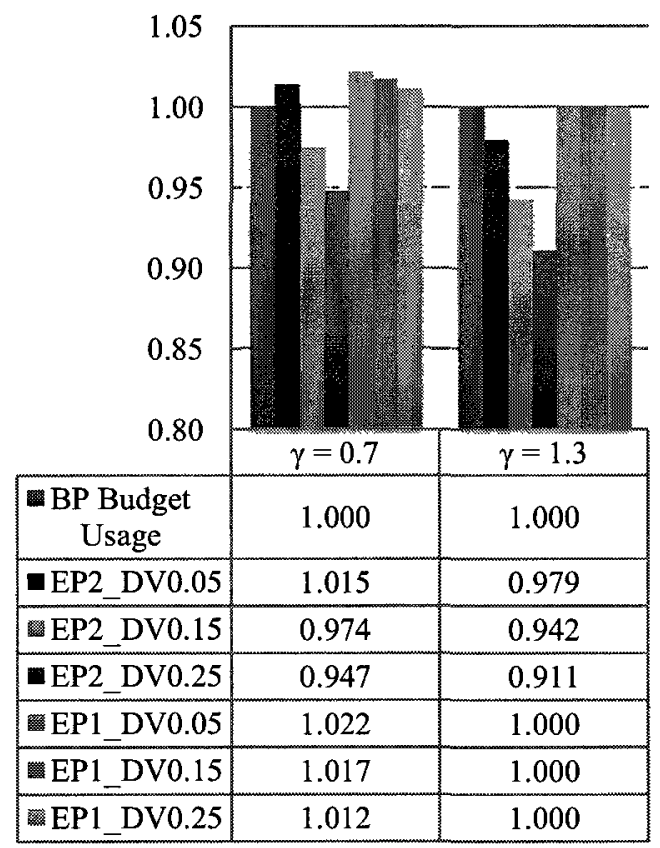

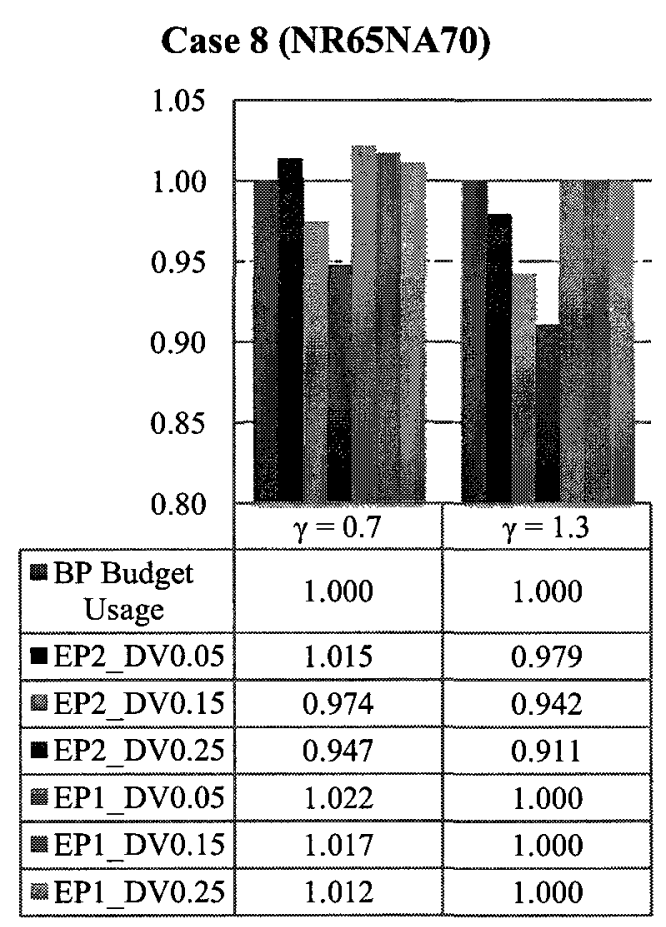

(b) with Normally Distributed Demand

\begin{tabular}{|c|c|c|}
\hline \multicolumn{3}{|c|}{ Case 8 (NR65NA70) } \\
\hline 1.05 & & \\
\hline 1.00 & & \\
\hline 0.95 & & \\
\hline 0.90 & & \\
\hline 0.85 & & \\
\hline 0.80 & $\gamma=0.7$ & $\gamma=1.3$ \\
\hline $\begin{array}{c}\text { BP Budget } \\
\text { Usage }\end{array}$ & 1.000 & 1.000 \\
\hline -EP2_DV0.1 & 1.032 & 0.994 \\
\hline EP2_DV1.0 & 0.973 & 0.949 \\
\hline -EP2_DV1.8 & 0.949 & 0.916 \\
\hline EP1_DV0.1 & 1.023 & 1.000 \\
\hline EP1_DV1.0 & 1.013 & 1.000 \\
\hline EP1_DV1.8 & 1.006 & 1.000 \\
\hline
\end{tabular}

\subsubsection{More on Management Insights from Liability Control Analysis}

The budget usage reported in Sections 6.4.1 - 6.4.4 may, in some cases, exceed the maximum amount of budget available with a targeted liability ratio (see the budget ratio of Type II in Tables $6.15,6.17$, and 6.19). In those cases, the simplest way for the LRP host to meet their targeted liability is to seek an additional budget in the ranges suggested by our models. However, in practice, this may not always be possible for the following reasons:

- The extra budget is not available 
- The LRP host does not want to spend more to buy additional rewards

- The extra amount of rewards are not available due to LRP capacity constraints

In that regard, the LRP host may resort to some managerial policies (e.g., change the redemption scheme to charge more points for redeeming a reward) to increase the overall redemption and achieve the targeted liability level without increasing the budget usage. Our models provide an analytical tool for LRP host firms to detect whether there is any conflict between meeting the target liability level and satisfying various resource and management constraints at the planning stage and to decide which managerial policy would be the most appropriate to apply under different scenarios. We illustrate this under BP setting (similar analysis can be performed under EP1 and the EP2 settings).

Let $q_{J}^{R b}$ represent the estimated optimal ordering quantities of rewards from partner $R_{J}$ given the targeted liability ratio $L_{U B}$ and relaxed budget and capacity constraints (i.e., constraints (4.43), (4.51), (4.64), (4.42), (4.50) and (4.61)), and let $q_{j}^{R}$ represents the estimated optimal ordering quantity of rewards from partner $R_{J}$ given budget and capacity constraints and a relaxed liability constraint. Since $q_{\text {, }}^{R}$ are the ordering quantities of rewards under budget and capacity constraints, we have $q_{J}^{R} \leq q_{J}^{R b}$ for $j=1, \ldots, J ; q_{j}^{R b}$ can be understood as the targeted redemption level that satisfies the targeted liability control requirement and $q_{j}^{R}$ can be understood as the maximum redemption level with the budget and capacity constraints. 
However, as mentioned in Chapter 3, what really matters is the actual redemption level rather than the maximum redemption level. Therefore, let $q_{j}^{R a}$ represent the actual redemption level for rewards from partner $R_{J}$, then $q_{J}^{R a}=\min \left(q_{J}^{R}, D_{j}^{R}\right)$, which indicates that the value of $q_{J}^{R a}$ depends on $q_{j}^{R}$ as well as $D_{j}^{R}$ and $q_{j}^{R a} \leq q_{j}^{R}$. Therefore, in order to achieve the targeted redemption level $q_{j}^{R b}$ and to satisfy the liability control requirement as well as the budget and capacity constraints at the same time, several managerial policies can be applied to make $q_{j}^{R a}$ as close to $q_{j}^{R b}$ as possible.

Case 1: When $q_{j}^{R}<D_{j}^{R}<q_{j}^{R b}$ or $q_{j}^{R}<q_{j}^{R b}<D_{j}^{R}$, in other words, $q_{j}^{R a}=q_{j}^{R}<q_{j}^{R b}$, the LRP manager may apply managerial policies such as charging more points for redeeming a reward to increase the actual redemption $\left(q_{j}^{R a}\right)$ of rewards to the target redemption level $q_{J}^{R b}$ without violating the budget and capacity constraints.

Case 2: When $D_{J}^{R}<q_{j}^{R}<q_{J}^{R b}$, in other words, $q_{J}^{R a}<q_{J}^{R}<q_{J}^{R b}$, the LRP manager may need to boost redemption demand (e.g., through a promotion) to first make $D_{J}^{R}$ as close to $q_{J}^{R}$ as possible. Then managerial policies should be applied (e.g., charging more points for redeeming a reward) to increase the redemption $\left(q_{j}^{R}\right)$ of rewards from the redemption partner $j$ so that the target redemption level $q_{j}^{R b}$ can be reached without violating the budget and capacity constraints. However, the LRP manager should pay more attention to the managerial policies to apply in this case because some of them (e.g., charging more points for redeeming a reward) may discourage 
customer redemption behavior and, therefore, have a negative impact on enhancing redemption demand to the maximum redemption level $q_{j}^{R}$.

\subsubsection{Summary}

In short, based on the above computational results and analyses, we conclude that:

(1) Increasing the liability control tightness will reduce LRP profitability no matter which model setting is used.

(2) Increasing the liability control tightness results in higher target redemption and an increase in the supply of rewards. Therefore, in order to meet a liability control target, the costs for rewards (i.e., the budget usage) may also increase and sometimes even exceed the maximum redemption budget available.

(3) Under EP1 setting, since the LRP host firm offers bonus points to accumulation partners, a higher accumulation may be expected. Therefore, to meet the same target level of liability, a higher redemption is required with a bonus points offering (i.e., EP1) than without a bonus points offering (i.e., BP and EP2).

(4) In comparison with BP and EP1 models, it seems that EP2 model does not have advantages in dealing with the changes in liability control, although with the decreases in demand variability, EP2 model performs better. This indicates that the notable advantages of using option contracts have not been observed in meeting the liability control requirements. 
In addition, if meeting the target liability requires the spending for rewards to exceed the maximum redemption budget available, various managerial policies can be used to deal with that conflict. Using our analytical models, the LRP host firm can detect whether there is any conflict between meeting the targeted liability level and satisfying various resource and management constraints at the planning stage. 


\section{Chapter 7 Conclusions and Future Research Directions}

In this study, we have intended to develop quantitative analytical models for rewards-supply aggregate planning in loyalty rewards programs (LRPs) that operate as profit centers. We have investigated the LRP host's rewards-supply planning decisions in the presence of multiple LRP accumulation partners, multiple redemption partners, multiple resource constraints (e.g., budget, capacity), and multiple management concerns including LRP profitability, liability control, and demand uncertainties.

We have proposed three analytical models to examine the impacts of internal dynamics (budget tightness and liability control tightness), and external uncertainties (demand uncertainties) on LRP host's rewards-ordering decisions and LRP performance under different system settings. The first model (Problem BP) is developed under the setting where only wholesale price contracts are used in both points-sale contracting and rewards-supply contracting. The second model (Problem EPI) extends the first model by adding the consideration of a common cooperative advertising practice (i.e., through bonus points) in the rewards-supply planning decisions. The third model (Problem EP2) extends the first model by considering option contracts in the rewards-supply contracting. We have captured, with each of these models, some of the relationships among independent variables and dependent variables. The independent variables refer to model inputs such as maximum available budget, target liability ratio, and demand variability. The dependent variables refer to model outputs such as LRP profitability, budget usage, liability ratio, ordering quantities of rewards and bonus points offering, number of units of options to purchase, and number of units of options to exercise. 
A heuristic-based and SAA scheme-based stochastic programming solution procedure is developed to obtain solutions for the three analytical models. Using a computer simulation, numerical experiments, and statistical analysis, we have analyzed the impacts of the internal dynamics and external uncertainties under each model setting as well as across model settings considering different realistically sized problems. This process has yielded a number of managerial insights about which system setting is more effective in different situations.

\subsection{Findings and Implications}

Our theoretical framework, computational results, and analysis lead to three important findings and managerial implications.

The first finding is that demand uncertainties have negative impacts on LRP performance (i.e., LRP profitability, liability ratio, and budget usage). When demand variability is low, EP1 model generates higher LRP profitability, but also higher liability. When demand variability is high, EP2 model is better than the other two models. Furthermore, with the increases in demand variability, the LRP host's estimated optimal ordering quantities of rewards increase, but the estimated optimal amount of bonus points offered to accumulation partners decreases. In addition, EP2 model results in lower budget usage than BP and EP1 models; that difference is more obvious when demand variability is high. However, EP2 model's advantage in terms of budget usage seems to weaken in large-size problems. These results indicate that option contract does play a role in coping with demand uncertainties. This finding is consistent with the findings in the previous studies on option contracts (e.g., Barnes-Schuster et al., 2002; Erkoc and Wu, 2005; Jin and $\mathrm{Wu}, 2007)$. To an LRP host, adopting an option contract in rewards-supply 
contracting results in relatively lower liability and relatively higher profitability. The advantage of using the option contract is more obvious when demand variability is high. In contrast, offering bonus points seems not to be preferred when demand variability is high. Indeed, bonus points are a double-edged sword to the LRP host. On one hand, offering bonus points may bring in higher LRP profitability, especially when demand variability is low. On the other hand, offering bonus points will result in higher liability no matter what the changes in demand variability.

One implication of this finding is that when an LRP host faces high demand uncertainties, an effective strategy to reduce the negative impacts of those uncertainties is to adopt option contracts in rewards-supply contracting. In addition to that, in order to take the best advantage of option contracts, the LRP host should pay attention to the design of the option contracts, especially when there is a large number of LRP redemption partners involved in the business. Offering bonus points may not be a good strategy to deal with demand uncertainties. A LRP host should be conservative in using bonus points when demand uncertainty is high.

The second finding is that a tight budget, an internal dynamic, has an impact on LRP profitability and liability, and on the LRP host's rewards-supply decisions. Although this is an intuitive finding, the corresponding computational experiments demonstrate that with given model inputs, we can examine how likely and at what level the impact of a tight budget will be. Moreover, with tight budget constraints, the responses of each model to the changes in demand variability are consistent with the responses obtained with loose budget constraints. This indicates that although a tight budget (i.e., a smaller available budget) weakens the effectiveness of using 
option contracts to reduce the negative impact of demand variability; in comparison with wholesale-price-only contracts, option contracts are still better. Besides that, no matter what the changes in the budget tightness, an LRP host's decisions on offering bonus points are made independently from rewards-ordering decisions.

One implication of the second finding is that through our analytical models, an LRP host has the capability to evaluate the impact of changes in the available budget for purchasing rewards on the profitability and liability as long as all the model input parameters can be determined or estimated. In a case where an LRP host has a tight budget for purchasing rewards, option contracts are still a better choice to cope with demand uncertainties and reduce the negative effect of the uncertainties on LRP performance in rewards-supply contracting.

The third finding is that increasing the liability control level results in increases in the ordering quantity of rewards and budget usages, and decrease in LRP profitability. In order to meet the same target level of liability control, a higher redemption is required under EP1 setting (with bonus points offering) than under BP and EP2 settings (i.e., without bonus points offering). Furthermore, in comparison to wholesale-price-only contracts, our numerical study does not indicate that option contracts are better for supporting the liability control target, although LRP performance improves in EP2 model with the increases in demand variability. Therefore, supply contracts (i.e., wholesale price contracts and option contracts) and bonus points may not be helpful in achieving a liability control target. 
One implementation of the third finding is that adding a liability control target in the rewardssupply planning may help the LRP host to detect potential conflicts between controlling liability and satisfying resource constraints, such as budget and capacity constraints. Moreover, as long as all model input parameters can be determined or estimated, an LRP host can use the model outputs to evaluate the impact of changes in liability control on other LRP constraints, and then make further managerial decisions on how to deal with the issues either through increasing the redemption budget (e.g., our models help in determining the range of the required budget) or adopting other managerial practices (e.g., change redemption scheme).

\subsection{Limitations and Contributions}

A few limitations of this study should be recognized. These limitations also point to some future research opportunities on this topic.

a. In this study, we assume that redemption and accumulation demands follow either normal or uniform distributions. Other types of theoretical demand distributions (e.g., exponential, bi-normal, lognormal, etc.) should also be examined in order to generalize the findings of the study.

b. The analysis and findings are based on solutions and outputs from our analytical models with given inputs. Therefore, if our models are used with individual data, the values of all input parameters should be determined and estimated accordingly.

c. The focus in this study is solely on the LRP host's profitability in rewards-supply planning decisions. The profitability of LRP partners is not considered. The model can be further extended to consider the coordination between the LRP host and the LRP 
partners by improving the overall rewards-points supply chain profitability (e.g., the sum of the profitability of an LRP host and its LRP partners).

d. We have examined bonus points and option contracts separately. We did not explore settings that consider both bonus points and option contracts. However, by combining problem EPI and problem EP2, a model that includes simultaneously bonus points and option contracts can be developed.

Despite these limitations, this study contributes to the development of the literature on LRP from the following four perspectives:

1. Our research synthesizes and extends the concept of supply chain management in the context of loyalty reward programs. LRPs are analyzed as a rewards-points supply chain with some unique features. A comprehensive typology framework for LRP classification is proposed that provides a guideline for academics and practitioners to understand the variety of LRPs in today's marketplace. Furthermore, based on the framework, 39 well known LRPs in the Canadian marketplace were examined to provide illustrative examples for different types of LRPs. This research focuses on a specific type of LRP that has not been studied in previous LRP literature.

2. This is the first study of its kind to investigate rewards-supply planning decisions in LRP management through a quantitative modeling approach. Constrained newsvendor-based stochastic linear programming (SLP) models were developed to model the rewardssupply planning decisions with multiple managerial concerns and different system 
settings. Moreover, a heuristic-based solution procedure based on Sample Average Approximation scheme was proposed to find the best solutions. Using SLP and its corresponding solution method to solve the operational issues in LRPs is also a novel application to the existing LRP literature.

3. This research provides numerical evidence of the relationships between internal dynamics, external uncertainties, and an LRP host firm's rewards-supply planning decisions and LRP profitability, respectively. To expand current knowledge, this study evaluates the impacts of internal dynamics and external uncertainties on the LRP host's decisions and LRP performance under three different system settings. These system settings relate to two LRP practices: rewards-supply contracting and cooperative advertising. We demonstrate that a supply contract (e.g., an option contract) plays a significant role in coping with demand uncertainties in rewards-supply aggregate planning. This finding is consistent with previous analytical studies on supply contracts. In regards to cooperative advertising through bonus points, it is a common practice in LRP operations to improve cooperation between the LRP host firm and its commerical partners. This study provides numerical evidence and management insights on conditions under which offering bonus points is a good choice.

4. This research makes a significant contribution to enhancing a better understanding of LRPs. We investigate the type of LRP system that is much closer to the actual examples deployed in the current marketplace than the LRP systems discussed in other literature. Consequently, we bring forward valuable managerial insights for LRP host firms to support their decisions on rewards-supply with multiple managerial concerns such as 
LRP profitability, liability control, demand uncertainties, and budget usage. This study also provides tested analytical models for judging how the internal dynamics and external uncertainties impact the LRP host's rewards-supply decisions and LRP profitability for real applications. In addition, the presented analysis may guide LRP host firms in the negotiation process with their commercial partners regarding rewards-supply contracts and help them to seek the possibility of improving cooperation in the partnerships to achieve win-win solutions.

\subsection{Future Research Directions}

A number of lines of research could be undertaken to provide a better understanding of LPR operations and the rewards/points-supply planning problems. For instance, some possible future research directions are:

Single-period model vs. multiple-period model

In this study, the analytical models that we propose are single-period models, in which all the input parameters except of the liability ratio are assumed to be independent from the parameters in either the previous or the following time periods. Our single-period models can be applied to solve multiple-period rewards-supply planning problems directly as long as the assumption of independence (all the input parameters in each time period are independent from the parameters in the other time periods) is still valid. In the case where this assumption is not valid any more, we need to extend our models to consider the dependency among parameters in different time periods. The common dependencies among parameters in the multi-period LRP rewards-supply planning problems are: 
(1) The accumulation and redemption demands in each time period may influence the demands in the subsequent time periods.

(2) Offering bonus points in a certain time period may have a delayed effect on accumulation and redemption demands. These delayed effects could occur in the following time periods rather than the current time period.

(3) The inventory of some imperishable rewards in the current time period can be used as part of the rewards-supply in following time periods.

Supply Contracts The present study assumes that the relationships between the LRP host and accumulation partners are governed by the wholesale-price contracts. Signing a wholesaleprice contract is more suitable in the case where the LRP host wants to keep a simple relationship with accumulation partners. However, in the case where the LRP host wants to have a closer relationship with LRP accumulation partners, other types of contracts may be more suitable to govern the relationships between the LRP host and the accumulation partners. For instance, the LRP host and an accumulation partner can sign a revenue-sharing type of contract. The LRP host will decide the ordering quantity of points for the accumulation partner and commit on the partner's minimum revenue increase. But at the same time, the accumulation partner has to share the increased revenue with the LRP host. Signing revenue-sharing type of contracts may allow the LRP host to have more flexibility in managing the accumulation business. In comparison with the wholesale-price contracts, applying revenue-sharing type of contracts requires more managerial efforts and expenses. An extension of Problem BP that includes the consideration of revenue-sharing contracts in accumulation business can be formulated as Problem BPI (See Appendix E.1 for the model notations and formulation). The 
same structure of revenue sharing types of contracts can be embedded in Problem EP1 and Problem EP2 models.

In addition, in the redemption business we consider option contracts with a simple structure that has been discussed in the existing literature. We think that the option structure with multi-layers can be applied to improve the flexibility in the rewards-supply contracting. For example, a threelayer option structure can be designed as follows:

Option level 1: $0 \leq m_{J}^{R_{1}} \leq M_{J}^{R_{1}}$, unit option price is $w_{J}^{R_{1}}$, and unit exercise price is $e_{J}^{R_{1}}$

Option level 2: $0 \leq m_{J}^{R_{2}} \leq M_{j}^{R_{2}}$, unit option price is $w_{J}^{R_{2}}$, and unit exercise price is $e_{J}^{R_{2}}$

Option level 3: $0 \leq m_{j}^{R_{3}} \leq M_{j}^{R_{3}}$, unit option price is $w_{j}^{R_{3}}$, and unit exercise price is $e_{j}^{R_{3}}$

where $M_{J}^{R_{3}} \geq M_{J}^{R_{2}} \geq M_{J}^{R_{1}}$ and $w_{J}^{R_{3}} \geq w_{J}^{R_{2}} \geq w_{J}^{R_{1}}$. Different upper bounds for the number of options are offered in the contract so that the buyer (i.e., LRP host $H$ ) can have different choices. To incorporate multi-layer option contracts, our analytical models should be modified as stochastic mixed integer programming models. A mathematical model for Problem EP2 with multi-layer option contracts is formulated as Problem EP3 (see Appendix E.2 for the model notations and formulation). Decomposition approaches may need to be developed in order to solve large-sized problems within a reasonable amount of computational time.

Aggregate model vs. disaggregate model We have developed rewards-supply planning models at the aggregate level in this study. In other words, we consider the LRP host's decisions on aggregate supply of rewards based on aggregate demands for accumulation and redemption in the planning horizon. The aggregate planning models are suitable for long-term and intermediate-term planning. However, for short-term rewards-supply planning problems, 
disaggregate planning models are necessary. Disaggregate planning models can include more customer-based demand varieties and partner-based varieties of the supply of rewards: for example, to incorporate LRP member segmentation or LRP partner segmentation in the decisions on dealing with redemption timing (i.e., when to redeem) and redemption structure (i.e., where and how much to redeem).

Modeling random parameters

In this study, redemption and accumulation demands are assumed to be random parameters following certain theoretical probability distributions (i.e., uniform, normal). To the best of our knowledge, no one has discussed in the literature on what would be the most representative distributions of accumulation and redemption demands. An empirical study can be undertaken to find out whether there are representative distributions of accumulation and redemption demands for various types of loyalty reward programs.

Explore other solution methods Although in the study, we did not discuss the case that the random parameters follow discrete probability distributions, our SAA-based heuristic solution procedure is still applicable for that case. Alternatively, exact approach (e.g., Birge and Louveaux, 1997) can be explored to solve some special cases of the rewards-supply planning problem described in the study. Discrete-event simulation is another suitable approach that can be used to deal with the rewards-supply planning problem. Hence, it offers another avenue that can be pursued and the solutions obtained can then be compared to those from the approximate approach implemented in this study. 


\section{References}

Ahmed, N.U. 1984. An efficient 0-1 integer programming algorithm for advertising media selection. Journal of the Academy of Marketing Science 12(2), 191-204.

Ağrali, S., J. Geunes. 2009. Solving knapsack problems with $S$-curve return functions. European Journal of Operational Research 193, 605-615.

Ailawadi, K.L., J.P. Beauchamp, N. Donthu, D.K. Gauri, V. Shankar. 2009. Communication and promotion decisions in retailing: a review and directions for future research. Journal of Retailing 85(1), 42-55.

Anupindi, R., Y. Bassok. 1998. Approximations for multiproduct contracts with stochastic demands and business volume discounts: single supplier case. IIE Transactions 30, 723-734.

Atalık,Ö. 2005. The impact of the reward program elements on airlines customers: case of Turkish airlines. Elektronik Sosyal Bilimler Dergisi 14(4), 17-33.

Armstrong, M.K. 1991. Retail responses to trade promotion: an incremental analysis of forward buying and retail promotion. Ph.D. Dissertation, University of Texas, Dallas, TX.

Agrawal, D. 1996. Effect of brand loyalty on advertising and trade promotions: a game theoretic analysis with empirical evidence. Marketing Science 15(1), 86-108.

Bagdonienè L., R. Jakštaitè. 2006. Customer loyalty programs: purpose, means and Development. Organizaciju vadyba:sisteminiai tyrimai 37, 21-35.

Barnes-Schuster, D., Y. Bassok, R. Anupindi. 2002. Coordination and flexibility in supply contracts with options. Manufacturing Service Operations Management 4, 171-207.

Banasiewicz, A. 2005. Loyalty program planning and analytics. Journal of Consumer Marketing $22(6), 332-339$.

Banerjee, A., L.H. Summers. 1987. On frequent-flyer programs and other frequency-inducing economic arrangements. Discussion Paper no. 1337, Harvard Institute of Economic Research.

Basu, A.K., R. Batra. 1988. ADSPLIT: a multi-brand advertising budget allocation model. Journal of Advertising 17(1), 44-51.

Beale, E.M. 1955. On minimizing a convex function subject to linear inequalities. Journal of the Royal Statistical Society 17B, 173-184.

Bergen, M., G. John. 1997. Understanding cooperative advertising participation rates in conventional channels. Journal of Marketing Research 34, 357-369. 
Berman, B. 2006. Developing an effective customer loyalty program. California Management Review 49(1), 123-150.

Birge, J.R., F.V. Louveaux. 1997. Introduction to stochastic programming. New York, NY: Springer.

Bockstael, N.E., I.E. Strand, D.W. Lipton. 1992. Pulsed generic advertising: the case of common property. Marine Resource Economics 7, 189-208.

Brown, A.O., H.L. Lee. 1997. Optimal pay to delay capacity reservation with application to the semiconductor industry. Working paper, Stanford University.

Burnetas A., P. Ritchken. 2005. Option pricing with downward-sloping demand curve: the case of supply chain options. Management Science 51(4), 566-580.

Buzacott, J., H. Yan, H. Zhang. 2011. Risk analysis of commitment-option contracts with forecast updates. IIE Transactions 43, 415-431.

Cachon, G.P. 2003. Supply chain coordination with contracts. In: Handbooks in Operations Research and Management Science: Supply Chain Management. Steve Graves and Ton de Lok (Eds.), North-Holland.

Cachon, G.P., M.A. Lariviere. 2005. Supply chain coordination with revenue-sharing contracts: strengths and limitations. Management Science 51(1), 30-44.

Caminal, R., C. Matutes. 1990. Endogenous switching costs in a duopoly model. International Journal of Industrial Organization 8, 353-373.

Carlsson, F., A. Löfgren. 2006. Airline choice, switching costs and frequent flyer programs. Applied Economics, 38(13), 1469-1475.

Chang, C. 1996. Semiconductor contract manufacturing. Report \#SCMS-WW-FR-9601, Dataquest Corporation.

Chen-Ritzo, C., T. Ervolina, T.P. Harrison, B. Gupta. 2010. Sales and operations planning in systems with order configuration uncertainty. European Journal of Operational Research 205 (3), 604-614.

Cheng, F., M. Ettl, G.Y. Lin, M. Schwarz, D.D. Yao. 2009. Designing flexible supply chain contracts with options. In Planning Production and Inventories in the Extended Enterprise, International Series in Operations Research and Management Science 152, K.G. Kempf et al. (Eds.), 207-229.

Cole, J. 1998. Boeing's surplus lot filling up. Seattle Times, Oct. 4, 1998. 
Corbett, C.J., G.A. DeCroix, A. Ha. 2005. Optimal shared-savings contracts in supply chains: linear contracts and double moral hazard. European Journal of Operational Research 163(3), 653-667.

Corbett, C.J., C.S. Tang. 1999. Designing supply contracts: Contract Type and Information Asymmetry. In: Quantitative models for supply chain management. S. Tayur, M. Magazine, R. Ganesan (Eds.), 269-298. Boston: Kluwer Adademic Publishing.

Crimmins, E.C. 1984. Cooperative Advertising. New York: Gene Wolfe and Co.

Dantzig, G.B. 1955. Linear programming under uncertainty. Management Science 1, 197-206.

Delft, C., J. Vial. 2004. A practical implementation of stochastic programming: an application to evaluation of option contracts in supply chains. Automatica 40, 743-756.

Diaby, M., A.L. Nsakanda. 2008. Coping with revenue recognition in the loyalty reward programs industry: A stochastic modeling approach. Proceedings of the American Conference on Applied Mathematics, Cambridge, Massachusetts, 79-86.

Dick, A.S., K. Basu. 1994. Customer loyalty: toward an integrated conceptual framework. Journal of the Academy of Marketing Science 22(2), 99-113.

Doyle, P., J. Saunders. 1990. Multiproduct advertising budgeting. Marketing Science 9(2), 97113.

Dowling, G.R., M. Uncles. 1997. Do customer loyalty programs really work? Sloan Management Review 38 (4), 71-82.

Duffy, D.L. 1998. Customer loyalty strategies. Journal of Consumer Marketing 15(5), 435-448.

Ebert, J. 2003. Psychological approaches to future rewards: sequences, valuation, effort, and frequency programs. Advances in Consumer Research 30, 68-71.

Emmons H., S.M. Gilbert. 1998. The role of returns policies in pricing and inventory decisions for catalogue goods. Management Science 44(2), 276-283.

Eppen, G.D., A.V. Iyer. 1997. Backup agreements in fashion buying-the value of upstream flexibility. Management Science 43(11), 1469-1484.

Erkoc, M., S.D. Wu. 2005. Managing high-tech capacity expansion via reservation contracts. Production and Operations Management 14(2), 232-251.

Feng, Y., A. Martel, S. D'Amours, R. Beauregard. 2010. A stochastic programming approach for coordinated contract decisions in a make-to-order manufacturing supply chain. Working paper, CIRRELT-2010-11, Interuniveristy Research Centre, Montreal. 
Gandomi, A., S. Zolfaghari. 2011. A stochastic model on the profitability of loyalty programs. Computers \& Industrial Engineering, (in press). Published online, DOI: 10.1016/j.cie.2011.04.002.

Golovachkina, N., J. Bradley. 2003. Supplier-manufacturer relationship under forced compliance contracts. Manufacturing \& Service Operations Management 5(1), 67-69.

Gudmundsson, S.V., E.R. de Boer, C. Lechner. 2002. Integrating frequent-flier programs in multilateral airline alliances. Journal of Air Transport Management 8, 409-417.

He, X., A. Prasad, S.P. Sethi. 2009. Cooperative advertising and pricing in a dynamic stochastic supply chain: feedback Stackelberg strategies. Production and Operations Management 18(1), $78-94$.

He, X., A. Krishnamoorthy, A. Prasad, S.P. Sethi. 2011. Retail competition and cooperative advertising. Operations Research Letters 39, 11-16.

Higle, J.L., S. Sen. 1996. Stochastic decomposition: a statistical method for large scale stochastic linear programming. Kluwer Academic Publishers, Dordrecht.

Ho, R., L. Huang, S. Huang, T. Lee, A. Rosten, C.S. Tang. 2009. An approach to develop effective customer loyalty programs: The VIP program at T\&T Supermarkets Inc. Managing Service Quality 19(6), 702-720.

Hofer, D. 2008. Reinforcing the value of frequent flyer miles. White paper, Loylogic Inc.

Holthausen, D.M., G. Assmus. 1982. Advertising budget allocation under uncertainty. Management Science 28(5), 487-499.

Huang, Z., S.X. Li, V. Mahajan. 2002. An analysis of manufacturer-retailer supply chain coordination in cooperative advertising. Decision Science 33(1), 469-494.

Ivanuskiene, N., V. Auruskeviciene. 2009. Loyalty programs challenges in retail banking industry. Economics \& Management 14, 407-412.

Jain, D., S.S. Singh. 2002. Customer lifetime value research in marketing: a review and further directions. Journal of Interactive Marketing 16(2), 34-46.

Jin, M., S.D. Wu. 2007. Capacity reservation contracts for high-tech industry. European Journal of Operational Research 176 (3), 1659-1677.

Kadar, M., B. Kotanko. 2001. Designing loyalty programs to enhance value growth. Mercer on Transport and Travel 8(2), 28-33.

Kall P., S.W. Wallace. 1994. Stochastic Programming. Wiley, Chichester. 
Kall, P., J. Mayer. 2005. Building and solving stochastic linear programming models with SLPIOR. In: Applications of Stochastic Programming. S.W.Wallance and W.T. Ziemba (Eds.), MPSSIAM-Series in Optimization, 79-93.

Karray, S., G. Zaccour. 2007. Effectiveness of coop advertising programs in competitive distribution channels. International Game Theory Review 9(2), 151-167.

Keh, H.T., Y.H. Lee. 2006. Do reward programs build loyalty for services? The moderating effect of satisfaction on type and timing of rewards. Journal of Retailing 82(2), 127-136.

Kim B., M. Shi, K. Srinivasan. 2001. Reward programs and tacit collusion. Marketing Science 20(2), 99-120.

Kim, B., M. Shi, K. Srinivasan. 2004. Managing capacity through reward programs. Management Science 50(4), 503-520.

Kim, S.Y., R. Staelin. 1999. Manufacturer allowances and retailer pass-through rates in a competitive environment. Marketing Science 18(1), 59-76.

Kinnucan, H.W., Ø. Myrland. 2002. Optimal seasonal allocation of generic advertising expenditures with product substitution: salmon in France. Marine Resource Economics 17, 102120.

Kivetz, R., I. Simonson. 2002. Earning the right to indulge: effort as a determinant of customer preferences toward frequency program rewards. Journal of Marketing Research 39(2), 155-170.

Klemperer, P. 1995. Competition when consumers have switching costs: an overview with applications to industrial organization, macroeconomics, and international trade. Review of Economic Studies 62, 515-539.

Klemperer, P. 1987. The competitiveness of markets with switching costs. The Rand Journal of Economics 18(1), 138-150.

Kleywegt, A.J., A. Shapiro, T. Homeme-De-Mello. 2001. The sample average approximation method for stochastic discrete optimization. SIAM Journal of Optimization 12(2), 479-502.

Labbi A., C. Berrospi. 2007. Optimizing marketing planning and budgeting using Markov decision processes: An airline case study. IBM Journal of Research and Development 51(3/4), 421-431.

Lariviere, M.A. 1999. Supply chain contracting and coordination with stochastic demand. In: Quantitative models for supply chain management, S. Tayur, M. Magazine, R. Ganesan (Eds.), 233-268. Boston: Kluwer Adademic Publishing.

Lewis, M. 2004. The influence of loyalty programs and short-term promotions on customer retention. Journal of Marketing 41(3), 281-292. 
Lewis, M. 2005. Incorporating strategic consumer behaviour into customer valuation. Journal of Marketing 69(4), 230-238.

Li, C.L., P. Kouvelis. 1999. Flexible and risk-sharing supply contracts under price uncertainty. Management Science 45(10), 1378-1398.

Liu, Y., Y. Zhang, Z. Zhao. 2005. The survey on supply chain coordination with contracts. Proceedings of the 7th International Conference on Electronic Commerce, 807-809.

Liu, Y. 2007. Long-term impact of loyalty programs on consumer purchase behavior \& loyalty. Journal of Marketing 71(4) 19-35.

MacClaren, R.C. 1992. Trade marketing vs. trade spending: the new marketing chic. Promo, Nov. 56.

Mak, W., D.P. Morton, R. K. Wood. 1999. Monte Carlo bounding techniques for determining solution quality in stochastic programs. Operations Research Letters 24, 47-56.

Martínez-de-Albéniz, V., D. Simchi-Levi. 2009. Competition in the Supply Option Market. Operations Research 57(5), 1082-1097.

Mantrala, M. K., P. Sinha, A.A. Zoltners. 1992. Impact of resource allocation rules on marketing investment-level decisions and profitability. Journal of Marketing Research 29, 162-175.

Meyer-Waarden, L. 2008. The influence of loyalty programme membership on customer purchase behaviour. European Journal of Marketing 42(1/2), 87-114.

Naert, P.A., P.S. Leeflang. 1978. Building implementable marketing models. Leiden/Boston: Martinus Nijhoff Social Sciences Division, Kluwer Academic Publishers.

Neslin, S. A. 2006. Sales Promotion. Marketing Science Institute.

Nsakanda, A.L., M. Diaby, Y. Cao. 2006. Predictive modeling in reward loyalty programs: an aggregate inventory model. CORS/Optimization Days Annual Conference, May 8-10, 2006, Montreal (Canada).

Nsakanda, A.L., M. Diaby, Y. Cao. 2010. An aggregate inventory-based model for predicting redemption and liability in loyalty reward programs industry. Information System Frontiers, (in press). Published online, DOI 10.1007/s10796-010-9247-z

Nunes, J.C., X. Drèze. 2006. Your loyalty program is betraying you. Harvard Business Review 84(4), 124-128.

Nunes, J.C., C.W. Park. 2003. Incommensurate resources: not just more of the same. Journal of Marketing Research 40, 26-38. 
Oliver, R.L. 1999. Whence customer loyalty. Journal of Marketing 63, 33-44.

O'Malley, L. 1998. Can loyalty schemes really build loyalty? Marketing Intelligence \& Planning $16(1), 47-55$.

O'Brien, L., C. Jones. 1995. Do rewards really create loyalty? Harvard Business Review, May/June, 75-82.

Palmer, A. 1996. Relationship marketing: a universal paradigm or management fad. The Learning Organization 3(3), 18-25.

Park, J., 2010. The effect of frequent flyer programs: A case study of the Korean airline industry. Journal of Air Transport Management 16 (5), 287-288.

Park, M., S. Park, F.D. Mele, I.E. Grossmann, 2006. Modeling of purchase and sales contract in supply chain optimization. Industrial \& Engineering Chemistry Research 45(14), 5013-5026.

Perakis, G., M. Zaretsky. 2008. Multiperiod models with capacities in competitive supply chain. Production and Operations Management 17(4), 439-454.

Ritchen, P., C. Tapiero. 1986. Contingent claims contracting for purchasing decisions in inventory management. Operations Research 34, 864-870.

Ruszczyński, A., A. Shapiro. 2003. Monte Carlo Sampling Methods. In: Handbooks in OR \& MS Vol. 10, A. Ruszczyński, A. Shapiro (Eds.), Elasevier Science B.V.

Ruszczyński, A., A. Swietanowshi. 1997. Accelerating the regularized decomposition method for two-stage stochastic linear problems. European Journal of Operational Research 101, 328-342.

Santoso, T., S. Ahmed, M. Goetschalckx, A. Shapiro. 2005. A stochastic programming approach for supply chain network design under uncertainty. European Journal of Operational Research 167, 96-115.

Sen, S., J.L. Higle. 1999. An introductory tutorial on stochastic linear programming models. Interfaces 29 (2), 33-61.

Serel, D.A., M. Dada, H. Monskowitz. 2001. Sourcing decisions with capacity reservation contracts. European Journal of Operational Research 131(3), 635-648.

Shapiro, A., T. Homem-de-Mello. 1998. A simulation-based approach to two-stage stochastic programming with recourse. Mathematical Programming 81(3), 301-325.

Sharp, B., A., Sharp. 1997. Loyalty programs and their impact on repeat-purchase loyalty patterns. International Journal of Research in Marketing, 14, 473-486. 
Shi, M.Z., D. Soman. 2004. Multi-medium reward programs.

http://www.rotman.utoronto.ca/bicpapers/pdf/04-07.pdf, [Accessed Oct. 10, 2008]

Shugan, S.M. 2005. Brand loyalty programs: are they shames? Marketing Science 24(2), 185193.

Simon, J.L., J. Arndt. 1980. The shape of the advertising response function. Journal of Advertising Research 20, 11-28.

Singh, S.S., D.C. Jain, T.V. Krishnan. 2008. Customer loyalty programs: are they profitable? Management Science 54(6), 1205-1211.

Spinler, S., A. Hunchzermeier. 2006. The valuation of options on capacity with cost and demand uncertainty. European Journal of Operational Research 171(3), 915-934.

Stewart, D.W., M.A. Kamins. 2002. Marketing communications. In: Handbook of Marketing, Weitz Barton and Wesley Robin (Eds.), Sage Publications, 282-309.

Taylor, G.A., S.A. Neslin. 2005. The current and future sales impact of a retail frequency reward program. Journal of Retailing 81(4), 293-305.

Tsay, A.A., N. Steven, A. Narendra. 1999. Modeling supply chain contracts: a review. In: Quantitative models for supply chain management, S. Tayur, M. Magazine, R. Ganesan (Eds.), 299-336. Boston: Kluwer Adademic Publishing.

Tsay, A.A. 2002. Risk sensitivity in distribution channel partnerships: implications for manufacturer return policies. Journal of Retailing 78, 147-160.

Tirole, J. 1988. The Theory of Industrial Organization. MIT Press, Cambridge, MA.

Toktas, B., J.W. Yen, Z.B. Zabinsky. 2006. Addressing capacity uncertainty in resourceconstrained assignment problems. Computers and Operational Research 33(3), 724-745.

Tull, D.S., V.R. Wood, D. Duhan, T. Gillpatrick, K.R. Robertson, J.G. Helgeson. 1986. Leveraged decision making in advertising: the flat maximum principle and its implications. Journal of Marketing Research 23, 25-32.

Vakratsas, D., F.M. Feinberg, F.M. Bass. 2004. The shape of advertising response functions revisited: a model of dynamic probabilistic thresholds. Marketing Science 23(1), 109-119.

Vande Kamp, P.R., H.M. Kaiser. 2000. Optimal temporal policies in fluid milk advertising. American Journal of Agricultural Economics 82, 274-286.

Wang, K.J., S.M. Wang, J.C. Chen. 2008. A resource portfolio planning model using samplingbased stochastic programming and genetic algorithm. European Journal of Operational Research 184, 327-340. 
Wang, X., L. Liu. 2007. Coordination in a retailer-led supply chain through option contract. International Journal of Production Economics 110, 115-127.

Wu, D.J., P. Kleindorfer, J. E. Zhang. 2002. Optimal bidding and contracting strategies for capital-intensive goods. European Journal of Operations Research 137(3), 657-676.

Wu, D.J., P.R. Kleindorfer. 2005. Competitive options, supply contracting, and electronic markets. Management Science 51(3), 452-466.

Wu, S.D., M. Erkoc, S. Karabuk. 2005. Managing capacity in the high-tech industry: a review of literature. The Engineering Economist 50, 125-158.

Xie, J., J.C. Wei. 2009. Coordinating advertising and pricing in a manufacturer-retailer channel. European Journal of Operational Research 197 (2), 785-791.

$\mathrm{Xu}$, N.X., L., Nozick. 2009. Modeling supplier selection and the use of option contracts for global supply chain design. Computers \& Operation Research 36(10), 2786-2800.

$\mathrm{Yi}, \mathrm{Y} ., \mathrm{H}$. Jeon. 2003. Effects of loyalty programs on value perception, program loyalty, and brand loyalty. Journal of the Academy of Marketing Science 31(3), 229-240.

Young, R.F., S.A. Greyser. 1982. Cooperative advertising: practices and problems. MSI Report No. 82-105. Cambridge, MA: Marketing Science Institute.

Yue, J., A.J. Wang, M. Huang. 2006. Coordination of cooperative adverting in a two-level supply chain when manufacturer offers discount. European Journal of Operational Research $168,65-85$.

Zanjani, M.K., M. Nourelfath, D. Ait-Kadi. 2007. A stochastic programming approach for production planning in a manufacturing environment with random yield. Working paper, CIRRELT-2007-58, Interuniversity Research Centre, Montreal.

Zanjani, M.K., D. Ait-Kadi, M. Nourelfath. 2007. Robust production planning in a manufacturing environment with random yield: a case in sawmill production planning. European Journal of Operational Research 201(3), 882-891.

Zhang, Z.J., A. Krishna, S.K. Dhar. 2000. The optimal choice of promotional vehicles: frontloaded or rear-loaded incentives? Management Science 46 (3), 348-362.

Zhao, Y., S. Wang, T.C. Cheng, X. Yang, Z. Huang. 2010. Coordination of Supply chains by option contracts. A cooperative game theory approach. European Journal of Operational Research 207, 668-675. 
Appendix A 
Appendix A.1: Sample of LRPs in today's marketplace in Canada

\begin{tabular}{|c|c|c|c|c|c|}
\hline & Name of LRP & $\begin{array}{l}\text { Organizational } \\
\text { Structure }\end{array}$ & Reward Scheme & Industry base & $\begin{array}{l}\text { Profit or Cost } \\
\text { center }\end{array}$ \\
\hline 1 & Aeroplan & Type B-IV & $\begin{array}{l}\text { Dynamic/ one- } \\
\text { medium/ direct / use } \\
\text { timing/ amount- } \\
\text { based, frequency- } \\
\text { based and portfolio- } \\
\text { based }\end{array}$ & Travel & Profit center \\
\hline 2 & Air Miles & Type B-IV & $\begin{array}{l}\text { Dynamic/ one- } \\
\text { medium/ direct / no } \\
\text { timing/ amount- } \\
\text { based }\end{array}$ & Travel & Profit center \\
\hline 3 & PC points & Type A-I & $\begin{array}{l}\text { Static/one-medium/ } \\
\text { direct/ no timing/ } \\
\text { amount-based }\end{array}$ & Grocery & Cost center \\
\hline 4 & $\begin{array}{l}\text { Shoppers } \\
\text { Optimum Program }\end{array}$ & Type A-I & $\begin{array}{l}\text { Static/one-medium/ } \\
\text { indirect/ no timing/ } \\
\text { amount-based }\end{array}$ & Grocery & Cost center \\
\hline 5 & $\begin{array}{l}\text { Canadian Tire } \\
\text { Money }\end{array}$ & Type A-I & $\begin{array}{l}\text { Static/one-medium/ } \\
\text { indirect/ no timing/ } \\
\text { amount-based }\end{array}$ & Dept. store & Cost center \\
\hline 6 & $\begin{array}{l}\text { American Airline } \\
\text { AAdvantage }\end{array}$ & $\begin{array}{l}\text { Type B-(I, II, or } \\
\text { III) }\end{array}$ & $\begin{array}{l}\text { Dynamic/one- } \\
\text { medium/direct/use } \\
\text { timing/portfolio- } \\
\text { based }\end{array}$ & Travel & $\begin{array}{l}\text { Semi-cost } \\
\text { center }\end{array}$ \\
\hline 7 & $\begin{array}{l}\text { United Airline } \\
\text { Mileage Plus }\end{array}$ & $\begin{array}{l}\text { Type B-(I, II, or } \\
\text { III) }\end{array}$ & $\begin{array}{l}\text { Dynamic/one- } \\
\text { medium/direct/ use } \\
\text { timing / amount- } \\
\text { based, frequency- } \\
\text { based, and portfolio- } \\
\text { based }\end{array}$ & Travel & $\begin{array}{l}\text { Semi-cost } \\
\text { center }\end{array}$ \\
\hline 8 & Save-On-More & Type C & $\begin{array}{l}\text { Dynamic/one- } \\
\text { medium/direct/use } \\
\text { timing/amount- } \\
\text { based }\end{array}$ & Grocery & $\begin{array}{l}\text { Semi-cost } \\
\text { center }\end{array}$ \\
\hline 9 & $\begin{array}{l}\text { Casino Niagara } \\
\text { Players Prestige } \\
\text { Club }\end{array}$ & Type A-I & $\begin{array}{l}\text { Static/one-medium } \\
\text { /mixed /no timing/ } \\
\text { portfolio-based }\end{array}$ & $\begin{array}{l}\text { Entertainment } \\
\text { (Casino) }\end{array}$ & Cost center \\
\hline 10 & $\begin{array}{l}\text { Casino Rama } \\
\text { Player's Passport } \\
\text { program }\end{array}$ & Type A-I & $\begin{array}{l}\text { Static/one-medium/ } \\
\text { indirect/ no timing/ } \\
\text { portfolio-based }\end{array}$ & $\begin{array}{l}\text { Entertainment } \\
\text { (Casino) }\end{array}$ & Cost center \\
\hline 11 & $\begin{array}{l}\text { Casino Windsor } \\
\text { Players Prestige } \\
\text { Club }\end{array}$ & \multicolumn{4}{|c|}{ The same as (9) } \\
\hline 12 & $\begin{array}{l}\text { Blockbuster } \\
\text { Reward }\end{array}$ & Type A-I & $\begin{array}{l}\text { Static/ one-medium } \\
\text { /mixed / use timing/ } \\
\text { frequency-based }\end{array}$ & $\begin{array}{l}\text { Entertainment } \\
\text { (Video rent) }\end{array}$ & Cost center \\
\hline 13 & Delta Privilege & Type A-I & $\begin{array}{l}\text { Static/ no-medium } \\
\text { /direct / no timing / } \\
\text { frequency-based }\end{array}$ & Hotel & Cost center \\
\hline
\end{tabular}


Appendix A.1: Sample of LRPs in today's marketplace in Canada (Cont.)

\begin{tabular}{|c|c|c|c|c|c|}
\hline 14 & Esso Extra & $\begin{array}{l}\text { Type A-(I, II, or } \\
\text { III)* }\end{array}$ & $\begin{array}{l}\text { Static/one-medium } \\
\text { /direct / no timing } \\
\text { /amount-based }\end{array}$ & Gas & Cost center \\
\hline 15 & $\begin{array}{l}\text { Best Western } \\
\text { Rewards }\end{array}$ & $\begin{array}{l}\text { Type B-(I, II, or } \\
\text { III) }\end{array}$ & $\begin{array}{l}\text { Static/ one-medium } \\
\text { (with option) /mixed } \\
\text { / no timing /amount- } \\
\text { based }\end{array}$ & Hotel & $\begin{array}{l}\text { Semi-cost } \\
\text { center }\end{array}$ \\
\hline 16 & $\begin{array}{l}\text { Carlson Hotels } \\
\text { Worldwide } \\
\text { Goldpointsplus } \\
\end{array}$ & $\begin{array}{l}\text { Type B-(II or } \\
\text { III)* }\end{array}$ & $\begin{array}{l}\text { Static/one-medium } \\
\text { /mixed / no timing } \\
\text { /amount-based }\end{array}$ & Hotel & $\begin{array}{l}\text { Semi-cost } \\
\text { center }\end{array}$ \\
\hline 17 & HBC Rewards & $\begin{array}{l}\text { Type B-(I, II, or } \\
\text { III)* }\end{array}$ & $\begin{array}{l}\text { Dynamic (for } \\
\text { rewards other than } \\
\text { gift card)/ one- } \\
\text { medium / mixed /no } \\
\text { timing / amount- } \\
\text { based }\end{array}$ & Dept. store & $\begin{array}{l}\text { Semi-cost } \\
\text { center }\end{array}$ \\
\hline 18 & Hilton Hhonours & $\begin{array}{l}\text { Type B-(I, II, or } \\
\text { III)* }\end{array}$ & $\begin{array}{l}\text { Dynamic/ two- } \\
\text { medium (with } \\
\text { option)/ mixed / no } \\
\text { timing/ portfolio }\end{array}$ & Hotel & $\begin{array}{l}\text { Semi-cost } \\
\text { center }\end{array}$ \\
\hline 19 & CAA Dollar & $\begin{array}{l}\text { Type B-(I, II, or } \\
\text { III)* }\end{array}$ & $\begin{array}{l}\text { Static/ one-medium/ } \\
\text { mixed / no timing / } \\
\text { portfolio-based }\end{array}$ & Travel & Cost center \\
\hline 20 & iCoke & $\begin{array}{l}\text { Type B-(I, II or } \\
\text { III)* }\end{array}$ & $\begin{array}{l}\text { Dynamic/ one- } \\
\text { medium/ mixed / no } \\
\text { timing / portfolio- } \\
\text { based }\end{array}$ & Food/beverage & Cost center \\
\hline 21 & $\begin{array}{l}\text { Futura Rewards } \\
\text { Program }\end{array}$ & Type B-IV & $\begin{array}{l}\text { Dynamic/no- } \\
\text { medium/ indirect / } \\
\text { no timing /amount- } \\
\text { based }\end{array}$ & LRP service & Profit center \\
\hline 22 & Marriott Rewards & $\begin{array}{l}\text { Type B-(I, II, or } \\
\text { III)* }\end{array}$ & $\begin{array}{l}\text { Dynamic/ one- } \\
\text { medium (with } \\
\text { option) / mixed/ no } \\
\text { timing / portfolio- } \\
\text { based }\end{array}$ & Hotel & $\begin{array}{l}\text { Semi-cost } \\
\text { center }\end{array}$ \\
\hline 23 & M \& M Max & $\begin{array}{l}\text { Type A-( I, II, } \\
\text { or III)* }\end{array}$ & $\begin{array}{l}\text { Dynamic/ no } \\
\text { medium / mixed / no } \\
\text { timing / amount- } \\
\text { based }\end{array}$ & Grocery & Cost center \\
\hline 24 & $\begin{array}{l}\text { OLGC Winner's } \\
\text { Circle }\end{array}$ & Type A-I & $\begin{array}{l}\text { Static/ one-medium/ } \\
\text { mixed/no timing / } \\
\text { amount-based }\end{array}$ & $\begin{array}{l}\text { Entertainment } \\
\text { (Casino) }\end{array}$ & Cost center \\
\hline 25 & $\begin{array}{l}\text { Pennington PS } \\
\text { Club Advantage }\end{array}$ & Type A-I & $\begin{array}{l}\text { Static/no-medium/ } \\
\text { direct/ no timing/ } \\
\text { amount-based }\end{array}$ & Dept. store & Cost center \\
\hline 26 & Petro-Points & $\begin{array}{l}\text { Type B-(I, II or } \\
\text { III)* }\end{array}$ & $\begin{array}{l}\text { Static/ one-medium/ } \\
\text { mixed/ no timing / } \\
\text { amount-based }\end{array}$ & Gas & Cost center \\
\hline 27 & $\begin{array}{l}\text { The Shoe } \\
\text { Company web } \\
\text { points }\end{array}$ & $\begin{array}{l}\text { Type A-(I, II, or } \\
\text { III)* }\end{array}$ & $\begin{array}{l}\text { Static/one-medium/ } \\
\text { indirect/ no timing/ } \\
\text { amount-based }\end{array}$ & Specialty store & Cost center \\
\hline
\end{tabular}


Appendix A.1: Sample of LRPs in today's marketplace in Canada (Cont.)

\begin{tabular}{|c|c|c|c|c|c|}
\hline 28 & RBC Rewards & $\begin{array}{l}\text { Type B-(I, II, or } \\
\text { III) }\end{array}$ & $\begin{array}{l}\text { Static/ two-medium/ } \\
\text { mixed/ no timing / } \\
\text { amount-based }\end{array}$ & Finance & $\begin{array}{l}\text { Semi-cost } \\
\text { center }\end{array}$ \\
\hline 29 & $\begin{array}{l}\text { Reservation- } \\
\text { Rewards }\end{array}$ & Type B-IV & $\begin{array}{l}\text { Static/ no-medium/ } \\
\text { indirect/ no timing/ } \\
\text { amount-based }\end{array}$ & LRP service & Profit center \\
\hline 30 & Sears Club & Type A-I & $\begin{array}{l}\text { Static/ one-medium/ } \\
\text { direct/ no timing/ } \\
\text { amount-based }\end{array}$ & Dept. store & Cost center \\
\hline 31 & $\begin{array}{l}\text { Scotia Bank } \\
\text { SCENE }\end{array}$ & Type C & $\begin{array}{l}\text { Static/ one-medium/ } \\
\text { mixed/ no timing/ } \\
\text { amount-based }\end{array}$ & $\begin{array}{l}\text { Finance \& } \\
\text { Entertainment }\end{array}$ & $\begin{array}{l}\text { Semi-cost } \\
\text { center }\end{array}$ \\
\hline 32 & $\begin{array}{l}\text { Starwood } \\
\text { Preferred Guest }\end{array}$ & $\begin{array}{l}\text { Type B-(I, II, or } \\
\text { III)* }\end{array}$ & \begin{tabular}{|l|} 
Dynamic/one- \\
medium/ mixed/ no \\
timing/ portfolio- \\
based
\end{tabular} & Hotel & $\begin{array}{l}\text { Semi-cost } \\
\text { center }\end{array}$ \\
\hline 33 & ZipRewards & Type A-I & $\begin{array}{l}\text { Static/one-medium/ } \\
\text { direct/ no timing/ } \\
\text { portfolio-based }\end{array}$ & $\begin{array}{l}\text { Entertainment } \\
\text { (video rent) }\end{array}$ & Cost center \\
\hline 34 & $\begin{array}{l}\text { American Express } \\
\text { Membership } \\
\text { Rewards }\end{array}$ & $\begin{array}{l}\text { Type B-( I or } \\
\text { II)* }\end{array}$ & $\begin{array}{l}\text { Dynamic/ one- } \\
\text { medium/ mixed / no } \\
\text { timing/ portfolio- } \\
\text { based }\end{array}$ & Finance & $\begin{array}{l}\text { Semi-cost } \\
\text { center }\end{array}$ \\
\hline 35 & Asia Miles & Type B-IV & $\begin{array}{l}\text { Dynamic/ one } \\
\text { medium/ mixed / no } \\
\text { timing / portfolio- } \\
\text { based } \\
\end{array}$ & LRP service & Profit center \\
\hline 36 & $\begin{array}{l}\text { Harrah's Total } \\
\text { Rewards }\end{array}$ & $\begin{array}{l}\text { Type B-(I, II, or } \\
\text { III)* }\end{array}$ & $\begin{array}{l}\text { Dynamic/ one- } \\
\text { medium/ direct/ use } \\
\text { timing/ portfolio- } \\
\text { based }\end{array}$ & $\begin{array}{l}\text { Entertainment } \\
\text { (Casino) }\end{array}$ & Cost center \\
\hline 37 & $\begin{array}{l}\text { Best Buy's Reward } \\
\text { Zone }\end{array}$ & $\begin{array}{l}\text { Type A-(I, II or } \\
\text { III)* }\end{array}$ & $\begin{array}{l}\text { Static/ one-medium/ } \\
\text { direct/ no timing/ } \\
\text { amount-based }\end{array}$ & Speciality store & Cost center \\
\hline 38 & ETR Rewards & Type A-I & $\begin{array}{l}\text { Static/no-medium/ } \\
\text { direct/ use timing/ } \\
\text { amount-based }\end{array}$ & Transportation & Cost center \\
\hline 39 & Club Sobeys & $\begin{array}{l}\text { Type A-(I, II or } \\
\text { III)* }\end{array}$ & $\begin{array}{l}\text { Static/one-medium/ } \\
\text { direct/ use timing/ } \\
\text { amount-based }\end{array}$ & Grocery & Cost center \\
\hline
\end{tabular}


Among these 39 LRPs:

\begin{tabular}{l|l|c}
\hline Industry & \multicolumn{1}{|c|}{ LRPs } & Total \\
\hline Travel & $1,2,6,7,19$ & 5 \\
\hline Hotel & $13,15,16,18,22,32$ & 6 \\
\hline Finance & $28,31,34$ & 3 \\
\hline Grocery & $3,4,8,23$ & 4 \\
\hline Dept. store & $5,17,25,30,39$ & 5 \\
\hline Entertainment & $9,10,11,12,24,31,33,36$ & 8 \\
\hline Specialty store & 27,37 & 2 \\
\hline Gas & 14,26 & 2 \\
\hline Food/beverage & 20 & 1 \\
\hline LRP service & $21,29,35$ & 3 \\
\hline Transportation & 38 & 1 \\
\hline
\end{tabular}

\begin{tabular}{l|l|c}
\hline Type of LRPs & \multicolumn{1}{|c}{ LRPs } & Total \\
\hline Type A & $\begin{array}{l}\mid \\
\text { A-I: } 3,4,5,9,10,11,12,13,24,25,30,33,38 \\
\text { A-I, II or III: } 14,23,37,39\end{array}$ & 17 \\
\hline Type B & $\begin{array}{l}\text { B-I, II or III: 6,7, 15,16, 17, 18, 19, 20, 22, 26, 27, 28 } \\
32,34,36 \\
\text { B-IV: } 1,2,21,29,35\end{array}$ & 20 \\
\hline Type C & 8,31 & 2 \\
\hline
\end{tabular}


Appendix B 
Appendix B.1: Computational outputs for examining BP model solvability and determining sample size and sample replications

(a) with Uniformly Distributed Demand

\begin{tabular}{|c|c|c|c|c|}
\hline $\begin{array}{l}\text { TC } \\
\text { No. }\end{array}$ & $\begin{array}{c}\text { Problem } \\
\text { No. }\end{array}$ & $\begin{array}{c}\text { MeanG } \\
(\%)\end{array}$ & $\begin{array}{l}\mathrm{CIU} \\
(\%)\end{array}$ & $\begin{array}{c}\mathrm{CPU} \\
\text { (mins) }\end{array}$ \\
\hline \multirow{4}{*}{1} & 1.1 & 0.132 & 0.179 & 0.003 \\
\hline & 1.2 & 0.082 & 0.116 & 0.003 \\
\hline & 1.3 & 0.083 & 0.107 & 0.004 \\
\hline & 1.4 & 0.063 & 0.081 & 0.005 \\
\hline \multirow{4}{*}{2} & 2.1 & 0.119 & 0.152 & 0.004 \\
\hline & 2.2 & 0.069 & 0.091 & 0.005 \\
\hline & 2.3 & 0.07 & 0.086 & 0.007 \\
\hline & 2.4 & 0.053 & 0.065 & 0.009 \\
\hline \multirow{4}{*}{3} & 3.1 & 0.022 & 0.028 & 0.008 \\
\hline & 3.2 & 0.011 & 0.015 & 0.014 \\
\hline & 3.3 & 0.012 & 0.015 & 0.019 \\
\hline & 3.4 & 0.007 & 0.009 & 0.030 \\
\hline \multirow{4}{*}{4} & 4.1 & 0.072 & 0.083 & 0.017 \\
\hline & 4.2 & 0.039 & 0.045 & 0.031 \\
\hline & 4.3 & 0.04 & 0.045 & 0.043 \\
\hline & 4.4 & 0.028 & 0.032 & 0.073 \\
\hline \multirow{4}{*}{5} & 5.1 & 0.017 & 0.020 & 0.022 \\
\hline & 5.2 & 0.009 & 0.010 & 0.040 \\
\hline & 5.3 & 0.009 & 0.010 & 0.055 \\
\hline & 5.4 & 0.006 & 0.007 & 0.093 \\
\hline \multirow{4}{*}{6} & 6.1 & 0.043 & 0.049 & 0.043 \\
\hline & 6.2 & 0.022 & 0.025 & 0.078 \\
\hline & 6.3 & 0.021 & 0.024 & 0.105 \\
\hline & 6.4 & 0.016 & 0.017 & 0.184 \\
\hline \multirow{4}{*}{7} & 7.1 & 0.067 & 0.073 & 0.078 \\
\hline & 7.2 & 0.035 & 0.038 & 0.142 \\
\hline & 7.3 & 0.034 & 0.037 & 0.203 \\
\hline & 7.4 & 0.024 & 0.026 & 0.334 \\
\hline \multirow{4}{*}{8} & 8.1 & 0.048 & 0.053 & 0.085 \\
\hline & 8.2 & 0.025 & 0.027 & 0.158 \\
\hline & 8.3 & 0.025 & 0.027 & 0.223 \\
\hline & 8.4 & 0.018 & 0.020 & 0.370 \\
\hline
\end{tabular}

(b) with Normally Distributed Demand

\begin{tabular}{|c|c|c|c|c|}
\hline $\begin{array}{l}\text { TC } \\
\text { No. }\end{array}$ & $\begin{array}{c}\text { Problem } \\
\text { No. }\end{array}$ & $\begin{array}{c}\text { MeanG } \\
(\%)\end{array}$ & $\begin{array}{l}\text { CIU } \\
(\%)\end{array}$ & $\begin{array}{c}\mathrm{CPU} \\
(\mathrm{mins})\end{array}$ \\
\hline \multirow{4}{*}{1} & 1.1 & 0.199 & 0.273 & 0.003 \\
\hline & 1.2 & 0.119 & 0.166 & 0.002 \\
\hline & 1.3 & 0.129 & 0.169 & 0.002 \\
\hline & 1.4 & 0.094 & 0.123 & 0.003 \\
\hline \multirow{4}{*}{2} & 2.1 & 0.175 & 0.223 & 0.004 \\
\hline & 2.2 & 0.106 & 0.136 & 0.005 \\
\hline & 2.3 & 0.109 & 0.134 & 0.007 \\
\hline & 2.4 & 0.082 & 0.101 & 0.011 \\
\hline \multirow{4}{*}{3} & 3.1 & 0.125 & 0.148 & 0.008 \\
\hline & 3.2 & 0.072 & 0.085 & 0.015 \\
\hline & 3.3 & 0.073 & 0.085 & 0.019 \\
\hline & 3.4 & 0.053 & 0.061 & 0.031 \\
\hline \multirow{4}{*}{4} & 4.1 & 0.119 & 0.136 & 0.017 \\
\hline & 4.2 & 0.066 & 0.076 & 0.032 \\
\hline & 4.3 & 0.069 & 0.077 & 0.041 \\
\hline & 4.4 & 0.048 & 0.054 & 0.073 \\
\hline \multirow{4}{*}{5} & 5.1 & 0.029 & 0.035 & 0.022 \\
\hline & 5.2 & 0.015 & 0.018 & 0.041 \\
\hline & 5.3 & 0.015 & 0.017 & 0.054 \\
\hline & 5.4 & 0.01 & 0.012 & 0.093 \\
\hline \multirow{4}{*}{6} & 6.1 & 0.078 & 0.088 & 0.044 \\
\hline & 6.2 & 0.041 & 0.047 & 0.079 \\
\hline & 6.3 & 0.042 & 0.046 & 0.106 \\
\hline & 6.4 & 0.032 & 0.036 & 0.184 \\
\hline \multirow{4}{*}{7} & 7.1 & 0.114 & 0.124 & 0.079 \\
\hline & 7.2 & 0.062 & 0.068 & 0.144 \\
\hline & 7.3 & 0.061 & 0.065 & 0.202 \\
\hline & 7.4 & 0.043 & 0.046 & 0.334 \\
\hline \multirow{4}{*}{8} & 8.1 & 0.077 & 0.085 & 0.087 \\
\hline & 8.2 & 0.041 & 0.045 & 0.159 \\
\hline & 8.3 & 0.040 & 0.044 & 0.221 \\
\hline & 8.4 & 0.030 & 0.033 & 0.371 \\
\hline
\end{tabular}


Appendix B.1: Computational outputs for examining BP model solvability and determining sample size and sample replications

(Cont.)

(a) with Uniformly Distributed Demand

\begin{tabular}{|c|c|c|c|c|}
\hline $\begin{array}{c}\text { TC } \\
\text { No. }\end{array}$ & $\begin{array}{c}\text { Problem } \\
\text { No. }\end{array}$ & $\begin{array}{c}\text { MeanG } \\
(\%)\end{array}$ & $\begin{array}{c}\text { CIU } \\
(\%)\end{array}$ & $\begin{array}{c}\text { CPU } \\
\text { (mins) }\end{array}$ \\
\hline & 9.1 & 0.041 & 0.045 & 0.119 \\
9 & 9.2 & 0.020 & 0.022 & 0.218 \\
& 9.3 & 0.020 & 0.022 & 0.301 \\
& 9.4 & 0.014 & 0.016 & 0.512 \\
\hline \multirow{4}{*}{10} & 10.1 & 0.038 & 0.041 & 0.171 \\
& 10.2 & 0.019 & 0.021 & 0.311 \\
& 10.3 & 0.019 & 0.021 & 0.426 \\
& 10.4 & 0.014 & 0.015 & 0.729 \\
\hline
\end{tabular}

(b) with Normally Distributed Demand

\begin{tabular}{|c|c|c|c|c|}
\hline $\begin{array}{c}\text { TC } \\
\text { No. }\end{array}$ & $\begin{array}{c}\text { Problem } \\
\text { No. }\end{array}$ & $\begin{array}{c}\text { MeanG } \\
(\%)\end{array}$ & $\begin{array}{c}\text { CIU } \\
(\%)\end{array}$ & $\begin{array}{c}\text { CPU } \\
\text { (mins) }\end{array}$ \\
\hline & 9.1 & 0.070 & 0.077 & 0.120 \\
9 & 9.2 & 0.036 & 0.040 & 0.219 \\
& 9.3 & 0.036 & 0.039 & 0.304 \\
& 9.4 & 0.026 & 0.028 & 0.520 \\
\hline \multirow{4}{*}{10} & 10.1 & 0.068 & 0.073 & 0.172 \\
& 10.2 & 0.036 & 0.039 & 0.313 \\
& 10.3 & 0.037 & 0.039 & 0.428 \\
& 10.4 & 0.026 & 0.027 & 0.731 \\
\hline
\end{tabular}

Appendix B.2: Computational outputs for examining EP1 model solvability and determining sample size and sample replications

(a) with Uniformly Distributed Demand

\begin{tabular}{|c|c|c|c|c|}
\hline $\begin{array}{c}\text { TC } \\
\text { No. }\end{array}$ & $\begin{array}{c}\text { Problem } \\
\text { No. }\end{array}$ & $\begin{array}{c}\text { MeanG } \\
(\%)\end{array}$ & $\begin{array}{c}\text { CIU } \\
(\%)\end{array}$ & $\begin{array}{c}\text { CPU } \\
(\text { mins })\end{array}$ \\
\hline \multirow{4}{*}{1} & 1.1 & 0.244 & 0.303 & 0.002 \\
& 1.2 & 0.144 & 0.184 & 0.004 \\
& 1.3 & 0.148 & 0.179 & 0.005 \\
& 1.4 & 0.104 & 0.126 & 0.007 \\
\hline \multirow{4}{*}{2} & 2.1 & 0.320 & 0.366 & 0.006 \\
& 2.2 & 0.175 & 0.204 & 0.010 \\
& 2.3 & 0.177 & 0.199 & 0.013 \\
& 2.4 & 0.125 & 0.141 & 0.018 \\
\hline \multirow{4}{*}{3} & 3.1 & 0.226 & 0.249 & 0.017 \\
& 3.2 & 0.115 & 0.127 & 0.027 \\
& 3.3 & 0.116 & 0.126 & 0.038 \\
& 3.4 & 0.078 & 0.085 & 0.063 \\
\hline \multirow{4}{*}{4} & 4.1 & 0.163 & 0.177 & 0.033 \\
& 4.2 & 0.084 & 0.092 & 0.059 \\
& 4.3 & 0.085 & 0.091 & 0.080 \\
& 4.4 & 0.059 & 0.063 & 0.135 \\
\hline
\end{tabular}

(b) with Normally Distributed Demand

\begin{tabular}{|c|c|c|c|c|}
\hline $\begin{array}{c}\text { TC } \\
\text { No. }\end{array}$ & $\begin{array}{c}\text { Problem } \\
\text { No. }\end{array}$ & $\begin{array}{c}\text { MeanG } \\
(\%)\end{array}$ & $\begin{array}{c}\text { CIU } \\
(\%)\end{array}$ & $\begin{array}{c}\text { CPU } \\
\text { (mins) }\end{array}$ \\
\hline & 1.1 & 0.843 & 0.946 & 0.002 \\
1 & 1.2 & 0.655 & 0.743 & 0.002 \\
& 1.3 & 0.726 & 0.797 & 0.003 \\
& 1.4 & 0.688 & 0.749 & 0.006 \\
\hline & 2.1 & 2.211 & 2.337 & 0.006 \\
& 2.2 & 1.980 & 2.103 & 0.009 \\
& 2.3 & 2.088 & 2.188 & 0.013 \\
& 2.4 & 1.856 & 1.944 & 0.018 \\
\hline \multirow{4}{*}{3} & 3.1 & 2.149 & 2.25 & 0.016 \\
& 3.2 & 1.847 & 1.937 & 0.028 \\
& 3.3 & 2.043 & 2.116 & 0.038 \\
& 3.4 & 1.873 & 1.941 & 0.063 \\
\hline \multirow{4}{*}{4} & 4.1 & 1.029 & 1.068 & 0.032 \\
& 4.2 & 0.862 & 0.893 & 0.060 \\
& 4.3 & 0.916 & 0.943 & 0.082 \\
& 4.4 & 0.803 & 0.828 & 0.136 \\
\hline
\end{tabular}


Appendix B.2: Computational outputs for examining EP1 model solvability and determining sample size and sample replications

(Cont.)

(a) with Uniformly Distributed Demand

\begin{tabular}{|c|c|c|c|c|}
\hline $\begin{array}{l}\text { TC } \\
\text { No. }\end{array}$ & $\begin{array}{c}\text { Problem } \\
\text { No. }\end{array}$ & $\begin{array}{c}\text { MeanG } \\
(\%)\end{array}$ & $\begin{array}{l}\mathrm{CIU} \\
(\%) \\
\end{array}$ & $\begin{array}{c}\mathrm{CPU} \\
\text { (mins) }\end{array}$ \\
\hline \multirow{4}{*}{5} & 5.1 & 0.178 & 0.190 & 0.059 \\
\hline & 5.2 & 0.092 & 0.097 & 0.108 \\
\hline & 5.3 & 0.092 & 0.097 & 0.146 \\
\hline & 5.4 & 0.061 & 0.064 & 0.250 \\
\hline \multirow{4}{*}{6} & 6.1 & 0.187 & 0.197 & 0.106 \\
\hline & 6.2 & 0.093 & 0.098 & 0.199 \\
\hline & 6.3 & 0.094 & 0.098 & 0.271 \\
\hline & 6.4 & 0.064 & 0.067 & 0.483 \\
\hline \multirow{4}{*}{7} & 7.1 & 0.186 & 0.194 & 0.188 \\
\hline & 7.2 & 0.095 & 0.099 & 0.363 \\
\hline & 7.3 & 0.095 & 0.098 & 0.490 \\
\hline & 7.4 & 0.065 & 0.068 & 0.846 \\
\hline \multirow{4}{*}{8} & 8.1 & 0.205 & 0.213 & 0.250 \\
\hline & 8.2 & 0.105 & 0.109 & 0.478 \\
\hline & 8.3 & 0.106 & 0.109 & 0.644 \\
\hline & 8.4 & 0.073 & 0.075 & 1.126 \\
\hline \multirow{4}{*}{9} & 9.1 & 0.168 & 0.174 & 0.341 \\
\hline & 9.2 & 0.085 & 0.089 & 0.640 \\
\hline & 9.3 & 0.086 & 0.089 & 0.858 \\
\hline & 9.4 & 0.058 & 0.060 & 1.479 \\
\hline \multirow{4}{*}{10} & 10.1 & 0.167 & 0.173 & 0.496 \\
\hline & 10.2 & 0.087 & 0.090 & 0.889 \\
\hline & 10.3 & 0.087 & 0.089 & 1.187 \\
\hline & 10.4 & 0.058 & 0.060 & 2.013 \\
\hline
\end{tabular}

(b) with Normally Distributed Demand

\begin{tabular}{|c|c|c|c|c|}
\hline $\begin{array}{l}\mathrm{TC} \\
\text { No. }\end{array}$ & $\begin{array}{c}\text { Problem } \\
\text { No. }\end{array}$ & $\begin{array}{c}\text { MeanG } \\
(\%)\end{array}$ & $\begin{array}{l}\mathrm{CIU} \\
(\%)\end{array}$ & $\begin{array}{c}\mathrm{CPU} \\
\text { (mins) }\end{array}$ \\
\hline \multirow{4}{*}{5} & 5.1 & 1.693 & 1.733 & 0.060 \\
\hline & 5.2 & 1.469 & 1.508 & 0.110 \\
\hline & 5.3 & 1.592 & 1.624 & 0.152 \\
\hline & 5.4 & 1.473 & 1.502 & 0.262 \\
\hline \multirow{4}{*}{6} & 6.1 & 1.557 & 1.591 & 0.110 \\
\hline & 6.2 & 1.316 & 1.347 & 0.203 \\
\hline & 6.3 & 1.415 & 1.44 & 0.284 \\
\hline & 6.4 & 1.314 & 1.339 & 0.473 \\
\hline \multirow{4}{*}{7} & 7.1 & 1.313 & 1.34 & 0.192 \\
\hline & 7.2 & 1.127 & 1.149 & 0.357 \\
\hline & 7.3 & 1.196 & 1.214 & 0.492 \\
\hline & 7.4 & 1.093 & 1.11 & 0.838 \\
\hline \multirow{4}{*}{8} & 8.1 & 1.687 & 1.715 & 0.256 \\
\hline & 8.2 & 1.466 & 1.491 & 0.475 \\
\hline & 8.3 & 1.56 & 1.58 & 0.650 \\
\hline & 8.4 & 1.474 & 1.493 & 1.124 \\
\hline \multirow{4}{*}{9} & 9.1 & 1.368 & 1.392 & 0.346 \\
\hline & 9.2 & 1.171 & 1.191 & 0.638 \\
\hline & 9.3 & 1.247 & 1.264 & 0.864 \\
\hline & 9.4 & 1.149 & 1.165 & 1.480 \\
\hline \multirow{4}{*}{10} & 10.1 & 1.398 & 1.418 & 0.487 \\
\hline & 10.2 & 1.198 & 1.217 & 0.887 \\
\hline & 10.3 & 1.285 & 1.301 & 1.189 \\
\hline & 10.4 & 1.179 & 1.193 & 2.019 \\
\hline
\end{tabular}


Appendix B.3: Computational outputs for examining EP2 model solvability and determining sample size and sample replications

(a) with Uniformly Distributed Demand

\begin{tabular}{|c|c|c|c|c|}
\hline $\begin{array}{c}\text { TC } \\
\text { No. }\end{array}$ & $\begin{array}{c}\text { Problem } \\
\text { No. }\end{array}$ & $\begin{array}{c}\text { MeanG } \\
(\%)\end{array}$ & $\begin{array}{c}\text { CIU } \\
(\%)\end{array}$ & $\begin{array}{c}\text { CPU } \\
\text { (mins) }\end{array}$ \\
\hline & 1.1 & 0.132 & 0.170 & 0.005 \\
1 & 1.2 & 0.076 & 0.100 & 0.007 \\
& 1.3 & 0.079 & 0.097 & 0.009 \\
& 1.4 & 0.058 & 0.070 & 0.015 \\
\hline & 2.1 & 0.103 & 0.126 & 0.009 \\
& 2.2 & 0.058 & 0.072 & 0.014 \\
2 & 2.3 & 0.061 & 0.072 & 0.021 \\
& 2.4 & 0.045 & 0.053 & 0.033 \\
\hline & 3.1 & 0.067 & 0.076 & 0.039 \\
3 & 3.2 & 0.036 & 0.041 & 0.069 \\
& 3.3 & 0.036 & 0.040 & 0.091 \\
& 3.4 & 0.026 & 0.030 & 0.156 \\
\hline & 4.1 & 0.085 & 0.093 & 0.104 \\
4 & 4.2 & 0.048 & 0.052 & 0.179 \\
& 4.3 & 0.047 & 0.051 & 0.237 \\
& 4.4 & 0.034 & 0.037 & 0.431 \\
\hline \multirow{4}{*}{5} & 5.1 & 0.102 & 0.114 & 0.176 \\
& 5.2 & 0.055 & 0.062 & 0.307 \\
& 5.3 & 0.056 & 0.061 & 0.397 \\
& 5.4 & 0.042 & 0.046 & 0.679 \\
\hline \multirow{4}{*}{6} & 6.1 & 0.068 & 0.073 & 0.345 \\
& 6.2 & 0.036 & 0.039 & 0.621 \\
& 6.3 & 0.037 & 0.039 & 0.750 \\
& 6.4 & 0.026 & 0.028 & 1.301 \\
\hline & 7.1 & 0.079 & 0.084 & 0.702 \\
& 7.2 & 0.043 & 0.046 & 1.151 \\
& 7.3 & 0.042 & 0.045 & 1.469 \\
& 7.4 & 0.031 & 0.032 & 2.553 \\
\hline
\end{tabular}

(b) with Normally Distributed Demand

\begin{tabular}{|c|c|c|c|c|}
\hline $\begin{array}{l}\text { TC } \\
\text { No. }\end{array}$ & $\begin{array}{c}\text { Problem } \\
\text { No. }\end{array}$ & $\begin{array}{c}\text { MeanG } \\
(\%)\end{array}$ & $\begin{array}{l}\text { CIU } \\
(\%)\end{array}$ & $\underset{\text { (mins) }}{\mathrm{CPU}}$ \\
\hline \multirow{4}{*}{1} & 1.1 & 0.182 & 0.241 & 0.006 \\
\hline & 1.2 & 0.100 & 0.137 & 0.010 \\
\hline & 1.3 & 0.111 & 0.141 & 0.012 \\
\hline & 1.4 & 0.078 & 0.100 & 0.021 \\
\hline \multirow{4}{*}{2} & 2.1 & 0.15 & 0.187 & 0.017 \\
\hline & 2.2 & 0.086 & 0.108 & 0.027 \\
\hline & 2.3 & 0.088 & 0.105 & 0.037 \\
\hline & 2.4 & 0.063 & 0.077 & 0.061 \\
\hline \multirow{4}{*}{3} & 3.1 & 0.111 & 0.128 & 0.078 \\
\hline & 3.2 & 0.060 & 0.070 & 0.131 \\
\hline & 3.3 & 0.061 & 0.069 & 0.173 \\
\hline & 3.4 & 0.042 & 0.048 & 0.291 \\
\hline \multirow{4}{*}{4} & 4.1 & 0.161 & 0.179 & 0.244 \\
\hline & 4.2 & 0.088 & 0.098 & 0.392 \\
\hline & 4.3 & 0.090 & 0.098 & 0.504 \\
\hline & 4.4 & 0.065 & 0.072 & 0.855 \\
\hline \multirow{4}{*}{5} & 5.1 & 0.117 & 0.13 & 0.410 \\
\hline & 5.2 & 0.061 & 0.068 & 0.636 \\
\hline & 5.3 & 0.064 & 0.071 & 0.807 \\
\hline & 5.4 & 0.047 & 0.052 & 1.354 \\
\hline \multirow{4}{*}{6} & 6.1 & 0.129 & 0.14 & 0.747 \\
\hline & 6.2 & 0.071 & 0.077 & 1.208 \\
\hline & 6.3 & 0.071 & 0.076 & 1.553 \\
\hline & 6.4 & 0.052 & 0.056 & 2.710 \\
\hline \multirow{4}{*}{7} & 7.1 & 0.146 & 0.157 & 1.450 \\
\hline & 7.2 & 0.079 & 0.085 & 2.324 \\
\hline & 7.3 & 0.077 & 0.082 & 2.957 \\
\hline & 7.4 & 0.054 & 0.057 & 5.360 \\
\hline
\end{tabular}


Appendix B.3: Computational outputs for examining EP2 model solvability and determining sample size and sample replications

(Cont.)

(a) with Uniformly Distributed Demand

\begin{tabular}{|c|c|c|c|c|}
\hline $\begin{array}{c}\text { TC } \\
\text { No. }\end{array}$ & $\begin{array}{c}\text { Problem } \\
\text { No. }\end{array}$ & $\begin{array}{c}\text { MeanG } \\
(\%)\end{array}$ & $\begin{array}{c}\text { CIU } \\
(\%)\end{array}$ & $\begin{array}{c}\text { CPU } \\
\text { (mins) }\end{array}$ \\
\hline \multirow{8}{*}{8} & 8.1 & 0.069 & 0.073 & 0.809 \\
& 8.2 & 0.037 & 0.039 & 1.298 \\
& 8.3 & 0.037 & 0.039 & 1.673 \\
& 8.4 & 0.026 & 0.028 & 2.858 \\
\hline \multirow{4}{*}{9} & 9.1 & 0.060 & 0.064 & 1.279 \\
& 9.2 & 0.032 & 0.034 & 1.990 \\
& 9.3 & 0.032 & 0.034 & 2.500 \\
& 9.4 & 0.023 & 0.024 & 4.323 \\
\hline \multirow{4}{*}{10} & 10.1 & 0.057 & 0.060 & 1.862 \\
& 10.2 & 0.030 & 0.032 & 2.933 \\
& 10.3 & 0.030 & 0.032 & 3.652 \\
& 10.4 & 0.022 & 0.023 & 6.352 \\
\hline
\end{tabular}

(b) with Normally Distributed Demand

\begin{tabular}{|c|c|c|c|c|}
\hline $\begin{array}{c}\text { TC } \\
\text { No. }\end{array}$ & $\begin{array}{c}\text { Problem } \\
\text { No. }\end{array}$ & $\begin{array}{c}\text { MeanG } \\
(\%)\end{array}$ & $\begin{array}{c}\text { CIU } \\
(\%)\end{array}$ & $\begin{array}{c}\text { CPU } \\
\text { (mins) }\end{array}$ \\
\hline & 8.1 & 0.122 & 0.132 & 1.674 \\
8 & 8.2 & 0.065 & 0.070 & 2.684 \\
& 8.3 & 0.065 & 0.069 & 3.404 \\
& 8.4 & 0.047 & 0.05 & 6.036 \\
\hline \multirow{4}{*}{9} & 9.1 & 0.104 & 0.111 & 2.399 \\
& 9.2 & 0.053 & 0.057 & 3.853 \\
& 9.3 & 0.053 & 0.056 & 4.885 \\
& 9.4 & 0.038 & 0.040 & 8.726 \\
\hline \multirow{4}{*}{10} & 10.1 & 0.106 & 0.113 & 3.496 \\
& 10.2 & 0.055 & 0.058 & 5.581 \\
& 10.3 & 0.055 & 0.058 & 7.078 \\
& 10.4 & 0.039 & 0.041 & 12.485 \\
\hline
\end{tabular}




\section{Appendix C}


Appendix C.1: Summary table of bonus points to offer with different levels of demand variability under EP1 Setting

(a) with Uniformly Distributed demand

\begin{tabular}{|c|c|c|c|c|}
\hline $\begin{array}{l}\text { TC } \\
\text { No. }\end{array}$ & DV & TQA & TBA & $\frac{\mathrm{TBA}}{\mathrm{TQA}}(\%)$ \\
\hline \multirow{5}{*}{1} & 0.05 & 45.00 & 3.43 & $7.61 \%$ \\
\hline & 0.10 & 45.00 & 3.10 & $6.89 \%$ \\
\hline & 0.15 & 45.00 & 2.78 & $6.17 \%$ \\
\hline & 0.20 & 45.00 & 2.45 & $5.45 \%$ \\
\hline & 0.25 & 45.00 & 2.13 & $4.73 \%$ \\
\hline \multirow{5}{*}{2} & 0.05 & 110.00 & 9.71 & $8.82 \%$ \\
\hline & 0.10 & 110.00 & 8.79 & $7.99 \%$ \\
\hline & 0.15 & 110.00 & 7.87 & $7.15 \%$ \\
\hline & 0.20 & 110.00 & 6.95 & $6.32 \%$ \\
\hline & 0.25 & 110.00 & 6.03 & $5.48 \%$ \\
\hline \multirow{5}{*}{3} & 0.05 & 222.50 & 19.13 & $8.60 \%$ \\
\hline & 0.10 & 222.50 & 17.31 & $7.78 \%$ \\
\hline & 0.15 & 222.50 & 15.50 & $6.97 \%$ \\
\hline & 0.20 & 222.50 & 13.69 & $6.15 \%$ \\
\hline & 0.25 & 222.50 & 11.88 & $5.34 \%$ \\
\hline \multirow{5}{*}{4} & 0.05 & 257.50 & 23.69 & $9.20 \%$ \\
\hline & 0.10 & 257.50 & 21.45 & $8.33 \%$ \\
\hline & 0.15 & 257.50 & 19.21 & $7.46 \%$ \\
\hline & 0.20 & 257.50 & 16.96 & $6.59 \%$ \\
\hline & 0.25 & 257.50 & 14.72 & $5.72 \%$ \\
\hline \multirow{5}{*}{5} & 0.05 & 675.00 & 59.95 & $8.88 \%$ \\
\hline & 0.10 & 675.00 & 54.27 & $8.04 \%$ \\
\hline & 0.15 & 675.00 & 48.59 & $7.20 \%$ \\
\hline & 0.20 & 675.00 & 42.91 & $6.36 \%$ \\
\hline & 0.25 & 675.00 & 37.24 & $5.52 \%$ \\
\hline \multirow{5}{*}{6} & 0.05 & 900.00 & 78.79 & $8.75 \%$ \\
\hline & 0.10 & 900.00 & 71.33 & $7.93 \%$ \\
\hline & 0.15 & 900.00 & 63.86 & $7.10 \%$ \\
\hline & 0.20 & 900.00 & 56.40 & $6.27 \%$ \\
\hline & 0.25 & 900.00 & 48.94 & $5.44 \%$ \\
\hline
\end{tabular}

(b) with Normally Distributed Demand

\begin{tabular}{|c|c|c|c|c|}
\hline $\begin{array}{l}\text { TC } \\
\text { No. }\end{array}$ & DV & TQA & TBA & $\frac{\mathrm{TBA}}{\mathrm{TQA}}(\%)$ \\
\hline \multirow{5}{*}{1} & 0.1 & 45.00 & 3.59 & $7.97 \%$ \\
\hline & 0.5 & 45.00 & 2.93 & $6.50 \%$ \\
\hline & 1.0 & 45.00 & 2.10 & $4.67 \%$ \\
\hline & 1.5 & 45.00 & 1.28 & $2.84 \%$ \\
\hline & 1.8 & 45.00 & 0.79 & $1.75 \%$ \\
\hline \multirow{5}{*}{2} & 0.1 & 110.00 & 10.12 & $9.20 \%$ \\
\hline & 0.5 & 110.00 & 8.09 & $7.35 \%$ \\
\hline & 1.0 & 110.00 & 5.55 & $5.04 \%$ \\
\hline & 1.5 & 110.00 & 3.11 & $2.82 \%$ \\
\hline & 1.8 & 110.00 & 1.98 & $1.80 \%$ \\
\hline \multirow{5}{*}{3} & 0.1 & 222.50 & 19.90 & $8.94 \%$ \\
\hline & 0.5 & 222.50 & 15.74 & $7.07 \%$ \\
\hline & 1.0 & 222.50 & 10.54 & $4.74 \%$ \\
\hline & 1.5 & 222.50 & 5.76 & $2.59 \%$ \\
\hline & 1.8 & 222.50 & 3.46 & $1.55 \%$ \\
\hline \multirow{5}{*}{4} & 0.1 & 257.50 & 24.57 & $9.54 \%$ \\
\hline & 0.5 & 257.50 & 19.08 & $7.41 \%$ \\
\hline & 1.0 & 257.50 & 12.22 & $4.75 \%$ \\
\hline & 1.5 & 257.50 & 6.29 & $2.44 \%$ \\
\hline & 1.8 & 257.50 & 3.90 & $1.51 \%$ \\
\hline \multirow{5}{*}{5} & 0.1 & 675.00 & 62.57 & $9.27 \%$ \\
\hline & 0.5 & 675.00 & 50.35 & $7.46 \%$ \\
\hline & 1.0 & 675.00 & 35.08 & $5.20 \%$ \\
\hline & 1.5 & 675.00 & 20.80 & $3.08 \%$ \\
\hline & 1.8 & 675.00 & 13.25 & $1.96 \%$ \\
\hline \multirow{5}{*}{6} & 0.1 & 900.00 & 82.11 & $9.12 \%$ \\
\hline & 0.5 & 900.00 & 65.55 & $7.28 \%$ \\
\hline & 1.0 & 900.00 & 44.85 & $4.98 \%$ \\
\hline & 1.5 & 900.00 & 25.52 & $2.84 \%$ \\
\hline & 1.8 & 900.00 & 15.94 & $1.77 \%$ \\
\hline
\end{tabular}


Appendix C.1: Summary Table of Bonus Points to Offer with Different Levels of Demand Variability under EP1 Setting

(Cont.)

(a) with Uniformly Distributed demand

\begin{tabular}{|c|c|c|c|c|}
\hline $\begin{array}{l}\text { TC } \\
\text { No. }\end{array}$ & DV & TQA & TBA & $\frac{\mathrm{TBA}}{\mathrm{TQA}}(\%)$ \\
\hline \multirow{5}{*}{7} & 0.05 & 1172.50 & 104.19 & $8.89 \%$ \\
\hline & 0.10 & 1172.50 & 94.33 & $8.04 \%$ \\
\hline & 0.15 & 1172.50 & 84.46 & $7.20 \%$ \\
\hline & 0.20 & 1172.50 & 74.59 & $6.36 \%$ \\
\hline & 0.25 & 1172.50 & 64.72 & $5.52 \%$ \\
\hline \multirow{5}{*}{8} & 0.05 & 1730.00 & 148.44 & $8.58 \%$ \\
\hline & 0.10 & 1730.00 & 134.38 & $7.77 \%$ \\
\hline & 0.15 & 1730.00 & 120.32 & $6.95 \%$ \\
\hline & 0.20 & 1730.00 & 106.26 & $6.14 \%$ \\
\hline & 0.25 & 1730.00 & 92.20 & $5.33 \%$ \\
\hline \multirow{5}{*}{9} & 0.05 & 1770.00 & 154.15 & $8.71 \%$ \\
\hline & 0.10 & 1770.00 & 139.55 & $7.88 \%$ \\
\hline & 0.15 & 1770.00 & 124.95 & $7.06 \%$ \\
\hline & 0.20 & 1770.00 & 110.35 & $6.23 \%$ \\
\hline & 0.25 & 1770.00 & 95.75 & $5.41 \%$ \\
\hline \multirow{5}{*}{10} & 0.05 & 1997.50 & 177.84 & $8.90 \%$ \\
\hline & 0.10 & 1997.50 & 161.00 & $8.06 \%$ \\
\hline & 0.15 & 1997.50 & 144.16 & $7.22 \%$ \\
\hline & 0.20 & 1997.50 & 127.31 & $6.37 \%$ \\
\hline & 0.25 & 1997.50 & 110.47 & $5.53 \%$ \\
\hline
\end{tabular}

(b) with Normally Distributed Demand

\begin{tabular}{|c|c|c|c|c|}
\hline $\begin{array}{l}\text { TC } \\
\text { No. }\end{array}$ & DV & TQA & TBA & $\frac{\mathrm{TBA}}{\mathrm{TQA}}(\%)$ \\
\hline \multirow{5}{*}{7} & 0.1 & 1172.50 & 108.55 & $9.26 \%$ \\
\hline & 0.5 & 1172.50 & 86.51 & $7.38 \%$ \\
\hline & 1.0 & 1172.50 & 58.95 & $5.03 \%$ \\
\hline & 1.5 & 1172.50 & 33.19 & $2.83 \%$ \\
\hline & 1.8 & 1172.50 & 20.67 & $1.76 \%$ \\
\hline \multirow{5}{*}{8} & 0.1 & 1730.00 & 154.82 & $8.95 \%$ \\
\hline & 0.5 & 1730.00 & 124.12 & $7.17 \%$ \\
\hline & 1.0 & 1730.00 & 85.74 & $4.96 \%$ \\
\hline & 1.5 & 1730.00 & 49.60 & $2.87 \%$ \\
\hline & 1.8 & 1730.00 & 30.87 & $1.78 \%$ \\
\hline \multirow{5}{*}{9} & 0.1 & 1770.00 & 160.49 & $9.07 \%$ \\
\hline & 0.5 & 1770.00 & 127.43 & $7.20 \%$ \\
\hline & 1.0 & 1770.00 & 86.11 & $4.87 \%$ \\
\hline & 1.5 & 1770.00 & 47.62 & $2.69 \%$ \\
\hline & 1.8 & 1770.00 & 29.74 & $1.68 \%$ \\
\hline \multirow{5}{*}{10} & 0.1 & 1997.50 & 185.27 & $9.28 \%$ \\
\hline & 0.5 & 1997.50 & 147.61 & $7.39 \%$ \\
\hline & 1.0 & 1997.50 & 100.54 & $5.03 \%$ \\
\hline & 1.5 & 1997.50 & 56.76 & $2.84 \%$ \\
\hline & 1.8 & 1997.50 & 35.69 & $1.79 \%$ \\
\hline
\end{tabular}


Appendix C.2: Summary table of options to purchase and to exercise with different demand variability under EP2 Setting

(a) with Uniformly Distributed Demand

\begin{tabular}{|c|c|c|c|c|c|c|}
\hline $\begin{array}{l}\mathrm{TC} \\
\text { No. }\end{array}$ & DV & TQR & TOR & TOE & $\frac{\mathrm{TOR}}{\mathrm{TQR}}(\%)$ & $\frac{\mathrm{TOE}}{\mathrm{TOR}}(\%)$ \\
\hline \multirow{5}{*}{1} & 0.05 & 46.22 & 2.44 & 0.46 & $5.28 \%$ & $18.93 \%$ \\
\hline & 0.10 & 47.44 & 4.88 & 0.92 & $10.29 \%$ & $18.91 \%$ \\
\hline & 0.15 & 48.66 & 7.32 & 1.39 & $15.04 \%$ & $18.92 \%$ \\
\hline & 0.20 & 49.88 & 9.76 & 1.85 & $19.57 \%$ & $18.91 \%$ \\
\hline & 0.25 & 51.10 & 12.20 & 2.31 & $23.88 \%$ & $18.92 \%$ \\
\hline \multirow{5}{*}{2} & 0.05 & 79.70 & 4.00 & 0.75 & $5.02 \%$ & $18.85 \%$ \\
\hline & 0.10 & 81.90 & 8.00 & 1.51 & $9.77 \%$ & $18.85 \%$ \\
\hline & 0.15 & 83.98 & 12.12 & 2.30 & $14.43 \%$ & $18.98 \%$ \\
\hline & 0.20 & 85.94 & 16.26 & 3.13 & $18.92 \%$ & $19.27 \%$ \\
\hline & 0.25 & 87.92 & 19.88 & 3.91 & $22.61 \%$ & $19.65 \%$ \\
\hline \multirow{5}{*}{3} & 0.05 & 167.07 & 8.11 & 1.58 & $4.86 \%$ & $19.44 \%$ \\
\hline & 0.10 & 171.61 & 16.25 & 3.17 & $9.47 \%$ & $19.49 \%$ \\
\hline & 0.15 & 176.12 & 24.14 & 4.75 & $13.70 \%$ & $19.67 \%$ \\
\hline & 0.20 & 180.12 & 32.01 & 6.43 & $17.77 \%$ & $20.07 \%$ \\
\hline & 0.25 & 182.07 & 38.41 & 8.40 & $21.10 \%$ & $21.88 \%$ \\
\hline \multirow{5}{*}{4} & 0.05 & 511.09 & 26.51 & 5.03 & $5.19 \%$ & $18.97 \%$ \\
\hline & 0.10 & 523.98 & 53.71 & 10.35 & $10.25 \%$ & $19.26 \%$ \\
\hline & 0.15 & 536.60 & 79.77 & 15.70 & $14.87 \%$ & $19.68 \%$ \\
\hline & 0.20 & 548.55 & 103.43 & 20.85 & $18.86 \%$ & $20.16 \%$ \\
\hline & 0.25 & 562.01 & 121.79 & 24.91 & $21.67 \%$ & $20.45 \%$ \\
\hline
\end{tabular}

(b) with Normally Distributed Demand

\begin{tabular}{|c|c|c|c|c|c|c|}
\hline $\begin{array}{c}\text { TC } \\
\text { No. }\end{array}$ & DV & TQR & TOR & TOE & $\frac{\text { TOR }}{\text { TQR }}(\%)$ & $\frac{\text { TOE }}{\text { TOR }}(\%)$ \\
\hline \multirow{5}{*}{1} & 0.1 & 45.25 & 0.99 & 0.13 & $2.19 \%$ & $13.29 \%$ \\
\cline { 2 - 7 } & 0.5 & 46.23 & 4.97 & 0.66 & $10.74 \%$ & $13.27 \%$ \\
\cline { 2 - 7 } & 1.0 & 47.47 & 9.93 & 1.32 & $20.92 \%$ & $13.27 \%$ \\
\cline { 2 - 7 } & 1.5 & 49.66 & 13.33 & 1.66 & $26.85 \%$ & $12.42 \%$ \\
\cline { 2 - 7 } & 1.8 & 52.51 & 13.44 & 1.46 & $25.60 \%$ & $10.85 \%$ \\
\hline \multirow{5}{*}{2} & 0.1 & 77.95 & 1.50 & 0.22 & $1.92 \%$ & $14.56 \%$ \\
\cline { 2 - 7 } & 0.5 & 79.76 & 7.49 & 1.09 & $9.38 \%$ & $14.58 \%$ \\
\cline { 2 - 7 } & 1.0 & 81.96 & 14.68 & 2.18 & $17.92 \%$ & $14.87 \%$ \\
\cline { 2 - 7 } & 1.5 & 84.20 & 20.59 & 3.16 & $24.45 \%$ & $15.32 \%$ \\
\cline { 2 - 7 } & 1.8 & 86.97 & 21.76 & 3.25 & $25.01 \%$ & $14.96 \%$ \\
\hline \multirow{5}{*}{3} & 0.1 & 163.43 & 2.90 & 0.47 & $1.78 \%$ & $16.09 \%$ \\
\cline { 2 - 7 } & 0.5 & 167.02 & 14.61 & 2.38 & $8.75 \%$ & $16.29 \%$ \\
\cline { 2 - 7 } & 1.0 & 171.67 & 26.81 & 4.59 & $15.62 \%$ & $17.11 \%$ \\
\hline & 1.5 & 177.99 & 35.51 & 6.10 & $19.95 \%$ & $17.18 \%$ \\
\hline & 1.8 & 182.48 & 38.01 & 6.64 & $20.83 \%$ & $17.47 \%$ \\
\hline \multirow{5}{*}{4} & 0.1 & 500.43 & 9.77 & 1.40 & $1.95 \%$ & $14.29 \%$ \\
\hline & 0.5 & 511.41 & 49.60 & 7.26 & $9.70 \%$ & $14.63 \%$ \\
\hline & 1.0 & 525.07 & 95.75 & 14.55 & $18.24 \%$ & $15.20 \%$ \\
\hline & 1.5 & 546.97 & 123.80 & 18.82 & $22.63 \%$ & $15.20 \%$ \\
\hline \multirow{4}{*}{1.8} & 565.11 & 127.63 & 19.53 & $22.58 \%$ & $15.30 \%$ \\
\hline
\end{tabular}


Appendix C.2: Summary table of options to purchase and to exercise with different demand variability under EP2 setting (Cont.)

(a) with Uniformly Distributed Demand

\begin{tabular}{|c|c|c|c|c|c|c|}
\hline $\begin{array}{l}\mathrm{TC} \\
\text { No. }\end{array}$ & DV & TQR & TOR & TOE & $\frac{\mathrm{TOR}}{\mathrm{TQR}}(\%)$ & $\frac{\mathrm{TOE}}{\mathrm{TOR}}(\%)$ \\
\hline \multirow{5}{*}{5} & 0.05 & 546.98 & 27.60 & 5.38 & $5.04 \%$ & $19.51 \%$ \\
\hline & 0.10 & 560.81 & 55.84 & 11.02 & $9.96 \%$ & $19.74 \%$ \\
\hline & 0.15 & 573.75 & 83.42 & 16.89 & $14.54 \%$ & $20.24 \%$ \\
\hline & 0.20 & 577.52 & 114.64 & 25.28 & $19.85 \%$ & $22.05 \%$ \\
\hline & 0.25 & 574.67 & 128.14 & 33.11 & $22.30 \%$ & $25.84 \%$ \\
\hline \multirow{5}{*}{6} & 0.05 & 936.22 & 45.18 & 8.56 & $4.83 \%$ & $18.95 \%$ \\
\hline & 0.10 & 961.26 & 91.53 & 17.54 & $9.52 \%$ & $19.16 \%$ \\
\hline & 0.15 & 985.13 & 138.78 & 26.97 & $14.09 \%$ & $19.43 \%$ \\
\hline & 0.20 & 1008.16 & 181.51 & 36.24 & $18.00 \%$ & $19.97 \%$ \\
\hline & 0.25 & 1032.78 & 213.43 & 43.87 & $20.67 \%$ & $20.55 \%$ \\
\hline \multirow{5}{*}{7} & 0.05 & 1504.32 & 71.99 & 13.88 & $4.79 \%$ & $19.27 \%$ \\
\hline & 0.10 & 1542.71 & 147.41 & 29.06 & $9.56 \%$ & $19.71 \%$ \\
\hline & 0.15 & 1578.86 & 224.55 & 45.14 & $14.22 \%$ & $20.10 \%$ \\
\hline & 0.20 & 1614.46 & 288.62 & 60.11 & $17.88 \%$ & $20.83 \%$ \\
\hline & 0.25 & 1653.52 & 343.11 & 72.31 & $20.75 \%$ & $21.08 \%$ \\
\hline \multirow{5}{*}{8} & 0.05 & 1495.61 & 73.40 & 14.25 & $4.91 \%$ & $19.42 \%$ \\
\hline & 0.10 & 1533.67 & 149.35 & 29.47 & $9.74 \%$ & $19.73 \%$ \\
\hline & 0.15 & 1569.13 & 226.24 & 45.59 & $14.42 \%$ & $20.15 \%$ \\
\hline & 0.20 & 1602.70 & 293.30 & 61.10 & $18.30 \%$ & $20.83 \%$ \\
\hline & 0.25 & 1642.94 & 341.60 & 72.32 & $20.79 \%$ & $21.17 \%$ \\
\hline
\end{tabular}

(b) with Normally Distributed Demand

\begin{tabular}{|c|c|c|c|c|c|c|}
\hline $\begin{array}{c}\text { TC } \\
\text { No. }\end{array}$ & DV & TQR & TOR & TOE & $\frac{\text { TOR }}{\text { TQR }}(\%)$ & $\frac{\text { TOE }}{\text { TOR }}(\%)$ \\
\hline \multirow{5}{*}{5} & 0.1 & 535.62 & 9.90 & 1.51 & $1.85 \%$ & $15.27 \%$ \\
\cline { 2 - 7 } & 0.5 & 547.37 & 50.26 & 7.87 & $9.18 \%$ & $15.65 \%$ \\
\cline { 2 - 7 } & 1.0 & 562.56 & 94.88 & 15.42 & $16.87 \%$ & $16.25 \%$ \\
\cline { 2 - 7 } & 1.5 & 577.37 & 123.40 & 21.86 & $21.37 \%$ & $17.72 \%$ \\
\cline { 2 - 7 } & 1.8 & 579.63 & 127.44 & 26.08 & $21.99 \%$ & $20.46 \%$ \\
\hline \multirow{5}{*}{6} & 0.1 & 915.12 & 15.53 & 2.43 & $1.70 \%$ & $15.64 \%$ \\
\cline { 2 - 7 } & 0.5 & 935.44 & 77.75 & 12.20 & $8.31 \%$ & $15.69 \%$ \\
\cline { 2 - 7 } & 1.0 & 960.92 & 153.73 & 24.31 & $16.00 \%$ & $15.81 \%$ \\
\cline { 2 - 7 } & 1.5 & 993.54 & 210.73 & 33.77 & $21.21 \%$ & $16.03 \%$ \\
\cline { 2 - 7 } & 1.8 & 1020.64 & 224.80 & 36.39 & $22.03 \%$ & $16.19 \%$ \\
\hline \multirow{5}{*}{7} & 0.1 & 1471.21 & 25.68 & 4.04 & $1.75 \%$ & $15.75 \%$ \\
\cline { 2 - 7 } & 0.5 & 1505.31 & 129.12 & 20.47 & $8.58 \%$ & $15.86 \%$ \\
\cline { 2 - 7 } & 1.0 & 1546.43 & 254.67 & 41.47 & $16.47 \%$ & $16.28 \%$ \\
\hline & 1.5 & 1598.21 & 342.65 & 57.66 & $21.44 \%$ & $16.83 \%$ \\
\hline & 1.8 & 1643.63 & 363.96 & 61.78 & $22.14 \%$ & $16.97 \%$ \\
\hline \multirow{5}{*}{8.1} & 1463.51 & 26.01 & 4.11 & $1.78 \%$ & $15.79 \%$ \\
\hline & 0.5 & 1496.85 & 130.73 & 20.79 & $8.73 \%$ & $15.90 \%$ \\
\hline & 1.5 & 1595.02 & 335.66 & 55.98 & $21.04 \%$ & $16.68 \%$ \\
\hline & 1.8 & 1636.92 & 354.99 & 60.74 & $21.69 \%$ & $17.11 \%$ \\
\hline
\end{tabular}


Appendix C.2: Summary table of options to purchase and to exercise with different demand variability under EP2 setting (Cont.)

(a) with Uniformly Distributed Demand

\begin{tabular}{|c|c|c|c|c|c|c|}
\hline $\begin{array}{c}\text { TC } \\
\text { No. }\end{array}$ & DV & TQR & TOR & TOE & $\frac{\text { TOR }}{\text { TQR }}(\%)$ & $\frac{\text { TOE }}{\text { TOR }}(\%)$ \\
\hline \multirow{4}{*}{9} & 0.05 & 2075.51 & 103.35 & 20.10 & $4.98 \%$ & $19.45 \%$ \\
\cline { 2 - 7 } & 0.10 & 2126.52 & 211.20 & 41.92 & $9.93 \%$ & $19.85 \%$ \\
\cline { 2 - 7 } & 0.15 & 2170.88 & 322.80 & 66.09 & $14.87 \%$ & $20.47 \%$ \\
\cline { 2 - 7 } & 0.20 & 2211.25 & 410.88 & 89.05 & $18.58 \%$ & $21.67 \%$ \\
\cline { 2 - 7 } & 0.25 & 2235.72 & 481.14 & 112.26 & $21.52 \%$ & $23.33 \%$ \\
\hline \multirow{4}{*}{10} & 0.05 & 2383.81 & 119.59 & 23.16 & $5.02 \%$ & $19.36 \%$ \\
\cline { 2 - 7 } & 0.10 & 2442.07 & 244.74 & 48.42 & $10.02 \%$ & $19.79 \%$ \\
\cline { 2 - 7 } & 0.15 & 2493.27 & 372.71 & 76.07 & $14.95 \%$ & $20.41 \%$ \\
\cline { 2 - 7 } & 0.20 & 2540.43 & 472.51 & 102.07 & $18.60 \%$ & $21.60 \%$ \\
\cline { 2 - 7 } & 0.25 & 2583.61 & 543.53 & 124.19 & $21.04 \%$ & $22.85 \%$ \\
\hline
\end{tabular}

(b) with Normally Distributed Demand

\begin{tabular}{|c|c|c|c|c|c|c|}
\hline $\begin{array}{c}\text { TC } \\
\text { No. }\end{array}$ & DV & TQR & TOR & TOE & $\frac{\text { TOR }}{\text { TQR }}(\%)$ & $\frac{\text { TOE }}{\text { TOR }}(\%)$ \\
\hline \multirow{4}{*}{9} & 0.1 & 2031.57 & 35.00 & 5.47 & $1.72 \%$ & $15.62 \%$ \\
\cline { 2 - 7 } & 0.5 & 2075.79 & 177.04 & 28.13 & $8.53 \%$ & $15.89 \%$ \\
\cline { 2 - 7 } & 1.0 & 2129.22 & 348.82 & 56.96 & $16.38 \%$ & $16.33 \%$ \\
\cline { 2 - 7 } & 1.5 & 2188.31 & 460.90 & 80.83 & $21.06 \%$ & $17.54 \%$ \\
\cline { 2 - 7 } & 1.8 & 2235.09 & 484.77 & 88.70 & $21.69 \%$ & $18.30 \%$ \\
\hline \multirow{4}{*}{10} & 0.1 & 2333.11 & 41.14 & 6.34 & $1.76 \%$ & $15.42 \%$ \\
\cline { 2 - 7 } & 0.5 & 2383.29 & 207.93 & 32.60 & $8.72 \%$ & $15.68 \%$ \\
\cline { 2 - 7 } & 1.0 & 2443.82 & 409.45 & 65.93 & $16.75 \%$ & $16.10 \%$ \\
\cline { 2 - 7 } & 1.5 & 2524.06 & 523.59 & 89.61 & $20.74 \%$ & $17.12 \%$ \\
\cline { 2 - 7 } & 1.8 & 2578.33 & 551.69 & 99.18 & $21.40 \%$ & $17.98 \%$ \\
\hline
\end{tabular}


Appendix C.3: LRP profitability comparisons across BP, EP1, and EP2

(a) with Uniformly Distributed Demand

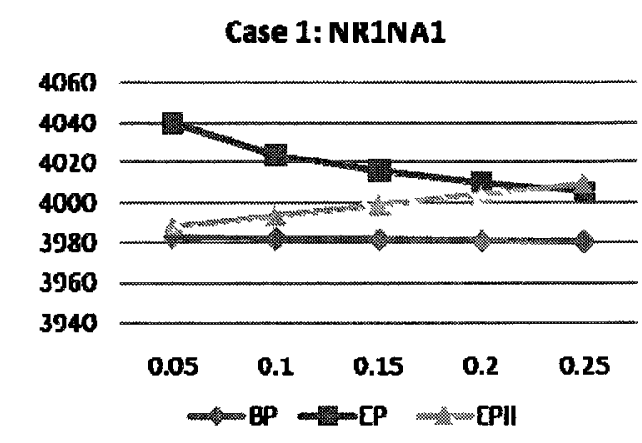

Case 2: NR3NAS

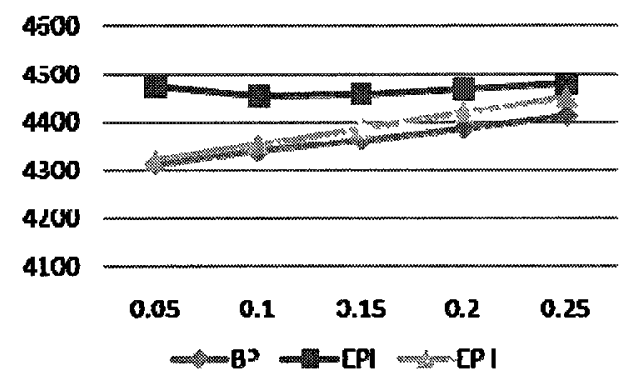

Case 3: NR1ONA10

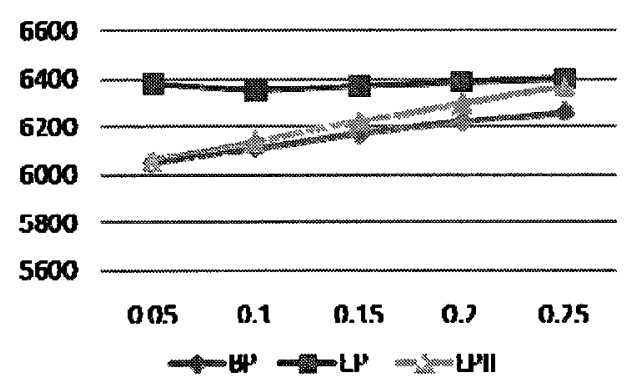

Case 4: NR2ONA15

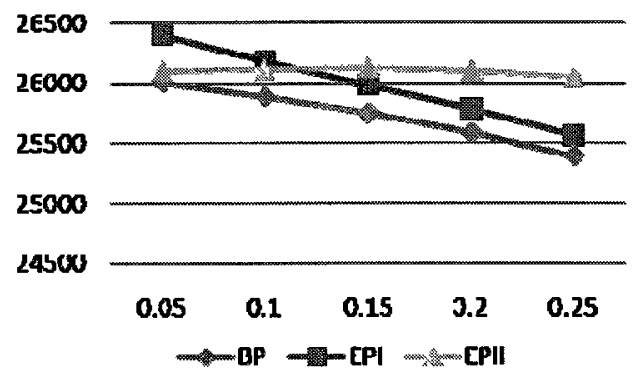

(b) with Normally Distributed Demand

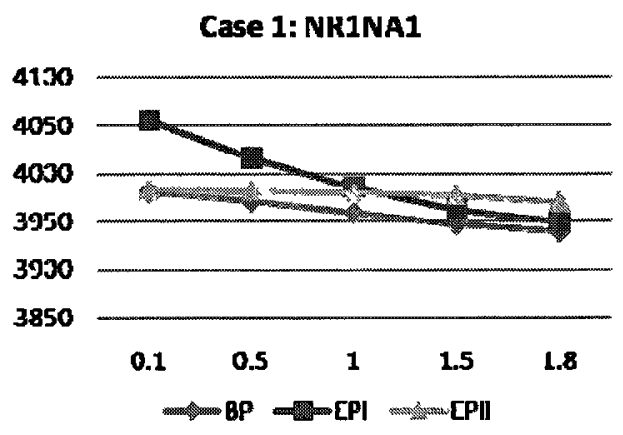

Case 2: NR3NA5

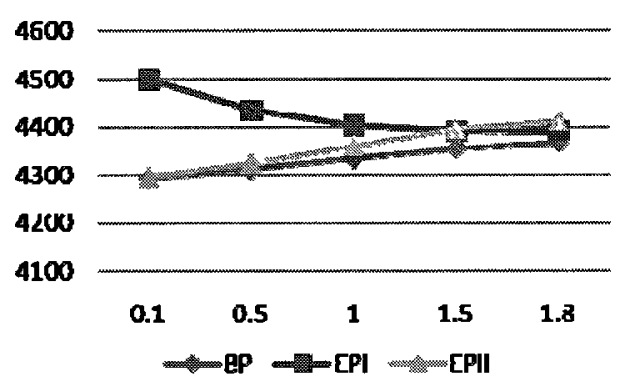

Case 3: NR1ONA10
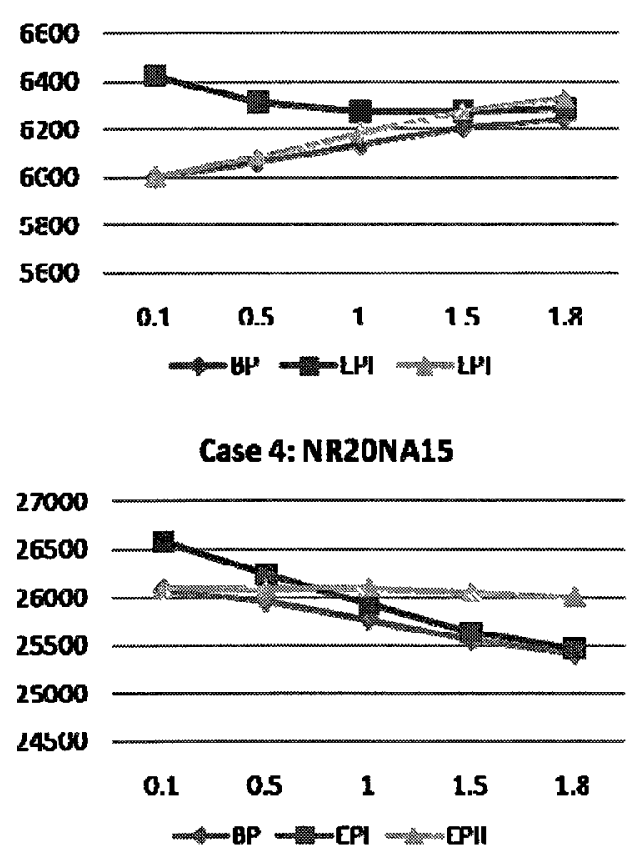
Appendix C.3: LRP profitability comparisons across BP, EP1, and EP2 (Cont.)

(a) with Uniformly Distributed Demand

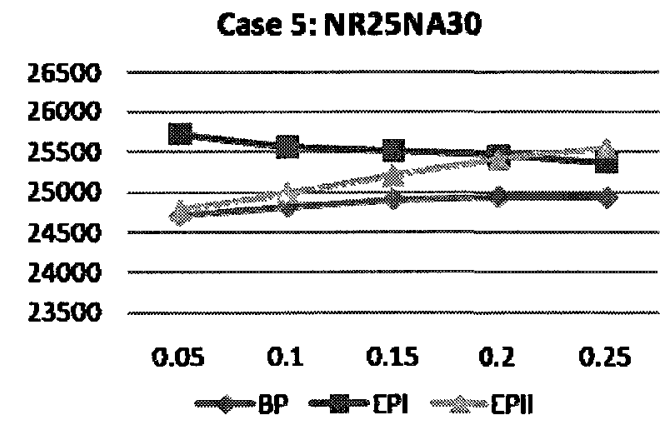

Case 6: NR4ONA40

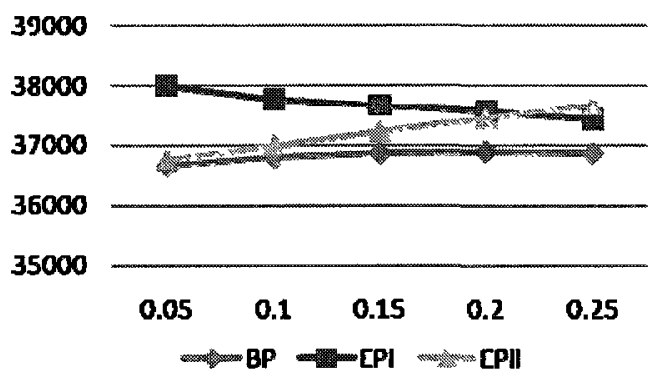

\section{Case 7: NR60NA55}

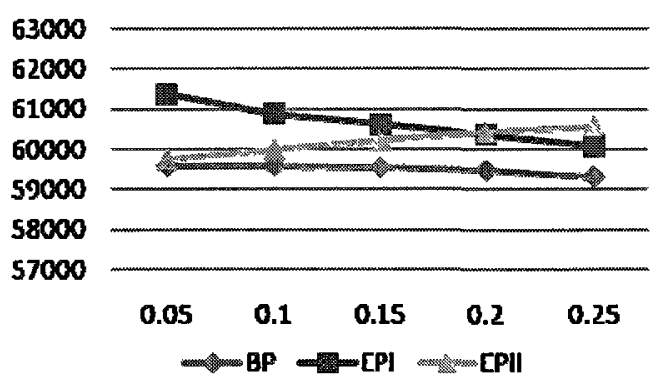

Case 8: NR65NA70

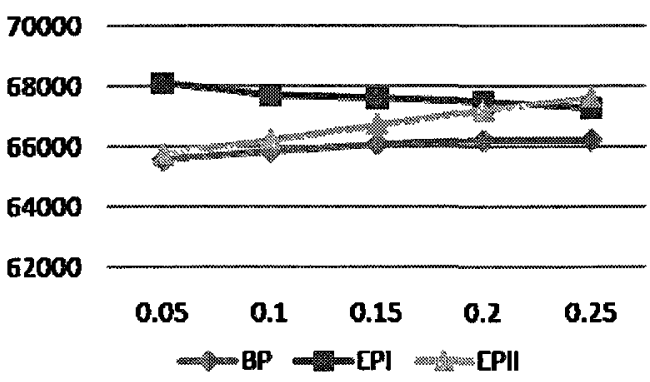

(b) with Normally Distributed Demand
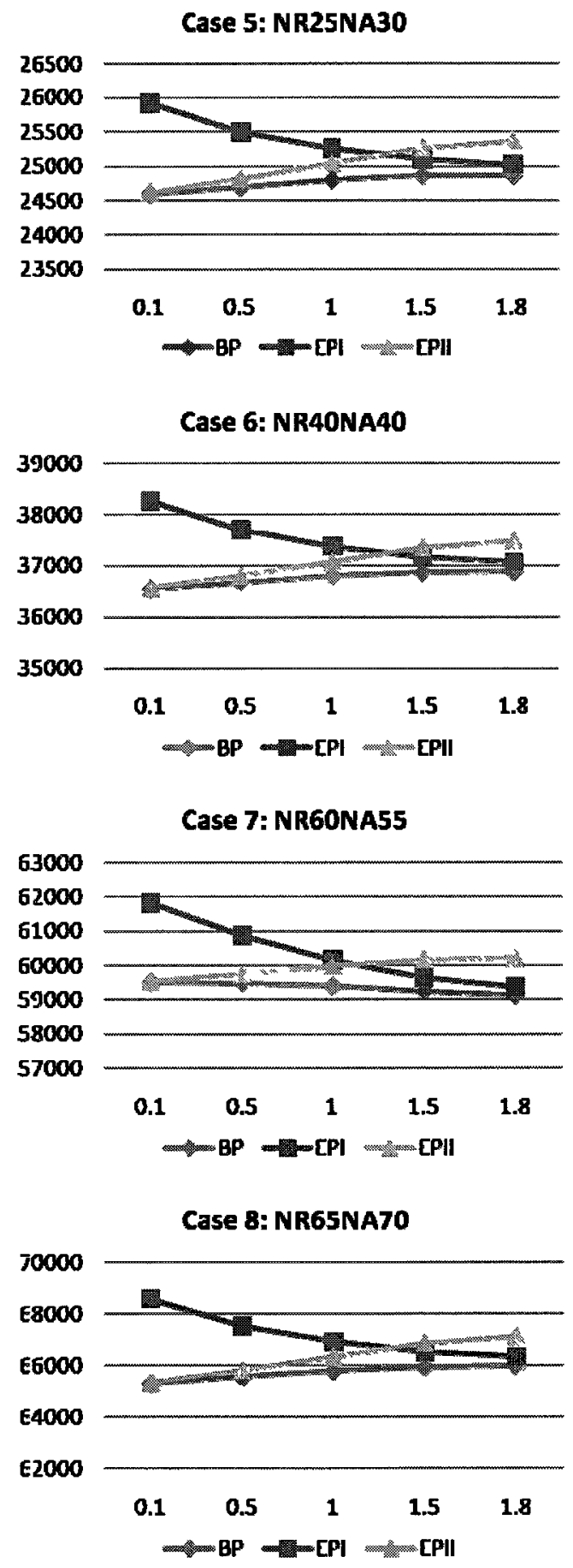
Appendix C.3: LRP profitability comparisons across BP, EP1, and EP2 (Cont.)

(a) with Uniformly Distributed Demand
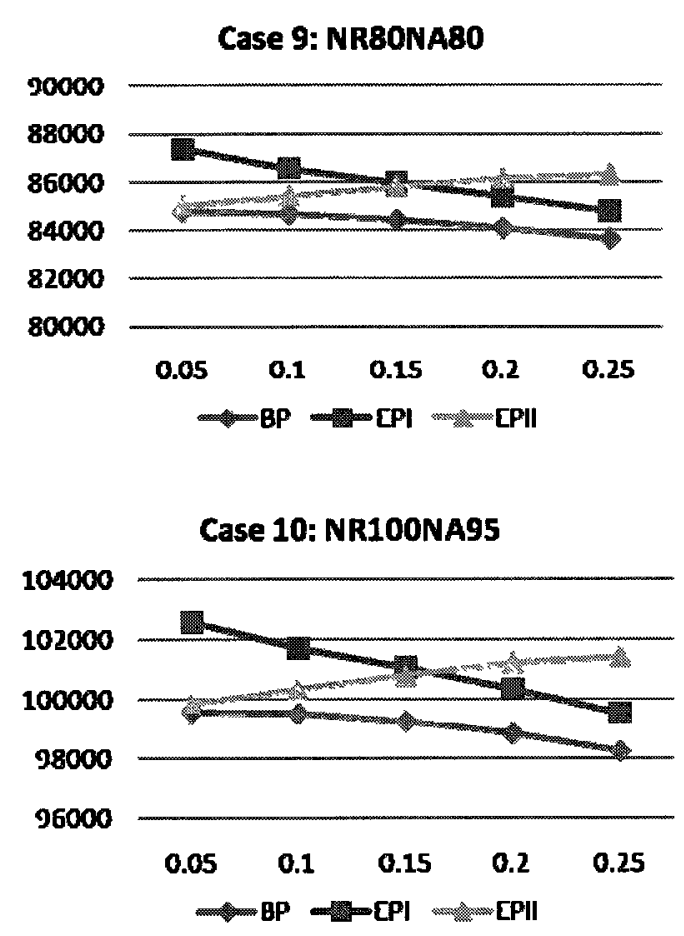

(b) with Normally Distributed Demand
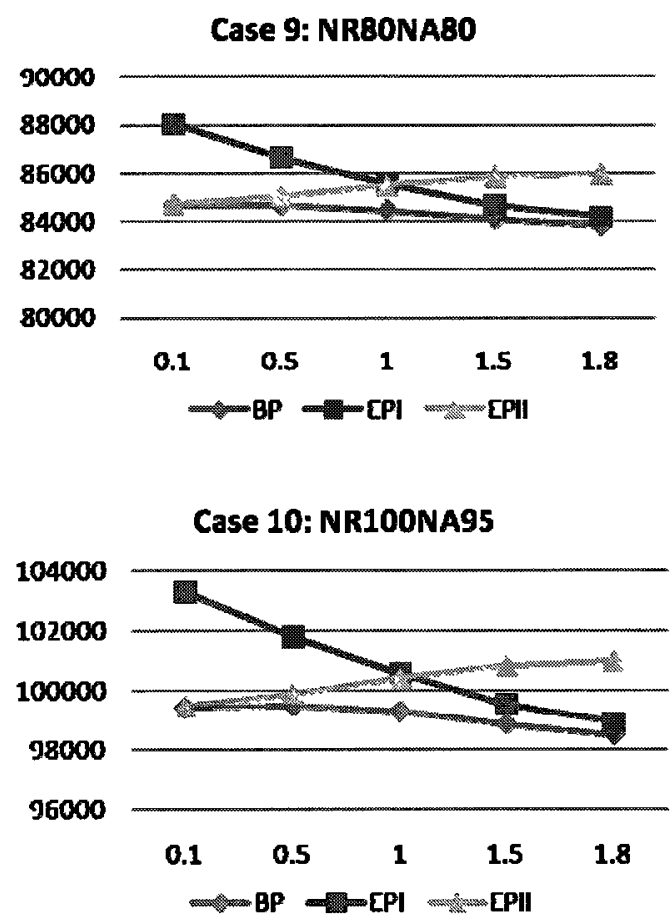
Appendix C.4: Liability ratio comparisons across BP, EP1, and EP2

(a) with Uniformly Distributed Demand

Case1: NR1NA1

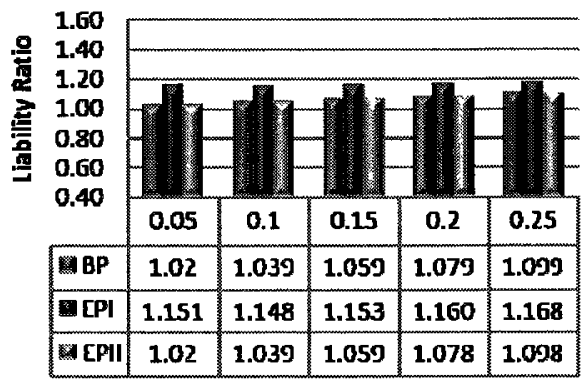

Case2: NR3NA5

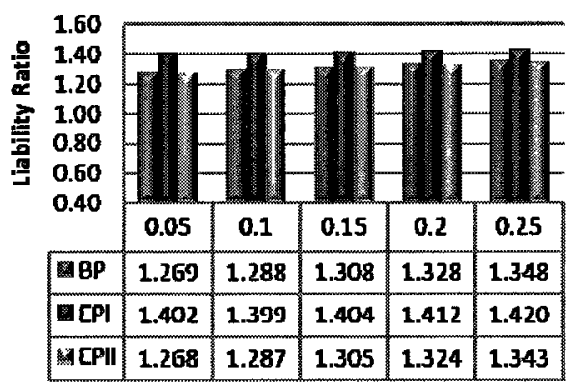

\section{Case3: NR1ONA10}

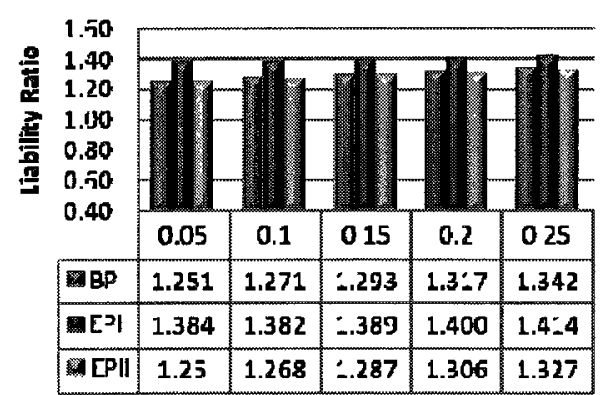

Case4: NR2ONA15

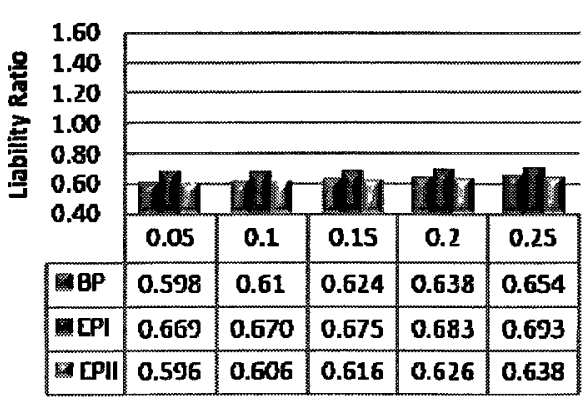

(b) with Normally Distributed Demand

Case1: NR1NA1

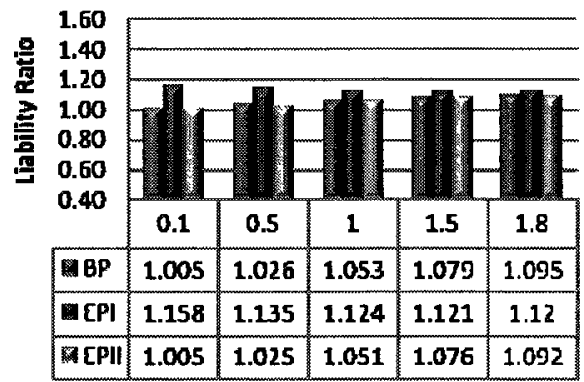

Case2: NR3NA5

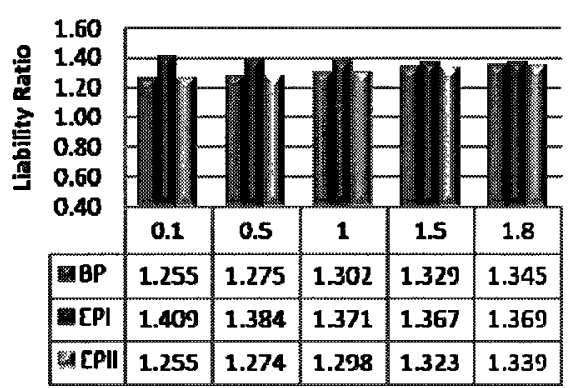

Case3: NR1ONA10

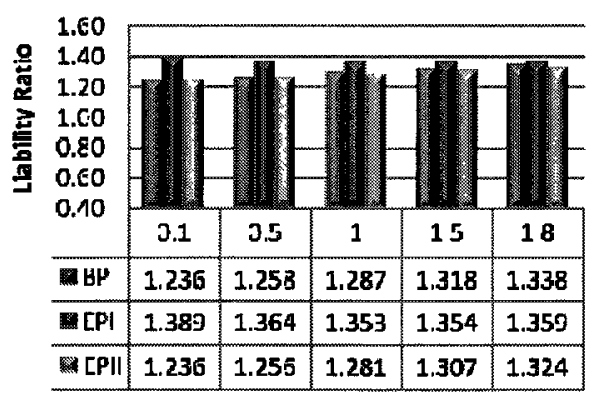

Case4: NR2ONA15

\begin{tabular}{|c|c|c|c|c|c|}
\hline & & & & & \\
\hline $\begin{array}{l}1.00 \\
1.40\end{array}$ & & & & & \\
\hline $\begin{array}{l}0.80 \\
0.60\end{array}$ & 28 & & & & \\
\hline & 0.1 & 0.5 & 1 & 1.5 & 1.8 \\
\hline BPP & 0.589 & 0.603 & 0.621 & 0.641 & 0.654 \\
\hline If & 0.671 & 0.659 & 0.655 & 0.658 & 0.664 \\
\hline [PII & 0.589 & 0.6 & 0.614 & 0.629 & 0.638 \\
\hline
\end{tabular}


Appendix C.4: Liability ratio comparisons across BP, EP1, and EP2

(Cont.)

(a) with Uniformly Distributed Demand

Case5: NR25NA30

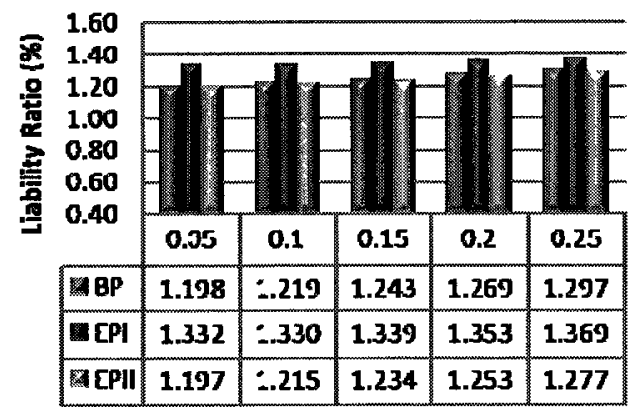

Case6: NR40NA40

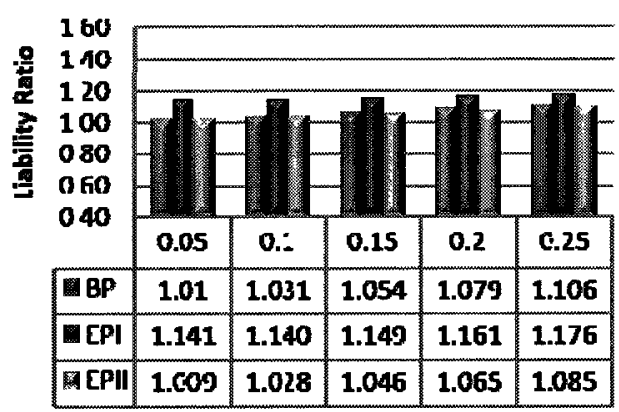

Case7: NR60NA55

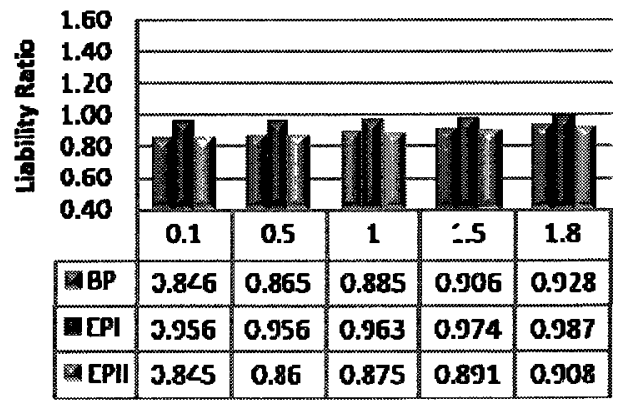

(b) with Normally Distributed Demand

Case5: NR25NA30

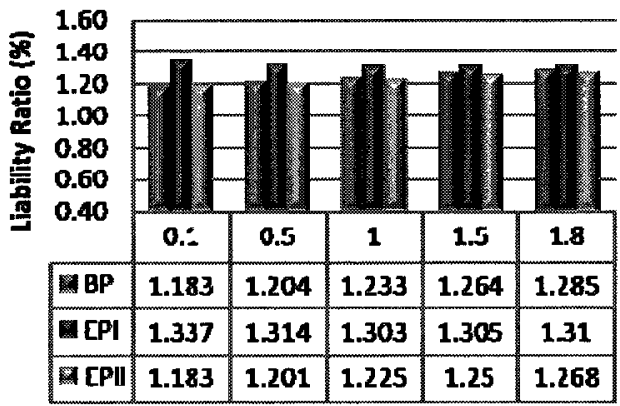

Case6: NR40NA40

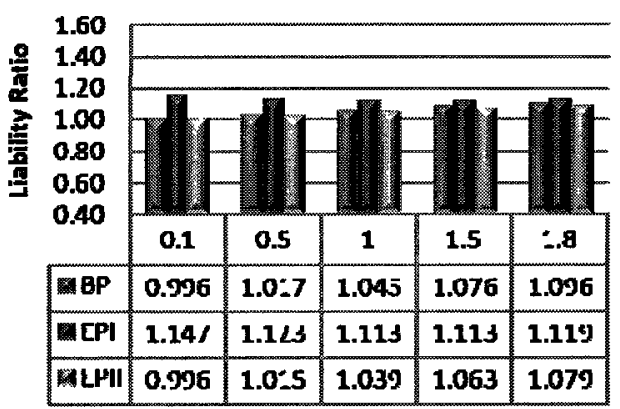

\section{Case7: NR60NA55}

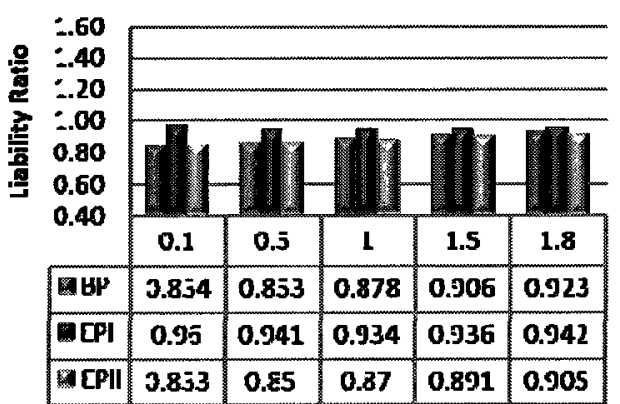


Appendix C.4: Liability ratio comparisons across BP, EP1, and EP2

(Cont.)

(a) with Uniformly Distributed Demand

Case8: NR65NA70

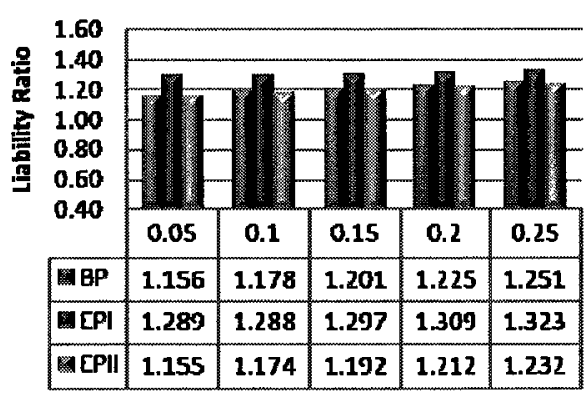

Case9: NR80NA80

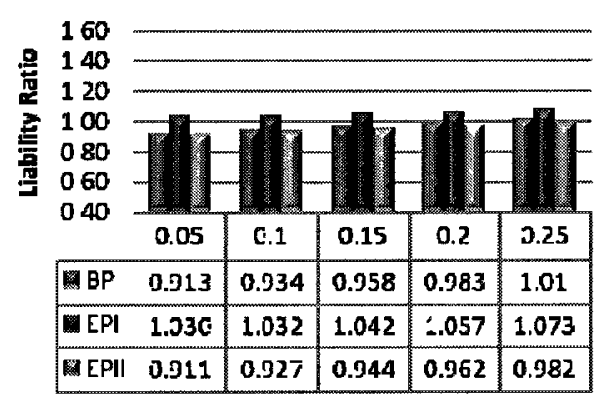

\section{Case10: NR100NA95}

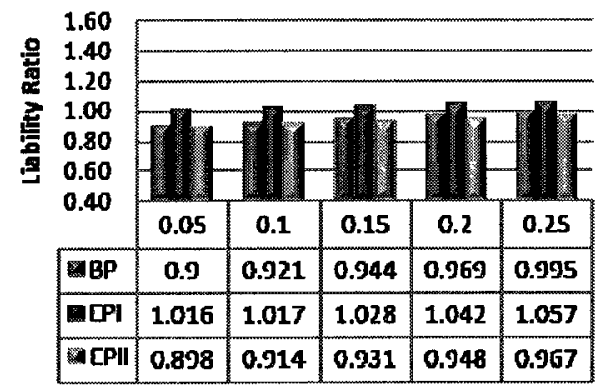

(b) with Normally Distributed Demand

Case8: NR65NA70

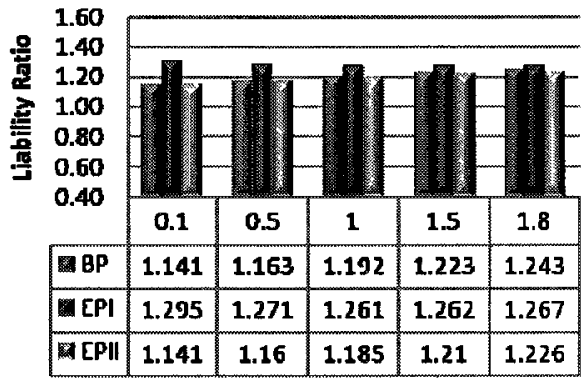

Case9: NR80NA80

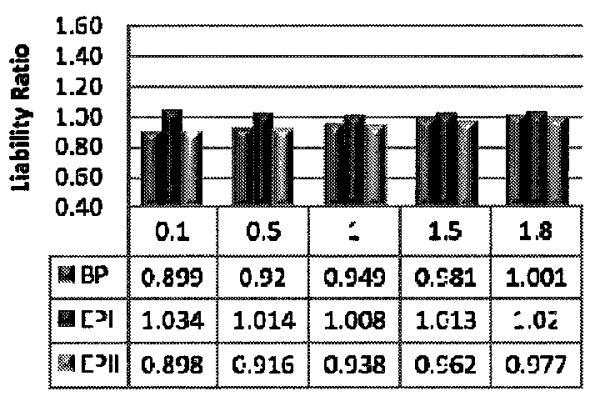

Case10: NR100NA95

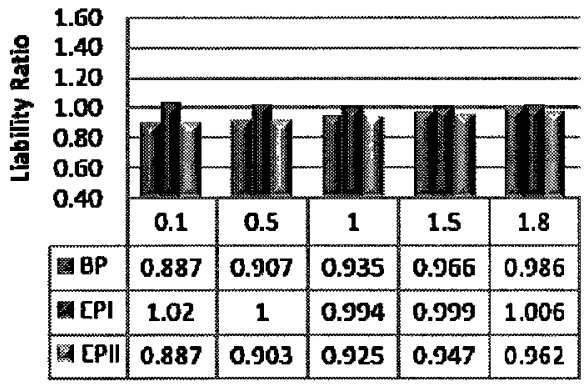


Appendix C.5: Budget usage comparisons across BP, EP1, and EP2

(a) with Uniformly Distributed Demand

Case1: NR1NA1

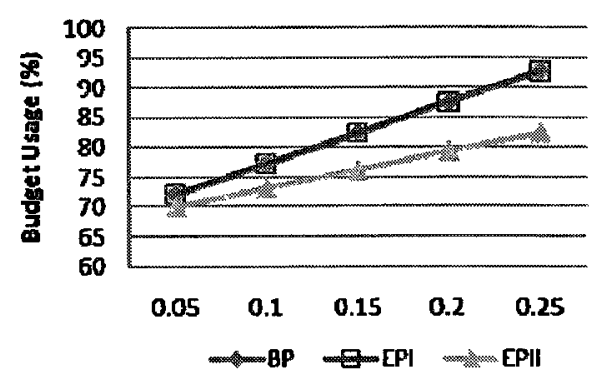

Case2: NR3NA5

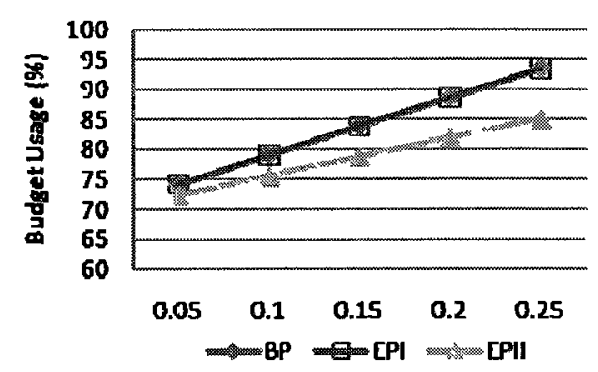

Case3: NR1ONA10

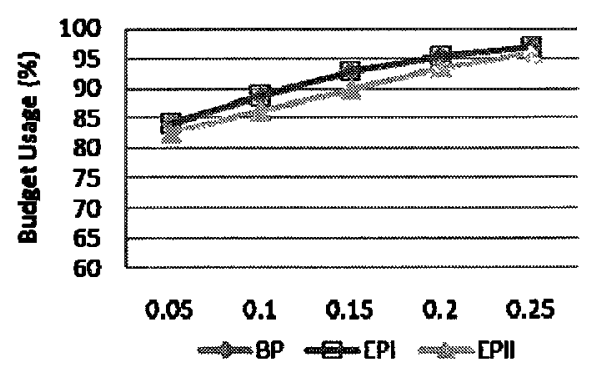

Case4: NR2ONA15

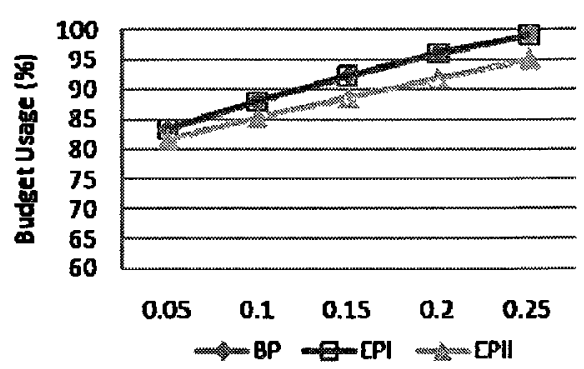

(b) with Normally Distributed Demand

Case1: NR1NA1

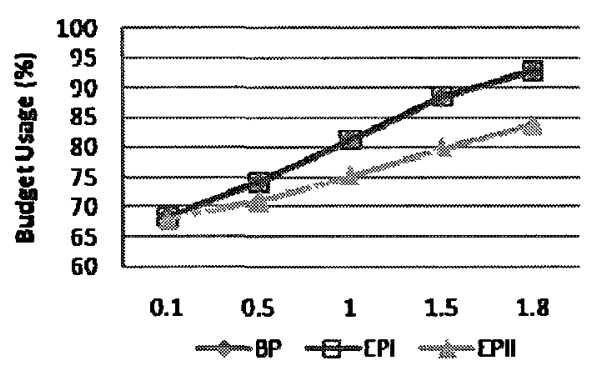

Case2: NR3NA5

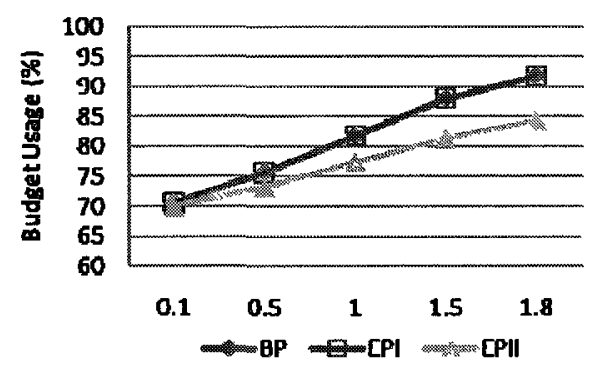

Case3: NR1ONA10

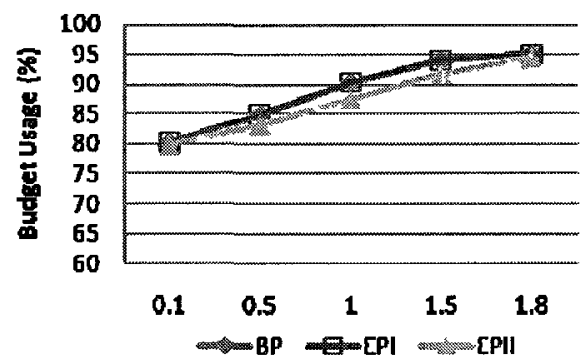

Case4: NR2ONA15

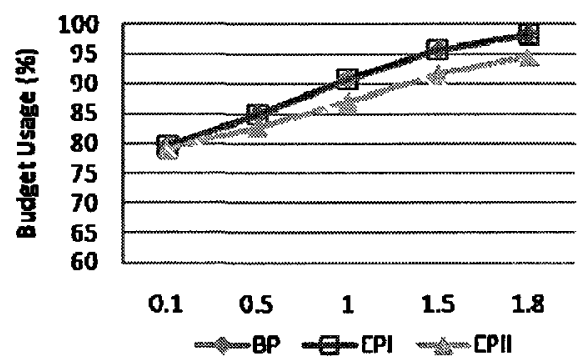


Appendix C.5: Budget usage comparisons across BP, EP1, and EP2

(Cont.)

(a) with Uniformly Distributed Demand

Case5: NR25NA30

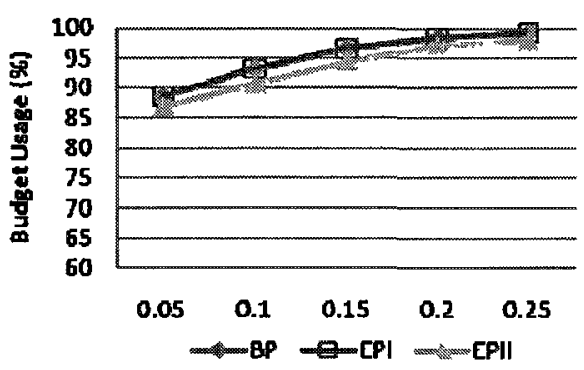

Case6: NR4ONA40

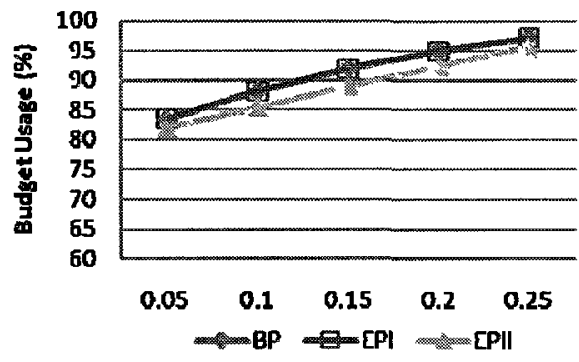

Case 7: NR60NA55

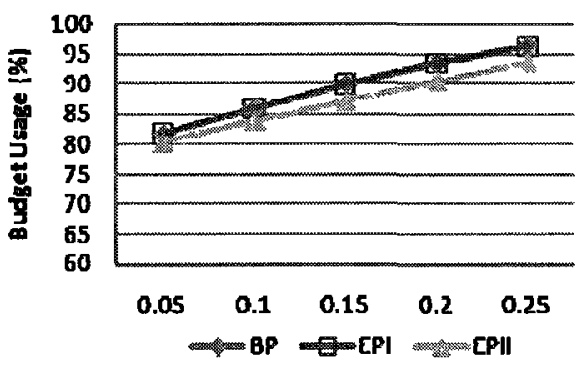

Case8: NR65NA70

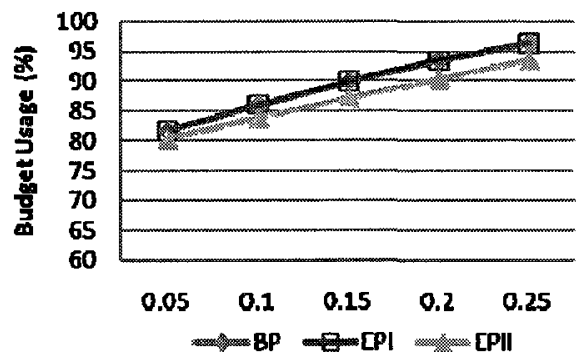

(b) with Normally Distributed Demand

Case5: NR25NA30

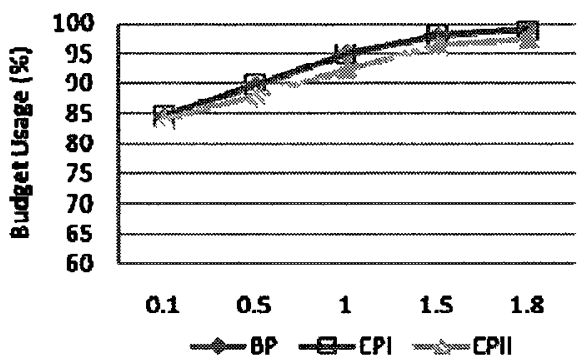

Case6: NR40NA40

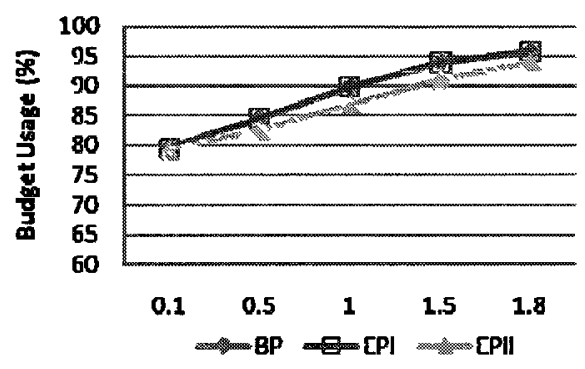

Case7: NR60NA55

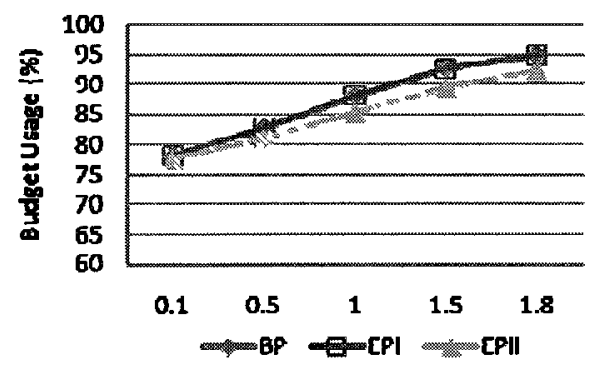

Case8: NR65NA70

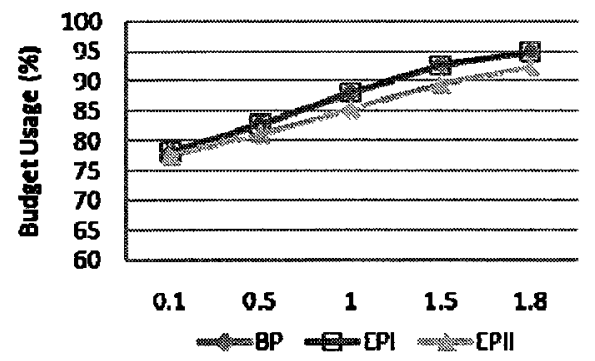


Appendix C.5: Budget usage comparisons across BP, EP1, and EP2

(Cont.)

(a) with Uniformly Distributed Demand

\section{Case9:NR80NA80}

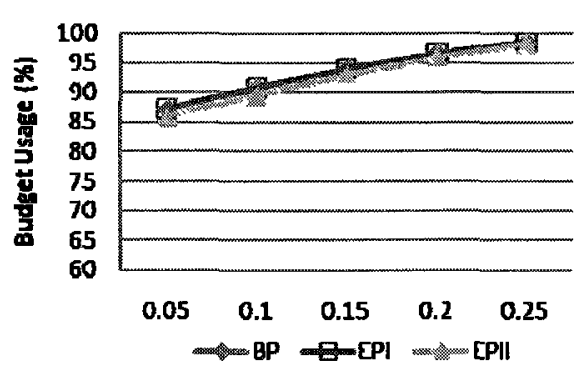

Case10: NR100NA95

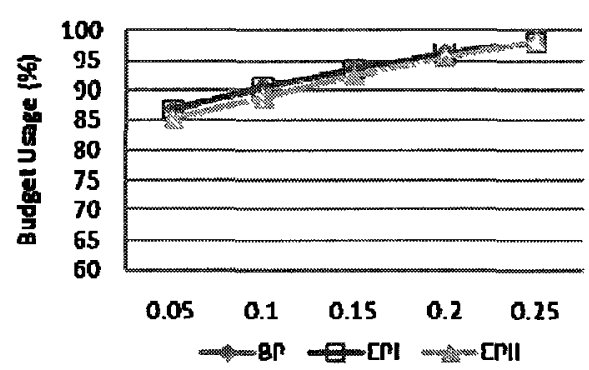

(b) with Normally Distributed Demand

\section{Case9: NR80NA80}

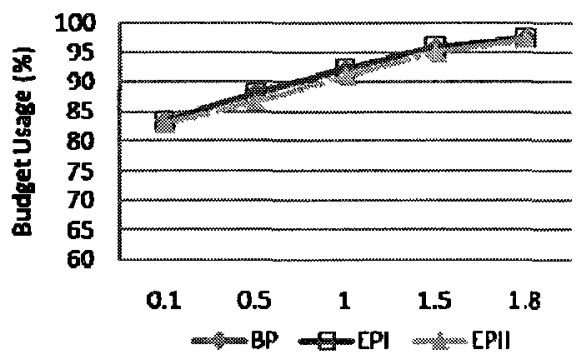

Case10: NR100NA95

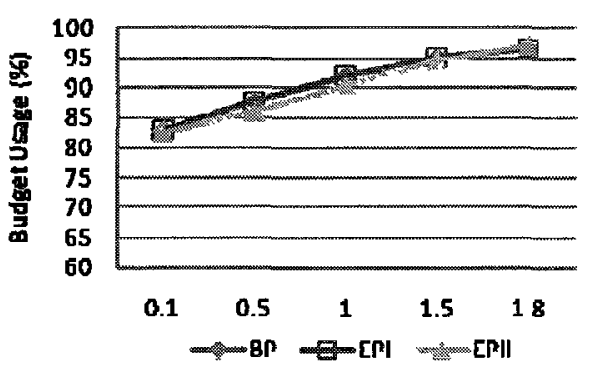


Appendix D 
Appendix D.1: Comparison of the impacts of budget tightness with a given level of demand variability under BP setting (Confidence intervals for $\mathrm{H1}, \mathrm{H} 2$, and $\mathrm{H3},\left(U_{i 2}-U_{i 1}\right) \pm$ half-width)

(a) with Normally Distributed Demand and Low Level of Demand Variability (Normal, DV-L)

\begin{tabular}{|cc|c|}
\hline \multicolumn{3}{|c|}{ Case 4 } \\
\hline \multirow{3}{*}{$i_{1}$} & BG-H \\
\hline Profitability & BG-L & $-1503.22 \pm 1.079$ \\
\hline
\end{tabular}
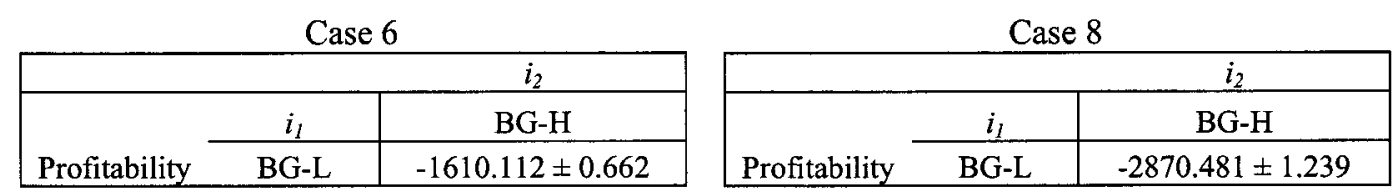

\begin{tabular}{|cc|c|}
\hline & \multicolumn{1}{c|}{$i_{2}$} \\
\hline $\begin{array}{c}\text { Liability } \\
\text { Ratio }\end{array}$ & $i_{1}$ & BG-H \\
\cline { 2 - 3 } & BG-L & $0.115 \pm 0.0012$ \\
\hline
\end{tabular}

\begin{tabular}{|cc|c|}
\hline & & $i_{2}$ \\
\hline $\begin{array}{c}\text { Liability } \\
\text { Ratio }\end{array}$ & $i_{1}$ & BG-H \\
\cline { 2 - 3 } & BG-L & $0.098 \pm 0.0001$ \\
\hline
\end{tabular}

\begin{tabular}{|c|c|c|}
\hline \multirow{3}{*}{ Liability } & \multicolumn{2}{|r|}{$\underline{i_{2}}$} \\
\hline & $i_{1}$ & BG-H \\
\hline & BG-L & $0.086 \pm 0.0001$ \\
\hline
\end{tabular}

\begin{tabular}{|lc|c|}
\hline & & $i_{2}$ \\
\hline Ordering & $i_{1}$ & BG-H \\
\cline { 2 - 3 } Quantity & BG-L & $-75.392 \pm 0.635$ \\
\hline
\end{tabular}

\begin{tabular}{|cc|c|}
\hline & & \multicolumn{1}{c|}{$i_{2}$} \\
\hline Ordering & $i_{1}$ & BG-H \\
\cline { 2 - 3 } Quantity & BG-L & $-117.298 \pm 0.197$ \\
\hline
\end{tabular}

\begin{tabular}{|c|c|c|}
\hline & & $i_{2}$ \\
\hline \multirow{2}{*}{ Ordering } & $i_{l}$ & BG-H \\
\hline & BG-L & $-191.099 \pm 0.105$ \\
\hline
\end{tabular}

(b) with Uniformly Distributed Demand and Low Level of Demand Variability (Uniform, DV-L)
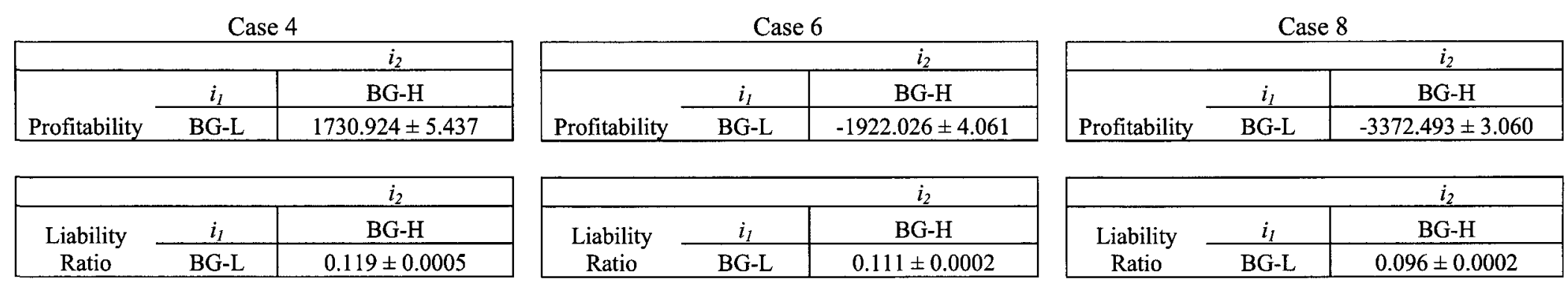

\begin{tabular}{|lc|c|}
\hline & & $i_{2}$ \\
\hline $\begin{array}{l}\text { Ordering } \\
\text { Quantity }\end{array}$ & $i_{1}$ & BG-L \\
\cline { 2 - 3 } & BG-H & $-97.746 \pm 0.205$ \\
\hline
\end{tabular}

\begin{tabular}{|lc|c|}
\hline & & $i_{2}$ \\
\hline Ordering & $i_{1}$ & BG-H \\
\cline { 2 - 3 } Quantity & BG-L & $-161.101 \pm 0.206$ \\
\hline
\end{tabular}

\begin{tabular}{|lc|c|}
\hline & & $i_{2}$ \\
\hline \multirow{2}{*}{$\begin{array}{l}\text { Ordering } \\
\text { Quantity }\end{array}$} & $i_{1}$ & BG-L \\
\cline { 2 - 3 }
\end{tabular}


Appendix D.1: Comparison of the impacts of budget tightness with a given level of demand variability under BP setting (Confidence intervals for $\mathrm{H} 1, \mathrm{H} 2$, and $\mathrm{H3},\left(U_{i 2}-U_{i l}\right) \pm$ half-width) (Cont.)

(c) with Normally Distributed Demand and Medium Level of Demand Variability (Normal, DV-M)
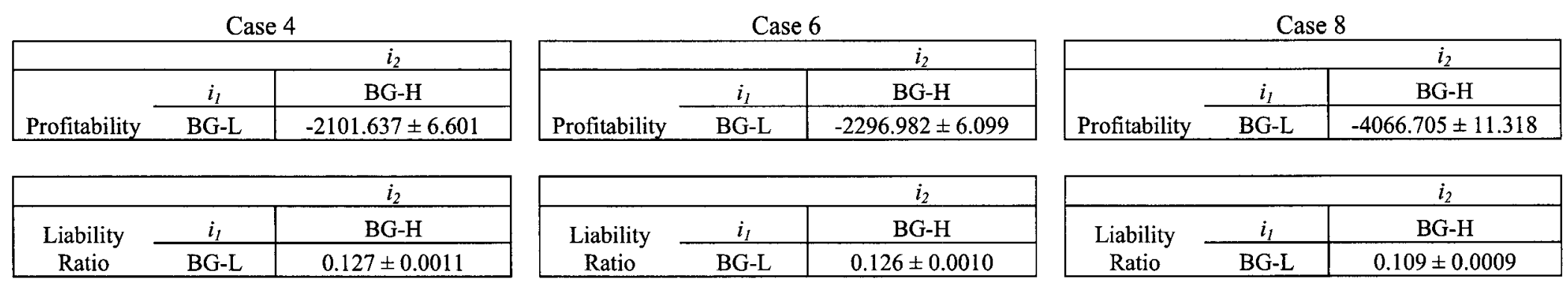

\begin{tabular}{|cc|c|}
\hline & \multicolumn{1}{c|}{$i_{2}$} \\
\hline \multirow{2}{*}{$\begin{array}{c}\text { Ordering } \\
\text { Quantity }\end{array}$} & $i_{1}$ & BG-H \\
\cline { 2 - 3 } & BG-L & $-146.470 \pm 2.208$ \\
\hline
\end{tabular}
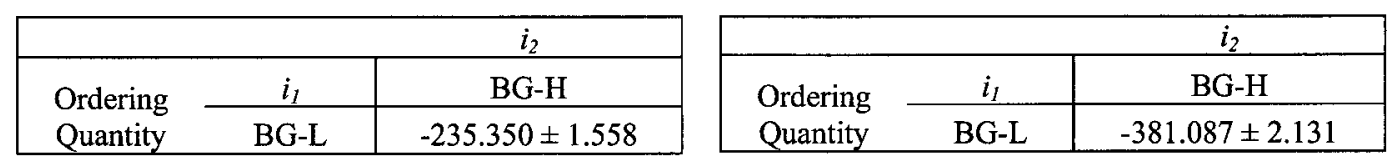

(d) with Uniformly Distributed Demand and Medium Level of Demand Variability (Uniform, DV-M)
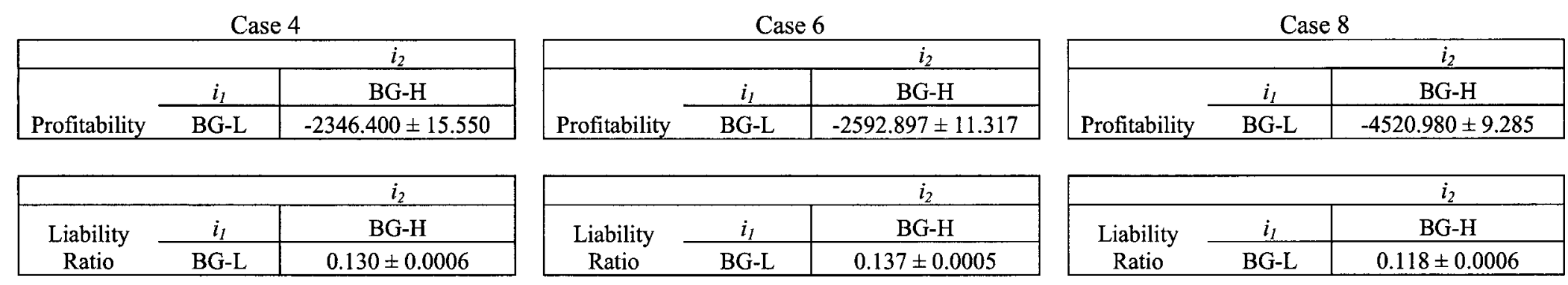

\begin{tabular}{|c|c|c|}
\hline & & $i_{2}$ \\
\hline \multirow{2}{*}{$\begin{array}{l}\text { Ordering } \\
\text { Quantity }\end{array}$} & $i_{1}$ & BG-H \\
\hline & BG-L & $-152.966 \pm 0.534$ \\
\hline
\end{tabular}

\begin{tabular}{|cc|c|}
\hline & \multicolumn{1}{c|}{$i_{2}$} \\
\hline Ordering & $i_{1}$ & BG-H \\
\cline { 2 - 3 } Quantity & BG-L & $-256.846 \pm 0.770$ \\
\hline
\end{tabular}

\begin{tabular}{|lc|c|}
\hline & \multicolumn{1}{c|}{$i_{2}$} \\
\hline Ordering & $i_{1}$ & BG-H \\
\cline { 2 - 3 } Quantity & BG-L & $-408.947 \pm 1.942$ \\
\hline
\end{tabular}


Appendix D.1: Comparison of the impacts of budget tightness with a given level of demand variability under BP setting (Confidence intervals for $\mathrm{H} 1, \mathrm{H} 2$, and $\mathrm{H3},\left(U_{i z^{-}}-U_{i 1}\right) \pm$ half-width) (Cont.)

(e) with Normally Distributed Demand and High Level of Demand Variability (Normal, DV-H)
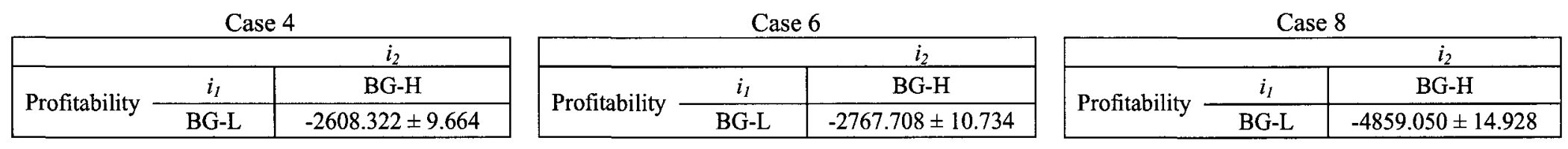

\begin{tabular}{|cc|c|}
\hline & \multicolumn{2}{c|}{$i_{2}$} \\
\hline $\begin{array}{c}\text { Liability } \\
\text { Ratio }\end{array}$ & $i_{1}$ & BG-H \\
\cline { 2 - 3 } & BG-L & $0.145 \pm 0.0010$ \\
\hline
\end{tabular}

\begin{tabular}{|cc|c|}
\hline & \multicolumn{1}{c|}{$i_{2}$} \\
\hline $\begin{array}{c}\text { Liability } \\
\text { Ratio }\end{array}$ & $i_{1}$ & BG-H \\
\cline { 2 - 3 } & BG-L & $0.136 \pm 0.0013$ \\
\hline
\end{tabular}

\begin{tabular}{|cc|c|}
\hline & & $i_{2}$ \\
\hline $\begin{array}{c}\text { Liability } \\
\text { Ratio }\end{array}$ & $i_{I}$ & BG-H \\
\cline { 2 - 3 } & BG-L & $0.119 \pm 0.0010$ \\
\hline
\end{tabular}

\begin{tabular}{|lc|c|}
\hline & \multicolumn{1}{c|}{$i_{2}$} \\
\hline $\begin{array}{l}\text { Ordering } \\
\text { Quantity }\end{array}$ & $i_{1}$ & BG-H \\
\cline { 2 - 3 } & BG-L & $-193.901 \pm 3.072$ \\
\hline
\end{tabular}

\begin{tabular}{|lc|c|}
\hline & \multicolumn{2}{c|}{$i_{2}$} \\
\hline $\begin{array}{l}\text { Ordering } \\
\text { Quantity }\end{array}$ & $i_{1}$ & BG-H \\
\cline { 2 - 3 } & BG-L & $-293.409 \pm 2.041$ \\
\hline
\end{tabular}

\begin{tabular}{|lc|c|}
\hline & \multicolumn{1}{c|}{$i_{2}$} \\
\hline $\begin{array}{l}\text { Ordering } \\
\text { Quantity }\end{array}$ & $i_{1}$ & BG-H \\
\cline { 2 - 3 } & BG-L & $-467.749 \pm 1.503$ \\
\hline
\end{tabular}

(f) with Uniformly Distributed Demand and High Level of Demand Variability (Uniform, DV-H)
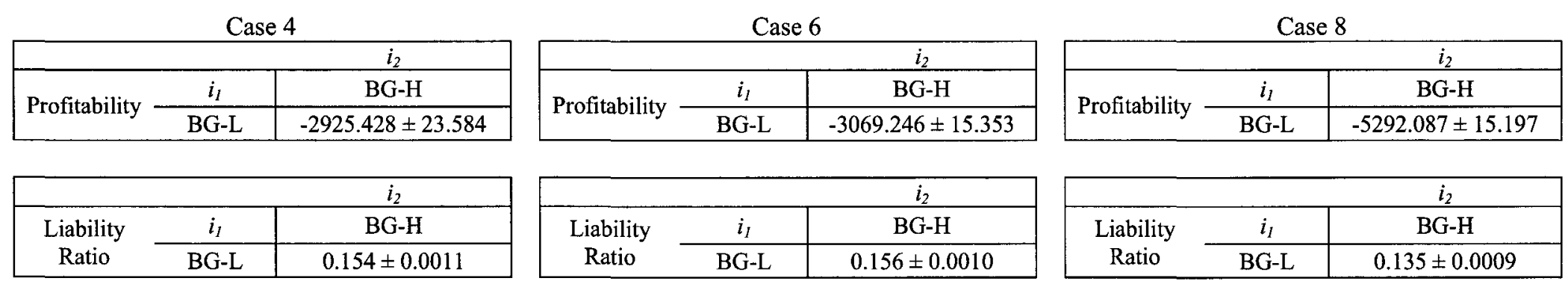

\begin{tabular}{|lc|c|}
\hline & \multicolumn{2}{c|}{$i_{2}$} \\
\hline Ordering & $i_{1}$ & BG-H \\
\cline { 2 - 3 } Quantity & $\mathrm{BG}-\mathrm{L}$ & $-198.226 \pm 1.112$ \\
\hline
\end{tabular}

\begin{tabular}{|c|c|c|}
\hline & & $i_{2}$ \\
\hline \multirow{2}{*}{$\begin{array}{l}\text { Ordering } \\
\text { Quantity }\end{array}$} & $i_{1}$ & BG-H \\
\hline & BG-L & $-315.105+1.060$ \\
\hline
\end{tabular}

\begin{tabular}{|lc|c|}
\hline & \multicolumn{1}{c|}{$i_{2}$} \\
\hline Ordering & $i_{1}$ & BG-H \\
\cline { 2 - 3 } Quantity & $\mathrm{BG}-\mathrm{L}$ & $-504.648 \pm 2.574$ \\
\hline
\end{tabular}


Appendix D.2: Comparison of the impacts of demand variability with a given level of budget tightness under BP setting (Confidence intervals for $\mathrm{H4}, \mathrm{H5}$, and $\mathrm{H6},\left(U_{i 2}-U_{i I}\right) \pm$ half-width)

(a) with Normally Distributed Demand and Low Level of Budget Tightness (Normal, BG-L)

\begin{tabular}{|cccc|}
\hline \multicolumn{3}{c}{ Case 4 } \\
\hline \multirow{4}{*}{ Profitability } & \multicolumn{2}{c|}{$j_{2}$} \\
\cline { 2 - 4 } & $j_{l}$ & DV-M & DV-H \\
\cline { 2 - 4 } & DV-L & -304.184 & -652.959 \\
\cline { 2 - 4 } & & \pm 15.793 & \pm 15.793 \\
\cline { 2 - 4 } & DV-M & & -348.775 \\
& & \pm 15.793 \\
\hline
\end{tabular}

\begin{tabular}{|c|c|c|c|}
\hline \multicolumn{4}{|c|}{ Case 6} \\
\hline & & \multicolumn{2}{|c|}{$j_{2}$} \\
\hline \multirow{3}{*}{ Profitability } & $j_{l}$ & DV-M & DV-H \\
\hline & DV-L & $\begin{array}{r}252.709 \\
\pm 15.481 \\
\end{array}$ & $\begin{array}{r}336.531 \\
\pm 15.481 \\
\end{array}$ \\
\hline & DV-M & & $\begin{array}{c}83.822 \\
\pm 15.481 \\
\end{array}$ \\
\hline & & \multicolumn{2}{|c|}{$j_{2}$} \\
\hline \multirow{3}{*}{$\begin{array}{c}\text { Liability } \\
\text { Ratio }\end{array}$} & $j_{1}$ & DV-M & DV-H \\
\hline & DV-L & $\begin{array}{c}0.049 \pm \\
0.0007 \\
\end{array}$ & $\begin{array}{c}0.1 \pm \\
0.0007\end{array}$ \\
\hline & DV-M & & $\begin{array}{c}0.051 \\
\pm 0.0007 \\
\end{array}$ \\
\hline
\end{tabular}

\begin{tabular}{|c|c|c|c|}
\hline \multicolumn{4}{|c|}{ Case 8} \\
\hline & & \multicolumn{2}{|c|}{$j_{2}$} \\
\hline \multirow{3}{*}{ Profitability } & $j_{1}$ & DV-M & DV-H \\
\hline & DV-L & $\begin{array}{r}489.689 \\
\pm 18.967\end{array}$ & $\begin{array}{r}657.949 \\
+18967\end{array}$ \\
\hline & \multicolumn{2}{|l|}{ DV-M } & $\begin{array}{c}168.26 \\
\pm 18.967 \\
\end{array}$ \\
\hline & & \multicolumn{2}{|c|}{$j_{2}$} \\
\hline \multirow{3}{*}{$\begin{array}{c}\text { Liability } \\
\text { Ratio }\end{array}$} & $j_{l}$ & DV-M & DV-H \\
\hline & DV-L & $\begin{array}{l}0.051 \pm \\
0.0003\end{array}$ & $\begin{array}{c}0.102 \\
\pm 0.0003 \\
\end{array}$ \\
\hline & DV-M & \multicolumn{2}{|c|}{$\begin{array}{c}0.051 \\
\pm 0.0003 \\
\end{array}$} \\
\hline & & \multicolumn{2}{|c|}{$\dot{j}_{2}$} \\
\hline \multirow{3}{*}{$\begin{array}{l}\text { Ordering } \\
\text { Quantity }\end{array}$} & $j_{I}$ & DV-M & DV-H \\
\hline & DV-L & $\begin{array}{r}191.048 \\
\pm 1.512 \\
\end{array}$ & $\begin{array}{r}290.167 \\
\pm 1.512 \\
\end{array}$ \\
\hline & DV-M & & $\begin{array}{r}99.119 \\
\pm 1.512 \\
\end{array}$ \\
\hline
\end{tabular}


Appendix D.2: Comparison of the impacts of demand variability with a given level of budget tightness under BP setting (Confidence intervals for $\mathrm{H4}, \mathrm{H5}$, and $\mathrm{H6},\left(U_{i 2}-U_{i 1}\right) \pm$ half-width)

(Cont.)

(b) with Uniformly Distributed Demand and Low Level of Budget Tightness (Uniform, BG-L)

\begin{tabular}{|c|c|c|c|}
\hline \multicolumn{4}{|c|}{ Case 4} \\
\hline & & \multicolumn{2}{|c|}{$j_{2}$} \\
\hline \multirow{3}{*}{ Profitability } & $j_{1}$ & DV-M & DV-H \\
\hline & DV-L & $\begin{array}{r}-257.953 \\
+31658\end{array}$ & $\begin{array}{l}-609.661 \\
+31658\end{array}$ \\
\hline & DV-M & & $\begin{array}{r}-351.708 \\
+31.658\end{array}$ \\
\hline
\end{tabular}

\begin{tabular}{|cccc|}
\hline \multicolumn{3}{c}{ Case 6 } \\
\hline & \multicolumn{3}{c|}{$j_{2}$} \\
\hline \multirow{4}{*}{ Profitability } & $j_{1}$ & DV-M & DV-H \\
\cline { 2 - 4 } & DV-L & $\begin{array}{c}197.547 \\
\pm 21.008\end{array}$ & $\begin{array}{c}631.42 \\
\pm 13.034\end{array}$ \\
\cline { 2 - 4 } & & & $\mathbf{- 1 . 9 1 4}$ \\
& DV-M & & $\pm \mathbf{2 1 . 0 0 8 *}$ \\
\hline
\end{tabular}

\begin{tabular}{|c|c|c|c|}
\hline \multicolumn{4}{|c|}{ Case 8} \\
\hline & & & \\
\hline \multirow{3}{*}{ Profitability } & $j_{1}$ & DV-M & DV-H \\
\hline & DV-L & $\begin{array}{l}504.495 \\
\pm 13.034\end{array}$ & $\begin{array}{c}631.42 \\
\pm 13.034\end{array}$ \\
\hline & DV-M & & $\begin{array}{r}126.925 \\
\pm 13.034 \\
\end{array}$ \\
\hline
\end{tabular}

\begin{tabular}{|cccc|}
\hline & \multicolumn{3}{c|}{$j_{2}$} \\
\hline & $j_{1}$ & DV-M & DV-H \\
\cline { 2 - 4 } Liability & DV-L & 0.026 & 0.056 \\
Ratio & \pm 0.0011 & \pm 0.0011 \\
\cline { 2 - 4 } & DV-M & & 0.03 \\
& & \pm 0.0011 \\
\hline
\end{tabular}
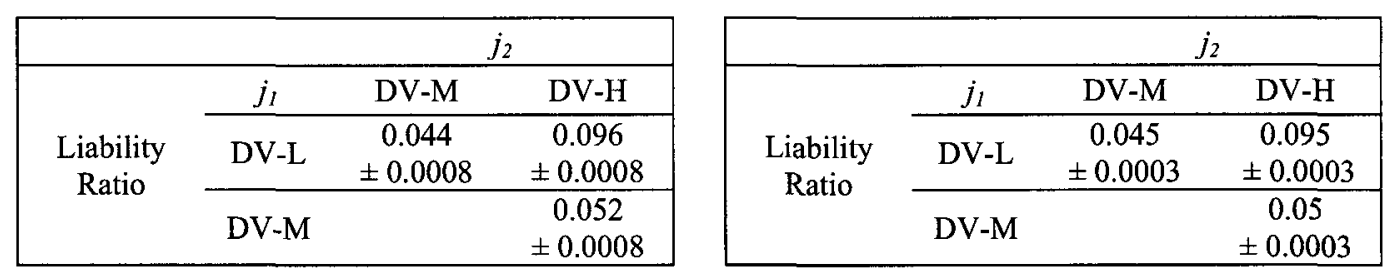

\begin{tabular}{|cccc|}
\hline & \multicolumn{3}{c|}{$j_{2}$} \\
\hline & $j_{1}$ & DV-M & DV-H \\
\cline { 2 - 4 } Ordering & \multirow{2}{*}{ DV-L } & 59.03 & 104.493 \\
Quantity & \pm 0.894 & \pm 0.894 \\
\cline { 2 - 4 } & \multirow{2}{*}{ DV-M } & & 45.463 \\
& & \pm 0.894 \\
\hline
\end{tabular}

\begin{tabular}{|cccc|}
\hline & & \multicolumn{2}{c|}{$j_{2}$} \\
\hline & $j_{1}$ & DV-M & DV-H \\
\cline { 2 - 4 } Ordering & \multirow{2}{*}{ DV-L } & $96.953 \pm$ & $156.155 \pm$ \\
Quantity & 1.342 & 1.342 \\
\cline { 2 - 4 } & \multirow{2}{*}{ DV-M } & & $59.202 \pm$ \\
& & 1.342 \\
\hline
\end{tabular}

\begin{tabular}{|cccc|}
\hline & \multicolumn{3}{c}{$j_{2}$} \\
\hline & $j_{1}$ & DV-M & DV-H \\
\cline { 2 - 4 } Ordering & \multirow{2}{*}{$\mathrm{DV}-\mathrm{L}$} & 150.539 & 245.695 \\
Quantity & \pm 2.775 & \pm 2.775 \\
\cline { 2 - 4 } & \multirow{2}{*}{$\mathrm{DV}-\mathrm{M}$} & & 95.156 \\
& & \pm 2.775 \\
\hline
\end{tabular}


Appendix D.2: Comparison of the impacts of demand variability with a given level of budget tightness under BP setting (Confidence intervals for $\mathrm{H4}, \mathrm{H5}$, and $\mathrm{H6},\left(U_{i 2}-U_{i 1}\right) \pm$ half-width)

(Cont.)

(c) with Normally Distributed Demand and High Level of Budget Tightness (Normal, BG-H)

\begin{tabular}{|cccc|}
\hline \multicolumn{4}{c|}{ Case 4 } \\
\hline \multirow{4}{*}{ Profitability } & \multicolumn{3}{c|}{$j_{2}$} \\
\cline { 2 - 4 } & $j_{1}$ & DV-M & DV-H \\
\cline { 2 - 4 } & DV-L & $\begin{array}{c}-902.601 \\
\pm 7.496\end{array}$ & $\begin{array}{c}-1758.061 \\
\pm 7.496\end{array}$ \\
\cline { 2 - 4 } & \multirow{2}{*}{ DV-M } & & $\begin{array}{c}-855.46 \\
\pm 7.496\end{array}$ \\
\hline
\end{tabular}

\begin{tabular}{|c|c|c|c|}
\hline \multicolumn{4}{|c|}{ Case 6} \\
\hline & & \multicolumn{2}{|c|}{$j_{2}$} \\
\hline \multirow{3}{*}{ Profitability } & $j_{1}$ & DV-M & DV-H \\
\hline & DV-L & $\begin{array}{l}-434.161 \\
\pm 12.969\end{array}$ & $\begin{array}{l}-821.065 \\
\pm 12.969\end{array}$ \\
\hline & DV-M & & $\begin{array}{l}-386.904 \\
\pm 12.969\end{array}$ \\
\hline & & \multicolumn{2}{|c|}{$j_{2}$} \\
\hline \multirow{3}{*}{$\begin{array}{c}\text { Liability } \\
\text { Ratio }\end{array}$} & $j_{1}$ & DV-M & DV-H \\
\hline & DV-L & $\begin{array}{c}0.077 \pm \\
0.0017\end{array}$ & $\begin{array}{l}0.138 \pm \\
0.0017\end{array}$ \\
\hline & DV-M & & $\begin{array}{c}0.061 \pm \\
0.0017\end{array}$ \\
\hline
\end{tabular}

\begin{tabular}{|c|c|c|c|}
\hline \multicolumn{4}{|c|}{ Case 8} \\
\hline & & \multicolumn{2}{|c|}{$j_{2}$} \\
\hline \multirow{3}{*}{ Profitability } & $j_{1}$ & DV-M & DV-H \\
\hline & DV-L & $\begin{array}{l}-706.535 \\
\pm 13.299\end{array}$ & $\begin{array}{l}-1330.62 \\
\pm 13.299\end{array}$ \\
\hline & DV-M & & $\begin{array}{c}-624.085 \\
\pm 13.299 \\
\end{array}$ \\
\hline & & \multicolumn{2}{|c|}{$\dot{j}_{2}$} \\
\hline \multirow{3}{*}{$\begin{array}{c}\text { Liability } \\
\text { Ratio }\end{array}$} & $j_{l}$ & DV-M & DV-H \\
\hline & DV-L & $\begin{array}{c}0.074 \pm \\
0.0012\end{array}$ & $\begin{array}{l}0.135 \pm \\
0.0012\end{array}$ \\
\hline & DV-M & & $\begin{array}{l}0.061 \pm \\
0.0012 \\
\end{array}$ \\
\hline
\end{tabular}

\begin{tabular}{|cccc|}
\hline & & \multicolumn{2}{c|}{$j_{2}$} \\
\hline & $j_{1}$ & DV-M & DV-H \\
\cline { 2 - 4 } Ordering & \multirow{2}{*}{ DV-L } & $3.633 \pm$ & $4.441 \pm$ \\
Quantity & 0.788 & 0.788 \\
\cline { 2 - 4 } & \multirow{2}{*}{ DV-M } & & $0.808 \pm$ \\
& & & 0.788 \\
\hline
\end{tabular}

\begin{tabular}{|cccc|}
\hline & \multicolumn{3}{c}{$j_{2}$} \\
\hline & $j_{1}$ & DV-M & DV-H \\
\cline { 2 - 4 } Ordering & \multirow{2}{*}{ DV-L } & $2.13 \pm$ & $11.299 \pm$ \\
Quantity & & 1.398 & 1.398 \\
\cline { 2 - 4 } & \multirow{2}{*}{ DV-M } & & $9.169 \pm$ \\
& & & 1.398 \\
\hline
\end{tabular}

\begin{tabular}{|lccc|}
\hline & \multicolumn{3}{c}{$\dot{j}_{2}$} \\
\hline & $j_{1}$ & $\mathrm{DV}-\mathrm{M}$ & $\mathrm{DV}-\mathrm{H}$ \\
\cline { 2 - 4 } Ordering & \multirow{2}{*}{$\mathrm{DV}-\mathrm{L}$} & $\mathbf{1 . 0 6} \pm$ & $13.517 \pm$ \\
Quantity & $\mathbf{1 . 9 1 5 ^ { * }}$ & 1.915 \\
\cline { 2 - 4 } & \multirow{2}{*}{$\mathrm{DV}-\mathrm{M}$} & & $12.457 \pm$ \\
& & 1.915 \\
\hline
\end{tabular}


Appendix D.2: Comparison of the impacts of demand variability with a given level of budget tightness under BP setting (Confidence intervals for $\mathrm{H4}, \mathrm{H5}$, and $\mathrm{H6},\left(U_{i 2}-U_{i l}\right) \pm$ half-width)

(Cont.)

(d) with Uniformly Distributed Demand and High Level of Budget Tightness (Uniform, BG-H)

\begin{tabular}{|cccc|}
\hline \multicolumn{4}{c}{ Case 4 } \\
\hline \multirow{4}{*}{ Profitability } & \multicolumn{3}{c|}{$j_{2}$} \\
\cline { 2 - 4 } & $j_{1}$ & DV-M & DV-H \\
\cline { 2 - 4 } & \multirow{2}{*}{ DV-L } & -873.429 & -1804.165 \\
& & \pm 9.715 & \pm 9.715 \\
\cline { 2 - 4 } & \multirow{2}{*}{ DV-M } & & -930.736 \\
& & \pm 9.715 \\
\hline
\end{tabular}
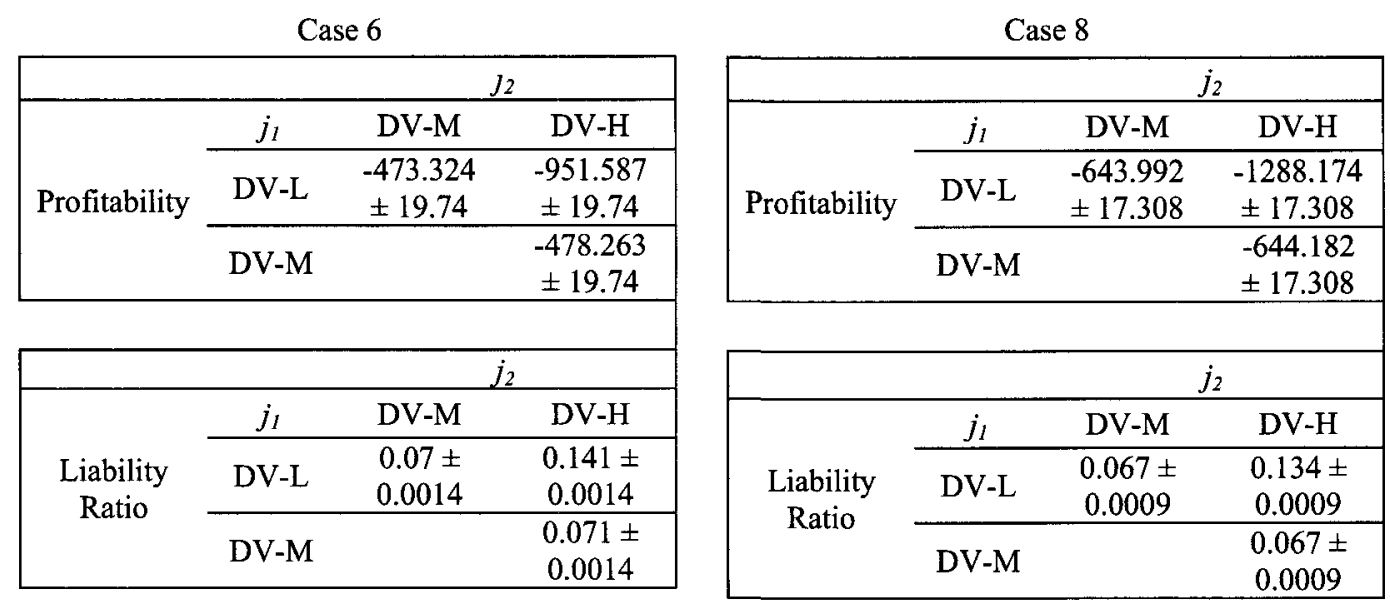

\begin{tabular}{|cccc|}
\hline & \multicolumn{3}{c|}{$j_{2}$} \\
\hline & $j_{1}$ & DV-M & DV-H \\
\cline { 2 - 4 } Ordering & \multirow{2}{*}{ DV-L } & 3.81 & 4.013 \\
Quantity & \pm 0.455 & \pm 0.455 \\
\cline { 2 - 4 } & \multirow{2}{*}{ DV-M } & & $\mathbf{0 . 2 0 3}$ \\
& & $\pm 0.455^{*}$ \\
\hline
\end{tabular}

\begin{tabular}{|cccc|}
\hline & \multicolumn{3}{c}{$j_{2}$} \\
\hline & \multirow{2}{*}{$J_{l}$} & $\mathrm{DV}-\mathrm{M}$ & $\mathrm{DV}-\mathrm{H}$ \\
\cline { 2 - 4 } Ordering & \multirow{2}{*}{$\mathrm{DV}-\mathrm{L}$} & $1.208 \pm$ & $2.151 \pm$ \\
Quantity & 0.565 & 0.565 \\
\cline { 2 - 4 } & \multirow{2}{*}{$\mathrm{DV}-\mathrm{M}$} & & $0.943 \pm$ \\
& & & 0.565 \\
\hline
\end{tabular}

\begin{tabular}{|cccc|}
\hline & \multicolumn{3}{c|}{$j_{2}$} \\
\hline & $J_{1}$ & DV-M & DV-H \\
\cline { 2 - 4 } Ordering & \multirow{2}{*}{ DV-L } & $\mathbf{0 . 0 3 7 \pm}$ & $\mathbf{- 0 . 5 0 8 \pm}$ \\
Quantity & $\mathbf{0 . 8 9 8 ^ { * }}$ & $\mathbf{0 . 8 9 8 ^ { * }}$ \\
\cline { 2 - 4 } & \multirow{2}{*}{ DV-M } & & $\mathbf{0 . 5 4 5 \pm}$ \\
& & & $\mathbf{0 . 8 9 8 ^ { * }}$ \\
\hline
\end{tabular}


Appendix D.3: Comparison of the impacts of budget tightness with a given level of demand variability under EP1 setting (Confidence intervals for $\mathrm{H1}, \mathrm{H} 2$, and $\mathrm{H3},\left(U_{i 2}-U_{i 1}\right) \pm$ half-width)

(a) with Normally Distributed Demand and Low Level of Demand Variability (Normal, DV-L)
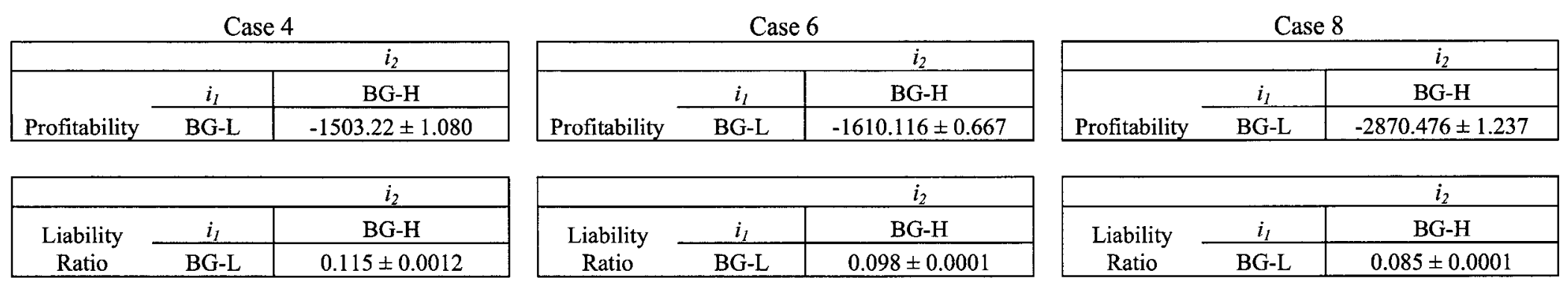

\begin{tabular}{|cc|c|}
\hline & \multicolumn{1}{c|}{$i_{2}$} \\
\hline \multirow{2}{*}{$\begin{array}{l}\text { Ordering } \\
\text { Quantity }\end{array}$} & $i_{1}$ & BG-H \\
\cline { 2 - 3 } & BG-L & $-75.384 \pm 0.667$ \\
\hline
\end{tabular}

\begin{tabular}{|lc|c|}
\hline & \multicolumn{2}{c|}{$i_{2}$} \\
\hline $\begin{array}{l}\text { Ordering } \\
\text { Quantity }\end{array}$ & $i_{1}$ & BG-H \\
\cline { 2 - 3 } & BG-L & $-117.298 \pm 0.197$ \\
\hline
\end{tabular}

\begin{tabular}{|lc|c|}
\hline & \multicolumn{2}{c|}{$i_{2}$} \\
\hline \multirow{2}{*}{$\begin{array}{l}\text { Ordering } \\
\text { Quantity }\end{array}$} & $i_{1}$ & BG-H \\
\cline { 2 - 3 } & BG-L & $-191.099 \pm 0.105$ \\
\hline
\end{tabular}

(b) with Uniformly Distributed Demand and Low Level of Demand Variability (Uniform, DV-L)

Case 4

\begin{tabular}{|cc|c|}
\hline \multicolumn{3}{|c|}{ Case 4 } \\
\hline & $i_{l}$ & BG-H \\
\cline { 2 - 3 } Profitability & BG-L & $-1730.924 \pm 5.437$ \\
\hline
\end{tabular}

\begin{tabular}{|cc|c|}
\hline & & $i_{2}$ \\
\hline $\begin{array}{c}\text { Liability } \\
\text { Ratio }\end{array}$ & $i_{1}$ & BG-H \\
\cline { 2 - 3 } & BG-L & $0.119 \pm 0.0004$ \\
\hline
\end{tabular}

\begin{tabular}{|c|c|c|}
\hline & & $i_{2}$ \\
\hline \multirow{2}{*}{$\begin{array}{l}\text { Ordering } \\
\text { Quantity }\end{array}$} & $i_{I}$ & BG-H \\
\hline & BG-L & $-97.746 \pm 0.205$ \\
\hline
\end{tabular}
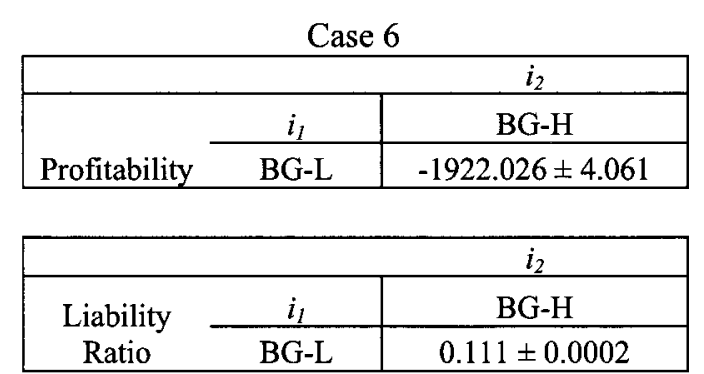

\begin{tabular}{|c|c|c|}
\hline & & $i_{2}$ \\
\hline \multirow{2}{*}{$\begin{array}{l}\text { Ordering } \\
\text { Quantity }\end{array}$} & $i_{1}$ & BG-H \\
\hline & BG-L & $-161.101 \pm 0.206$ \\
\hline
\end{tabular}

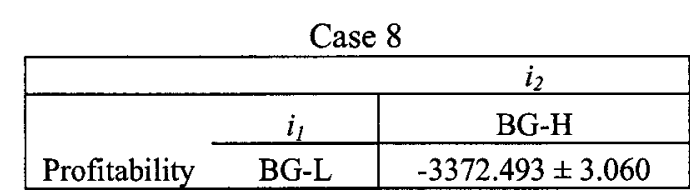

\begin{tabular}{|cc|c|}
\hline & & $i_{2}$ \\
\hline $\begin{array}{c}\text { Liability } \\
\text { Ratio }\end{array}$ & $i_{1}$ & BG-H \\
\cline { 2 - 3 } & BG-L & $0.096 \pm 0.0002$ \\
\hline
\end{tabular}

\begin{tabular}{|lc|c|}
\hline & \multicolumn{2}{c|}{$i_{2}$} \\
\hline Ordering & $i_{1}$ & BG-H \\
\cline { 2 - 3 } Quantity & BG-L & $-258.445 \pm 0.709$ \\
\hline
\end{tabular}


Appendix D.3: Comparison of the impacts of budget tightness with a given level of demand variability under EP1 setting (Confidence intervals for $\mathrm{H} 1, \mathrm{H} 2$, and $\mathrm{H3},\left(U_{i 2}-U_{i 1}\right) \pm$ half-width) (Cont.)

(c) with Normally Distributed Demand and Medium Level of Demand Variability (Normal, DV-M)
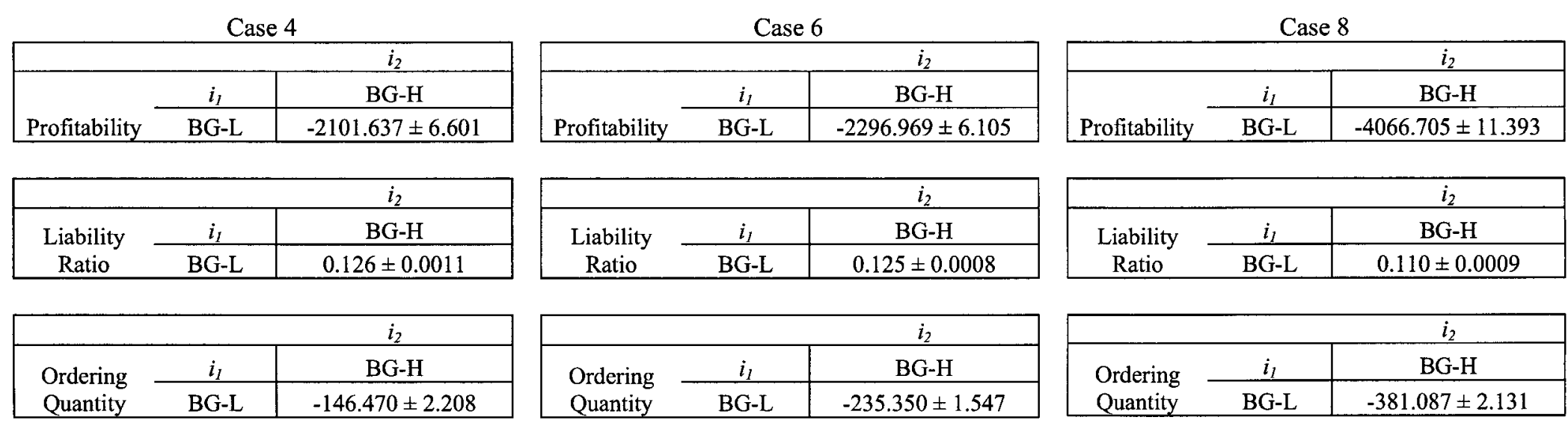

\begin{tabular}{|cc|c|}
\hline & \multicolumn{1}{c|}{$i_{2}$} \\
\hline Ordering & $i_{l}$ & BG-H \\
\cline { 2 - 3 } Quantity & BG-L & $-381.087 \pm 2.131$ \\
\hline
\end{tabular}

(d) with Uniformly Distributed Demand and Medium Level of Demand Variability (Uniform, DV-M)
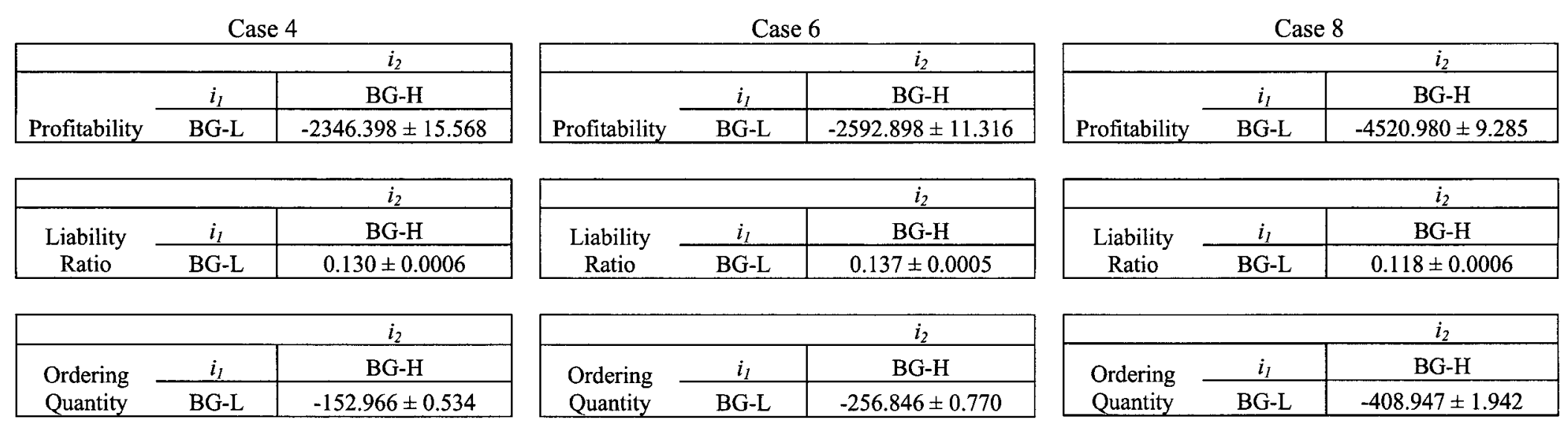

\begin{tabular}{|cc|c|}
\hline & & $i_{2}$ \\
\hline Ordering & $i_{1}$ & BG-H \\
\cline { 2 - 3 } Quantity & BG-L & $-256.846 \pm 0.770$ \\
\hline
\end{tabular}

\begin{tabular}{|c|c|c|}
\hline & & $i_{2}$ \\
\hline \multirow{2}{*}{$\begin{array}{l}\text { Ordering } \\
\text { Quantity }\end{array}$} & $i_{1}$ & $\mathrm{BG}-\mathrm{H}$ \\
\hline & BG-L & $-408.947 \pm 1.942$ \\
\hline
\end{tabular}


Appendix D.3: Comparison of the impacts of budget tightness with a given level of demand variability under EP1 setting (Confidence intervals for $\mathrm{H1}, \mathrm{H} 2$, and $\mathrm{H3},\left(U_{i 2}-U_{i I}\right) \pm$ half-width) (Cont.)

(e) with Normally Distributed Demand and High Level of Demand Variability ( Normal, DV-H)
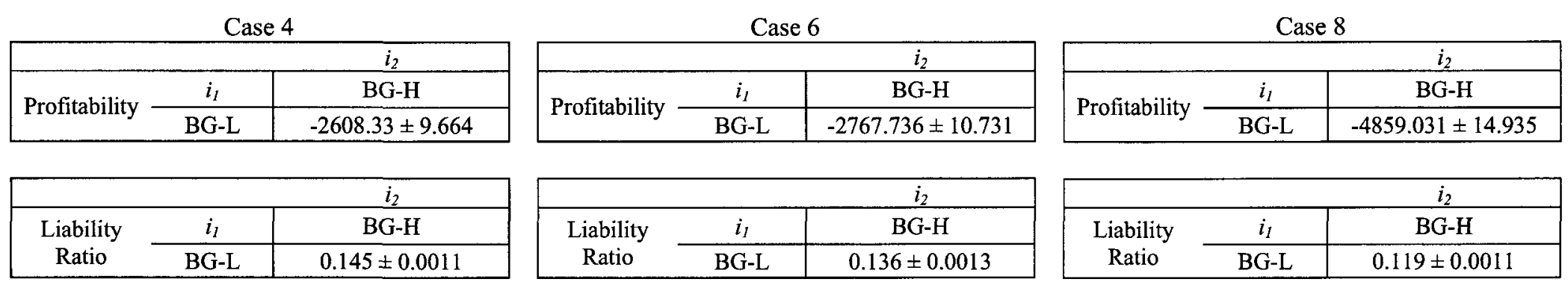

\begin{tabular}{|c|c|c|}
\hline & & $i_{2}$ \\
\hline \multirow{2}{*}{$\begin{array}{l}\text { Ordering } \\
\text { Quantity }\end{array}$} & $i_{I}$ & BG-H \\
\hline & BG-L & $-193.901 \pm 3.072$ \\
\hline
\end{tabular}

\begin{tabular}{|lc|c|}
\hline & \multicolumn{2}{c|}{$i_{2}$} \\
\hline Ordering & $i_{1}$ & BG-H \\
\cline { 2 - 3 } Quantity & BG-L & $-293.409 \pm 2.041$ \\
\hline
\end{tabular}

\begin{tabular}{|c|c|c|}
\hline & & $i_{2}$ \\
\hline \multirow{2}{*}{$\begin{array}{l}\text { Ordering } \\
\text { Quantity }\end{array}$} & $i_{I}$ & BG-H \\
\hline & BG-L & $-467.749 \pm 1.503$ \\
\hline
\end{tabular}

(f) with Uniformly Distributed Demand and High Level of Demand Variability (Uniform, DV-H)
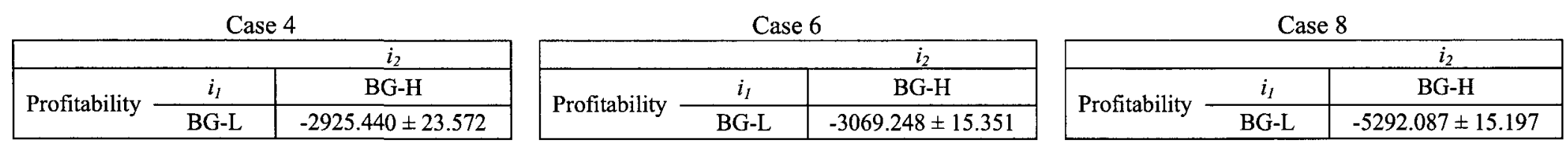

\begin{tabular}{|cc|c|}
\hline & & $i_{2}$ \\
\hline $\begin{array}{c}\text { Liability } \\
\text { Ratio }\end{array}$ & $i_{I}$ & BG-H \\
\cline { 2 - 3 } & BG-L & $0.153 \pm 0.0011$ \\
\hline
\end{tabular}

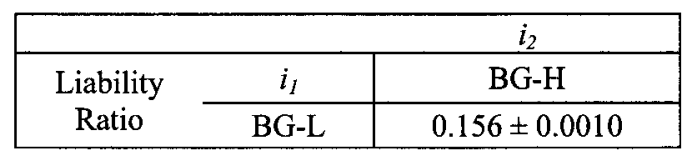

\begin{tabular}{|cc|c|}
\hline & \multicolumn{1}{c|}{$i_{2}$} \\
\hline $\begin{array}{c}\text { Liability } \\
\text { Ratio }\end{array}$ & $i_{I}$ & BG-H \\
\cline { 2 - 3 } & BG-L & $0.135 \pm 0.0009$ \\
\hline
\end{tabular}

\begin{tabular}{|lc|c|}
\hline & & $i_{2}$ \\
\hline $\begin{array}{l}\text { Ordering } \\
\text { Quantity }\end{array}$ & $i_{1}$ & BG-H \\
\cline { 2 - 3 } & BG-L & $-198.226 \pm 1.112$ \\
\hline
\end{tabular}

\begin{tabular}{|lc|c|}
\hline & \multicolumn{2}{c|}{$i_{2}$} \\
\hline $\begin{array}{l}\text { Ordering } \\
\text { Quantity }\end{array}$ & $i_{l}$ & BG-H \\
\cline { 2 - 3 } & BG-L & $-315.105 \pm 1.060$ \\
\hline
\end{tabular}

\begin{tabular}{|c|c|c|}
\hline & & $i_{2}$ \\
\hline \multirow{2}{*}{$\begin{array}{l}\text { Ordering } \\
\text { Quantity }\end{array}$} & $i_{1}$ & BG-H \\
\hline & BG-L & $-504.648 \pm 2.574$ \\
\hline
\end{tabular}


Appendix D.4: Comparison of the impacts of demand variability with a given level of budget tightness under EP1 setting (Confidence intervals for $\mathrm{H4}, \mathrm{H5}$, and $\mathrm{H6},\left(U_{i 2}-U_{i 1}\right) \pm$ half-width)

(a) with Normally Distributed Demand and Low Level of Budget Tightness (Normal, BG-L)
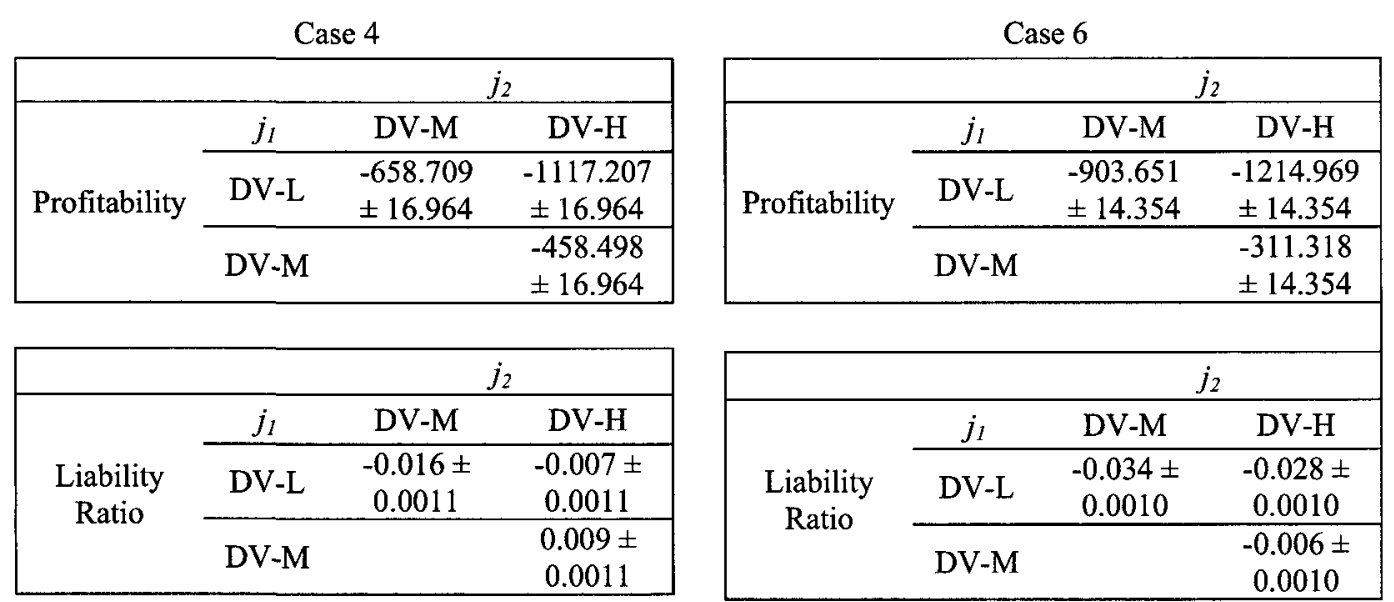

\begin{tabular}{|c|c|c|c|}
\hline \multicolumn{4}{|c|}{ Case 8} \\
\hline & & \multicolumn{2}{|c|}{$j_{2}$} \\
\hline \multirow{3}{*}{ Profitability } & $\dot{j}_{1}$ & DV-M & DV-H \\
\hline & DV-L & $\begin{array}{c}-1676.737 \\
\pm 18.263\end{array}$ & $\begin{array}{c}-2263.113 \\
\pm 18.263\end{array}$ \\
\hline & \multicolumn{2}{|l|}{ DV-M } & $\begin{array}{l}-586.376 \\
\pm 18.263\end{array}$ \\
\hline & & \multicolumn{2}{|c|}{$\dot{j}_{2}$} \\
\hline \multirow{3}{*}{$\begin{array}{c}\text { Liability } \\
\text { Ratio }\end{array}$} & $j_{1}$ & DV-M & DV-H \\
\hline & DV-L & $\begin{array}{c}-0.034 \pm \\
0.0009\end{array}$ & $\begin{array}{c}-0.028 \pm \\
0.0009\end{array}$ \\
\hline & DV-M & & $\begin{array}{c}-0.006 \pm \\
0.0009\end{array}$ \\
\hline & & \multicolumn{2}{|c|}{$j_{2}$} \\
\hline \multirow{3}{*}{$\begin{array}{l}\text { Ordering } \\
\text { Quantity }\end{array}$} & $j_{1}$ & DV-M & DV-H \\
\hline & DV-L & $\begin{array}{r}191.048 \\
\pm 1.512\end{array}$ & $\begin{array}{l}290.167 \\
\pm 1.512\end{array}$ \\
\hline & DV-M & & $\begin{array}{r}99.119 \\
\pm 1.512\end{array}$ \\
\hline
\end{tabular}


Appendix D.4: Comparison of the impacts of demand variability with a given level of budget tightness under EP1 setting (Confidence intervals for $\mathrm{H4}, \mathrm{H5}$, and $\mathrm{H6},\left(U_{i 2}-U_{i I}\right) \pm$ half-width)

(Cont.)

(b) with Uniformly Distributed Demand and Low Level of Budget Tightness (Uniform, BG-L)

\begin{tabular}{|cccc|}
\hline \multicolumn{4}{c}{ Case 4 } \\
\hline \multirow{4}{*}{ Profitability } & \multicolumn{3}{c|}{$j_{2}$} \\
\cline { 2 - 4 } & $j_{1}$ & DV-M & DV-H \\
\cline { 2 - 4 } & \multirow{2}{*}{ DV-L } & -416.917 & -837.081 \\
& & \pm 32.006 & \pm 32.006 \\
\cline { 2 - 4 } & DV-M & & -420.164 \\
& & \pm 32.006 \\
\hline
\end{tabular}

\begin{tabular}{|cccc|}
\hline \multicolumn{4}{c}{ Case 6 } \\
\hline \multirow{4}{*}{ Profitability } & \multicolumn{3}{c|}{$j_{2}$} \\
\cline { 2 - 4 } & \multirow{2}{*}{ DV-L } & DV-M & DV-H \\
\cline { 2 - 4 } & & \pm 21.443 & -571.184 \\
& \multirow{2}{*}{ DV-M } & & $-21.443 .8 \pm$ \\
& & 21.443 \\
\hline
\end{tabular}

\begin{tabular}{|cccc|}
\hline \multicolumn{4}{c|}{ Case 8 } \\
\hline \multirow{4}{*}{ Profitability } & \multicolumn{3}{c|}{$j_{2}$} \\
\cline { 2 - 4 } & $j_{1}$ & DV-M & DV-H \\
\cline { 2 - 4 } & \multirow{2}{*}{ DV-L } & -504.412 & -814.107 \\
& \multirow{2}{*}{ DV-M } & & $\begin{array}{c}\text {-309.695 } \\
\end{array}$ \\
& & & \pm 11.842 \\
\hline
\end{tabular}

\begin{tabular}{|cccc|}
\hline & & \multicolumn{2}{c|}{$j_{2}$} \\
\hline & $j_{1}$ & DV-M & DV-H \\
\cline { 2 - 4 } Liability & \multirow{2}{*}{ DV-L } & $0.006 \pm$ & $0.024 \pm$ \\
Ratio & & 0.0012 & 0.0012 \\
\cline { 2 - 4 } & \multirow{2}{*}{ DV-M } & & $0.018 \pm$ \\
& & & 0.0012 \\
\hline
\end{tabular}
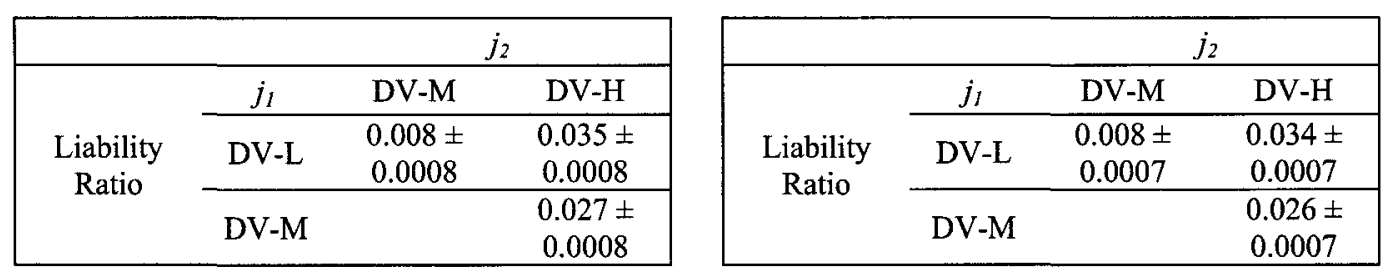

\begin{tabular}{|cccc|}
\hline & \multicolumn{3}{c|}{$j_{2}$} \\
\hline & $j_{1}$ & DV-M & DV-H \\
\cline { 2 - 4 } Ordering & \multirow{2}{*}{ DV-L } & 59.03 & 104.493 \\
Quantity & \pm 0.894 & \pm 0.894 \\
\cline { 2 - 4 } & \multirow{2}{*}{ DV-M } & & 45.463 \\
& & \pm 0.894 \\
\hline
\end{tabular}

\begin{tabular}{|cccc|}
\hline & \multicolumn{3}{c|}{$j_{2}$} \\
\hline & $j_{1}$ & DV-M & DV-H \\
\cline { 2 - 4 } Ordering & DV-L & $96.953 \pm$ & $156.155 \pm$ \\
Quantity & 1.342 & 1.342 \\
\cline { 2 - 4 } & DV-M & & $59.202 \pm$ \\
& & 1.342 \\
\hline
\end{tabular}

\begin{tabular}{|cccc|}
\hline & & \multicolumn{3}{c|}{$j_{2}$} \\
\hline & $j_{1}$ & DV-M & DV-H \\
\cline { 2 - 4 } Ordering & DV-L & 150.539 & 245.695 \\
Quantity & & \pm 2.775 & \pm 2.775 \\
\cline { 2 - 4 } & DV-M & & 95.156 \\
& & & \pm 2.775 \\
\hline
\end{tabular}


Appendix D.4: Comparison of the impacts of demand variability with a given level of budget tightness under EP1 setting (Confidence intervals for $\mathrm{H4}, \mathrm{H5}$, and $\mathrm{H6},\left(U_{i 2}-U_{i 1}\right) \pm$ half-width)

(Cont.)

(c) with Normally Distributed Demand and High Level of Budget Tightness (Normal, BG-H)

\begin{tabular}{|cccc|}
\hline \multicolumn{4}{c}{ Case 4 } \\
\hline \multirow{4}{*}{ Profitability } & \multicolumn{3}{c|}{$j_{2}$} \\
\cline { 2 - 4 } & $j_{1}$ & DV-M & DV-H \\
\cline { 2 - 4 } & DV-L & -1257.126 & -2222.317 \\
& & \pm 9.843 & \pm 9.843 \\
\cline { 2 - 4 } & \multirow{2}{*}{ DV-M } & & -965.191 \\
& & \pm 9.843 \\
\hline
\end{tabular}

\begin{tabular}{|cccc|}
\hline \multicolumn{4}{c|}{ Case 6 } \\
\hline & \multicolumn{3}{c|}{$j_{2}$} \\
\hline \multirow{4}{*}{ Profitability } & $j_{1}$ & DV-M & DV-H \\
\cline { 2 - 4 } & \multirow{2}{*}{ DV-L } & -1590.504 & -2372.589 \\
& & \pm 11.330 & \pm 11.330 \\
\cline { 2 - 4 } & \multirow{2}{*}{ DV-M } & & -782.085 \\
& & \pm 11.330 \\
\hline
\end{tabular}
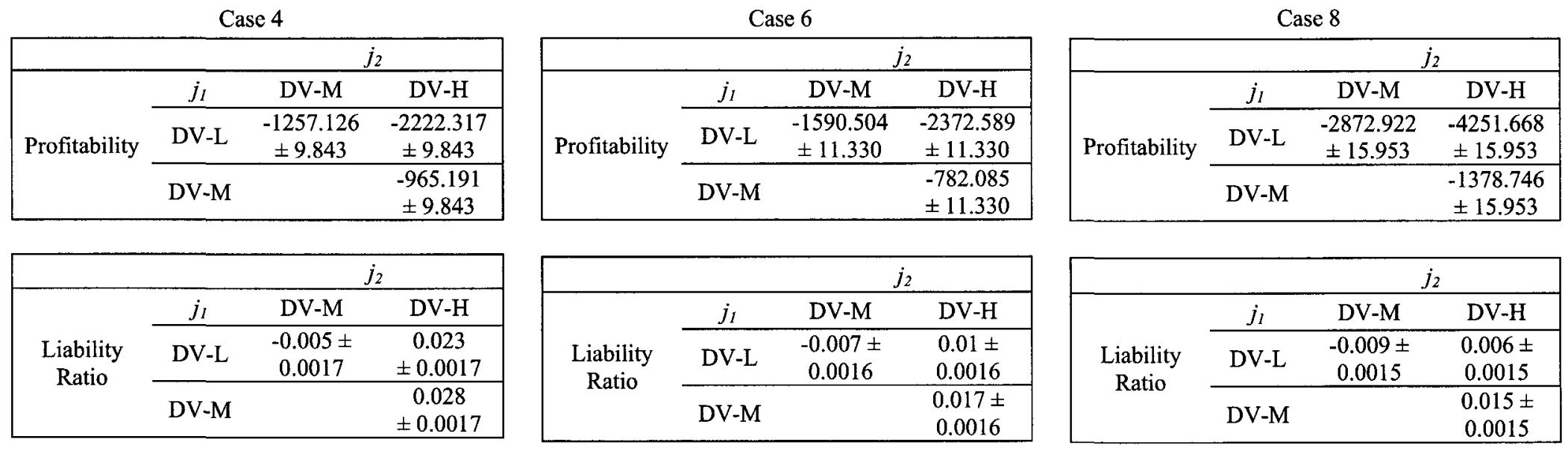

\begin{tabular}{|cccc|}
\hline & \multicolumn{3}{c}{$j_{2}$} \\
\hline & $j_{1}$ & DV-M & DV-H \\
\cline { 2 - 4 } Ordering & \multirow{2}{*}{ DV-L } & $3.633 \pm$ & $4.441 \pm$ \\
Quantity & 0.788 & 0.788 \\
\cline { 2 - 4 } & \multirow{2}{*}{ DV-M } & & $0.808 \pm$ \\
& & & 0.788 \\
\hline
\end{tabular}

\begin{tabular}{|cccc|}
\hline & & \multicolumn{3}{c}{$j_{2}$} \\
\hline & $j_{1}$ & $\mathrm{DV}-\mathrm{M}$ & $\mathrm{DV}-\mathrm{H}$ \\
\cline { 2 - 4 } Ordering & \multirow{2}{*}{$\mathrm{DV}-\mathrm{L}$} & $1.06 \pm$ & $13.517 \pm$ \\
Quantity & 1.915 & 1.915 \\
\cline { 2 - 4 } & \multirow{2}{*}{$\mathrm{DV}-\mathrm{M}$} & & $12.457 \pm$ \\
& & & 1.915 \\
\hline
\end{tabular}

\begin{tabular}{|cccc|}
\hline & \multicolumn{3}{c}{$j_{2}$} \\
\hline & $j_{1}$ & DV-M & DV-H \\
\cline { 2 - 4 } Ordering & \multirow{2}{*}{ DV-L } & $1.06 \pm$ & $13.517 \pm$ \\
Quantity & $1.915^{*}$ & 1.915 \\
\cline { 2 - 4 } & \multirow{2}{*}{ DV-M } & & $12.457 \pm$ \\
& & & 1.915 \\
\hline
\end{tabular}


Appendix D.4: Comparison of the impacts of demand variability with a given level of budget tightness under EP1 setting (Confidence intervals for $\mathrm{H} 4, \mathrm{H5}$, and $\mathrm{H6},\left(U_{i 2}-U_{i 1}\right) \pm$ half-width)

(Cont.)

(d) with Uniformly Distributed Demand and High Level of Budget Tightness (Uniform, BG-H)
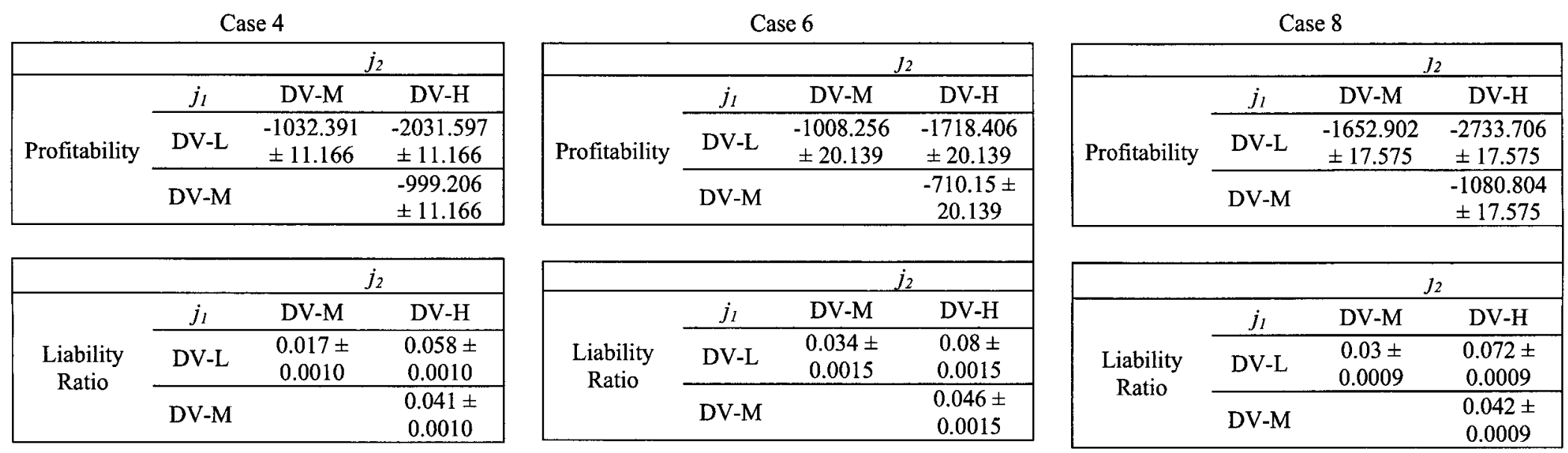

\begin{tabular}{|cccc|}
\hline & \multicolumn{3}{c|}{$j_{2}$} \\
\hline & $j_{1}$ & DV-M & DV-H \\
\cline { 2 - 4 } Ordering & \multirow{2}{*}{ DV-L } & 3.81 & 4.013 \\
Quantity & \pm 0.455 & \pm 0.455 \\
\cline { 2 - 4 } & \multirow{2}{*}{ DV-M } & & $\begin{array}{c}\mathbf{0 . 2 0 3} \\
\pm \mathbf{0 . 4 5 5}\end{array}$ \\
\hline
\end{tabular}

\begin{tabular}{|cccc|}
\hline & \multicolumn{3}{c}{$j_{2}$} \\
\hline & $j_{1}$ & DV-M & DV-H \\
\cline { 2 - 4 } Ordering & \multirow{2}{*}{ DV-L } & $1.208 \pm$ & $2.151 \pm$ \\
Quantity & 0.565 & 0.565 \\
\cline { 2 - 4 } & \multirow{2}{*}{ DV-M } & & $0.943 \pm$ \\
& & & 0.565 \\
\hline
\end{tabular}

\begin{tabular}{|cccc|}
\hline & & \multicolumn{2}{c|}{$j_{2}$} \\
\hline & $j_{1}$ & DV-M & DV-H \\
\cline { 2 - 4 } Ordering & \multirow{2}{*}{ DV-L } & $\mathbf{0 . 0 3 7 \pm}$ & $\mathbf{0 . 5 0 8 \pm}$ \\
Quantity & & $\mathbf{0 . 8 9 8 ^ { * }}$ & $\mathbf{0 . 8 9 8 ^ { * }}$ \\
\cline { 2 - 4 } & \multirow{2}{*}{ DV-M } & & $\mathbf{0 . 5 4 5 \pm}$ \\
& & & $\mathbf{0 . 8 9 8 ^ { * }}$ \\
\hline
\end{tabular}




\section{Appendix D.5: Comparison of the impacts of budget tightness with a given level of demand variability under EP2 setting}

(Confidence intervals for $\mathrm{H1}, \mathrm{H} 2$, and $\mathrm{H3},\left(U_{i 2}-U_{i 1}\right) \pm$ half-width)

(a) with Normally Distributed Demand and Low Level of Demand Variability (Normal, DV-L)
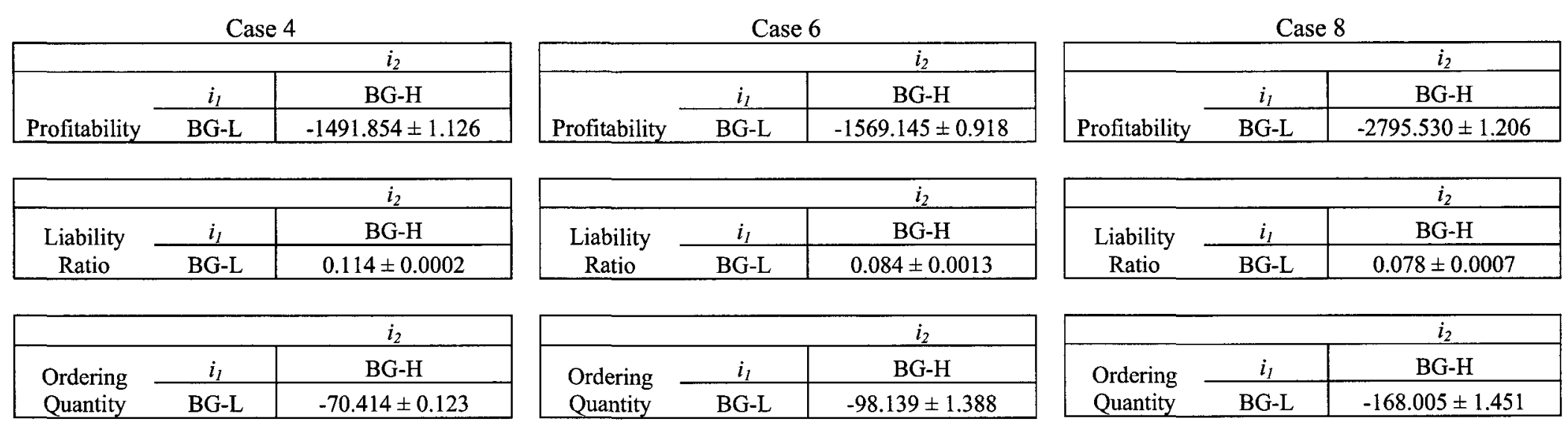

(b) with Uniformly Distributed Demand and Low Level of Demand Variability (Uniform, DV-L)

Case 4

\begin{tabular}{|cc|c|}
\hline & \multicolumn{3}{c|}{$i_{2}$} \\
\hline & $i_{1}$ & BG-H \\
\cline { 2 - 3 } Profitability & BG-L & $-1640.414 \pm 5.174$ \\
\hline
\end{tabular}

\begin{tabular}{|cc|c|}
\hline & \multicolumn{1}{c|}{$i_{2}$} \\
\hline $\begin{array}{c}\text { Liability } \\
\text { Ratio }\end{array}$ & $i_{1}$ & BG-H \\
\cline { 2 - 3 } & BG-L & $0.117 \pm 0.0002$ \\
\hline
\end{tabular}

\begin{tabular}{|c|c|c|}
\hline & & $i_{2}$ \\
\hline \multirow{2}{*}{$\begin{array}{l}\text { Ordering } \\
\text { Quantity }\end{array}$} & $i_{1}$ & BG-H \\
\hline & BG-L & $-85.984 \pm 0.183$ \\
\hline
\end{tabular}

Case 6

\begin{tabular}{|cc|c|}
\hline & \multicolumn{2}{c|}{$i_{2}$} \\
\hline \multirow{2}{*}{ Profitability } & $i_{1}$ & BG-H \\
\cline { 2 - 3 } & BG-L & $-1684.855 \pm 4.061$ \\
\hline
\end{tabular}
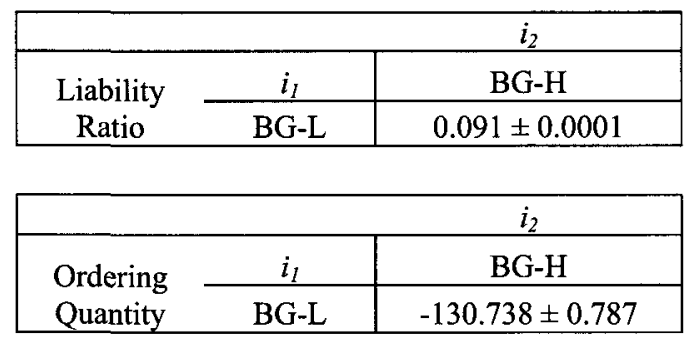

\begin{tabular}{|cc|c|}
\hline \multicolumn{3}{|c|}{ Case 8 } \\
\hline \multirow{3}{*}{ Profitability } & $i_{l}$ & $i_{2}$ \\
\cline { 2 - 3 } & BG-L & $-2976.170 \pm 4.197$ \\
\hline
\end{tabular}

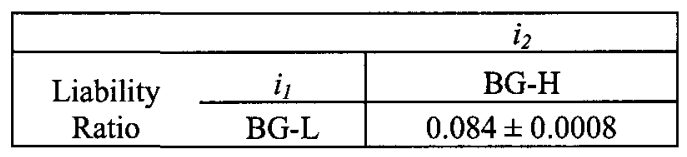

\begin{tabular}{|cc|c|}
\hline & \multicolumn{1}{c|}{$i_{2}$} \\
\hline Ordering & $i_{1}$ & BG-H \\
\cline { 2 - 3 } Quantity & BG-L & $-220.639 \pm 1.825$ \\
\hline
\end{tabular}


Appendix D.5: Comparison of the impacts of budget tightness with a given level of demand variability under EP2 setting (Confidence intervals for $\mathrm{H1}, \mathrm{H} 2$, and $\mathrm{H3},\left(U_{i 2}-U_{i l}\right) \pm$ half-width) (Cont.)

(c) with Normally Distributed Demand and Medium Level of Demand Variability (Normal, DV-M)
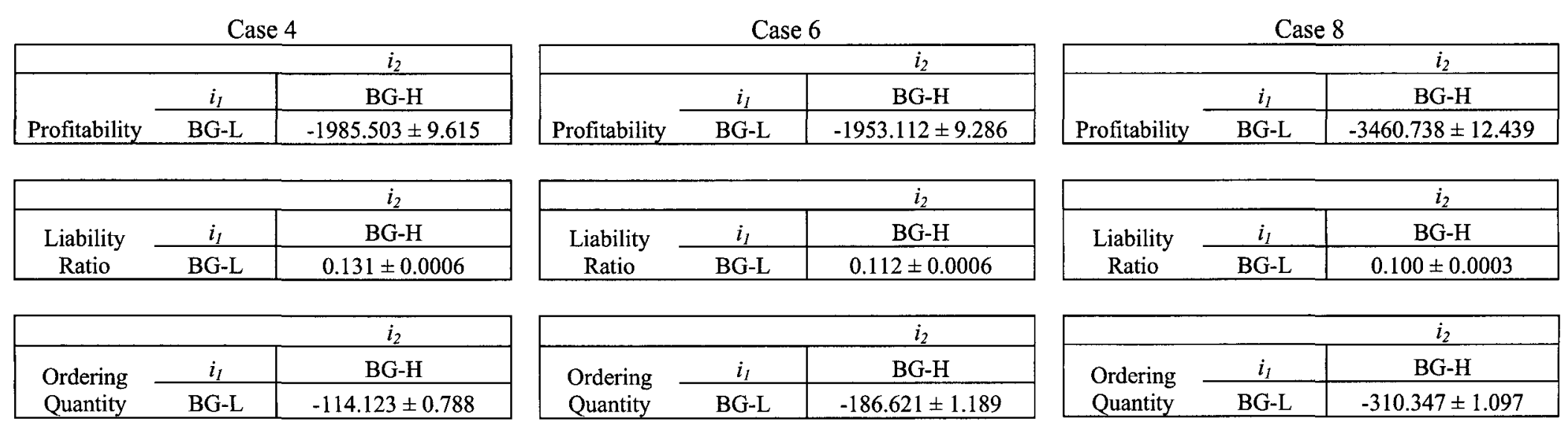

(d) with Uniformly Distributed Demand and Medium Level of Demand Variability (Uniform, DV-M)
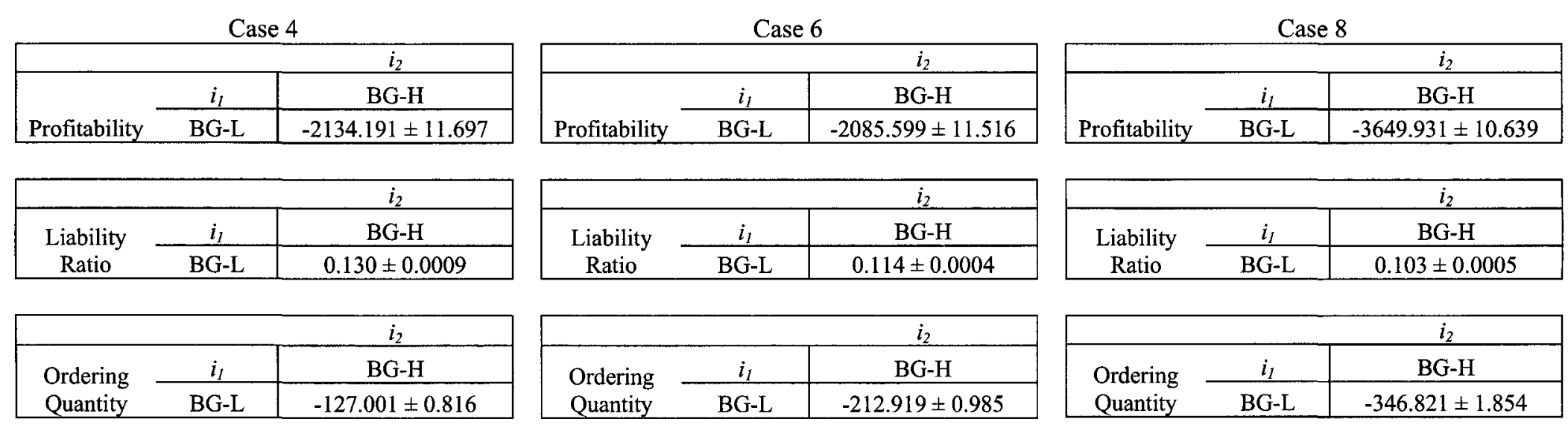

\begin{tabular}{|lc|c|}
\hline & \multicolumn{2}{c|}{$i_{2}$} \\
\hline Ordering & $i_{1}$ & BG-H \\
\cline { 2 - 3 } Quantity & BG-L & $-346.821 \pm 1.854$ \\
\hline
\end{tabular}


Appendix D.5: Comparison of the impacts of budget tightness with a given level of demand variability under EP2 setting (Confidence intervals for $\mathrm{H1}, \mathrm{H} 2$, and $\mathrm{H3},\left(U_{i 2}-U_{i 1}\right) \pm$ half-width) (Cont.)

(e) with Normally Distributed Demand and High Level of Demand Variability (Normal, DV-H)
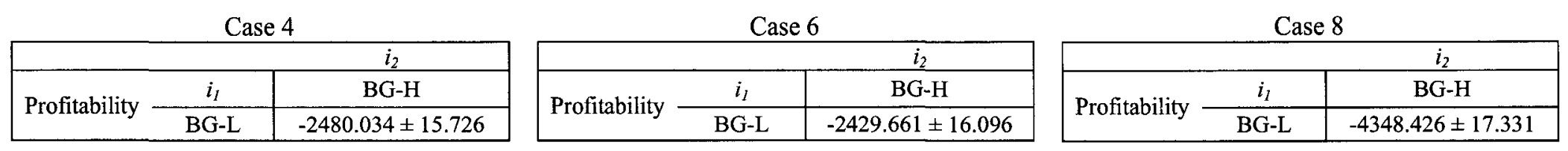

\begin{tabular}{|cc|c|}
\hline & & $i_{2}$ \\
\hline $\begin{array}{c}\text { Liability } \\
\text { Ratio }\end{array}$ & $i_{l}$ & BG-H \\
\cline { 2 - 3 } & BG-L & $0.145 \pm 0.0010$ \\
\hline
\end{tabular}

\begin{tabular}{|cc|c|}
\hline & \multicolumn{2}{c|}{$i_{2}$} \\
\hline $\begin{array}{c}\text { Liability } \\
\text { Ratio }\end{array}$ & $i_{1}$ & BG-H \\
\cline { 2 - 3 } & $\mathrm{BG}-\mathrm{L}$ & $0.128 \pm 0.0011$ \\
\hline
\end{tabular}

\begin{tabular}{|cc|c|}
\hline & \multicolumn{1}{c|}{$i_{2}$} \\
\hline $\begin{array}{c}\text { Liability } \\
\text { Ratio }\end{array}$ & $i_{1}$ & BG-H \\
\cline { 2 - 3 } & BG-L & $0.114 \pm 0.0004$ \\
\hline
\end{tabular}

\begin{tabular}{|c|c|c|}
\hline & & $i_{2}$ \\
\hline \multirow{2}{*}{$\begin{array}{l}\text { Ordering } \\
\text { Quantity }\end{array}$} & $i_{1}$ & BG-H \\
\hline & BG-L & $-224.332 \pm 2.036$ \\
\hline
\end{tabular}

\begin{tabular}{|lc|c|}
\hline & \multicolumn{2}{c|}{$i_{2}$} \\
\hline $\begin{array}{l}\text { Ordering } \\
\text { Quantity }\end{array}$ & $i_{l}$ & BG-H \\
\cline { 2 - 3 } & $\mathrm{BG}-\mathrm{L}$ & $-350.884 \pm 2.195$ \\
\hline
\end{tabular}

\begin{tabular}{|lc|c|}
\hline & \multicolumn{1}{c|}{$i_{2}$} \\
\hline $\begin{array}{l}\text { Ordering } \\
\text { Quantity }\end{array}$ & $i_{1}$ & BG-H \\
\cline { 2 - 3 } & $\mathrm{BG}-\mathrm{L}$ & $-567.059 \pm 1.388$ \\
\hline
\end{tabular}

(f) with Uniformly Distributed Demand and High Level of Demand Variability (Uniform, DV-H)

\section{Case 4}

\begin{tabular}{|cc|c|}
\hline \multicolumn{2}{|c|}{$i_{2}$} \\
\hline \multirow{2}{*}{ Profitability } & $i_{1}$ & BG-H \\
\cline { 2 - 3 } & BG-L & $-2755.038 \pm 26.015$ \\
\hline
\end{tabular}

\begin{tabular}{|cc|c|}
\hline & \multicolumn{2}{c}{$i_{2}$} \\
\hline $\begin{array}{c}\text { Liability } \\
\text { Ratio }\end{array}$ & $i_{1}$ & BG-H \\
\cline { 2 - 3 } & BG-L & $0.148 \pm 0.0007$ \\
\hline
\end{tabular}

\begin{tabular}{|cc|c|}
\hline & \multicolumn{2}{c|}{$i_{2}$} \\
\hline Ordering & $i_{1}$ & BG-H \\
\cline { 2 - 3 } Quantity & BG-L & $-163.822 \pm 1.187$ \\
\hline
\end{tabular}
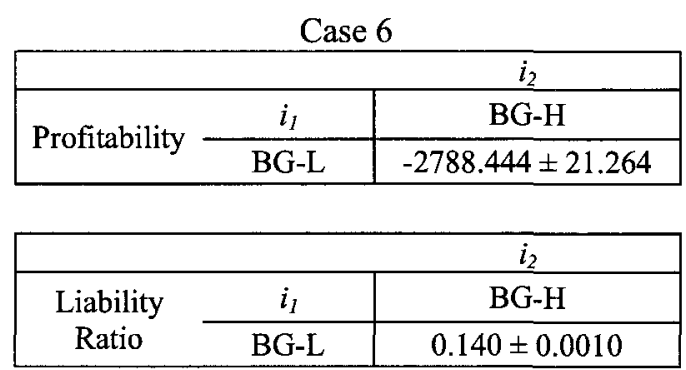

\begin{tabular}{|cc|c|}
\hline & \multicolumn{2}{c|}{$i_{2}$} \\
\hline Ordering & $i_{1}$ & BG-H \\
\cline { 2 - 3 } Quantity & BG-L & $-282.085 \pm 1.264$ \\
\hline
\end{tabular}

Case 8

\begin{tabular}{|cc|c|}
\hline \multicolumn{3}{|c|}{ Case 8 } \\
\hline \multirow{2}{*}{ Profitability } & $i_{1}$ & BG-H \\
\cline { 2 - 3 } & BG-L & $-4863.801 \pm 22.869$ \\
\hline
\end{tabular}

\begin{tabular}{|cc|c|}
\hline & \multicolumn{2}{c|}{$i_{2}$} \\
\hline $\begin{array}{c}\text { Liability } \\
\text { Ratio }\end{array}$ & $i_{1}$ & BG-H \\
\cline { 2 - 3 } & BG-L & $0.125 \pm 0.0008$ \\
\hline
\end{tabular}

\begin{tabular}{|lc|c|}
\hline & \multicolumn{2}{c|}{$i_{2}$} \\
\hline Ordering & $i_{1}$ & $\mathrm{BG}-\mathrm{H}$ \\
\cline { 2 - 3 } Quantity & $\mathrm{BG}-\mathrm{L}$ & $-453 . .849 \pm 1.992$ \\
\hline
\end{tabular}




\section{Appendix D.6: Comparison of the impacts of demand variability with a given level of budget tightness under EP2 setting}

(Confidence intervals for $\mathrm{H4}, \mathrm{H5}$, and $\mathrm{H6},\left(U_{i 2}-U_{i 1}\right) \pm$ half-width)

(a) with Normally Distributed Demand and Low Level of Budget Tightness (Normal, BG-L)

Case 4

\begin{tabular}{|cccc|}
\hline & & \multicolumn{2}{c|}{$j_{2}$} \\
\hline \multirow{4}{*}{ Profitability } & $j_{1}$ & DV-M & DV-H \\
\cline { 2 - 4 } & \multirow{2}{*}{ DV-L } & $\mathbf{4 . 6 7 4 \pm}$ & $-91.065 \pm$ \\
& & $\mathbf{2 2 . 2 5 9 *}$ & 22.259 \\
\cline { 2 - 4 } & \multirow{2}{*}{ DV-M } & & $-95.739 \pm$ \\
& & 22.259 \\
\hline
\end{tabular}

\begin{tabular}{|cccc|}
\hline & & \multicolumn{2}{c}{$j_{2}$} \\
\hline & $j_{1}$ & DV-M & DV-H \\
\cline { 2 - 4 } Liability & \multirow{2}{*}{ DV-L } & $0.025 \pm$ & $0.049 \pm$ \\
Ratio & 0.0008 & 0.0008 \\
\cline { 2 - 4 } & \multirow{2}{*}{ DV-M } & & $0.024 \pm$ \\
& & & 0.0008 \\
\hline
\end{tabular}

\begin{tabular}{|cccc|}
\hline & \multicolumn{3}{c}{$j_{2}$} \\
\hline & $j_{I}$ & DV-M & DV-H \\
\cline { 2 - 4 } Ordering & DV-L & $37.794 \pm$ & $82.812 \pm$ \\
Quantity & 1.761 & 1.761 \\
\cline { 2 - 4 } & & & $45.018 \pm$ \\
& DV-M & & 1.761 \\
\hline
\end{tabular}

Case 6

\begin{tabular}{|c|c|c|c|}
\hline & & \multicolumn{2}{|c|}{$j_{2}$} \\
\hline \multirow{3}{*}{ Profitability } & $j_{1}$ & DV-M & DV-H \\
\hline & DV-L & $\begin{array}{c}512.181 \pm \\
15.825\end{array}$ & $\begin{array}{c}919.077 \pm \\
15.825\end{array}$ \\
\hline & DV-M & & $\begin{array}{c}406.896 \pm \\
15.825 \\
\end{array}$ \\
\hline & & \multicolumn{2}{|c|}{$j_{2}$} \\
\hline \multirow{3}{*}{$\begin{array}{c}\text { Liability } \\
\text { Ratio }\end{array}$} & $j_{1}$ & DV-M & DV-H \\
\hline & DV-L & $\begin{array}{c}0.043 \pm \\
0.0008\end{array}$ & $\begin{array}{c}0.083 \pm \\
0.0008\end{array}$ \\
\hline & DV-M & & $\begin{array}{l}0.04 \pm \\
0.0008\end{array}$ \\
\hline
\end{tabular}

\begin{tabular}{|cccc|}
\hline & & \multicolumn{2}{c}{$j_{2}$} \\
\hline & $j_{1}$ & DV-M & DV-H \\
\cline { 2 - 4 } Ordering & \multirow{2}{*}{ DV-L } & $67.683 \pm$ & $139.48 \pm$ \\
Quantity & & 1.717 & 1.717 \\
\cline { 2 - 4 } & \multirow{2}{*}{ DV-M } & & $71.797 \pm$ \\
& & & 1.717 \\
\hline
\end{tabular}

Case 8

\begin{tabular}{|c|c|c|c|}
\hline & & \multicolumn{2}{|c|}{$j_{2}$} \\
\hline \multirow{3}{*}{ Profitability } & $j_{1}$ & DV-M & DV-H \\
\hline & DV-L & $\begin{array}{l}1000.526 \\
\pm 24.262\end{array}$ & $\begin{array}{l}1000.526 \\
\pm 24.262\end{array}$ \\
\hline & \multicolumn{2}{|l|}{ DV-M } & $\begin{array}{c}767.138 \pm \\
24.262\end{array}$ \\
\hline & & \multicolumn{2}{|c|}{$j_{2}$} \\
\hline \multirow{3}{*}{$\begin{array}{c}\text { Liability } \\
\text { Ratio }\end{array}$} & $j_{l}$ & DV-M & DV-H \\
\hline & DV-L & $\begin{array}{c}0.044 \pm \\
0.0002\end{array}$ & $\begin{array}{c}0.085 \pm \\
0.0002\end{array}$ \\
\hline & DV-M & & $\begin{array}{c}0.041 \pm \\
0.0002\end{array}$ \\
\hline
\end{tabular}

\begin{tabular}{|cccc|}
\hline & \multicolumn{3}{c}{$j_{2}$} \\
\hline & $j_{1}$ & $\mathrm{DV}-\mathrm{M}$ & $\mathrm{DV}-\mathrm{H}$ \\
\cline { 2 - 4 } Ordering & $\mathrm{DV}-\mathrm{L}$ & $112.061 \pm$ & $230.047 \pm$ \\
Quantity & 1.264 & 1.264 \\
\cline { 2 - 4 } & & & $117.986 \pm$ \\
& $\mathrm{DV}-\mathrm{M}$ & & 1.264 \\
\hline
\end{tabular}




\section{Appendix D.6: Comparison of the impacts of demand variability with a given level of budget tightness under EP2 setting}

(Confidence intervals for $\mathrm{H4}, \mathrm{H5}$, and $\mathrm{H6},\left(U_{i 2}-U_{i 1}\right) \pm$ half-width)

(Cont.)

(b) with Uniformly Distributed Demand and Low Level of Budget Tightness (Uniform, BG-L)

\begin{tabular}{|cccc|}
\hline \multicolumn{4}{c|}{ Case 4 } \\
\hline \multirow{4}{*}{ Profitability } & \multicolumn{3}{c|}{$j_{2}$} \\
\cline { 2 - 4 } & $j_{1}$ & DV-M & DV-H \\
\cline { 2 - 4 } & DV-L & $\mathbf{2 1 . 9 1 4 \pm}$ & $\mathbf{- 5 3 . 6 8 9 \pm}$ \\
\cline { 2 - 4 } & DV-M & & 32.025 \\
& & $-75.603 \pm$ \\
& & 32.025 \\
\hline
\end{tabular}

\begin{tabular}{|cccc|}
\hline \multicolumn{4}{c}{ Case 6 } \\
\hline \multirow{4}{*}{ Profitability } & \multicolumn{3}{c|}{$j_{2}$} \\
\cline { 2 - 4 } & $j_{1}$ & DV-M & DV-H \\
\cline { 2 - 4 } & \multirow{2}{*}{ DV-L } & $478.774 \pm$ & $910.509 \pm$ \\
& & 23.126 & 23.126 \\
\cline { 2 - 4 } & DV-M & & $431.735 \pm$ \\
& & 23.126 \\
\hline
\end{tabular}

\begin{tabular}{|cccc|}
\hline \multicolumn{4}{c}{ Case 8 } \\
\hline \multirow{4}{*}{ Profitability } & \multicolumn{3}{c|}{$j_{2}$} \\
\cline { 2 - 4 } & \multirow{2}{*}{$j_{1}$} & DV-M & DV-H \\
\cline { 2 - 4 } & \multirow{2}{*}{ DV-L } & $16.9047 \pm$ & 1874.283 \\
& \multirow{2}{*}{ DV-M } & & $882.236 \pm$ \\
& & 16.905 \\
\hline
\end{tabular}

\begin{tabular}{|cccc|}
\hline & & \multicolumn{2}{c|}{$j_{2}$} \\
\hline & $j_{I}$ & DV-M & DV-H \\
\cline { 2 - 4 } Liability & \multirow{2}{*}{ DV-L } & $0.02 \pm$ & $0.042 \pm$ \\
Ratio & & 0.0012 & 0.0012 \\
\cline { 2 - 4 } & \multirow{2}{*}{ DV-M } & & $0.022 \pm$ \\
& & & 0.0012 \\
\hline
\end{tabular}

\begin{tabular}{|cccc|}
\hline & \multicolumn{3}{c}{$j_{2}$} \\
\hline & $j_{1}$ & DV-M & DV-H \\
\cline { 2 - 4 } Liability & \multirow{2}{*}{ DV-L } & $0.037 \pm$ & $0.076 \pm$ \\
Ratio & & 0.0009 & 0.0009 \\
\cline { 2 - 4 } & \multirow{2}{*}{ DV-M } & & $0.039 \pm$ \\
& & & 0.0009 \\
\hline
\end{tabular}

\begin{tabular}{|cccc|}
\hline & \multicolumn{3}{c}{$J_{2}$} \\
\hline & $\dot{j}_{1}$ & $\mathrm{DV}-\mathrm{M}$ & $\mathrm{DV}-\mathrm{H}$ \\
\cline { 2 - 4 } Liability & \multirow{2}{*}{$\mathrm{DV}-\mathrm{L}$} & $0.037 \pm$ & $0.077 \pm$ \\
Ratio & 0.0006 & 0.0006 \\
\cline { 2 - 4 } & \multirow{2}{*}{$\mathrm{DV}-\mathrm{M}$} & & $0.04 \pm$ \\
& & & 0.0006 \\
\hline
\end{tabular}

\begin{tabular}{|cccc|}
\hline & & \multicolumn{2}{c|}{$j_{2}$} \\
\hline & $j_{1}$ & DV-M & DV-H \\
\cline { 2 - 4 } Ordering & \multirow{2}{*}{ DV-L } & 36.381 & 70.806 \\
Quantity & \pm 1.156 & \pm 1.156 \\
\cline { 2 - 4 } & \multirow{2}{*}{ DV-M } & & 34.625 \\
& & \pm 1.156 \\
\hline
\end{tabular}

\begin{tabular}{|cccc|}
\hline & \multicolumn{3}{c|}{$j_{2}$} \\
\hline & $j_{1}$ & DV-M & DV-H \\
\cline { 2 - 4 } Ordering & DV-L & $67.313 \pm$ & $131.862 \pm$ \\
Quantity & 1.359 & 1.359 \\
\cline { 2 - 4 } & \multirow{2}{*}{ DV-M } & & $64.549 \pm$ \\
& & 1.359 \\
\hline
\end{tabular}

\begin{tabular}{|cccc|}
\hline & & \multicolumn{2}{c|}{$j_{2}$} \\
\hline & $j_{I}$ & DV-M & DV-H \\
\cline { 2 - 4 } Ordering & \multirow{2}{*}{ DV-L } & 104.854 & 205.405 \\
Quantity & \pm 1.978 & \pm 1.978 \\
\cline { 2 - 4 } & \multirow{2}{*}{ DV-M } & & 100.551 \\
& & \pm 1.978 \\
\hline
\end{tabular}


Appendix D.6: Comparison of the impacts of demand variability with a given level of budget tightness under EP2 setting (Confidence intervals for $\mathrm{H} 4, \mathrm{H5}$, and $\mathrm{H6},\left(U_{i 2}-U_{i I}\right) \pm$ half-width)

(Cont.)

(c) with Normally Distributed Demand and High Level of Budget Tightness (Normal, BG-H)

Case 4

\begin{tabular}{|cccc|}
\hline & & \multicolumn{2}{c|}{$j_{2}$} \\
\hline \multirow{4}{*}{ Profitability } & $j_{1}$ & DV-M & DV-H \\
\cline { 2 - 4 } & \multirow{2}{*}{ DV-L } & -488.975 & -1079.245 \\
& & \pm 11.932 & \pm 11.932 \\
\cline { 2 - 4 } & \multirow{2}{*}{ DV-M } & & $-590.27 \pm$ \\
& & 11.932 \\
\hline
\end{tabular}

\begin{tabular}{|cccc|}
\hline & & \multicolumn{2}{c|}{$j_{2}$} \\
\hline \multirow{4}{*}{ Liability } & $j_{1}$ & DV-M & DV-H \\
\cline { 2 - 4 } Ratio & \multirow{2}{*}{ DV-L } & $0.042 \pm$ & $0.08 \pm$ \\
& & 0.0013 & 0.0013 \\
\cline { 2 - 4 } & \multirow{2}{*}{ DV-M } & & $0.038 \pm$ \\
& & & 0.0013 \\
\hline
\end{tabular}

\begin{tabular}{|cccc|}
\hline & \multicolumn{3}{c}{$j_{2}$} \\
\hline & $j_{1}$ & DV-M & DV-H \\
\cline { 2 - 4 } Ordering & \multirow{2}{*}{ DV-L } & $-5.915 \pm$ & $-71.106 \pm$ \\
Quantity & 0.471 & 0.471 \\
\cline { 2 - 4 } & \multirow{2}{*}{ DV-M } & & $-65.191 \pm$ \\
& & & 0.471 \\
\hline
\end{tabular}

Case 6

\begin{tabular}{|c|c|c|c|}
\hline & & \multicolumn{2}{|c|}{$j_{2}$} \\
\hline \multirow{3}{*}{ Profitability } & $j_{1}$ & DV-M & DV-H \\
\hline & DV-L & $\begin{array}{c}128.214 \pm \\
13.552\end{array}$ & $\begin{array}{c}58.561 \pm \\
13.552\end{array}$ \\
\hline & DV-M & & $\begin{array}{c}-69.653 \pm \\
13.552\end{array}$ \\
\hline & & \multicolumn{2}{|c|}{$j_{2}$} \\
\hline \multirow{3}{*}{$\begin{array}{l}\text { Liability } \\
\text { Ratio }\end{array}$} & $j_{I}$ & DV-M & DV-H \\
\hline & DV-L & $\begin{array}{c}0.071 \pm \\
0.0017\end{array}$ & $\begin{array}{l}0.127 \pm \\
0.0017\end{array}$ \\
\hline & DV-M & & $\begin{array}{c}0.056 \pm \\
0.0017\end{array}$ \\
\hline
\end{tabular}

\begin{tabular}{|cccc|}
\hline & \multicolumn{3}{c}{$j_{2}$} \\
\hline & $j_{1}$ & DV-M & DV-H \\
\cline { 2 - 4 } Ordering & \multirow{2}{*}{ DV-L } & $-20.799 \pm$ & -113.265 \\
Quantity & 1.714 & \pm 1.714 \\
\cline { 2 - 4 } & \multirow{2}{*}{ DV-M } & & $-92.466 \pm$ \\
& & & 1.714 \\
\hline
\end{tabular}

Case 8

\begin{tabular}{|c|c|c|c|}
\hline & & \multicolumn{2}{|c|}{$j_{2}$} \\
\hline \multirow{3}{*}{ Profitability } & $j_{l}$ & DV-M & DV-H \\
\hline & DV-L & $\begin{array}{c}335.318 \pm \\
13.058 \\
\end{array}$ & $\begin{array}{c}214.768 \pm \\
13.058 \\
\end{array}$ \\
\hline & DV-M & & $\begin{array}{c}-120.55 \pm \\
13.058 \\
\end{array}$ \\
\hline & & \multicolumn{2}{|c|}{$j_{2}$} \\
\hline \multirow{3}{*}{$\begin{array}{c}\text { Liability } \\
\text { Ratio }\end{array}$} & $j_{1}$ & DV-M & $\mathrm{DV}-\mathrm{H}$ \\
\hline & $D V-L$ & $\begin{array}{c}0.066 \pm \\
0.0007 \\
\end{array}$ & $\begin{array}{l}0.121 \pm \\
0.0007\end{array}$ \\
\hline & DV-M & & $\begin{array}{c}0.055 \pm \\
0.0007\end{array}$ \\
\hline & & \multicolumn{2}{|c|}{$i_{i}$} \\
\hline \multirow{3}{*}{$\begin{array}{l}\text { Ordering } \\
\text { Quantity }\end{array}$} & $j_{I}$ & DV-M & DV-H \\
\hline & DV-L & $\begin{array}{c}-30.281 \pm \\
1.617\end{array}$ & $\begin{array}{c}-169.007 \\
\pm 1.617\end{array}$ \\
\hline & DV-M & & $\begin{array}{c}-138.726 \\
\pm 1.617 \\
\end{array}$ \\
\hline
\end{tabular}


Appendix D.6: Comparison of the impacts of demand variability with a given level of budget tightness under EP2 setting (Confidence intervals for $\mathrm{H4}, \mathrm{H5}$, and $\mathrm{H6},\left(U_{i 2}-U_{i I}\right) \pm$ half-width)

(Cont.)

(d) with Uniformly Distributed Demand and High Level of Budget Tightness (Uniform, BG-H)

\begin{tabular}{|cccc|}
\hline \multicolumn{3}{c}{ Case 4 } \\
\hline \multirow{4}{*}{ Profitability } & \multicolumn{2}{c|}{$j_{2}$} \\
\cline { 2 - 4 } & $j_{1}$ & DV-M & DV-H \\
\cline { 2 - 4 } & DV-L & -471.863 & -1168.313 \\
\cline { 2 - 4 } & & \pm 11.768 & \pm 11.768 \\
\cline { 2 - 4 } & DV-M & & $-696.45 \pm$ \\
& & 11.768 \\
\hline
\end{tabular}

\begin{tabular}{|c|c|c|c|}
\hline \multicolumn{4}{|c|}{ Case 6} \\
\hline & & & \\
\hline \multirow{3}{*}{ Profitabılity } & $j_{l}$ & DV-M & DV-H \\
\hline & DV-L & $\begin{array}{l}78.03 \pm \\
18.312\end{array}$ & $\begin{array}{c}-193.08 \pm \\
18.312\end{array}$ \\
\hline & DV-M & & $\begin{array}{c}-271.11 \pm \\
18.312 \\
\end{array}$ \\
\hline & & \multicolumn{2}{|c|}{$j_{2}$} \\
\hline \multirow{3}{*}{$\begin{array}{c}\text { Liability } \\
\text { Ratio }\end{array}$} & $j_{I}$ & DV-M & DV-H \\
\hline & DV-L & $\begin{array}{l}0.06 \pm \\
0.0010\end{array}$ & $\begin{array}{c}0.125 \pm \\
0.0010 \\
\end{array}$ \\
\hline & DV-M & & $\begin{array}{c}0.065 \pm \\
0.0010\end{array}$ \\
\hline
\end{tabular}

\begin{tabular}{|c|c|c|c|}
\hline \multicolumn{4}{|c|}{ Case 8} \\
\hline & & \multicolumn{2}{|c|}{$j_{2}$} \\
\hline \multirow{3}{*}{ Profitability } & $j_{I}$ & DV-M & DV-H \\
\hline & DV-L & $\begin{array}{c}318.286 \pm \\
16.294\end{array}$ & $\begin{array}{r}-13.348= \\
16.294^{*}\end{array}$ \\
\hline & \multicolumn{2}{|l|}{ DV-M } & $\begin{array}{r}-331.634 \\
\pm 16.294 \\
\end{array}$ \\
\hline & & \multicolumn{2}{|c|}{$j_{2}$} \\
\hline \multirow{3}{*}{$\begin{array}{c}\text { Liability } \\
\text { Ratio }\end{array}$} & $\overline{j_{I}}$ & DV-M & DV-H \\
\hline & DV-L & $\begin{array}{c}0.056 \pm \\
0.0011 \\
\end{array}$ & $\begin{array}{c}0.118 \pm \\
0.0011 \\
\end{array}$ \\
\hline & DV-M & & $\begin{array}{c}0.062 \pm \\
0.0011 \\
\end{array}$ \\
\hline
\end{tabular}

\begin{tabular}{|cccc|}
\hline & \multicolumn{3}{c}{$j_{2}$} \\
\hline & $j_{1}$ & DV-M & DV-H \\
\cline { 2 - 4 } Ordering & \multirow{2}{*}{ DV-L } & -4.836 & -7.032 \\
Quantity & \pm 0.437 & \pm 0.437 \\
\cline { 2 - 4 } & \multirow{2}{*}{ DV-M } & & -2.196 \\
& & \pm 0.437 \\
\hline
\end{tabular}

\begin{tabular}{|cccc|}
\hline & \multicolumn{3}{c}{$j_{2}$} \\
\hline & $j_{l}$ & DV-M & DV-H \\
\cline { 2 - 4 } Ordering & \multirow{2}{*}{ DV-L } & $-14.868 \pm$ & $-19.485 \pm$ \\
Quantity & & 0.574 & 0.574 \\
\cline { 2 - 4 } & \multirow{2}{*}{ DV-M } & & $-4.617 \pm$ \\
& & & 0.574 \\
\hline
\end{tabular}

\begin{tabular}{|cccc|}
\hline & & \multicolumn{2}{c|}{$j_{2}$} \\
\hline & $j_{1}$ & DV-M & DV-H \\
\cline { 2 - 4 } Ordering & \multirow{2}{*}{ DV-L } & -21.328 & -27.805 \\
Quantity & \pm 1.895 & \pm 1.895 \\
\cline { 2 - 4 } & \multirow{2}{*}{ DV-M } & & -6.477 \\
& & \pm 1.895 \\
\hline
\end{tabular}




\section{Appendix D.7: Comparison of the impacts of the model settings with a given level of budget tightness and}

a given level of demand variability (Confidence intervals for $\mathrm{H7}, \mathrm{H8}$, and $\mathrm{H9},\left(U_{i 2}-U_{i 1}\right) \pm$ half-width)

(a) with Normally Distributed Demand and Low Level of budget tightness and low level of Demand Variability (Normal, BG-L, DV-L)
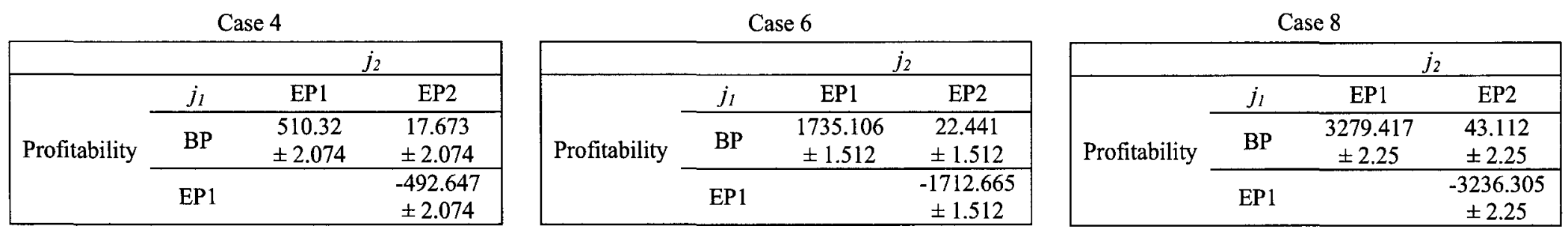

\begin{tabular}{|cccc|}
\hline & & \multicolumn{2}{c|}{$J_{2}$} \\
\hline & $j_{1}$ & $\mathrm{EP} 1$ & $\mathrm{EP} 2$ \\
\cline { 2 - 4 } Liability & $\mathrm{BP}$ & $0.082 \pm$ & $\mathbf{0 . 0 0 0 \pm}$ \\
Ratio & & 0.00004 & $\mathbf{0 . 0 0 0 0 4}$ \\
\cline { 2 - 4 } & $\mathrm{EP} 1$ & & $-0.082 \pm$ \\
& & & $\mathbf{0 . 0 0 0 0 4}$ \\
\hline
\end{tabular}

\begin{tabular}{|cccc|}
\hline & \multicolumn{3}{c}{$j_{2}$} \\
\hline & $j_{1}$ & EP1 & EP2 \\
\cline { 2 - 4 } Liability & BP & $0.151 \pm$ & $\mathbf{0 . 0 0 0 \pm}$ \\
Ratio & & 0.00006 & $\mathbf{0 . 0 0 0 0 6}$ \\
\cline { 2 - 4 } & EP1 & & $-0.151 \pm$ \\
& & & 0.00006 \\
\hline
\end{tabular}

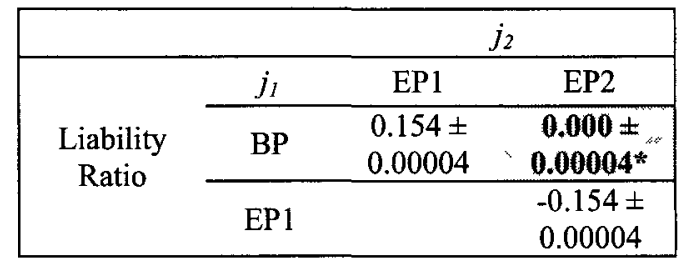

\begin{tabular}{|cccc|}
\hline & \multicolumn{3}{c|}{$j_{2}$} \\
\hline & $j_{1}$ & $\mathrm{EP} 1$ & $\mathrm{EP} 2$ \\
\cline { 2 - 4 } Ordering & $\mathrm{BP}$ & $\mathbf{0 . 0 0 0}$ & -5.234 \\
Quantity & & $\pm \mathbf{0 . 2 4 2 *}$ & \pm 0.242 \\
\cline { 2 - 4 } & $\mathrm{EP} 1$ & & -5.234 \\
& & \pm 0.242 \\
\hline
\end{tabular}

\begin{tabular}{|cccc|}
\hline & \multicolumn{3}{c|}{$j_{2}$} \\
\hline & $j_{1}$ & $\mathrm{EP} 1$ & $\mathrm{EP} 2$ \\
\cline { 2 - 4 } Ordering & $\mathrm{BP}$ & $\mathbf{0 . 0 0 0}$ & -7.743 \\
Quantity & & $\pm \mathbf{0 . 2 8 3 ^ { * }}$ & \pm 0.283 \\
\cline { 2 - 4 } & $\mathrm{EP} 1$ & & $\begin{array}{c}-7.743 \\
\pm 0.283\end{array}$ \\
\hline
\end{tabular}

\begin{tabular}{|cccc|}
\hline & \multicolumn{3}{c}{$j_{2}$} \\
\hline & $j_{1}$ & $E P 1$ & $E P 2$ \\
\cline { 2 - 4 } Ordernng & $\mathrm{BP}$ & $\mathbf{0 . 0 0 0}$ & -12.706 \\
Quantity & & $\mathbf{0 . 1 5 9 ^ { * }}$ & \pm 0.159 \\
\cline { 2 - 4 } & $\mathrm{EP} 1$ & & -12.706 \\
& & \pm 0.159 \\
\hline
\end{tabular}


Appendix D.7: Comparison of the impacts of the model settings with a given level of budget tightness and a given level of demand variability (Confidence intervals for $\mathrm{H7}, \mathrm{H8}$, and $\mathrm{H9},\left(U_{i 2}-U_{i 1}\right) \pm$ half-width)

$$
\text { (Cont.) }
$$

(b) with Uniformly Distributed Demand and Low Level of budget tightness and low level of Demand Variability (Uniform, BG-L, DV-L)

\begin{tabular}{|cccc|}
\hline \multicolumn{4}{c}{ Case 4 } \\
\hline & \multicolumn{3}{c|}{$j_{2}$} \\
\hline \multirow{4}{*}{ Profitability } & $j_{I}$ & EP1 & EP2 \\
\cline { 2 - 4 } & \multirow{2}{*}{ BP } & 397.180 & 98.222 \\
& & \pm 9.577 & \pm 9.577 \\
\cline { 2 - 4 } & \multirow{2}{*}{ EP1 } & & -298.958 \\
& & \pm 9.577 \\
\hline
\end{tabular}

\begin{tabular}{|cccc|}
\hline \multicolumn{4}{c}{ Case 6 } \\
\hline \multirow{4}{*}{ Profitability } & \multicolumn{3}{c|}{$J_{2}$} \\
\cline { 2 - 4 } & $j_{1}$ & $\mathrm{EP} 1$ & $\mathrm{EP} 2$ \\
\cline { 2 - 4 } & $\mathrm{BP}$ & $\begin{array}{c}1341.436 \\
\pm 6.494\end{array}$ & $\begin{array}{c}78.050 \\
\pm 6.494\end{array}$ \\
\cline { 2 - 4 } & \multirow{2}{*}{$\mathrm{EP} 1$} & & $\begin{array}{c}-1263.386 \\
\pm 6.494\end{array}$ \\
\hline
\end{tabular}

\begin{tabular}{|cccc|}
\hline \multicolumn{4}{c}{ Case 8 } \\
\hline & \multicolumn{3}{c|}{$j_{2}$} \\
\hline & $j_{1}$ & $\mathrm{EP} 1$ & $\mathrm{EP} 2$ \\
\cline { 2 - 4 } Profitability & \multirow{2}{*}{$\mathrm{BP}$} & $\begin{array}{c}2527.869 \\
\pm 4.288\end{array}$ & $\begin{array}{c}162.885 \\
\pm 4.288\end{array}$ \\
\cline { 2 - 4 } & \multirow{2}{*}{$\mathrm{EP} 1$} & & $\begin{array}{c}-2364.984 \\
\pm 4.288\end{array}$ \\
\hline
\end{tabular}
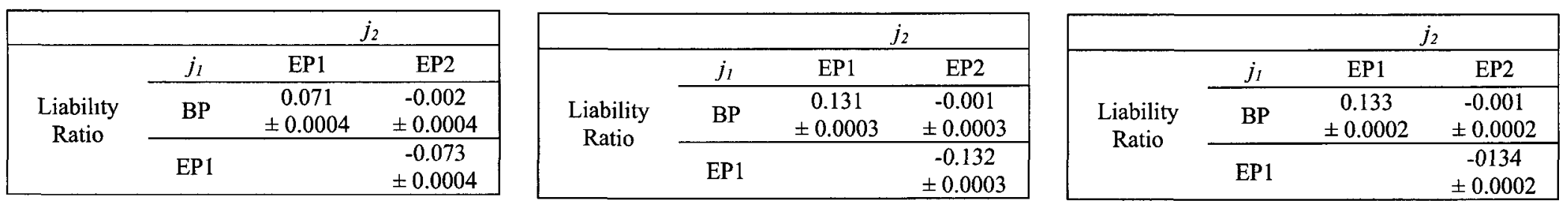

\begin{tabular}{|cccc|}
\hline & \multicolumn{3}{c|}{$j_{2}$} \\
\hline & $j_{1}$ & $\mathrm{EP} 1$ & $\mathrm{EP} 2$ \\
\cline { 2 - 4 } Ordering & \multirow{2}{*}{$\mathrm{BP}$} & $\mathbf{0 . 0 0 0}$ & -14.944 \\
Quantity & & $\mathbf{0 . 2 6 1 ^ { * }}$ & $\pm \mathbf{0 . 2 6 1}$ \\
\cline { 2 - 4 } & \multirow{2}{*}{$\mathrm{EP} 1$} & & -14.944 \\
& & \pm 0.261 \\
\hline
\end{tabular}

\begin{tabular}{|cccc|}
\hline & \multicolumn{3}{c|}{$j_{2}$} \\
\hline & $j_{1}$ & $\mathrm{EP} 1$ & $\mathrm{EP} 2$ \\
\cline { 2 - 4 } Ordering & $\mathrm{BP}$ & $\mathbf{0 . 0 0 0}$ & -24.951 \\
Quantity & & $\mathbf{0 . 4 7 9 *}$ & \pm 0.479 \\
\cline { 2 - 4 } & $\mathrm{EP} 1$ & & -24.951 \\
& & & \pm 0.479 \\
\hline
\end{tabular}

\begin{tabular}{|lccc|}
\hline & \multicolumn{3}{c}{$j_{2}$} \\
\hline & $j_{1}$ & EP1 & EP2 \\
\cline { 2 - 4 } Ordering & \multirow{2}{*}{$\mathrm{BP}$} & $\mathbf{0 . 0 0 0}$ & $\mathbf{- 3 7 . 9 2 8}$ \\
Quantity & & $\mathbf{1 . 0 1 5}$ & \pm 1.015 \\
\cline { 2 - 4 } & \multirow{2}{*}{$\mathrm{EP1}$} & & $\begin{array}{c}\text { *37.928 } \\
\end{array}$ \\
& & & \pm 1.015 \\
\hline
\end{tabular}


Appendix D.7: Comparison of the impacts of the model settings with a given level of budget tightness and a given level of demand variability (Confidence intervals for $\mathrm{H7}, \mathrm{H8}$, and $\mathrm{H9},\left(U_{i 2}-U_{i 1}\right) \pm$ half-width)

\section{(Cont.)}

(c) with Normally Distributed Demand and Low Level of budget tightness and Medium level of Demand Variability (Normal, BG-L, DV-M)

\begin{tabular}{|cccc|}
\multicolumn{4}{c}{ Case 4 } \\
\hline \multirow{4}{*}{$j_{1}$} & \multicolumn{3}{c|}{$j_{2}$} \\
\cline { 2 - 4 } PP1 & EP2 \\
\cline { 2 - 4 } Profitability & BP & 155.795 & 326.531 \\
& & \pm 17.077 & \pm 17.077 \\
\cline { 2 - 4 } & EP1 & & 170.736 \\
& & \pm 17.077 \\
\hline
\end{tabular}

\begin{tabular}{|cccc|}
\hline \multicolumn{4}{c}{ Case 6 } \\
\hline \multirow{4}{*}{ Profitability } & \multicolumn{3}{c|}{$j_{2}$} \\
\cline { 2 - 4 } & $j_{1}$ & $\mathrm{EP} 1$ & $\mathrm{EP} 2$ \\
\cline { 2 - 4 } & $\mathrm{BP}$ & 578.746 & 281.913 \\
& & \pm 13.739 & \pm 13.739 \\
\cline { 2 - 4 } & $\mathrm{EP} 1$ & & -296.833 \\
& & \pm 13.739 \\
\hline
\end{tabular}

\begin{tabular}{|cccc|}
\multicolumn{4}{c}{ Case 8 } \\
\hline \multirow{4}{*}{$j_{1}$} & \multicolumn{2}{c|}{$j_{2}$} \\
\hline \multirow{4}{*}{ Profitability } & EP2 \\
\cline { 2 - 4 } & BP & 1112.991 & 553.949 \\
& & \pm 18.781 & \pm 18.781 \\
\cline { 2 - 4 } & EP1 & & -559.042 \\
& & \pm 18.781 \\
\hline
\end{tabular}

\begin{tabular}{|cccc|}
\hline & \multicolumn{3}{c|}{$j_{2}$} \\
\hline & $j_{1}$ & $\mathrm{EP} 1$ & $\mathrm{EP} 2$ \\
\cline { 2 - 4 } Liability & $\mathrm{BP}$ & $0.034 \pm$ & $-0.007 \pm$ \\
Ratio & & 0.0009 & 0.0009 \\
\cline { 2 - 4 } & $\mathrm{EP} 1$ & & $\begin{array}{c}-0.041 \pm \\
0.0009\end{array}$ \\
\hline
\end{tabular}

\begin{tabular}{|cccc|}
\hline & \multicolumn{3}{c}{$j_{2}$} \\
\hline & $j_{1}$ & $\mathrm{EP} 1$ & $\mathrm{EP} 2$ \\
\cline { 2 - 4 } Liability & $\mathrm{BP}$ & $0.068 \pm$ & $-0.006 \pm$ \\
Ratio & & 0.0008 & 0.0008 \\
\cline { 2 - 4 } & $\mathrm{EP} 1$ & & $-0.074 \pm$ \\
& & & 0.0008 \\
\hline
\end{tabular}

\begin{tabular}{|cccc|}
\hline & \multicolumn{3}{c}{$j_{2}$} \\
\hline & $j_{1}$ & $\mathrm{EP} 1$ & $\mathrm{EP} 2$ \\
\cline { 2 - 4 } Liability & \multirow{2}{*}{ BP } & $0.069 \pm$ & $-0.007 \pm$ \\
Ratio & & 0.0007 & 0.0007 \\
\cline { 2 - 4 } & $\mathrm{EP} 1$ & & $\begin{array}{c}-0.076 \pm \\
0.0007\end{array}$ \\
\hline
\end{tabular}

\begin{tabular}{|cccc|}
\hline & \multicolumn{3}{c}{$j_{2}$} \\
\hline & $j_{1}$ & $\mathrm{EP} 1$ & $\mathrm{EP} 2$ \\
\cline { 2 - 4 } Ordering & $\mathrm{BP}$ & $\mathbf{0 . 0 0 0}$ & -42.151 \\
Quantity & & $\mathbf{2 . 6 2 4 ^ { * }}$ & \pm 2.624 \\
\cline { 2 - 4 } & & & -42.151 \\
& $\mathrm{EP} 1$ & & \pm 2.624 \\
\hline
\end{tabular}

\begin{tabular}{|cccc|}
\hline & \multicolumn{3}{c}{$j_{2}$} \\
\hline & $j_{1}$ & EP1 & EP2 \\
\cline { 2 - 4 } Ordering & BP & $\mathbf{0 . 0 0 0}$ & -60.242 \\
Quantity & & $\pm \mathbf{2 . 6 0 4} *$ & \pm 2.604 \\
\cline { 2 - 4 } & \multirow{2}{*}{$\mathrm{EP} 1$} & & -60.242 \\
& & \pm 2.604 \\
\hline
\end{tabular}

\begin{tabular}{|c|c|c|c|}
\hline & & \multicolumn{2}{|c|}{$\overline{j_{2}}$} \\
\hline \multirow{3}{*}{$\begin{array}{l}\text { Ordering } \\
\text { Quantity }\end{array}$} & $j_{l}$ & EP1 & EP2 \\
\hline & BP & $\begin{aligned} & 0.0000^{3 \times x^{*}} \\
\pm & 1.474^{*}\end{aligned}$ & $\begin{array}{l}-91.693 \\
\pm 1.474\end{array}$ \\
\hline & EP1 & & $\begin{array}{l}-91.693 \\
\pm 1.474\end{array}$ \\
\hline
\end{tabular}


Appendix D.7: Comparison of the impacts of the model settings with a given level of budget tightness and a given level of demand variability (Confidence intervals for $\mathrm{H} 7, \mathrm{H8}$, and $\mathrm{H9},\left(U_{i 2}-U_{i 1}\right) \pm$ half-width)

(Cont.)

(d) with Uniformly Distributed Demand and Low Level of budget tightness and Medium level of Demand Variability (Uniform, BG-L, DV-M)

\begin{tabular}{|cccc|}
\hline \multicolumn{4}{c}{ Case 4 } \\
\hline \multirow{4}{*}{ Profitability } & \multicolumn{3}{c|}{$j_{2}$} \\
\cline { 2 - 4 } & \multirow{2}{*}{$\mathrm{BP}$} & 238.216 & $\mathrm{EP} 2$ \\
\cline { 2 - 4 } & \multirow{2}{*}{$\mathrm{EP} 1$} & & 378.089 \\
& & & 139.873 \\
& & & \pm 28.185 \\
\hline
\end{tabular}

\begin{tabular}{|cccc|}
\hline \multicolumn{4}{c}{ Case 6 } \\
\hline & \multicolumn{3}{c|}{$J_{2}$} \\
\hline \multirow{4}{*}{ Profitability } & $j_{l}$ & $\mathrm{EP} 1$ & $\mathrm{EP} 2$ \\
\cline { 2 - 4 } & $\mathrm{BP}$ & $\begin{array}{c}806.505 \\
\pm 19.637\end{array}$ & $\begin{array}{c}359.277 \\
\pm 19.637\end{array}$ \\
\cline { 2 - 4 } & \multirow{2}{*}{$\mathrm{EP} 1$} & & $\begin{array}{c}-447.228 \\
\pm 19.637\end{array}$ \\
\hline
\end{tabular}

\begin{tabular}{|cccc|}
\hline \multicolumn{4}{c}{ Case 8 } \\
\hline \multirow{4}{*}{ Profitability } & \multicolumn{3}{c|}{$J_{2}$} \\
\cline { 2 - 4 } & $j_{1}$ & EP1 & EP2 \\
\cline { 2 - 4 } & \multirow{2}{*}{ BP } & 1518.962 & 650.437 \\
& \multirow{2}{*}{ EP1 } & & $\begin{array}{c}\mathbf{8} 2.668 .525 \\
\end{array}$ \\
& & & \pm 12.665 \\
\hline
\end{tabular}

\begin{tabular}{|cccc|}
\hline & \multicolumn{3}{c}{$j_{2}$} \\
\hline & $j_{l}$ & $\mathrm{EP} 1$ & $\mathrm{EP} 2$ \\
\cline { 2 - 4 } Liability & \multirow{2}{*}{$\mathrm{BP}$} & $0.051 \pm$ & $-0.008 \pm$ \\
Ratio & & 0.0011 & 0.0011 \\
\cline { 2 - 4 } & \multirow{2}{*}{$\mathrm{EP} 1$} & & $-0.059 \pm$ \\
& & & 0.0011 \\
\hline
\end{tabular}

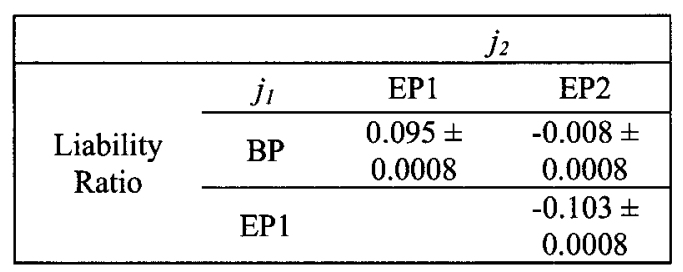

\begin{tabular}{|cccc|}
\hline & & \multicolumn{2}{c|}{$j_{2}$} \\
\hline & $j_{I}$ & $\mathrm{EP} 1$ & $\mathrm{EP} 2$ \\
\cline { 2 - 4 } Liability & \multirow{2}{*}{$\mathrm{BP}$} & $0.096 \pm$ & $-0.009 \pm$ \\
Ratio & & 0.0006 & 0.0006 \\
\cline { 2 - 4 } & \multirow{2}{*}{$\mathrm{EP} 1$} & & $-0.105 \pm$ \\
& & & 0.0006 \\
\hline
\end{tabular}

\begin{tabular}{|cccc|}
\hline & \multicolumn{3}{c}{$j_{2}$} \\
\hline & $j_{1}$ & $\mathrm{EP} 1$ & $\mathrm{EP} 2$ \\
\cline { 2 - 4 } Ordering & \multirow{2}{*}{$\mathrm{BP}$} & $\mathbf{0 . 0 0 0}$ & -37.793 \\
Quantity & & $\mathbf{0 . 8 0 9 *}$ & \pm 0.809 \\
\cline { 2 - 4 } & \multirow{2}{*}{$\mathrm{EP} 1$} & & -37.793 \\
& & \pm 0.809 \\
\hline
\end{tabular}

\begin{tabular}{|cccc|}
\hline & \multicolumn{3}{c|}{$j_{2}$} \\
\hline & $j_{I}$ & EP1 & EP2 \\
\cline { 2 - 4 } Ordering & \multirow{2}{*}{$\mathrm{BP}$} & $\mathbf{0 . 0 0 0}$ & $\mathbf{- 5 4 . 5 9 1}$ \\
Quantity & $\mathbf{1 . 4 7 5 ^ { * }}$ & \pm 1.475 \\
\cline { 2 - 4 } & \multirow{2}{*}{$\mathrm{EP} 1$} & & -54.591 \\
& & \pm 1.475 \\
\hline
\end{tabular}

\begin{tabular}{|cccc|}
\hline & \multicolumn{3}{c}{$J_{2}$} \\
\hline & $j_{1}$ & $\mathrm{EP} 1$ & $\mathrm{EP} 2$ \\
\cline { 2 - 4 } Ordering & \multirow{2}{*}{$\mathrm{BP}$} & $\mathbf{0 . 0 0 0}$ & -83.613 \\
Quantity & & $\pm \mathbf{2 . 7 3 5 *}$ & \pm 2.735 \\
\cline { 2 - 4 } & \multirow{2}{*}{$\mathrm{EP} 1$} & & -83.613 \\
& & & \pm 2.735 \\
\hline
\end{tabular}


Appendix D.7: Comparison of the impacts of the model settings with a given level of budget tightness and a given level of demand variability (Confidence intervals for $\mathrm{H7}, \mathrm{H8}$, and $\mathrm{H9},\left(U_{i 2}-U_{i 1}\right) \pm$ half-width)

(Cont.)

(e) with Normally Distributed Demand and Low Level of budget tightness and High level of Demand Variability (Normal, BG-L, DV-H)

\begin{tabular}{|c|c|c|c|}
\hline \multicolumn{4}{|c|}{ Case 4} \\
\hline & & & \\
\hline \multirow{3}{*}{ Profitability } & $j_{I}$ & EP1 & $\mathrm{EP} 2$ \\
\hline & $\mathrm{BP}$ & $\begin{array}{r}46.072 \\
+27.143\end{array}$ & $\begin{array}{r}579.567 \\
+27143\end{array}$ \\
\hline & EP1 & & $\begin{array}{r}533.495 \\
\pm 27.143\end{array}$ \\
\hline
\end{tabular}
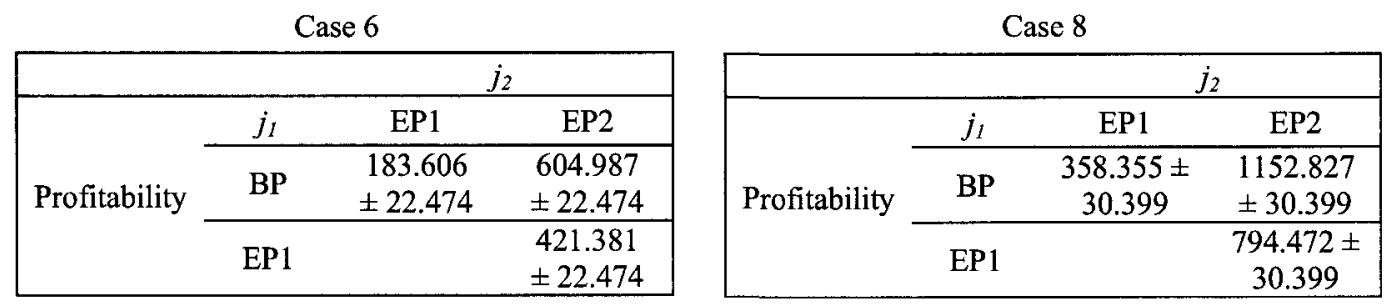

\begin{tabular}{|cccc|}
\hline & \multicolumn{3}{c|}{$j_{2}$} \\
\hline & $j_{1}$ & $\mathrm{EP} 1$ & $\mathrm{EP} 2$ \\
\cline { 2 - 4 } Liability & $\mathrm{BP}$ & $0.010 \pm$ & $-0.016 \pm$ \\
Ratio & & 0.0013 & 0.0013 \\
\cline { 2 - 4 } & $\mathrm{EP} 1$ & & $-0.026 \pm$ \\
& & & 0.0013 \\
\hline
\end{tabular}
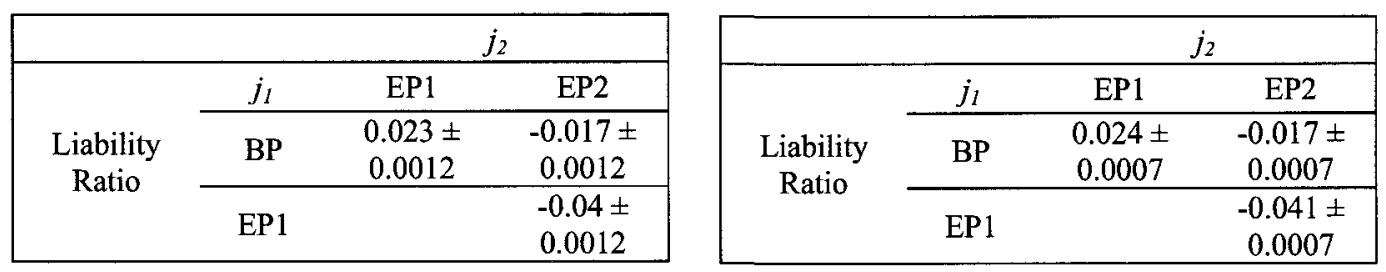

\begin{tabular}{|cccc|}
\hline & \multicolumn{3}{c|}{$j_{2}$} \\
\hline & $j_{1}$ & $\mathrm{EP} 1$ & $\mathrm{EP} 2$ \\
\cline { 2 - 4 } Ordering & $\mathrm{BP}$ & $\mathbf{0 . 0 0 0}$ & $\begin{array}{c}-45.372 \\
\mathbf{1 3 . 9 8 *}\end{array}$ \\
Quantity & & $\mathbf{\pm 3 . 9 8}$ \\
\cline { 2 - 4 } & $\mathrm{EP} 1$ & & $\begin{array}{c}-45.372 \\
\pm 3.98\end{array}$ \\
\hline
\end{tabular}

\begin{tabular}{|cccc|}
\hline & \multicolumn{3}{c|}{$j_{2}$} \\
\hline & $j_{1}$ & EP1 & EP2 \\
\cline { 2 - 4 } Ordering & \multirow{2}{*}{$\mathrm{BP}$} & $\mathbf{0 . 0 0 0}$ & $\mathbf{- 5 5 . 6 7 3}$ \\
Quantity & & $\mathbf{2 . 7 1 2 ^ { * }}$ & \pm 2.712 \\
\cline { 2 - 4 } & \multirow{2}{*}{$\mathrm{EP1}$} & & $\mathbf{5 5 . 6 7 3}$ \\
& & \pm 2.712 \\
\hline
\end{tabular}

\begin{tabular}{|cccc|}
\hline & \multicolumn{3}{c|}{$j_{2}$} \\
\hline & $j_{1}$ & $\mathrm{EP} 1$ & $\mathrm{EP} 2$ \\
\cline { 2 - 4 } Ordering & \multirow{2}{*}{$\mathrm{BP}$} & $\mathbf{0 . 0 0 0}$ & $-\mathbf{7 2 . 8 2 6 \pm}$ \\
Quantity & & $\pm \mathbf{1 . 9 9 3}^{*}$ & 1.993 \\
\cline { 2 - 4 } & \multirow{2}{*}{$\mathrm{EP} 1$} & & $-72.826 \pm$ \\
& & 1.993 \\
\hline
\end{tabular}


Appendix D.7: Comparison of the impacts of the model settings with a given level of budget tightness and a given level of demand variability (Confidence intervals for $\mathrm{H} 7, \mathrm{H8}$, and $\mathrm{H9},\left(U_{i 2}-U_{i l}\right) \pm$ half-width)

(Cont.)

(f) with Uniformly Distributed Demand and Low Level of Budget Tightness and High Level of Demand Variability (Uniform, BG-L, DV-H)

\begin{tabular}{|cccc|}
\hline \multicolumn{4}{c}{ Case 4 } \\
\hline & \multicolumn{3}{c}{$j_{2}$} \\
\hline \multirow{3}{*}{ Profitability } & BP1 & EP2 \\
\cline { 2 - 4 } & \multirow{2}{*}{ BP } & 169.760 & 654.194 \\
& & \pm 46.541 & \pm 46.541 \\
\cline { 2 - 4 } & EP1 & & 484.434 \\
& & \pm 46.541 \\
\hline
\end{tabular}

\begin{tabular}{|cccc|}
\hline \multicolumn{4}{c}{ Case 6 } \\
\hline & \multicolumn{3}{c|}{$j_{2}$} \\
\cline { 2 - 4 } Profitability & $j_{1}$ & EP1 & EP2 \\
\cline { 2 - 4 } & \multirow{2}{*}{ BP } & 574.619 & 792.926 \\
& & \pm 31.751 & \pm 31.751 \\
\cline { 2 - 4 } & \multirow{2}{*}{ EP1 } & & 218.307 \\
& & \pm 31.751 \\
\hline
\end{tabular}

\begin{tabular}{|cccc|}
\hline \multicolumn{4}{c}{ Case 8 } \\
\hline \multirow{4}{*}{ Profitability } & \multicolumn{3}{c|}{$j_{2}$} \\
\cline { 2 - 4 } & BP & $\begin{array}{l}1082.342 \\
\pm 20.424\end{array}$ & $\begin{array}{c}1405.748 \\
\pm 20.424\end{array}$ \\
\cline { 2 - 4 } & & & $\begin{array}{c}323.406 \pm \\
\text { EP1 }\end{array}$ \\
& & 20.424 \\
\hline
\end{tabular}

\begin{tabular}{|cccc|}
\hline & \multicolumn{3}{c}{$j_{2}$} \\
\hline & $j_{1}$ & EP1 & EP2 \\
\cline { 2 - 4 } Liability & \multirow{2}{*}{$\mathrm{BP}$} & $0.039 \pm$ & $-0.016 \pm$ \\
Rat10 & & 0.0017 & 0.0017 \\
\cline { 2 - 4 } & \multirow{2}{*}{$\mathrm{EP} 1$} & & $-0.055 \pm$ \\
& & & 0.0017 \\
\hline
\end{tabular}
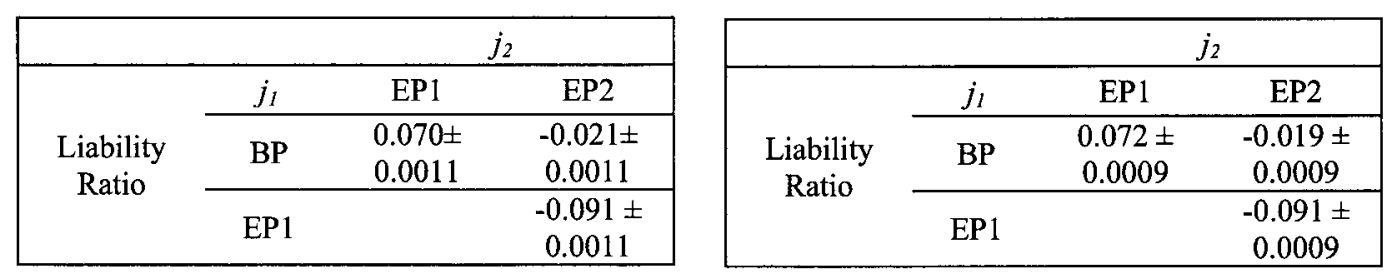

\begin{tabular}{|cccc|}
\hline & \multicolumn{3}{c}{$j_{2}$} \\
\hline & \multirow{3}{*}{$J_{1}$} & $\mathrm{EP} 1$ & $\mathrm{EP} 2$ \\
\cline { 2 - 4 } Ordering & $\mathrm{BP}$ & $\mathbf{0 . 0 0 0}$ & $-48.631 \pm$ \\
Quantity & $\mathbf{1 . 4 8 8 ^ { * }}$ & 1.488 \\
\cline { 2 - 4 } & \multirow{2}{*}{$\mathrm{EP} 1$} & & $-48.631 \pm$ \\
& & 1.488 \\
\hline
\end{tabular}

\begin{tabular}{|cccc|}
\hline & \multicolumn{3}{c|}{$j_{2}$} \\
\hline & $j_{1}$ & $\mathrm{EP} 1$ & $\mathrm{EP} 2$ \\
\cline { 2 - 4 } Ordering & \multirow{2}{*}{$\mathrm{BP}$} & $\mathbf{0 . 0 0 0}$ & -49.244 \\
Quantity & & $\mathbf{1 . 7 4 4 *}$ & \pm 1.744 \\
\cline { 2 - 4 } & \multirow{2}{*}{$\mathrm{EP1}$} & & -49.244 \\
& & \pm 1.744 \\
\hline
\end{tabular}

\begin{tabular}{|cccc|}
\hline & \multicolumn{3}{c}{$j_{2}$} \\
\hline & $j_{1}$ & $\mathrm{EP} 1$ & $\mathrm{EP} 2$ \\
\cline { 2 - 4 } Ordering & \multirow{2}{*}{$\mathrm{BP}$} & $\mathbf{0 . 0 0 0}$ & -78.218 \\
Quantity & & $\mathbf{\mathbf { 3 . 2 8 6 }}$ & \pm 3.286 \\
\cline { 2 - 4 } & \multirow{2}{*}{$\mathrm{EP} 1$} & & -78.218 \\
& & & \pm 3.286 \\
\hline
\end{tabular}


Appendix D.7: Comparison of the impacts of the model settings with a given level of budget tightness and a given level of demand variability (Confidence intervals for $\mathrm{H7}$, $\mathrm{H8}$, and $\mathrm{H} 9,\left(U_{i 2}-U_{i 1}\right) \pm$ half-width)

$$
\text { (Cont.) }
$$

(g) with Normally Distributed Demand and High Level of Budget Tightness and Low Level of Demand Variability (Normal, BG-H, DV-L)

\begin{tabular}{|c|c|c|c|}
\hline \multicolumn{4}{|c|}{ Case 4} \\
\hline & & & \\
\hline \multirow{3}{*}{ Profitability } & $j_{l}$ & EP1 & EP2 \\
\hline & $\mathrm{BP}$ & $\begin{array}{l}510.320 \\
\pm 0.984\end{array}$ & $\begin{array}{r}29.039 \\
\pm 0.984\end{array}$ \\
\hline & EP1 & & $\begin{array}{c}-481.281 \\
\pm 0.984\end{array}$ \\
\hline
\end{tabular}

\begin{tabular}{|cccc|}
\hline \multicolumn{4}{c}{ Case 6 } \\
\hline \multirow{4}{*}{ Profitability } & \multicolumn{3}{c|}{$j_{2}$} \\
\cline { 2 - 4 } & \multirow{2}{*}{$\mathrm{BP}$} & 1735.102 & $\mathrm{EP} 2$ \\
& & \pm 1.200 & \pm 1.200 \\
\cline { 2 - 4 } & $\mathrm{EP} 1$ & & -1671.69 \\
& & & \pm 1.200 \\
\hline
\end{tabular}

\begin{tabular}{|cccc|}
\hline \multicolumn{4}{c}{ Case 8 } \\
\hline \multirow{4}{*}{ Profitability } & \multicolumn{3}{c|}{$j_{2}$} \\
\cline { 2 - 4 } & \multirow{2}{*}{ BP } & $\begin{array}{c}3279.422 \\
\pm 1.901\end{array}$ & $\begin{array}{c}118.063 \\
\pm 1.901\end{array}$ \\
\cline { 2 - 4 } & \multirow{2}{*}{ EP1 } & & $\begin{array}{c}-3161.36 \\
\pm 1.901\end{array}$ \\
\hline
\end{tabular}

\begin{tabular}{|cccc|}
\hline & \multicolumn{3}{c}{$j_{2}$} \\
\hline & $j_{1}$ & $\mathrm{EP} 1$ & $\mathrm{EP} 2$ \\
\cline { 2 - 4 } Liability & $\mathrm{BP}$ & $0.082 \pm$ & $-0.001 \pm$ \\
Ratio & & 0.0014 & 0.0014 \\
\cline { 2 - 4 } & $\mathrm{EP} 1$ & & $-0.083 \pm$ \\
& & & 0.0014 \\
\hline
\end{tabular}
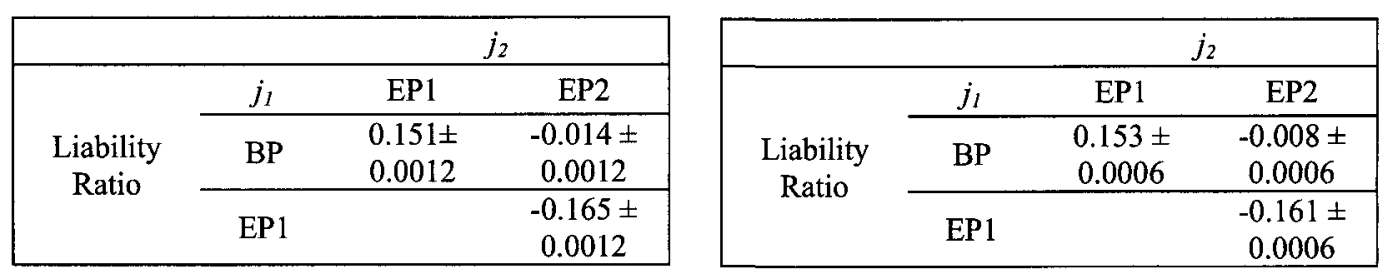

\begin{tabular}{|lccc|}
\hline & \multicolumn{3}{c|}{$j_{2}$} \\
\hline & $j_{1}$ & $\mathrm{EP} 1$ & $\mathrm{EP} 2$ \\
\cline { 2 - 4 } Ordering & $\mathrm{BP}$ & $\mathbf{0 . 0 0 0}$ & $\mathbf{0 . 0 . 2 5 6}$ \\
Quantity & $\mathbf{0 . 8 6 3 ^ { * }}$ & $\mathbf{\pm 0 . 8 6 3 ^ { * }}$ \\
\cline { 2 - 5 } & $\mathrm{EP} 1$ & & $\mathbf{0 . 2 5 6}$ \\
& & $\mathbf{0 . 8 6 3 ^ { * }}$ \\
\hline
\end{tabular}

\begin{tabular}{|cccc|}
\hline & \multicolumn{3}{c|}{$j_{2}$} \\
\hline & $j_{1}$ & $\mathrm{EP} 1$ & $\mathrm{EP} 2$ \\
\cline { 2 - 4 } Ordering & \multirow{2}{*}{$\mathrm{BP}$} & $\mathbf{0 . 0 0 0}$ & 11.416 \\
Quantity & & $\mathbf{1 . 3 0 8 ^ { * }}$ & \pm 1.308 \\
\cline { 2 - 4 } & \multirow{2}{*}{$\mathrm{EP1}$} & & $\begin{array}{c}11.416 \\
\end{array}$ \\
& & & \pm 1.308 \\
\hline
\end{tabular}

\begin{tabular}{|c|c|c|c|}
\hline & & \multicolumn{2}{|c|}{$j_{2}$} \\
\hline \multirow{3}{*}{$\begin{array}{l}\text { Ordering } \\
\text { Quantity }\end{array}$} & $\dot{j}_{1}$ & EP1 & EP2 \\
\hline & BP & $\begin{array}{c}0.000 \\
\pm 1.314^{*}\end{array}$ & $\begin{array}{r}10.388 \\
\pm 1.314\end{array}$ \\
\hline & EP1 & & $\begin{array}{r}10.388 \\
\pm 1.314\end{array}$ \\
\hline
\end{tabular}


Appendix D.7: Comparison of the impacts of the model settings with a given level of budget tightness and a given level of demand variability (Confidence intervals for $\mathrm{H} 7, \mathrm{H8}$, and $\mathrm{H9},\left(U_{i 2}-U_{i 1}\right) \pm$ half-width)

(Cont.)

(h) with Uniformly Distributed Demand and High Level of Budget Tightness and Low Level of Demand Variability (Uniform, BG-H, DV-L)

\begin{tabular}{|cccc|}
\hline \multicolumn{4}{c}{ Case 4 } \\
\hline \multirow{4}{*}{ Profitability } & \multicolumn{3}{c|}{$j_{2}$} \\
\cline { 2 - 4 } & $j_{1}$ & EP1 & EP2 \\
\cline { 2 - 4 } & \multirow{2}{*}{ BP } & 397.180 & 188.732 \\
& & \pm 3.094 & \pm 3.094 \\
\cline { 2 - 4 } & EP1 & & -208.448 \\
& & & \pm 3.094 \\
\hline
\end{tabular}

\begin{tabular}{|cccc|}
\hline \multicolumn{4}{c}{ Case 6 } \\
\hline & \multicolumn{3}{c|}{$j_{2}$} \\
\hline \multirow{4}{*}{ Profitability } & BP & $\begin{array}{c}1341.436 \\
\pm 6.211\end{array}$ & $\begin{array}{c}315.221 \\
\pm 6.211\end{array}$ \\
\cline { 2 - 4 } & \multirow{2}{*}{ EP1 } & & $\begin{array}{c}-1026.22 \\
\pm 6.211\end{array}$ \\
\cline { 2 - 4 } & & & \pm 6
\end{tabular}

\begin{tabular}{|cccc|}
\hline \multicolumn{4}{c}{ Case 8 } \\
\hline & \multicolumn{3}{c|}{$j_{2}$} \\
\cline { 2 - 4 } Profitability & $j_{1}$ & EP1 & EP2 \\
\cline { 2 - 4 } & \multirow{2}{*}{$\mathrm{BP}$} & 2527.87 & 559.208 \\
& & \pm 4.708 & \pm 4.708 \\
\cline { 2 - 4 } & \multirow{2}{*}{$\mathrm{EP} 1$} & & -1968.66 \\
& & \pm 4.708 \\
\hline
\end{tabular}

\begin{tabular}{|cccc|}
\hline & \multicolumn{3}{c}{$j_{2}$} \\
\hline & $j_{1}$ & EP1 & EP2 \\
\cline { 2 - 4 } Liability & \multirow{2}{*}{$\mathrm{BP}$} & $0.071 \pm$ & $-0.004 \pm$ \\
Ratio & \multirow{2}{*}{0.0007} & 0.0007 \\
\cline { 2 - 4 } & \multirow{2}{*}{$\mathrm{EP} 1$} & & $-0.075 \pm$ \\
& & 0.0007 \\
\hline
\end{tabular}

\begin{tabular}{|cccc|}
\hline & \multicolumn{3}{c}{$j_{2}$} \\
\hline & $j_{1}$ & $\mathrm{EP} 1$ & $\mathrm{EP} 2$ \\
\cline { 2 - 4 } Liability & \multirow{2}{*}{$\mathrm{BP}$} & $0.131 \pm$ & $-0.021 \pm$ \\
Ratio & & 0.0004 & 0.0004 \\
\cline { 2 - 4 } & \multirow{2}{*}{$\mathrm{EP} 1$} & & $-0.152 \pm$ \\
& & & 0.0004 \\
\hline
\end{tabular}

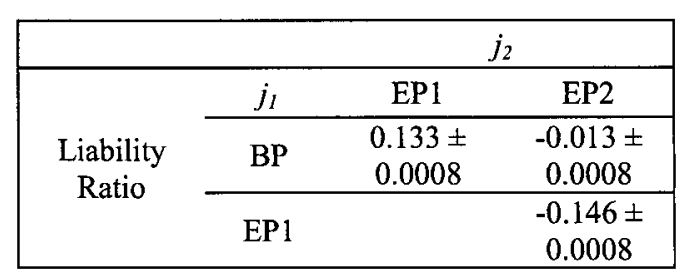

\begin{tabular}{|cccc|}
\hline & \multicolumn{3}{c|}{$j_{2}$} \\
\hline & $j_{1}$ & $\mathrm{EP} 1$ & $\mathrm{EP} 2$ \\
\cline { 2 - 4 } Ordering & \multirow{2}{*}{$\mathrm{BP}$} & $\mathbf{0 . 0 0 0}$ & -3.182 \\
Quantity & $\mathbf{\mathbf { 0 . 2 0 7 * }}$ & \pm 0.207 \\
\cline { 2 - 4 } & \multirow{2}{*}{$\mathrm{EP} 1$} & & -3.182 \\
& & & \pm 0.207 \\
\hline
\end{tabular}

\begin{tabular}{|cccc|}
\hline & \multicolumn{3}{c|}{$j_{2}$} \\
\hline & $j_{1}$ & $\mathrm{EP} 1$ & $\mathrm{EP} 2$ \\
\cline { 2 - 4 } Ordering & \multirow{2}{*}{$\mathrm{BP}$} & $\mathbf{0 . 0 0 0}$ & 5.412 \\
Quantity & $\mathbf{0 . 1 7 0 ^ { * }}$ & \pm 0.170 \\
\cline { 2 - 4 } & \multirow{2}{*}{$\mathrm{EP1}$} & & 5.412 \\
& & \pm 0.170 \\
\hline
\end{tabular}

\begin{tabular}{|cccc|}
\hline & \multicolumn{3}{c}{$j_{2}$} \\
\hline & $j_{1}$ & $\mathrm{EP} 1$ & $\mathrm{EP} 2$ \\
\cline { 2 - 4 } Ordering & $\mathrm{BP}$ & $\mathbf{0 . 0 0 0}$ & $\mathbf{- 0 . 1 2 2}$ \\
Quantity & $\mathbf{1 . 4 7 5 ^ { * }}$ & \pm 1.475 \\
\cline { 2 - 4 } & \multirow{2}{*}{$\mathrm{EP} 1$} & & $\begin{array}{c}-0.122 \\
\end{array}$ \\
& & & \pm 1.475 \\
\hline
\end{tabular}


Appendix D.7: Comparison of the impacts of the model settings with a given level of budget tightness and a given level of demand variability (Confidence intervals for $\mathrm{H7}, \mathrm{H8}$, and $\mathrm{H9},\left(U_{i 2}-U_{i 1}\right) \pm$ half-width)

$$
\text { (Cont.) }
$$

(i) with Normally Distributed Demand and High Level of Budget Tightness and Medium Level of Demand Variability (Normal, BG-H, DV-M)

\begin{tabular}{|c|c|c|c|}
\hline \multicolumn{4}{|c|}{ Case 4} \\
\hline & & & \\
\hline \multirow{3}{*}{ Profitability } & $j_{I}$ & EP1 & EP2 \\
\hline & $\mathrm{BP}$ & $\begin{array}{r}155.795 \\
\pm 9.445 \\
\end{array}$ & $\begin{array}{r}442.665 \\
\pm 9.445 \\
\end{array}$ \\
\hline & EP1 & & $\begin{array}{l}286.870 \\
\pm 9.445\end{array}$ \\
\hline
\end{tabular}
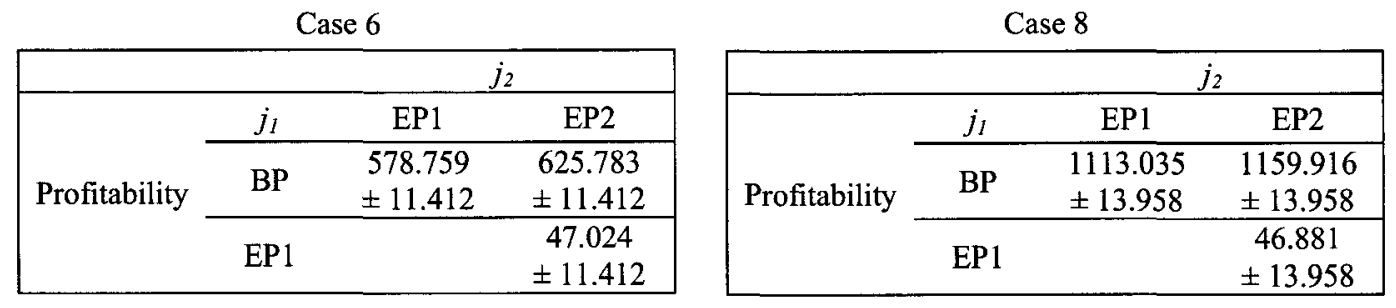

\begin{tabular}{|cccc|}
\hline & \multicolumn{3}{c|}{$j_{2}$} \\
\hline & $j_{1}$ & $\mathrm{EP} 1$ & $\mathrm{EP} 2$ \\
\cline { 2 - 4 } Liability & \multirow{2}{*}{$\mathrm{BP}$} & $0.033 \pm$ & $-0.003 \pm$ \\
Ratio & & 0.0014 & 0.0014 \\
\cline { 2 - 4 } & \multirow{2}{*}{$\mathrm{EP} 1$} & & $-0.036 \pm$ \\
& & & 0.0014 \\
\hline
\end{tabular}
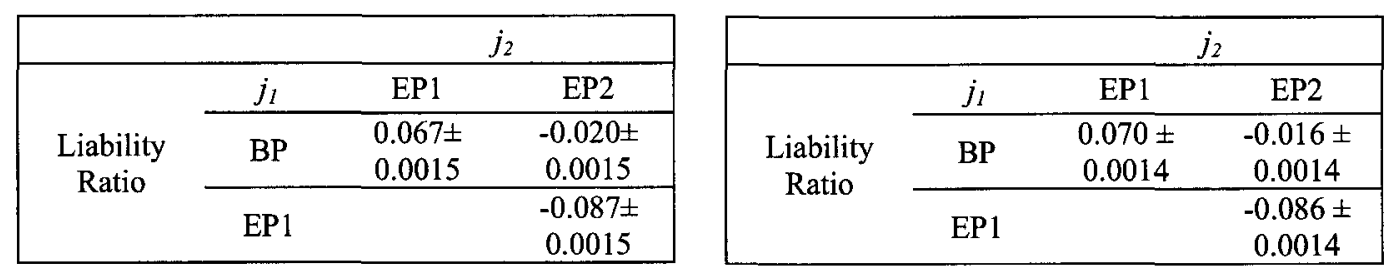

\begin{tabular}{|c|c|c|c|}
\hline & & \multicolumn{2}{|c|}{$j_{2}$} \\
\hline \multirow{3}{*}{$\begin{array}{l}\text { Ordering } \\
\text { Quantity }\end{array}$} & $j_{1}$ & EP1 & EP2 \\
\hline & BP & $\begin{array}{c}0.000 \\
\pm \mathbf{0 . 5 3 3}\end{array}$ & $\begin{array}{r}-9.804 \\
\pm 0.533 \\
\end{array}$ \\
\hline & EP1 & & $\begin{array}{c}-9.804 \\
\pm 0.533\end{array}$ \\
\hline
\end{tabular}

\begin{tabular}{|cccc|}
\hline & \multicolumn{3}{c|}{$j_{2}$} \\
\hline & $j_{1}$ & EP1 & EP2 \\
\cline { 2 - 4 } Ordering & \multirow{2}{*}{ BP } & $\mathbf{0 . 0 0 0}$ & -11.613 \\
Quantity & $\mathbf{1 . 1 1 2 *}$ & \pm 1.112 \\
\cline { 2 - 4 } & \multirow{2}{*}{ EP1 } & & -11.613 \\
& & \pm 1.112 \\
\hline
\end{tabular}

\begin{tabular}{|cccc|}
\hline & \multicolumn{3}{c}{$j_{2}$} \\
\hline & $j_{1}$ & EP1 & EP2 \\
\cline { 2 - 4 } Ordering & \multirow{2}{*}{$\mathrm{BP}$} & $\mathbf{0 . 0 0 0}$ & -20.953 \\
Quantity & & $\mathbf{2 . 1 7 0 ^ { * }}$ & \pm 2.170 \\
\cline { 2 - 4 } & \multirow{2}{*}{$\mathrm{EP1}$} & & -20.953 \\
& & \pm 2.170 \\
\hline
\end{tabular}


Appendix D.7: Comparison of the impacts of the model settings with a given level of budget tightness and a given level of demand variability (Confidence intervals for $\mathrm{H} 7, \mathrm{H8}$, and $\mathrm{H} 9,\left(U_{i 2}-U_{i 1}\right) \pm$ half-width)

$$
\text { (Cont.) }
$$

(j) with Uniformly Distributed Demand and High Level of Budget Tightness and Medium Level of Demand Variability (Uniform, BG-H, DV-M)

\begin{tabular}{|cccc|}
\hline \multicolumn{4}{c}{ Case 4 } \\
\hline & \multicolumn{3}{c|}{$j_{2}$} \\
\hline & $j_{1}$ & EP1 & EP2 \\
\cline { 2 - 4 } Profitability & \multirow{2}{*}{ BP } & 238.218 & 590.298 \\
& & \pm 10.571 & \pm 10.571 \\
\cline { 2 - 4 } & \multirow{2}{*}{ EP1 } & & 352.08 \\
& & \pm 10.571 \\
\hline
\end{tabular}

\begin{tabular}{|cccc|}
\hline \multicolumn{4}{c}{ Case 6 } \\
\hline & \multicolumn{3}{c|}{$j_{2}$} \\
\cline { 2 - 4 } Profitability & EP1 & EP2 \\
\cline { 2 - 4 } & \multirow{2}{*}{ BP } & 806.504 & 866.575 \\
& & \pm 16.723 & \pm 16.723 \\
\cline { 2 - 4 } & \multirow{2}{*}{ EP1 } & & 60.071 \\
& & \pm 16.723 \\
\hline
\end{tabular}

\begin{tabular}{|cccc|}
\hline \multicolumn{4}{c}{ Case 8 } \\
\hline & \multicolumn{3}{c|}{$j_{2}$} \\
\hline & $j_{1}$ & EP1 & EP2 \\
\cline { 2 - 4 } Profitability & \multirow{2}{*}{ BP } & 1518.96 & 1521.486 \\
& & \pm 13.838 & \pm 13.838 \\
\cline { 2 - 4 } & \multirow{2}{*}{ EP1 } & & 2.526 \\
& & \pm 13.838 \\
\hline
\end{tabular}

\begin{tabular}{|cccc|}
\hline & \multicolumn{3}{c|}{$j_{2}$} \\
\hline & $j_{1}$ & $\mathrm{EP} 1$ & $\mathrm{EP} 2$ \\
\cline { 2 - 4 } Liability & \multirow{2}{*}{$\mathrm{BP}$} & $0.051 \pm$ & $-0.008 \pm$ \\
Ratio & \multirow{2}{*}{$\mathrm{0.0008}$} & 0.0008 \\
\cline { 2 - 4 } & \multirow{2}{*}{$\mathrm{EP} 1$} & & $-0.059 \pm$ \\
& & & 0.0008 \\
\hline
\end{tabular}

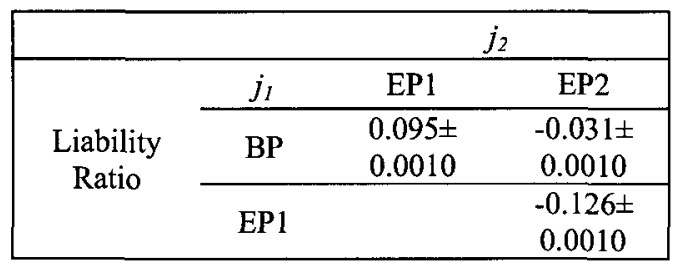

\begin{tabular}{|cccc|}
\hline & \multicolumn{3}{c}{$j_{2}$} \\
\hline & $j_{1}$ & $\mathrm{EP} 1$ & $\mathrm{EP} 2$ \\
\cline { 2 - 4 } Liability & \multirow{2}{*}{$\mathrm{BP}$} & $0.096 \pm$ & $-0.024 \pm$ \\
Ratio & & 0.0008 & 0.0008 \\
\cline { 2 - 4 } & \multirow{2}{*}{$\mathrm{EP} 1$} & & $-0.120 \pm$ \\
& & & 0.0008 \\
\hline
\end{tabular}

\begin{tabular}{|lccc|}
\hline & \multicolumn{3}{c}{$j_{2}$} \\
\hline & \multirow{2}{*}{$j_{1}$} & EP1 & EP2 \\
\cline { 2 - 4 } Ordering & \multirow{2}{*}{ BP } & $\mathbf{0 . 0 0 0}$ & $-11.828 \pm$ \\
Quantity & & $\pm \mathbf{0 . 4 4 9 *}$ & 0.449 \\
\cline { 2 - 4 } & \multirow{2}{*}{ EP1 } & & $-11.828 \pm$ \\
& & & 0.449 \\
\hline
\end{tabular}

\begin{tabular}{|cccc|}
\hline & \multicolumn{3}{c}{$j_{2}$} \\
\hline & $j_{1}$ & $\mathrm{EP} 1$ & $\mathrm{EP} 2$ \\
\cline { 2 - 4 } Ordering & \multirow{2}{*}{$\mathrm{BP}$} & $\mathbf{0 . 0 0 0}$ & $-10.664 \pm$ \\
Quantity & & $\mathbf{0 . 5 4 1 *}$ & 0.541 \\
\cline { 2 - 4 } & $\mathrm{EP} 1$ & & $-10.664 \pm$ \\
& & & 0.541 \\
\hline
\end{tabular}

\begin{tabular}{|lccc|}
\hline & \multicolumn{3}{c|}{$J_{2}$} \\
\hline & $j_{1}$ & $\mathrm{EP} 1$ & $\mathrm{EP} 2$ \\
\cline { 2 - 4 } Ordering & \multirow{2}{*}{$\mathrm{BP}$} & $\mathbf{0 . 0 0 0}$ & $\mathbf{- 2 1 . 4 8 7 \pm}$ \\
Quantity & $\mathbf{1 . 0 9 8 ^ { * }}$ & 1.098 \\
\cline { 2 - 4 } & \multirow{2}{*}{$\mathrm{EP} 1$} & & $-21.487 \pm$ \\
& & & 1.098 \\
\hline
\end{tabular}


Appendix D.7: Comparison of the impacts of the model settings with a given level of budget tightness and a given level of demand variability (Confidence intervals for $\mathrm{H7}, \mathrm{H8}$, and $\mathrm{H9},\left(U_{i 2}-U_{i 1}\right) \pm$ half-width)

(Cont.)

(k) with Normally Distributed Demand and High Level of Budget Tightness and High Level of Demand Variability (Normal, BG-H, DV-H)
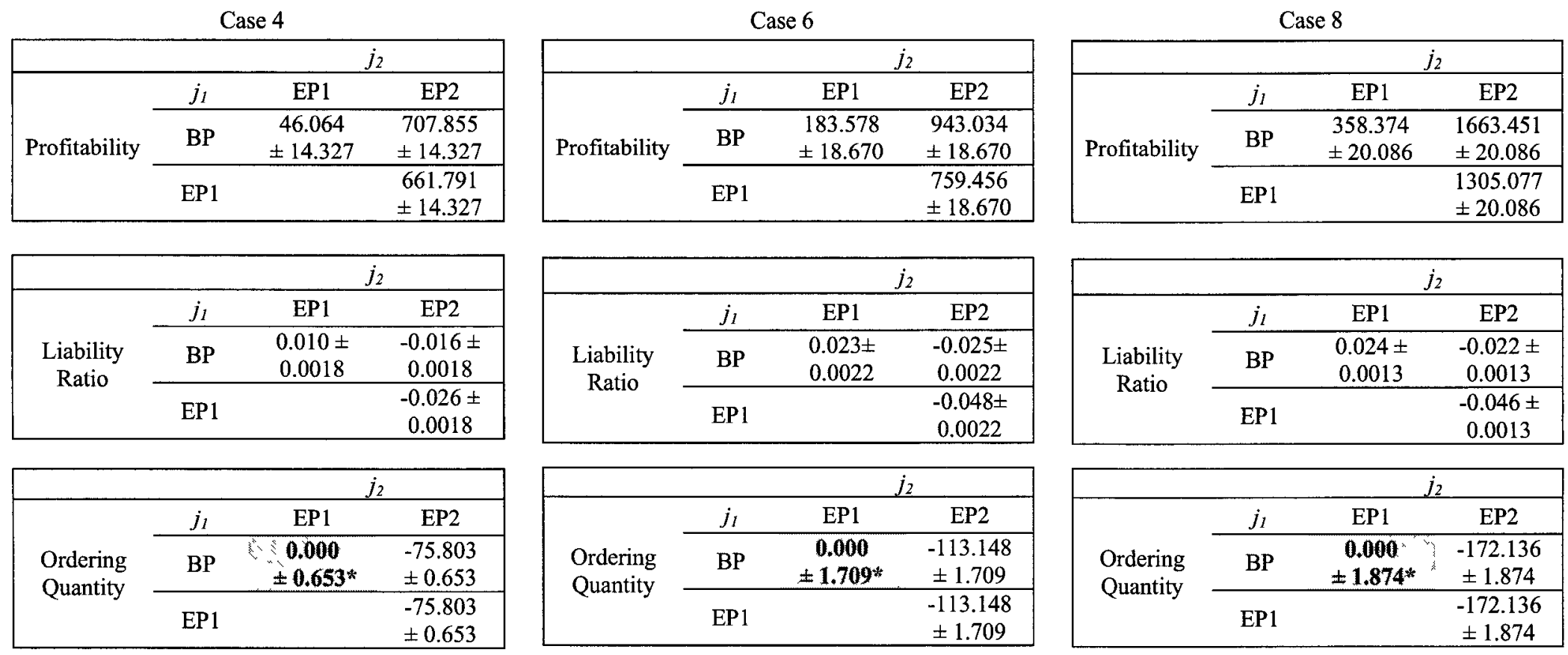

\begin{tabular}{|cccc|}
\hline & \multicolumn{3}{c|}{$j_{2}$} \\
\hline & $j_{1}$ & $\mathrm{EP} 1$ & $\mathrm{EP} 2$ \\
\cline { 2 - 4 } Ordering & $\mathrm{BP}$ & $\mathbf{0 . 0 0 0}$ & $\mathbf{1 7 2 . 1 3 6}$ \\
Quantity & & $\mathbf{1 . 8 7 4 ^ { * }}$ & \pm 1.874 \\
\cline { 2 - 4 } & $\mathrm{EP} 1$ & & $\begin{array}{c}-172.136 \\
\pm 1.874\end{array}$ \\
\hline
\end{tabular}


Appendix D.7: Comparison of the impacts of the model settings with a given level of budget tightness and a given level of demand variability (Confidence intervals for $\mathrm{H} 7, \mathrm{H8}$, and $\mathrm{H9},\left(U_{i 2}-U_{i 1}\right) \pm$ half-width)

(Cont.)

(1) with Uniformly Distributed Demand and High Level of Budget Tightness and High Level of Demand Variability (Uniform, BG-H, DV-H)

\begin{tabular}{|cccc|}
\multicolumn{4}{c}{ Case 4 } \\
\hline \multirow{4}{*}{$\begin{array}{c}\text { EP1 } \\
\text { Profitability }\end{array}$} & \multicolumn{2}{c|}{ EP2 } \\
\cline { 2 - 4 } & BP & $\begin{array}{r}169.748 \\
\pm 15.369\end{array}$ & $\begin{array}{c}824.584 \\
\pm 15.369\end{array}$ \\
\cline { 2 - 4 } & & & 654.836 \\
& EP1 & & \pm 15.369 \\
\hline
\end{tabular}

\begin{tabular}{|cccc|}
\hline \multicolumn{4}{c}{ Case 6 } \\
\hline & \multicolumn{3}{c|}{$j_{2}$} \\
\cline { 2 - 4 } Profitability & $j_{1}$ & EP1 & EP2 \\
\cline { 2 - 4 } & \multirow{2}{*}{ BP } & 574.617 & 1073.728 \\
& & \pm 28.501 & \pm 28.501 \\
\cline { 2 - 4 } & \multirow{2}{*}{ EP1 } & & 499.111 \\
& & \pm 28.501 \\
\hline
\end{tabular}

\begin{tabular}{|cccc|}
\multicolumn{4}{c}{ Case 8 } \\
\hline & \multicolumn{3}{c|}{$j_{2}$} \\
\hline & $j_{1}$ & EP1 & EP2 \\
\cline { 2 - 4 } Profitability & \multirow{2}{*}{ BP } & 1082.338 & 1834.034 \\
& & \pm 25.697 & \pm 25.697 \\
\cline { 2 - 4 } & \multirow{2}{*}{ EP1 } & & 751.696 \\
& & \pm 25.697 \\
\hline
\end{tabular}

\begin{tabular}{|cccc|}
\hline & \multicolumn{3}{c}{$j_{2}$} \\
\hline & $j_{l}$ & $\mathrm{EP} 1$ & $\mathrm{EP} 2$ \\
\cline { 2 - 4 } Liability & \multirow{2}{*}{$\mathrm{BP}$} & $0.038 \pm$ & $-0.022 \pm$ \\
Ratio & & 0.0011 & 0.0011 \\
\cline { 2 - 4 } & \multirow{2}{*}{$\mathrm{EP} 1$} & & $-0.060 \pm$ \\
& & & 0.0011 \\
\hline
\end{tabular}
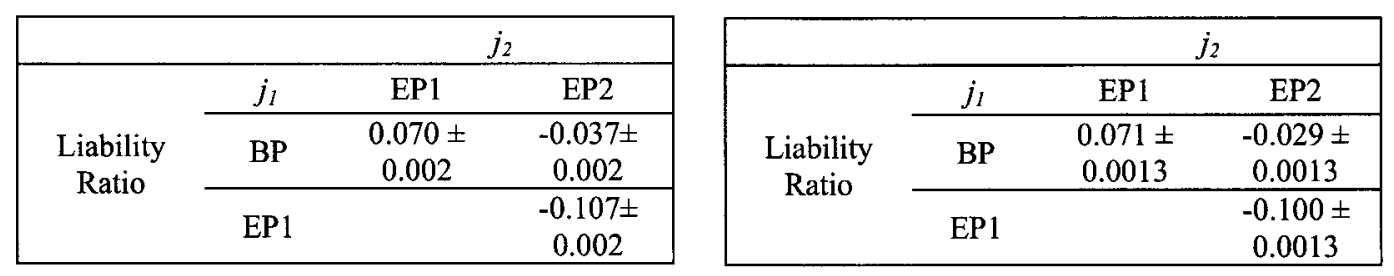

\begin{tabular}{|cccc|}
\hline & \multicolumn{3}{c}{$j_{2}$} \\
\hline & $j_{1}$ & $\mathrm{EP1}$ & $\mathrm{EP} 2$ \\
\cline { 2 - 4 } Ordering & \multirow{2}{*}{$\mathrm{BP}$} & $\mathbf{0 . 0 0 0}$ & $-14.227 \pm$ \\
Quantity & & $\mathbf{\mathbf { 0 . 6 0 1 }}{ }^{*}$ & $\mathbf{0 . 6 0 1}$ \\
\cline { 2 - 4 } & \multirow{2}{*}{$\mathrm{EP1}$} & & $-14.227 \pm$ \\
& & & $\mathbf{0 . 6 0 1}$ \\
\hline
\end{tabular}

\begin{tabular}{|cccc|}
\hline & \multicolumn{3}{c|}{$j_{2}$} \\
\hline & $j_{1}$ & $\mathrm{EP} 1$ & $\mathrm{EP} 2$ \\
\cline { 2 - 4 } Ordering & \multirow{2}{*}{$\mathrm{BP}$} & $\mathbf{0 . 0 0 0}$ & -16.224 \\
Quantity & $\mathbf{0 . 8 0 3} \boldsymbol{*}^{*}$ & \pm 0.803 \\
\cline { 2 - 4 } & \multirow{2}{*}{$\mathrm{EP} 1$} & & -16.224 \\
& & \pm 0.803 \\
\hline
\end{tabular}

\begin{tabular}{|c|c|c|c|}
\hline & & $j_{2}$ & \\
\hline \multirow{3}{*}{$\begin{array}{l}\text { Ordering } \\
\text { Quantity }\end{array}$} & $\dot{j}_{1}$ & EP1 & EP2 \\
\hline & BP & $\begin{array}{r}0.000^{\prime} \\
\pm 1.350^{*} \\
\end{array}$ & $\begin{array}{l}-27.419 \\
\pm 1.350 \\
\end{array}$ \\
\hline & EP1 & & $\begin{array}{r}-27.419 \\
\pm 1.350 \\
\end{array}$ \\
\hline
\end{tabular}


Appendix E 
Appendix E.1: Extended mathematical model for Problem $B P$ with revenue-sharing type of contracts

The additional notations used in the extended model are defined as follows:

(1) Indices:

$A_{1} \quad$ LRP accumulation partners who choose a wholesale price contract

$A_{21} \quad$ LRP accumulation partners who choose a revenue-sharing type contract

(2) Parameters in objective function:

$D_{1}^{A} \quad$ LPR members' accumulation demand towards LRP accumulation partner $A_{1}$.

$D_{2 \imath}^{A} \quad$ LRP members' accumulation demand towards LRP accumulation partner $A_{21}$.

$q_{1 \imath}^{A} \quad$ LRP accumulation partner $A_{I_{l}}$ 's ordering quantity of points.

$q_{2 \imath}^{A} \quad$ LRP accumulation partner $A_{2 \imath}$ 's ordering quantity of points.

$w_{l_{1}}^{A} \quad$ Wholesale price per unit of points that LRP host $H$ charges LRP accumulation partner $A_{1 !}$.

$w_{l,}^{\prime A} \quad$ Price per unit of points that LPR host $H$ charges LRP accumulation partner $A_{l,}$ when accumulation demand is more than LRP partner $A_{l}$ 's ordering quantity.

$w_{2 l}^{A} \quad$ Revenue sharing in terms of the price per unit of points that LRP host $H$ charges partner $A_{2 t}$.

$w_{2 i}^{\prime A} \quad$ Extra revenue sharing in terms of the price per unit of points that LRP host $H$ charges LRP partner $A_{2 l}$ when the actual revenue increase is higher than that to which $H$ has committed.

$s_{2 l}^{A} \quad$ Penalty per unit of points that LRP host $H$ pays to LRP partner $A_{2 l}$ when the actual revenue increase is lower than that to which $H$ has committed. 
The model is formulated as follows ((here after Problem BP1):

$$
\begin{aligned}
& \Pi_{H}\left(q_{j}^{R} ; D_{J}^{R}, D_{11}^{A}, D_{2 \imath}^{A}\right)=\max E\left[\pi_{H(R)}\left(q_{J}^{R} ; D_{J}^{R}\right)+\pi_{H(A)}\left(; D_{12}^{A}, D_{22}^{A}\right)\right] \\
& =\max E\left[\sum_{j=1}^{J}\left(p_{J}^{R} \times \min \left\{q_{J}^{R}, D_{J}^{R}\right\}-w_{J}^{R} \times q_{J}^{R}-v_{J}^{R} \times\left[D_{J}^{R}-q_{J}^{R}\right]_{+}+s_{j}^{R} \times\left[q_{J}^{R}-D_{J}^{R}\right]_{+}\right)\right]
\end{aligned}
$$

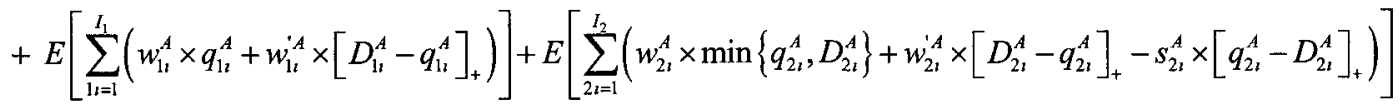

subject to:

(1) Liability control constraints:

$$
\begin{gathered}
\frac{l}{l_{0}} \leq L_{U B} \\
\text { where } l=l_{0}+\sum_{l \imath=1}^{I_{1}}\left(q_{1 \imath}^{A}+\left[D_{1 \imath}^{A}-q_{1 \imath}^{A}\right]_{+}\right)+\sum_{2 \imath=1}^{I_{2}}\left(\min \left\{q_{2 \imath}^{A}, D_{2 \imath}^{A}\right\}+\left[D_{2 \imath}^{A}-q_{2 \imath}^{A}\right]_{+}\right)-\sum_{j=1}^{J}\left(\min \left\{q_{J}^{R}, D_{\jmath}^{R}\right\}\right)
\end{gathered}
$$

(2) LRP redemption partners' capacity limitations on rewards supply:

$$
q_{j}^{R} \leq Q_{J}^{R}, \text { for } j=1, \ldots, J
$$

(3) LRP host's budget constraint on purchasing rewards:

$$
\sum_{j=1}^{J}\left(w_{J}^{R} \times q_{J}^{R}\right) \leq W^{R}
$$

(4) Non-negative constraints:

$$
q_{J}^{R} \geq 0, \text { for } j=1, \ldots, J
$$

The objective function in Problem BPI consists of two parts: the LRP host's profitability from the redemption business, $\pi_{H(R)}\left(q_{J}^{R} ; D_{J}^{R}\right)$, and the LRP host's profitability from the accumulation business, $\pi_{H(A)}\left(; D_{1 \imath}^{A}, D_{2 l}^{A}\right)$. The profitability function $\pi_{H(R)}\left(q_{j}^{R} ; D_{j}^{R}\right)$ in Problem BPI is the same as the profitability function in Problem BP. At the accumulation side, the LRP host's profitability comes from points-related business with both LRP accumulation partners $A_{1 l}$ and partners $A_{21}$ : 


$$
\begin{aligned}
\pi_{H(A)}\left(; D_{1 \imath}^{A}, D_{2 l}^{A}\right)= & \sum_{1 \imath=1}^{I_{1}}\left(w_{1 \imath}^{A} \times q_{1 \imath}^{A}+w_{1 \imath}^{\prime A} \times\left[D_{1 \imath}^{A}-q_{1 l}^{A}\right]_{+}\right) \\
& +\sum_{2 \imath=1}^{I_{2}}\left(w_{2 l}^{A} \times \min \left\{q_{2 l}^{A}, D_{2 l}^{A}\right\}+w_{2 l}^{\prime A} \times\left[D_{2 l}^{A}-q_{2 l}^{A}\right]_{+}\right)-\sum_{2 l=1}^{I_{2}}\left(s_{2 l}^{A} \times\left[q_{2 l}^{A}-D_{2 l}^{A}\right]_{+}\right)
\end{aligned}
$$

LRP host $H$ offers a wholesale-price contract to partners $A_{l l}$. Therefore, the meaning of the first two terms in that profitability function is the same as the meaning of (BP-A). LRP host $H$ offers a "revenue-sharing" type of contract to LRP accumulation partner $A_{2 l}$, in which $H$ commits to increase $A_{2 l}$ 's revenue to a minimum level during a specified time period. In return, $A_{2 l}$ will commit to pay back to $H$ a share of revenue increase. The third term, $w_{2 l}^{A} \times \min \left\{q_{2 i}^{A}, D_{2 t}^{A}\right\}$, refers to the share of revenue increase that $A_{2 t}$ pays back to host $H$. The amount of the share is converted in terms of the points that have been accumulated. The fourth term, $w_{2 t}^{A A} \times\left[D_{2 t}^{A}-q_{2 t}^{A}\right]_{+}$, refers to the amount of extra share of the revenue increase when $D_{2 i}^{A}>q_{2 l}^{A}$. It indicates the situation where an actual increase in revenue is higher than the minimum committed amount. If the actual revenue increase is lower than the amount that $H$ committed (i.e., $\left[q_{2 l}^{A}-D_{2 i}^{A}\right]_{+}$), then LRP host $H$ will pay a unit penalty cost of $s_{2 l}^{A}$. The last term in the function, $s_{2 l}^{A} \times\left[q_{2 l}^{A}-D_{2 l}^{A}\right]_{+}$, refers to the overall penalty cost that LRP host $H$ pays to partner $A_{2 \iota}$ when $D_{2 \iota}^{A}<q_{2 \imath}^{A}$. 


\section{Appendix E.2: Extended mathematical model for Problem EP2 with multi-layer of contracts}

The following are additional notations used in the extended model:

(1) Additional indices:

$k \quad$ Option layer offered by partner $R_{\jmath} . k=1,2, \ldots, K$

(2) Additional decision variables:

$m_{j}^{R_{k}} \quad$ Number of layer $k$ options that LRP host $H$ purchases from redemption partner $R_{J}$.

$q_{j}^{R_{k}} \quad$ Number of layer $k$ options that LRP host $H$ exercises.

$y_{j}^{R_{k}} \quad$ Whether LRP host $H$ chooses layer $k$ option from redemption partner $R_{J}$.

(3) Additional parameters in objective function and constraints:

$w_{J}^{R_{k}} \quad$ Option price per unit of points that redemption partner $R_{J}$ charges LRP host $H$ for option $k$.

$e_{J}^{R_{k}} \quad$ Exercise price per unit of points that redemption partner $R_{\jmath}$ charges LRP host $H$ when $H$ exercises the option $k$.

The model is formulated as follows (here after Problem EP3):

$$
\begin{aligned}
& \Pi_{H}\left(q_{J}^{R_{0}}, m_{J}^{R_{k}}, q_{J}^{R_{k}} ; D_{J}^{R}, D_{\imath}^{A}\right)=\max E\left[\pi_{H(A)}\left(; D_{\imath}^{A}\right)+\pi_{H(R)}\left(q_{J}^{R_{0}}, m_{J}^{R_{k}}, q_{J}^{R_{k}} ; D_{J}^{R}\right)\right] \\
& =\max \left\{\begin{array}{l}
\sum_{\imath=1}^{I}\left(w_{\imath}^{A} \times q_{\imath}^{A}\right)-\sum_{J=1}^{J}\left[w_{J}^{R_{0}} \times q_{J}^{R_{0}}+\sum_{k=1}^{K}\left(w_{J}^{R_{k}} m_{J}^{R_{k}}+e_{J}^{R_{k}} q_{J}^{R_{k}}\right)\right]+E\left[\sum_{\imath=1}^{I} w_{\imath}^{\prime A} \times\left[D_{\imath}^{A}-q_{\imath}^{A}\right]_{+}\right]+ \\
\left.E\left[\sum_{j=1}^{J} p_{J}^{R} \times \min \left\{q_{J}^{R_{0}}+\sum_{k=1}^{K} q_{\jmath}^{R_{k}}, D_{J}^{R}\right\}-\sum_{J=1}^{J} v_{J}^{R} \times\left[D_{J}^{R}-\left(q_{J}^{R_{0}}+\sum_{k=1}^{K} q_{J}^{R_{k}}\right)\right]_{+}+\sum_{J=1}^{J} s_{J}^{R} \times\left[q_{J}^{R_{0}}-D_{J}^{R}\right]_{+}\right]\right\}
\end{array}\right.
\end{aligned}
$$

subject to:

(1) Liability constraints: 


$$
\frac{l}{l_{0}} \leq L_{U B}
$$

$$
\text { where } l=l_{0}+\sum_{i=1}^{I}\left(q_{i}^{A}+\left[D_{i}^{A}-q_{i}^{A}\right]_{+}\right)-\sum_{j=1}^{J}\left(q_{j}^{R_{0}}+\sum_{k=1}^{K} q_{j}^{R_{k}}\right)
$$

(2) Redemption partners' capacity limitations on rewards supply:

$$
q_{j}^{R_{0}} \leq Q_{j}^{R_{0}}
$$

(3) Host $H$ 's overall budget constraint on purchasing rewards:

$$
\sum_{j=1}^{J}\left(w_{J}^{R_{0}} \times q_{J}^{R_{0}}+\sum_{k=1}^{K}\left(w_{J}^{R_{k}} m_{J}^{R_{k}}+e_{J}^{R_{k}} q_{J}^{R_{k}}\right)\right) \leq W^{R}
$$

(4) Upper bound of option quantity that host $H$ is allowed to purchase for each option layer $k$ :

$$
m_{J}^{R_{k}} \leq M_{J}^{R_{k}} y_{j}^{R_{k}}, \text { for } k=1, \ldots K, j=1, \ldots, J
$$

(5) Logic constraints:

$$
\begin{aligned}
& \sum_{k=1}^{K} y_{J}^{R_{k}}=1, \text { for } j=1, \ldots J \\
& q_{J}^{R_{k}} \leq m_{J}^{R_{k}}, \text { for } k=1, \ldots K
\end{aligned}
$$

(6) Non-negativity constraints:

$$
\begin{aligned}
& q_{J}^{R_{0}}, m_{J}^{R_{k}} \geq 0 \\
& q_{J}^{R_{k}} \geq 0 \\
& y_{J}^{R_{k}}=0 \text { or } 1
\end{aligned}
$$

In this model, the meaning of each term in the objective function is the same as the meaning of each term in the objective function of Problem EP2. The meanings of constraints (7.7) (7.10) and (7.12) are the same as those in problem EP2. In constraints (7.10) and (7.12), we define a binary variable $y_{j}^{R_{k}}$, for each redemption partner $R_{J}$, and each option level $k$, to 
define a condition in which although each partner $R_{j}$ offers multiple options, LRP host $H$ is allowed to choose only one option layer. Therefore, Constraint (7.12) represents the condition and Constraint (7.10) defines the upper bound of option quantity that LRP host $H$ is allowed to purchase for each option $k$ from partner $R_{j}$. 